\title{
Boron-Catalyzed Hydroarylation of 1,3-Dienes with Arylamines
}

Gautam Kumar[a], Zheng-Wang Qu[b]*, Stefan Grimme ${ }^{[b]}$ and Indranil Chatterjee ${ }^{[a]^{*}}$

${ }^{[a]}$ Department of Chemistry, Indian Institute of Technology Ropar

Ropar, Punjab-140001, India

Indranil.chatterjee@iitrpr.ac.in

${ }^{[b]}$ Mulliken Center for Theoretical Chemistry, Institut für Physikalische und Theoretische Chemie, Universität Bonn

Beringstraße 4, D-53115, Bonn (Germany)

qu@thch.uni-bonn.de

\section{Supporting Information}

\section{Table of Contents}

$1.0 \quad$ General Information $\quad$ S4

2.0 Hydroarylation of olefins, Regioselective hydroarylation of 1,3-dienes and S5 challanges.

2.1 Optimization of Reaction Conditions for Hydroarylation S6

3.0 Effect of water content, Deuterium-labelling and Kinetic Isotope Effect S7 (KIE).

4.0 List of the Dienes and Arylamines Used $\quad$ S8

5.0 General Procedure for the Reaction of 1,3-Dienes and Arylamines (GP) $\quad$ S9

6.0 Experimental Details for the Synthesized Compounds $\quad \mathbf{S 1 0}$

$6.1(E)-N$-phenyl-4-(4-phenylbut-3-en-2-yl)aniline (3) S10

6.2 (E)-N-(4-(4-phenylbut-3-en-2-yl)phenyl)naphthalen-1-amine (4) S10

6.3 (E)-3-(4-phenylbut-3-en-2-yl)-9H-carbazole (5) S11

6.4 (E)-2-methyl-3-(4-phenylbut-3-en-2-yl)-1H-indole (6) S11 
6.5 (E)-N-isopropyl-4-(4-phenylbut-3-en-2-yl)aniline and (E)-N-isopropyl-4-(1- S12 phenylbut-2-en-1-yl)aniline $\left(7+7{ }^{\prime}\right)$

6.6 (E)-N-cyclohexyl-4-(4-phenylbut-3-en-2-yl)aniline and $(E)-N$-cyclohexyl-4-(1- S12 phenylbut-2-en-1-yl)aniline (8 + 8')

6.7 (E)-N,N-diphenyl-4-(4-phenylbut-3-en-2-yl)aniline (9) S13

6.8 (E)-N-methyl-N-phenyl-4-(4-phenylbut-3-en-2-yl)aniline and (E)-N-methyl- $N$ - S13 phenyl-4-(1-phenylbut-2-en-1-yl)aniline $(10$ + 10')

6.9 (E)-1-benzyl-3-(4-phenylbut-3-en-2-yl)-1H-pyrrole (11) S12

6.10 (E)-4-(4-(4-phenylbut-3-en-2-yl)phenyl)morpholine and $(E)-4-(4-(1-p h e n y l b u t-~ S 12$ 2-en-1-yl)phenyl)morpholine $(12+12 ')$

6.11 (E)-1-methyl-6-(4-phenylbut-3-en-2-yl)-1,2,3,4-tetrahydroquinoline and (E)-1- S15 methyl-6-(1-phenylbut-2-en-1-yl)-1,2,3,4-tetrahydroquinoline $(13$ + 13')

6.12 1-((2S)-1-methyl-6-((E)-4-phenylbut-3-en-2-yl)-1,2,3,4-tetrahydroquinolin-2- S16 yl)butan-2-ylium and (2S)-2-butyl-1-methyl-6-((E)-1-phenylbut-2-en-1-yl)1,2,3,4-tetrahydroquinoline $\left(14+14^{\prime}\right)$

$6.13(E)-N$-phenyl-4-(4-(p-tolyl)but-3-en-2-yl)aniline (15) S16

6.14 (E)-N-phenyl-4-(4-(o-tolyl)but-3-en-2-yl)aniline (16) S17

6.15 (E)-4-(4-(4-methoxyphenyl)but-3-en-2-yl)-N-phenylaniline (17) S17

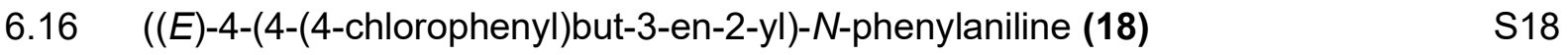

$6.17((E)-4-(4-($ naphthalen-2-yl)but-3-en-2-yl)-N-phenylaniline (19) S18

6.18 (E)-4-(4-(anthracen-9-yl)but-3-en-2-yl)-N-phenylaniline (20) S19

6.19 (E)-N-phenyl-4-(1-phenylpent-1-en-3-yl)aniline (21) S19

6.20 (E)-N-phenyl-4-(1-phenyloct-1-en-3-yl)aniline (22) S20

6.21 (E)-N-phenyl-4-(6-phenylhex-3-en-2-yl)aniline (23) S20

$6.22 \quad(E)-4-($ oct-3-en-2-yl)-N-phenylaniline (24) S21

$6.23(E)-4-(1-c y c l o h e x y l b u t-2-e n-1-y l)-N$-phenylaniline (25) S21

$6.24 \quad \mathrm{~N}$-phenyl-4-(4-phenylpent-3-en-2-yl)aniline (26) S22

6.25 4-(4,4-diphenylbut-3-en-2-yl)-N-phenylaniline (27) S22

6.26 4-(3-methylbut-2-en-1-yl)-N-phenylaniline (28) S23

6.27 (E)-4-(3,7-dimethylocta-2,6-dien-1-yl)-N-phenylaniline (29) S23

6.28 (E)-4-(2-methyl-4-phenylbut-3-en-2-yl)-N-phenylaniline and 4-(3-methyl-1- S24 phenylbut-2-en-1-yl)-N-phenylaniline $(\mathbf{3 0}+\mathbf{3 0}$ ') 
6.29 (E)-4-(3-methyl-1-phenylpent-1-en-3-yl)-N-phenylaniline and 4-(3-methyl-1- S24 phenylpent-2-en-1-yl)- $N$-phenylaniline $(31+31 ')$

6.30 4-(1,3-diphenylpent-2-en-1-yl)-N-phenylaniline (32) S25

6.31 (E)-4-(2-methyl-1,4-diphenylbut-3-en-2-yl)-N-phenylaniline and 4-(3-methyl- S26 1,4-diphenylbut-2-en-1-yl)- $N$-phenylaniline (33+33')

$6.32 \quad N$-phenyl-4-(1,3,4-triphenylbut-2-en-1-yl)aniline (34) S26

6.33 N-phenyl-1',2',3',4'-tetrahydro-[1,1'-biphenyl]-2-amine (35) S27

6.34 5-methyl-N-(p-tolyl)-1',2',3',4'-tetrahydro-[1,1'-biphenyl]-2-amine (36) S27

7.0 Gram scale synthesis $\quad$ S28

8.0 Detection of the contact ion pair 2aH ${ }^{+} \mathrm{HOBCF}^{-}$and $\mathrm{H}_{2} \mathrm{O} \cdot \mathrm{BCF}$ adduct $\quad \mathrm{S} 28$

8.1 Crystal Data and Structure Refinement for Complex $\left((2 \mathrm{a})_{2} \mathrm{H}^{+}(\mathrm{HOBCF})_{2} \mathrm{H}^{-}\right) \quad \mathrm{S} 30$ (CCDC 2111194)

9.0 Effect of Water Content $\quad$ S32

10.0 Control Experiment $\$ 33$

11.0 Deuterium labelling S33

11.1 (E)-N-(4-(4-phenylbut-3-en-2-yl)phenyl-2,6- $\left.d_{2}\right)$ benzen-2,4,6- $d_{3}$-amine (3- $\left.d_{5}\right) \quad$ S33

11.2 (E)-N-phenyl-4-(4-phenylbut-3-en-2-yl)aniline-d (3-d $\left.\mathbf{d}_{1}\right) \quad$ S34

11.3 (E)-N-(4-(4-phenylbut-3-en-2-yl-1-d)phenyl-2,6- $\left.d_{2}\right)$ benzen-2,3,4,5,6- $d_{5}$-amine- S34 $d\left(3-d_{7}\right)$

11.4 (E)- $N$-methyl- $N$-(4-(4-phenylbut-3-en-2-yl-1-d)phenyl-2,6- $\left.d_{2}\right)$ aniline-2,4,6- $d_{3} \quad S 35$ and $(E)-N$-methyl-N-(4-(1-phenylbut-2-en-1-yl-4-d)phenyl-2,6- $\left.d_{2}\right)$ aniline-2,4,6$d_{3}\left(10-d+10^{\prime}-d\right)$

12.0 Independent Kinetic isotope exchange (KIE) study $\$$

12.1 Independent Kinetic isotope exchange (KIE) study for secondary amine $\quad$ S36

12.2 Independent Kinetic isotope exchange (KIE) study for tertiary amine S37

$\begin{array}{lll}13.0 & \text { NMR Spectra } & \text { S39 }\end{array}$

$\begin{array}{lll}14.0 & \text { DFT Calculations } & \mathbf{S 7 7}\end{array}$

$\begin{array}{lll}14.1 & \text { Computational details } & \text { S77 }\end{array}$

14.2 Figure S2: DFT computed free energy paths for the reaction of $\mathbf{1 a}$ and $2 \mathrm{~h}$ S78 $\mathrm{Ph}_{2} \mathrm{NMe}$

14.3 Table S2: DFT-computed energies in 1,2-dichloroethane (DCE) solution $\quad$ S79

14.4 Table S3: DFT-optimized atomic Cartesian coordinates in DCE solution S85

$\begin{array}{lll}15.0 & \text { References } & \text { S134 }\end{array}$ 


\subsection{General Information}

Reactions were performed inside the argon filled glove box, using oven-dried glassware. Liquids and solutions were transferred with syringes. Solvents used were dried and purified by following standard procedures. Technical grade solvents for extraction or chromatography (ethyl acetate, and petroleum ether) were distilled prior to use. $\mathrm{CDCl}_{3}$ was stored over $4 \AA$ molecular sieves. Used chemicals were purchased from Sigma-Aldrich, TCl, Alfa-Aesar and Sisco Research Laboratories (SRL) used without further purification. All the liquid chemicals distilled freshly prior to use. Analytical thin-layer chromatography (TLC) was performed on silica gel $60 \mathrm{~F} 254$ glass plates from Merck. ${ }^{1} \mathrm{H},{ }^{13} \mathrm{C},{ }^{19} \mathrm{~F},{ }^{11} \mathrm{~B}$ NMR spectra was recorded in $\mathrm{CDCl}_{3}$ unless otherwise stated on JEOL JNM ECS-400 MHz and Bruker $500 \mathrm{MHz} / 400 \mathrm{MHz}$ instrument. Chemical shifts are reported in parts per million (ppm) and are referenced to the residual solvent resonance as the internal standard $\left(\mathrm{CDCl}_{3}: \delta=7.26 \mathrm{ppm}\right.$ for ${ }^{1} \mathrm{H} \mathrm{NMR}$ and $\mathrm{CDCl}_{3}: \delta=77.16 \mathrm{ppm}$ for ${ }^{13} \mathrm{C}$ NMR; 1,2-Dibromomethane was used as an internal standard to calculate NMR yields. Data are reported as follows: chemical shift, multiplicity (br = broad singlet, $\mathrm{s}=$ singlet, $\mathrm{d}=$ doublet, $\mathrm{dd}=$ doublet of doublets, $\mathrm{t}=$ triplet, $\mathrm{q}=$ quartet, sept = septet, $\mathrm{m}=$ multiplet), coupling constants $(\mathrm{Hz})$, and integration. All the HRMS/MS-MS data were recorded on XEVO G2-XS QTOF. The Single crystal recorded at Bruker D8 venture diffractometer. Dienes 1o, $\mathbf{1 p}, \mathbf{1 v}$ and arylamines $\mathbf{2 a}, \mathbf{2 b}, \mathbf{2 c}, \mathbf{2 d}, \mathbf{2 g}, \mathbf{2} \mathrm{h}, \mathbf{2 j}$ has been obtained

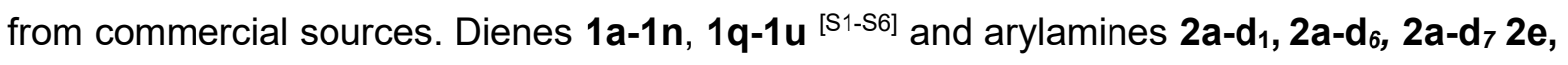
$\left.\mathbf{2 f}, \mathbf{2} \mathbf{h}, \mathbf{2} \mathbf{h}-\mathbf{d}_{6}, \mathbf{2} \mathbf{i}, \mathbf{2} \mathbf{k}, \mathbf{2}{ }^{[S 7,} \mathrm{s} 8, \mathrm{~s} 11-\mathrm{S} 13\right]$ were synthesized according to the procedure in the literature. 


\subsection{Hydroarylation of olefins, Regioselective hydroarylation of 1,3-dienes and}

challanges.

Transition-metal-free hydroarylation of olefins (prior work)

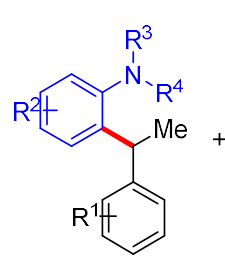<smiles>[R17]1ccccc1</smiles>
$\mathrm{R}^{3-1}$<smiles>[R]c1ccc(C(C)N([R])CC)cc1</smiles>

\section{Beller's work}

$\mathrm{HBF}_{4} \mathrm{Et}_{2} \mathrm{O}, 1999$

\section{Bergman's work}

$\mathrm{PhNH}_{3} \mathrm{~B}\left(\mathrm{C}_{6} \mathrm{~F}_{5}\right) \cdot \mathrm{Ft}_{2} \mathrm{O}, 2005$
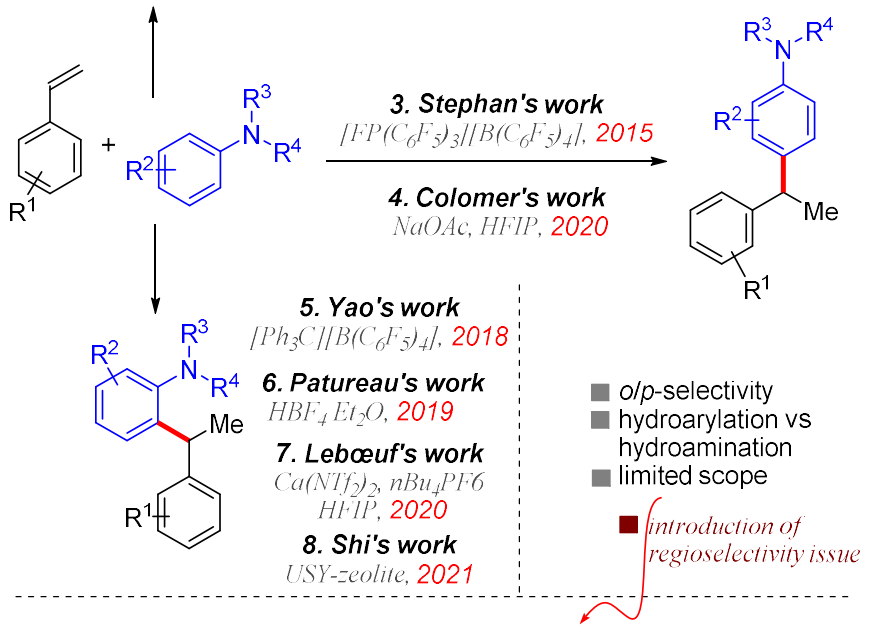

Transition-metal-free hydroarylation of conjugated dienes

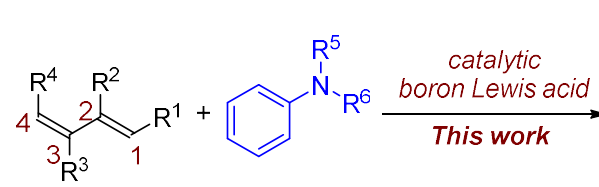<smiles>[R]C=C([R])C([R])([R])c1ccc(N([R7])[R])cc1</smiles>

4,3-hydroarylation in major cases

$\mathrm{R}^{5}$

mild reaction condition, scale up within short reaction tim

- broad substrate scope; terminal as well as internal dienes

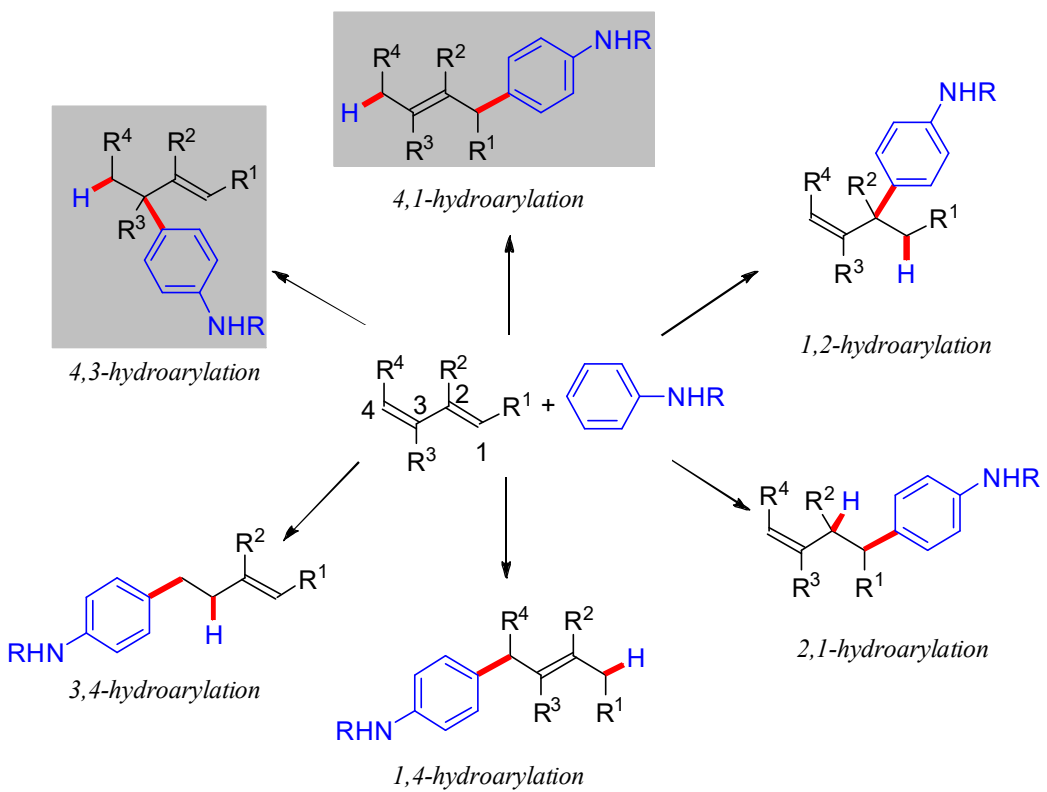




\subsection{Optimization of Reaction Conditions for Hydroarylation}

We commenced our study by reacting $(E)$-buta-1,3-dien-1-ylbenzene (1a, 1.0 equiv) as a model substrate with diphenylamine (2a, 1.5 equiv) in a series of solvents $(0.15 \mathrm{M})$ in the presence of $\mathrm{B}\left(\mathrm{C}_{6} \mathrm{~F}_{5}\right)_{3}$ as catalyst at $40^{\circ} \mathrm{C}$ for $4 \mathrm{~h}$ (Table S1). While solvent like THF provided only traces of conversion, $\mathrm{MeCN}, \mathrm{CHCl}_{3}, \mathrm{MeNO}_{2}$ promoted the reaction with moderate ${ }^{1} \mathrm{H}$ NMR yields (Table $S 1$, entries 1-4). The reaction in toluene and dichloromethane (DCM) resulted in a drastically improved reactivity delivering 3 in $62 \%$ and $72 \%$ yield respectively and complete regioselectivity for 4,3-hydroarylation (Table S1, entry 5-6). To our delight, using 1,2-dichloroethane (DCE) as a solvent, the desired product 3 was obtained in excellent isolated yield $(90 \%)$ and regioselectivity (>20:1 rr) (Table S1, entry 7). Lower catalyst loading provided low yield of $\mathbf{3}$ (Table S1, entries 913). Transition-metal-based catalysts $\mathrm{Cu}(\mathrm{OTf})_{2}, \mathrm{Fe}(\mathrm{OTf})_{3}$ and $\mathrm{Yb}(\mathrm{OTf})_{3}$ were unable to catalyze the reaction, whereas $\ln (\mathrm{OTf})_{3}, \mathrm{Sc}(\mathrm{OTf})_{3}$ catalyzed the reaction albeit in lower yield (Table S1, entries 14-16). Brønsted acid $\mathrm{Tf}_{2} \mathrm{NH}$ and other boron Lewis acids such as $\mathrm{BCl}_{3}$ and $\mathrm{BF}_{3} \mathrm{Et}_{2} \mathrm{O}$ provided 3 with lower ${ }^{1} \mathrm{H}$ NMR yields.

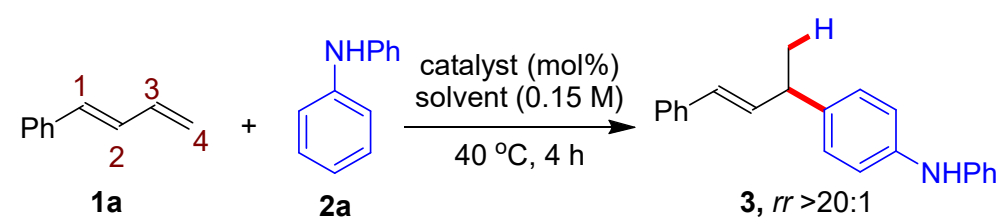

\begin{tabular}{|c|c|c|c|}
\hline Entry & catalyst (mol\%) & Solvent & Yield $\mathbf{3}(\%)^{[\mathrm{a}]}$ \\
\hline 1 & $\mathrm{~B}\left(\mathrm{C}_{6} \mathrm{~F}_{5}\right)_{3}(10)$ & THF & trace \\
\hline 2 & $\mathrm{~B}\left(\mathrm{C}_{6} \mathrm{~F}_{5}\right)_{3}(10)$ & $\mathrm{CH}_{3} \mathrm{CN}$ & 18 \\
\hline 3 & $\mathrm{~B}\left(\mathrm{C}_{6} \mathrm{~F}_{5}\right)_{3}(10)$ & $\mathrm{CHCl}_{3}$ & 40 \\
\hline 4 & $\mathrm{~B}\left(\mathrm{C}_{6} \mathrm{~F}_{5}\right)_{3}(10)$ & $\mathrm{MeNO}_{2}$ & 50 \\
\hline 5 & $\mathrm{~B}\left(\mathrm{C}_{6} \mathrm{~F}_{5}\right)_{3}(10)$ & Toluene & 62 \\
\hline 6 & $\mathrm{~B}\left(\mathrm{C}_{6} \mathrm{~F}_{5}\right)_{3}(10)$ & DCM & 72 \\
\hline 7 & $B\left(C_{6} F_{5}\right)_{3}(10)$ & 1,2-DCE & $>99(90)^{[b]}$ \\
\hline 8 & $\mathrm{~B}\left(\mathrm{C}_{6} \mathrm{~F}_{5}\right)_{3}(5)$ & 1,2-DCE & 40 \\
\hline 9 & $\mathrm{Cu}(\mathrm{OTf})_{2}(10)$ & 1,2-DCE & $\mathrm{nr}$ \\
\hline 10 & $\mathrm{Fe}(\mathrm{OTf})_{3}(10)$ & 1,2-DCE & trace \\
\hline 11 & $\mathrm{Yb}(\mathrm{OTf})_{3}(10)$ & 1,2-DCE & $\mathrm{nr}$ \\
\hline 12 & $\ln (\mathrm{OTf})_{3}(10)$ & 1,2-DCE & 27 \\
\hline 13 & $\mathrm{Sc}(\mathrm{OTf})_{3}(10)$ & 1,2-DCE & 19 \\
\hline 14 & $\mathrm{BCl}_{3}(10)^{[\mathrm{c}]}$ & 1,2-DCE & 45 \\
\hline 15 & $\mathrm{BF}_{3} \cdot \mathrm{Et}_{2} \mathrm{O}(10)$ & 1,2-DCE & 26 \\
\hline 16 & $\mathrm{Tf}_{2} \mathrm{NH}(10)$ & 1,2-DCE & 30 \\
\hline
\end{tabular}

Table S1 [a] Reaction condition 1a (1.0 equiv) and 2a (1.5 equiv) stirred in solvent at it for $4 \mathrm{~h}$. Yield determined by ${ }^{1} \mathrm{H}$ NMR using $\mathrm{CH}_{2} \mathrm{Br}_{2}$ as internal standard. [b] Isolated yield. [c] Used as $1 \mathrm{M}$ solution in DCM. $\mathrm{nr}=$ no reaction. $\mathrm{rr}=$ regioisomeric ratio, determined by ${ }^{1} \mathrm{H}$ NMR of crude reaction mixture. 


\subsection{Effect of water content, Deuterium-labelling and Kinetic Isotope Effect (KIE).} a) Effect of water content

$$
\text { (1.0 equiv) }
$$<smiles>C(=C/c1ccccc1)\CC(/C=C/c1ccccc1)c1ccc(Nc2ccccc2)cc1</smiles>

(i) 1,2-DCE (dry \& dist.) - $x=100 \mathrm{~mol} \%, 3$ (30\% yield)

(ii) 1,2-DCE (dry \& dist.) - $\mathrm{x}=10 \mathrm{~mol} \%, 3$ (50\% yield)

(iii) 1,2-DCE (normal without dist.) - $x=0$ mol\%, 3 (60\% yield)

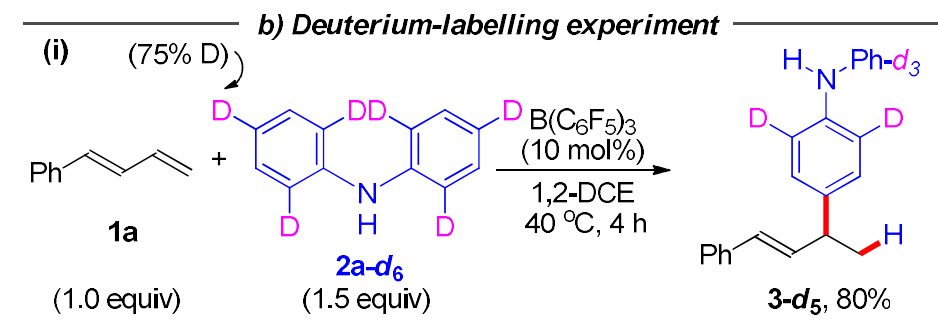

(ii)

$\left(32 \%\right.$ D) D-N $\mathrm{N}^{-\mathrm{Ph}}$

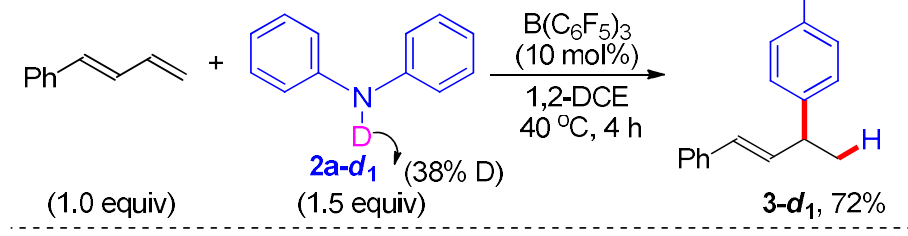

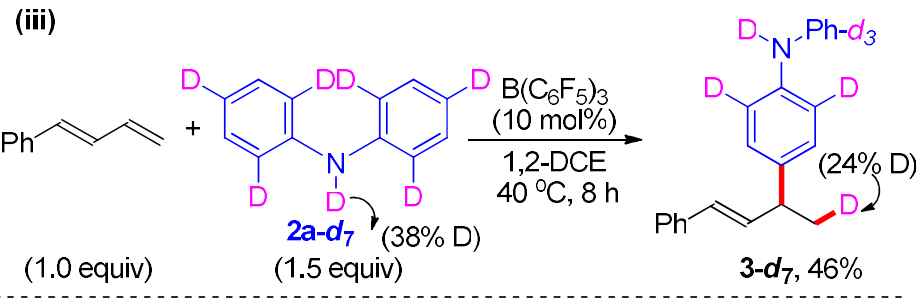

(iv)

$$
\text { (iv) }
$$

$\mathrm{Me}{ }_{\mathrm{N}^{-}}-\mathrm{Ph}-\mathrm{d}_{3}$

c) Kinetic Isotope Exchange (KIE)

(i)

$$
\begin{aligned}
& 1 \mathrm{a}+\mathrm{Ph}_{2} \mathrm{NH} \stackrel{\text { Standard Cond. }}{\longrightarrow} \mathbf{3} \\
& K_{H}=11.96 \\
& 1 \mathrm{a}+2 \mathrm{a}-d_{7} \stackrel{\text { Standard Cond. }}{\longrightarrow} \quad 3-d_{7} \\
& \frac{K_{H}}{K_{D}}=2.81 \\
& K_{D}=4.25
\end{aligned}
$$

(ii) $1 \mathrm{a}+\mathrm{Ph}_{2} \mathrm{NMe} \stackrel{\text { Standard Cond. }}{\longrightarrow} 10+10$

$$
K_{H}=11.25
$$

$1 \mathrm{a}+2 \mathrm{~h}-\mathrm{d}_{6} \stackrel{\text { Standard Cond. }}{\longrightarrow} 10-\mathrm{d}+10^{\prime}-\mathrm{d}$

$$
K_{D}=9.99
$$




\subsection{List of the Dienes and Arylamines Used}

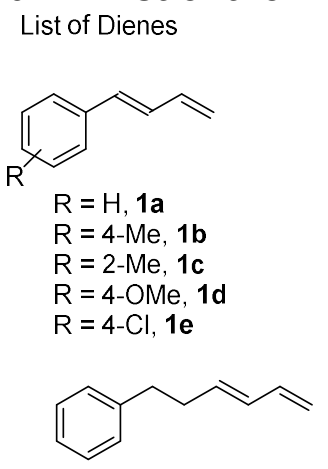

$1 \mathrm{j}$

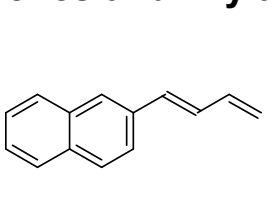

$1 f$

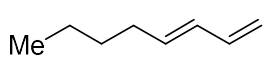

$1 \mathrm{k}$

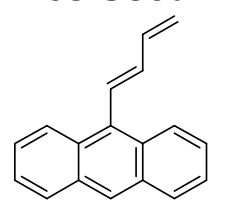

$1 \mathrm{~g}$<smiles>CC=CC=Cc1ccccc1</smiles>

$1 \mathrm{~h}$<smiles>CCCC/C=C/C=C/c1ccccc1</smiles>

$1 \mathrm{i}$<smiles>C=CC(=C)CCC=C(C)C</smiles><smiles>C(=C/c1ccccc1)\c1ccccc1</smiles><smiles>c1ccccc1</smiles>

1v

Successful Arylamines<smiles>c1ccc(Nc2ccccc2)cc1</smiles>

2a<smiles>Cc1cc2ccccc2[nH]1</smiles><smiles>CC(C)Nc1ccccc1</smiles>

$2 d$<smiles></smiles>

$2 \mathrm{i}$<smiles>[2H]N(c1ccccc1)c1ccccc1</smiles>
$2 \mathrm{a}-d_{1}$

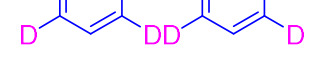
$2 \mathrm{a}-d_{6}$<smiles>c1ccc(NC2CCCCC2)cc1</smiles>

$2 f$<smiles>CN1CCCc2ccccc21</smiles>

$2 k$<smiles>[2H]c1cc([2H])c2c(c1)[10B]c1cc(Cl)cc([2H])c1N2[2H]</smiles>

$2 \mathrm{a}-d_{7}$<smiles>c1ccc(N(c2ccccc2)c2ccccc2)cc1</smiles>

$2 \mathrm{~g}$<smiles>CCCC[C@H]1CCc2ccccc2N1C</smiles>

2I<smiles>c1ccc(Nc2cccc3ccccc23)cc1</smiles>

$2 b$

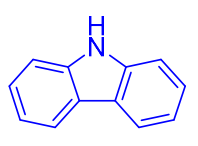

2c

Unsuccessful or traces of products formation Arylamines<smiles>Nc1ccccc1</smiles><smiles>CNc1ccccc1</smiles><smiles>CN(C)c1ccccc1</smiles><smiles>c1ccc2[nH]ccc2c1</smiles> 


\subsection{General Procedure for the Reaction of 1,3-Dienes and Arylamines (GP):}

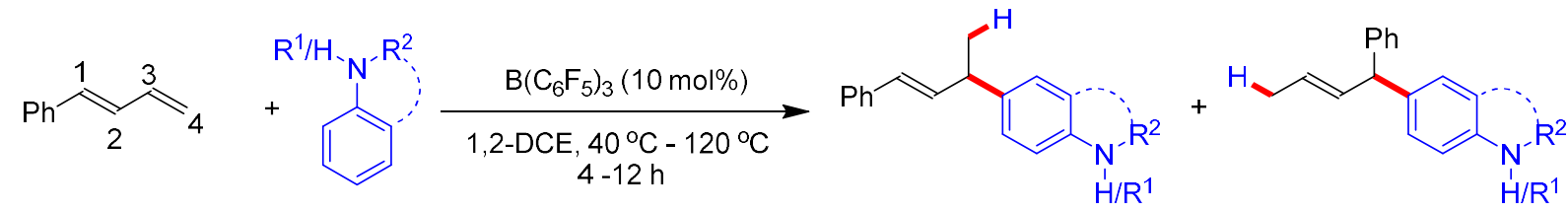

Procedure A : $\quad$ In an argon filled glove box, an oven-dried glass vial is charged with a magnetic stir bar, tris(pentafluorophenyl)borane $\left(B\left(\mathrm{C}_{6} \mathrm{~F}_{5}\right)_{3}\right)(10.0 \mathrm{~mol} \%)$ and 1,2-DCE $(0.15 \mathrm{M})$ (dry and distilled). Arylamine 2a-2m (1.5 equiv) and diene 1a-1v (1.0 equiv) simultaneously added to the mixture. The vial has been taken out from the glove box and stirred for four hours at preheated oil bath at $40{ }^{\circ} \mathrm{C}$ temperature. The crude reaction mixture is used to measure the regiomeric ratio ( $r r)$ and $E / Z$ ratio by ${ }^{1} \mathrm{H}$ NMR. The analytically pure product is obtained by column chromatography on silica gel using petroleum ether and dichloromethane (DCM) or EtOAc as eluent.

Procedure B: $\quad$ In an argon filled glove box, an oven-dried glass vial is charged with a magnetic stir bar, tris(pentafluorophenyl)borane $\left(B\left(\mathrm{C}_{6} \mathrm{~F}_{5}\right)_{3}\right)(10.0 \mathrm{~mol} \%)$ and 1,2-DCE $(0.15 \mathrm{M})$ (dry and distilled). Arylamine $\mathbf{2 a - 2 m}$ (1.5 equiv) and diene $\mathbf{1 a - 1 v}$ (1.0 equiv) simultaneously added to the mixture. The vial has been taken out from the glove box and stirred for four to twelve hours at preheated oil bath at $80{ }^{\circ} \mathrm{C}$ temperature. The crude reaction mixture is used to measure the regiomeric ratio $(r r)$ and $E / Z$ ratio by ${ }^{1} \mathrm{H}$ NMR. The analytically pure product is obtained by column chromatography on silica gel using petroleum ether and dichloromethane (DCM) or EtOAc as eluent.

Procedure C: In an argon filled glove box, an oven-dried sealed tube is charged with a magnetic stir bar, tris(pentafluorophenyl)borane $\left(B\left(\mathrm{C}_{6} \mathrm{~F}_{5}\right)_{3}\right)(10.0 \mathrm{~mol} \%)$ and 1,2-DCE $(0.15 \mathrm{M})$ (dry and distilled). Arylamine $\mathbf{2 a - 2 m ~ ( 1 . 5 ~ e q u i v ) ~ a n d ~ d i e n e ~} 1 \mathbf{a}-\mathbf{1} \mathbf{v}$ (1.0 equiv) simultaneously added to the mixture. The vial has been taken out from the glove box and stirred for four hours at preheated oil bath at $120^{\circ} \mathrm{C}$ temperature. The crude reaction mixture is used to measure the regiomeric ratio $(r r)$ and $E / Z$ ratio by ${ }^{1} \mathrm{H}$ NMR. The analytically pure product is obtained by column chromatography on silica gel using petroleum ether and dichloromethane (DCM) or EtOAc as eluent. 


\subsection{Experimental Details for the Synthesized Compounds}

\section{1. (E)-N-phenyl-4-(4-phenylbut-3-en-2-yl)aniline (3)}

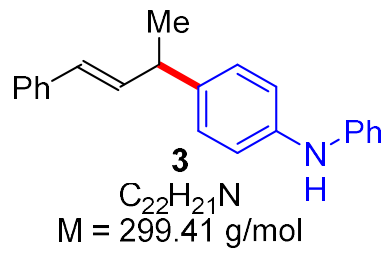

According to GP-A, using 1a (16.0 mg, $0.12 \mathrm{mmol}, 1.0$ equiv), 2a (30.0 mg, $0.18 \mathrm{mmol}, 1.5$ equiv), and tris(pentafluorophenyl)borane $\left(\mathrm{B}_{(}\left(\mathrm{C}_{6} \mathrm{~F}_{5}\right)_{3}\right)(6.0 \mathrm{mg}, 12 \mu \mathrm{mol}, 10 \mathrm{~mol} \%)$. Purification by column chromatography on silica gel using petroleum ether/DCM $(9: 1 \mathrm{v} / \mathrm{v})$ as eluent afforded the product 3 (32.0 mg, 90\%) as colorless oil. HRMS (ESI) m/z: [(M+H) $\left.{ }^{+}\right]$: calcd for $\mathrm{C}_{22} \mathrm{H}_{22} \mathrm{~N}^{+} 300.1747$; found 300.1771. ' ${ }^{1} \mathrm{H}$ NMR (400 MHz, $\left.\mathrm{CDCl}_{3}\right)$ ס 7.39-7.37 (m, 2H), 7.32-7.25 (m, 4H), 7.23-7.17 (m, 3H), 7.09-7.06 (m, 4H), 6.94-6.90 (m, 1H), 6.46-6.36 (m, 2H), 3.65-3.58 (m, 1H), $1.47(\mathrm{~d}, \mathrm{~J}=6.9 \mathrm{~Hz}$, $3 \mathrm{H}) .{ }^{13} \mathrm{C}$ NMR $\left(101 \mathrm{MHz}, \mathrm{CDCl}_{3}\right) \delta 143.5,141.1,138.8,137.7,135.6,129.5,128.6,128.4,128.3$, 127.1, 126.3, 120.9, 118.7, 117.5, 42.0, 21.4 .

\section{$6.2 \quad(E)-\mathrm{N}-(4-(4-p h e n y l b u t-3-e n-2-y l) p h e n y l) n a p h t h a l e n-1-a m i n e ~(4)$}

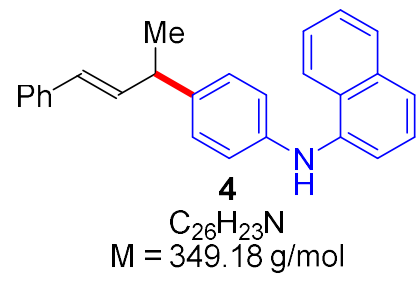

According to GP-A, using 1a (16.0 mg, 0.12 mmol, 1.0 equiv), 2b (39.0 mg, 0.18 mmol, 1.5 equiv), and tris(pentafluorophenyl)borane $\left(\mathrm{B}\left(\mathrm{C}_{6} \mathrm{~F}_{5}\right)_{3}\right)(6.0 \mathrm{mg}, 12 \mu \mathrm{mol}, 10 \mathrm{~mol} \%)$. Purification by column chromatography on silica gel using petroleum ether/DCM $(8: 2 \mathrm{v} / \mathrm{v})$ as eluent afforded the product 4 (41.0 mg, 98\%) as colorless oil. HRMS (ESI) $m / z$ : $\left[(\mathrm{M}+\mathrm{H})^{+}\right]$calcd for $\mathrm{C}_{26} \mathrm{H}_{24} \mathrm{~N}^{+} 350.1903$; found 350.1883. ' ${ }^{1} \mathrm{~N}$ NMR (400 MHz, DMSO-D $)$ ठ 8.19-8.16 (m, 1H), $8.14(\mathrm{~s}, 1 \mathrm{H}), 7.89-7.85(\mathrm{~m}, 1 \mathrm{H})$, 7.52-7.46 (m, 3H), 7.41-7.39 (m, 2H), $7.36(\mathrm{t}, J=7.8 \mathrm{~Hz}, 1 \mathrm{H}), 7.28(\mathrm{q}, J=7.4 \mathrm{~Hz}, 3 \mathrm{H}), 7.20$ (d, $J=7.3 \mathrm{~Hz}, 1 \mathrm{H}), 7.18-7.14(\mathrm{~m}, 2 \mathrm{H}), 7.04(\mathrm{~m}, 2 \mathrm{H}), 6.49-6.39(\mathrm{~m}, 2 \mathrm{H}), 3.60-3.54(\mathrm{~m}, 1 \mathrm{H}), 1.38(\mathrm{~d}$, $J=7.0 \mathrm{~Hz}, 3 \mathrm{H}) .{ }^{13} \mathrm{C}$ NMR $\left(101 \mathrm{MHz}, \mathrm{DMSO}-\mathrm{D}_{6}\right) \delta 142.9,139.9,137.2,136.8,135.8,134.4,128.6$, $128.1,127.7,127.5,127.0,126.6,126.2,126.0$ (2C), 124.9, 122.7, 120.8, 117.8, 112.9, 41.4, 21.4 


\section{3 (E)-3-(4-phenylbut-3-en-2-yl)-9H-carbazole (5)}

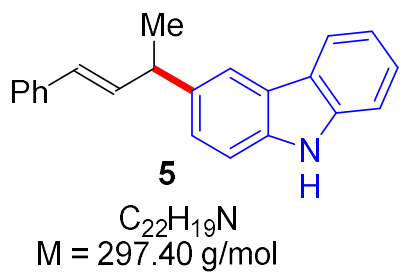

According to GP-B, using 1a (16.0 mg, $0.12 \mathrm{mmol}, 1.0$ equiv), 2c (30.0 mg, $0.18 \mathrm{mmol}, 1.5$ equiv),

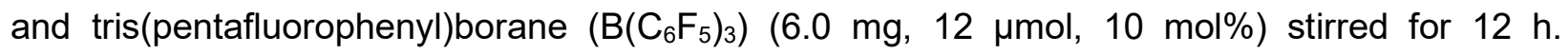
Purification by column chromatography on silica gel using petroleum ether/DCM (8:2 v/v) as eluent afforded the product $5(29.0 \mathrm{mg}, 82 \%)$ as white solid. Melting point: $142-143{ }^{\circ} \mathrm{C}$. HRMS (ESI) $\mathrm{m} / \mathrm{z}:\left[(\mathrm{M}+\mathrm{H})^{+}\right]$calcd for $\mathrm{C}_{22} \mathrm{H}_{20} \mathrm{~N}^{+}$298.1590; found 298.1589. ${ }^{1} \mathrm{H}$ NMR $\left(400 \mathrm{MHz}, \mathrm{CDCl}_{3}\right) \delta$ $8.07(\mathrm{~d}, J=7.3 \mathrm{~Hz}, 1 \mathrm{H}), 8.00(\mathrm{~s}, 1 \mathrm{H}), 7.97(\mathrm{~d}, J=1.6 \mathrm{~Hz}, 1 \mathrm{H}), 7.44-7.37(\mathrm{~m}, 5 \mathrm{H}), 7.35-7.32$ (m, $1 \mathrm{H}), 7.31-7.27(\mathrm{~m}, 2 \mathrm{H}), 7.24-7.18(\mathrm{~m}, 2 \mathrm{H}), 6.55-6.44(\mathrm{~m}, 2 \mathrm{H}), 3.87-3.80(\mathrm{~m}, 1 \mathrm{H}), 1.58(\mathrm{~d}, J=$ $7.8 \mathrm{~Hz}, 3 \mathrm{H}) .{ }^{13} \mathrm{C}$ NMR $\left(101 \mathrm{MHz}, \mathrm{CDCl}_{3}\right) \delta 140.0,138.3,137.8,136.9,136.2,128.6,128.2,127.1$, $126.3,125.9,125.8,123.6,123.4,120.4,119.4,118.7,110.7,110.6,42.7,21.9$.

\section{4 (E)-2-methyl-3-(4-phenylbut-3-en-2-yl)-1H-indole (6)}

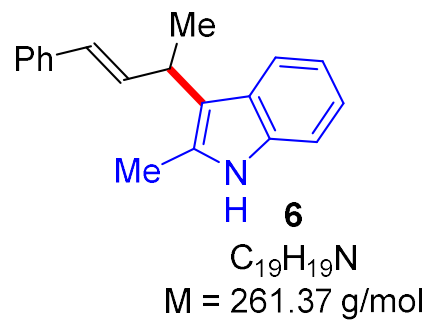

According to GP-B, using 1a (16.0 mg, $0.12 \mathrm{mmol}, 1.0$ equiv), 2d (30.0 mg, $0.18 \mathrm{mmol}, 1.5$ equiv),

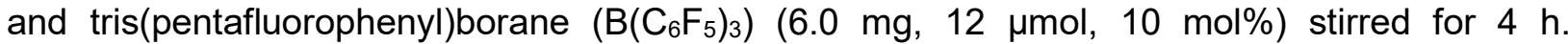
Purification by column chromatography on silica gel using petroleum ether/DCM (8:2 v/v) as eluent afforded the product $6(31.0 \mathrm{mg}, 98 \%)$ as colorless gel turned into blue colored at rt. HRMS (ESI) $\mathrm{m} / \mathrm{z}$ : $\left[(\mathrm{M}+\mathrm{H})^{+}\right]$calcd for $\mathrm{C}_{19} \mathrm{H}_{20} \mathrm{~N}^{+}$262.1590; found 262.1598. ${ }^{1} \mathrm{H}$ NMR $\left(400 \mathrm{MHz}, \mathrm{CDCl}_{3}\right) \delta$ $7.74(\mathrm{~s}, 1 \mathrm{H}), 7.62(\mathrm{~d}, J=8.0 \mathrm{~Hz}, 1 \mathrm{H}), 7.35(\mathrm{~d}, J=7.2 \mathrm{~Hz}, 2 \mathrm{H}), 7.29-7.26(\mathrm{~m}, 3 \mathrm{H}), 7.18(\mathrm{t}, J=7.3$ $\mathrm{Hz}, 1 \mathrm{H}), 7.11(\mathrm{t}, J=7.5 \mathrm{~Hz}, 1 \mathrm{H}), 7.04(\mathrm{t}, J=7.5 \mathrm{~Hz}, 1 \mathrm{H}), 6.61(\mathrm{dd}, J=16.0,5.5 \mathrm{~Hz}, 1 \mathrm{H}), 6.45$ (dd, $J=16.2,1.8 \mathrm{~Hz}, 1 \mathrm{H}), 3.95-3.89(\mathrm{~m}, 1 \mathrm{H}), 2.42(\mathrm{~s}, 3 \mathrm{H}), 1.58(\mathrm{~d}, J=7.3 \mathrm{~Hz}, 3 \mathrm{H}) .{ }^{13} \mathrm{C}$ NMR $(101$ $\left.\mathrm{MHz}, \mathrm{CDCl}_{3}\right) \delta 138.0,135.4,135.3,130.4,128.6,127.9,127.7,126.9,126.2,120.9,119.4,119.1$, 114.9, 110.4, 33.7, 20.4, 12.4 . 


\section{$6.5(E)$ - $N$-isopropyl-4-(4-phenylbut-3-en-2-yl)aniline and (E)-N-isopropyl-4-(1- phenylbut-2-en-1-yl)aniline $\left(7+7{ }^{\prime}\right)$}

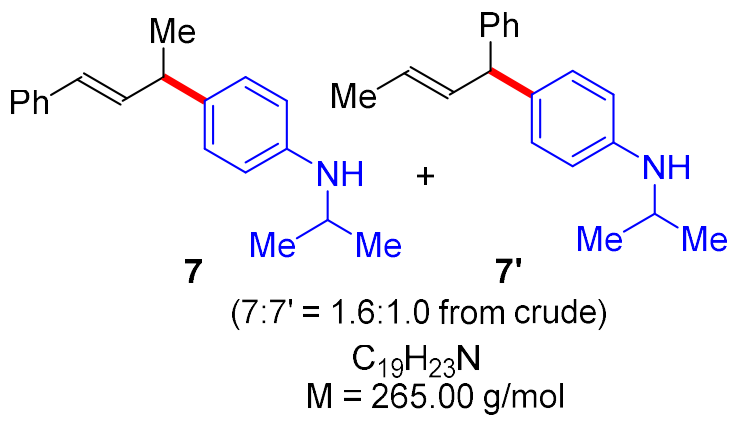

According to GP-C, using $1 \mathrm{a}$ ( $16.0 \mathrm{mg}, 0.12 \mathrm{mmol}, 1.0$ equiv), $2 \mathrm{e}(24.0 \mathrm{mg}, 0.18 \mathrm{mmol}, 1.5$ equiv), and tris(pentafluorophenyl)borane $\left(\mathrm{B}\left(\mathrm{C}_{6} \mathrm{~F}_{5}\right)_{3}\right)(6.0 \mathrm{mg}, 12 \mu \mathrm{mol}, 10 \mathrm{~mol} \%)$. Purification by column chromatography on silica gel using petroleum ether/EtOAc $(9.7: 0.3 \mathrm{v} / \mathrm{v})$ as eluent afforded the product $7+7^{\prime}(17.0 \mathrm{mg}, 54 \%)$ as colorless oil. HRMS (ESI) $\mathrm{m} / \mathrm{z}$ : [(M+H) $]$ calcd for $\mathrm{C}_{19} \mathrm{H}_{24} \mathrm{~N}^{+}$ 266.1903; found 266.1917. ' $\mathrm{H}$ NMR $\left(400 \mathrm{MHz}, \mathrm{CDCl}_{3}\right) \delta$ 7.36-7.34 (m, 2H), 7.32-7.25 (m, 5H), 7.22-7.16 (m, 3H), 7.11-7.07 (m, 2H), 7.01-6.97 (m, 2H), $6.63(\mathrm{dd}, J=20.5,8.1 \mathrm{~Hz}, 3 \mathrm{H}), 6.46-$ $6.32(\mathrm{~m}, 2 \mathrm{H}), 5.91-5.85(\mathrm{~m}, 1 \mathrm{H}), 5.44-5.38(\mathrm{~m}, 1 \mathrm{H}), 4.56(\mathrm{~d}, J=7.6 \mathrm{~Hz}, 1 \mathrm{H}), 3.63-3.51(\mathrm{~m}, 3 \mathrm{H})$, 1.73-1.70 (m, 3H), $1.42(\mathrm{~d}, J=6.9 \mathrm{~Hz}, 3 \mathrm{H}), 1.21(\mathrm{t}, J=6.3 \mathrm{~Hz}, 11 \mathrm{H}) .{ }^{13} \mathrm{C} \mathrm{NMR}\left(101 \mathrm{MHz}, \mathrm{CDCl}_{3}\right)$ $\delta 144.9,137.8,136.0,134.2,129.4,128.6,128.4,128.3,128.0,127.0,126.5,126.2,126.1,114.6$, 114.2, 53.3, 41.8, 29.9, $22.8(2 \mathrm{C}), 21.4,21.1,18.2$.

\section{6 (E)-N-cyclohexyl-4-(4-phenylbut-3-en-2-yl)aniline and (E)-N-cyclohexyl-4-(1-} phenylbut-2-en-1-yl)aniline $\left(8+8^{\prime}\right)$

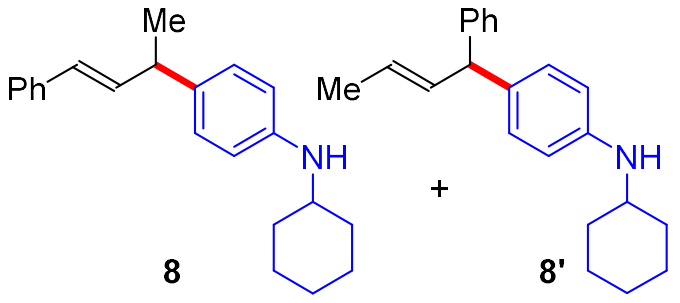

$\left(8: 8^{\prime}=1.6: 1.0\right.$ from crude)

$$
\begin{aligned}
& \mathrm{C}_{22} \mathrm{H}_{27} \mathrm{~N} \\
M= & 305.47 \mathrm{~g} / \mathrm{mol}
\end{aligned}
$$

According to GP-C, using 1a (16.0 mg, $0.12 \mathrm{mmol}, 1.0$ equiv), $2 \mathrm{f}$ ( $32.0 \mathrm{mg}, 0.18 \mathrm{mmol}, 1.5$ equiv), and tris(pentafluorophenyl)borane $\left(\mathrm{B}\left(\mathrm{C}_{6} \mathrm{~F}_{5}\right)_{3}\right)(6.0 \mathrm{mg}, 12 \mu \mathrm{mol}, 10 \mathrm{~mol} \%)$. Purification by column chromatography on silica gel using petroleum ether/EtOAc $(9.7: 0.3 \mathrm{v} / \mathrm{v})$ as eluent afforded the product $8+\mathbf{8}^{\prime}(20.0 \mathrm{mg}, 54 \%$,) as colorless oil. 
HRMS (ESI) $\mathrm{m} / \mathrm{z}$ : $\left[(\mathrm{M}+\mathrm{H})^{+}\right]$calcd for $\mathrm{C}_{22} \mathrm{H}_{28} \mathrm{~N}^{+}$306.2216; found 306.2209. ${ }^{1} \mathrm{H}$ NMR (400 MHz, $\left.\mathrm{CDCl}_{3}\right)$ ठ 7.36-7.23 (m, 4H), 7.21-7.14 (m, 4H), $7.09(\mathrm{~d}, J=8.0 \mathrm{~Hz}, 0.44 \mathrm{H}), 6.98(\mathrm{~d}, J=8.1 \mathrm{~Hz}$, 2H), 6.70-6.58 (m, 3H), 6.41-6.31 (m, 0.48H), 5.91-5.84 (m, 1H), 5.46-5.37 (m, 1H), $4.56(\mathrm{~d}, J$ $=7.6 \mathrm{~Hz}, 1 \mathrm{H}), 3.57-3.51(\mathrm{~m}, 0.30 \mathrm{H}), 3.24-3.16(\mathrm{~m}, 1 \mathrm{H}), 2.06-2.01(\mathrm{~m}, 3 \mathrm{H}), 1.77-1.70(\mathrm{~m}, 7 \mathrm{H})$, 1.64-1.60 (m, 2H), $1.42(\mathrm{~d}, J=7.0 \mathrm{~Hz}, 1 \mathrm{H}), 1.38-1.27(\mathrm{~m}, 4 \mathrm{H}), 1.24-1.13(\mathrm{~m}, 4 \mathrm{H}) .{ }^{13} \mathrm{C}$ NMR $(101$ $\left.\mathrm{MHz}, \mathrm{CDCl}_{3}\right) \delta 144.9,136.0,134.2,129.9,129.4,128.7,128.6,128.4,128.3,127.0,126.5,126.2$, $126.1,114.5,114.4,53.4,41.8,33.2,25.9,25.1,18.1$.

\section{7 (E)-N,N-diphenyl-4-(4-phenylbut-3-en-2-yl)aniline (9)}

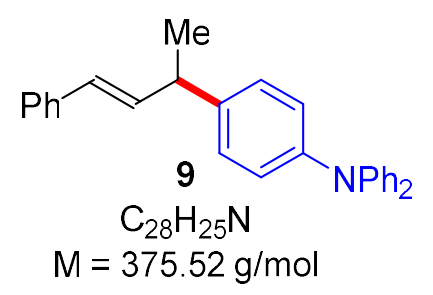

According to GP-A, using $1 \mathrm{a}$ ( $16.0 \mathrm{mg}, 0.12 \mathrm{mmol}, 1.0$ equiv), $\mathbf{2 g}(44.0 \mathrm{mg}, 0.18 \mathrm{mmol}, 1.5$ equiv), and tris(pentafluorophenyl)borane $\left(\mathrm{B}_{(}\left(\mathrm{C}_{6} \mathrm{~F}_{5}\right)_{3}\right)(6.0 \mathrm{mg}, 12 \mu \mathrm{mol}, 10 \mathrm{~mol} \%)$. Purification by column chromatography on silica gel using petroleum ether/DCM $(8: 2 \mathrm{v} / \mathrm{v})$ as eluent afforded the product 9 (40.0 mg, 88\%) as colorless gel. HRMS (ESI) m/z: [(M+H) $\left.{ }^{+}\right]$calcd for $\mathrm{C}_{28} \mathrm{H}_{26} \mathrm{~N}^{+} 376.2060$; found 376.2030. ${ }^{1} \mathrm{H}$ NMR $\left(400 \mathrm{MHz}, \mathrm{CDCl}_{3}\right) \delta 7.40-7.38(\mathrm{~m}, 2 \mathrm{H}), 7.31(\mathrm{t}, J=7.6 \mathrm{~Hz}, 2 \mathrm{H}), 7.27-7.21$ (m, 5H), 7.18-7.15 (m, 2H), 7.10 (d, J = 8.2 Hz, 4H), 7.07-7.04 (m, 2H), $7.00(\mathrm{t}, J=7.4 \mathrm{~Hz}, 2 \mathrm{H})$, 6.47-6.36 (m, 2H), 3.65-3.58 (m, 1H), $1.48(\mathrm{~d}, J=7.1 \mathrm{~Hz}, 3 \mathrm{H}) .{ }^{13} \mathrm{C} \mathrm{NMR}\left(101 \mathrm{MHz}, \mathrm{CDCl}_{3}\right) \delta$ 148.0, 146.0, 140.2, 137.7, 135.5, 129.3, 128.6, 128.4, 128.1, 127.2, 126.3, 124.6, 124.0, 122.5, 42.2, 21.3.

\section{8 (E)-N-methyl-N-phenyl-4-(4-phenylbut-3-en-2-yl)aniline and $(E)-N$-methyl- $N$-phenyl-} 4-(1-phenylbut-2-en-1-yl)aniline $\left(10+10^{\prime}\right)$

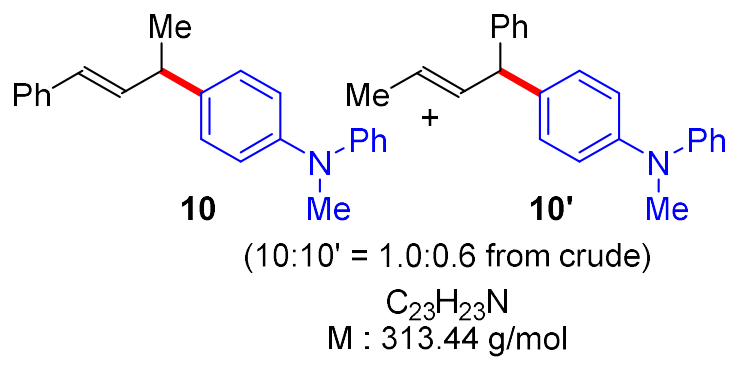

According to GP-A, using $1 \mathrm{a}$ ( $16.0 \mathrm{mg}, 0.12 \mathrm{mmol}, 1.0$ equiv), $2 \mathrm{~h}$ (33.0 mg, $0.18 \mathrm{mmol}, 1.5$ equiv), and tris(pentafluorophenyl)borane $\left(\mathrm{B}_{(}\left(\mathrm{C}_{6} \mathrm{~F}_{5}\right)_{3}\right)(6.0 \mathrm{mg}, 12 \mu \mathrm{mol}, 10 \mathrm{~mol} \%)$. Purification by column chromatography on silica gel using petroleum ether/DCM $(8: 2 \mathrm{v} / \mathrm{v})$ as eluent afforded the product 
$10+10^{\prime}(32.0 \mathrm{mg}, 86 \%)$ as colorless oil. HRMS (ESI) $\mathrm{m} / \mathrm{z}$ : $\left[(\mathrm{M}+\mathrm{H})^{+}\right]$calcd for $\mathrm{C}_{23} \mathrm{H}_{24} \mathrm{~N}^{+} 314.1903$; found 314.1904. ${ }^{1} \mathrm{H}$ NMR (400 MHz, $\left.\mathrm{CDCl}_{3}\right)$ ठ 7.40-7.37 (m, 2H), 7.32-7.18 (m, 7H), 7.04-6.97 (m, 4H), 6.94-6.90 (m, 1H), 6.46-6.36 (m, 2H), 3.65-3.59 (m, 1H), $3.32(\mathrm{~s}, 3 \mathrm{H}), 1.47(\mathrm{~d}, J=6.9$ $\mathrm{Hz}, 3 \mathrm{H}) .{ }^{13} \mathrm{C}$ NMR $\left(101 \mathrm{MHz}, \mathrm{CDCl}_{3}\right) \delta 149.2,147.3,139.2,137.8,135.6,129.2,128.6,128.4$, 128.2, 127.1, 126.3, 121.7, 120.6, 119.4, 42.1, 40.4, 21.4.

\section{9 (E)-1-benzyl-3-(4-phenylbut-3-en-2-yl)-1 H-pyrrole (11)}

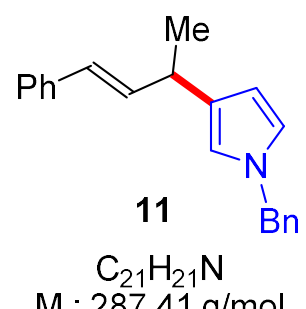

According to GP-B, using 1a (16.0 mg, $0.12 \mathrm{mmol}, 1.0$ equiv), $2 \mathbf{i}$ ( $29.0 \mathrm{mg}, 0.18 \mathrm{mmol}, 1.5$ equiv), and tris(pentafluorophenyl)borane $\left(\mathrm{B}\left(\mathrm{C}_{6} \mathrm{~F}_{5}\right)_{3}\right)(6.0 \mathrm{mg}, 12 \mu \mathrm{mol}, 10 \mathrm{~mol} \%)$ stirred for $12 \mathrm{~h}$. Purification by column chromatography on silica gel using petroleum ether/DCM $(9: 1 \mathrm{v} / \mathrm{v})$ as eluent afforded the product $11(20.0 \mathrm{mg}, 58 \%)$ as colorless oil. HRMS $(\mathrm{ESI}) \mathrm{m} / \mathrm{z}:\left[(\mathrm{M}+\mathrm{H})^{+}\right]$calcd for $\mathrm{C}_{21} \mathrm{H}_{22} \mathrm{~N}^{+}$288.1747; found 288.1750. ${ }^{1} \mathrm{H}$ NMR $\left(400 \mathrm{MHz}, \mathrm{CDCl}_{3}\right) \delta 7.31-7.18(\mathrm{~m}, 8 \mathrm{H}), 6.98-$ $6.95(\mathrm{~m}, 2 \mathrm{H}), 6.66-6.60(\mathrm{~m}, 1 \mathrm{H}), 6.26-6.17(\mathrm{~m}, 3 \mathrm{H}), 6.10-6.08(\mathrm{~m}, 1 \mathrm{H}), 5.17-5.02(\mathrm{~m}, 2 \mathrm{H}), 3.51-$ $3.44(\mathrm{~m}, 1 \mathrm{H}), 1.40(\mathrm{~d}, J=7.0 \mathrm{~Hz}, 3 \mathrm{H}) .{ }^{13} \mathrm{C}$ NMR $\left(101 \mathrm{MHz}, \mathrm{CDCl}_{3}\right) \delta 138.7,137.4,136.0,134.7$, 128.8, 128.7, 128.6, 127.4, 127.2, 126.4, 126.3, 121.8, 107.2, 105.5, 50.4, 34.8, 20.6.

\subsection{0 (E)-4-(4-(4-phenylbut-3-en-2-yl)phenyl)morpholine and (E)-4-(4-(1-phenylbut-2-en-1- yl)phenyl)morpholine $(12+12 ')$}

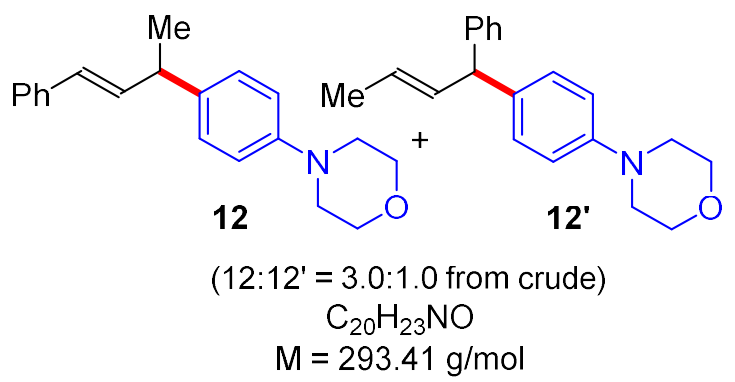

According to GP-C, using 1a (16.0 mg, $0.12 \mathrm{mmol}, 1.0$ equiv), $2 \mathrm{j}$ (29.0 mg, $0.18 \mathrm{mmol}, 1.5$ equiv), and tris(pentafluorophenyl)borane $\left(\mathrm{B}_{(}\left(\mathrm{C}_{6} \mathrm{~F}_{5}\right)_{3}\right)(6.0 \mathrm{mg}, 12 \mu \mathrm{mol}, 10 \mathrm{~mol} \%)$. Purification by column chromatography on silica gel using petroleum ether/DCM $(8: 2 \mathrm{v} / \mathrm{v})$ as eluent afforded the product 12 + 12' (19 mg, $57 \%$,) as colorless oil. HRMS (ESI) $\mathrm{m} / \mathrm{z}:\left[(\mathrm{M}+\mathrm{H})^{+}\right]$calcd for $\mathrm{C}_{20} \mathrm{H}_{24} \mathrm{NO}^{+} 294.1853$; 
found 294.1845. ${ }^{1} \mathrm{H}$ NMR (400 MHz, $\left.\mathrm{CDCl}_{3}\right) \delta$ 7.36-7.33 (m, 2H), 7.31-7.28 (m, 2H), 7.21-7.17 $(\mathrm{m}, 3.54 \mathrm{H}), 7.10-7.08(\mathrm{~m}, 0.50 \mathrm{H}), 6.90-6.88(\mathrm{~m}, 2 \mathrm{H}), 6.86-6.84(\mathrm{~m}, 0.49 \mathrm{H}), 6.43-6.33(\mathrm{~m}, 2 \mathrm{H})$, 5.92-5.86 (m, 0.31H), 5.45-5.40 (m, 0.31H), $4.60(\mathrm{~d}, J=7.7 \mathrm{~Hz}, 0.26 \mathrm{H}), 3.87-3.84(\mathrm{~m}, 4.60 \mathrm{H})$, 3.61-3.55 (m, 1H), 3.15-3.11 (m, 4.64H), 1.73-1.71 (m, 0.71H), $1.44(\mathrm{~d}, J=7.0 \mathrm{~Hz}, 3 \mathrm{H}) .{ }^{13} \mathrm{C}$ NMR $\left(101 \mathrm{MHz}, \mathrm{CDCl}_{3}\right) \delta 149.8,137.8,137.3,135.8,133.9,129.3,128.6(2 \mathrm{C}), 128.4,128.3$, $128.1,127.1,126.7,126.2(2 \mathrm{C}), 116.0,115.8,67.1,53.3,49.7,49.6,41.8,21.3$.

\subsection{1 (E)-1-methyl-6-(4-phenylbut-3-en-2-yl)-1,2,3,4-tetrahydroquinoline and (E)-1-methyl-} 6-(1-phenylbut-2-en-1-yl)-1,2,3,4-tetrahydroquinoline (13 + 13')

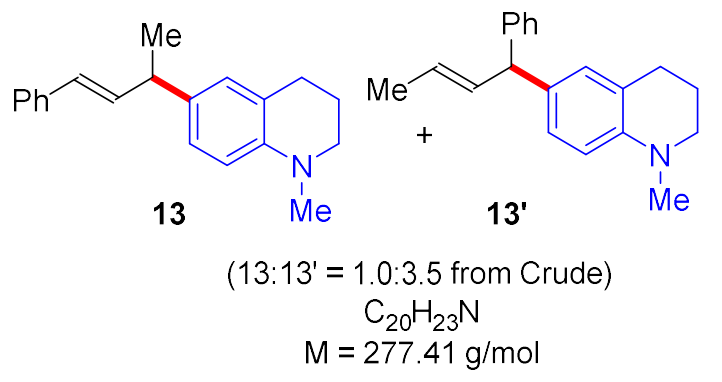

According to GP-C, using $1 \mathrm{a}$ (16.0 mg, $0.12 \mathrm{mmol}, 1.0$ equiv), 2k (28.0 mg, $0.18 \mathrm{mmol}, 1.5$ equiv), and tris(pentafluorophenyl)borane $\left(\mathrm{B}\left(\mathrm{C}_{6} \mathrm{~F}_{5}\right)_{3}\right)(6.0 \mathrm{mg}, 12 \mu \mathrm{mol}, 10 \mathrm{~mol} \%)$. Purification by column chromatography on silica gel using petroleum ether/DCM $(8: 2 \mathrm{v} / \mathrm{v})$ as eluent afforded the product 13 + 13' (19 mg, $57 \%$ ) as colorless oil. HRMS (ESI) $\mathrm{m} / \mathrm{z}$ : $\left[(\mathrm{M}+\mathrm{H})^{+}\right]$calcd for $\mathrm{C}_{20} \mathrm{H}_{24} \mathrm{~N}^{+} 278.1903$; found 278.1896. ${ }^{1} \mathrm{H}$ NMR $\left(400 \mathrm{MHz}, \mathrm{CDCl}_{3}\right) \delta 7.39-7.37(\mathrm{~m}, 0.31 \mathrm{H}), 7.32-7.28(\mathrm{~m}, 2.14 \mathrm{H}), 7.23-$ $7.16(\mathrm{~m}, 3 \mathrm{H}), 7.01(\mathrm{dd}, J=8.5,2.3 \mathrm{~Hz}, 0.16 \mathrm{H}), 6.90(\mathrm{dd}, J=8.3,2.3 \mathrm{~Hz}, 1 \mathrm{H}), 6.83(\mathrm{~d}, J=2.1 \mathrm{~Hz}$, $0.11 \mathrm{H}), 6.79(\mathrm{~d}, J=2.1 \mathrm{~Hz}, 1 \mathrm{H}), 6.61(\mathrm{~d}, J=8.5 \mathrm{~Hz}, 0.19 \mathrm{H}), 6.57(\mathrm{~d}, J=8.4 \mathrm{~Hz}, 1 \mathrm{H}), 6.58-6.56$ $(\mathrm{m}, 0.30 \mathrm{H}), 5.94-5.88(\mathrm{~m}, 1 \mathrm{H}), 5.59-5.40(\mathrm{~m}, 1 \mathrm{H}), 4.55(\mathrm{~d}, J=7.8 \mathrm{~Hz}, 1 \mathrm{H}), 3.56-3.50(\mathrm{~m}, 0.16 \mathrm{H})$, 3.23-3.19 (m, 2.61H), $2.89(\mathrm{~s}, 0.47 \mathrm{H}), 2.87(\mathrm{~s}, 3 \mathrm{H}), 2.80-2.70(\mathrm{~m}, 2.32 \mathrm{H}), 2.04-1.95(\mathrm{~m}, 2.76 \mathrm{H})$, 1.76-1.73 (m, 3H), $1.44(\mathrm{~d}, J=7.2 \mathrm{~Hz}, 0.48 \mathrm{H}) .{ }^{13} \mathrm{C}$ NMR $\left(101 \mathrm{MHz}, \mathrm{CDCl}_{3}\right) \delta$ 145.2, 138.0, 136.3, 134.4, 132.2, 129.0, 128.6, 128.3, 128.0, 127.8, 127.0, 126.2 (2C), 126.0, 125.8, 125.6, 123.2, $123.0,111.5,111.2,53.4,51.4,41.7,40.1,39.4,38.6,34.2,27.9,22.8,22.6,21.4,18.2$. 
6.12 1-((2S)-1-methyl-6-((E)-4-phenylbut-3-en-2-yl)-1,2,3,4-tetrahydroquinolin-2-yl)butan2-ylium and (2S)-2-butyl-1-methyl-6-((E)-1-phenylbut-2-en-1-yl)-1,2,3,4-tetrahydroquinoline $(14+14 ')$

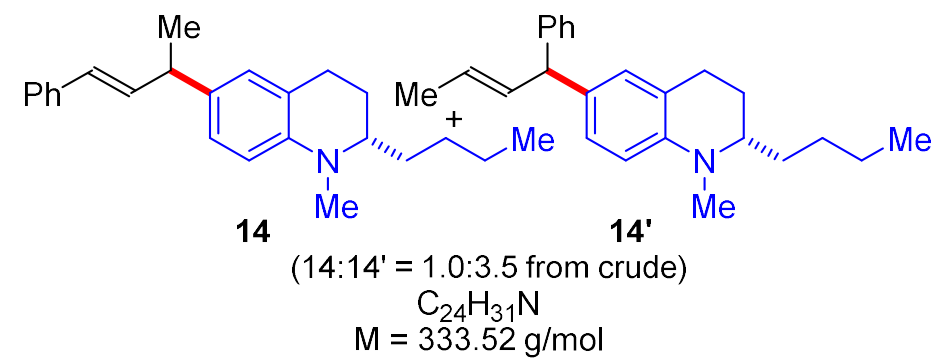

According to GP-C, using 1a (16.0 mg, $0.12 \mathrm{mmol}, 1.0$ equiv), $2 \mathrm{~m}$ (34.0 mg, $0.18 \mathrm{mmol}, 1.5$ equiv), and tris(pentafluorophenyl)borane $\left(\mathrm{B}_{(}\left(\mathrm{C}_{6} \mathrm{~F}_{5}\right)_{3}\right)(6.0 \mathrm{mg}, 12 \mu \mathrm{mol}, 10 \mathrm{~mol} \%)$. Purification by column chromatography on silica gel using petroleum ether/DCM (7:3 v/v) as eluent afforded the product 14' (19 mg, 57\%) as Yellow oil. HRMS (ESI) $\mathrm{m} / \mathrm{z}$ : $\left[(\mathrm{M}+\mathrm{H})^{+}\right]$calcd for $\mathrm{C}_{24} \mathrm{H}_{32} \mathrm{~N}^{+} 334.2530$; found 334.2537. ${ }^{1} \mathrm{H}$ NMR $\left(400 \mathrm{MHz}, \mathrm{CDCl}_{3}\right) \delta 7.30-7.26(\mathrm{~m}, 2 \mathrm{H}), 7.22-7.16(\mathrm{~m}, 3 \mathrm{H}), 6.87(\mathrm{~d}, \mathrm{~J}=$ $6.8 \mathrm{~Hz}, 1 \mathrm{H}), 6.78(\mathrm{t}, J=3.1 \mathrm{~Hz}, 1 \mathrm{H}), 6.45(\mathrm{~d}, J=8.3 \mathrm{~Hz}, 1 \mathrm{H}), 5.93-5.87(\mathrm{~m}, 1 \mathrm{H}), 5.48-5.39(\mathrm{~m}$, $1 \mathrm{H}), 4.53(\mathrm{~d}, J=7.9 \mathrm{~Hz}, 1 \mathrm{H}), 3.20(\mathrm{~d}, J=8.3 \mathrm{~Hz}, 1 \mathrm{H}), 2.90(\mathrm{~s}, 3 \mathrm{H}), 2.80-2.72(\mathrm{~m}, 1 \mathrm{H}), 2.60(\mathrm{~d}, J$ $=16.5 \mathrm{~Hz}, 1 \mathrm{H}), 1.89-1.85(\mathrm{~m}, 2 \mathrm{H}), 1.72(\mathrm{dd}, J=6.6,1.4 \mathrm{~Hz}, 3 \mathrm{H}), 1.44-1.22(\mathrm{~m}, 6 \mathrm{H}), 0.91(\mathrm{t}, J=$ $6.7 \mathrm{~Hz}, 3 \mathrm{H}) .{ }^{13} \mathrm{C}$ NMR $\left(101 \mathrm{MHz}, \mathrm{CDCl}_{3}\right) \delta 145.3,143.9,134.5,130.9,128.8,128.6,128.3,127.0$, 126.0 (2C), 121.9, 110.3, 59.1, 53.4, 38.1, 31.0, 28.4, 24.6, 23.8, 23.0, 18.2, 14.3.

\subsection{3 (E)-N-phenyl-4-(4-(p-tolyl)but-3-en-2-yl)aniline (15)}

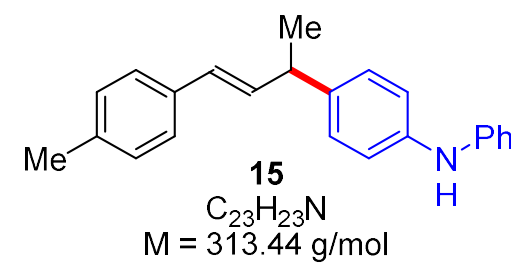

According to GP-A, using 1b (17.0 mg, $0.12 \mathrm{mmol}, 1.0$ equiv), 2a (30.0 mg, $0.18 \mathrm{mmol}, 1.5$ equiv), and tris(pentafluorophenyl)borane $\left(\mathrm{B}\left(\mathrm{C}_{6} \mathrm{~F}_{5}\right)_{3}\right)(6.0 \mathrm{mg}, 12 \mu \mathrm{mol}, 10 \mathrm{~mol} \%)$. Purification by column chromatography on silica gel using petroleum ether/DCM $(9: 1 \mathrm{v} / \mathrm{v})$ as eluent afforded the product 15 (34.0 mg, 90\%) as colorless oil. HRMS (ESI) $m / z$ : $\left[(\mathrm{M}+\mathrm{H})^{+}\right]$calcd for $\mathrm{C}_{23} \mathrm{H}_{24} \mathrm{~N}^{+} 314.1903$; found 314.1910. ${ }^{1} \mathrm{H}$ NMR $\left(400 \mathrm{MHz}, \mathrm{CDCl}_{3}\right) \delta 7.29-7.25(\mathrm{~m}, 4 \mathrm{H}), 7.19(\mathrm{~d}, J=8.3 \mathrm{~Hz}, 2 \mathrm{H}), 7.12(\mathrm{~d}, J=$ $7.8 \mathrm{~Hz}, 2 \mathrm{H}), 7.09-7.06(\mathrm{~m}, 4 \mathrm{H}), 6.92(\mathrm{t}, \mathrm{J}=7.3 \mathrm{~Hz}, 1 \mathrm{H}), 6.42-6.30(\mathrm{~m}, 2 \mathrm{H}), 3.63-3.57(\mathrm{~m}, 1 \mathrm{H})$, $2.34(\mathrm{~s}, 3 \mathrm{H}), 1.46(\mathrm{~d}, J=7.4 \mathrm{~Hz}, 3 \mathrm{H}) .{ }^{13} \mathrm{C}$ NMR $\left(101 \mathrm{MHz}, \mathrm{CDCl}_{3}\right) \delta$ 143.5, 141.0, 138.9, 136.8, $134.9,134.6,129.5,129.3,128.3,128.2,126.1,120.8,118.7,117.4,42.0,21.4,21.3$. 


\subsection{4 (E)-N-phenyl-4-(4-(o-tolyl)but-3-en-2-yl)aniline (16)}

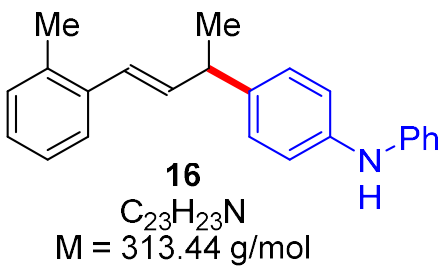

According to GP-A, using 1c (17.0 mg, $0.12 \mathrm{mmol}, 1.0$ equiv), 2a (30.0 mg, $0.18 \mathrm{mmol}, 1.5$ equiv), and tris(pentafluorophenyl)borane $\left(\mathrm{B}_{(}\left(\mathrm{C}_{6} \mathrm{~F}_{5}\right)_{3}\right)(6.0 \mathrm{mg}, 12 \mu \mathrm{mol}, 10 \mathrm{~mol} \%)$. Purification by column chromatography on silica gel using petroleum ether/DCM $(9: 1 \mathrm{v} / \mathrm{v})$ as eluent afforded the product 16 (33.0 mg, 85\%) as colorless oil. HRMS (ESI) $\mathrm{m} / \mathrm{z}$ : $\left[(\mathrm{M}+\mathrm{H})^{+}\right]$calcd for $\mathrm{C}_{23} \mathrm{H}_{24} \mathrm{~N}^{+} 314.1903$, found 314.1903. ${ }^{1} \mathrm{H}$ NMR (500 MHz, $\left.\mathrm{CDCl}_{3}\right) \delta$ 7.41-7.39 (m, 1H), 7.23-7.20 (m, 2H), 7.16 (d, J = 8.1 $\mathrm{Hz}, 2 \mathrm{H}), 7.11-7.09(\mathrm{~m}, 3 \mathrm{H}), 7.03-7.00(\mathrm{~m}, 4 \mathrm{H}), 6.87(\mathrm{t}, J=7.3 \mathrm{~Hz}, 1 \mathrm{H}), 6.59(\mathrm{~d}, J=15.7 \mathrm{~Hz}, 1 \mathrm{H})$, 6.24-6.19 (m, 1H), $5.62(\mathrm{~s}, 1 \mathrm{H}), 3.62-3.56(\mathrm{~m}, 1 \mathrm{H}), 2.31(\mathrm{~s}, 3 \mathrm{H}), 1.44(\mathrm{~d}, J=7.0 \mathrm{~Hz}, 3 \mathrm{H}) .{ }^{13} \mathrm{C}$ NMR $\left(126 \mathrm{MHz} \mathrm{CDCl}_{3}\right) \delta 143.7,141.2,138.7,137.1,136.9,135.2,130.3,129.4,128.2,127.1,126.2$, 126.1, 125.6, 120.7, 118.6, 117.4, 42.3, 21.5, 20.0.

\subsection{5 (E)-4-(4-(4-methoxyphenyl)but-3-en-2-yl)-N-phenylaniline (17)}

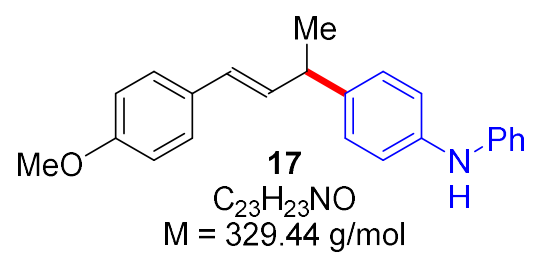

According to GP-A, using 1d (19.0 mg, $0.12 \mathrm{mmol}, 1.0$ equiv), 2a (30.0 mg, $0.18 \mathrm{mmol}, 1.5$ equiv), and tris(pentafluorophenyl)borane $\left(\mathrm{B}\left(\mathrm{C}_{6} \mathrm{~F}_{5}\right)_{3}\right)(6.0 \mathrm{mg}, 12 \mu \mathrm{mol}, 10 \mathrm{~mol} \%)$. Purification by column chromatography on silica gel using petroleum ether/DCM $(8: 2 \mathrm{v} / \mathrm{v})$ as eluent afforded the product 17 (32.0 mg, 80\%) as colorless oil. HRMS (ESI) $\mathrm{m} / \mathrm{z}$ : $\left[(\mathrm{M}+\mathrm{H})^{+}\right]$calcd for $\mathrm{C}_{23} \mathrm{H}_{24} \mathrm{NO}^{+} 330.1853$; found 330.1855. ${ }^{1} \mathrm{H}$ NMR (400 MHz, $\left.\mathrm{CDCl}_{3}\right) \delta$ 7.32-7.24 (m, 4H), 7.20-7.15 (m, 2H), 7.10-7.03 $(\mathrm{m}, 4 \mathrm{H}), 6.93-6.90(\mathrm{~m}, 1 \mathrm{H}), 6.86-6.82(\mathrm{~m}, 2 \mathrm{H}), 6.36(\mathrm{~d}, J=15.8 \mathrm{~Hz}, 1 \mathrm{H}), 6.27-6.21(\mathrm{~m}, 1 \mathrm{H}), 3.80$ (s, 3H), 3.62-3.55 (m, 1H), 1.45 (d, J = 7.0, $1.5 \mathrm{~Hz}, 3 \mathrm{H}) .{ }^{13} \mathrm{C}$ NMR (101 MHz, CDCl $) \delta 158.9$, 143.6, 141.0, 139.1, 133.6, 130.6, 129.5, 128.3, 127.7, 127.4, 120.9, 118.7, 117.5, 114.0, 55.4, 42.0, 21.5 . 


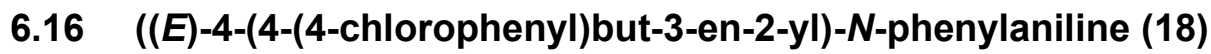

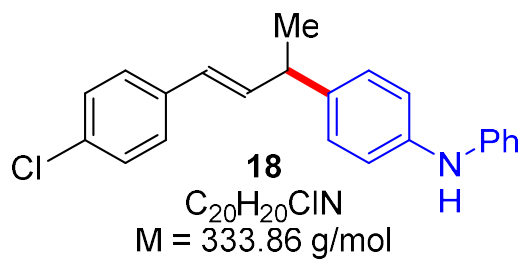

According to GP-A, using 1e (20.0 mg, $0.12 \mathrm{mmol}, 1.0$ equiv), 2a (30.0 mg, $0.18 \mathrm{mmol}, 1.5$ equiv), and tris(pentafluorophenyl)borane $\left(\mathrm{B}\left(\mathrm{C}_{6} \mathrm{~F}_{5}\right)_{3}\right)(6.0 \mathrm{mg}, 12 \mu \mathrm{mol}, 10 \mathrm{~mol} \%)$. Purification by column chromatography on silica gel using petroleum ether/DCM $(8: 2 \mathrm{v} / \mathrm{v})$ as eluent afforded the product $18(32.0 \mathrm{mg}, 80 \%)$ as colorless oil. HRMS (ESI) $\mathrm{m} / \mathrm{z}$ : $\left[(\mathrm{M}+\mathrm{H})^{+}\right]$calcd for $\mathrm{C}_{20} \mathrm{H}_{21} \mathrm{CIN}^{+} 334.1357$; found 334.1361. ${ }^{1} \mathrm{H}$ NMR (400 MHz, $\left.\mathrm{CDCl}_{3}\right) \delta$ 7.30-7.24 (m, 6H), 7.18-7.15 (m, 2H), 7.10-7.04 $(\mathrm{m}, 4 \mathrm{H}), 6.92(\mathrm{t}, J=7.4 \mathrm{~Hz}, 1 \mathrm{H}), 6.40-6.31(\mathrm{~m}, 2 \mathrm{H}), 3.63-3.56(\mathrm{~m}, 1 \mathrm{H}), 1.46(\mathrm{~d}, J=7.2 \mathrm{~Hz}, 3 \mathrm{H})$. ${ }^{13} \mathrm{C}$ NMR $\left(101 \mathrm{MHz}, \mathrm{CDCl}_{3}\right) \delta 143.4,141.3,138.3,136.4,136.2,132.6,129.5,128.7,128.3,127.5$, $127.2,120.9,118.6,117.5,42.0,21.3$.

\subsection{7 (E)-4-(4-(naphthalen-2-yl)but-3-en-2-yl)-N-phenylaniline (19)}

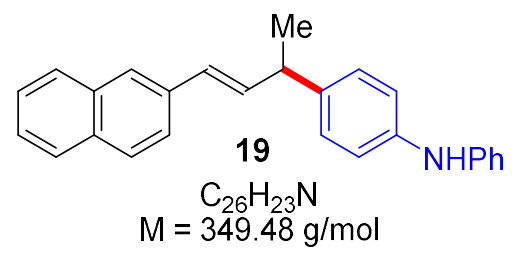

According to GP-A, using $1 \mathrm{f}$ (22.0 mg, $0.12 \mathrm{mmol}, 1.0$ equiv), 2a (30.0 mg, $0.18 \mathrm{mmol}, 1.5$ equiv), and tris(pentafluorophenyl)borane $\left(\mathrm{B}\left(\mathrm{C}_{6} \mathrm{~F}_{5}\right)_{3}\right)(6.0 \mathrm{mg}, 12 \mu \mathrm{mol}, 10 \mathrm{~mol} \%)$. Purification by column chromatography on silica gel using petroleum ether/DCM $(8: 2 \mathrm{v} / \mathrm{v})$ as eluent afforded the product 19 (36.0 mg, 85\%) as colorless oil. HRMS (ESI) $\mathrm{m} / \mathrm{z}$ : $\left[(\mathrm{M}+\mathrm{H})^{+}\right]$calcd for $\mathrm{C}_{26} \mathrm{H}_{24} \mathrm{~N}^{+} 350.1903$; found 350.1901. ${ }^{1} \mathrm{H}$ NMR (400 MHz, $\left.\mathrm{CDCl}_{3}\right) \delta 7.80-7.75(\mathrm{~m}, 3 \mathrm{H}), 7.71(\mathrm{~d}, J=1.7 \mathrm{~Hz}, 1 \mathrm{H}), 7.60$ (dd, $J=$ 8.5, $1.8 \mathrm{~Hz}, 1 \mathrm{H}), 7.47-7.40(\mathrm{~m}, 2 \mathrm{H}), 7.30-7.19(\mathrm{~m}, 4 \mathrm{H}), 7.14-7.03(\mathrm{~m}, 4 \mathrm{H}), 6.95-6.91(\mathrm{~m}, 1 \mathrm{H})$, 6.60-6.48 (m, 2H), 3.70-3.63 (m, 1H), $1.50(\mathrm{~d}, J=6.9 \mathrm{~Hz}, 3 \mathrm{H}) .{ }^{13} \mathrm{C}$ NMR $\left(101 \mathrm{MHz}, \mathrm{CDCl}_{3}\right) \delta$ 143.4, 141.1, 138.8, 136.1, 135.2, 133.8, 132.8, 129.5, 128.5, 128.3, 128.2, 128.0, 127.8, 126.3, $125.9,125.7,123.8,121.0,118.7,117.6,42.2,21.4$. 


\subsection{8 (E)-4-(4-(anthracen-9-yl)but-3-en-2-yl)-N-phenylaniline (20)}

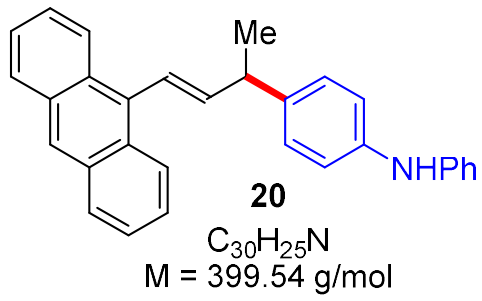

According to GP-A, using $1 \mathrm{~g}$ (28.0 mg, $0.12 \mathrm{mmol}, 1.0$ equiv), 2a (30.0 mg, $0.18 \mathrm{mmol}, 1.5$ equiv), and tris(pentafluorophenyl)borane $\left(\mathrm{B}\left(\mathrm{C}_{6} \mathrm{~F}_{5}\right)_{3}\right)(6.0 \mathrm{mg}, 12 \mu \mathrm{mol}, 10 \mathrm{~mol} \%)$. Purification by column chromatography on silica gel using petroleum ether/DCM $(7: 3 \mathrm{v} / \mathrm{v})$ as eluent afforded the product 20 (41.0 mg, 85\%) as yellow oil. HRMS (ESI) $\mathrm{m} / \mathrm{z}$ : $\left[(\mathrm{M}+\mathrm{H})^{+}\right]$calcd for $\mathrm{C}_{30} \mathrm{H}_{26} \mathrm{~N}^{+}$400.2060; found 400.2065. ' ${ }^{1} \mathrm{~N}$ NMR (400 MHz, DMSO-D 6 ) 8.51 (s, 1H), 8.26-8.22 (m, 2H), 8.09-8.05 (m, 2H), 7.52-7.48 (m, 4H), 7.34-7.30 (m, 2H), 7.23-7.18 (m, 3H), $7.14(\mathrm{~s}, 1 \mathrm{H}), 7.12-7.09(\mathrm{~m}, 2 \mathrm{H}), 7.06$ $(\mathrm{d}, J=7.9 \mathrm{~Hz}, 2 \mathrm{H}), 6.78(\mathrm{t}, J=7.2 \mathrm{~Hz}, 1 \mathrm{H}), 6.13(\mathrm{dd}, J=16.1,6.9 \mathrm{~Hz}, 1 \mathrm{H}), 3.93-3.86(\mathrm{~m}, 1 \mathrm{H})$, $1.58(\mathrm{~d}, J=7.0 \mathrm{~Hz}, 3 \mathrm{H}) .{ }^{13} \mathrm{C}$ NMR (101 MHz, DMSO-D 6 ) $\delta 144.3,143.8,141.5,136.6,132.7$, $131.0,129.2$, 128.9, 128.6, 127.9, 125.8, 125.7, 125.6, 125.3, 123.2, 119.3, 117.4, 116.3, 42.1, 21.4 .

\subsection{9 (E)-N-phenyl-4-(1-phenylpent-1-en-3-yl)aniline (21)}

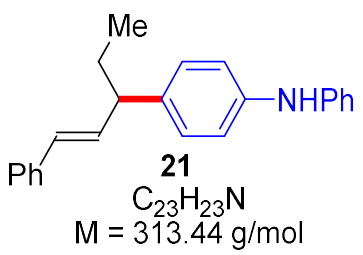

According to GP-A, using $1 \mathrm{~h}$ ( $24.0 \mathrm{mg}, 0.12 \mathrm{mmol}, 1.0$ equiv), 2a (30.0 mg, $0.18 \mathrm{mmol}, 1.5$ equiv), and tris(pentafluorophenyl)borane $\left(\mathrm{B}\left(\mathrm{C}_{6} \mathrm{~F}_{5}\right)_{3}\right)(6.0 \mathrm{mg}, 12 \mu \mathrm{mol}, 10 \mathrm{~mol} \%)$. Purification by column chromatography on silica gel using petroleum ether/DCM $(9: 1 \mathrm{v} / \mathrm{v})$ as eluent afforded the product 21 (30.0 mg, 80\%) as colorless oil. HRMS (ESI) $\mathrm{m} / \mathrm{z}$ : $\left[(\mathrm{M}+\mathrm{H})^{+}\right]$calcd for $\mathrm{C}_{23} \mathrm{H}_{24} \mathrm{~N}^{+} 314.1903$; found 314.1895. ${ }^{1} \mathrm{H}$ NMR $\left(400 \mathrm{MHz}, \mathrm{CDCl}_{3}\right) \delta$ 7.37-735 (m, 2H), 7.31-7.24 (m, 4H), 7.21-7.08 (m, 7H), $6.95(\mathrm{t}, J=7.3 \mathrm{~Hz}, 1 \mathrm{H}), 6.42-6.28(\mathrm{~m}, 2 \mathrm{H}), 3.27(\mathrm{q}, J=7.5 \mathrm{~Hz}, 1 \mathrm{H}), 1.85-1.77(\mathrm{~m}, 2 \mathrm{H}), 0.92(\mathrm{t}, J$ $=7.3 \mathrm{~Hz}, 3 \mathrm{H}) .{ }^{13} \mathrm{C}$ NMR $\left(101 \mathrm{MHz}, \mathrm{CDCl}_{3}\right) \delta 143.2,140.7,138.1,137.8,134.5,129.5,129.3$, $128.7,128.6,127.1,126.2,121.3,118.9,117.8,50.4,28.9,12.5$. 


\subsection{0 (E)-N-phenyl-4-(1-phenyloct-1-en-3-yl)aniline (22)}

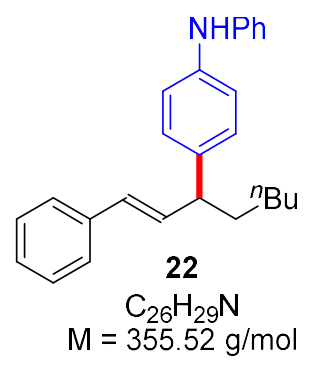

According to GP-A, using $1 \mathrm{i}$ (22.0 mg, $0.12 \mathrm{mmol}, 1.0$ equiv), 2a (30.0 mg, $0.18 \mathrm{mmol}, 1.5$ equiv), and tris(pentafluorophenyl)borane $\left(\mathrm{B}\left(\mathrm{C}_{6} \mathrm{~F}_{5}\right)_{3}\right)(6.0 \mathrm{mg}, 12 \mu \mathrm{mol}, 10 \mathrm{~mol} \%)$. Purification by column chromatography on silica gel using petroleum ether/DCM $(9: 1 \mathrm{v} / \mathrm{v})$ as eluent afforded the product 22 (34.0 mg, 80\%) as colorless oil. HRMS (ESI) $\mathrm{m} / \mathrm{z}$ : $\left[(\mathrm{M}+\mathrm{H})^{+}\right]$calcd for $\mathrm{C}_{26} \mathrm{H}_{30} \mathrm{~N}^{+} 356.2373$; found 356.2378. ${ }^{1} \mathrm{H}$ NMR $\left(400 \mathrm{MHz}, \mathrm{CDCl}_{3}\right) \delta 7.41-7.38(\mathrm{~m}, 2 \mathrm{H}), 7.34-7.26(\mathrm{~m}, 4 \mathrm{H}), 7.24-7.17(\mathrm{~m}, 3 \mathrm{H})$, 7.11-7.06 (m, 4H), 6.96-6.91 (m, 1H), 6.45-6.32 (m, 2H), 3.42-3.36 (m, 1H), 1.86-1.78 (m, 2H), 1.40-1.29 (m, 6H), 0.94-0.90 (m, 3H).). ${ }^{13} \mathrm{C}$ NMR (101 MHz, $\left.\mathrm{CDCl}_{3}\right) \delta 143.6,141.1,137.8(2 \mathrm{C})$, 134.9, 129.4, 129.1, 128.6 (2C), 127.1, 126.2, 120.7, 118.6, 117.4, 48.6, 36.0, 32.0, 27.5, 22.7, 14.3.

\subsection{1 (E)-N-phenyl-4-(6-phenylhex-3-en-2-yl)aniline (23)}

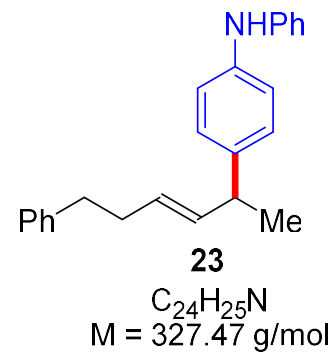

According to GP-A, using 1j (15.0 mg, $0.12 \mathrm{mmol}, 1.0$ equiv), 2a (30.0 mg, $0.18 \mathrm{mmol}, 1.5$ equiv), and tris(pentafluorophenyl)borane $\left(\mathrm{B}_{(}\left(\mathrm{C}_{6} \mathrm{~F}_{5}\right)_{3}\right)(6.0 \mathrm{mg}, 12 \mu \mathrm{mol}, 10 \mathrm{~mol} \%)$. Purification by column chromatography on silica gel using petroleum ether/DCM $(9: 1 \mathrm{v} / \mathrm{v})$ as eluent afforded the product 23 (31.0 mg, 80\%) as colorless oil. HRMS (ESI) m/z: [(M+H) $\left.{ }^{+}\right]$calcd for $\mathrm{C}_{24} \mathrm{H}_{26} \mathrm{~N}^{+} 328.2060$; found 328.2054. ' ${ }^{1} \mathrm{~N} M R\left(400 \mathrm{MHz}, \mathrm{CDCl}_{3}\right) \delta$ 7.31-7.25 (m, 4H), 7.21-7.17 (m, 3H), 7.13-7.05 (m, 6H), $6.92(\mathrm{t}, J=7.3 \mathrm{~Hz}, 1 \mathrm{H}), 5.63-5.57(\mathrm{~m}, 1 \mathrm{H}), 5.53-5.44(\mathrm{~m}, 1 \mathrm{H}), 3.20(\mathrm{q}, J=7.4 \mathrm{~Hz}, 1 \mathrm{H}), 2.66-$ $2.52(\mathrm{~m}, 2 \mathrm{H}), 2.00(\mathrm{q}, J=7.8 \mathrm{~Hz}, 2 \mathrm{H}), 1.70(\mathrm{~d}, J=6.1 \mathrm{~Hz}, 3 \mathrm{H}) \cdot{ }^{13} \mathrm{C}$ NMR $\left(101 \mathrm{MHz}, \mathrm{CDCl}_{3}\right) \delta$ $143.5,142.6,140.9,138.3,135.3,129.5,128.6,128.5,128.4,125.8,124.9,120.8,118.7,117.5$, $47.8,37.8,33.9,18.2$. 


\subsection{2 (E)-4-(oct-3-en-2-yl)-N-phenylaniline (24)}

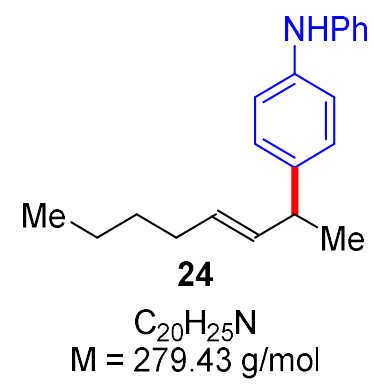

According to GP-A, using $1 \mathbf{k}(13.0 \mathrm{mg}, 0.12 \mathrm{mmol}, 1.0$ equiv), 2a (30.0 mg, $0.18 \mathrm{mmol}, 1.5$ equiv), and tris(pentafluorophenyl)borane $\left(\mathrm{B}\left(\mathrm{C}_{6} \mathrm{~F}_{5}\right)_{3}\right)(6.0 \mathrm{mg}, 12 \mu \mathrm{mol}, 10 \mathrm{~mol} \%)$. Purification by column chromatography on silica gel using petroleum ether/DCM $(9: 1 \mathrm{v} / \mathrm{v})$ as eluent afforded the product 24 (20.0 mg, 60\%) as colorless oil. HRMS (ESI) $m / z$ : $\left[(\mathrm{M}+\mathrm{H})^{+}\right]$calcd for $\mathrm{C}_{20} \mathrm{H}_{26} \mathrm{~N}^{+} 280.2060$; found 280.2044. ${ }^{1} \mathrm{H}$ NMR (400 MHz, DMSO-D 6$) \delta 8.03(\mathrm{~s}, 1 \mathrm{H}), 7.21-7.16(\mathrm{~m}, 2 \mathrm{H}), 7.05-6.94(\mathrm{~m}, 6 \mathrm{H})$, $6.76(\mathrm{t}, J=7.3 \mathrm{~Hz}, 1 \mathrm{H}), 5.56-5.49(\mathrm{~m}, 1 \mathrm{H}), 5.42-5.34(\mathrm{~m}, 1 \mathrm{H}), 3.11-3.04(\mathrm{~m}, 1 \mathrm{H}), 1.62-1.53(\mathrm{~m}$, $5 \mathrm{H}), 1.29-1.09(\mathrm{~m}, 4 \mathrm{H}), 0.83(\mathrm{t}, J=7.1 \mathrm{~Hz}, 3 \mathrm{H}) .{ }^{13} \mathrm{C}$ NMR $\left(101 \mathrm{MHz}, \mathrm{DMSO}-\mathrm{D}_{6}\right) \delta$ 143.9, 141.1, 136.6, 136.0, 129.1, 127.8, 123.2, 119.1, 117.3, 116.0, 47.6, 35.4, 29.4, 22.1, 17.7, 14.0.

\section{23 (E)-4-(1-cyclohexylbut-2-en-1-yl)-N-phenylaniline (25)}

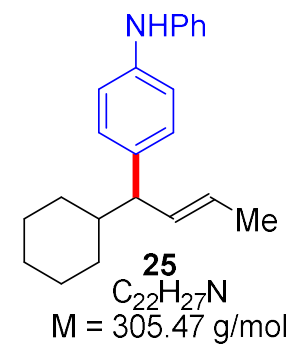

According to GP-A, using 1 ( $16.0 \mathrm{mg}, 0.12 \mathrm{mmol}, 1.0$ equiv), 2a (30.0 mg, $0.18 \mathrm{mmol}, 1.5$ equiv), and tris(pentafluorophenyl)borane $\left(\mathrm{B}\left(\mathrm{C}_{6} \mathrm{~F}_{5}\right)_{3}\right)(6.0 \mathrm{mg}, 12 \mu \mathrm{mol}, 10 \mathrm{~mol} \%)$. Purification by column chromatography on silica gel using petroleum ether/DCM $(9: 1 \mathrm{v} / \mathrm{v})$ as eluent afforded the product 25 (22.0 mg, 60\%) as colorless oil. HRMS (ESI) $\mathrm{m} / \mathrm{z}$ : $\left[(\mathrm{M}+\mathrm{H})^{+}\right]$calcd for $\mathrm{C}_{22} \mathrm{H}_{28} \mathrm{~N}^{+} 306.2216$; found 306.2212. ${ }^{1} \mathrm{H}$ NMR $\left(400 \mathrm{MHz}, \mathrm{CDCl}_{3}\right)$ ס 7.27-7.23 (m, 2H), 7.08-7.01 (m, 6H), $6.90(\mathrm{t}, \mathrm{J}=7.3 \mathrm{~Hz}$, $1 \mathrm{H}), 5.63-5.56(\mathrm{~m}, 1 \mathrm{H}), 5.47-5.38(\mathrm{~m}, 1 \mathrm{H}), 2.84(\mathrm{t}, \mathrm{J}=9.0 \mathrm{~Hz}, 1 \mathrm{H}), 1.91-1.86(\mathrm{~m}, 1 \mathrm{H}), 1.77-1.63$ $(\mathrm{m}, 5 \mathrm{H}), 1.54-1.43(\mathrm{~m}, 2 \mathrm{H}), 1.34-1.10(\mathrm{~m}, 4 \mathrm{H}), 0.95-0.75(\mathrm{~m}, 2 \mathrm{H}) .{ }^{13} \mathrm{C} \mathrm{NMR}\left(101 \mathrm{MHz}, \mathrm{CDCl}_{3}\right) \delta$ 143.7, 140.6, 138.3, 134.2, 129.4, 128.8, 125.2, 120.7, 118.7, 117.3, 55.8, 42.7, 31.6, 31.5, 26.7, 26.6, 18.2 . 


\subsection{N-phenyl-4-(4-phenylpent-3-en-2-yl)aniline (26)}
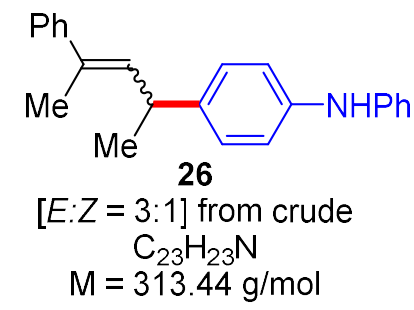

According to GP-A, using $1 \mathrm{~m}$ (17.0 mg, $0.12 \mathrm{mmol}, 1.0$ equiv), $2 \mathrm{a}$ (30.0 mg, $0.18 \mathrm{mmol}, 1.5$ equiv), and tris(pentafluorophenyl)borane $\left(\mathrm{B}_{(}\left(\mathrm{C}_{6} \mathrm{~F}_{5}\right)_{3}\right)(6.0 \mathrm{mg}, 12 \mu \mathrm{mol}, 10 \mathrm{~mol} \%)$. Purification by column chromatography on silica gel using petroleum ether/DCM $(8: 2 \mathrm{v} / \mathrm{v})$ as eluent afforded the product 26 (30.0 mg, 80\%) as colorless oil. HRMS (ESI) m/z: [(M+H) calcd for $\mathrm{C}_{23} \mathrm{H}_{24} \mathrm{~N}^{+}$ 314.1903; found 314.1907. ' ${ }^{1} \mathrm{H}$ NMR $\left(400 \mathrm{MHz}, \mathrm{CDCl}_{3}\right) \delta 7.43-7.40(\mathrm{~m}, 2 \mathrm{H}), 7.34-7.19(\mathrm{~m}, 7 \mathrm{H})$, 7.04-7.01 (m, 4H), 6.93-6.89 (m, 1H), 5.92-5.90 (m, 1H), 3.87-3.79 (m, 1H), 2.12 (d, J = 1.5 Hz, $3 \mathrm{H}), 1.42(\mathrm{~d}, J=6.9 \mathrm{~Hz}, 3 \mathrm{H}) .{ }^{13} \mathrm{C}$ NMR $\left(101 \mathrm{MHz}, \mathrm{CDCl}_{3}\right) \delta 143.9,143.7,140.9,139.7,133.8$, $133.8,129.4,128.3,127.9,126.8,125.9,120.7,118.7,117.4,38.1,22.5,16.2$.

\subsection{4-(4,4-diphenylbut-3-en-2-yl)-N-phenylaniline (27)}

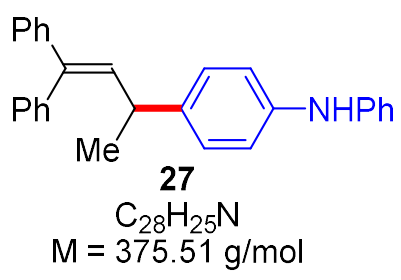

According to GP-A, using 1n (25.0 mg, $0.12 \mathrm{mmol}, 1.0$ equiv), 2a (30.0 mg, $0.18 \mathrm{mmol}, 1.5$ equiv), and tris(pentafluorophenyl)borane $\left(\mathrm{B}_{(}\left(\mathrm{C}_{6} \mathrm{~F}_{5}\right)_{3}\right)(6.0 \mathrm{mg}, 12 \mu \mathrm{mol}, 10 \mathrm{~mol} \%)$. Purification by column chromatography on silica gel using petroleum ether/DCM $(8: 2 \mathrm{v} / \mathrm{v})$ as eluent afforded the product 27 (36.0 mg, 80\%) as colorless oil. HRMS (ESI) $\mathrm{m} / \mathrm{z}$ : $\left[(\mathrm{M}+\mathrm{H})^{+}\right]$calcd for $\mathrm{C}_{28} \mathrm{H}_{26} \mathrm{~N}^{+} 376.2060$; found 376.2071. ' ${ }^{1} \mathrm{H}$ NMR (400 MHz, $\left.\mathrm{CDCl}_{3}\right)$ ס 7.42-7.33 (m, 3H), 7.28-7.19 (m, 9H), 7.13-7.09 (m, 2H), 7.06-7.01 (m, 4H), 6.92-6.88 (m, 1H), $6.20(\mathrm{~d}, J=10.3 \mathrm{~Hz}, 1 \mathrm{H}), 5.64(\mathrm{~s}, 1 \mathrm{H}), 3.60-3.52(\mathrm{~m}, 1 \mathrm{H})$, $1.38(\mathrm{~d}, J=6.9 \mathrm{~Hz}, 3 \mathrm{H}) .{ }^{13} \mathrm{C}$ NMR $\left(101 \mathrm{MHz}, \mathrm{CDCl}_{3}\right) \delta 142.5,140.2,140.0,134.5,130.0,129.8$, $129.5,128.8,128.4,128.2,128.0,127.4,127.2,127.1,126.0,121.3,119.0,117.8,38.7,22.4$. 


\subsection{4-(3-methylbut-2-en-1-yl)-N-phenylaniline (28)}

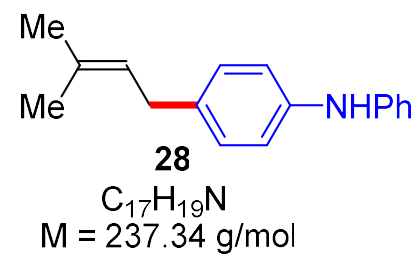

According to GP-A, using 10 (8.0 mg, $0.12 \mathrm{mmol}, 1.0$ equiv), 2a (30.0 mg, $0.18 \mathrm{mmol}, 1.5$ equiv), and tris(pentafluorophenyl)borane $\left(\mathrm{B}_{(}\left(\mathrm{C}_{6} \mathrm{~F}_{5}\right)_{3}\right)(6.0 \mathrm{mg}, 12 \mu \mathrm{mol}, 10 \mathrm{~mol} \%)$. Purification by column chromatography on silica gel using petroleum ether/DCM $(9: 1 \mathrm{v} / \mathrm{v})$ as eluent afforded the product 28 (16.0 mg, 55\%) as colorless oil. HRMS (ESI) $\mathrm{m} / \mathrm{z}$ : $\left[(\mathrm{M}+\mathrm{H})^{+}\right]$calcd for $\mathrm{C}_{17} \mathrm{H}_{20} \mathrm{~N}^{+}$238.1590; found 238.1597. ${ }^{1} \mathrm{H}$ NMR (400 MHz, $\left.\mathrm{CDCl}_{3}\right) \delta 7.27-7.23(\mathrm{~m}, 2 \mathrm{H}), 7.11-7.08(\mathrm{~m}, 2 \mathrm{H}), 7.05-7.03(\mathrm{~m}$, 4H), 6.90 (t, $J=7.4 \mathrm{~Hz}, 1 \mathrm{H}), 5.36-5.31(\mathrm{~m}, 1 \mathrm{H}), 3.31(\mathrm{~d}, J=7.3 \mathrm{~Hz}, 2 \mathrm{H}), 1.75(\mathrm{~d}, J=10.9 \mathrm{~Hz}$, $6 \mathrm{H}) .{ }^{13} \mathrm{C}$ NMR $\left(101 \mathrm{MHz}, \mathrm{CDCl}_{3}\right) \delta 143.8,140.7,135.1,132.4,129.4,129.3,123.6,120.6,119.0$, $117.2,33.8,25.9,18.0$.

\subsection{7 (E)-4-(3,7-dimethylocta-2,6-dien-1-yl)-N-phenylaniline (29)}

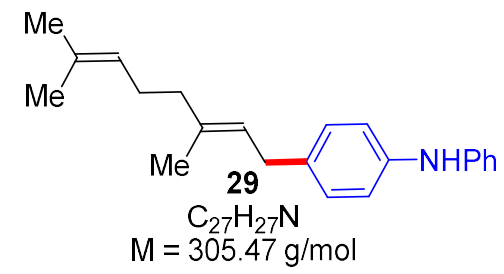

According to GP-A, using 1p (16.0 mg, $0.12 \mathrm{mmol}, 1.0$ equiv), 2a (30.0 mg, $0.18 \mathrm{mmol}, 1.5$ equiv), and tris(pentafluorophenyl)borane $\left(\mathrm{B}\left(\mathrm{C}_{6} \mathrm{~F}_{5}\right)_{3}\right)(6.0 \mathrm{mg}, 12 \mu \mathrm{mol}, 10 \mathrm{~mol} \%)$. Purification by column chromatography on silica gel using petroleum ether/DCM $(9: 1 \mathrm{v} / \mathrm{v})$ as eluent afforded the product 29 (20.0 mg, 55\%) as colorless oil. HRMS (ESI) $\mathrm{m} / \mathrm{z}$ : $\left[(\mathrm{M}+\mathrm{H})^{+}\right]$calcd for $\mathrm{C}_{27} \mathrm{H}_{28} \mathrm{~N}^{+} 306.2216$; found 306.2225. ${ }^{1} \mathrm{H}$ NMR (400 MHz, $\left.\mathrm{CDCl}_{3}\right)$ ס 7.26-7.22 (m, 2H), 7.11-7.08 (m, 2H), 7.04-7.00 (m, 4H), 6.91-6.87 (m, 1H), $5.62(\mathrm{~s}, 1 \mathrm{H}), 5.35-5.31(\mathrm{~m}, 1 \mathrm{H}), 5.18-5.11(\mathrm{~m}, 1 \mathrm{H}), 3.31(\mathrm{~d}, J=7.3 \mathrm{~Hz}, 2 \mathrm{H})$, 2.19-2.08 (m, 4H), $1.76(\mathrm{~d}, J=1.3 \mathrm{~Hz}, 3 \mathrm{H}), 1.70(\mathrm{~s}, 3 \mathrm{H}), 1.63(\mathrm{~s}, 3 \mathrm{H}) .{ }^{13} \mathrm{C} \mathrm{NMR}\left(101 \mathrm{MHz}, \mathrm{CDCl}_{3}\right)$ $\delta 144.0,140.8,136.1,135.0,131.9,129.4,129.3,124.4,124.3,120.5,118.9,117.1,33.5,32.1$, 26.8, 25.9, 23.6, 17.8. 

phenylbut-2-en-1-yl)-N-phenylaniline $\left(30+30^{\prime}\right)$

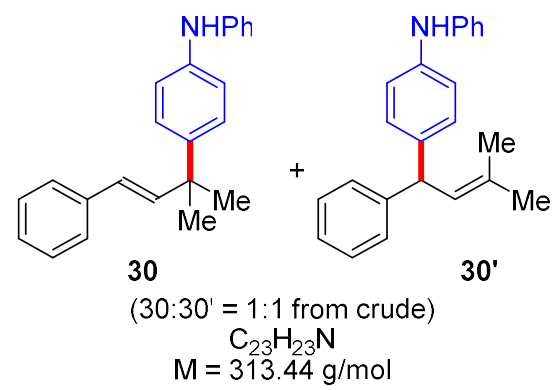

According to GP-A, using 1q (17.0 mg, $0.12 \mathrm{mmol}, 1.0$ equiv), 2a (30.0 mg, $0.18 \mathrm{mmol}, 1.5$ equiv), and tris(pentafluorophenyl)borane $\left(\mathrm{B}_{(}\left(\mathrm{C}_{6} \mathrm{~F}_{5}\right)_{3}\right)(6.0 \mathrm{mg}, 12 \mu \mathrm{mol}, 10 \mathrm{~mol} \%)$. Purification by column chromatography on silica gel using petroleum ether/DCM $(9: 1 \mathrm{v} / \mathrm{v})$ as eluent afforded the product $30+30^{\prime}(36.0 \mathrm{mg}, 95 \%)$ as colorless oil. HRMS (ESI) $\mathrm{m} / \mathrm{z}$ : $\left[(\mathrm{M}+\mathrm{H})^{+}\right]$calcd for $\mathrm{C}_{23} \mathrm{H}_{24} \mathrm{~N}^{+} 314.1903$; found 314.1905. ${ }^{1} \mathrm{H}$ NMR (400 MHz, $\left.\mathrm{CDCl}_{3}\right) \delta$ 7.41-7.38 (m, 2H), 7.33-7.17 (m, 14H), 7.11-7.02 $(\mathrm{m}, 10 \mathrm{H}), 6.94-6.90(\mathrm{~m}, 2 \mathrm{H}), 6.46-6.38(\mathrm{~m}, 2 \mathrm{H}), 5.64-5.60(\mathrm{~m}, 1 \mathrm{H}), 4.84(\mathrm{~d}, J=9.5 \mathrm{~Hz}, 1 \mathrm{H}), 1.80$ $(\mathrm{d}, J=1.5 \mathrm{~Hz}, 3 \mathrm{H}), 1.74(\mathrm{~d}, J=1.6 \mathrm{~Hz}, 3 \mathrm{H}), 1.51(\mathrm{~s}, 6 \mathrm{H}) \cdot{ }^{13} \mathrm{C}$ NMR $\left(101 \mathrm{MHz}, \mathrm{CDCl}_{3}\right) \delta 145.5$, $143.2,141.9,140.8,140.7,140.5,138.5,137.8,132.4,129.5,129.2,128.7,128.5,128.3,127.6$, $127.3,127.1,126.3,126.1,126.0,121.1$ (2C), 118.6, 118.2, 117.7 (2C), 48.9, 40.4, 28.9, 26.1, 18.2.

6.29 (E)-4-(3-methyl-1-phenylpent-1-en-3-yl)-N-phenylaniline and 4-(3-methyl-1phenylpent-2-en-1-yl)-N-phenylaniline (31 + 31')

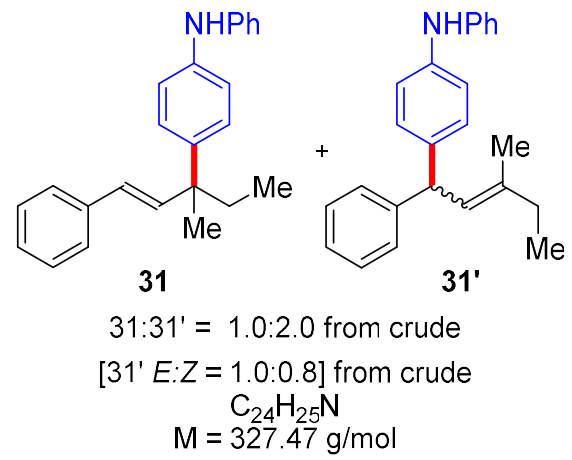

According to GP-A, using 1r (19.0 mg, $0.12 \mathrm{mmol}, 1.0$ equiv), 2a (30.0 mg, $0.18 \mathrm{mmol}, 1.5$ equiv), and tris(pentafluorophenyl)borane $\left(\mathrm{B}_{(}\left(\mathrm{C}_{6} \mathrm{~F}_{5}\right)_{3}\right)(6.0 \mathrm{mg}, 12 \mu \mathrm{mol}, 10 \mathrm{~mol} \%)$. Purification by column chromatography on silica gel using petroleum ether/DCM $(8: 2 \mathrm{v} / \mathrm{v})$ as eluent afforded the product $31+31 '(29.0 \mathrm{mg}, 75 \%)$ as colorless oil. HRMS (ESI) $\mathrm{m} / \mathrm{z}$ : $\left[(\mathrm{M}+\mathrm{H})^{+}\right]$calcd for $\mathrm{C}_{24} \mathrm{H}_{26} \mathrm{~N}^{+} 328.2060$; found 328.2068. ${ }^{1} \mathrm{H}$ NMR $\left(400 \mathrm{MHz}, \mathrm{CDCl}_{3}\right) \delta$ 7.42-7.39 (m, 2.33H), 7.33-7.17 (m, 26.25H), 
7.10-7.01 (m, 18.59H), 6.93-6.89 (m, 3.16H), $6.41(\mathrm{~d}, J=2.7 \mathrm{~Hz}, 2 \mathrm{H}), 5.63-5.60(\mathrm{~m}, 1.69 \mathrm{H})$, $5.57(\mathrm{~d}, J=9.9 \mathrm{~Hz}, 0.89 \mathrm{H}$ ), 4.86 (dd, $J=9.7,3.4 \mathrm{~Hz}, 2.58 \mathrm{H}$ ), 2.16 (q, $J=7.6 \mathrm{~Hz}, 1.82 \mathrm{H}$ ), 2.10 (q, $J=7.5 \mathrm{~Hz}, 3.64 \mathrm{H}), 1.95-1.83(\mathrm{~m}, 2 \mathrm{H}), 1.79(\mathrm{~d}, J=1.6 \mathrm{~Hz}, 2.57 \mathrm{H}), 1.72(\mathrm{~d}, J=1.6 \mathrm{~Hz}, 5.24 \mathrm{H})$, 1.45 (s, 3H), $1.06(\mathrm{t}, J=7.4 \mathrm{~Hz}, 5.32 \mathrm{H}), 0.99(\mathrm{t}, J=7.6 \mathrm{~Hz}, 2.55 \mathrm{H}), 0.85(\mathrm{t}, J=7.4 \mathrm{~Hz}, 3 \mathrm{H}) .{ }^{13} \mathrm{C}$ NMR $\left(101 \mathrm{MHz}, \mathrm{CDCl}_{3}\right) \delta 145.7,143.4,140.9,140.8,140.7,139.6,138.5,138.4,138.0,137.9$, 137.8, 129.4, 129.2, 128.7, 128.5, 128.4, 127.9, 127.1 (2C), 127.0, 126.3, 126.1 (2C), 120.9, $118.5,118.1,117.6,48.6,48.4,43.8,34.0,32.6,25.2,25.1,23.3,16.5,12.9,9.3$.

\subsection{4-(1,3-diphenylpent-2-en-1-yl)-N-phenylaniline (32)}

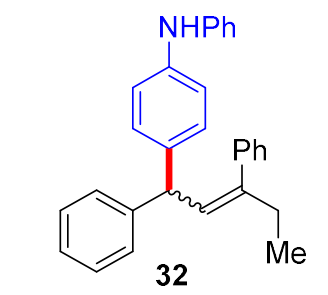

$[E: Z=1.0: 0.5]$ from crude

$$
\mathrm{M}=389.54 \mathrm{~g} / \mathrm{mol}
$$

According to GP-A, using $1 \mathrm{~s}$ ( $26.0 \mathrm{mg}, 0.12 \mathrm{mmol}, 1.0$ equiv), 2a (30.0 mg, $0.18 \mathrm{mmol}, 1.5$ equiv), and tris(pentafluorophenyl)borane $\left(\mathrm{B}_{(}\left(\mathrm{C}_{6} \mathrm{~F}_{5}\right)_{3}\right)(6.0 \mathrm{mg}, 12 \mu \mathrm{mol}, 10 \mathrm{~mol} \%)$. Purification by column chromatography on silica gel using petroleum ether/DCM $(8: 2 \mathrm{v} / \mathrm{v})$ as eluent afforded the product 32 (32.0 mg, 70\%) as colorless oil. HRMS (ESI) $\mathrm{m} / \mathrm{z}$ : $\left[(\mathrm{M}+\mathrm{H})^{+}\right]$calcd for $\mathrm{C}_{29} \mathrm{H}_{28} \mathrm{~N}^{+} 390.2216$; found 390.2149. ${ }^{1} \mathrm{H}$ NMR (400 MHz, $\left.\mathrm{CDCl}_{3}\right)$ ס 7.69-7.66 (m, 1.14H), 7.61-7.43 (m, 14.77H), 7.41-7.38 $(\mathrm{m}, 5.13 \mathrm{H}), 7.33-7.27(\mathrm{~m}, 4.47 \mathrm{H}), 7.18-7.14(\mathrm{~m}, 1.56 \mathrm{H}), 6.35(\mathrm{~d}, J=9.8 \mathrm{~Hz}, 0.58 \mathrm{H}), 6.15(\mathrm{dt}, J$ $=10.4,1.4 \mathrm{~Hz}, 1 \mathrm{H}), 5.92(\mathrm{~s}, 1 \mathrm{H}), 5.30(\mathrm{~d}, J=9.8 \mathrm{~Hz}, 0.56 \mathrm{H}), 4.86(\mathrm{~d}, J=10.5 \mathrm{~Hz}, 1 \mathrm{H}), 2.90(\mathrm{q}, J$ $=7.6 \mathrm{~Hz}, 1 \mathrm{H}), 2.72-2.66(\mathrm{~m}, 2 \mathrm{H}), 1.27(\mathrm{dt}, J=12.4,7.5 \mathrm{~Hz}, 4.62 \mathrm{H}) .{ }^{13} \mathrm{C} \mathrm{NMR}\left(101 \mathrm{MHz}, \mathrm{CDCl}_{3}\right)$ $\delta 145.5,145.2,143.5(2 \mathrm{C}), 143.1,142.7,141.9,141.3,141.2,141.1,138.0,137.7,130.6,129.4$, 129.3, 129.2, 128.6, 128.5, 128.4 (3C), 128.3, 128.2, 127.9, 127.0, 126.8, 126.7, 126.3, 126.1, 120.8 (2C), 118.3, 118.2, 117.5 (2C), 49.2, 49.0, 32.4, 23.4, 13.7, 13.1. 
6.31 (E)-4-(2-methyl-1,4-diphenylbut-3-en-2-yl)-N-phenylaniline and 4-(3-methyl-1,4diphenylbut-2-en-1-yl)-N-phenylaniline (33+33')

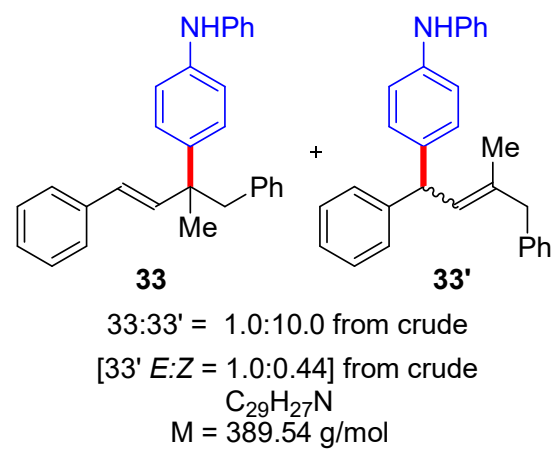

According to GP-A, using 1t (26.0 mg, $0.12 \mathrm{mmol}, 1.0$ equiv), 2a (30.0 mg, $0.18 \mathrm{mmol}, 1.5$ equiv), and tris(pentafluorophenyl)borane $\left(\mathrm{B}_{(}\left(\mathrm{C}_{6} \mathrm{~F}_{5}\right)_{3}\right)(6.0 \mathrm{mg}, 12 \mu \mathrm{mol}, 10 \mathrm{~mol} \%)$. Purification by column chromatography on silica gel using petroleum ether/DCM $(8: 2 \mathrm{v} / \mathrm{v})$ as eluent afforded the product 33 + 33' (37.0 mg, 80\%) as colorless oil. HRMS (ESI) $\mathrm{m} / \mathrm{z}$ : $\left[(\mathrm{M}+\mathrm{H})^{+}\right]$calcd for $\mathrm{C}_{29} \mathrm{H}_{28} \mathrm{~N}^{+} 390.2216$; found 390.2224. ${ }^{1} \mathrm{H}$ NMR $\left(400 \mathrm{MHz}, \mathrm{CDCl}_{3}\right) \delta$ 7.39-7.37 (m, 1H), 7.33-7.26 (m, 6H), 7.25-7.15 $(\mathrm{m}, 9 \mathrm{H}), 7.13-7.03(\mathrm{~m}, 7 \mathrm{H}), 6.96-6.89(\mathrm{~m}, 2 \mathrm{H}), 6.53(\mathrm{~d}, J=16.1 \mathrm{~Hz}, 0.26 \mathrm{H}), 6.35(\mathrm{~d}, J=16.3 \mathrm{~Hz}$, $0.27 \mathrm{H}), 5.81-5.77(\mathrm{~m}, 1.10 \mathrm{H}), 5.00(\mathrm{~d}, J=9.7 \mathrm{~Hz}, 0.10 \mathrm{H}), 4.88(\mathrm{~d}, J=9.5 \mathrm{~Hz}, 1 \mathrm{H}), 3.49(\mathrm{~s}, 0.20 \mathrm{H})$, $3.39(\mathrm{~s}, 2 \mathrm{H}), 3.14(\mathrm{~d}, J=3.1 \mathrm{~Hz}, 0.50 \mathrm{H}), 1.72(\mathrm{~d}, J=0.9 \mathrm{~Hz}, 0.31 \mathrm{H}), 1.65(\mathrm{~d}, J=0.9 \mathrm{~Hz}, 3 \mathrm{H}), 1.42$ (s, 1H). ${ }^{13} \mathrm{C}$ NMR $\left(101 \mathrm{MHz}, \mathrm{CDCl}_{3}\right) \delta 145.5,145.4,143.5,141.2$ (2C), 141.1, 140.8, 140.5, 140.2, 139.7, 139.4, 138.3, 138.0, 137.9 (2C), 135.2, 134.6, 130.9, 129.7, 129.5 (2C), 129.4, 129.3, 129.2, 129.1, 129.0, 128.8, 128.7, 128.6, 128.5 (2C), 128.4 (2C), 128.2 (2C), 128.1, 127.6, 127.2 (2C), 126.3, 126.2 (3C), 126.1, 125.9, 120.8, 118.4, 118.3, 118.1, 117.9, 117.5 (2C), 117.4, 48.9, $48.8,48.5,46.5,44.5,38.2,25.1,23.7,16.3$.

\subsection{N-phenyl-4-(1,3,4-triphenylbut-2-en-1-yl)aniline (34)}

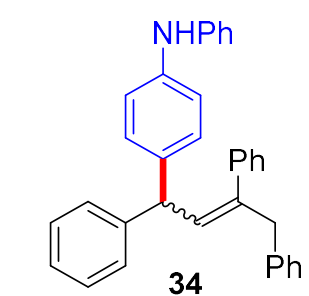

$[E: Z=3.0: 1.0]$ from crude
${ }^{C_{34} \mathrm{H}_{29} \mathrm{~N}}$
$M=451.61 \mathrm{~g} / \mathrm{mol}$

According to GP-A, using $1 \mathbf{u}(34.0 \mathrm{mg}, 0.12 \mathrm{mmol}, 1.0$ equiv), 2a (30.0 mg, $0.18 \mathrm{mmol}, 1.5$ equiv), and tris(pentafluorophenyl)borane $\left(\mathrm{B}_{(}\left(\mathrm{C}_{6} \mathrm{~F}_{5}\right)_{3}\right)(6.0 \mathrm{mg}, 12 \mu \mathrm{mol}, 10 \mathrm{~mol} \%)$. Purification by column chromatography on silica gel using petroleum ether/DCM $(8: 2 \mathrm{v} / \mathrm{v})$ as eluent afforded the product 
34 (38.0 mg, 70\%) as colorless oil. HRMS (ESI) m/z: $\left[(\mathrm{M}+\mathrm{H})^{+}\right]$calcd for $\mathrm{C}_{34} \mathrm{H}_{30} \mathrm{~N}^{+} 452.2373$; found 452.2381. ${ }^{1} \mathrm{H}$ NMR (400 MHz, DMSO-D 6 ) $\delta 8.11(\mathrm{~s}, 1 \mathrm{H}), 7.31-7.25(\mathrm{~m}, 4.80 \mathrm{H}), 7.24-7.16(\mathrm{~m}$, 7.18H), 7.13-7.09 (m, 5.34H), 7.07-7.02 (m, 5.33H), 7.01-6.96 (m, 3.65H), $6.78(\mathrm{t}, J=7.3 \mathrm{~Hz}$, $1.12 \mathrm{H}), 6.53(\mathrm{~d}, J=10.1 \mathrm{~Hz}, 0.12 \mathrm{H}), 6.16(\mathrm{~d}, J=10.6 \mathrm{~Hz}, 1 \mathrm{H}), 5.15(\mathrm{~d}, J=10.1 \mathrm{~Hz}, 0.12 \mathrm{H}), 4.55$ (d, $J=10.6 \mathrm{~Hz}, 1 \mathrm{H}), 4.03(\mathrm{~s}, 0.24 \mathrm{H}), 3.76(\mathrm{~s}, 2 \mathrm{H}) .{ }^{13} \mathrm{C}$ NMR $\left(101 \mathrm{MHz}, \mathrm{DMSO}_{-} \mathrm{D}_{6}\right) \delta$ 145.1, 143.6, 141.5, 139.6, 139.4, 139.3, 135.8, 130.9, 129.1, 128.7, 128.5, 128.4, 128.2, 128.1, 127.7, 127.0, $126.4,126.0(2 \mathrm{C}), 119.4,117.1,116.4,48.9,44.8$.

\section{$6.33 N$-phenyl-1',2',3',4'-tetrahydro-[1,1'-biphenyl]-2-amine (35)}

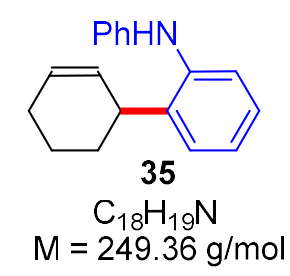

According to GP-A, using $1 \mathbf{v}$ ( $10.0 \mathrm{mg}, 0.12 \mathrm{mmol}, 1.0$ equiv), $2 \mathrm{a}$ ( $30.0 \mathrm{mg}, 0.18 \mathrm{mmol}, 1.5$ equiv), and tris(pentafluorophenyl)borane $\left(\mathrm{B}_{(}\left(\mathrm{C}_{6} \mathrm{~F}_{5}\right)_{3}\right)(6.0 \mathrm{mg}, 12 \mu \mathrm{mol}, 10 \mathrm{~mol} \%)$. Purification by column chromatography on silica gel using petroleum ether/DCM $(9: 1 \mathrm{v} / \mathrm{v})$ as eluent afforded the product 35 (18.0 mg, 60\%) as colorless oil. HRMS (ESI) $\mathrm{m} / \mathrm{z}$ : $\left[(\mathrm{M}+\mathrm{H})^{+}\right]$calcd for $\mathrm{C}_{18} \mathrm{H}_{20} \mathrm{~N}^{+} 250.1590$; found 250.1591. ${ }^{1} \mathrm{H}$ NMR $\left(400 \mathrm{MHz}, \mathrm{CDCl}_{3}\right)$ ס 7.30-7.27 (m, 1H), 7.26-7.21 (m, 3H), 7.18-7.14 (m 1H), 7.01-6.93 (m, 3H), $6.87(\mathrm{t}, J=7.3 \mathrm{~Hz}, 1 \mathrm{H}), 6.01-5.96(\mathrm{~m}, 1 \mathrm{H}), 5.76-5.72(\mathrm{~m}, 1 \mathrm{H}), 3.61-3.55(\mathrm{~m}$, 1H), 2.12-2.07 (m, 2H), 2.00-1.91 (m, 1H), 1.80-1.72 (m, 1H), 1.68-1.58 (m, 2H). ${ }^{13} \mathrm{C}$ NMR $(101$ $\left.\mathrm{MHz}, \mathrm{CDCl}_{3}\right) \delta 144.8,140.6,136.5,130.1,129.8,129.4,129.4,127.0,122.5,120.8,120.2,116.8$, 38.6, 29.7, 25.1, 21.6.

\subsection{5-methyl-N-(p-tolyl)-1',2',3',4'-tetrahydro-[1,1'-biphenyl]-2-amine (36)}

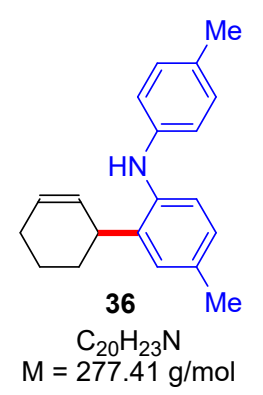

According to GP-A, using $1 \mathrm{v}$ (10.0 mg, $0.12 \mathrm{mmol}, 1.0$ equiv), $2 \mathrm{~m}$ (35.0 mg, $0.18 \mathrm{mmol}, 1.5$ equiv), and tris(pentafluorophenyl)borane $\left(\mathrm{B}\left(\mathrm{C}_{6} \mathrm{~F}_{5}\right)_{3}\right)(6.0 \mathrm{mg}, 12 \mu \mathrm{mol}, 10 \mathrm{~mol} \%)$. Purification by column chromatography on silica gel using petroleum ether/DCM $(9: 1 \mathrm{v} / \mathrm{v})$ as eluent afforded the 
product $36(20.0 \mathrm{mg}, 60 \%)$ as colorless oil. HRMS (ESI) $\mathrm{m} / \mathrm{z}$ : $\left[(\mathrm{M}+\mathrm{H})^{+}\right]$calcd for $\mathrm{C}_{20} \mathrm{H}_{24} \mathrm{~N}^{+}$ 278.1903; found 278.1916. ${ }^{1} \mathrm{H}$ NMR $\left(400 \mathrm{MHz}, \mathrm{CDCl}_{3}\right) \delta 7.15(\mathrm{~d}, J=8.0 \mathrm{~Hz}, 1 \mathrm{H}), 7.04-7.01(\mathrm{~m}$, $3 \mathrm{H}), 6.98-6.96(\mathrm{~m}, 1 \mathrm{H}), 6.82(\mathrm{~d}, J=8.1 \mathrm{~Hz}, 2 \mathrm{H}), 5.98-5.93(\mathrm{~m}, 1 \mathrm{H}), 5.74-5.70(\mathrm{~m}, 1 \mathrm{H}), 3.60-$ $3.54(\mathrm{~m}, 1 \mathrm{H}), 2.31(\mathrm{~s}, 3 \mathrm{H}), 2.28(\mathrm{~s}, 3 \mathrm{H}), 2.12-2.05(\mathrm{~m}, 2 \mathrm{H}), 1.98-1.91(\mathrm{~m}, 1 \mathrm{H}), 1.79-1.72(\mathrm{~m}, 1 \mathrm{H})$, 1.67-1.57 (m, 2H). ${ }^{13} \mathrm{C}$ NMR (101 MHz, $\left.\mathrm{CDCl}_{3}\right) \delta 142.9,138.4,136.8,131.9,130.3,130.2,129.9$, 129.1 (2C), 127.6, 121.3, 116.6, 38.4, 30.0, 25.1, 21.7, 21.0, 20.7.

\subsection{Gram scale synthesis}
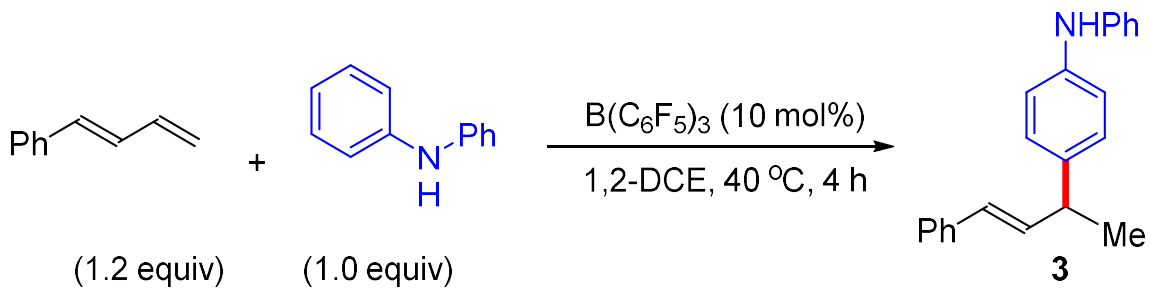

Gram scale synthesis has been done by using the GP-A, 1 a (550.0 mg, 4.22 mmol, 1.0 equiv), 2a (1057.11 mg, $6.33 \mathrm{mmol}, 1.5$ equiv), and tris(pentafluorophenyl)borane $\left(\mathrm{B}_{(}\left(\mathrm{C}_{6} \mathrm{~F}_{5}\right)_{3} 197.0\right.$ $\mathrm{mg}, 0.38 \mathrm{mmol}, 10.0 \mathrm{~mol} \%)$. Purification by column chromatography on silica gel using petroleum ether/DCM (9:1 v/v) as eluent afforded the product $3 \mathrm{a}(1.02 \mathrm{~g}, 81 \%)$ as colorless oil.

\subsection{Detection of the contact ion pair $2 \mathrm{aH}^{+} \mathrm{HOBCF}^{-}$and $\mathrm{H}_{2} \mathrm{O} \cdot \mathrm{BCF}$ adduct}

To identify the contact ion pair (in case of secondary amine) and $\mathrm{H}_{2} \mathrm{O}-\mathrm{BCF}$ adduct formation (in case of tertiary amine). Two separate experiments have been performed as follows-

(i) $\mathrm{BCF}+\mathrm{H}_{2} \mathrm{O}+2 \mathrm{a}\left(\mathrm{Ph}_{2} \mathrm{NH}\right)$ as 1:1:1 dissolved into DCE and recorded the ${ }^{19} \mathrm{~F},{ }^{11} \mathrm{~B}$ NMR with no solvent locking system in $400 \mathrm{MHz}$ JEOL JNM ECS400. ${ }^{1} \mathrm{H}$ NMR recorded in the $\mathrm{CDCl}_{3}$ solvent to check the shifting of $-\mathrm{OH}$ and $-\mathrm{NH}$ peaks. The peak for- $\mathrm{OH}$ and $-\mathrm{NH}$ is coming together at $5.67 \mathrm{ppm}$. The crystal for SC-XRD grown in DCE/n-pentane at room temperature for $12 \mathrm{~h}$.

${ }^{1} \mathrm{H}$ NMR $\left(400 \mathrm{MHz}, \mathrm{CDCl}_{3}\right)$ ठ 7.34-7.29 (m, 4H), 7.11-7.01 (m, 6H), $5.67(\mathrm{~s}, 3 \mathrm{H}) .{ }^{19} \mathrm{~F}$ NMR (376 MHz, NONE) $\delta-136.62,-160.17,-165.72 .{ }^{11} \mathrm{~B}$ NMR (128 MHz, NONE) $\delta-5.13$.

(ii) Similarly, for $\mathrm{BCF}+\mathrm{H}_{2} \mathrm{O}+2 \mathrm{~g}\left(\mathrm{Ph}_{3} \mathrm{~N}\right)$ recorded the ${ }^{19} \mathrm{~F},{ }^{11} \mathrm{~B}$ NMR to check the $\mathrm{H}_{2} \mathrm{O} \cdot \mathrm{BCF}$ adducts formation.

${ }^{19} \mathrm{~F}$ NMR (376 MHz, NONE) $\delta-136.08,-158.72,-165.42,{ }^{11} \mathrm{~B}$ NMR (128 MHz, NONE) $\delta$ 4.58 . 
Note- reference for spectral data of $\mathrm{BCF}-\mathrm{OH}$ and $\mathrm{BCF}-\mathrm{OH}_{2}{ }^{[\mathrm{S}]}$

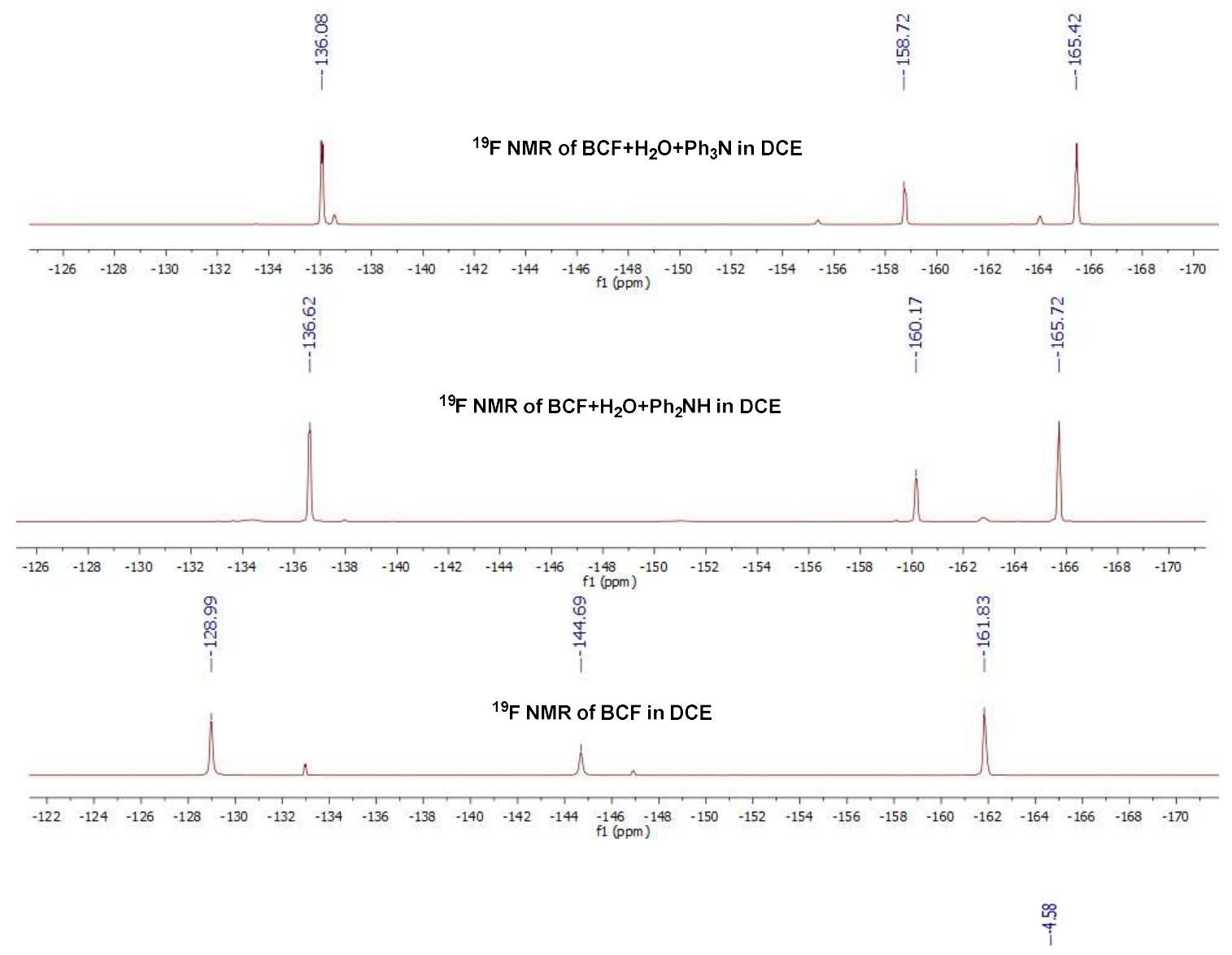

${ }^{11} \mathrm{~B} \mathrm{NMR}$ of $\mathrm{BCF}+\mathrm{H}_{2} \mathrm{O}+\mathrm{Ph}_{3} \mathrm{~N}$ in DCE
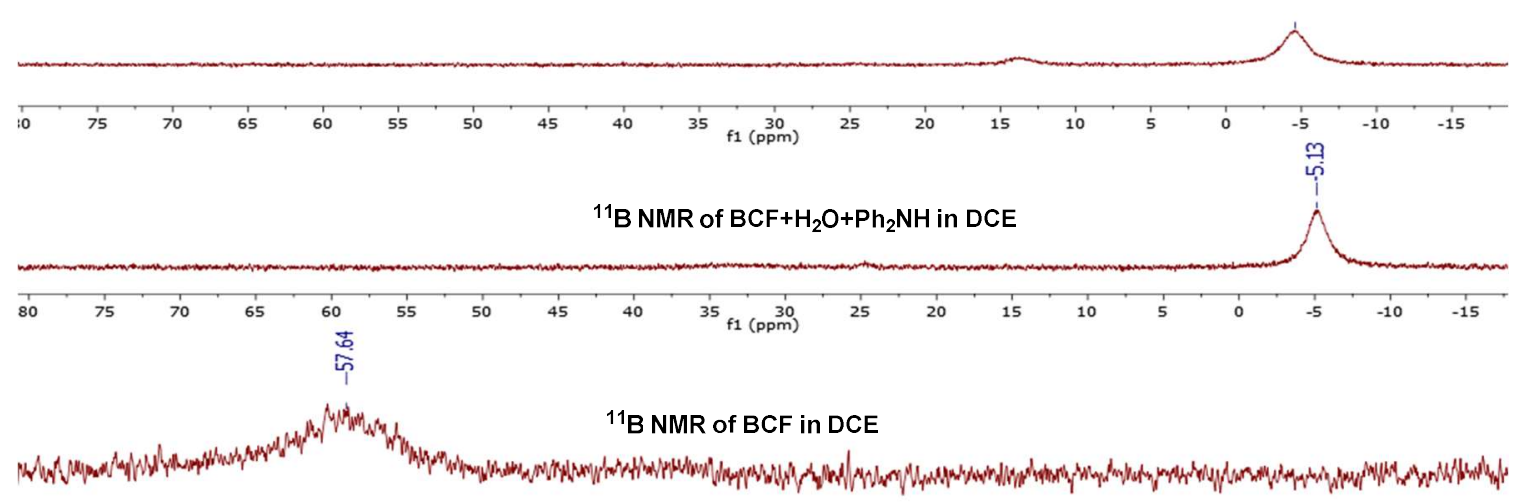

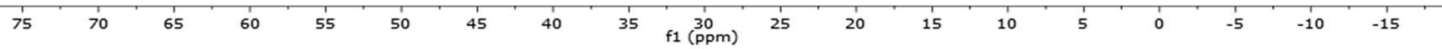



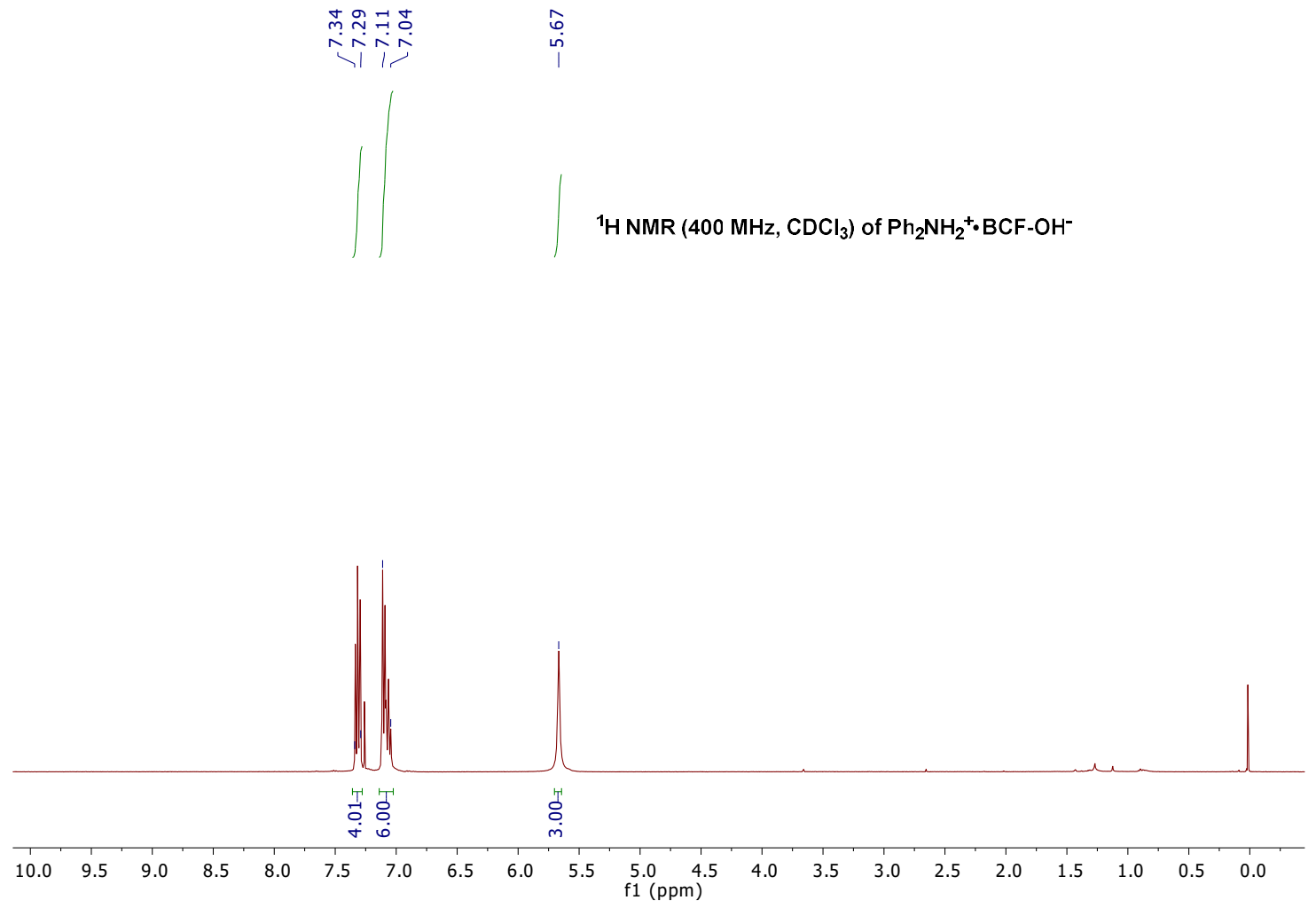

\subsection{Crystal Data and Structure Refinement for Complex $\left((2 \mathrm{a})_{2} \mathrm{H}^{+}(\mathrm{HOBCF})_{2} \mathrm{H}^{-}\right)$ $(\text { (CCDC2111194) })^{[S 10]}$}
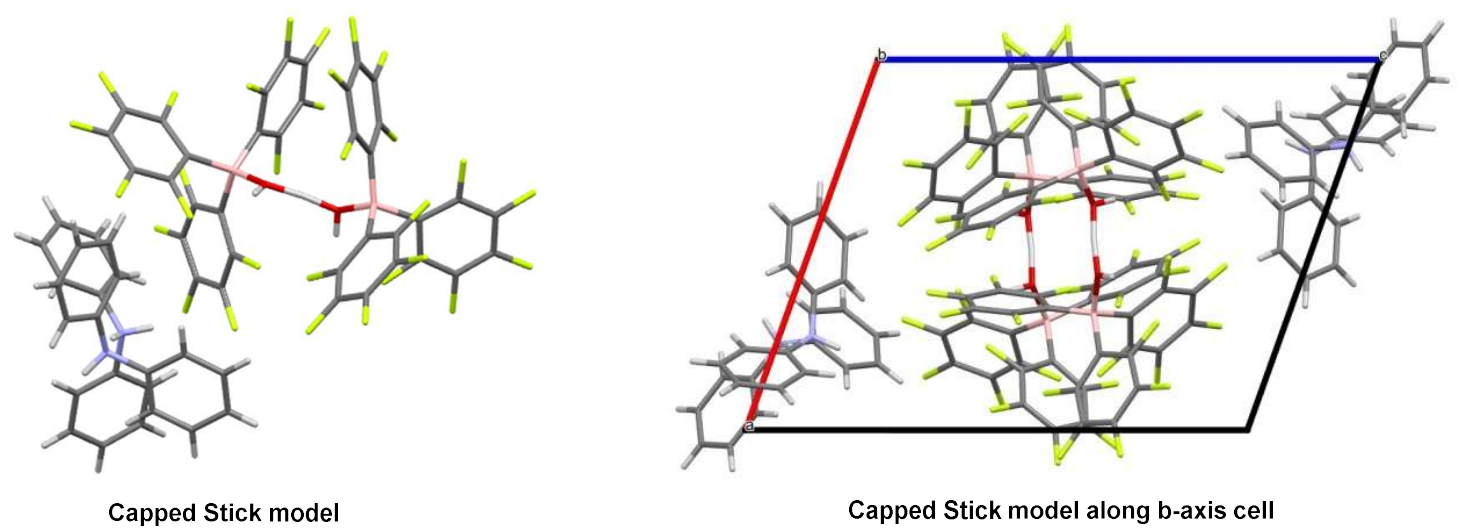

Atom color code: grey = carbon atom, white $=$ hydrogen atom, blue $=$ nitrogen atom, green $=$ fluorine, salmon $=$ boron atom and red $=$ oxygen atom. 


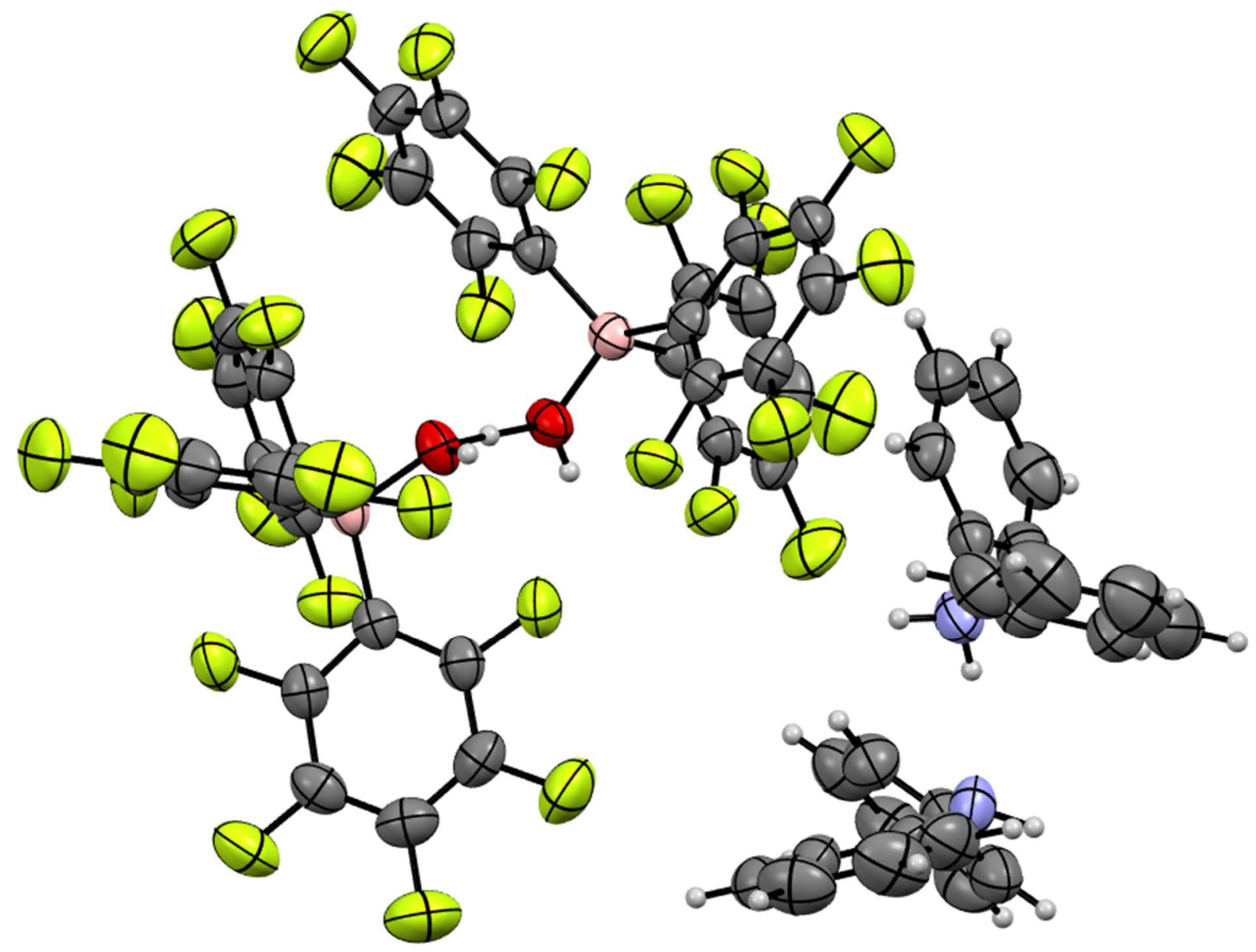

Figure S1 ORTEP drawing of $\left((2 \mathrm{a})_{2} \mathrm{H}^{+}(\mathrm{HOBCF})_{2} \mathrm{H}^{-}\right)$showing thermal ellipsoids at the $50 \%$ probability.

\begin{tabular}{|l|l|}
\hline \multicolumn{2}{|l|}{ Crystal data and structure refinement for Complex $\left((2 \mathrm{a})_{2} \mathrm{H}^{+}(\mathbf{H O B C F})_{2} \mathbf{H}^{-}\right)$(CCDC2111194) } \\
\hline Identification Code & icgk_03_409_0m-sr \\
\hline Ccdc no. & 2111194 \\
\hline Empirical formula & $\mathrm{C}_{30} \mathrm{H}_{13} \mathrm{BF}{ }_{15} \mathrm{NO}$ \\
\hline Formula weight & 699.22 \\
\hline Temperature/K & 273.15 \\
\hline Crystal system & triclinic \\
\hline Space group & $\mathrm{P}-1$ \\
\hline $\mathrm{a} / \AA$ & $12.9009(7)$ \\
\hline $\mathrm{b} / \AA$ & $14.2446(6)$ \\
\hline $\mathrm{c} / \AA$ & $16.8164(9)$ \\
\hline$\alpha / /^{\circ}$ & $102.2630(10)$ \\
\hline$\beta /{ }^{\circ}$ & $108.067(2)$ \\
\hline$\gamma^{\circ}$ & $95.055(2)$ \\
\hline &
\end{tabular}




\begin{tabular}{|l|l|}
\hline Volume $/ \AA^{3}$ & $2831.2(2)$ \\
\hline$Z$ & 4 \\
\hline$\rho_{\text {calcg }} / \mathrm{cm}^{3}$ & 1.640 \\
\hline$\mu / \mathrm{mm}^{-1}$ & 0.167 \\
\hline $\mathrm{F}(000)$ & 1392.0 \\
\hline Crystal size $/ \mathrm{mm}^{3}$ & $0.259 \times 0.215 \times 0.189$ \\
\hline Radiation & $\mathrm{MoK} \alpha(\lambda=0.71073)$ \\
\hline $2 \Theta$ range for data collection/ & \\
\hline Index ranges & 4.438 to 52.888 \\
\hline Reflections collected & $-16 \leq \mathrm{h} \leq 16,-17 \leq \mathrm{k} \leq 17,-21 \leq 1 \leq 21$ \\
\hline Independent reflections & 62353 \\
\hline Data/restraints/parameters & $11625\left[\mathrm{R}_{\text {int }}=0.0502, \mathrm{R}_{\text {sigma }}=0.0362\right]$ \\
\hline Goodness-of-fit on $\mathrm{F}^{2}$ & $11625 / 0 / 877$ \\
\hline Final R indexes $[\mathrm{I}>=2 \sigma(\mathrm{I})]$ & 1.082 \\
\hline Final $\mathrm{R}$ indexes [all data] & $\mathrm{R}_{1}=0.0436, \mathrm{wR}_{2}=0.1111$ \\
\hline Largest diff. peak/hole $/ \mathrm{e} \AA^{-3}$ & $\mathrm{R}_{1}=0.0704, \mathrm{wR}_{2}=0.1240$ \\
\hline & $0.32 /-0.22$ \\
\hline
\end{tabular}

\subsection{Effect of Water content}

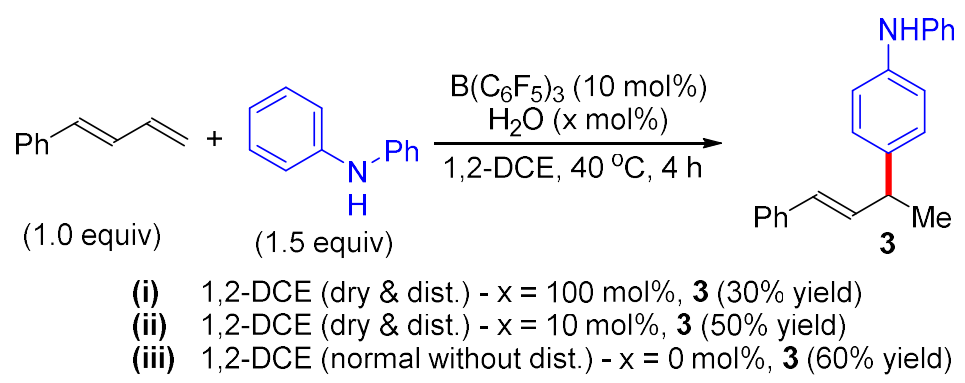

(i) Following modified (first $\mathrm{H}_{2} \mathrm{O}(2.2 \mathrm{mg}, 0.12 \mathrm{mmol}, 1.0$ equiv) taken into vial) GP-A, using 1a (16.0 mg, $0.12 \mathrm{mmol}, 1.0$ equiv), 2a (30.0 mg, $0.18 \mathrm{mmol}, 1.5$ equiv), and tris(pentafluorophenyl)borane $\left(\mathrm{B}_{(}\left(\mathrm{C}_{6} \mathrm{~F}_{5}\right)_{3}\right)(6.0 \mathrm{mg}, 12 \mu \mathrm{mol}, 10 \mathrm{~mol} \%)$. Purification by column chromatography on silica gel using petroleum ether/DCM $(9: 1 \mathrm{v} / \mathrm{v})$ as eluent afforded the product $3(11.0 \mathrm{mg}, 30 \%)$.

(ii) Following modified (first $\mathrm{H}_{2} \mathrm{O}(0.2 \mathrm{mg}, 12 \mu \mathrm{mmol}, 10 \mathrm{~mol} \%)$ taken into vial) GP-A, using 1a (16.0 mg, $0.12 \mathrm{mmol}, 1.0$ equiv), 2a (30.0 mg, $0.18 \mathrm{mmol}, 1.5$ equiv), and tris(pentafluorophenyl)borane $\left(\mathrm{B}_{(}\left(\mathrm{C}_{6} \mathrm{~F}_{5}\right)_{3}\right)(6.0 \mathrm{mg}, 12 \mu \mathrm{mol}, 10 \mathrm{~mol} \%)$. Purification by column chromatography on silica gel using petroleum ether/DCM $(9: 1 \mathrm{v} / \mathrm{v})$ as eluent afforded the product $3(18.0 \mathrm{mg}, 50 \%)$.

Note: $\mathrm{H}_{2} \mathrm{O}$ was used as:- $0.47 \mathrm{M}-\mathrm{H}_{2} \mathrm{O}$ solution in DCE, added $25 \mu \mathrm{l}\left(0.2 \mathrm{mg} \mathrm{H} \mathrm{H}_{2} \mathrm{O}\right)$. 
(iii) Following modified (the normal without distilled DCE has been used) GP-A, using 1a (16.0 mg, $0.12 \mathrm{mmol}, 1.0$ equiv), $2 \mathrm{a}(30.0 \mathrm{mg}, 0.18 \mathrm{mmol}, 1.5$ equiv), and tris(pentafluorophenyl)borane $\left.\left(\mathrm{B}_{(} \mathrm{C}_{6} \mathrm{~F}_{5}\right)_{3}\right)(6.0 \mathrm{mg}, 12 \mu \mathrm{mol}, 10 \mathrm{~mol} \%)$. Purification by column chromatography on silica gel using petroleum ether/DCM $(9: 1 \mathrm{v} / \mathrm{v})$ as eluent afforded the product $3(22.0 \mathrm{mg}, 60 \%)$.

\subsection{Control Experiment}

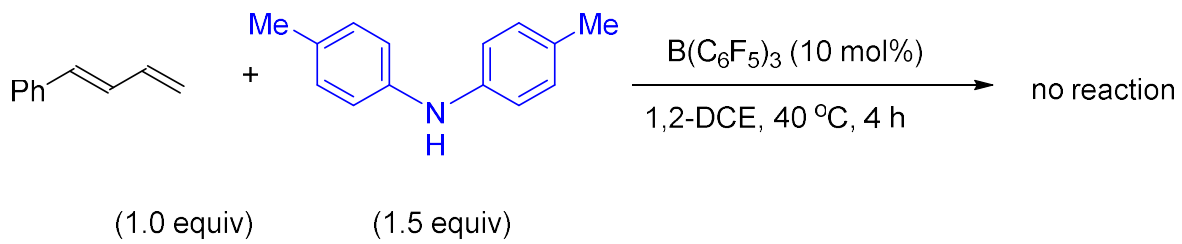

According to GP-A, using 1a (16.0 mg, $0.12 \mathrm{mmol}, 1.0$ equiv), $2 \mathrm{~m}(36.0 \mathrm{mg}, 0.18 \mathrm{mmol}, 1.5$ equiv), and tris(pentafluorophenyl)borane $\left(B\left(\mathrm{C}_{6} \mathrm{~F}_{5}\right)_{3}\right)(6.0 \mathrm{mg}, 12 \mu \mathrm{mol}, 10 \mathrm{~mol} \%)$. Product was not detected.

\subsection{Deuterium labeling}

\section{1 (E)-N-(4-(4-phenylbut-3-en-2-yl)phenyl-2,6- $\left.d_{2}\right)$ benzen-2,4,6- $d_{3}$-amine $\left(3-d_{5}\right)$}

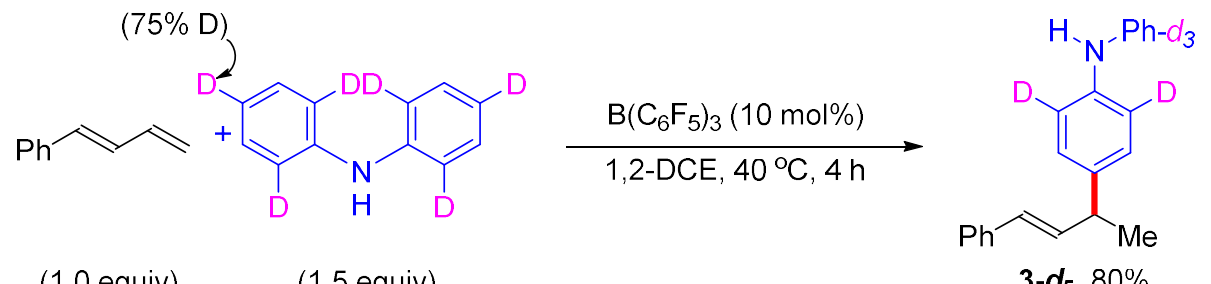

According to GP-A, using 1 a (16.0 mg, $0.12 \mathrm{mmol}, 1.0$ equiv), 2a- $\boldsymbol{d}_{6}(31.0 \mathrm{mg}, 0.18 \mathrm{mmol}, 1.5$ equiv), and tris(pentafluorophenyl)borane $\left(\mathrm{B}_{(}\left(\mathrm{C}_{6} \mathrm{~F}_{5}\right)_{3}\right)(6.0 \mathrm{mg}, 12 \mu \mathrm{mol}, 10 \mathrm{~mol} \%)$. Purification by column chromatography on silica gel using petroleum ether/DCM $(9: 1 \mathrm{v} / \mathrm{v})$ as eluent afforded the product 3- $\boldsymbol{d}_{\mathbf{5}}(22.0 \mathrm{mg}, 80 \%)$ as colorless oil. HRMS (ESI) $\mathrm{m} / \mathrm{z}$ : $\left[(\mathrm{M}+\mathrm{H})^{+}\right]$calcd for $\mathrm{C}_{22} \mathrm{H}_{17} \mathrm{D}_{5} \mathrm{~N}^{+}$ 305.2061; found 305.2047. ' $\mathrm{H}$ NMR $\left(400 \mathrm{MHz}, \mathrm{CDCl}_{3}\right) \delta$ 7.38-7.35 (m, 2H), 7.31-7.23 (m, 4H), 7.22-7.16 (m, 3H), 7.06-7.03 (m, 1H), $6.90(\mathrm{t}, J=7.3 \mathrm{~Hz}, 0.34 \mathrm{H}), 6.44-6.35(\mathrm{~m}, 2 \mathrm{H}), 5.66(\mathrm{~s}$, $1 \mathrm{H}), 3.63-3.57(\mathrm{~m}, 1 \mathrm{H}), 1.46(\mathrm{~d}, J=7.0 \mathrm{~Hz}, 3 \mathrm{H}) .{ }^{13} \mathrm{C}$ NMR $\left(101 \mathrm{MHz}, \mathrm{CDCl}_{3}\right) \delta 143.6,143.5$, 141.2, 141.1, 138.5, 137.7, 135.7, 129.4, 129.3, 129.2, 128.6, 128.3, 128.2 (2C), 127.1, 126.3, 120.7, 118.5, 117.3, 42.0,41.9, 21.4 . 


\section{2 (E)-N-phenyl-4-(4-phenylbut-3-en-2-yl)aniline-d (3- $\left.d_{1}\right)$}

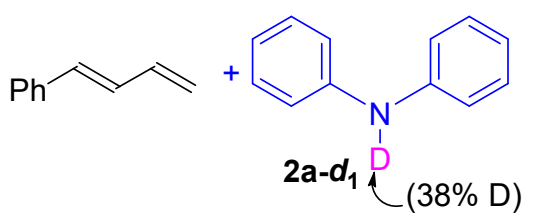

(1.0 equiv)

(1.5 equiv)

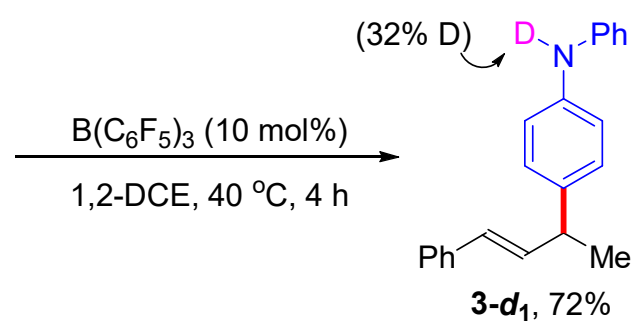

According to GP-A, using 1a (16.0 mg, $0.12 \mathrm{mmol}, 1.0$ equiv), 2a- $\boldsymbol{d}_{1}$ (30.0 mg, $0.18 \mathrm{mmol}, 1.5$ equiv), and tris(pentafluorophenyl)borane $\left(\mathrm{B}_{(}\left(\mathrm{C}_{6} \mathrm{~F}_{5}\right)_{3}\right)(6.0 \mathrm{mg}, 12 \mu \mathrm{mol}, 10 \mathrm{~mol} \%)$. Purification by column chromatography on silica gel using petroleum ether/DCM $(9: 1 \mathrm{v} / \mathrm{v})$ as eluent afforded the product (3- $\left.\boldsymbol{d}_{1}\right)(26.0 \mathrm{mg}, 72 \%)$ as colorless oil. HRMS (ESI) $\mathrm{m} / \mathrm{z}$ : $\left[(\mathrm{M}+\mathrm{H})^{+}\right]$calcd for $\mathrm{C}_{22} \mathrm{H}_{21} \mathrm{D}_{1} \mathrm{~N}^{+}$ 301.1810; found 301.1803. 1H NMR (400 MHz, $\left.\mathrm{CDCl}_{3}\right) \delta 7.37-7.34(\mathrm{~m}, 2 \mathrm{H}), 7.30-7.22(\mathrm{~m}, 4 \mathrm{H})$, 7.20-7.15 (m, 3H), 7.05-7.02 (m, 4H), 6.89-6.87 (m, 1H), 6.43-6.33 (m, 2H), $5.66(\mathrm{~s}, 0.68 \mathrm{H})$, $3.59(\mathrm{~m}, 1 \mathrm{H}), 1.45(\mathrm{~d}, J=6.9 \mathrm{~Hz}, 3 \mathrm{H}) .{ }^{13} \mathrm{C}$ NMR $\left(101 \mathrm{MHz}, \mathrm{CDCl}_{3}\right) \delta 143.7,141.2,138.6,137.7$, $135.7,129.4$, 128.6, 128.4, 128.3, 127.1, 126.3, 120.7, 118.6, 117.4, 42.0, 21.4.

\section{$11.3(E)-N-\left(4-(4-p h e n y l b u t-3-e n-2-y l-1-d)\right.$ phenyl-2,6- $\left.d_{2}\right)$ benzen-2,3,4,5,6- $d_{5}$-amine-d (3- $\left.d_{7}\right)$}

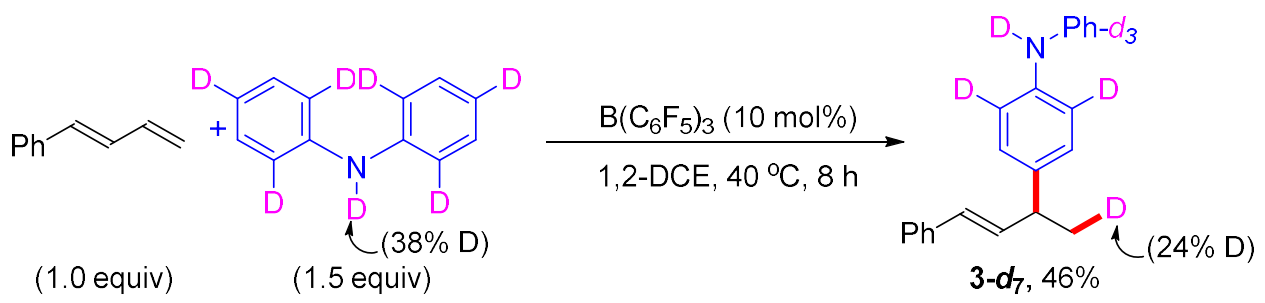

According to GP-A, using 1a (16.0 mg, $0.12 \mathrm{mmol}, 1.0$ equiv), 2a- $\mathbf{d}_{\mathbf{7}}$ (31.0 $\mathrm{mg}, 0.18 \mathrm{mmol}, 1.5$ equiv), and tris(pentafluorophenyl)borane $\left(\mathrm{B}_{(}\left(\mathrm{C}_{6} \mathrm{~F}_{5}\right)_{3}\right)(6.0 \mathrm{mg}, 12 \mu \mathrm{mol}, 10 \mathrm{~mol} \%)$ stirred for $8 \mathrm{~h}$. Purification by column chromatography on silica gel using petroleum ether/DCM $(9: 1 \mathrm{v} / \mathrm{v})$ as eluent afforded the product $\left(3-d_{7}\right)(17.0 \mathrm{mg}, 46 \%)$ as colorless oil. HRMS (ESI) $m / z:\left[(M+H)^{+}\right]$: calcd for $\mathrm{C}_{22} \mathrm{H}_{15} \mathrm{D}_{7} \mathrm{~N}^{+} 307.2187$; found 307.2206. ${ }^{1} \mathrm{H} \mathrm{NMR}\left(400 \mathrm{MHz}, \mathrm{CDCl}_{3}\right) \delta 7.38-7.35(\mathrm{~m}, 2 \mathrm{H})$, 7.31-7.23 (m, 4H), 7.22-7.17 (m, 3H), $7.05(\mathrm{~d}, J=8.7 \mathrm{~Hz}, 0.89 \mathrm{H}), 6.90(\mathrm{t}, J=7.4 \mathrm{~Hz}, 0.21 \mathrm{H})$, 6.45-6.35 (m, 2H), $5.66(\mathrm{~s}, 0.62 \mathrm{H}), 3.64-3.57(\mathrm{~m}, 1 \mathrm{H}), 1.45(\mathrm{t}, \mathrm{J}=6.8 \mathrm{~Hz}, 2.76 \mathrm{H}) .{ }^{13} \mathrm{C}$ NMR $(101$ $\left.\mathrm{MHz}_{\mathrm{CDCl}}\right) \delta 143.6,143.5,141.2,141.1,138.5,137.8,135.7,129.3,129.2,128.6,128.4,128.3$, 
128.2, 127.1, 126.3, 120.7, 118.5, 118.2, 117.9, 117.4, 117.3, 116.8, 42.0, 41.9, 21.4, 21.2, 21.1, 20.9.

Note: $\mathbf{2 a}-\boldsymbol{d}_{7}$ has been synthesized by following procedure ${ }^{[\mathrm{S13a}]}$ using the $\mathbf{2 a}-\boldsymbol{d}_{6} .92 \%$ yield, yellowish solid. Deuterium labeling measured by ${ }^{1} \mathrm{H}$ NMR.

11.4 (E)-N-methyl-N-(4-(4-phenylbut-3-en-2-yl-1-d)phenyl-2,6- $\left.d_{2}\right)$ aniline-2,4,6- $d_{3}$ and $(E)-$ $N$-methyl-N-(4-(1-phenylbut-2-en-1-yl-4-d)phenyl-2,6- $\left.d_{2}\right)$ aniline-2,4,6- $d_{3}(10-d+10$ '-d)

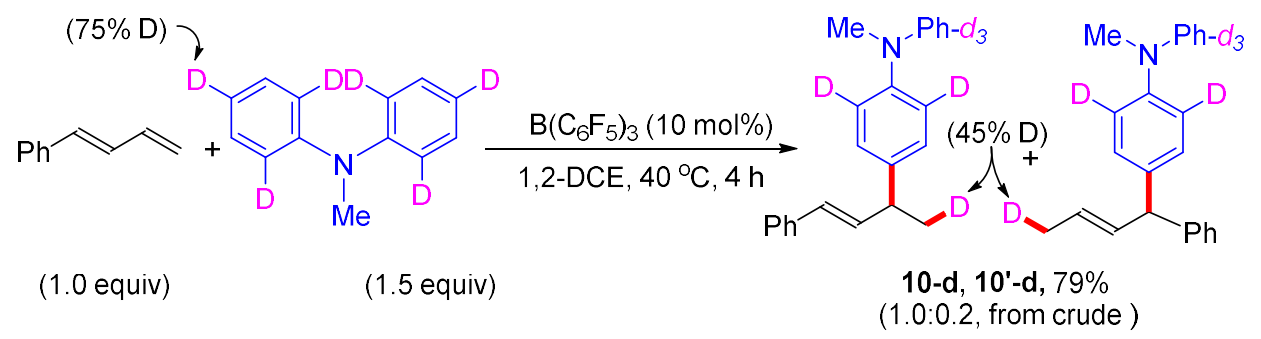

According to GP-A, using 1 a (16.0 mg, $0.12 \mathrm{mmol}, 1.0$ equiv), 2h- $\boldsymbol{d}_{6}$ (34.0 mg, $0.18 \mathrm{mmol}, 1.5$ equiv), and tris(pentafluorophenyl)borane $\left(\mathrm{B}_{(}\left(\mathrm{C}_{6} \mathrm{~F}_{5}\right)_{3}\right)(6.0 \mathrm{mg}, 12 \mu \mathrm{mol}, 10 \mathrm{~mol} \%)$. Purification by column chromatography on silica gel using petroleum ether/DCM $(9: 1 \mathrm{v} / \mathrm{v})$ as eluent afforded the product $10-d+10^{\prime}-d(30.0 \mathrm{mg}, 79 \%)$ as colorless oil. HRMS (ESI) $\mathrm{m} / \mathrm{z}$ : $\left[(\mathrm{M}+\mathrm{H})^{+}\right]$calcd for $\mathrm{C}_{23} \mathrm{H}_{18} \mathrm{D}_{6} \mathrm{~N}^{+} 320.2280$; found $320.2190 .{ }^{1} \mathrm{H}$ NMR $\left(400 \mathrm{MHz}, \mathrm{CDCl}_{3}\right) \delta 7.39-7.36(\mathrm{~m}, 2 \mathrm{H}), 7.32-$ $7.18(\mathrm{~m}, 7 \mathrm{H}), 7.03-6.98(\mathrm{~m}, 0.92 \mathrm{H}), 6.91(\mathrm{t}, \mathrm{J}=7.3 \mathrm{~Hz}, 0.21 \mathrm{H}), 6.46-6.36(\mathrm{~m}, 2 \mathrm{H}), 3.65-3.58(\mathrm{~m}$, $1 \mathrm{H}), 3.31(\mathrm{~s}, 3 \mathrm{H}), 1.46(\mathrm{t}, J=6.8 \mathrm{~Hz}, 2.55 \mathrm{H}) .{ }^{13} \mathrm{C}$ NMR $\delta$ 149.2, 149.1, $147.2(2 \mathrm{C}), 139.1,137.7$, 135.6, 129.1, 129.0, 128.6, 128.3, 128.1, 128.0, 127.1, 126.2, 121.6, 121.2, 121.0, 120.5, 120.3, 120.0, 119.4, 119.0, 118.8, 42.0 (2C), 40.4, 21.3, 21.2, 21.0, 20.8.

Note: $\mathbf{2} \mathbf{h}-\boldsymbol{d}_{\mathbf{6}}$ has been synthesized by following procedure ${ }^{[\mathrm{S13b]}}$ using the $\mathbf{2 a}-\boldsymbol{d}_{6} .90 \%$ yield, colorless liquid. Deuterium labeling measured by ${ }^{1} \mathrm{H}$ NMR. 


\subsection{Independent Kinetic isotope exchange (KIE) study}

Procedure: Following GP-A, eight sets of reactions was setup and stopped at 30, 60, 90, 120, $150,180,210$, and 240 minutes. The products have been isolated to calculate the yields at particular time. All the reactions repeated three times to minimize the error and average taken, graph plotted for yield versus time in excel. These experiments performed for non-deuterated and deuterated secondary as well as tertiary amines independently. And the $K_{H} / K_{D}$ ratio has been calculated.

\subsection{Independent Kinetic isotope exchange (KIE) study for secondary amine}

According to GP-A, using 1a (16.0 mg, $0.12 \mathrm{mmol}, 1.0$ equiv), 2a (30.0 mg, $0.18 \mathrm{mmol}, 1.5$ equiv), and tris(pentafluorophenyl)borane $\left(\mathrm{B}_{(}\left(\mathrm{C}_{6} \mathrm{~F}_{5}\right)_{3}\right)(6.0 \mathrm{mg}, 12 \mu \mathrm{mol}, 10 \mathrm{~mol} \%)$. Total eight sets of reactions performed each one has stopped at the particular time as $30,60,90,120,150,180$, 210, and 240 minutes. Purification by column chromatography on silica gel using petroleum ether/DCM (9:1 v/v) as eluent afforded the product 3 (Scheme i). Similarly, using $1 \mathrm{a}$ (16.0 mg, $0.12 \mathrm{mmol}, 1.0$ equiv), $2 \mathrm{a}-\boldsymbol{d}_{7}(31.0 \mathrm{mg}, 0.18 \mathrm{mmol}, 1.5$ equiv), and tris(pentafluorophenyl)borane $\left(\mathrm{B}\left(\mathrm{C}_{6} \mathrm{~F}_{5}\right)_{3}\right)(6.0 \mathrm{mg}, 12 \mu \mathrm{mol}, 10 \mathrm{~mol} \%)$. Total eight sets of reactions performed each one has stopped at the particular time as 30,60, 90, 120, 150, 180, 210, and 240 minutes. Purification by column chromatography on silica gel using petroleum ether/DCM $(9: 1 \mathrm{v} / \mathrm{v})$ as eluent afforded the product $\left(3-d_{7}\right)$ (Scheme ii). Hence the $K_{H} / K_{D}=2.81$.

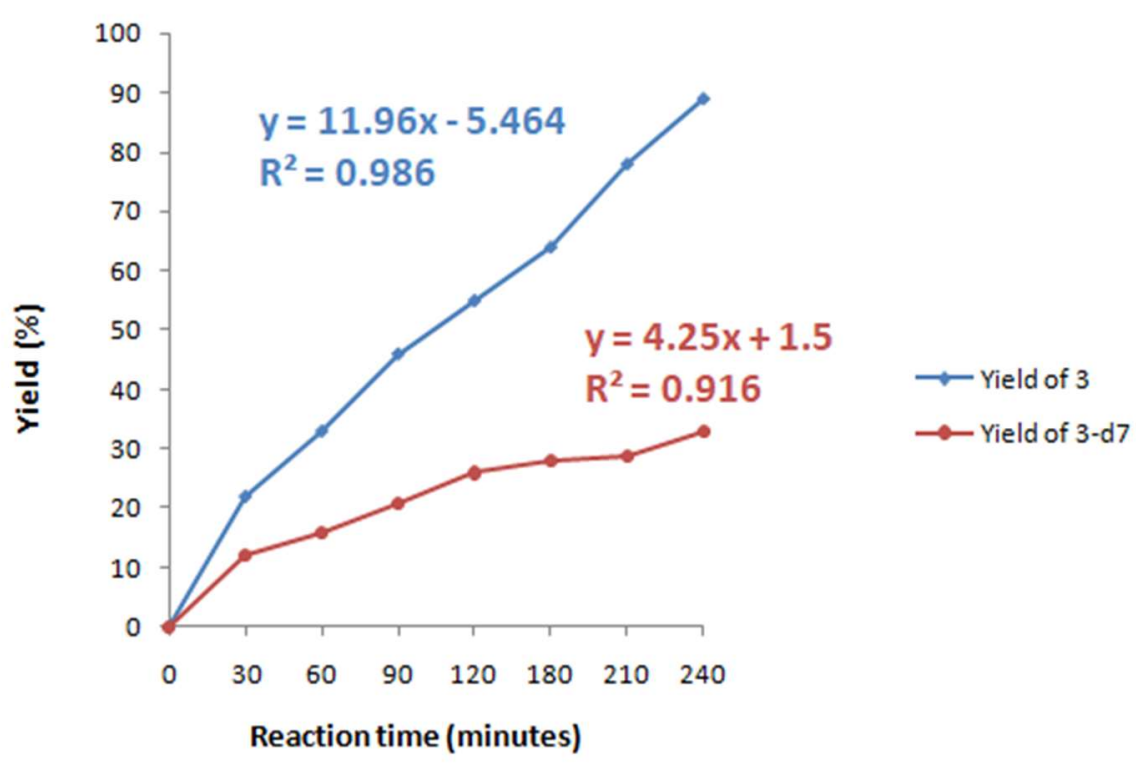




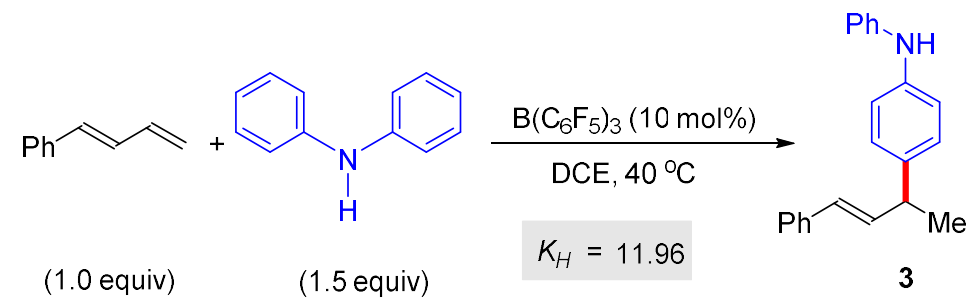

(i)
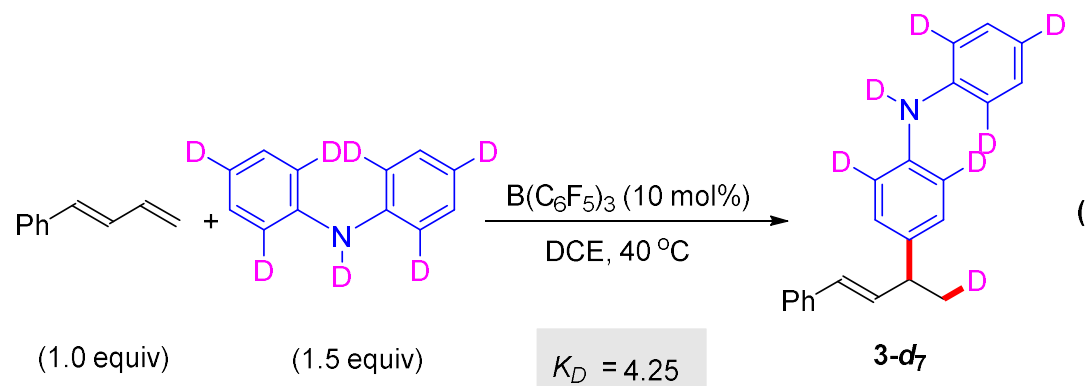

(1.0 equiv)

(1.5 equiv)

$$
K_{D}=4.25
$$

(ii)

$$
\frac{K_{H}}{K_{D}}=2.81
$$

\subsection{Independent Kinetic isotope exchange (KIE) study for tertiary amine}

According to GP-A, using 1a (16.0 mg, $0.12 \mathrm{mmol}, 1.0$ equiv), $2 \mathrm{~h}$ (33.0 mg, $0.18 \mathrm{mmol}, 1.5$ equiv), and tris(pentafluorophenyl)borane $\left(\mathrm{B}_{(}\left(\mathrm{C}_{6} \mathrm{~F}_{5}\right)_{3}\right)(6.0 \mathrm{mg}, 12 \mu \mathrm{mol}, 10 \mathrm{~mol} \%)$. Total eight sets of reactions performed each one has stopped at the particular time as $30,60,90,120,150,180$, 210, and 240 minutes. Purification by column chromatography on silica gel using petroleum ether/DCM (8:2 v/v) as eluent afforded the product $10+10^{\prime}$ (Scheme ii). Similiarly, using 1a (16.0 $\mathrm{mg}, \quad 0.12 \mathrm{mmol}, \quad 1.0$ equiv), $\mathbf{2} \mathbf{h}-\boldsymbol{d}_{6} \quad(34.0 \mathrm{mg}, \quad 0.18 \mathrm{mmol}, 1.5$ equiv), and tris(pentafluorophenyl)borane $\left(\mathrm{B}\left(\mathrm{C}_{6} \mathrm{~F}_{5}\right)_{3}\right)(6.0 \mathrm{mg}, 12 \mu \mathrm{mol}, 10 \mathrm{~mol} \%)$. Purification by column chromatography on silica gel using petroleum ether/DCM $(9: 1 \mathrm{v} / \mathrm{v})$ as eluent afforded the product 10-d + 10'-d (Scheme iv). Hence the $K_{H} / K_{D}=1.12$. 

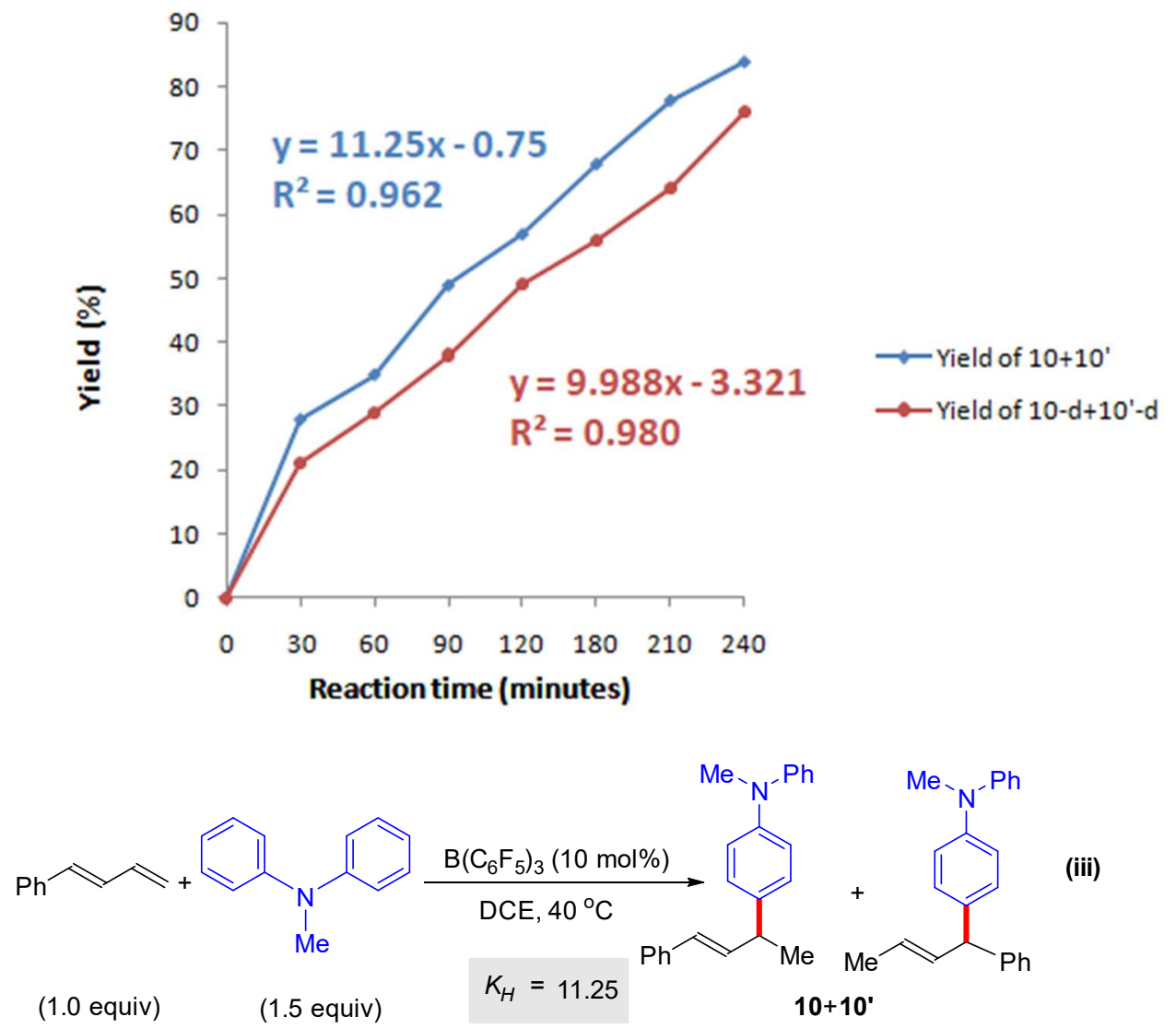<smiles>[2H]CC(=O)O[Ga]O[Na]</smiles>

$$
\frac{K_{H}}{K_{D}}=1.12
$$




\subsection{NMR Spectra}

(E)-N-phenyl-4-(4-phenylbut-3-en-2-yl)aniline (3)
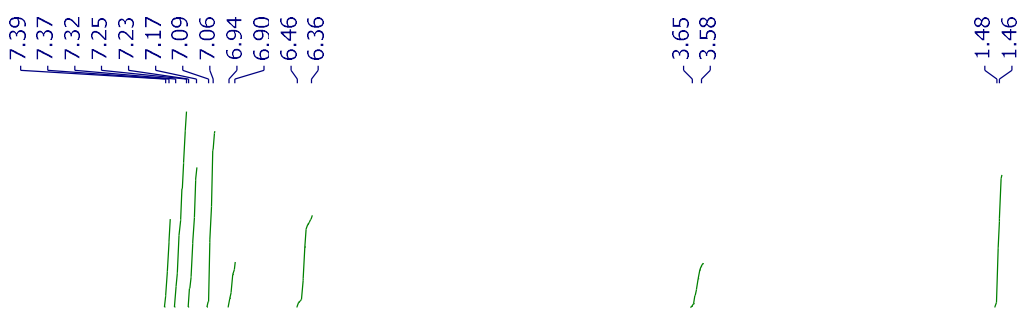

${ }^{1} \mathrm{H}$ NMR $\left(400 \mathrm{MHz}, \mathrm{CDCl}_{3}\right)$<smiles>CC(/C=C/c1ccccc1)c1ccc(Nc2ccccc2)cc1</smiles>

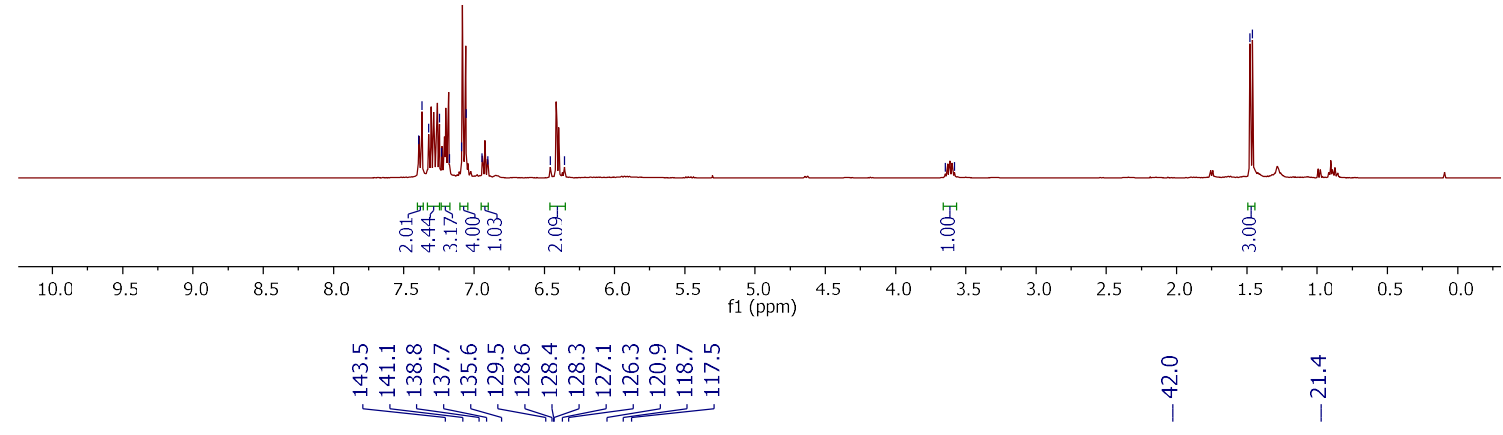

${ }^{13} \mathrm{C} \mathrm{NMR}\left(101 \mathrm{MHz}, \mathrm{CDCl}_{3}\right)$<smiles>CC(/C=C/c1ccccc1)c1ccc(Nc2ccccc2)cc1</smiles>

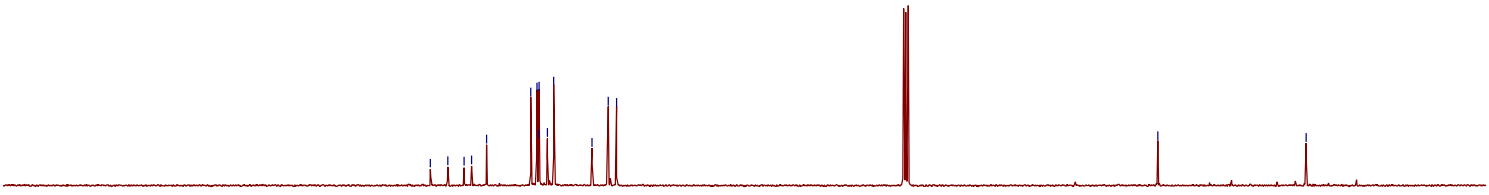

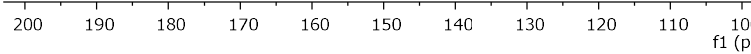


(E)-N-(4-(4-phenylbut-3-en-2-yl)phenyl)naphthalen-1-amine (4)
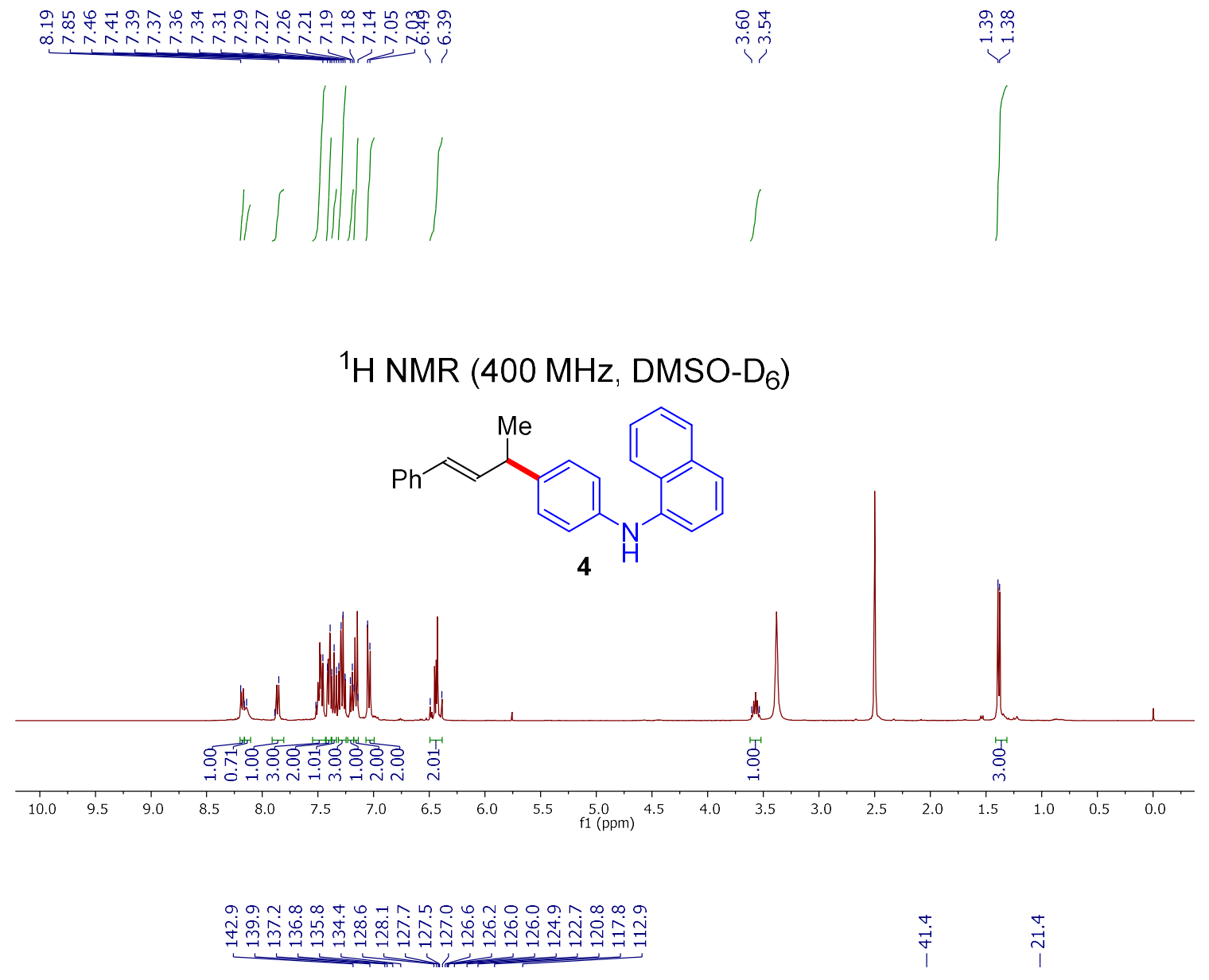

${ }^{13} \mathrm{C}$ NMR $\left(101 \mathrm{MHz}, \mathrm{DMSO}-\mathrm{D}_{6}\right)$<smiles>CC(/C=C/c1ccccc1)c1ccc(Nc2cccc3ccccc23)cc1</smiles>

|

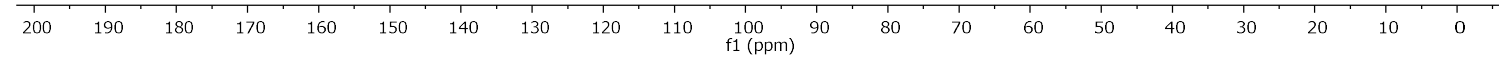


(E)-3-(4-phenylbut-3-en-2-yl)-9H-carbazole (5)

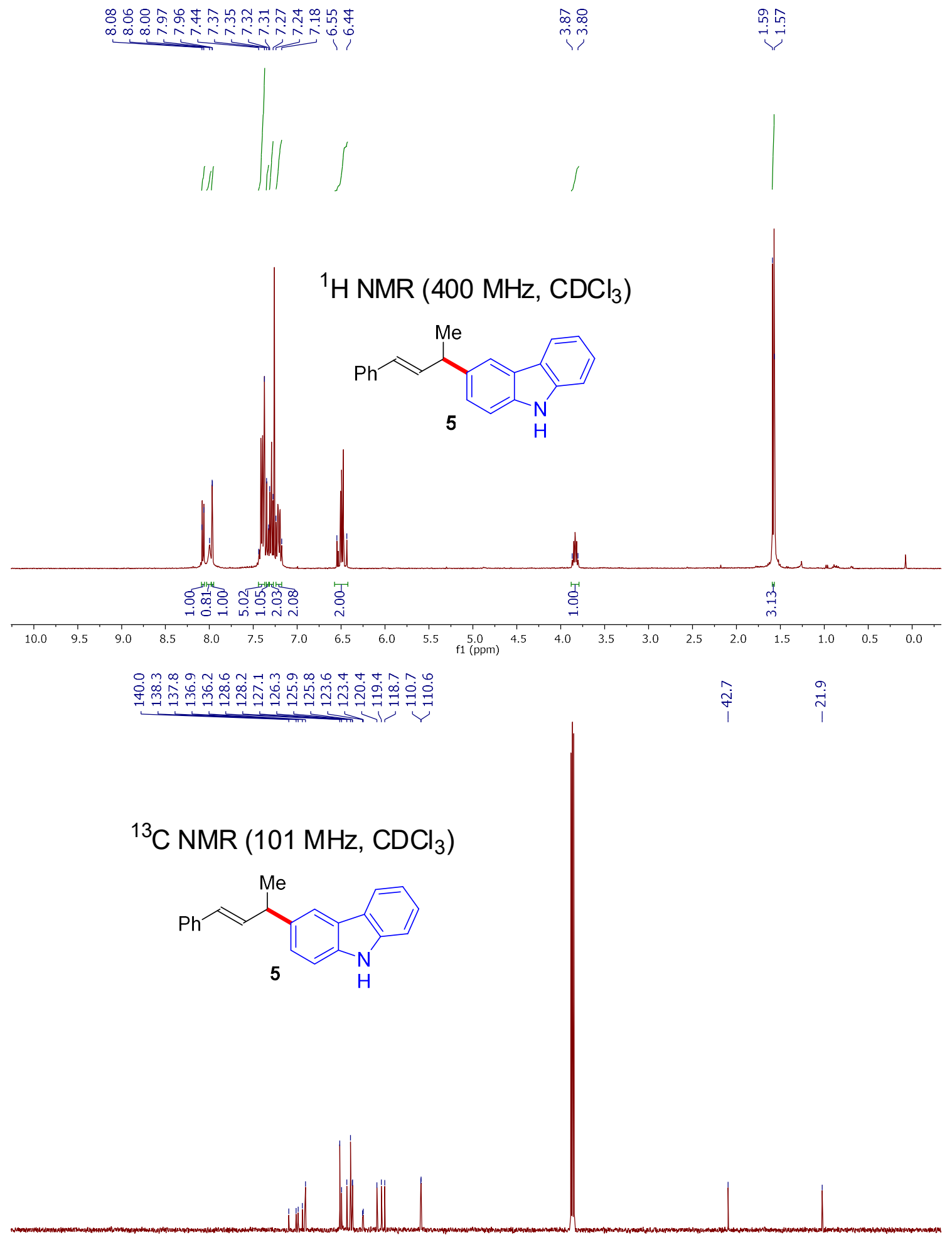


(E)-2-methyl-3-(4-phenylbut-3-en-2-yl)-1 $H$-indole (6)
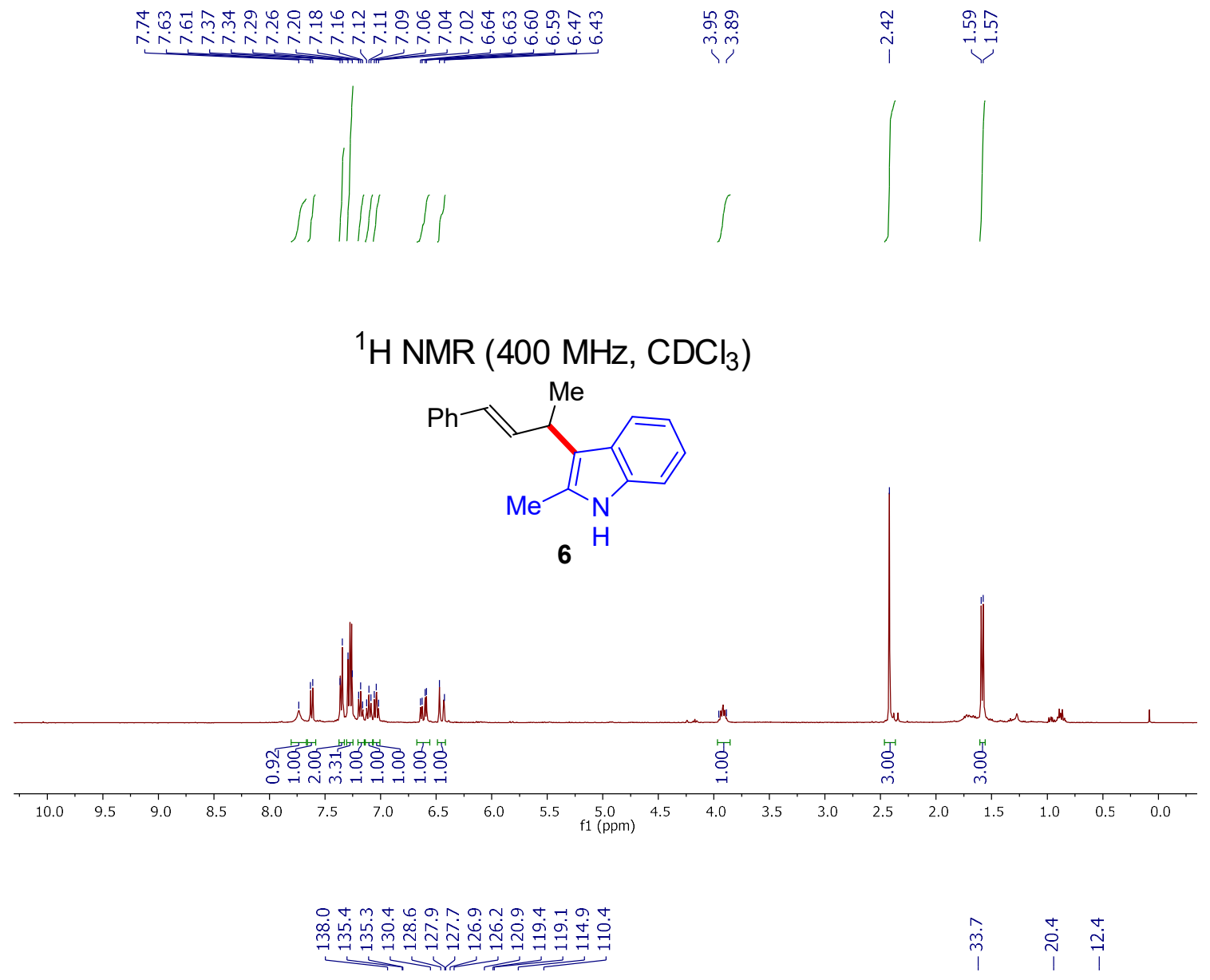

${ }^{13} \mathrm{C} \mathrm{NMR}\left(101 \mathrm{MHz}, \mathrm{CDCl}_{3}\right)$
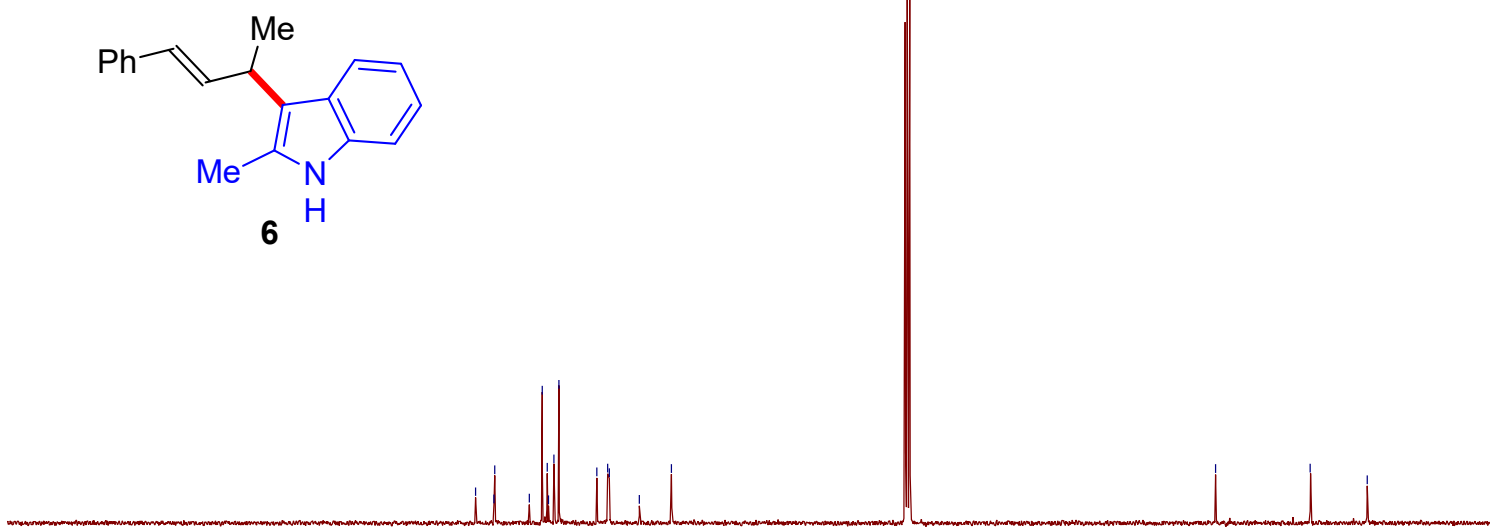

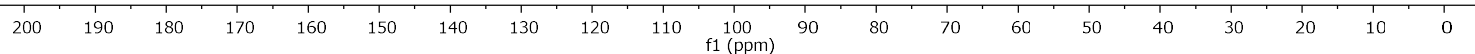


(E)-N-isopropyl-4-(4-phenylbut-3-en-2-yl)aniline and (E)-N-isopropyl-4-(1phenylbut-2-en-1-yl)aniline (7+7')

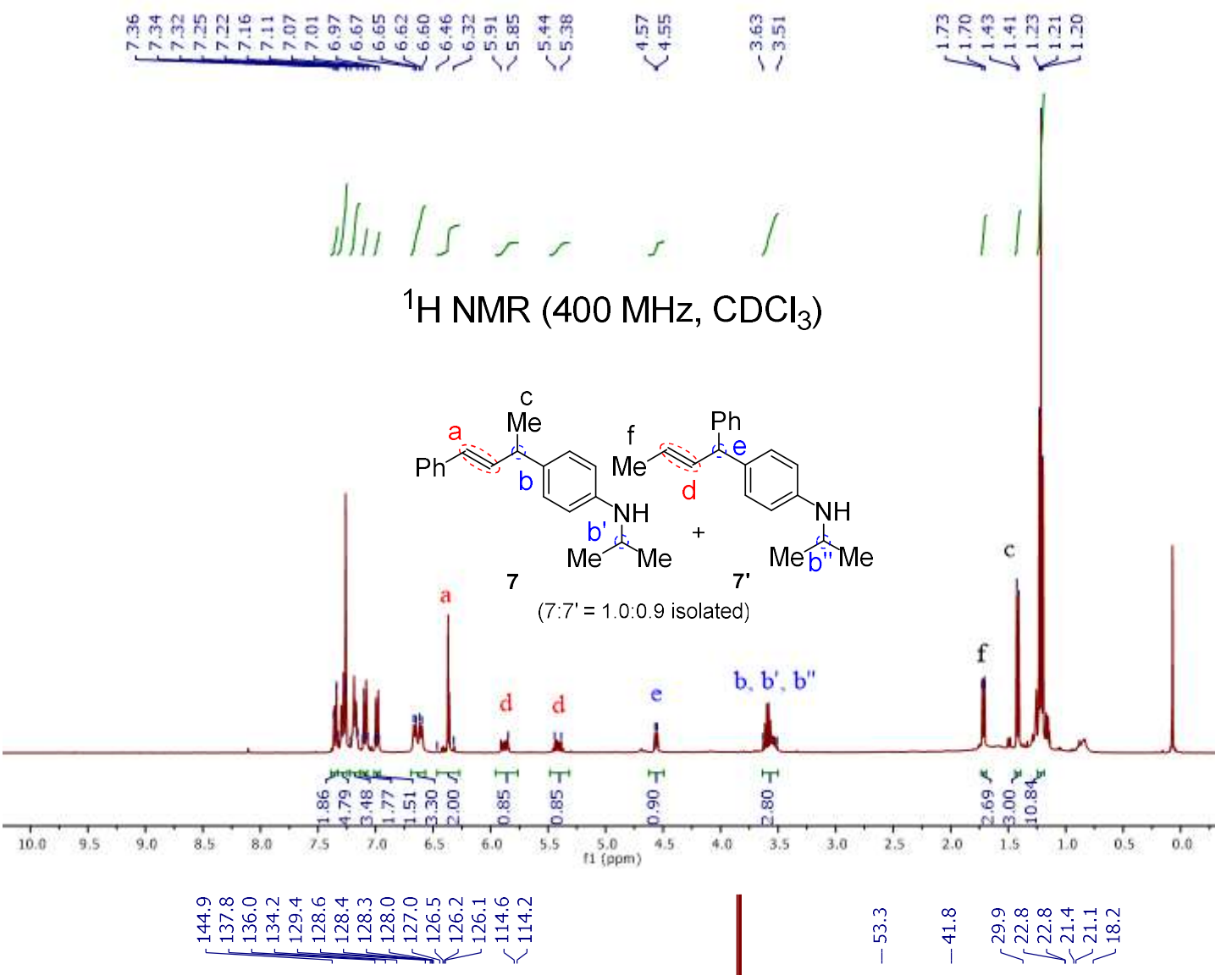

${ }^{13} \mathrm{C}$ NMR $\left(101 \mathrm{MHz}, \mathrm{CDCl}_{3}\right)$
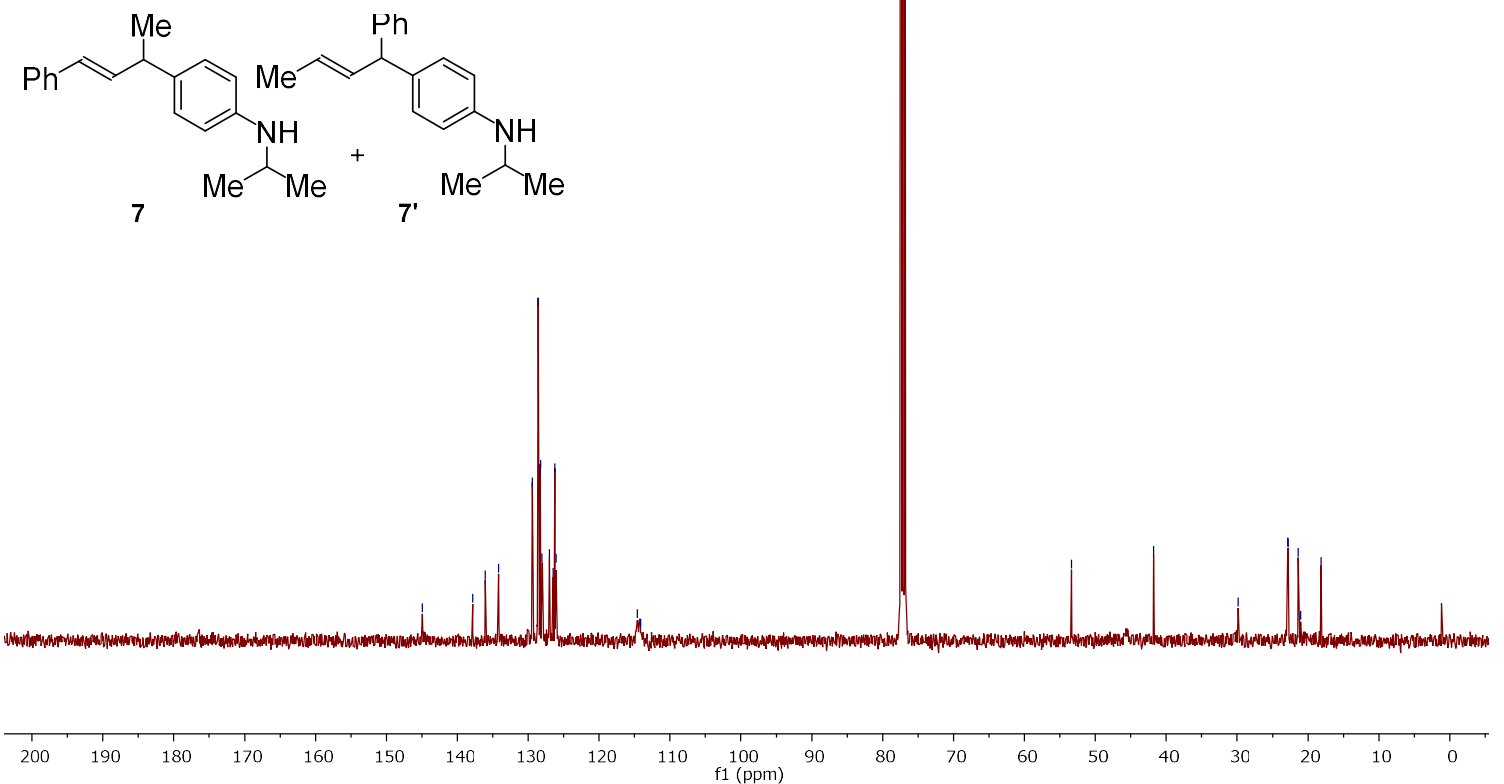

phenylbut-2-en-1-yl)aniline (8 + 8')
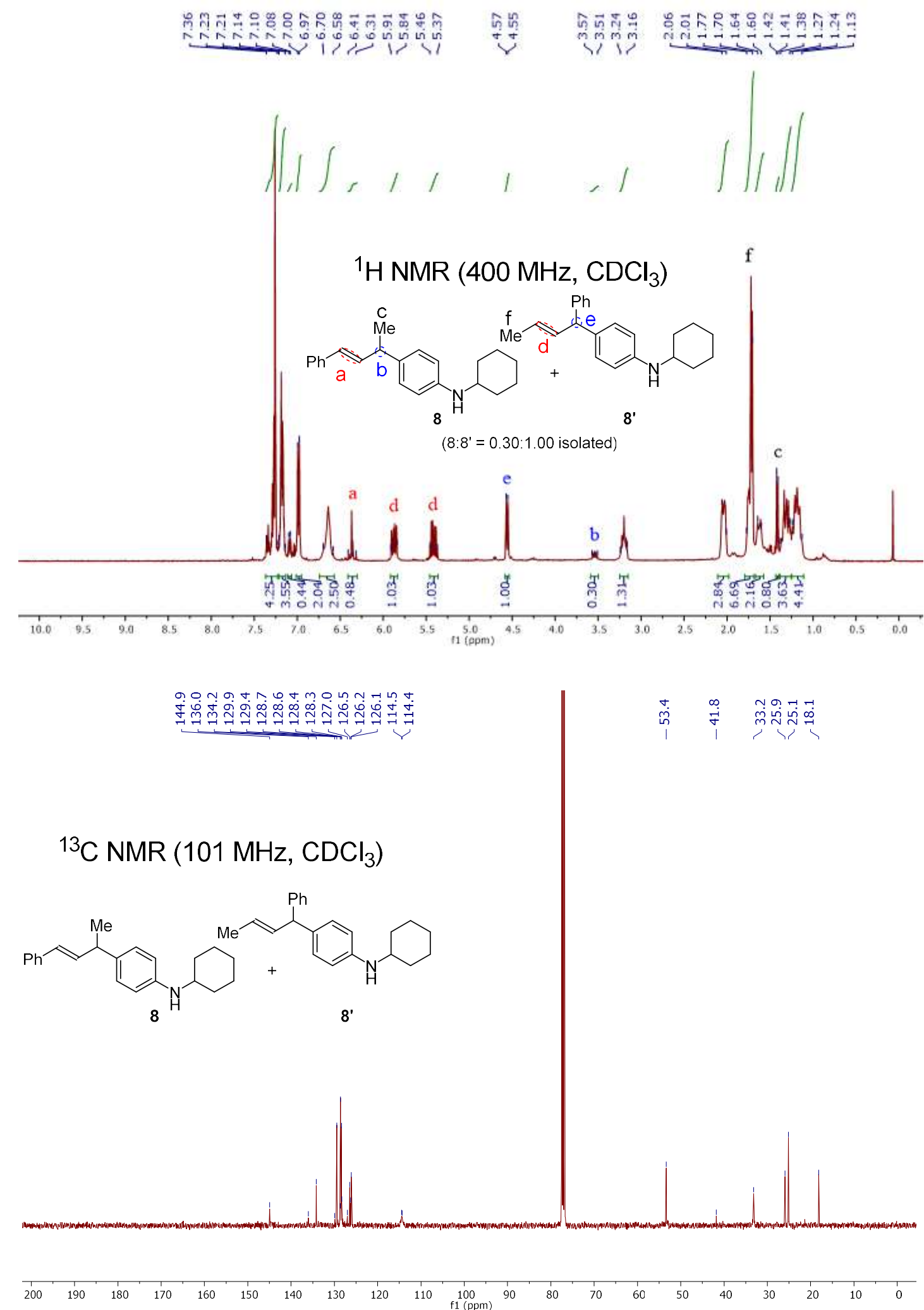
(E)-N,N-diphenyl-4-(4-phenylbut-3-en-2-yl)aniline (9)

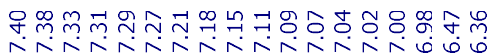

e

în

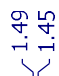

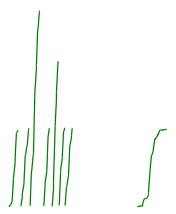

${ }^{1} \mathrm{H}$ NMR $\left(400 \mathrm{MHz}, \mathrm{CDCl}_{3}\right)$

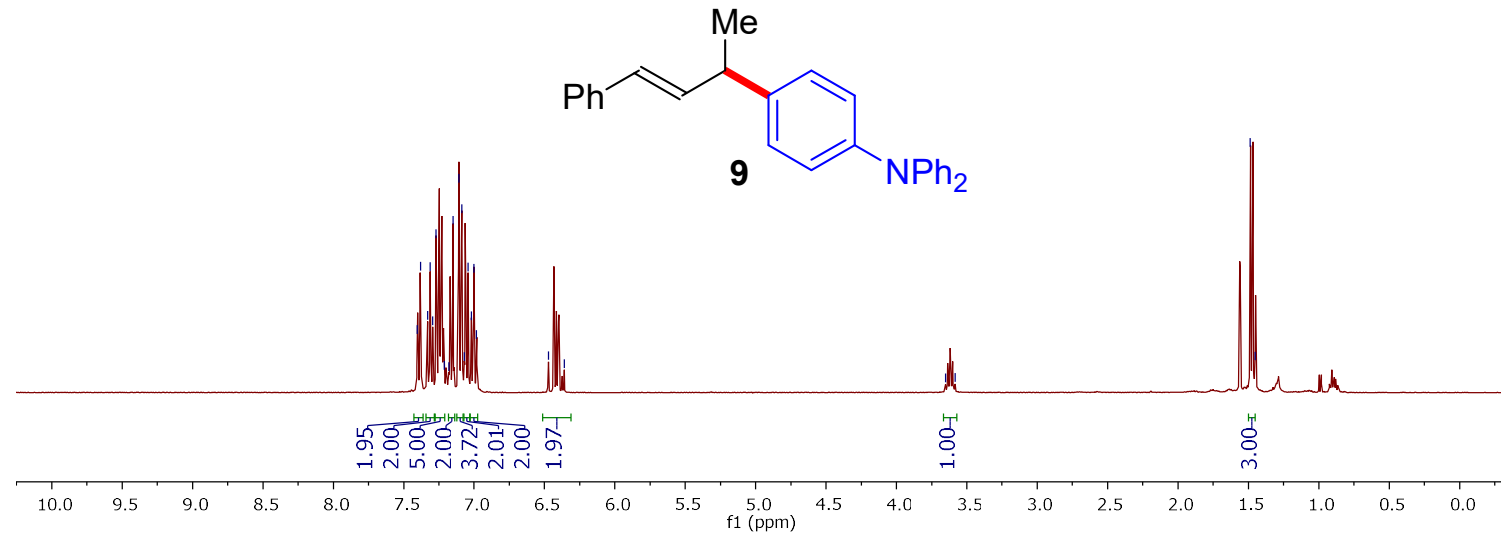

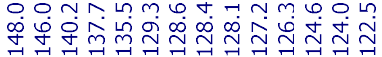

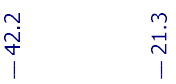

${ }^{13} \mathrm{C}$ NMR $\left(101 \mathrm{MHz}, \mathrm{CDCl}_{3}\right)$
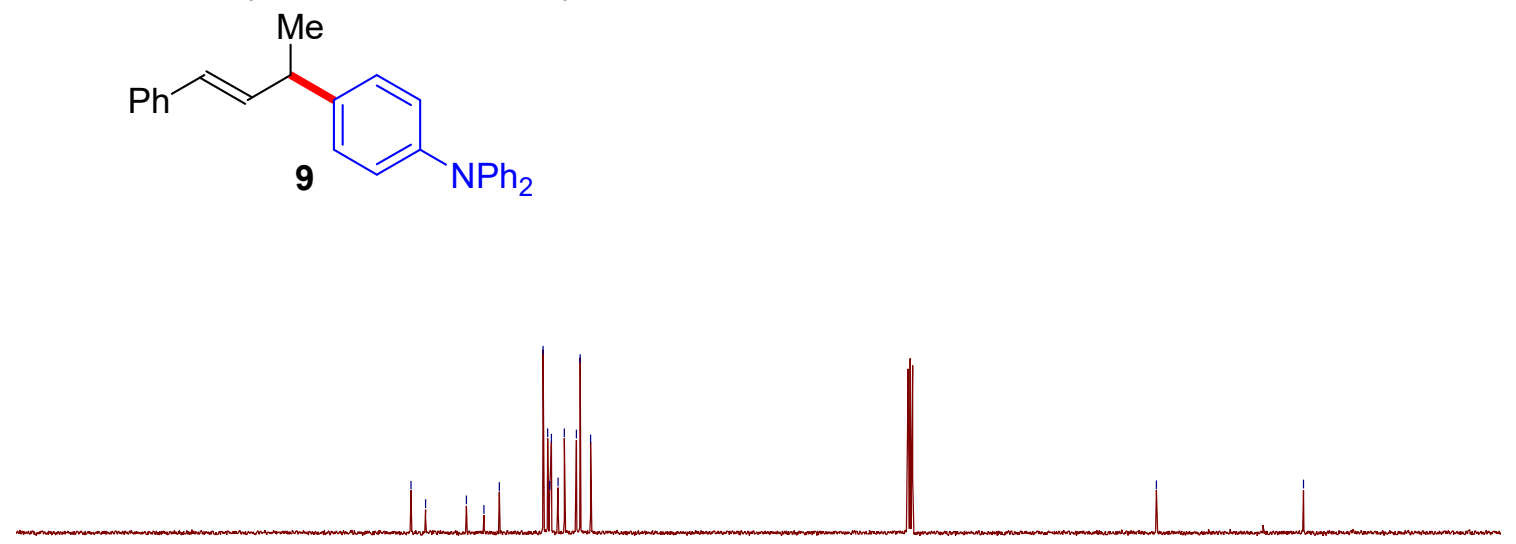

200

$150 \quad 14$

$100 \quad 90$ 
(E)-N-methyl-N-phenyl-4-(4-phenylbut-3-en-2-yl)aniline (10)

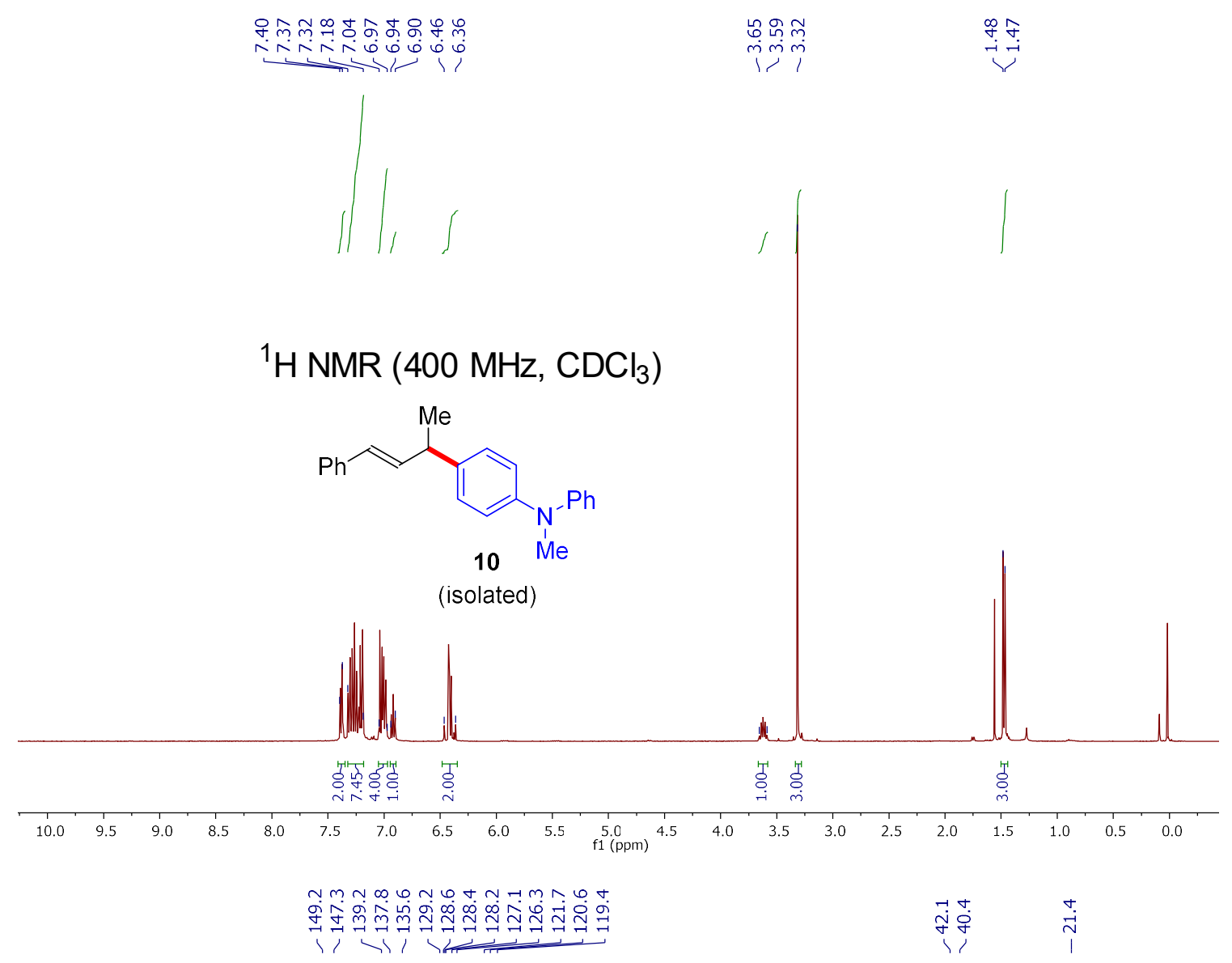

${ }^{13} \mathrm{C}$ NMR $\left(101 \mathrm{MHz}, \mathrm{CDCl}_{3}\right)$
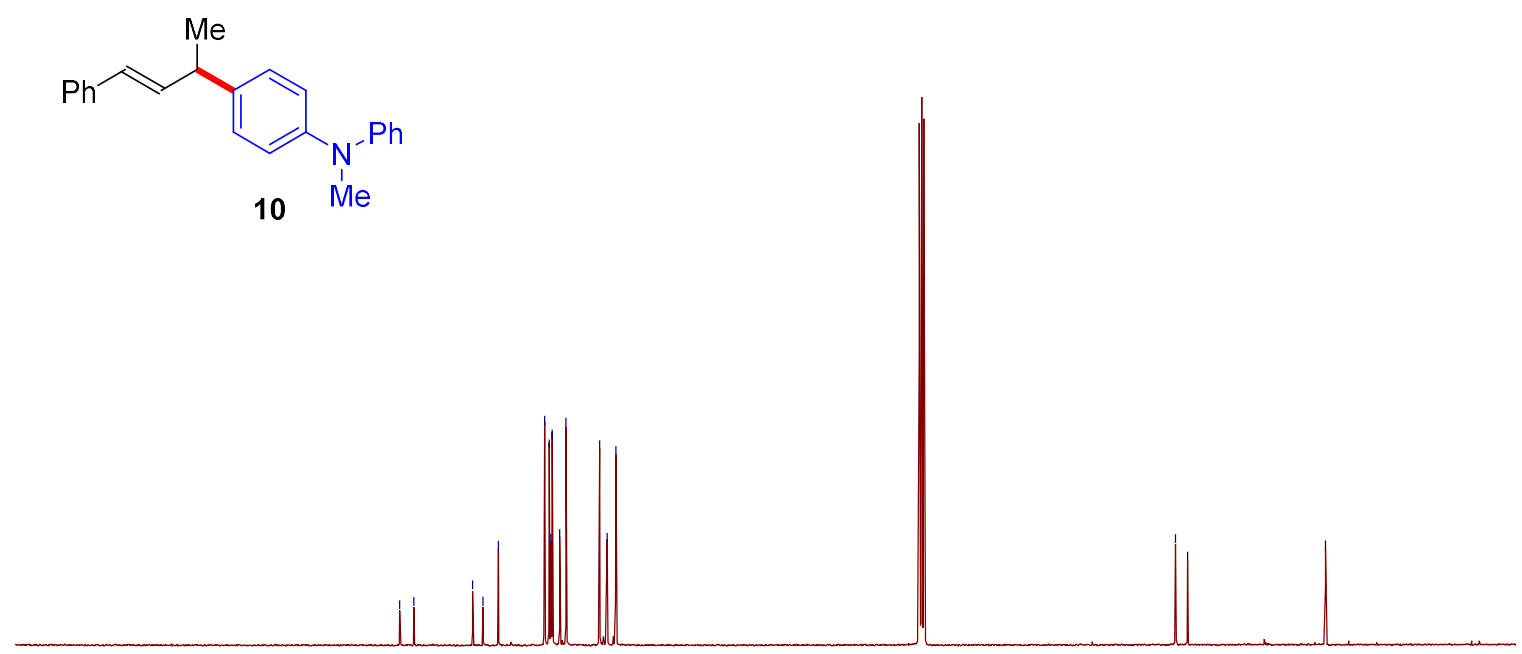

200

$200 \quad 190 \quad 180$

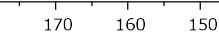

$150 \quad 140$ 
(E)-1-benzyl-3-(4-phenylbut-3-en-2-yl)-1H-pyrrole (11)
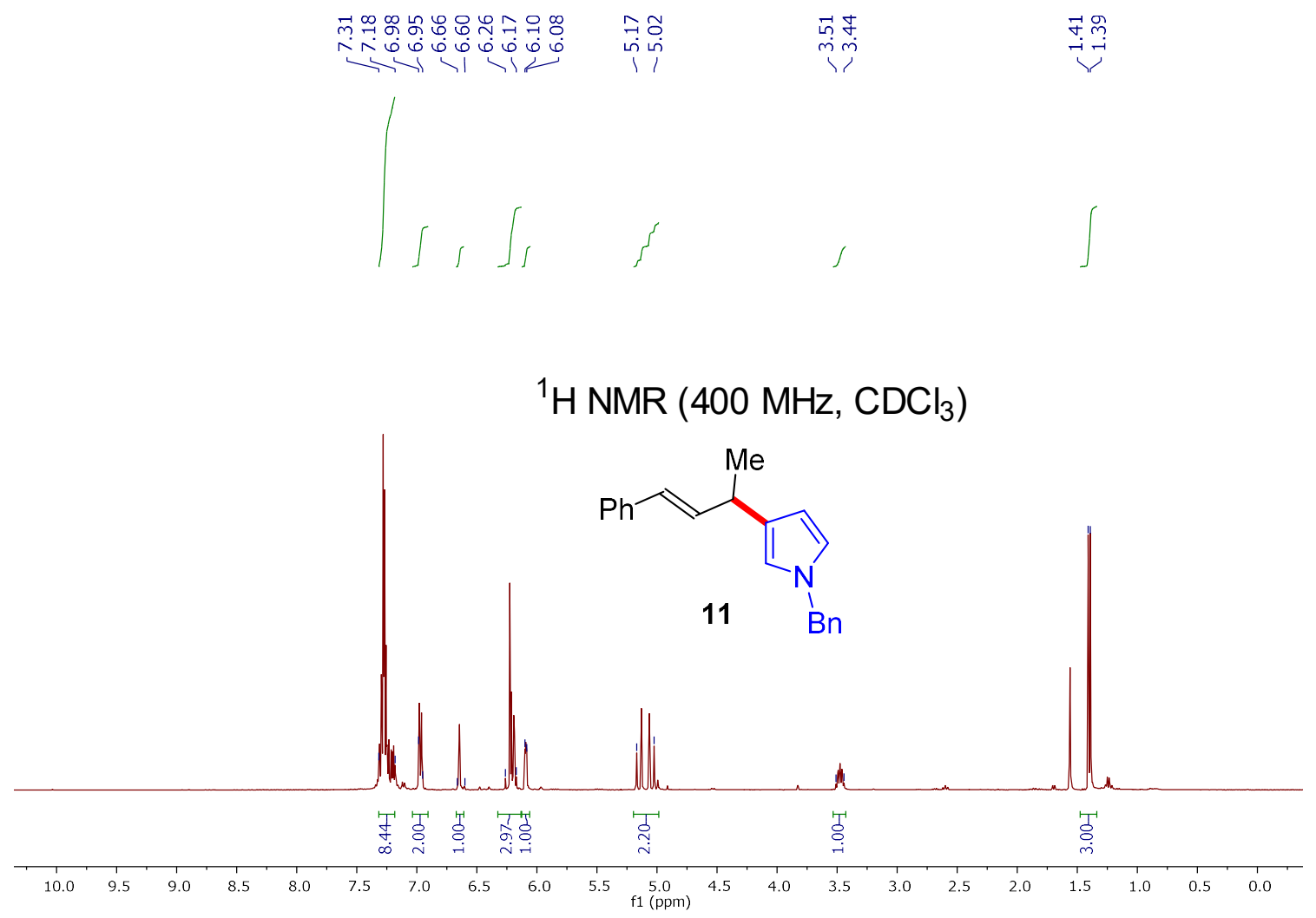

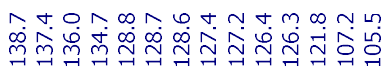

$\begin{array}{ccc}\forall & \infty & 0 \\ 0 & \dot{j} & \stackrel{0}{0} \\ 1 & 1 & 1\end{array}$

${ }^{13} \mathrm{C}$ NMR $\left(101 \mathrm{MHz}, \mathrm{CDCl}_{3}\right)$
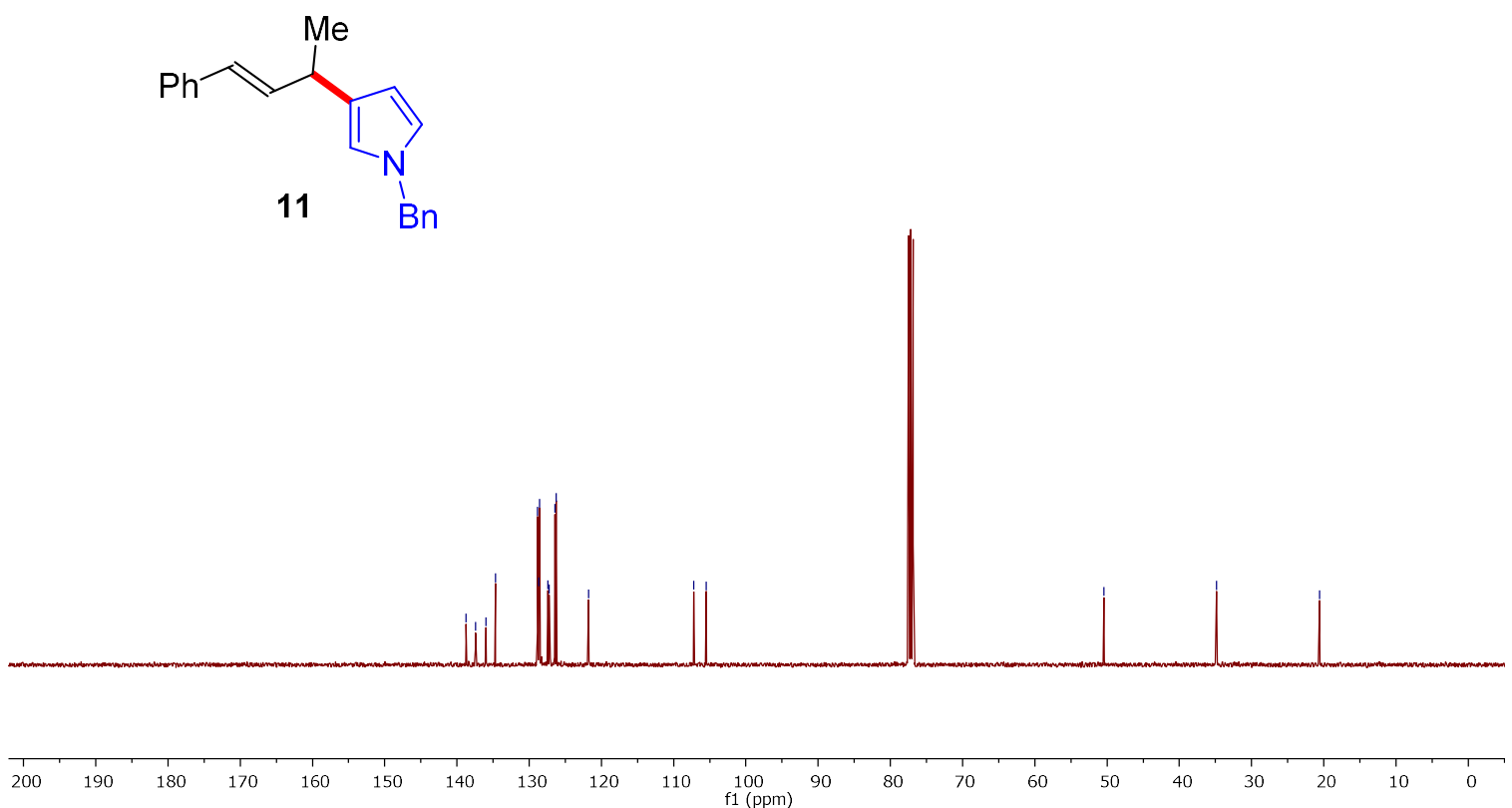
$(E)$-4-(4-(4-phenylbut-3-en-2-yl)phenyl)morpholine and $(E)$-4-(4-(1-phenylbut-2-en1-yl)phenyl)morpholine $\left(12+12^{\prime}\right)$

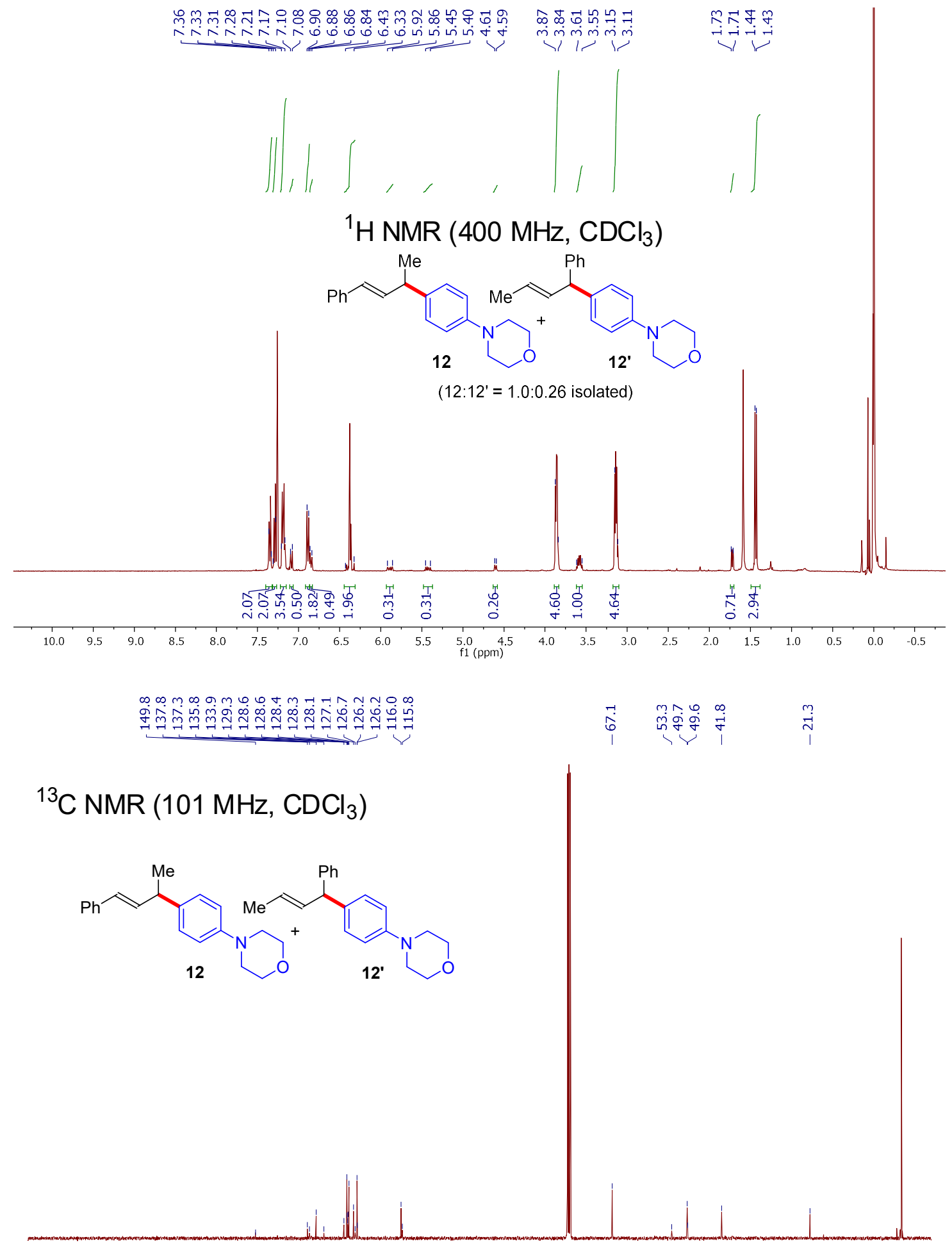

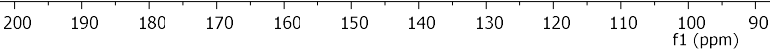


(E)-1-methyl-6-(4-phenylbut-3-en-2-yl)-1,2,3,4-tetrahydroquinoline and (E)-1methyl-6-(1-phenylbut-2-en-1-yl)-1,2,3,4-tetrahydroquinoline (13 + 13')

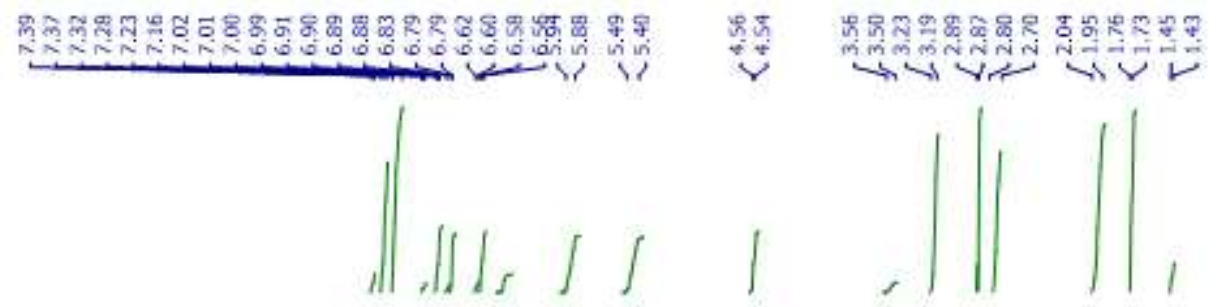

${ }^{1} \mathrm{H}$ NMR $\left(400 \mathrm{MHz}, \mathrm{CDCl}_{3}\right)$<smiles>CCC=C(Cc1ccccc1)C(Cl)c1ccc2c(c1)CCCN2C</smiles>

$\left(13: 13^{\prime}=0.16: 1.0\right.$ isolated $)$

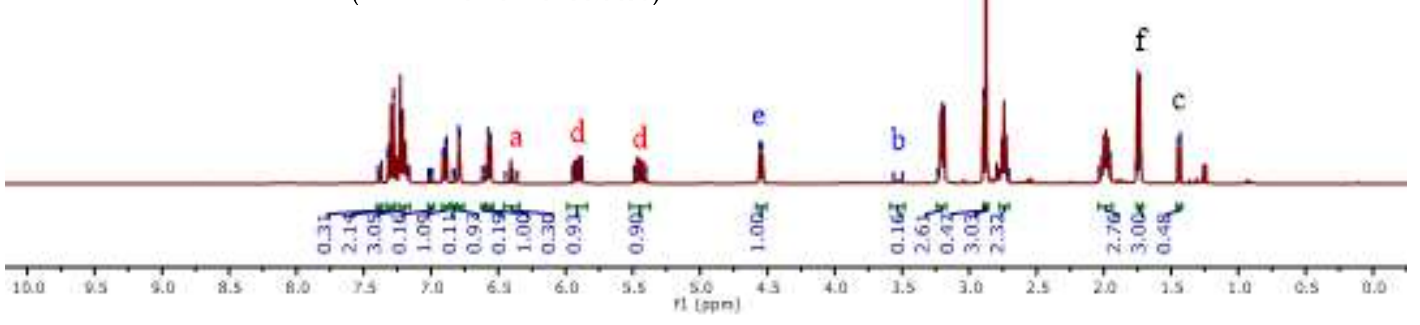

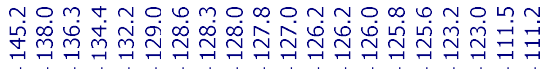

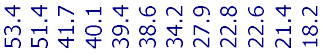

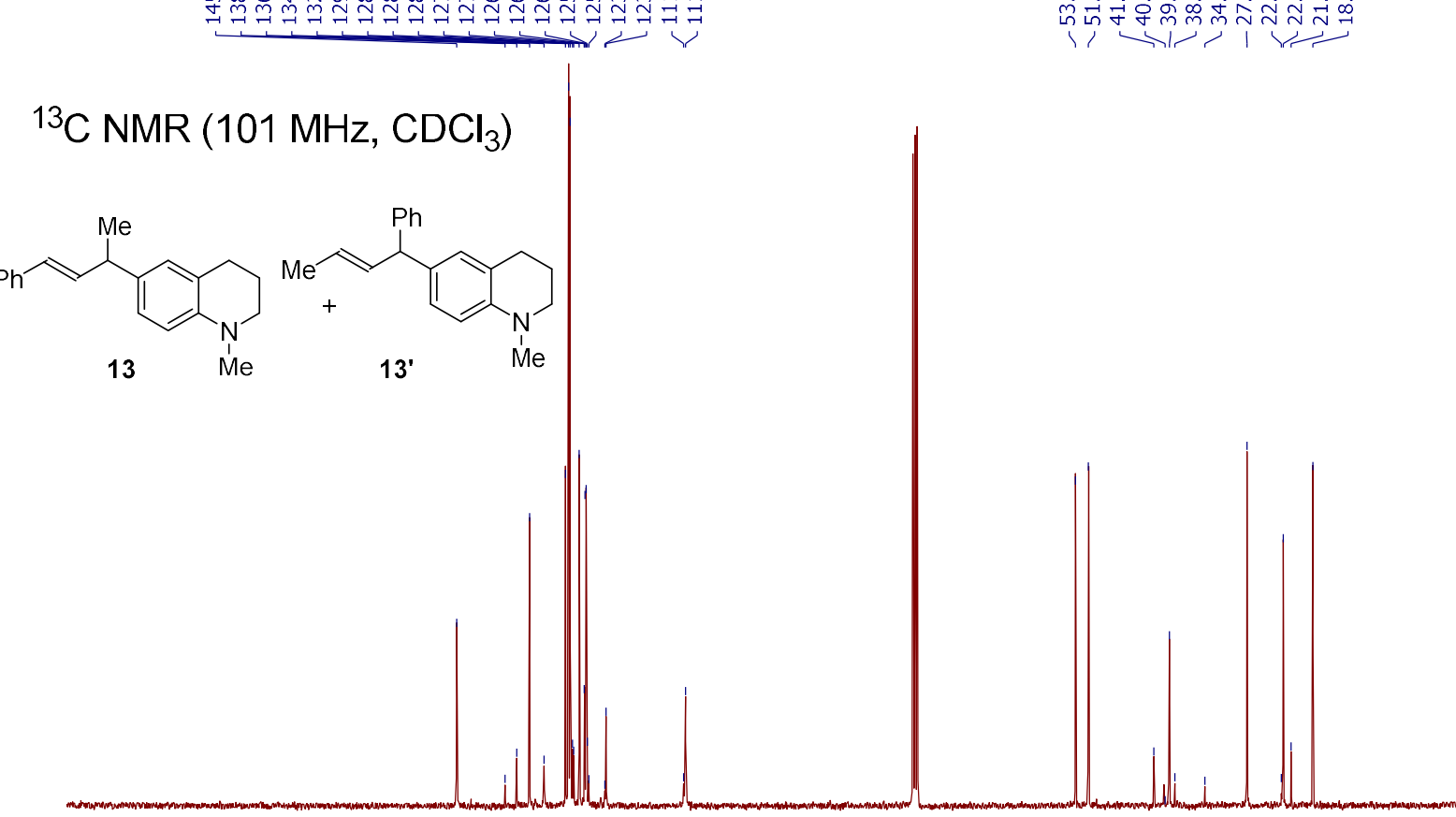

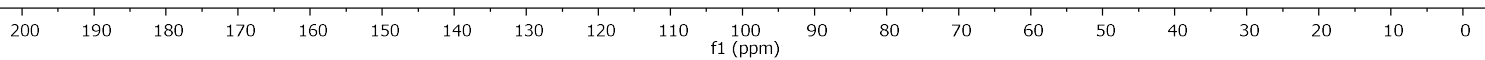


(2S)-2-butyl-1-methyl-6-((E)-1-phenylbut-2-en-1-yl)-1,2,3,4-tetrahydroquinoline (14')

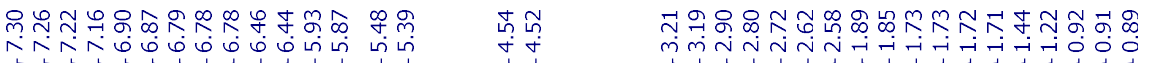

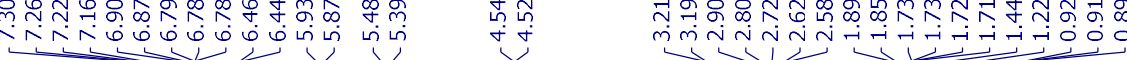

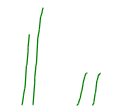

${ }^{1} \mathrm{H} \mathrm{NMR}\left(400 \mathrm{MHz}, \mathrm{CDCl}_{3}\right)$

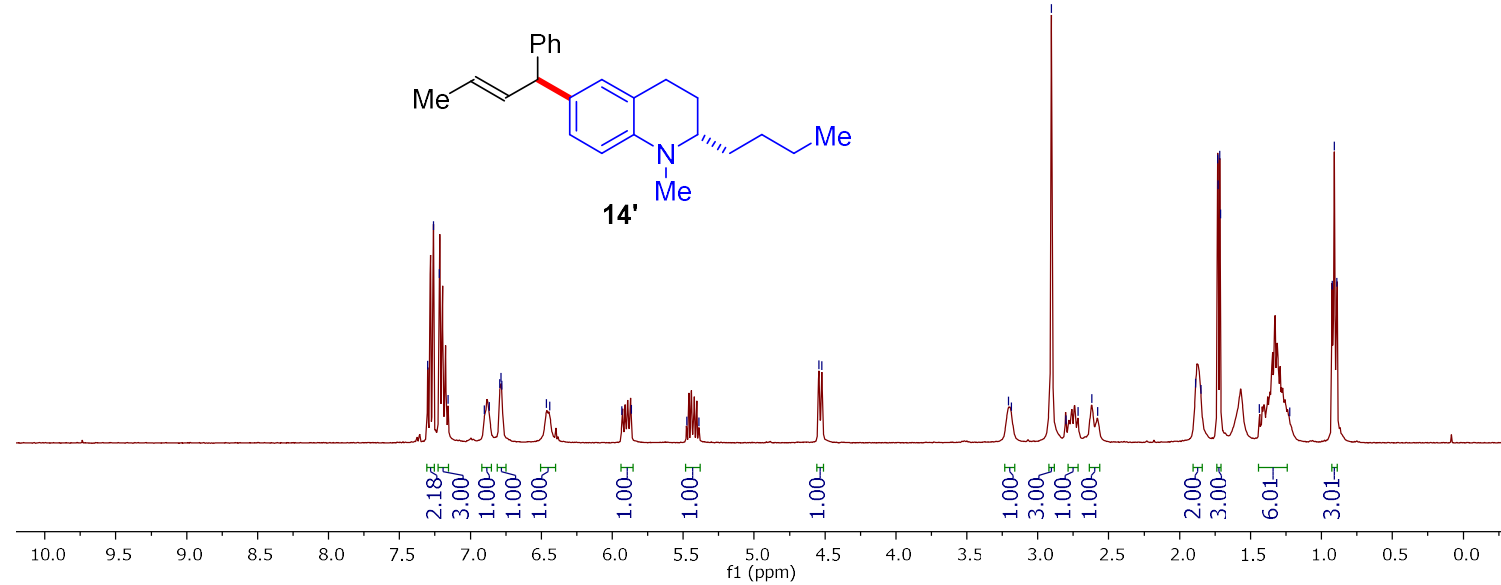

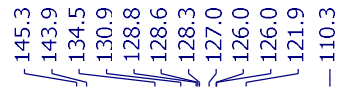

尚

${ }^{13} \mathrm{C}$ NMR $\left(101 \mathrm{MHz}, \mathrm{CDCl}_{3}\right)$
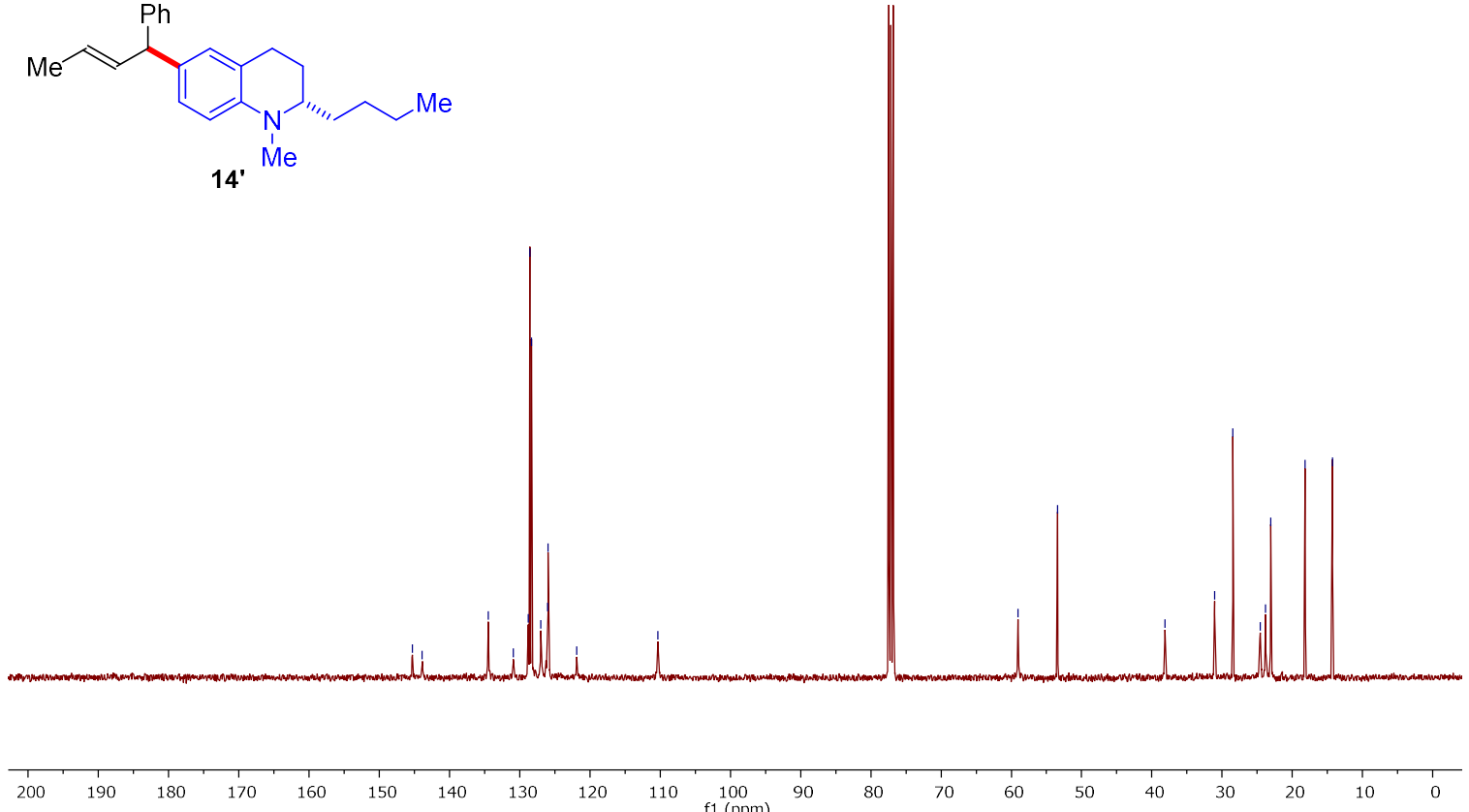
(E)-N-phenyl-4-(4-(p-tolyl)but-3-en-2-yl)aniline (15)

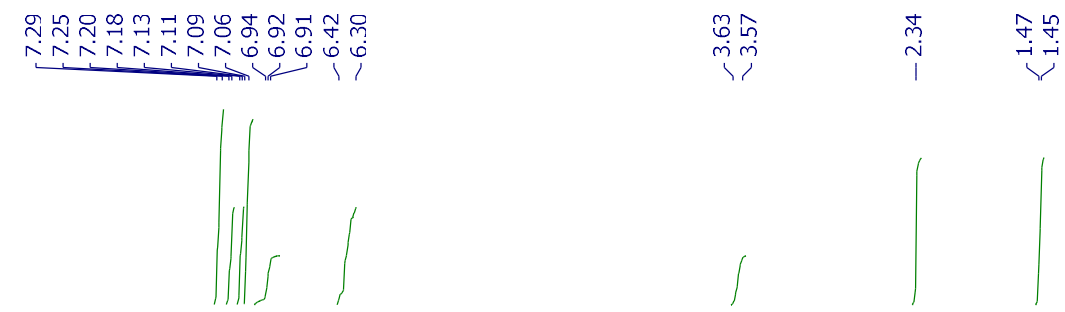

${ }^{1} \mathrm{H}$ NMR $\left(400 \mathrm{MHz}, \mathrm{CDCl}_{3}\right)$
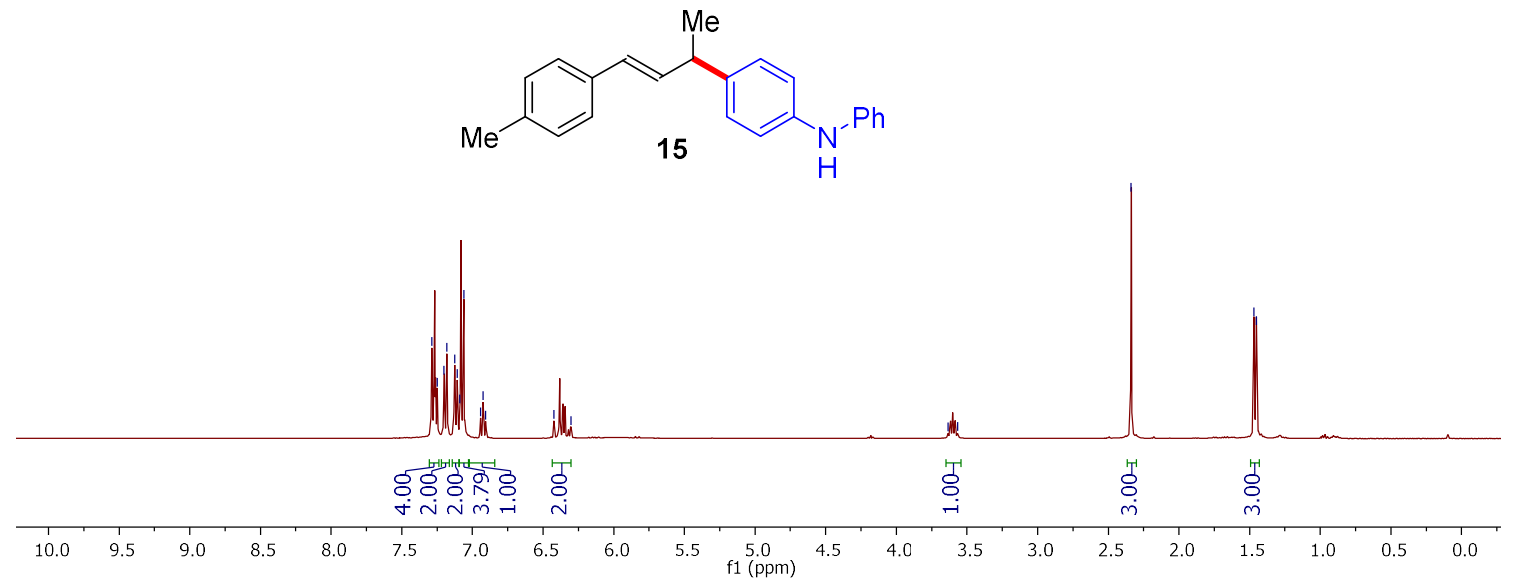

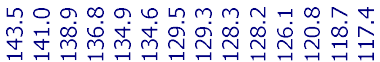

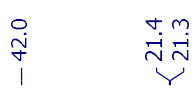

${ }^{13} \mathrm{C}$ NMR $\left(101 \mathrm{MHz}, \mathrm{CDCl}_{3}\right)$
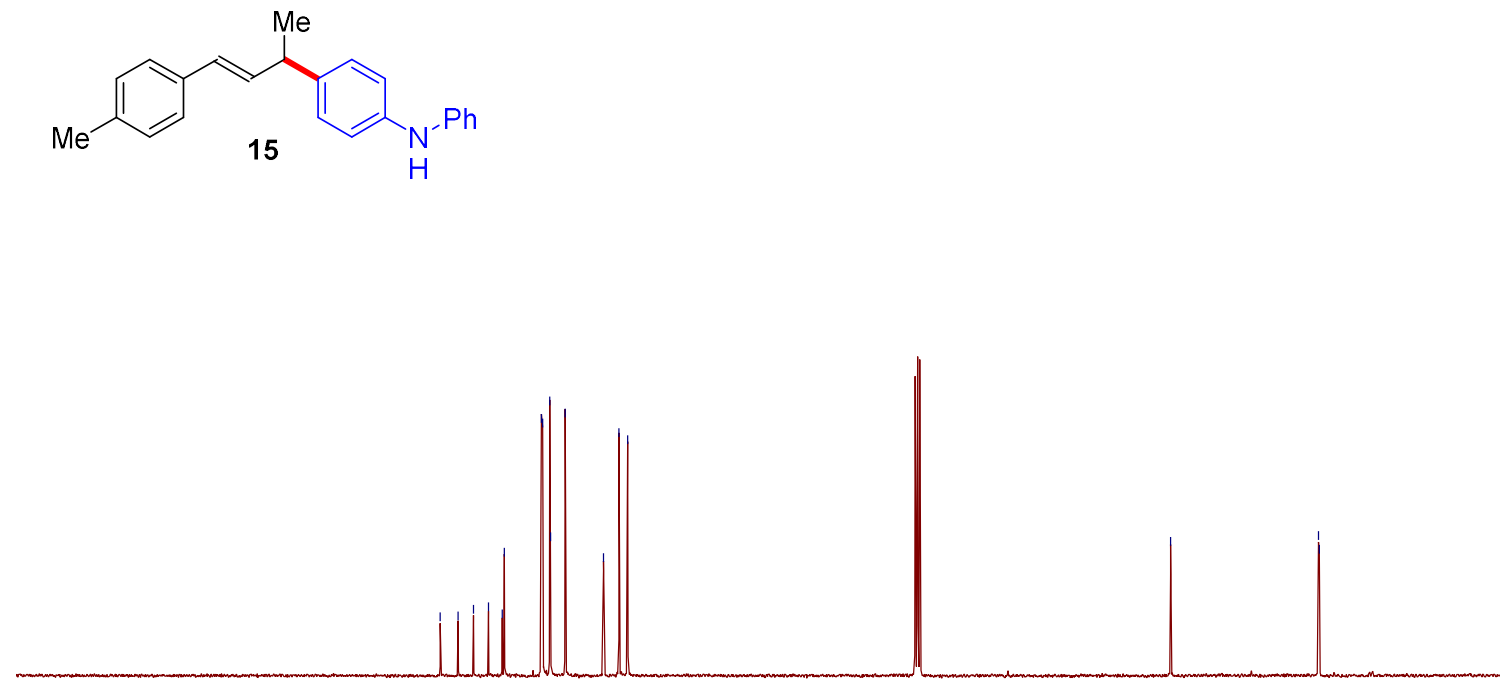

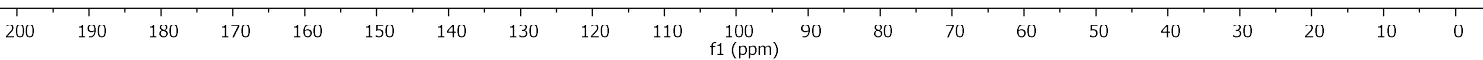


(E)-N-phenyl-4-(4-(0-tolyl)but-3-en-2-yl)aniline (16)
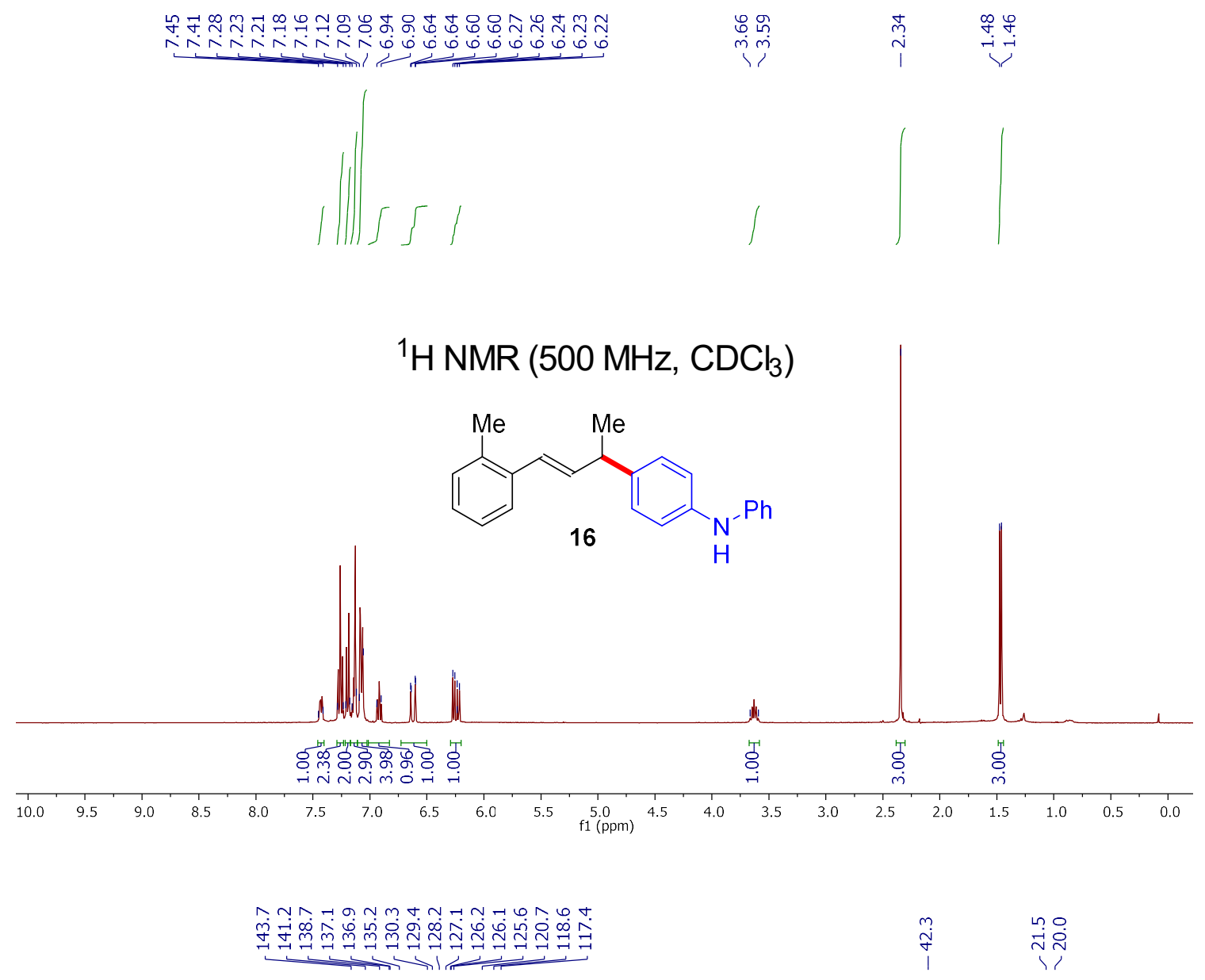

${ }^{13} \mathrm{C} \mathrm{NMR}\left(126 \mathrm{MHz}, \mathrm{CDCl}_{3}\right)$<smiles>Cc1ccccc1/C=C/C(C)c1ccc(Nc2ccccc2)cc1</smiles>
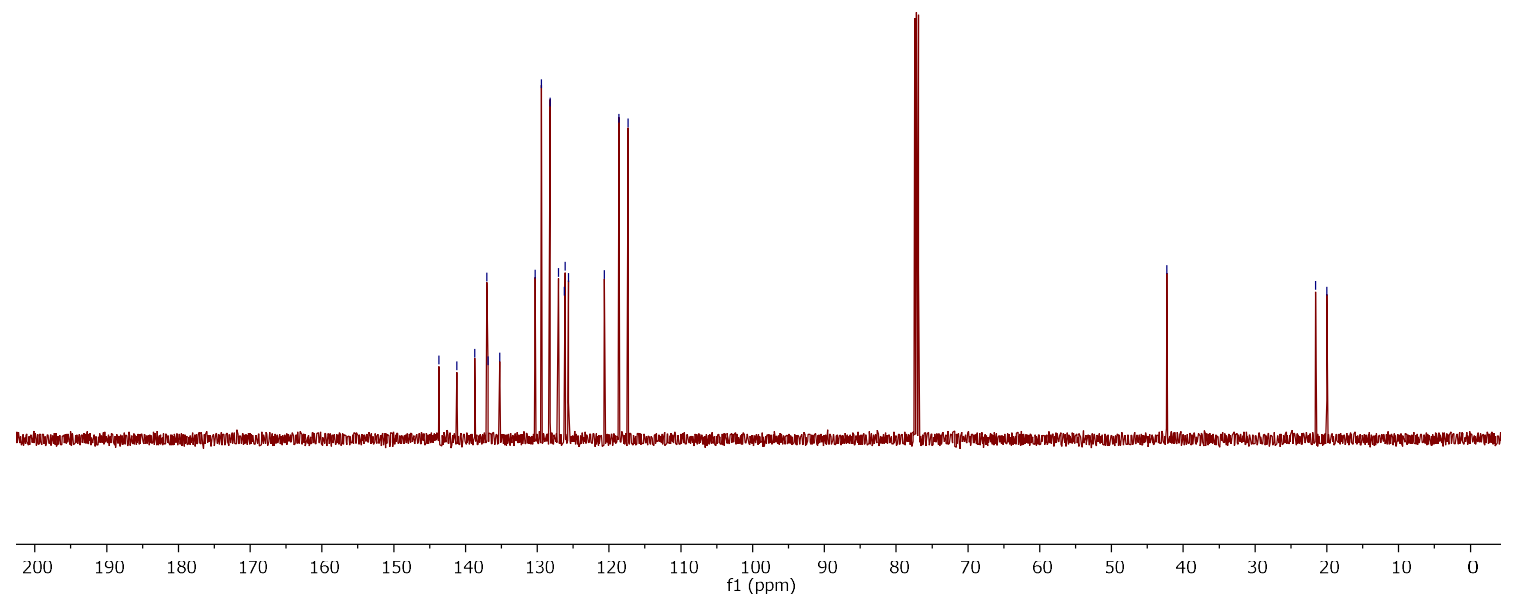
(E)-4-(4-(4-methoxyphenyl)but-3-en-2-yl)-N-phenylaniline (17)

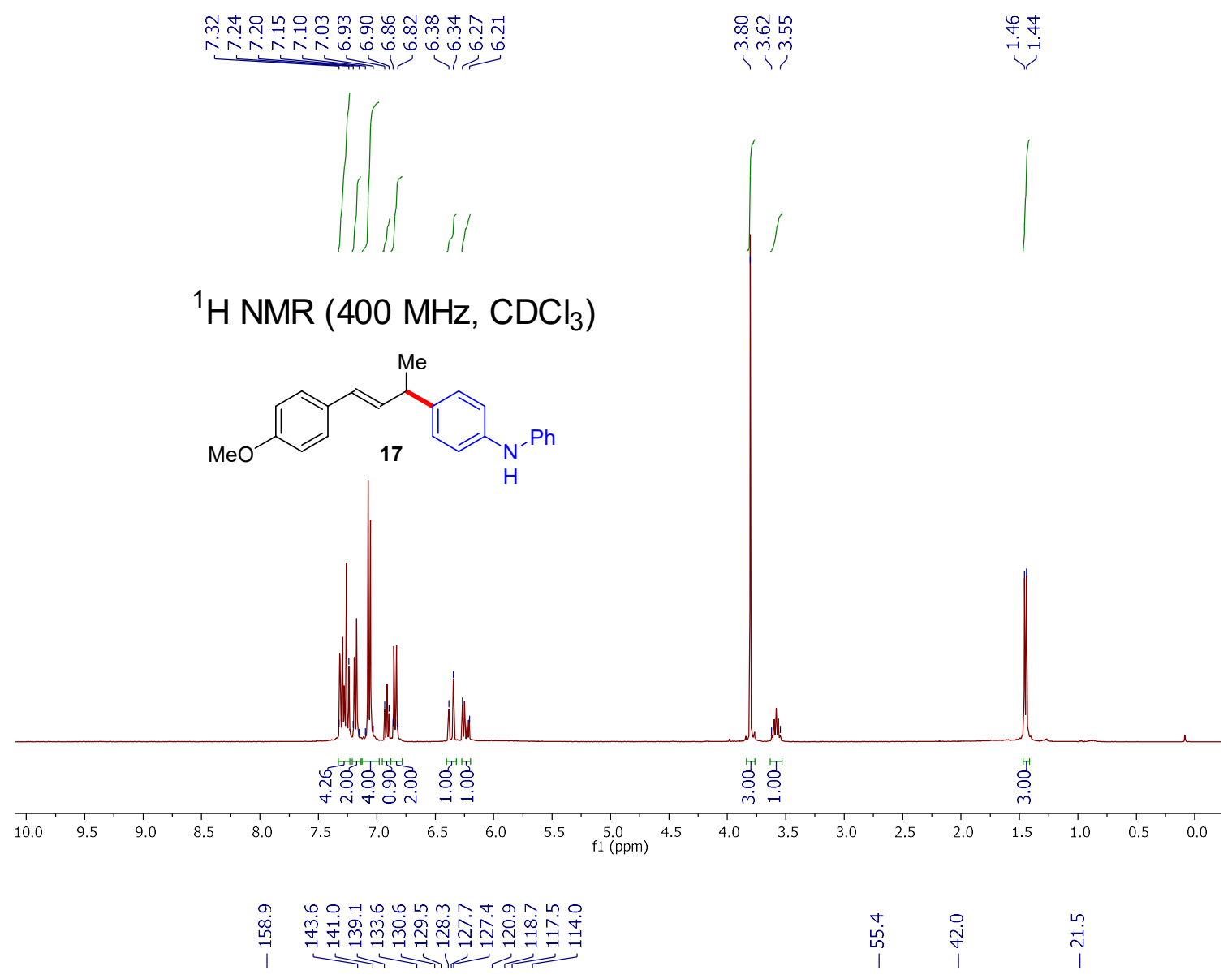

${ }^{13} \mathrm{C}$ NMR $\left(101 \mathrm{MHz}, \mathrm{CDCl}_{3}\right)$
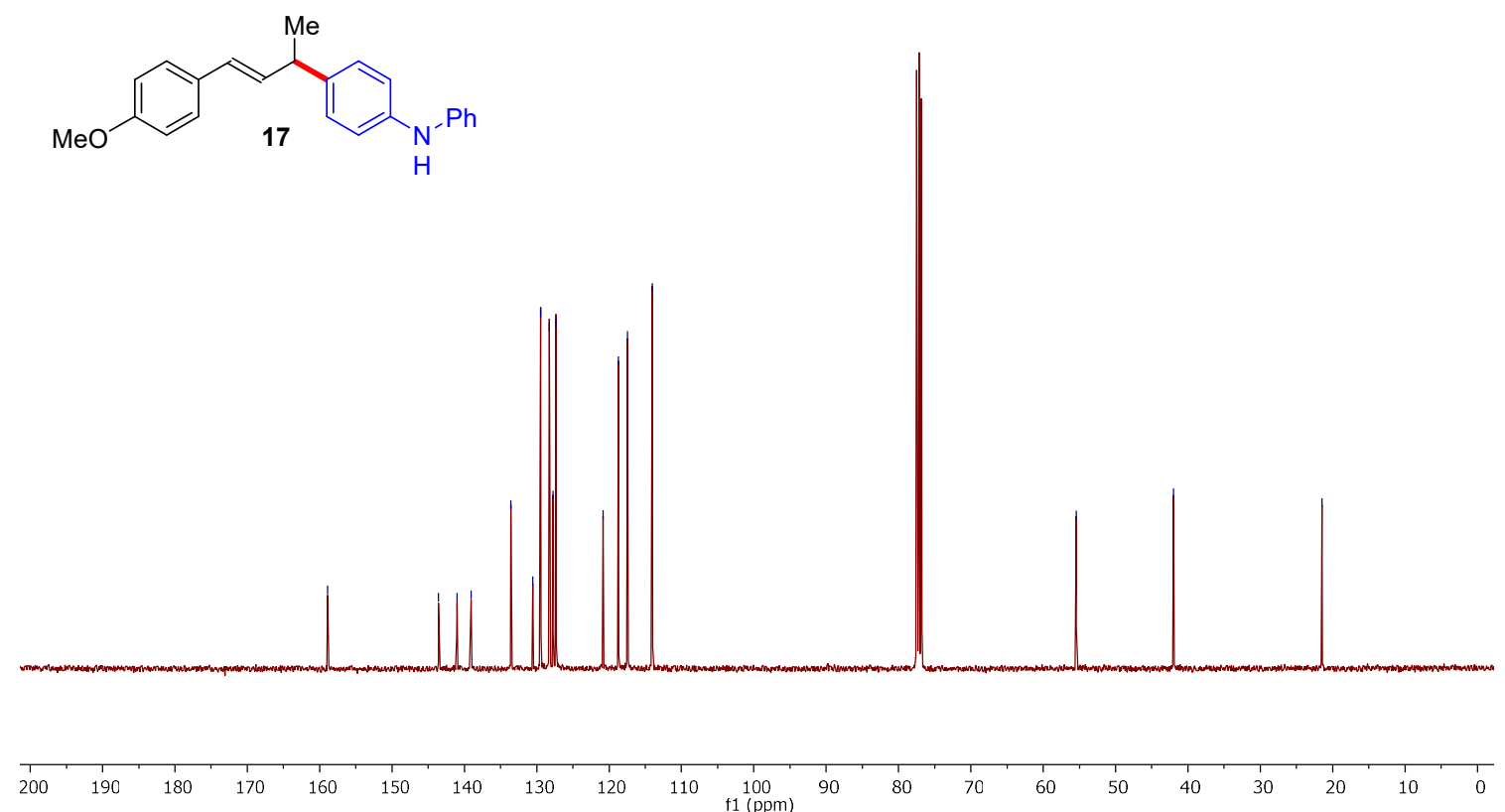
((E)-4-(4-(4-chlorophenyl)but-3-en-2-yl)-N-phenylaniline (18)

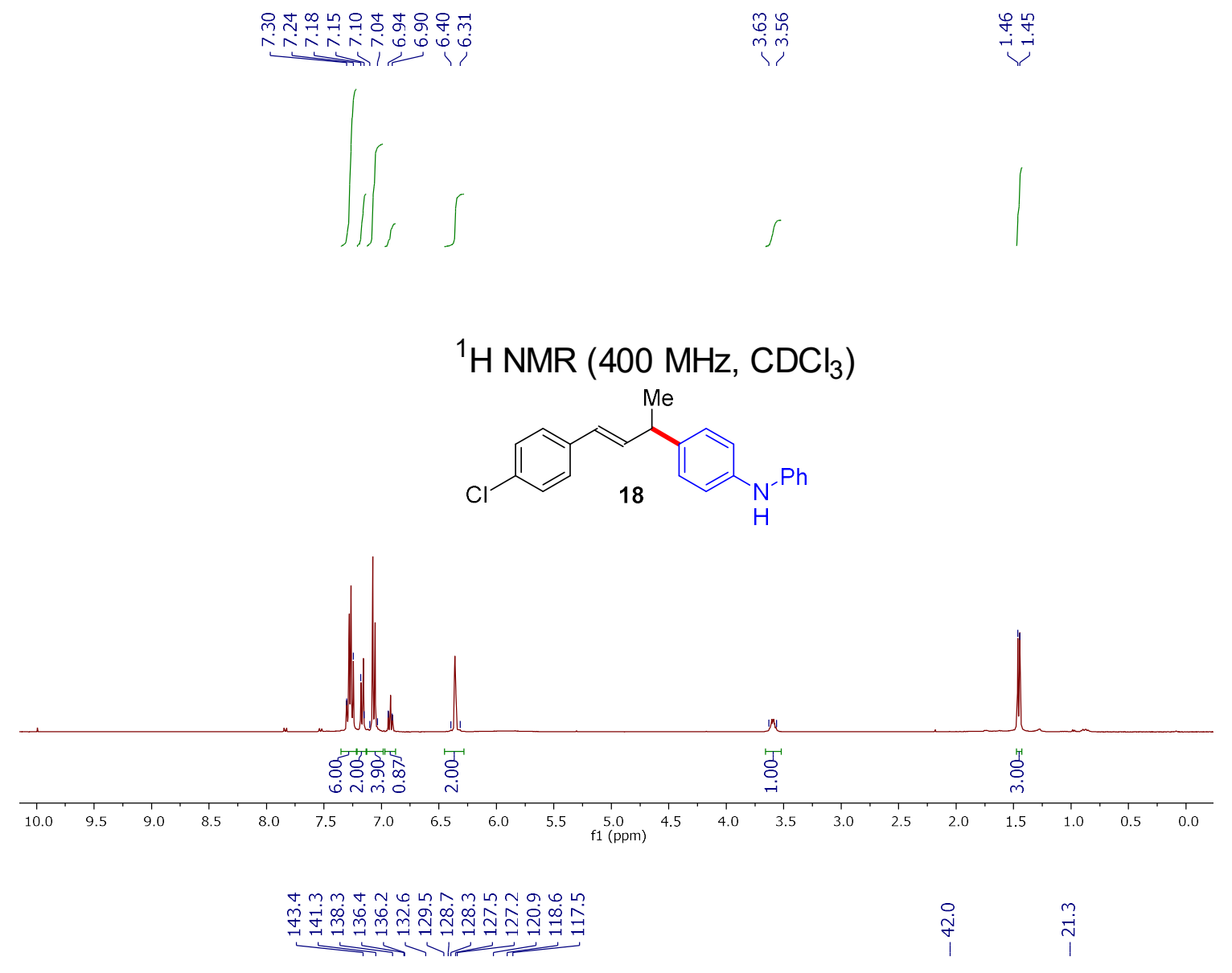

${ }^{13} \mathrm{C}$ NMR $\left(101 \mathrm{MHz}, \mathrm{CDCl}_{3}\right)$

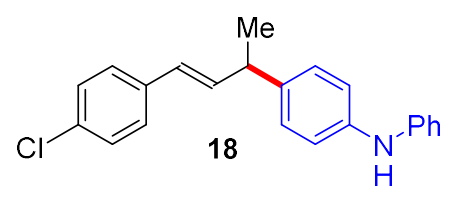


(E)-4-(4-(naphthalen-2-yl)but-3-en-2-yl)-N-phenylaniline (19)

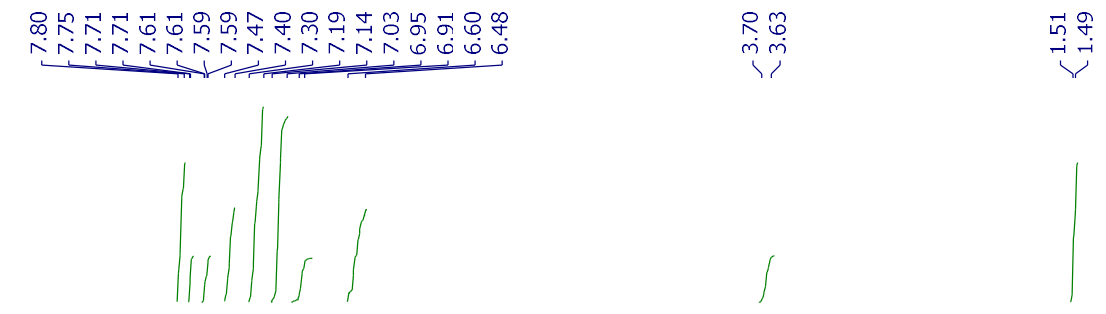

${ }^{1} \mathrm{H} \mathrm{NMR}\left(400 \mathrm{MHz}, \mathrm{CDCl}_{3}\right)$
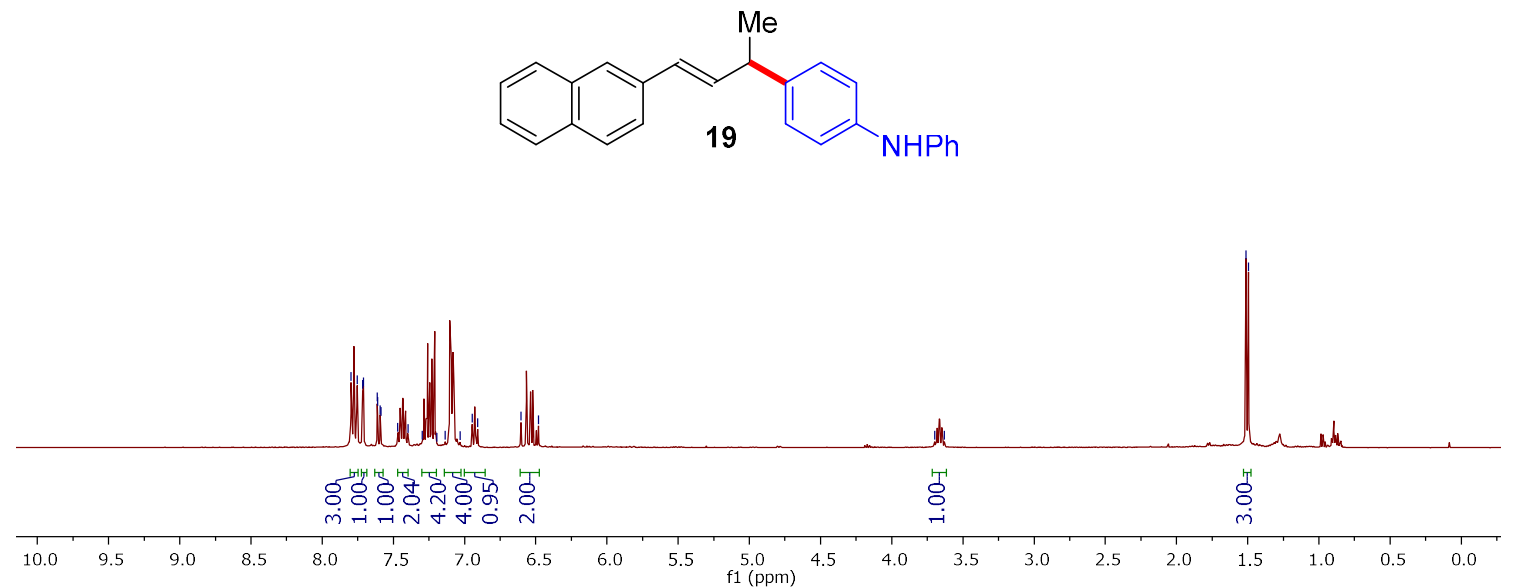

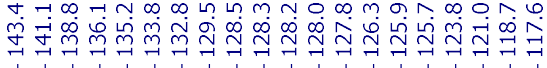

${ }^{13} \mathrm{C}$ NMR $\left(101 \mathrm{MHz}, \mathrm{CDCl}_{3}\right)$
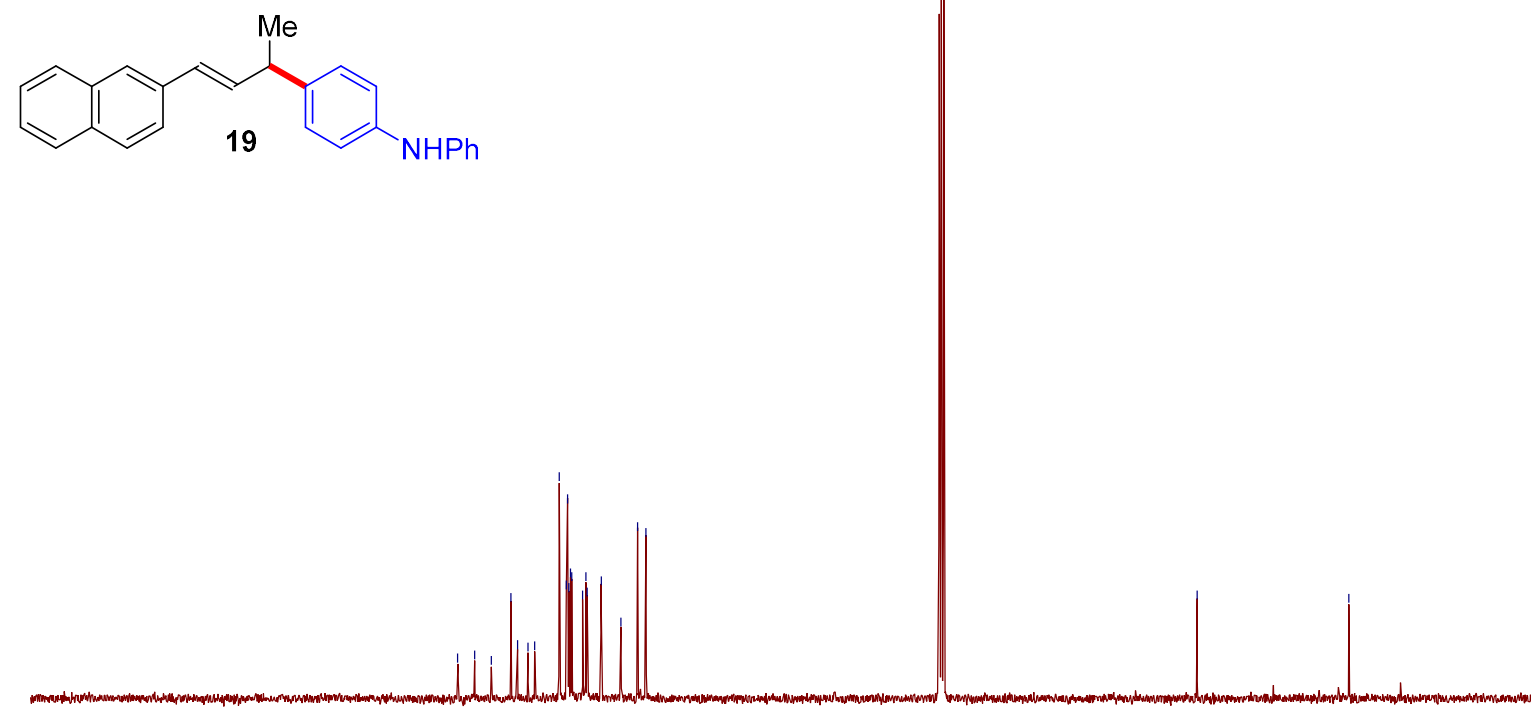
(E)-4-(4-(anthracen-9-yl)but-3-en-2-yl)-N-phenylaniline (20)

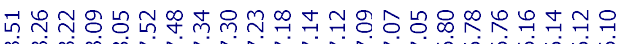

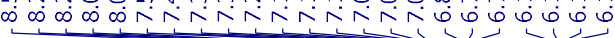

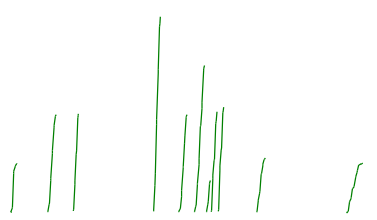

$m \infty$
$\dot{m} \infty$
$m$

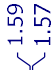

${ }^{1} \mathrm{H}$ NMR $\left(400 \mathrm{MHz}, \mathrm{DMSO}-\mathrm{D}_{6}\right)$
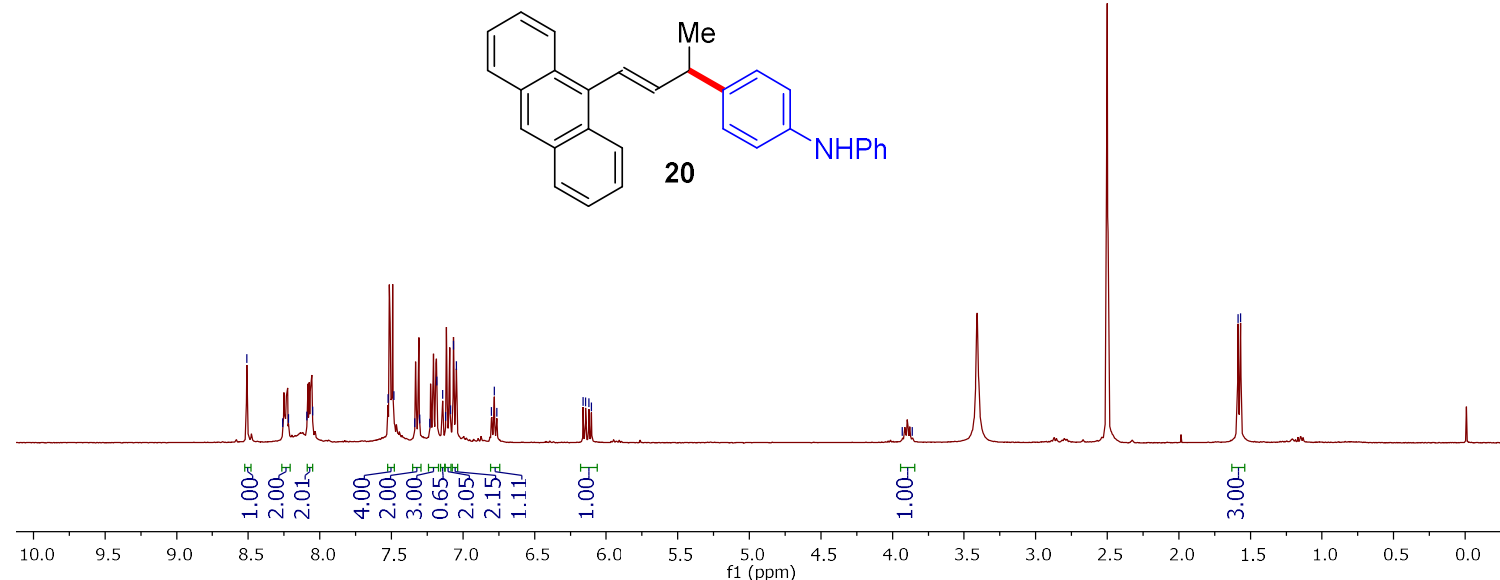

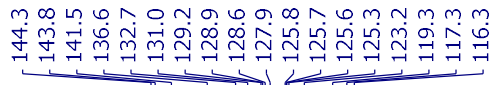

${ }^{13} \mathrm{C}$ NMR $\left(101 \mathrm{MHz}, \mathrm{DMSO}-\mathrm{D}_{6}\right)$
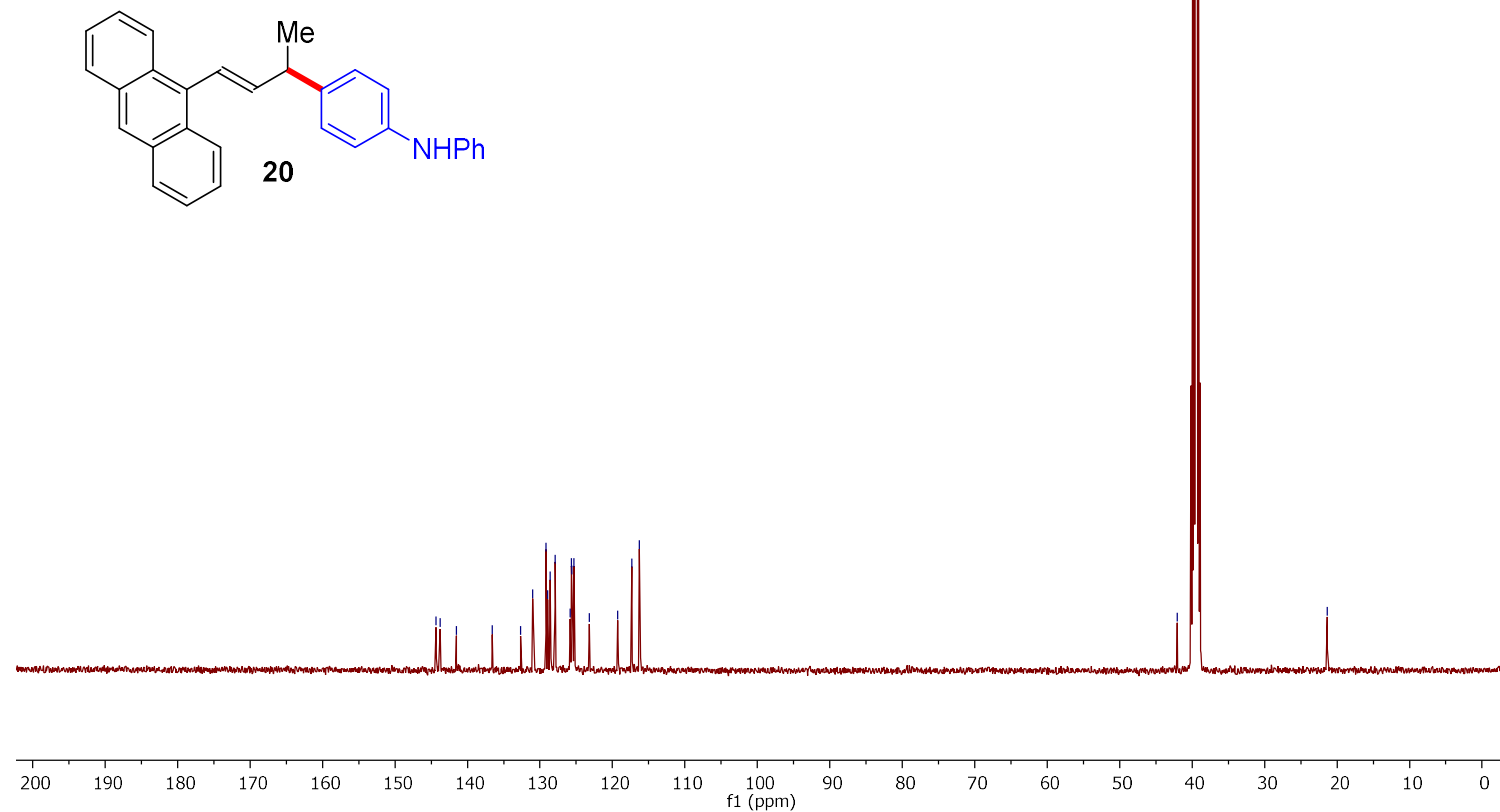
$(E)-N$-phenyl-4-(1-phenylpent-1-en-3-yl)aniline (21)

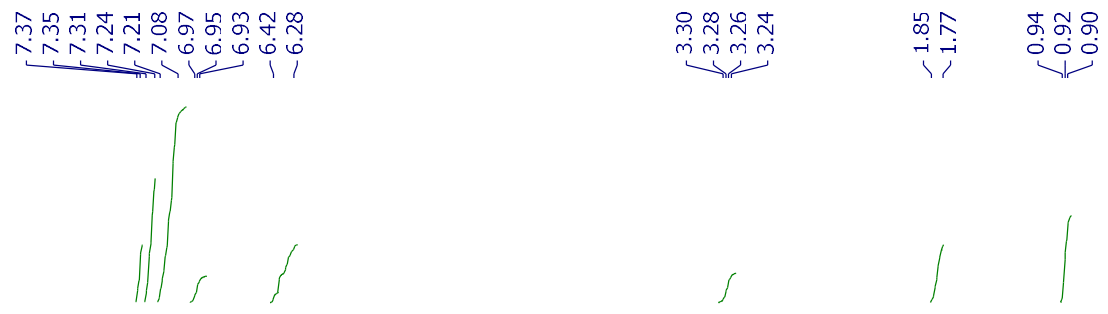

${ }^{1} \mathrm{H}$ NMR $\left(400 \mathrm{MHz}, \mathrm{CDCl}_{3}\right)$
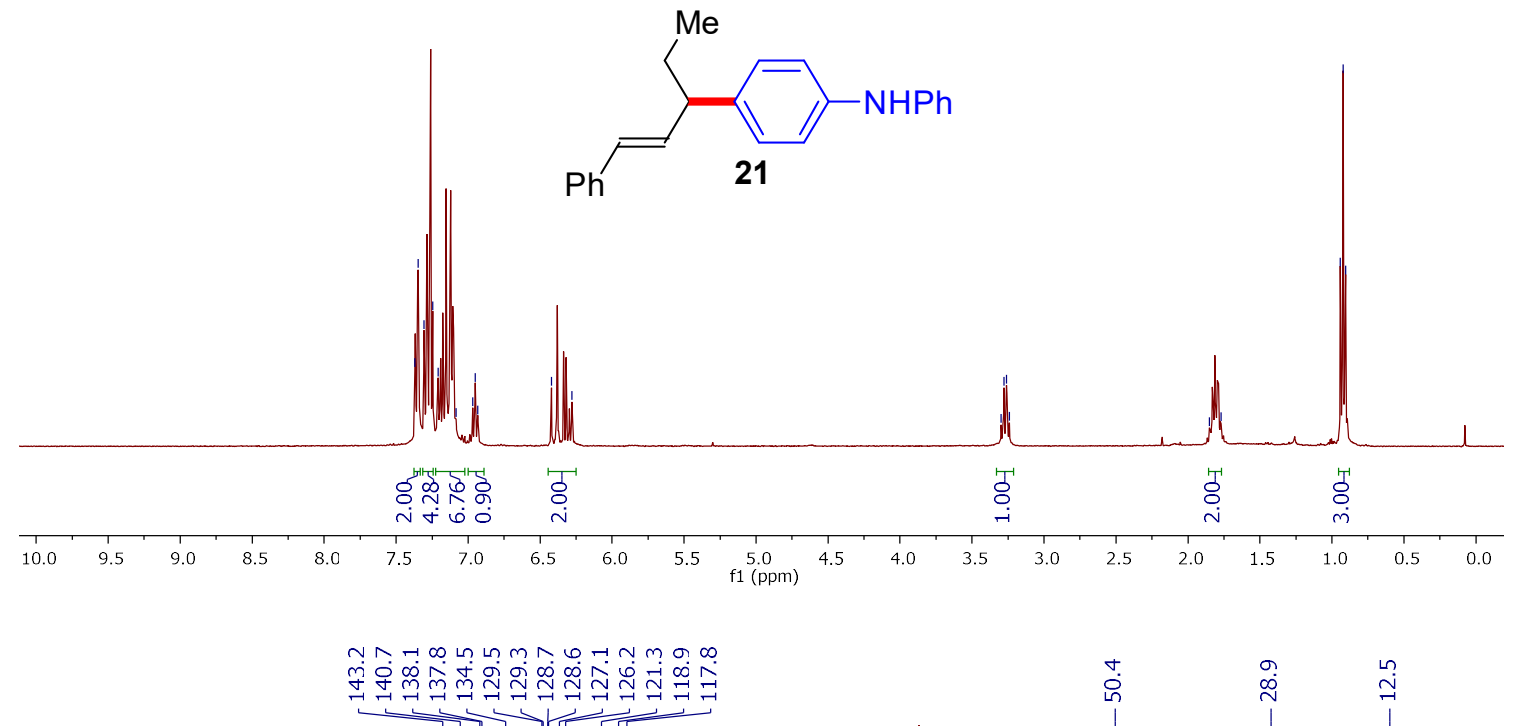

${ }^{13} \mathrm{C}$ NMR (101 MHz, $\mathrm{CDCl}_{3}$ )
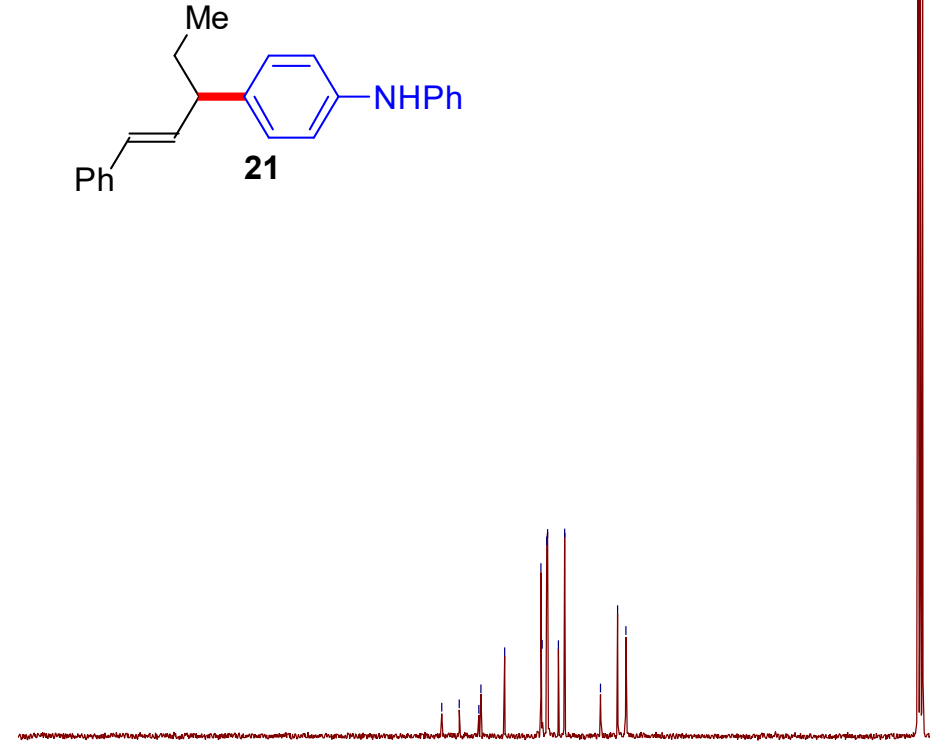

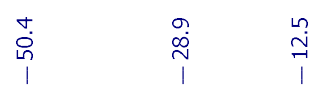

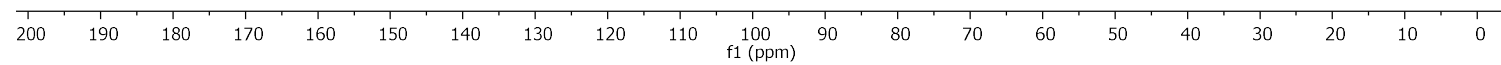


(E)-N-phenyl-4-(1-phenyloct-1-en-3-yl)aniline (22)
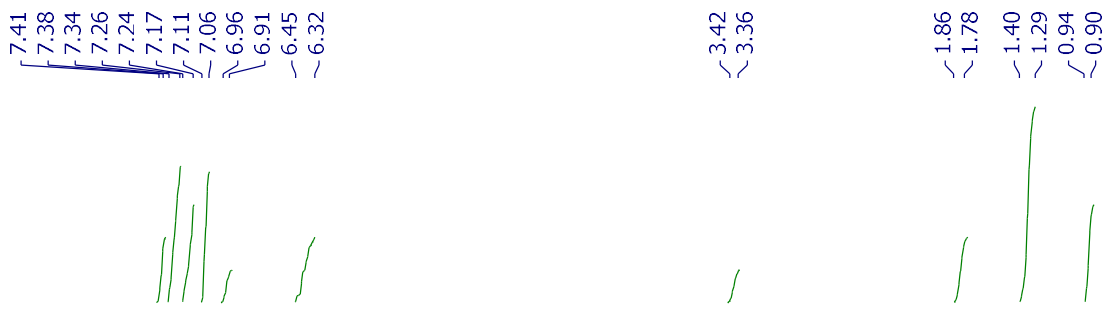

${ }^{1} \mathrm{H}$ NMR (400 MHz, $\left.\mathrm{CDCl}_{3}\right)$
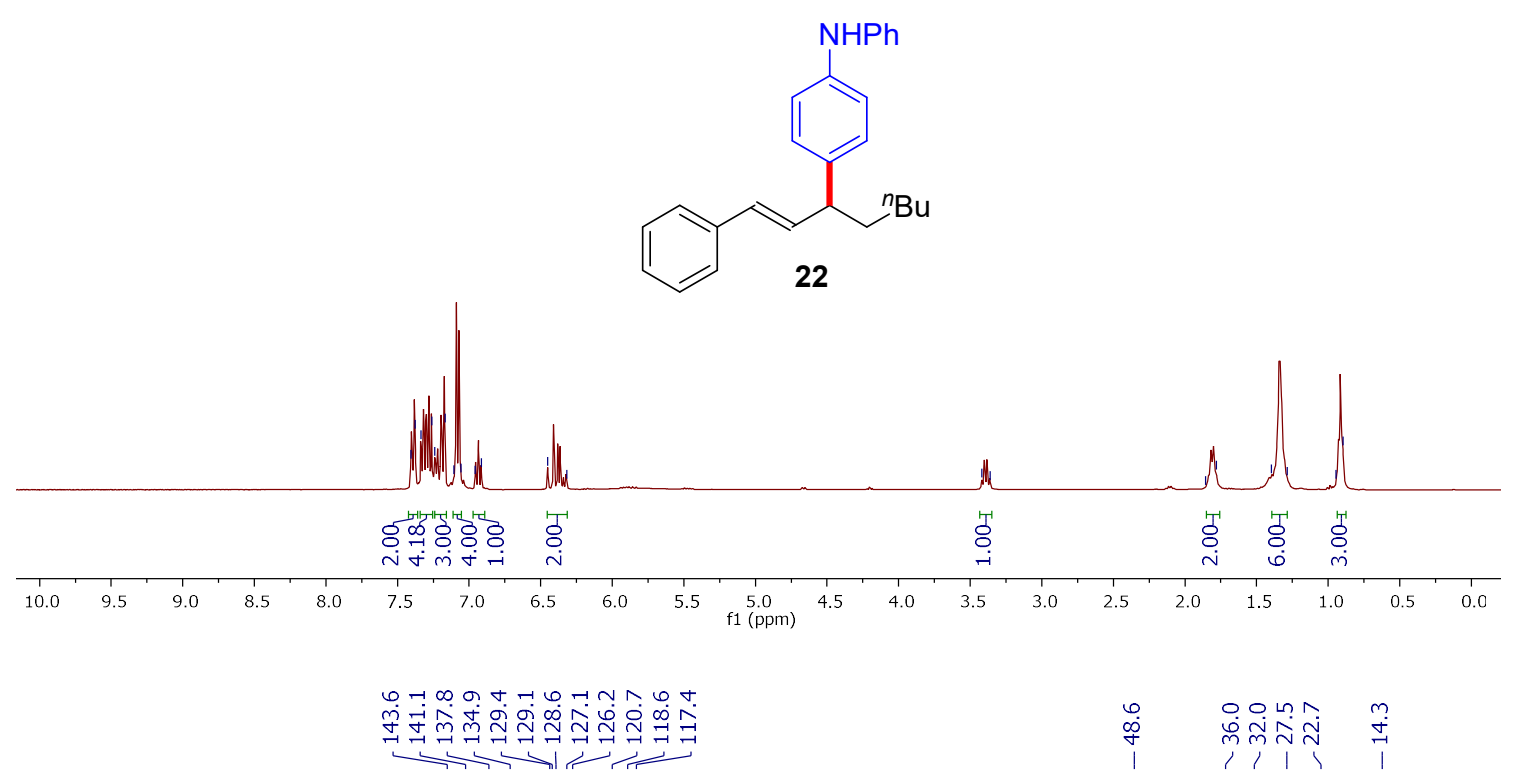

${ }^{13} \mathrm{C}$ NMR (101 MHz, $\mathrm{CDCl}_{3}$ )<smiles>CC(C)(C)CC(/C=C/c1ccccc1)c1ccc(Nc2ccccc2)cc1</smiles>
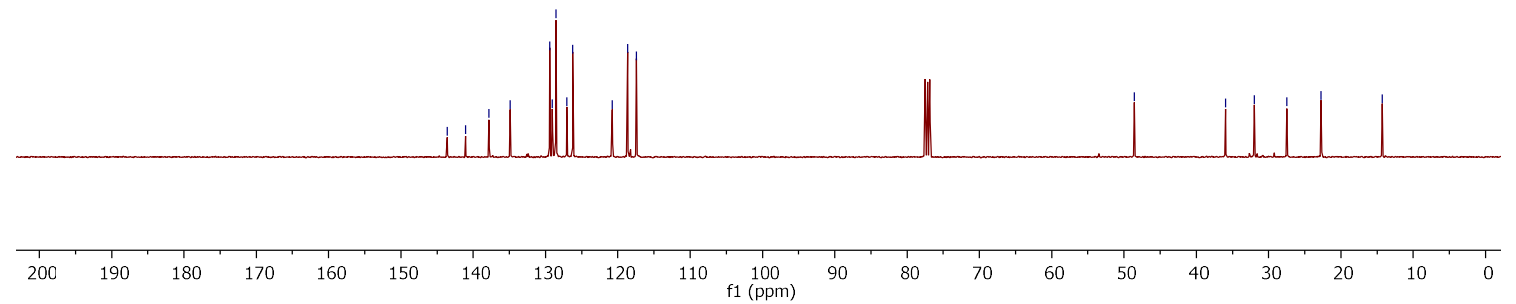
(E)-N-phenyl-4-(6-phenylhex-3-en-2-yl)aniline (23)

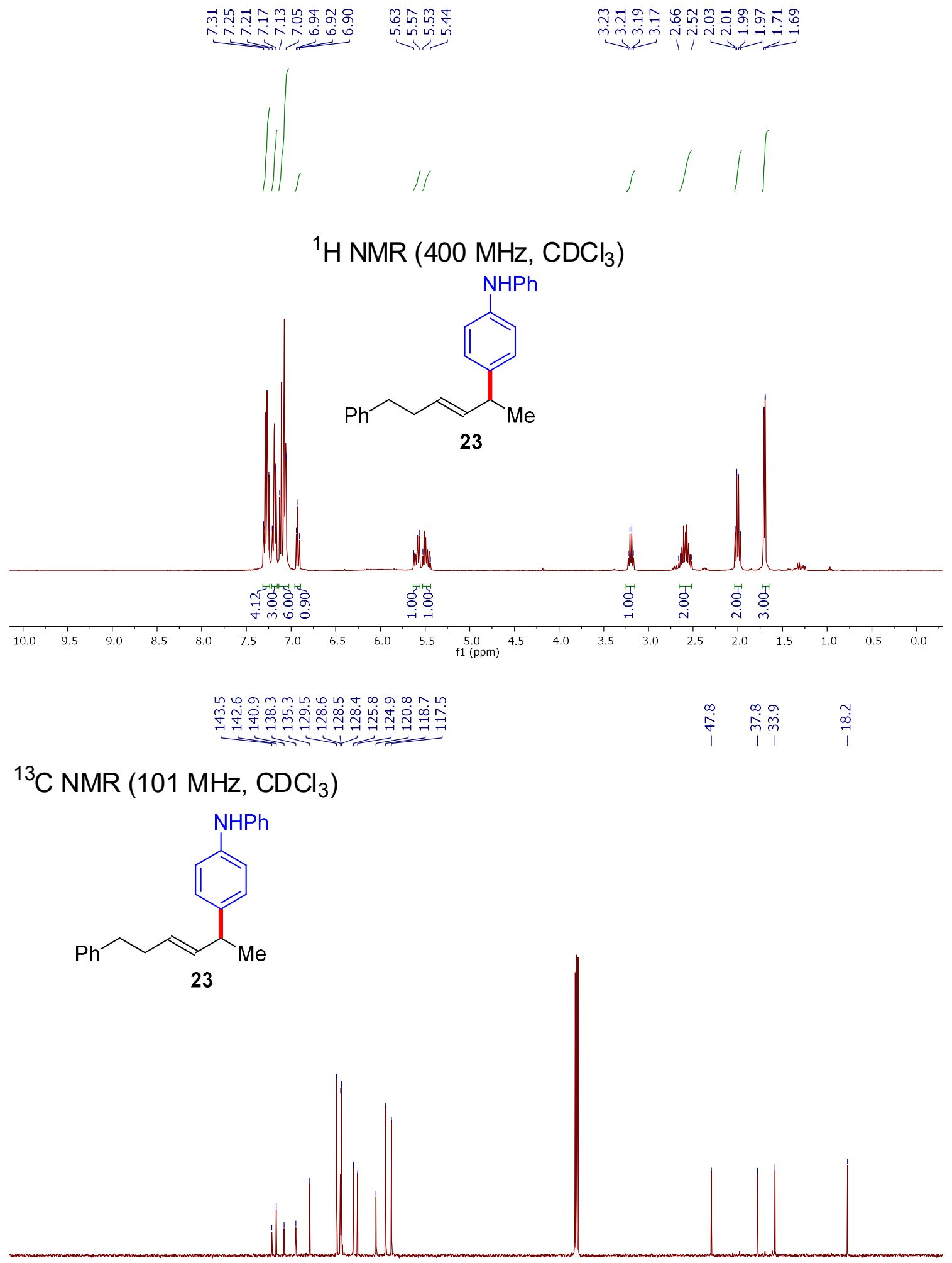

$\begin{array}{lllllllllllllllllllll}100 & 190 & 180 & 170 & 160 & 150 & 140 & 130 & 120 & 110 & 100 & 10 & 80 & 70 & 60 & 50 & 40 & 30 & 20 & 10 & 0\end{array}$ 
(E)-4-(oct-3-en-2-yl)-N-phenylaniline (24)

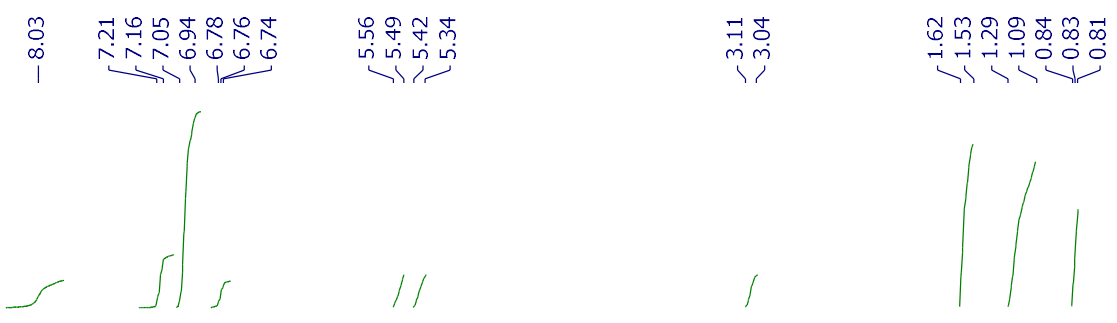

${ }^{1} \mathrm{H}$ NMR $(400 \mathrm{MHz}$, DMSO-D $)$

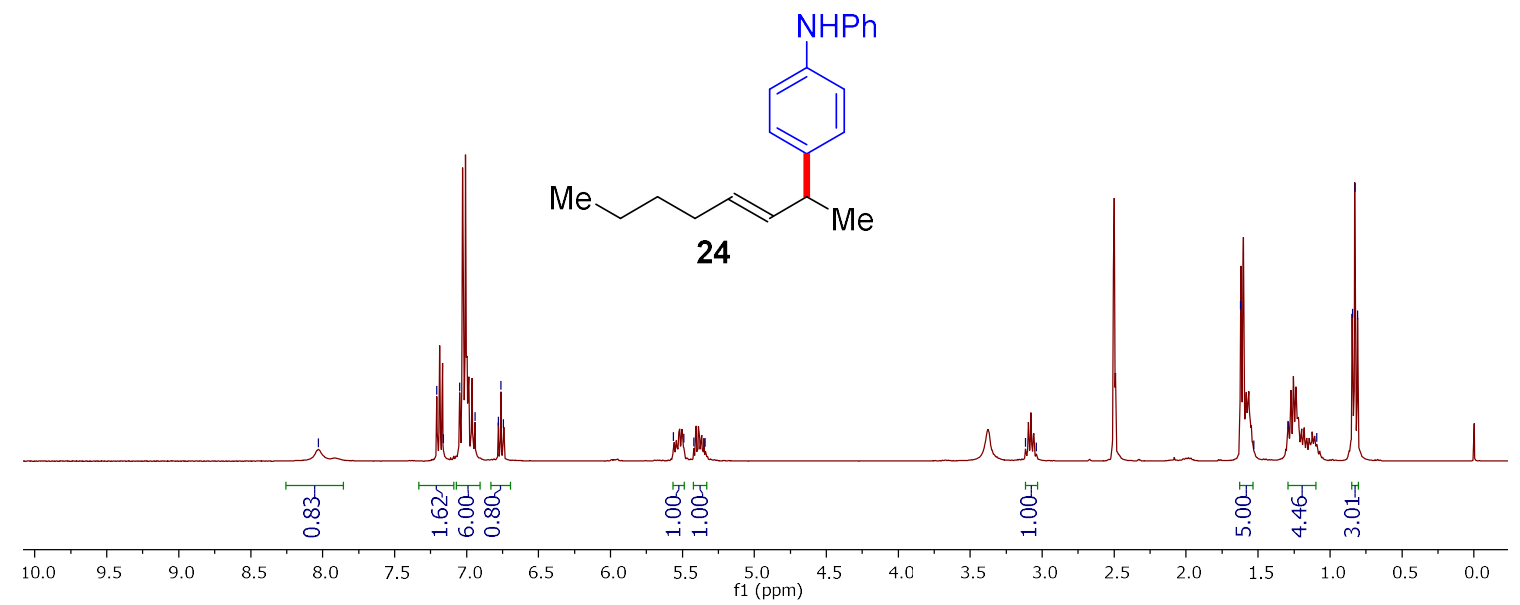

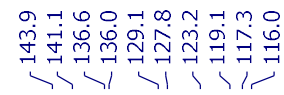

守 $\quad$ miñ

${ }^{13} \mathrm{C}$ NMR (101 MHz, DMSO-D 6 )

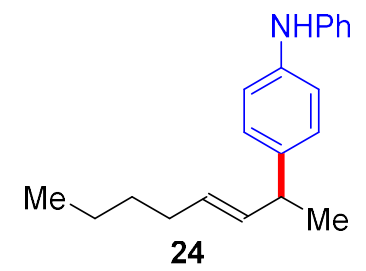

t ||$+\mid$

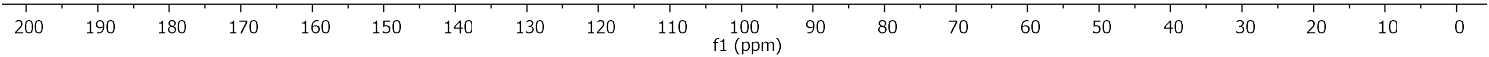


(E)-4-(1-cyclohexylbut-2-en-1-yl)-N-phenylaniline (25)

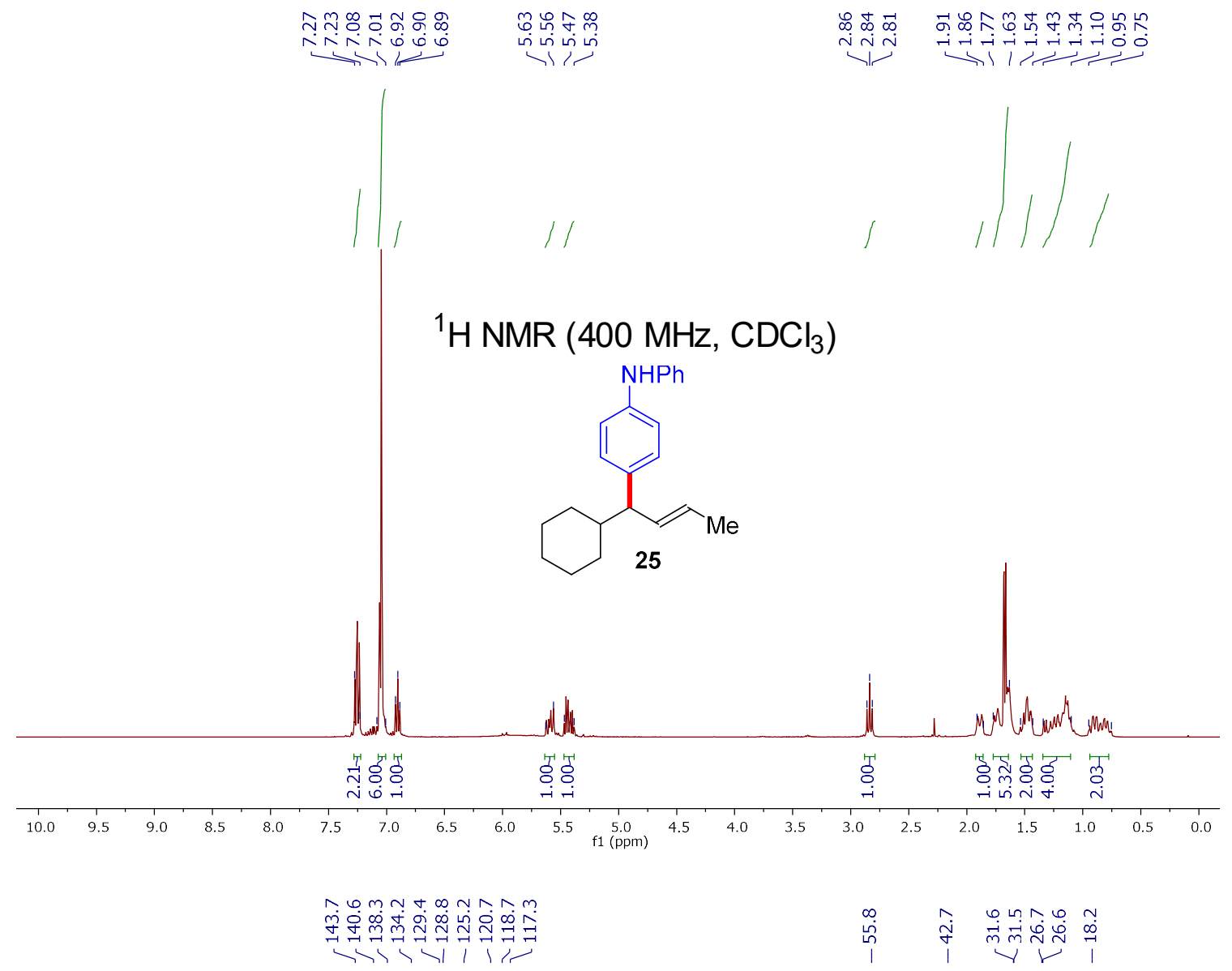

${ }^{13} \mathrm{C}$ NMR $\left(101 \mathrm{MHz}, \mathrm{CDCl}_{3}\right)$<smiles>C/C=C/C(c1ccc(Nc2ccccc2)cc1)C1CCCCC1</smiles>

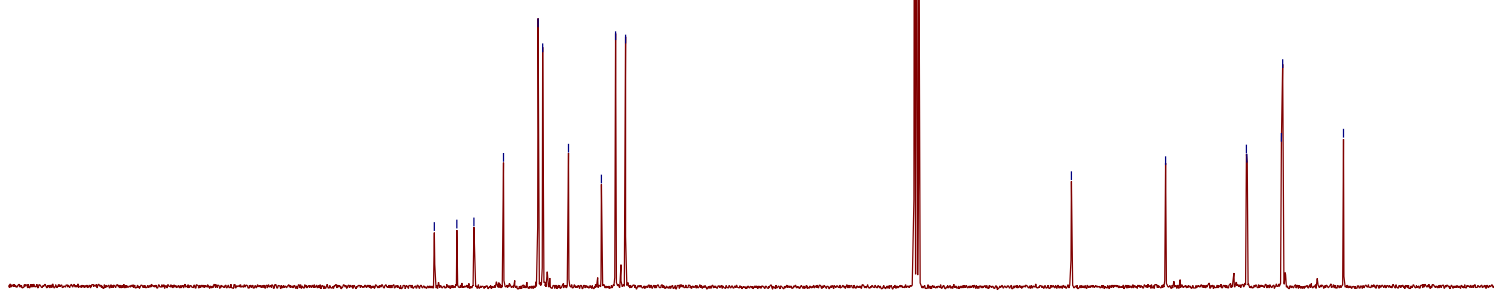

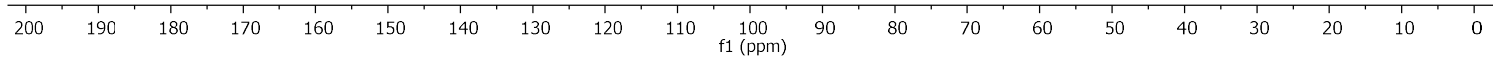


(E)-N-phenyl-4-(4-phenylpent-3-en-2-yl)aniline (26)

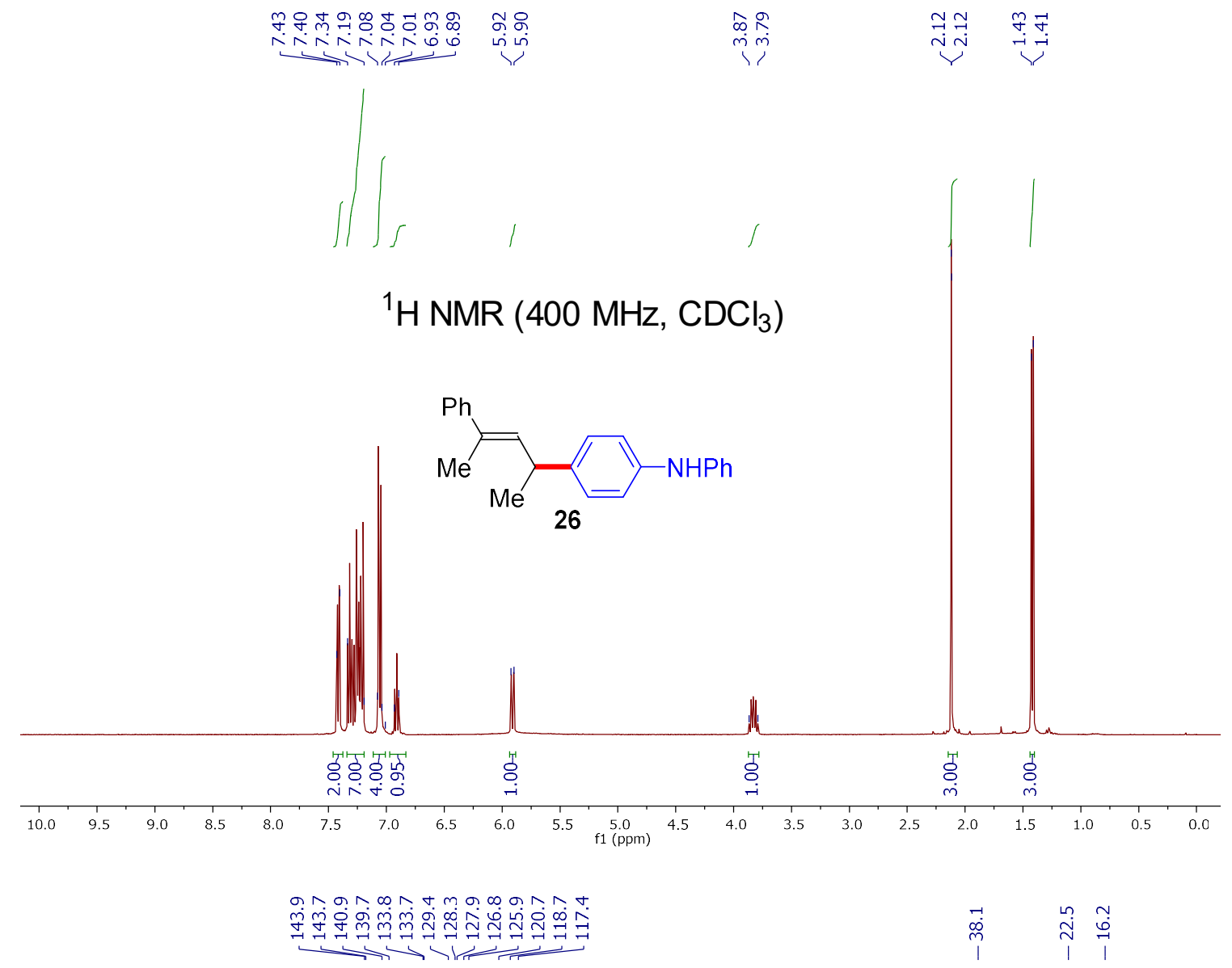

${ }^{13} \mathrm{C}$ NMR $\left(101 \mathrm{MHz}, \mathrm{CDCl}_{3}\right)$
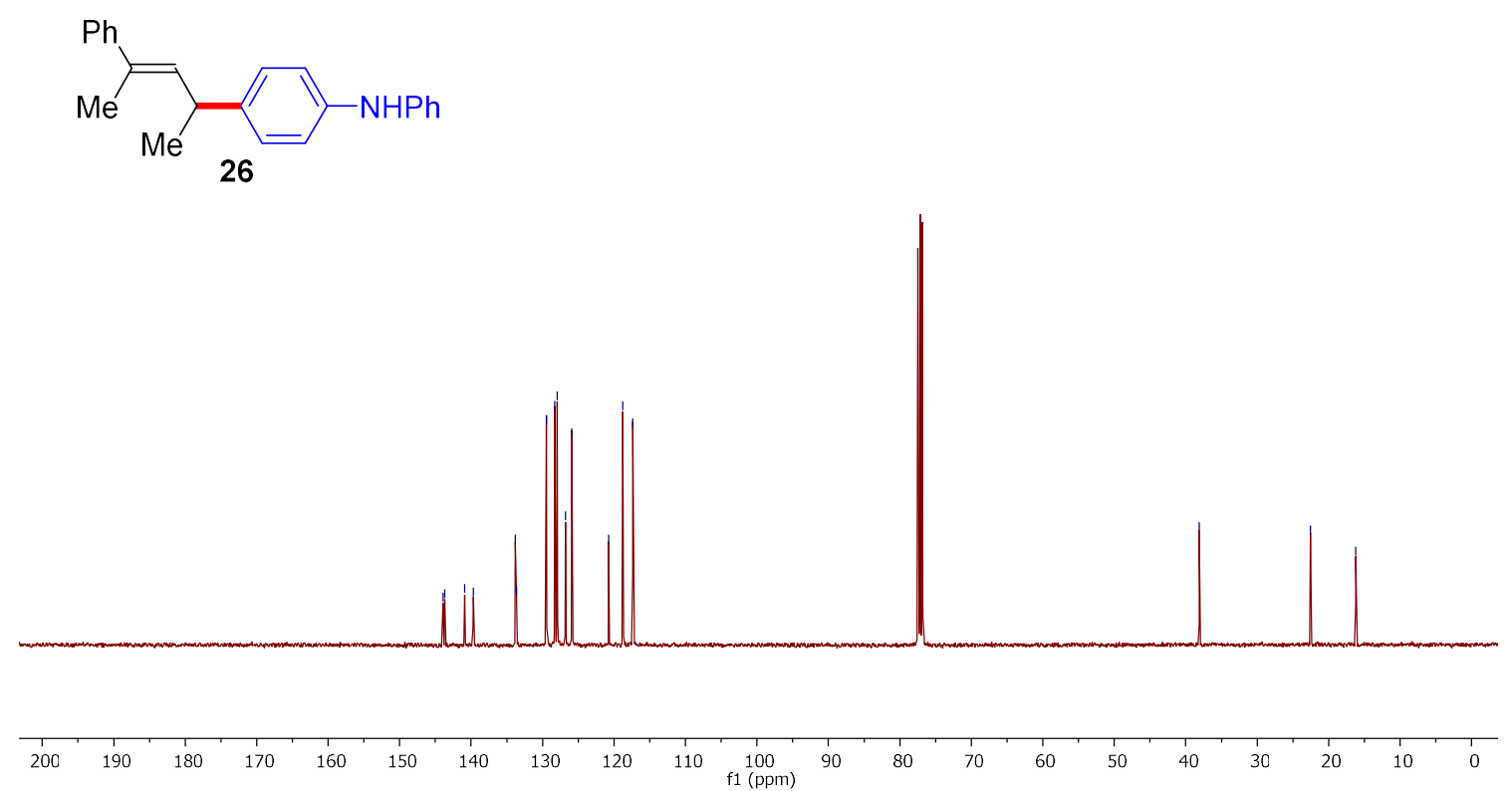
4-(4,4-diphenylbut-3-en-2-yl)-N-phenylaniline (27)
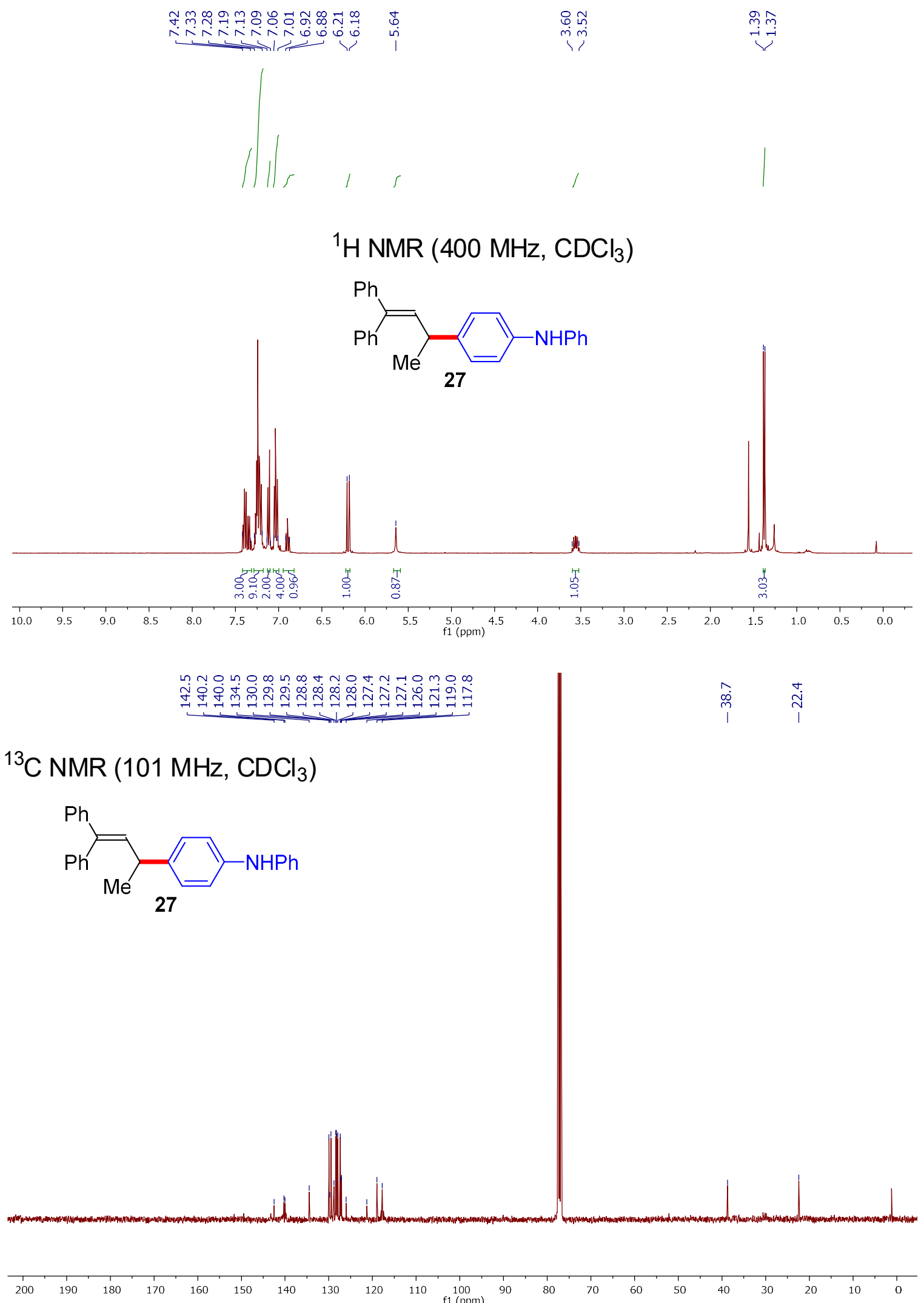
4-(3-methylbut-2-en-1-yl)-N-phenylaniline (28)

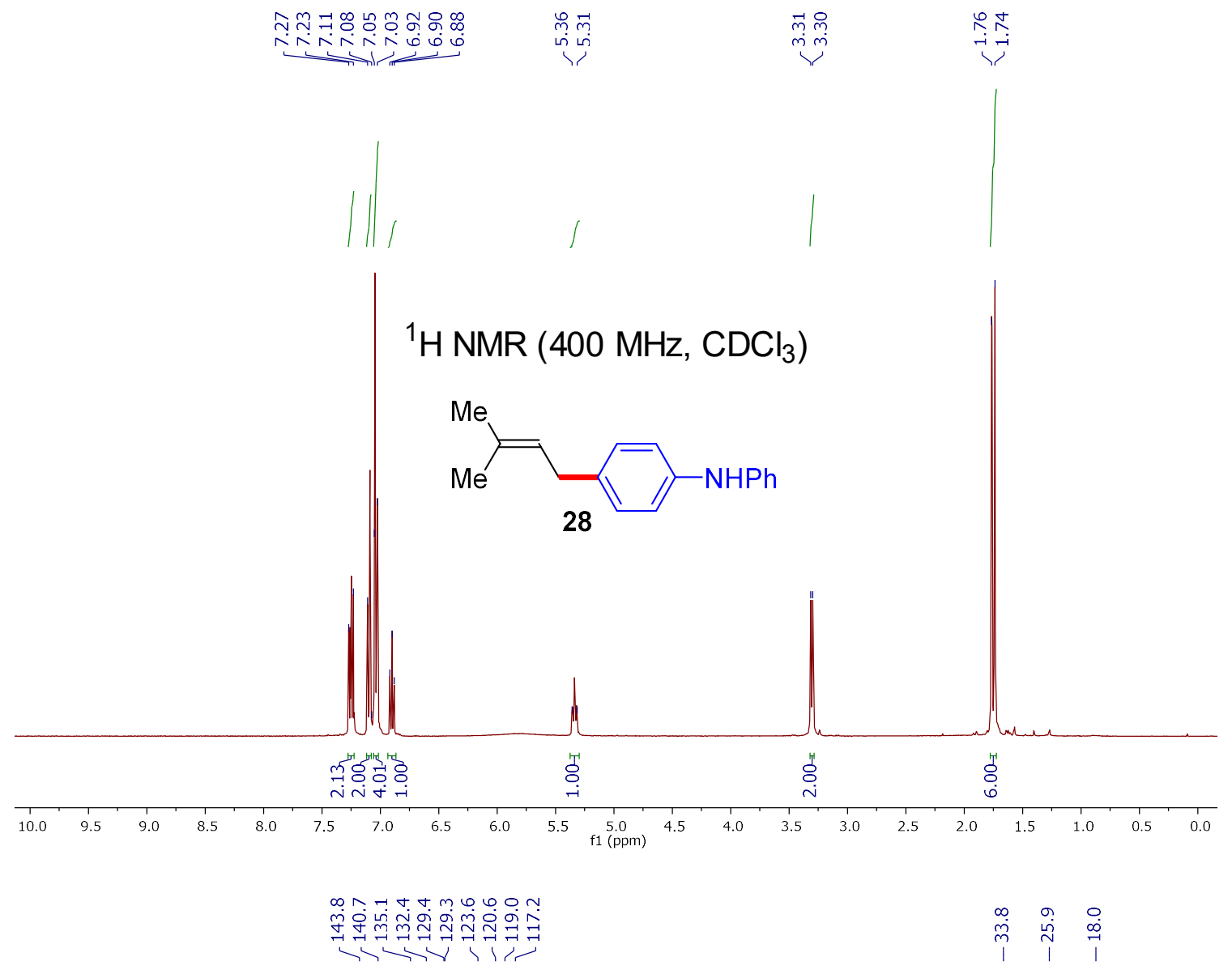

${ }^{13} \mathrm{C}$ NMR $\left(101 \mathrm{MHz}, \mathrm{CDCl}_{3}\right)$
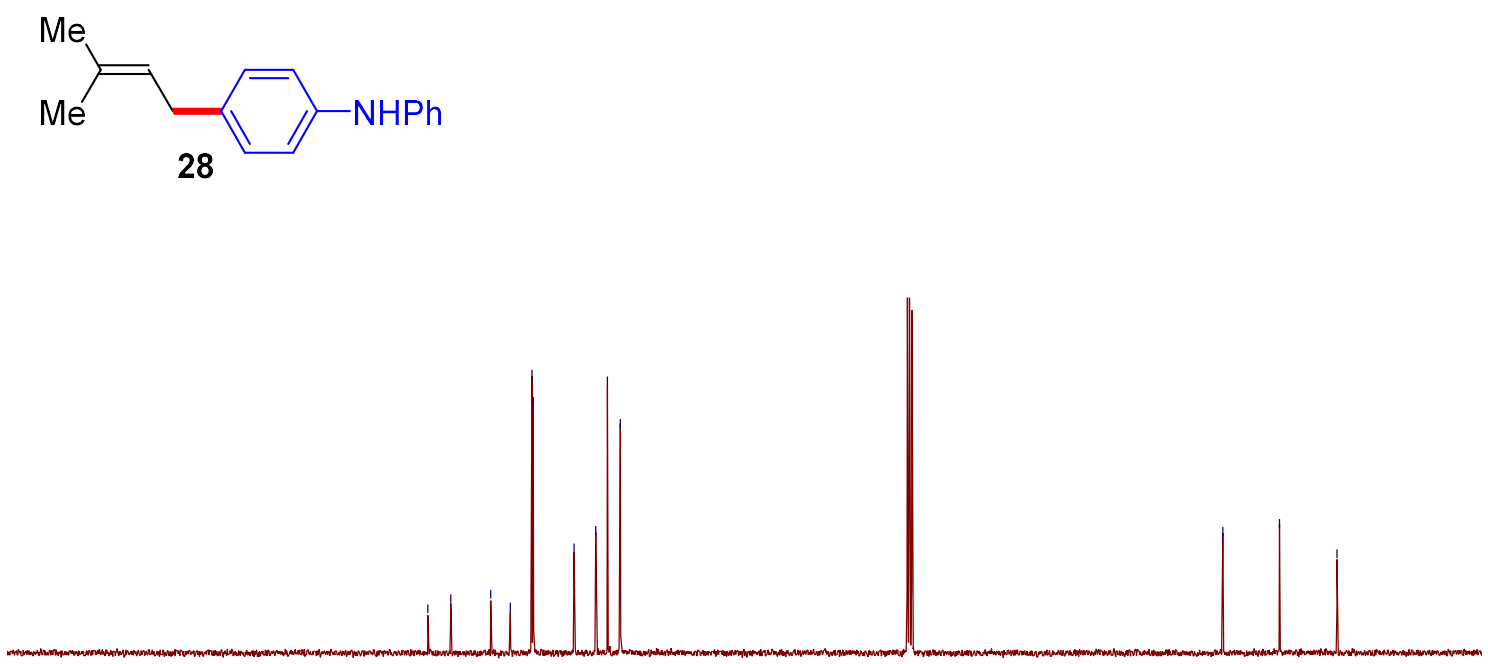

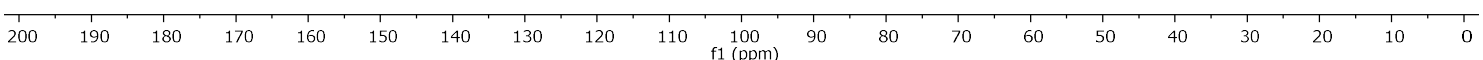


(E)-4-(3,7-dimethylocta-2,6-dien-1-yl)-N-phenylaniline (29)
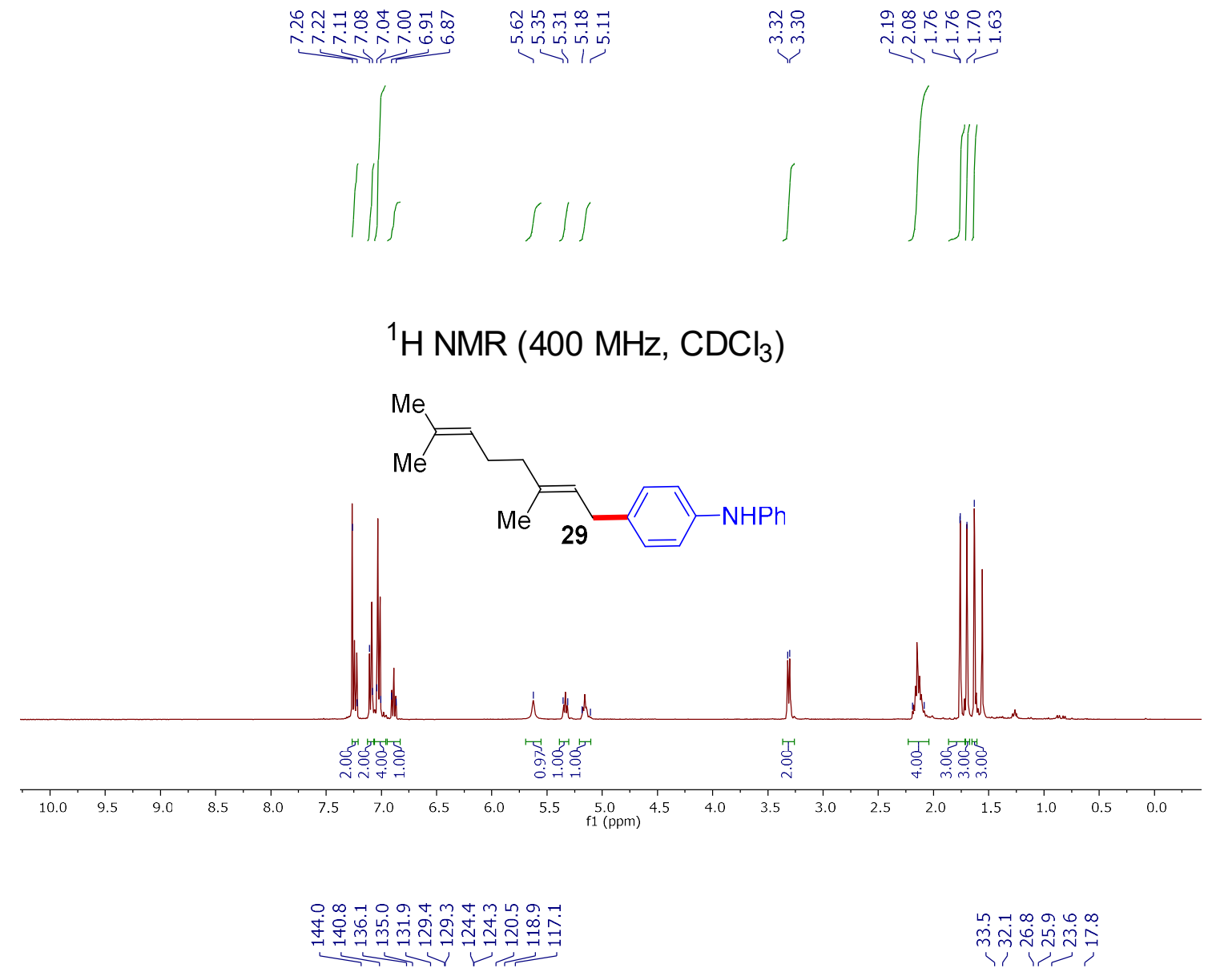

${ }^{13} \mathrm{C}$ NMR $\left(101 \mathrm{MHz}, \mathrm{CDCl}_{3}\right)$
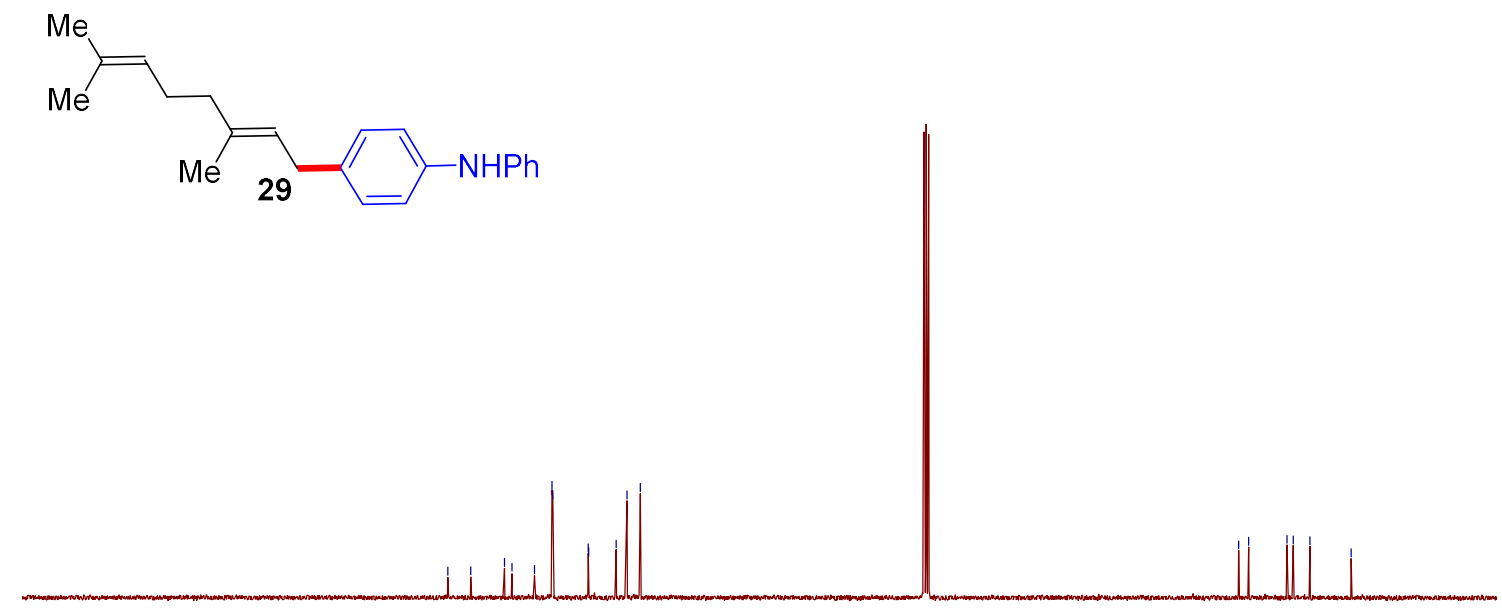

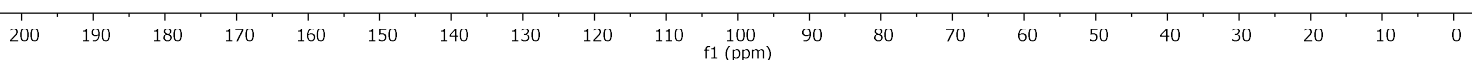


(E)-4-(2-methyl-4-phenylbut-3-en-2-yl)-N-phenylaniline

and

4-(3-methyl-1phenylbut-2-en-1-yl)-N-phenylaniline $\left(30+30^{\prime}\right)$

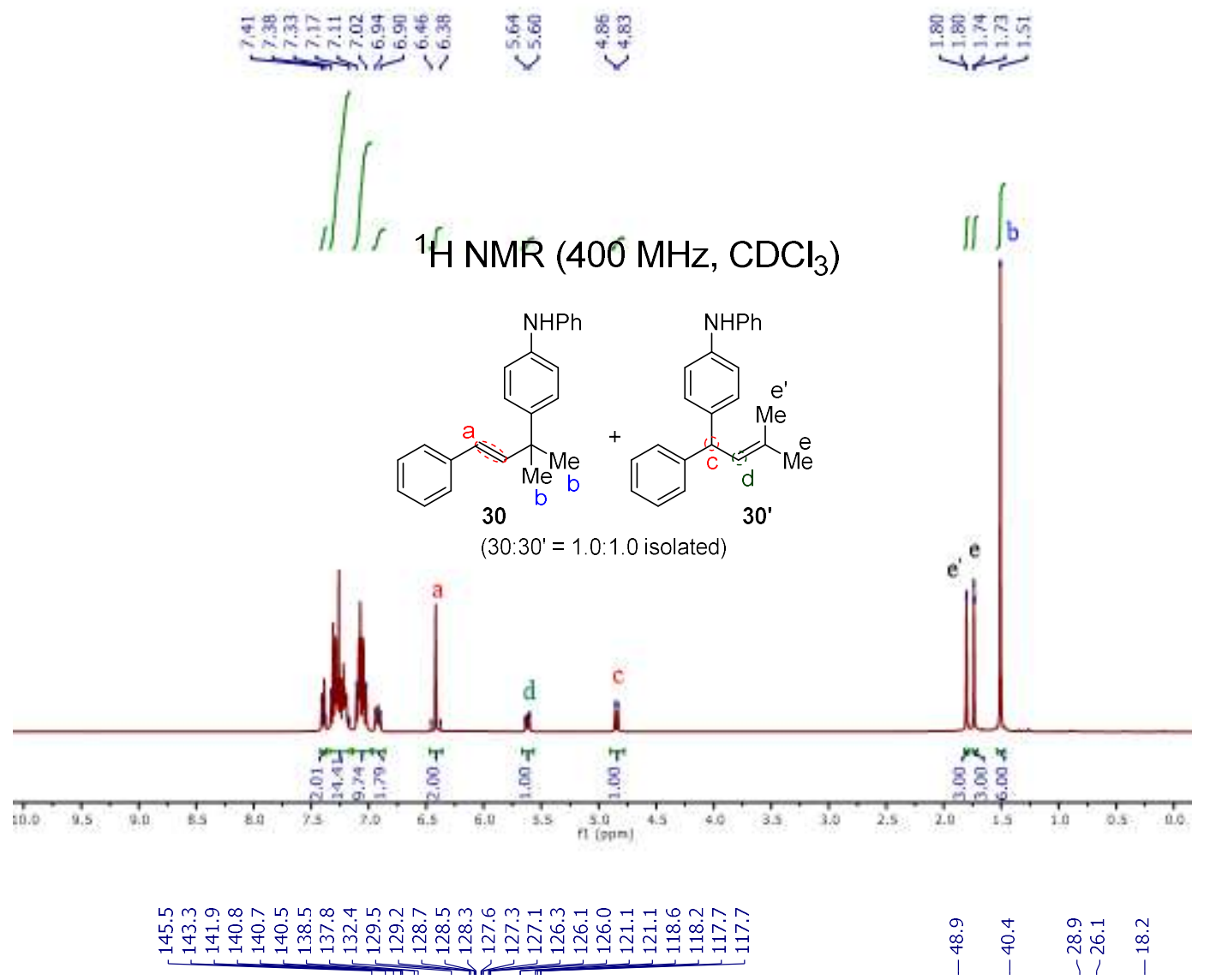

${ }^{13} \mathrm{C}$ NMR $\left(101 \mathrm{MHz}, \mathrm{CDCl}_{3}\right)$<smiles>CC(C)=CC(c1ccccc1)c1ccc(Nc2ccccc2)cc1</smiles>

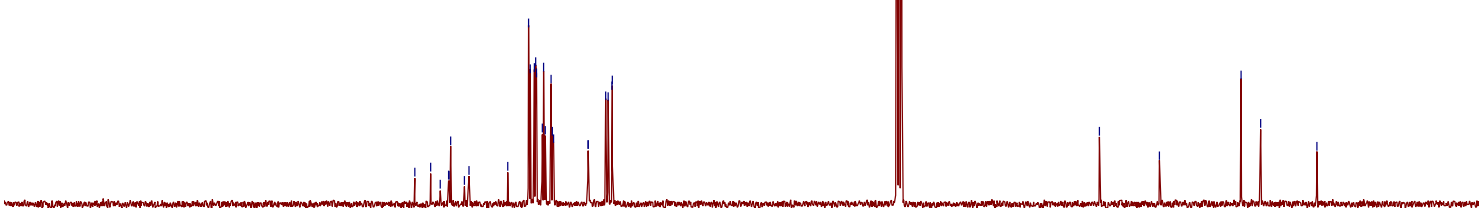

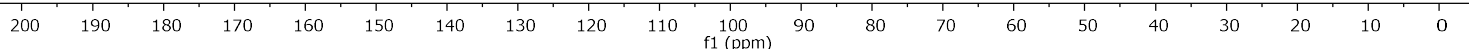


(E)-4-(3-methyl-1-phenylpent-1-en-3-yl)-N-phenylaniline and 4-(3-methyl-1phenylpent-2-en-1-yl)-N-phenylaniline $\left(31+31^{\prime}\right)$

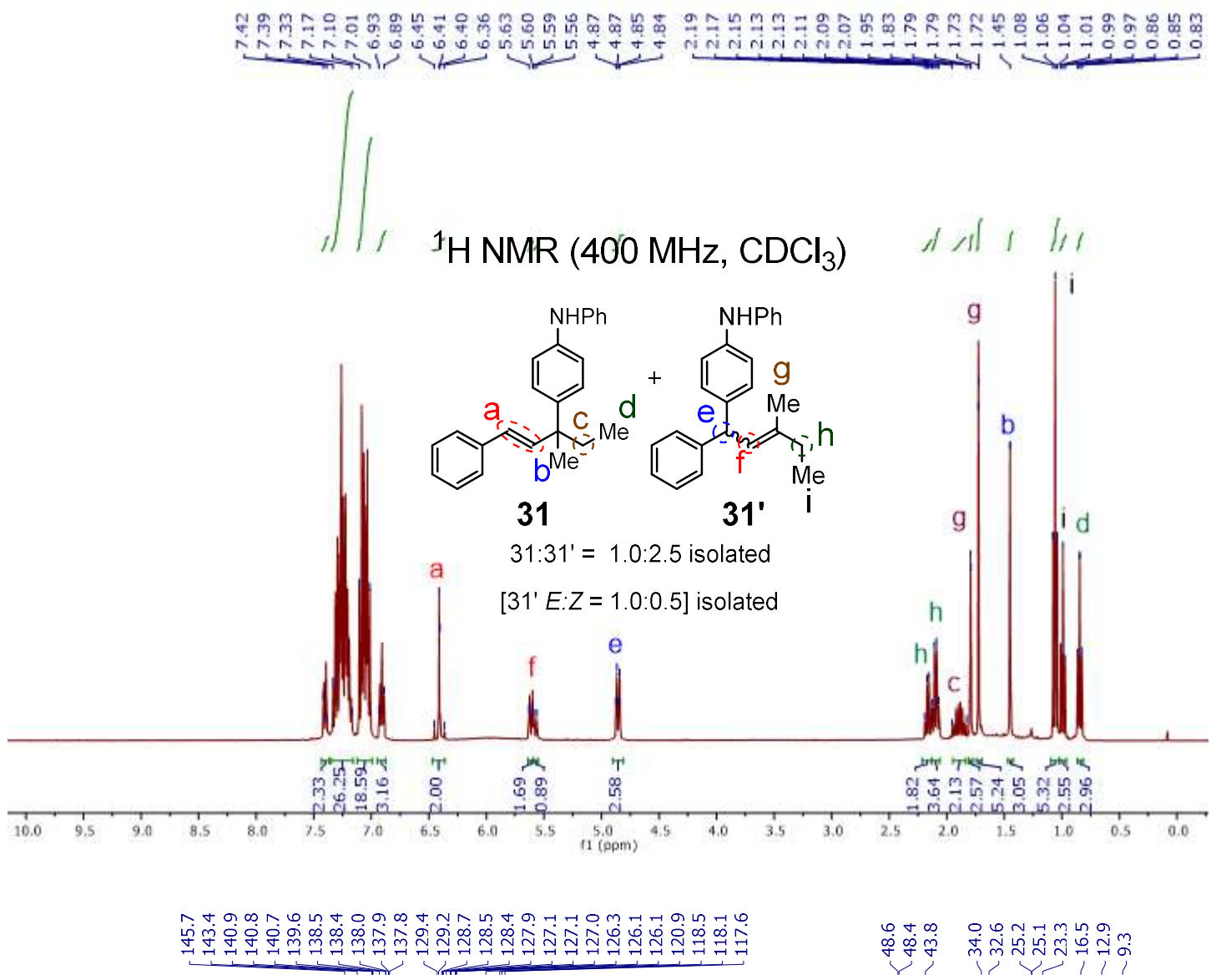

${ }^{13} \mathrm{C}$ NMR $\left(101 \mathrm{MHz}, \mathrm{CDCl}_{3}\right)$<smiles>CC(C)C=C(c1ccccc1)c1ccc(Nc2ccccc2)cc1</smiles>
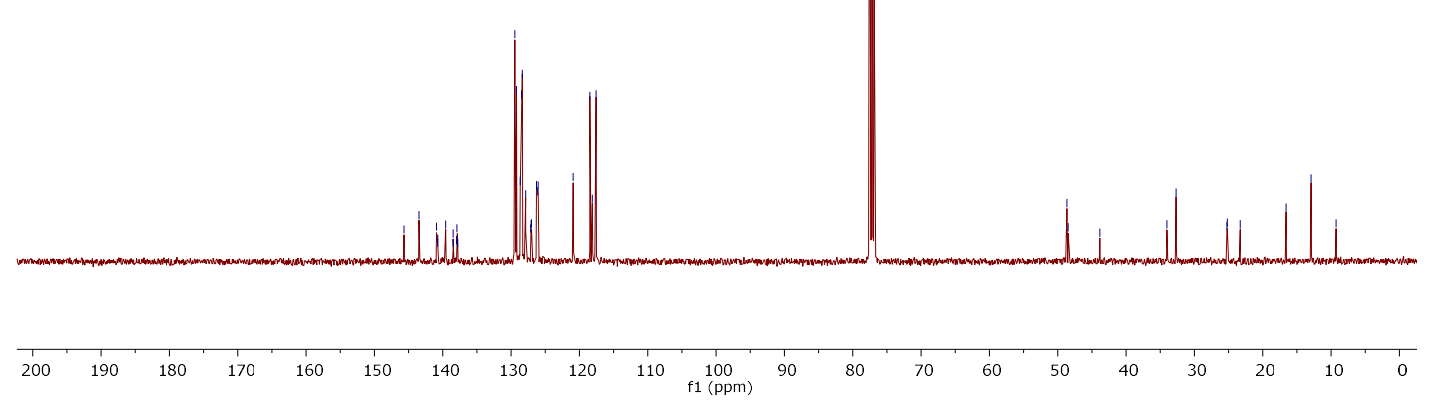


\section{4-(1,3-diphenylpent-2-en-1-yl)-N-phenylaniline (32)}

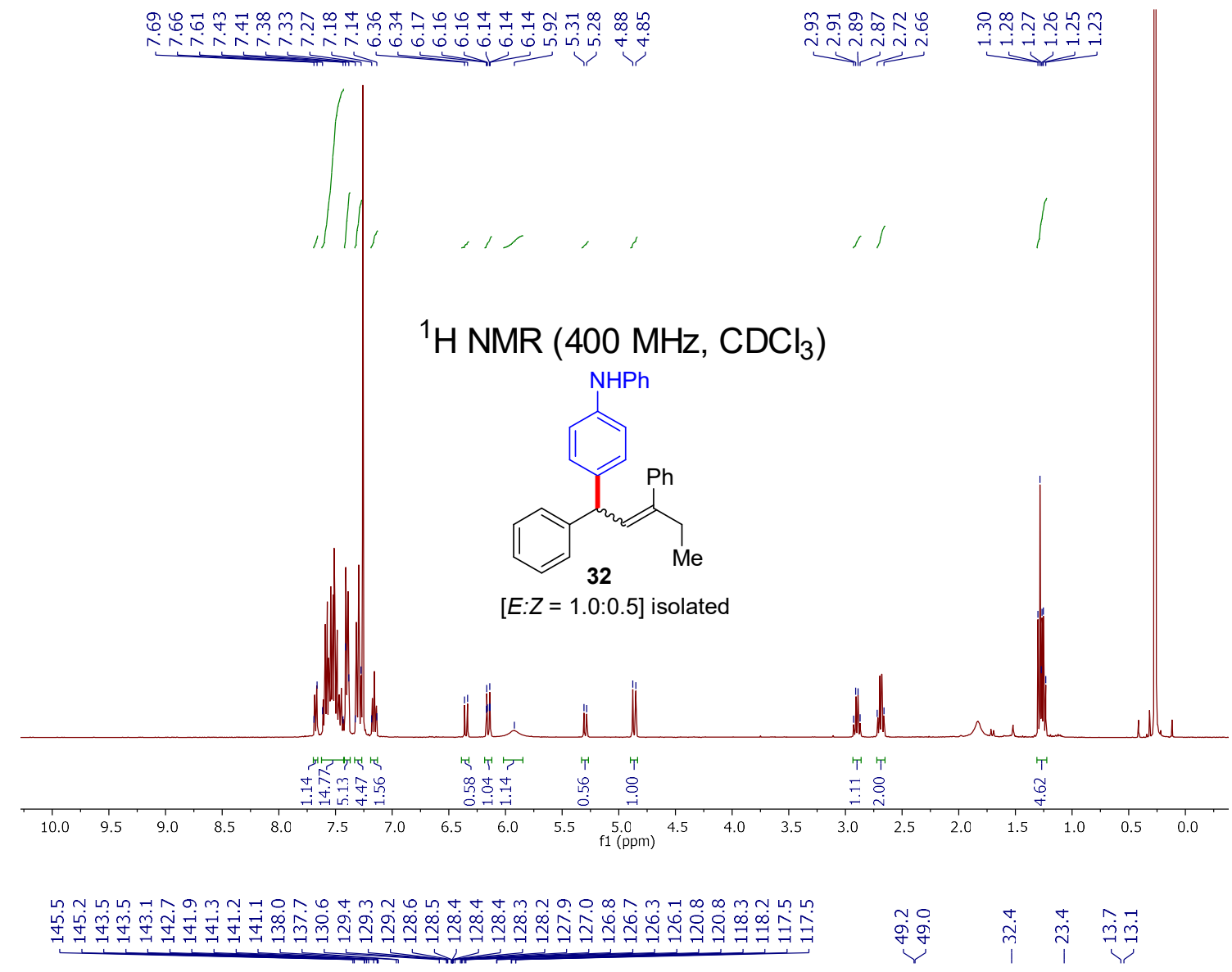

${ }^{13} \mathrm{C}$ NMR (101 MHz, CDCl 3 )

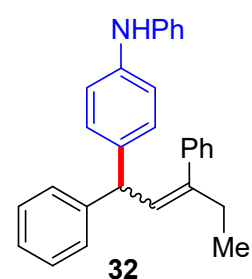

$[E: Z=1.0: 0.5]$ isolated

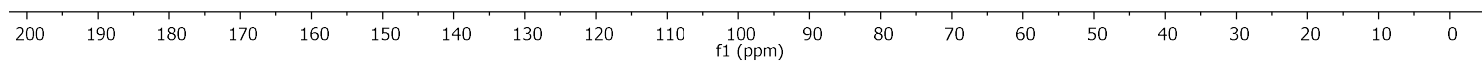


(E)-4-(2-methyl-1,4-diphenylbut-3-en-2-yl)-N-phenylaniline and 4-(3-methyl-1,4diphenylbut-2-en-1-yl)-N-phenylaniline (33+33')

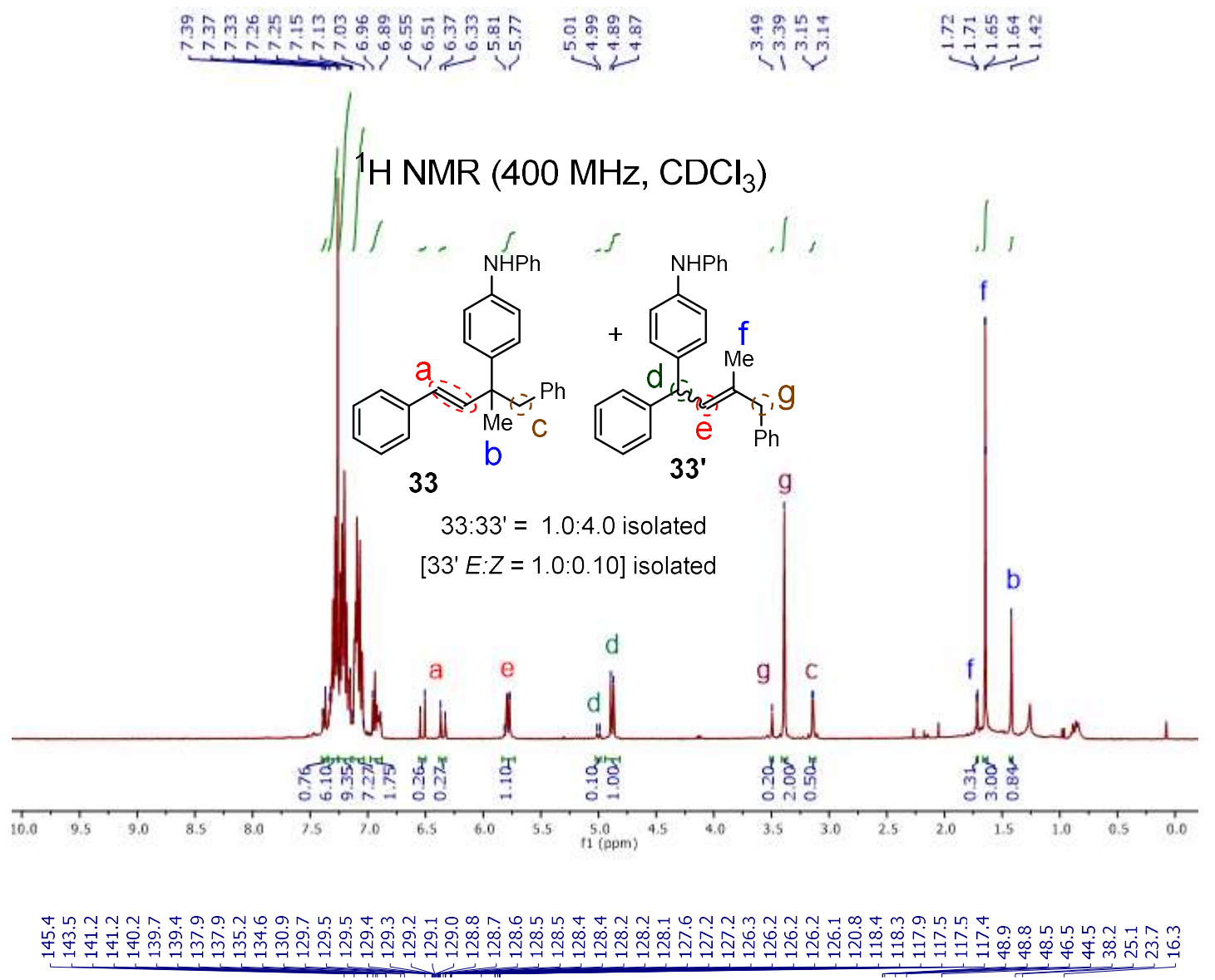

${ }^{13} \mathrm{C}$ NMR $\left(101 \mathrm{MHz}, \mathrm{CDCl}_{3}\right)$

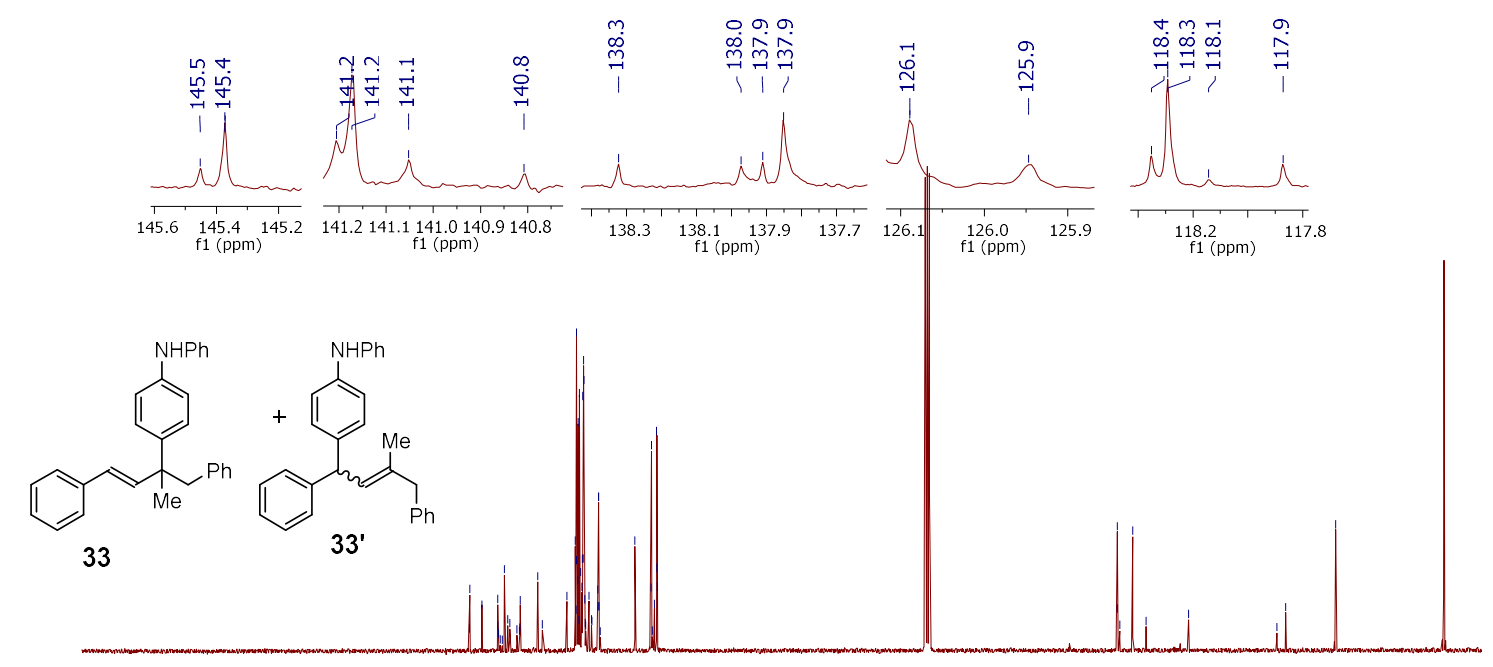

$\begin{array}{lllllllllllllllllllllll}1 & 1 \\ 200 & 190 & 180 & 170 & 160 & 150 & 140 & 130 & 120 & 110 & 100 & 10 & 10 & 70 & 60 & 50 & 40 & 30 & 20 & 10 & 0\end{array}$ 


\section{N-phenyl-4-(1,3,4-triphenylbut-2-en-1-yl)aniline (34)}

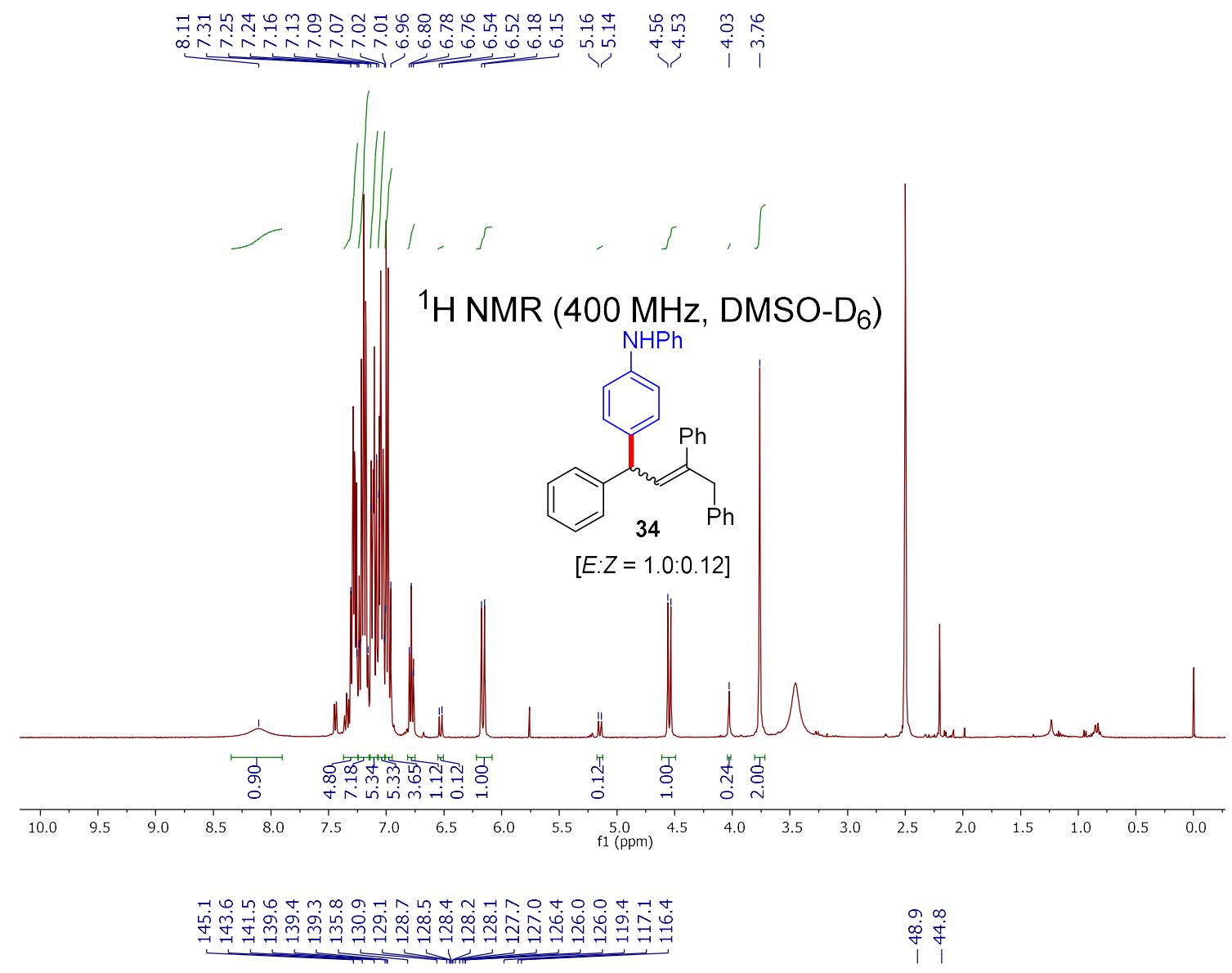

${ }^{13} \mathrm{C}$ NMR $\left(101 \mathrm{MHz}, \mathrm{DMSO}-\mathrm{D}_{6}\right)$<smiles>C(=C/c1ccccc1)\c1ccccc1</smiles>

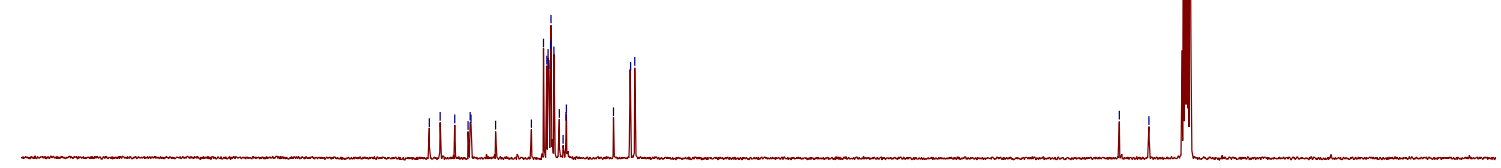

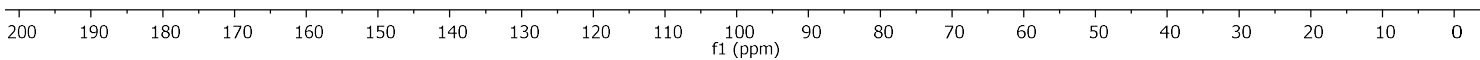




\section{N-phenyl-1',2',3',4'-tetrahydro-[1,1'-biphenyl]-2-amine (35)}
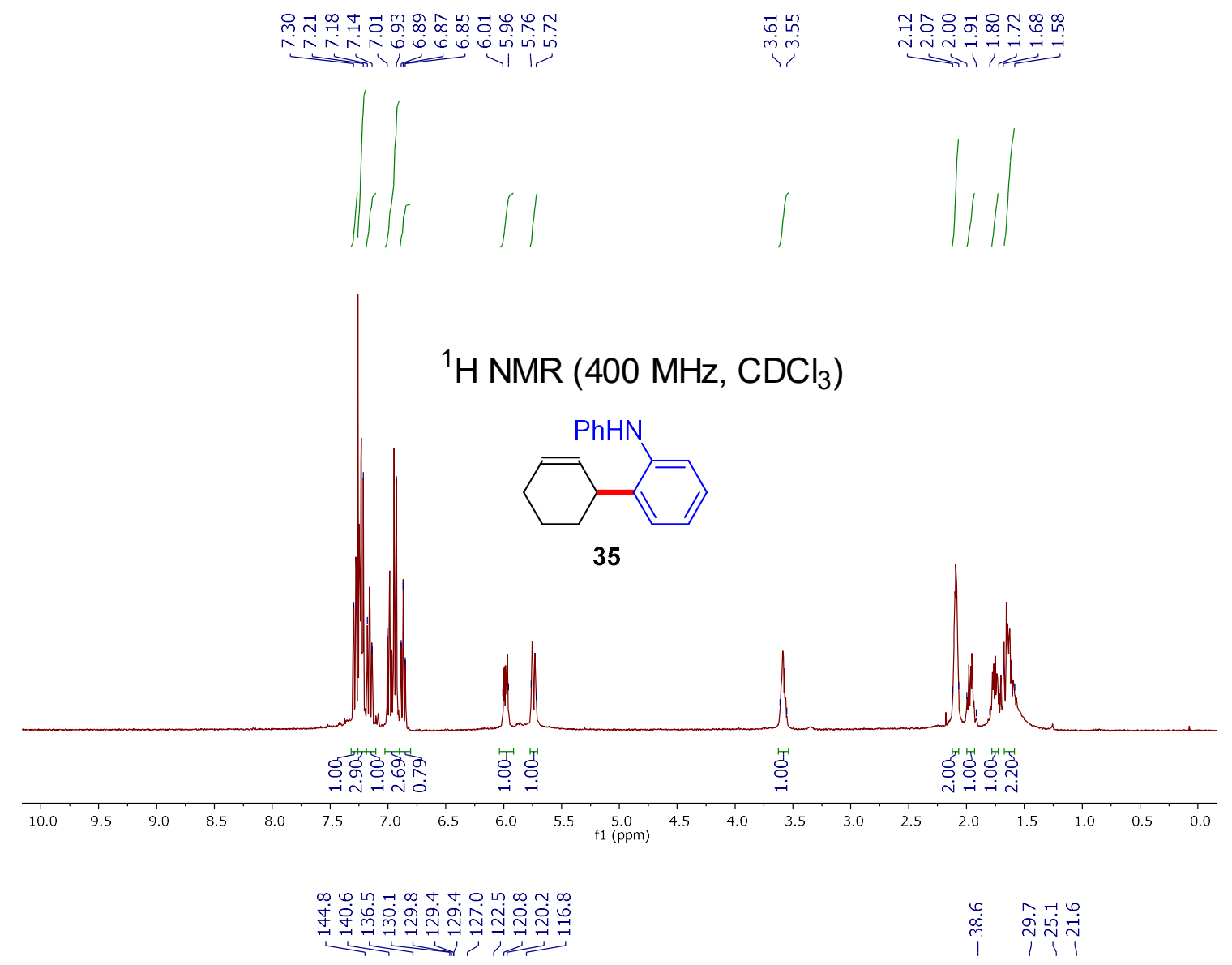

${ }^{13} \mathrm{C} \mathrm{NMR}\left(101 \mathrm{MHz}, \mathrm{CDCl}_{3}\right)$

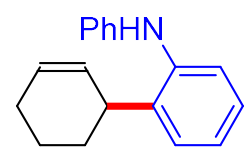

35

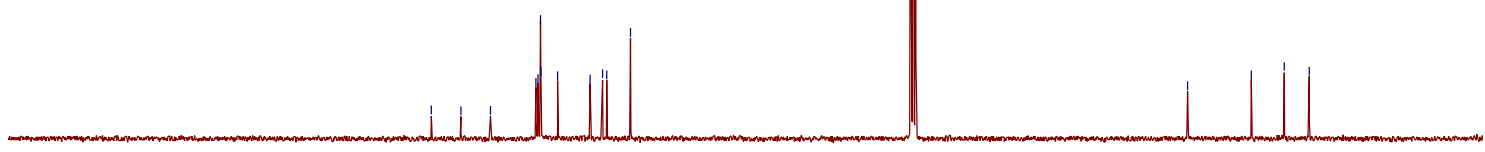

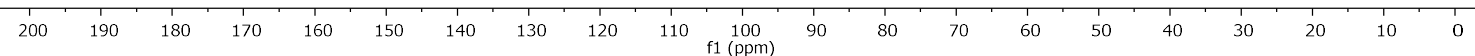




\section{5-methyl-N-(p-tolyl)-1',2',3',4'-tetrahydro-[1,1'-biphenyl]-2-amine (36)}

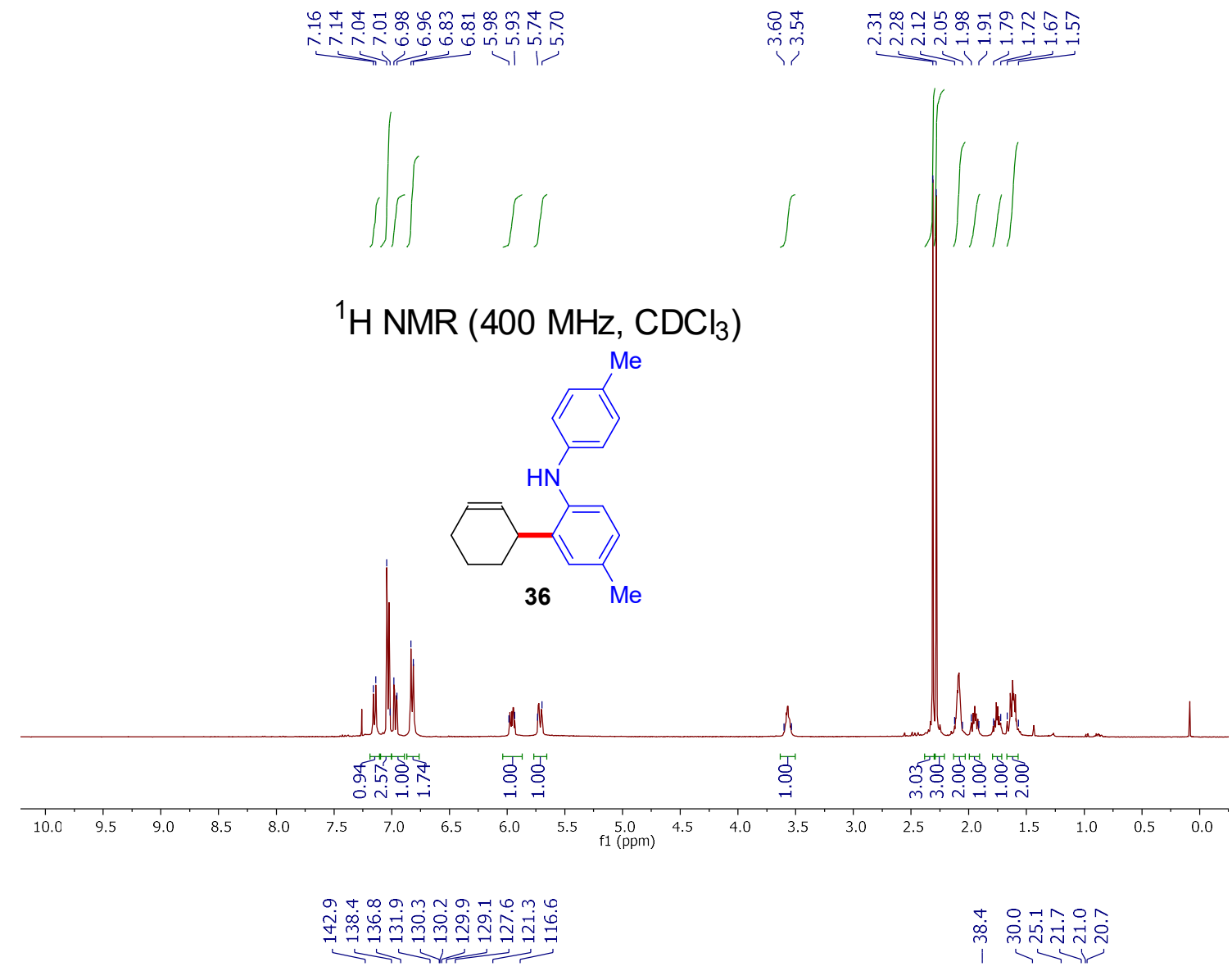

${ }^{13} \mathrm{C}$ NMR $\left(101 \mathrm{MHz}, \mathrm{CDCl}_{3}\right)$
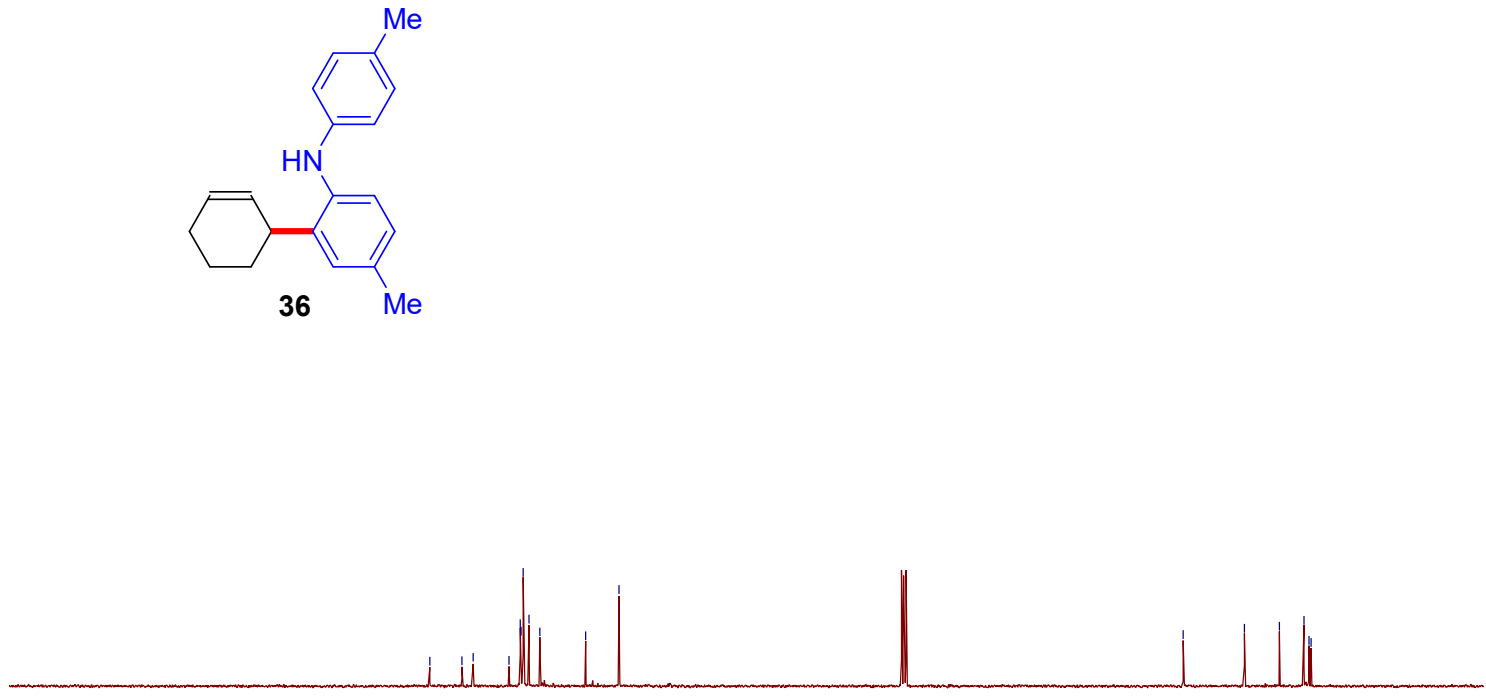

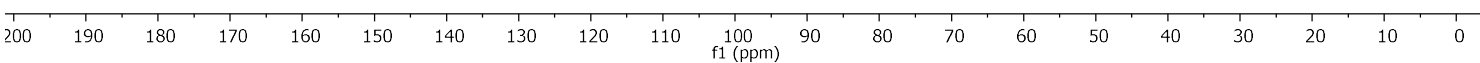


(E)-N-(4-(4-phenylbut-3-en-2-yl)phenyl-2,6- $\left.d_{2}\right)$ benzen-2,4,6- $d_{3}$-amine (3- $\left.d_{5}\right)$

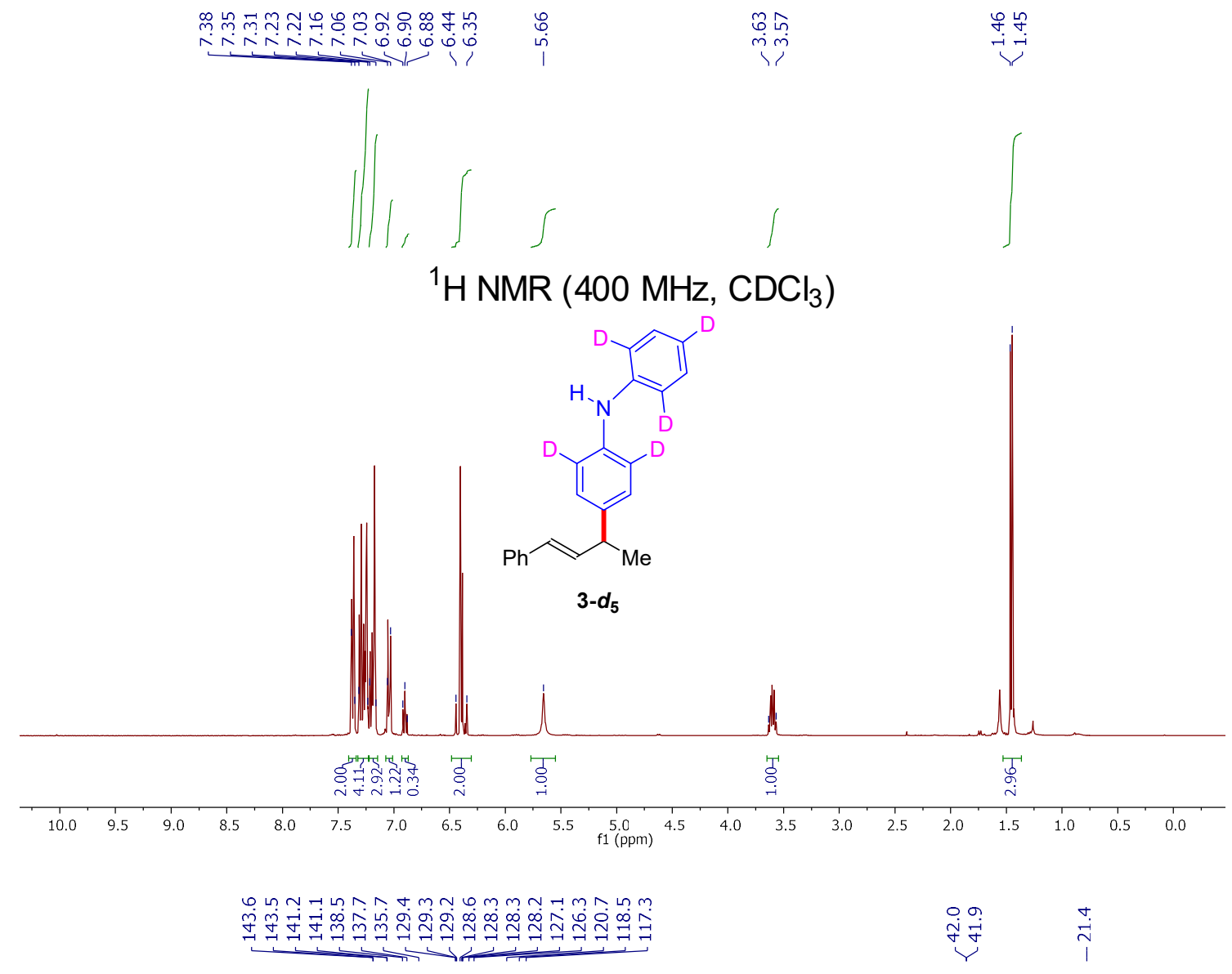

${ }^{13} \mathrm{C} \mathrm{NMR}\left(101 \mathrm{MHz}, \mathrm{CDCl}_{3}\right)$

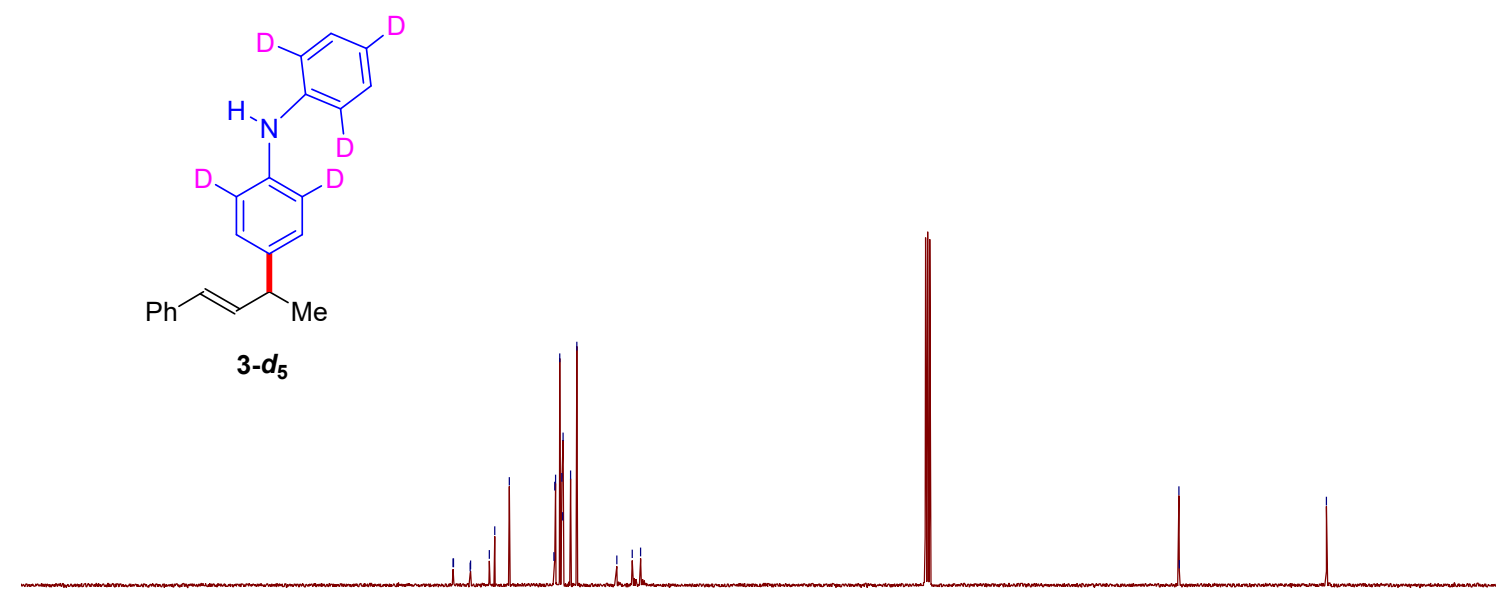

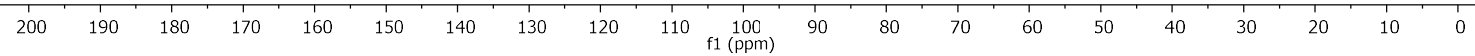


(E)-N-phenyl-4-(4-phenylbut-3-en-2-yl)aniline-d (3-d $\left.{ }_{1}\right)$

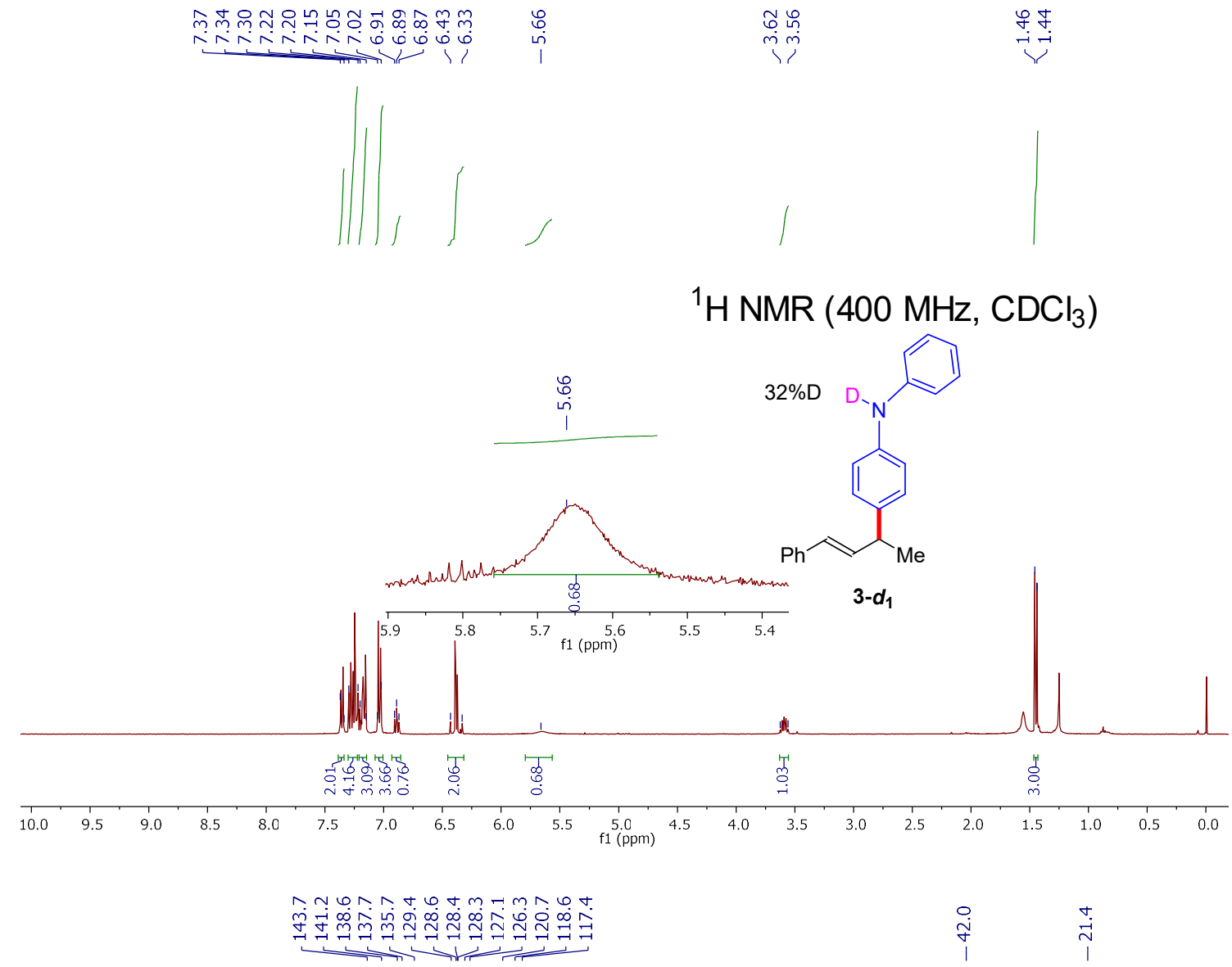

${ }^{13} \mathrm{C}$ NMR $\left(101 \mathrm{MHz}, \mathrm{CDCl}_{3}\right)$
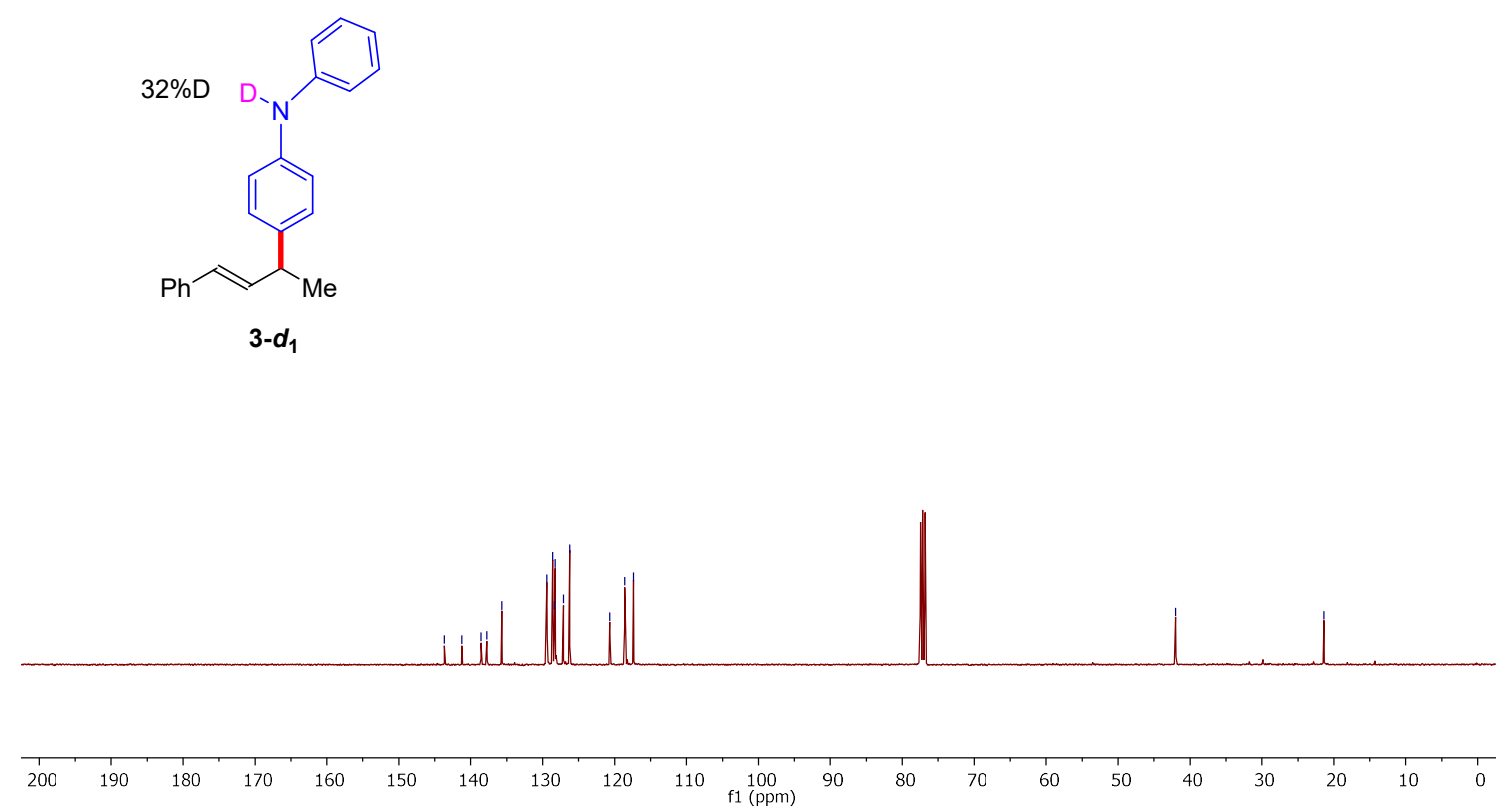
(E)-N-(4-(4-phenylbut-3-en-2-yl-1-d)phenyl-2,6- $\left.d_{2}\right)$ benzen-2,3,4,5,6- $d_{5}$-amine- $d\left(3-d_{7}\right)$
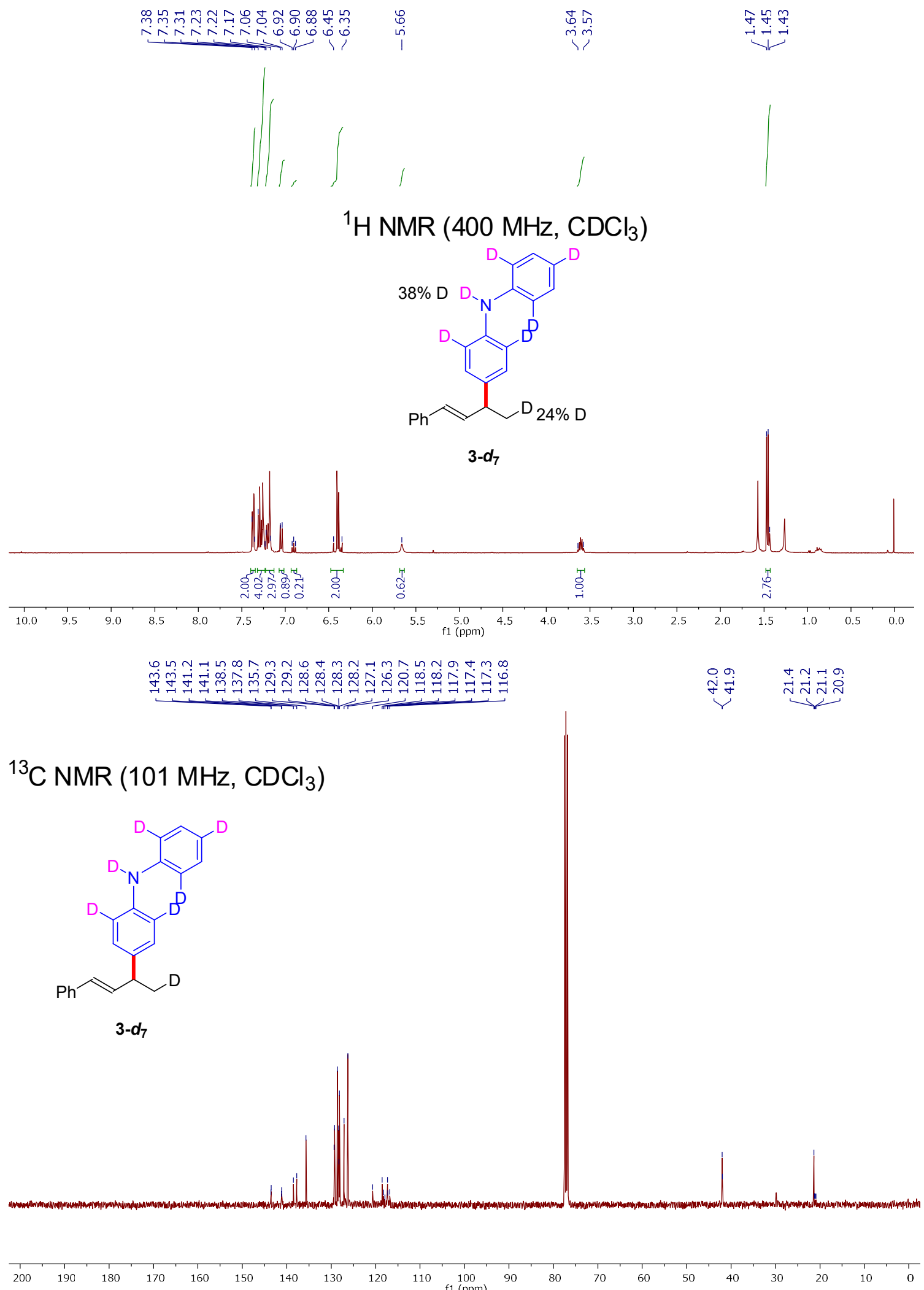
(E)-N-methyl-N-(4-(4-phenylbut-3-en-2-yl-1-d)phenyl-2,6- $\left.d_{2}\right)$ aniline-2,4,6- $d_{3}(10-d)$

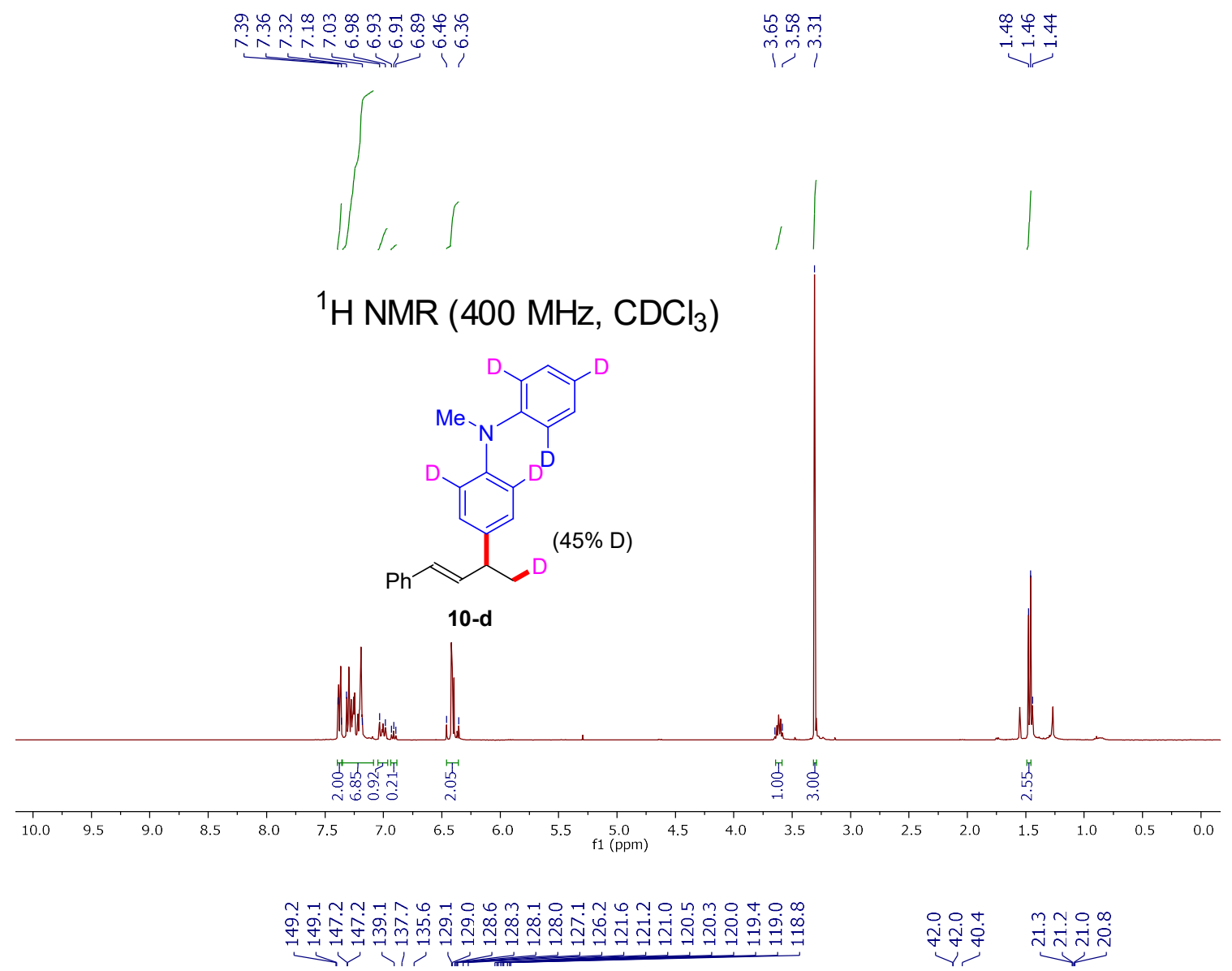

${ }^{13} \mathrm{C}$ NMR $\left(101 \mathrm{MHz}, \mathrm{CDCl}_{3}\right)$
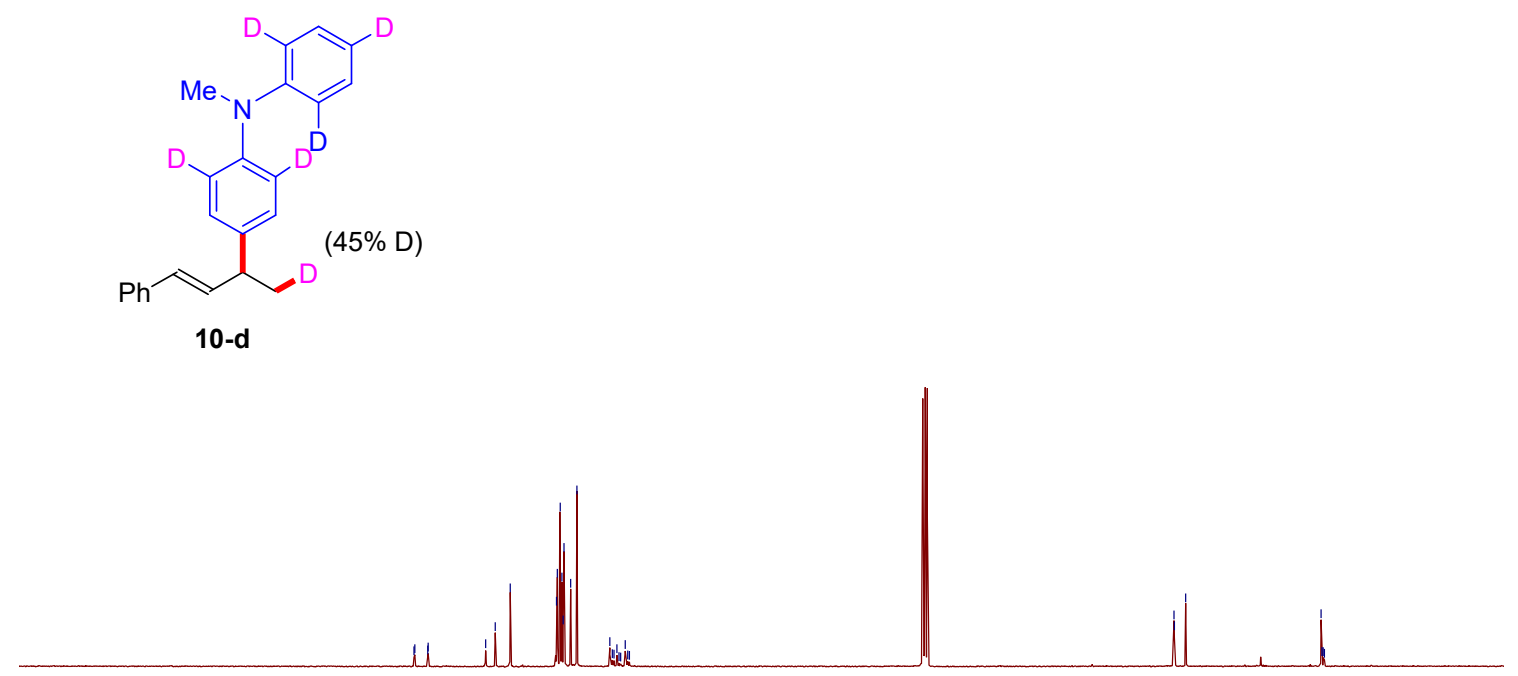

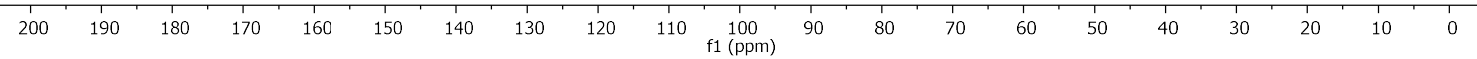




\section{DFT Calculations}

14.1. Computational Details: The quantum chemical DFT calculations have been performed with the TURBOMOLE 7.4 suite of programs ${ }^{[S 14]}$ The structures are fully optimized at the TPSS-D3/def2-TZVP + COSMO(DCE) level of theory, which combines the TPSS meta-GGA density functional[515] with the BJ-damped DFT-D3 dispersion correction ${ }^{[\mathrm{S} 16, \mathrm{~S} 17]}$ and the def2-TZVP basis set, ${ }^{[\mathrm{S} 18, \mathrm{~S} 19]}$ using the Conductor-like Screening Model (COSMO) continuum solvation mode ${ }^{[\mathrm{S} 20]}$ for 1 ,2-dichloroethane (DCE) solvent (dielectric constant $\varepsilon=10.5$ and solvent diameter $R_{\text {solv }}=3.36 \AA$ ). The densityfitting Rl-J approach ${ }^{[\mathrm{S} 18, \mathrm{S21}, \mathrm{S22]}}$ is used to accelerate the geometry optimization and numerical harmonic frequency calculations ${ }^{[\mathrm{S} 23]}$ in solution. The optimized structures are characterized by frequency analysis to identify the nature of located stationary points (no imaginary frequency for true minima and only one imaginary frequency for transition state) and to provide thermal corrections (at $298.15 \mathrm{~K}$ and $1 \mathrm{~atm}$ ) according to the modified ideal gas-rigid rotor-harmonic oscillator model. ${ }^{\left[{ }^{[24]}\right.}$ This choice of dispersion-corrected meta-GGA functional makes the efficient exploration of all potential reaction paths possible.

The final solvation free energies in DCE solution are computed with the COSMO-RS solvation model[ ${ }^{[\mathrm{25}]}$ (parameter file: BP_TZVP_19.ctd) using the COSMOtherm program package ${ }^{[\mathrm{S} 26]}$ on the above TPSS-D3 optimized structures, and corrected by $+1.89 \mathrm{kcal}^{-\mathrm{mol}^{-1}}$ to account for higher reference solute concentration of $1 \mathrm{~mol} \cdot \mathrm{L}^{-1}$ usually used in solution. To check the effects of the chosen DFT functional on the reaction energies and barriers, single-point calculations at both the meta-GGA TPSS-D3 ${ }^{[S 15]}$ and hybrid-meta-GGA PW6B95-D3 ${ }^{[S 26]}$ levels are performed using a larger def2-QZVP basis set. ${ }^{[S 19,}{ }^{\text {S28] }}$ The final reaction Gibbs free energies $(\Delta G)$ are determined from the electronic single-point energies plus TPSS-D3 thermal corrections and COSMO-RS solvation free energies. The computed reaction free energies from both DFT functionals are in good mutual agreement with the mean, mean absolute and standard deviations being $0.2,1.0$ and $1.5 \mathrm{kcal} / \mathrm{mol}$ for reaction energies and $4.3,4.3$ and $1.2 \mathrm{kcal} / \mathrm{mol}$ for transition barriers, respectively, with about $4.3 \mathrm{kcal} / \mathrm{mol}$ higher barriers found at the PW6B95-D3 level. In our discussion, higher-level PW6B95D3 Gibbs free energies (in $\mathrm{kcal} / \mathrm{mol}$, at $298.15 \mathrm{~K}$ and $1 \mathrm{~mol} / \mathrm{L}$ concentration) will be used in our discussion unless specified otherwise. The applied DFT methods in combination with the large AO basis set provide usually accurate electronic energies leading to errors for chemical energies

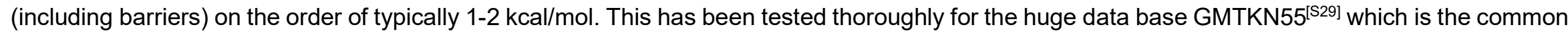
standard in the field of DFT benchmarking. 
14.2. Figure S2. DFT computed free energy paths for the reaction of $1 \mathrm{a}$ and tertiary amine $1 \mathrm{~h} \mathrm{Ph}_{2} \mathrm{NMe}$.

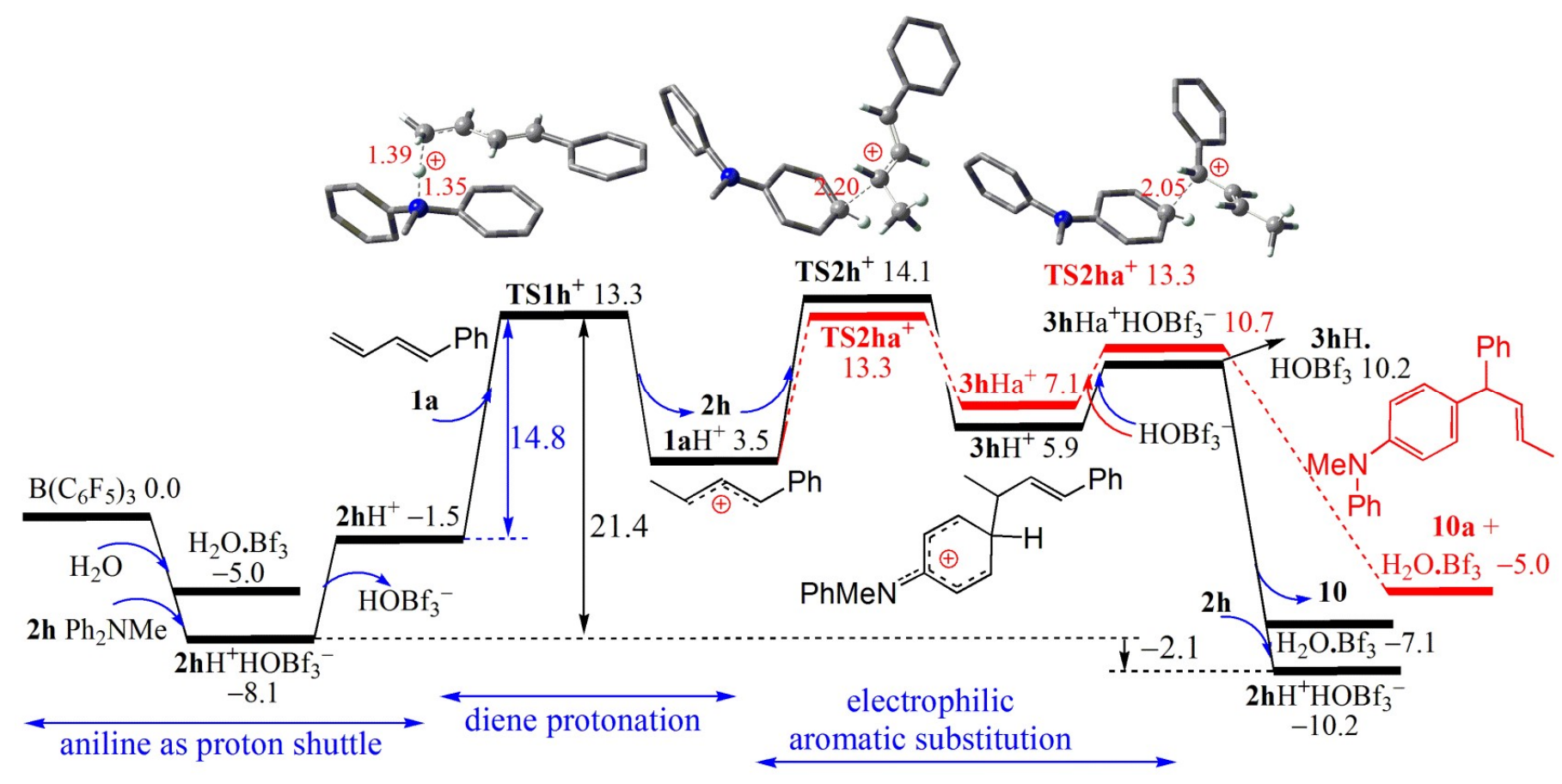

The $\mathrm{N}$-methylation of amine $\mathbf{2 a} \mathrm{Ph}_{2} \mathrm{NH}$ leads to methylated $\mathbf{2} \mathbf{h} \mathrm{Ph}_{2} \mathrm{NMe}$ with $3.9 \mathrm{kcal} / \mathrm{mol}$ higher proton affinity, leading to prefered protonation of $\mathbf{2 h}$ with acidic $\mathrm{H}_{2} \mathrm{O} \cdot \mathrm{Bf}_{3}$ complex. Further proton transfer to 1,3-diene $2 \mathbf{a}$ is $5.0 \mathrm{kcal} / \mathrm{mol}$ endergonic over a low barrier of $14.8 \mathrm{kcal} / \mathrm{mol}$ (via transition state $\mathbf{T S} \mathbf{h}^{+}$), followed by comepeting electrophilic attack of allyl cation $1 \mathrm{aH}+$ via either 3 or 1 position on the prefered phenyl para-position of amine $\mathbf{2 h}$ as well as even faster deprotonation with $\mathrm{HOBf}_{3}{ }^{-}$anion instead of neutral amine $\mathbf{2} \mathbf{h}$ (see Table S2). The attack via 1-position (red line) is kinetically $0.8 \mathrm{kcal} / \mathrm{mol}$ more favorable to form 4,1 -hydroarylation product $10 \mathrm{a}$, which is almost neutral in free energy from reactants of $1 \mathrm{a}$ and $2 \mathrm{~h}$, but thermodynamically $2.1 \mathrm{kcal} / \mathrm{mol}$ less stable than the 4,3-hydroarylation product 10. 
14.3 Table S2. TPSS-D3/def2-TZVP + COSMO computed imaginary frequency (ImF), zero-point energies (ZPE), gas-phase enthalpic (Hc) and Gibbs free-energy (Gc) corrections; the COSMO-RS computed solvation enthalpic (Hsol) and Gibbs free-energy (Gsol) corrections in dichloroethane (DCE); TPSS-D3/def2-QZVP and PW6B95-D3/def2-QZVP single-point energies (TPSS-D3 and PW6B95-D3); the total PW6B95-D3 free energies $\mathrm{G}_{\mathrm{P}}$; the relative electronic energies $\left(\Delta \mathrm{E}_{\mathrm{T}}\right.$ and $\left.\Delta \mathrm{E}_{\mathrm{P}}\right)$ and Gibbs free-energies $\left(\Delta \mathrm{G}_{\mathrm{T}}\right.$ and $\left.\Delta \mathrm{G}_{\mathrm{P}}\right)$ at the TPSS-D3 and PW6B95-D3 levels. See also maintext Figure $\mathbf{S 1}$ for most labellings of structures.

\begin{tabular}{|c|c|c|c|c|c|c|c|c|c|c|c|c|c|}
\hline $\begin{array}{l}\text { Reactions } \\
\text { in DCE solution }\end{array}$ & $\begin{array}{l}\operatorname{ImF} \\
\mathrm{cm}^{-1}\end{array}$ & $\begin{array}{l}\text { ZPE } \\
\mathrm{kcal} \\
/ \mathrm{mol}\end{array}$ & $\begin{array}{l}\mathrm{Hc} \\
\mathrm{kcal} \\
/ \mathrm{mol}\end{array}$ & $\begin{array}{l}\mathrm{Gc} \\
\mathrm{kcal} \\
/ \mathrm{mol}\end{array}$ & $\begin{array}{l}\text { Hsol } \\
\mathrm{kcal} \\
/ \mathrm{mol}\end{array}$ & $\begin{array}{l}\text { Gsol } \\
\mathrm{kcal} \\
/ \mathrm{mol}\end{array}$ & $\begin{array}{l}\text { TPSS-D3 } \\
\text { Eh }\end{array}$ & $\begin{array}{l}\text { PW6B95-D3 } \\
\text { Eh }\end{array}$ & $\begin{array}{l}\mathrm{G}_{\mathrm{P}} \\
\mathrm{Eh}\end{array}$ & $\begin{array}{l}\Delta \mathrm{E}_{\mathrm{T}} \\
\mathrm{kcal} \\
/ \mathrm{mol}\end{array}$ & $\begin{array}{l}\Delta \mathrm{E}_{\mathrm{p}} \\
\mathrm{kcal} \\
/ \mathrm{mol}\end{array}$ & $\begin{array}{l}\Delta G_{p} \\
\text { kcal } \\
/ \mathrm{mol}\end{array}$ & $\begin{array}{l}\Delta \mathrm{G}_{\mathrm{T}} \\
\mathrm{kcal} \\
/ \mathrm{mol} \\
\end{array}$ \\
\hline \multicolumn{14}{|l|}{$\mathrm{Hw}^{+}\left(\mathrm{H}_{2} \mathrm{O}+\mathrm{Bf}_{3}-\right.$} \\
\hline $\left.\mathrm{HOBf}_{3}^{-}\right)$ & 0 & 5.59 & 6.72 & -3.36 & 27.09 & 27.94 & -0.45807 & -0.45365 & -0.41148 & & & & \\
\hline 1a $\left(\mathrm{PhCH}=\mathrm{CHCH}=\mathrm{CH}_{2}\right)$ & 0 & 103.34 & 109.48 & 82.26 & -11.28 & -7.29 & -387.31229 & -387.73467 & -387.61220 & & & & \\
\hline 2a $\left(\mathrm{Ph}_{2} \mathrm{NH}\right)$ & 0 & 122.93 & 129.81 & 101.30 & -15.27 & -10.65 & -518.99784 & -519.56654 & -519.41907 & & & & \\
\hline $\mathrm{H}_{2} \mathrm{O}$ & 0 & 13.00 & 15.38 & 1.92 & -4.57 & -2.82 & -76.47338 & -76.54983 & -76.54826 & & & & \\
\hline $\mathrm{Bf}_{3}\left(\mathrm{~B}\left(\mathrm{C}_{6} \mathrm{~F}_{5}\right)_{3}\right)$ & 0 & 94.50 & 113.21 & 61.07 & -12.06 & -9.31 & -2209.62232 & -2211.87404 & -2211.78854 & & & & \\
\hline \multicolumn{14}{|c|}{ Proton affinity of 1-phenyl-1,3-diene $1 \mathrm{a}$ is $6.8,3.2$ and $5.6 \mathrm{kcal} / \mathrm{mol}$ higher than the product 3 , tertiary amine $P h_{3} N(2 \mathrm{~g})$, and cyclohexa-1,3-diene $1 \mathrm{w}$} \\
\hline $3 \mathrm{H}^{+}+\mathbf{1 a}$ & 0 & 341.64 & 360.25 & 292.50 & -71.35 & -57.82 & -1294.01362 & -1295.42234 & -1295.04234 & 0.00 & 0.00 & 0.00 & 0.00 \\
\hline $\mathbf{3}+\mathbf{1} \mathbf{a H}^{+}$ & 0 & 341.66 & 360.30 & 292.47 & -77.92 & -64.07 & -1294.01352 & -1295.42322 & -1295.05323 & 0.06 & -0.56 & -6.84 & -6.21 \\
\hline $2 \mathrm{gH}^{+}+1 \mathrm{a}$ & 0 & 283.07 & 299.23 & 236.50 & -65.81 & -53.77 & -1137.87784 & -1139.11694 & -1138.81972 & 0.00 & 0.00 & 0.00 & 0.00 \\
\hline $2 \mathrm{~g}+1 \mathrm{aH}^{+}$ & 0 & 284.17 & 300.12 & 238.49 & -71.34 & -59.35 & -1137.87644 & -1139.11625 & -1138.82474 & 0.88 & 0.44 & -3.15 & -2.71 \\
\hline $1 w^{+}+1 a$ & 0 & 187.03 & 196.97 & 148.31 & -69.35 & -59.14 & -621.22473 & -621.89594 & -621.74782 & 0.00 & 0.00 & 0.00 & 0.00 \\
\hline $\mathbf{2 a} \cdot \mathrm{Bf}_{3}+\mathbf{2 a}$ & 0 & 341.67 & 374.20 & 279.50 & -38.72 & -29.11 & -3247.63978 & -3251.03017 & -3250.62512 & 13.66 & 14.46 & 0.98 & 1.77 \\
\hline TS0a & $1262 \mathrm{i}$ & 338.90 & 371.66 & 292.09 & -33.47 & -26.94 & -3247.62272 & -3251.00809 & -3250.58255 & -2.96 & -0.61 & 27.69 & 25.34 \\
\hline $\mathbf{2} \mathbf{a H}{ }^{+}+\mathrm{Ph}_{2} \mathrm{NBf}_{3}^{-}$ & 0 & 341.33 & 373.91 & 279.21 & 106.80 & -94.98 & -3247.50150 & -3250.89174 & -3250.59213 & 73.11 & 72.41 & 21.68 & 22.38 \\
\hline $\mathbf{2} \mathbf{a} \cdot \mathrm{Bf}_{3 \mathrm{c}}+\mathbf{2 a}$ & 0 & 340.79 & 373.67 & 278.20 & -42.00 & -32.13 & -3247.61881 & -3251.00718 & -3250.60901 & -0.51 & -0.03 & 11.09 & 10.61 \\
\hline & & & & & & $3 / 9$ & & & & & & & \\
\hline
\end{tabular}




\begin{tabular}{|c|c|c|c|c|c|c|c|c|c|c|c|c|c|}
\hline $2 \mathbf{a H}^{+}+\mathrm{PhNHC}_{6} \mathrm{H}_{4} \mathrm{Bf}_{3}^{-}$ & $1495 \mathrm{i}$ & 337.50 & 374.07 & 288.99 & $\begin{array}{r}-40.10 \\
- \\
109.38 \\
\end{array}$ & -32.28 & -3247.60026 & -3250.98380 & -3250.57170 & 76.12 & 14.63 & 20.42 & 31.00 \\
\hline \multicolumn{14}{|c|}{ Protonation of amine $2 a \mathrm{Ph}_{2} \mathrm{NH}$ with the help of residual water $\mathrm{H}_{2} \mathrm{O}$} \\
\hline $\mathbf{2 a}+\mathrm{Bf}_{3}+\mathrm{H}_{2} \mathrm{O}$ & 0 & 230.43 & 258.40 & 164.29 & -31.90 & -22.78 & -2805.09355 & -2807.99042 & -2807.75587 & $\begin{array}{r}0.00 \\
-\end{array}$ & $\begin{array}{r}0.00 \\
-\end{array}$ & 0.00 & 0.00 \\
\hline $\mathbf{2 a}+\mathrm{H}_{2} \mathrm{O} \cdot \mathrm{Bf}_{3}$ & 0 & 233.68 & 260.48 & 176.46 & -32.92 & -25.21 & -2805.11433 & -2808.01085 & -2807.76379 & $\begin{array}{r}13.04 \\
-\end{array}$ & $\begin{array}{r}12.82 \\
-\end{array}$ & -4.97 & -5.19 \\
\hline TS0 & $1021 \mathrm{i}$ & 236.02 & 262.81 & 193.86 & -28.39 & -22.86 & -2805.14031 & -2808.03376 & -2807.75823 & $\begin{array}{r}29.35 \\
-\end{array}$ & $\begin{array}{r}27.20 \\
-\end{array}$ & -1.48 & -3.63 \\
\hline $\mathbf{2} \mathbf{a} \mathrm{H}^{+} \mathrm{HOBf}_{3}^{-}$ & 0 & 233.80 & 261.04 & 191.37 & $\begin{array}{r}-29.45 \\
-\end{array}$ & -23.87 & -2805.14163 & -2808.03643 & -2807.76650 & 30.17 & 28.87 & -6.67 & -7.97 \\
\hline \multicolumn{14}{|c|}{.followed by facile but $1.2 \mathrm{kcal} / \mathrm{mol}$ endergonic protonation of diene $1 \mathrm{a}$ with ammonium $2 \mathrm{aH} \mathrm{H}^{+}$} \\
\hline $\mathbf{1 a}+\mathbf{2 a}+\mathrm{Hw}^{+}$ & 0 & 231.85 & 246.01 & 180.20 & 0.54 & 9.99 & -906.76820 & -907.75487 & -907.44275 & 0.00 & 0.00 & 0.00 & 0.00 \\
\hline $\mathbf{2} \mathbf{a} \mathrm{H}^{+}+\mathbf{1 a}$ & 0 & 235.33 & 248.42 & 192.48 & -69.68 & -58.57 & -906.67037 & -907.65844 & -907.43902 & 61.39 & 60.51 & 2.34 & 3.22 \\
\hline TS1 $^{+}$ & $1349 \mathrm{i}$ & 231.82 & 245.12 & 202.79 & -57.21 & -48.30 & -906.68884 & -907.66727 & -907.41807 & 49.80 & 54.97 & 15.49 & 10.32 \\
\hline $\mathbf{2 a}+\mathbf{1} \mathbf{a} H^{+}$ & 0 & 234.99 & 247.97 & 192.41 & -68.34 & -56.86 & -906.67402 & -907.65922 & -907.43718 & 59.10 & 60.02 & 3.49 & 2.57 \\
\hline \multicolumn{14}{|c|}{..selective electrophilic aromatic substitution of allyl cation $\mathbf{1 a H} H^{+}$via 3-position on para-position of $\mathbf{2 a}$} \\
\hline $\mathbf{1 a}+\mathbf{2 a}+\mathrm{Hw}^{+}$ & 0 & 231.85 & 246.01 & 180.20 & 0.54 & 9.99 & -906.76820 & -907.75487 & -907.44275 & 0.00 & 0.00 & 0.00 & 0.00 \\
\hline 2a.1 $\mathbf{a H} H^{+} \quad$ (loose $\pi$-adduct $)$ & 0 & $\begin{array}{r}236.54 \\
234.3\end{array}$ & $\begin{array}{r}249.68 \\
247.7\end{array}$ & $\begin{array}{r}208.15 \\
205.0\end{array}$ & -56.74 & $\begin{array}{r}-48.09 \\
-\end{array}$ & -906.71123 & -907.68883 & -907.43074 & 35.75 & 41.44 & 7.53 & 1.85 \\
\hline $\mathbf{T S 2}^{+}$ & $169 \mathrm{i}$ & 8 & 4 & 0 & -56.86 & 47.60 & -906.69758 & -907.67519 & -907.42135 & 44.32 & 50.00 & 13.43 & 7.75 \\
\hline $3 \mathbf{a H}^{+} \quad$ (tight $\sigma$-adduct $)$ & 0 & $\begin{array}{r}238.30 \\
233.8\end{array}$ & $\begin{array}{r}250.77 \\
246.7\end{array}$ & $\begin{array}{r}210.12 \\
205.1\end{array}$ & -60.10 & -50.58 & -906.70160 & -907.68774 & -907.43049 & 41.79 & 42.12 & 7.69 & 7.36 \\
\hline TS2a $^{+} \quad$ (via 1-position) & $302 \mathrm{i}$ & 5 & 9 & 0 & -56.41 & 47.40 & -906.69361 & -907.67235 & -907.41802 & 46.81 & 51.78 & 15.52 & 10.55 \\
\hline $3 \mathbf{a H}^{+} 1$ & 0 & 237.22 & 249.96 & 208.84 & -58.66 & -49.40 & -906.69917 & -907.68614 & -907.42904 & 43.32 & 43.13 & 8.60 & 8.79 \\
\hline \multicolumn{14}{|c|}{.. and facile deprotonation with $2 a$} \\
\hline $\mathbf{1 a}+2 * \mathbf{2} \mathbf{a}+\mathrm{Hw}^{+}$ & 0 & 354.78 & 375.83 & 281.50 & -14.72 & -0.66 & -1425.76604 & -1427.32141 & -1426.86182 & 0.00 & 0.00 & 0.00 & 0.00 \\
\hline $\mathbf{3} \mathbf{a} H^{+}+\mathbf{2 a}$ & 0 & 361.23 & 380.58 & 311.42 & -75.37 & -61.23 & -1425.69944 & -1427.25429 & -1426.84956 & 41.79 & 42.12 & 7.69 & 7.36 \\
\hline $\mathrm{TS3}^{+}$ & $1159 \mathrm{i}$ & 355.89 & 376.53 & 319.14 & -66.57 & -55.11 & -1425.71275 & -1427.26218 & -1426.83840 & 33.44 & 37.17 & 14.69 & 10.97 \\
\hline
\end{tabular}




\begin{tabular}{|c|c|c|c|c|c|c|c|c|c|c|c|c|c|}
\hline $3+2 \mathrm{aH}^{+}$ & 0 & 361.59 & 381.08 & 311.58 & -83.25 & -69.14 & -1425.69542 & -1427.25431 & -1426.86194 & 44.32 & 42.10 & -0.08 & 2.14 \\
\hline $\mathbf{3}+\mathbf{2 a}+\mathrm{Hw}^{+}$ & 0 & 358.12 & 378.67 & 299.29 & -13.02 & -0.58 & -1425.79325 & -1427.35074 & -1426.86567 & 17.07 & 18.41 & -2.42 & -1.08 \\
\hline \multicolumn{14}{|c|}{ Deprotonation with $\mathrm{HOBf}_{3}{ }^{-}$anion is kinetically similarly favorable but limited by low concentration } \\
\hline $\mathbf{1 a}+\mathbf{2 a}+\mathrm{Bf}_{3}+\mathrm{H}_{2} \mathrm{O}$ & 0 & 333.77 & 367.88 & 246.55 & -43.18 & -30.07 & -3192.40584 & -3195.72509 & -3195.36807 & 0.00 & 0.00 & 0.00 & 0.00 \\
\hline $3 \mathbf{a H}^{+}+\mathrm{HOBf}_{3}^{-}$ & 0 & 340.21 & 372.63 & 276.47 & 103.83 & -90.64 & -3192.33924 & -3195.65797 & -3195.35581 & 41.79 & 42.12 & 7.69 & 7.36 \\
\hline $3 \mathrm{aH}^{+} \mathrm{HOBf}_{3}^{-}$ & 0 & 339.61 & 372.87 & 291.76 & -41.89 & -33.57 & -3192.44250 & -3195.75857 & -3195.34411 & 23.01 & 21.00 & 15.03 & 13.03 \\
\hline TS3a & $367 i$ & 338.74 & 372.22 & 289.25 & -42.04 & -33.71 & -3192.44163 & -3195.75660 & -3195.34636 & 22.46 & 9.77 & 13.62 & 10.93 \\
\hline $3+\mathrm{Bf}_{3}+\mathrm{H}_{2} \mathrm{O}$ & 0 & 337.10 & 370.73 & 264.34 & -41.48 & -29.99 & -3192.43305 & -3195.75442 & -3195.37192 & 17.07 & 18.41 & -2.42 & -1.08 \\
\hline \multicolumn{14}{|c|}{ Deprotonation with 1 a is kinetically $5.6 \mathrm{kcal} / \mathrm{mol}$ less efficient } \\
\hline $2^{*} \mathbf{1} \mathbf{a}+\mathbf{2 a}+\mathrm{Hw}^{+}$ & 0 & 335.19 & 355.50 & 262.46 & -10.74 & 2.70 & -1294.08049 & -1295.48954 & -1295.05495 & 0.00 & 0.00 & 0.00 & 0.00 \\
\hline $3 \mathbf{a H}^{+}+\mathbf{1 a}$ & 0 & 341.64 & 360.25 & 292.38 & -71.38 & -57.87 & -1294.01389 & -1295.42242 & -1295.04269 & 41.79 & 42.12 & 7.69 & 7.36 \\
\hline $\mathrm{TS} \mathbf{a}^{+}$ & $\begin{array}{r}1142 \mathrm{i} \\
1101\end{array}$ & $\begin{array}{r}336.48 \\
355.4\end{array}$ & $\begin{array}{r}356.10 \\
376.2\end{array}$ & $\begin{array}{r}300.88 \\
318.4\end{array}$ & -63.93 & -52.82 & -1294.02152 & -1295.42096 & -1295.02264 & 37.01 & 43.04 & 20.27 & 14.24 \\
\hline $\mathbf{T S} \mathbf{b}^{+}$ & $\mathrm{i}$ & 2 & 5 & 5 & -65.11 & 54.06 & 1425.71082 & 1427.26091 & 1426.83658 & 34.66 & 37.96 & 15.84 & 12.53 \\
\hline $\mathbf{3}+\mathbf{1 a H}^{+}$ & 0 & 341.66 & 360.30 & 292.47 & -77.92 & -64.07 & -1294.01352 & -1295.42322 & -1295.05323 & 42.03 & 41.62 & 1.08 & 1.49 \\
\hline \multicolumn{14}{|c|}{$\begin{array}{l}\text { Effects of } \mathrm{N} \text {-methylation of } \mathrm{Ph}{ }_{2} \mathrm{NH} \text { : more electron-rich phenyl within } \mathbf{2 h} \mathrm{Ph}_{2} \mathrm{NMe} \\
\text { The proton affinity of } \boldsymbol{2 h} \text { is } 3.8 \mathrm{kcal} / \text { mol higher than } 2 \boldsymbol{a}\end{array}$} \\
\hline $\mathbf{2 h H ^ { + } + 2 a}$ & 0 & 271.81 & 286.62 & 227.05 & -71.90 & -59.85 & -1077.69563 & 36924 & -1078.59677 & 0.00 & 0.00 & 0.00 & 0.00 \\
\hline $\mathbf{2 h}+\mathbf{2} \mathrm{aH}^{+}$ & 0 & 271.85 & 286.63 & 227.08 & -73.39 & -61.65 & -1077.68647 & -1078.86027 & -1078.59063 & 5.75 & 5.63 & 3.85 & 3.97 \\
\hline \multicolumn{14}{|l|}{ Preferred protonation } \\
\hline $\mathbf{2 h}+\mathrm{Bf}_{3}+\mathrm{H}_{2} \mathrm{O}$ & 0 & 247.36 & 276.27 & 179.84 & -31.62 & -22.50 & -2844.42410 & -2847.36038 & -2847.10061 & 0.00 & 0.00 & 0.00 & 0.00 \\
\hline $2 \mathbf{h}+\mathrm{H}_{2} \mathrm{O}_{3} \mathrm{Bf}_{3}$ & 0 & 250.61 & 278.36 & 192.01 & -32.64 & -24.93 & -2844.44488 & -2847.38081 & -2847.10853 & 13.04 & 12.82 & -4.97 & -5.19 \\
\hline $2 \mathbf{h H}^{+} \mathrm{HOBf}_{3}^{-}$ & 0 & 251.44 & 279.74 & 207.94 & -29.77 & -24.03 & -2844.47449 & -2847.40957 & -2847.11347 & 31.62 & 30.87 & -8.07 & -8.82 \\
\hline $\mathbf{2} \mathbf{h} \mathrm{H}^{+}+\mathrm{HOBf}_{3}^{-}$ & 0 & 250.79 & 278.67 & 192.10 & 100.36 & -89.26 & -2844.33543 & -2847.27292 & -2847.10301 & 55.64 & 54.88 & -1.51 & -0.75 \\
\hline
\end{tabular}




\begin{tabular}{|c|c|c|c|c|c|c|c|c|c|c|c|c|c|}
\hline $\mathbf{1 a}+\mathbf{2 h}+\mathrm{Hw}^{+}$ & 0 & 248.79 & 263.89 & 195.75 & 0.83 & 10.27 & -946.09875 & -947.12483 & -946.78749 & 0.00 & 0.00 & 0.00 & 0.00 \\
\hline \multirow[t]{2}{*}{$2 \mathrm{hH}^{+}+1 \mathbf{a}$} & 0 & 252.21 & 266.29 & 208.01 & -67.91 & -56.49 & -946.01008 & -947.03737 & -946.78989 & 55.64 & 54.88 & -1.51 & -0.75 \\
\hline & 1381 & 249.0 & 263.2 & 219.2 & & - & & & & & & & \\
\hline $\mathbf{T S 1 h}^{+}$ & $\mathrm{i}$ & 3 & 3 & 4 & -56.26 & 47.28 & -946.02611 & -947.04337 & -946.76632 & 45.58 & 51.12 & 13.28 & 7.75 \\
\hline $\mathbf{2 h}+\mathbf{1} \mathbf{a} \mathrm{H}^{+}$ & 0 & 251.92 & 265.84 & 207.97 & -68.06 & -56.59 & -946.00457 & -947.02917 & -946.78192 & 59.10 & 60.02 & 3.49 & 2.57 \\
\hline $\mathbf{T S 2 h}^{+}$ & $164 i$ & 251.29 & 265.70 & 220.82 & -55.52 & -46.26 & -946.03041 & -947.04619 & -946.76499 & 42.89 & 49.34 & 14.11 & 7.66 \\
\hline $3 \mathrm{hH}^{+}$ & 0 & 255.46 & 268.83 & 226.52 & -59.02 & -49.36 & -946.03794 & -947.06340 & -946.77807 & 38.16 & 38.54 & 5.91 & 5.52 \\
\hline TS2 $\mathrm{ha}^{+}$ & $286 \mathrm{i}$ & 251.01 & 265.55 & 220.44 & -55.52 & -46.42 & -946.02885 & -947.04669 & -946.76637 & 43.86 & 49.03 & 13.25 & 8.09 \\
\hline $3 \mathbf{h H a}{ }^{+}$ & 0 & 254.46 & 268.10 & 225.38 & -57.64 & -48.24 & -946.03545 & -947.06153 & -946.77624 & 39.72 & 39.72 & 7.06 & 7.06 \\
\hline \multicolumn{14}{|c|}{$\ldots 2 \boldsymbol{h} \mathrm{Ph}_{2} \mathrm{NMe}$ is less efficient for deprotonation due to higher steric hindrance at $\mathrm{N}$-center } \\
\hline $\mathbf{1 a}+2 * \mathbf{2} \mathbf{h}+\mathrm{Hw}^{+}$ & 0 & 388.65 & 411.58 & 312.60 & -14.16 & -0.11 & -1504.42714 & -1506.06133 & -1505.55129 & 0.00 & 0.00 & 0.00 & 0.00 \\
\hline TS3h $^{+}$ & $1432 \mathrm{i}$ & 390.75 & 413.07 & 353.06 & -61.48 & -50.57 & -1504.38221 & -1506.00997 & -1505.52489 & 28.20 & 32.23 & 16.57 & 12.53 \\
\hline TS3ha $^{+}$ & $1441 \mathrm{i}$ & 390.23 & 412.76 & 352.33 & -61.03 & -50.27 & -1504.37686 & -1506.00372 & -1505.51934 & 31.56 & 36.15 & 20.05 & 15.46 \\
\hline \multicolumn{14}{|c|}{. facile deprotonation with $\mathrm{HOBf}_{3}{ }^{-}$instead to form the product 10 and kinetically more favorable but less stable $\mathbf{1 0 a}$} \\
\hline $\mathbf{1 a}+\mathbf{2 h}+\mathrm{Bf}_{3}$ & 0 & 350.70 & 385.76 & 262.10 & -42.90 & -29.79 & -3231.73639 & -3235.09505 & -3234.71280 & 0.00 & 0.00 & 0.00 & 0.00 \\
\hline $3 \mathbf{h H}^{+}+\mathrm{HOBf}_{3}^{-}$ & 0 & 357.37 & 390.70 & 292.87 & $102.75^{-}$ & -89.43 & -3231.67558 & -3235.03363 & -3234.70339 & 38.16 & 38.54 & 5.91 & 5.52 \\
\hline $3 \mathbf{h H} \mathrm{H}^{+} \mathrm{HOBf}_{3}^{-}$ & 0 & 356.05 & 390.45 & 307.51 & -39.24 & -31.26 & -3231.78253 & -3235.13974 & -3234.69651 & $28.95^{-}$ & $28.04^{-}$ & 10.22 & 9.31 \\
\hline $10+\mathrm{H}_{2} \mathrm{O} \cdot \mathrm{Bf}_{3}$ & 0 & 357.17 & 390.62 & 292.36 & -42.16 & -32.10 & -3231.78452 & -3235.14493 & -3234.72417 & 30.20 & 31.30 & -7.13 & -6.03 \\
\hline $3 \mathbf{h H a}^{+}+\mathrm{HOBf}_{3}^{-}$ & 0 & 356.38 & 389.96 & 291.73 & 101.36 & -88.30 & -3231.67308 & -3235.03176 & -3234.70156 & 39.72 & 39.72 & 7.06 & 7.06 \\
\hline $3 \mathbf{h H a}{ }^{+} \mathrm{HOBf}_{3}^{-}$ & 0 & 357.23 & 391.26 & 308.96 & -39.95 & -31.77 & -3231.78450 & -3235.14047 & -3234.69574 & 30.19 & 28.50 & 10.71 & 9.02 \\
\hline $10 \mathbf{a}+\mathrm{H}_{2} \mathrm{O} \cdot \mathrm{Bf}_{3}$ & 0 & 356.27 & 389.98 & 291.39 & -41.37 & -31.59 & -3231.77993 & -3235.14065 & -3234.72061 & 27.32 & 28.61 & -4.90 & -3.61 \\
\hline
\end{tabular}

Effects of $\mathrm{H} / \mathrm{Ph}$ replacement of $2 \mathrm{a} \mathrm{Ph}_{2} \mathrm{NH}$ : less electron-rich phenyl ring within $2 \mathrm{~g} \mathrm{Ph}_{3} \mathrm{~N}$ with preferred phenyl para-position as $\mathrm{H}^{+}$acceptor

Tertiary amine $2 \mathrm{~g}$ cannot transfer proton to diene $1 \mathrm{a}$ and another $P h_{3} N$ with the help of $B f_{3}$ only.
$\mathbf{2 g}+\mathrm{Bf}_{3}+\mathbf{1 a}$
$\begin{array}{llll}0 & 369.95 & 404.66 & 290.71\end{array}$
$\begin{array}{lll}-41.61 & -29.73 & -3347.13487\end{array}$
$\begin{array}{ll}-3350.63229 & -3350.20737\end{array}$
$\begin{array}{llll}0.00 & 0.00 & 0.00 & 0.00\end{array}$
TSOg
$\begin{array}{llll}1456 \mathrm{i} & 367.19 & 402.62 & 317.28\end{array}$
$-39.79-32.28$
$-3347.11208$
$-3350.59782$
$-3350.14063$
$\begin{array}{llll}14.31 & 21.63 & 41.88 & 34.55\end{array}$ 


\begin{tabular}{|c|c|c|c|c|c|c|c|c|c|c|c|c|c|}
\hline $1 \mathrm{aH}^{+}+2 \mathrm{gcBf}_{3}^{-}$ & 0 & 371.24 & 406.21 & 306.34 & 105.48 & -92.82 & -3347.01973 & -3350.51651 & -3350.17023 & 72.26 & 72.65 & 23.31 & 22.91 \\
\hline $2 * 2 \mathrm{~g}+\mathrm{Bf}_{3}$ & 0 & 438.73 & 477.14 & 355.83 & -48.60 & -35.58 & -3710.02285 & -3713.92118 & -3713.40180 & 0.00 & 0.00 & 0.00 & 0.00 \\
\hline $2 \mathrm{gHc}^{+}+2 \mathrm{gcBf}_{3}^{-}$ & 0 & 438.91 & 477.80 & 369.47 & 106.94 & -93.08 & -3709.90910 & -3713.80610 & -3713.35963 & 71.37 & 72.22 & 26.46 & 25.62 \\
\hline $2 \mathrm{gHc}^{+}+1 \mathrm{a}$ & 0 & 283.07 & 299.23 & 236.50 & -65.81 & -53.77 & -1137.87784 & -1139.11694 & -1138.81972 & 0.00 & 0.00 & 0.00 & 0.00 \\
\hline $2 \mathrm{~g}+1 \mathrm{aH}^{+}$ & 0 & 284.17 & 300.12 & 238.49 & -71.34 & -59.35 & -1137.87644 & -1139.11625 & -1138.82474 & 0.88 & 0.44 & -3.15 & -2.71 \\
\hline $2 \mathrm{gHc}++\mathbf{2 a}$ & 0 & 302.66 & 319.56 & 255.54 & -69.80 & -57.12 & -1269.56339 & -1270.94881 & -1270.62659 & 0.00 & 0.00 & 0.00 & 0.00 \\
\hline 2g. $\mathrm{Bf}_{3} \quad$ (unstable $\pi$-adduct) & 0 & 267.95 & 297.04 & 224.22 & -25.18 & -19.92 & -2959.84085 & -2962.91594 & -2962.58734 & 11.46 & 11.50 & 4.91 & 4.95 \\
\hline 2g. $\mathrm{Bf}_{3} \mathrm{c}$ & 0 & 267.66 & 296.47 & 224.30 & -29.34 & -23.55 & -2959.82259 & -2962.89618 & -2962.57326 & 0.00 & 0.90 & 13.75 & 12.84 \\
\hline \multicolumn{14}{|c|}{..leading to selective protonation of $1 \mathbf{a}$ at first } \\
\hline $\mathbf{1 a}+\mathrm{Bf}_{3}+\mathrm{H}_{2} \mathrm{O}$ & 0 & 210.84 & 238.07 & 145.25 & -27.91 & -19.42 & -2673.40800 & -2676.15855 & -2675.94900 & 0.00 & 0.00 & 0.00 & 0.00 \\
\hline $\mathbf{1 a}+\mathrm{H}_{2} \mathrm{O} \cdot \mathrm{Bf}_{3}$ & 0 & 214.09 & 240.15 & 157.41 & -28.94 & -21.85 & -2673.42878 & -2676.17898 & -2675.95692 & 13.04 & 12.82 & -4.97 & -5.19 \\
\hline $\mathrm{HOBf}_{3}{ }^{-}+\mathbf{1 a H}^{+}$ & 0 & 213.97 & 240.02 & 157.46 & -96.79 & -86.27 & -2673.31381 & -2676.06290 & -2675.94343 & 59.10 & 60.02 & 3.49 & 2.57 \\
\hline \multicolumn{14}{|c|}{.followed by facile electrophilic aromatic substitution with allyl action 1 a $H^{+}$} \\
\hline $\mathbf{1 a}+\mathbf{2 g}+\mathrm{Hw}^{+}$ & 0 & 281.04 & 298.16 & 226.27 & -2.46 & 7.51 & -1137.97062 & -1139.21190 & -1138.83031 & 0.00 & 0.00 & 0.00 & 0.00 \\
\hline $2 \mathrm{~g}+1 \mathrm{aH}^{+}$ & 0 & 284.17 & 300.12 & 238.49 & -71.34 & -59.35 & -1137.87644 & -1139.11625 & -1138.82474 & 59.10 & 60.02 & 3.49 & 2.57 \\
\hline TS2g+ & $337 i$ & 283.56 & 299.93 & 250.98 & -57.98 & -47.96 & -1137.90077 & -1139.13291 & -1138.80637 & 43.84 & 49.57 & 15.02 & 9.29 \\
\hline $3 \mathbf{g H}^{+}$ & 0 & 287.51 & 302.94 & 256.53 & -59.88 & -49.63 & -1137.90875 & -1139.14848 & -1138.81576 & 38.83 & 39.80 & 9.13 & 8.16 \\
\hline \multicolumn{14}{|c|}{.. and deprotonation with $\mathrm{HOBf}_{3}{ }^{-}$anion to form product 9} \\
\hline $\mathbf{1 a}+\mathbf{2 g}+\mathrm{Bf}_{3}+\mathrm{H}_{2} \mathrm{O}$ & 0 & 382.95 & 420.03 & 292.62 & -46.18 & -32.55 & -3423.60826 & -3427.18212 & -3426.75563 & 0.00 & 0.00 & 0.00 & 0.00 \\
\hline $\mathrm{HOBf}_{3}{ }^{-}+\mathbf{3} \mathbf{g H}^{+}$ & 0 & 389.43 & 424.81 & 322.88 & 103.60 & -89.69 & -3423.54639 & -3427.11870 & -3426.74108 & 38.83 & 39.80 & 9.13 & 8.16 \\
\hline
\end{tabular}




\begin{tabular}{|c|c|c|c|c|c|c|c|c|c|c|c|c|c|}
\hline $3 \mathbf{g H}^{+} \mathrm{HOBf}_{3}^{-}$ & 0 & 389.13 & 425.36 & 338.76 & -42.78 & -34.10 & -3423.65085 & -3427.21960 & -3426.73108 & $26.73^{-}$ & $23.52^{-}$ & 15.40 & 12.19 \\
\hline $\mathbf{9}+\mathrm{H}_{2} \mathrm{O} \cdot \mathrm{Bf}_{3}$ & 0 & 389.23 & 424.79 & 322.27 & -45.66 & -35.06 & -3423.65649 & -3427.23207 & -3426.76836 & 30.27 & 31.35 & -7.99 & -6.91 \\
\hline
\end{tabular}

\begin{tabular}{|c|c|c|c|c|c|c|c|c|c|c|c|c|c|}
\hline $\mathrm{PhNH}_{2}+\mathrm{Bf}_{3}$ & 0 & 167.45 & 190.29 & 116.24 & -23.30 & -16.70 & -2497.41858 & -2499.98387 & -2499.81921 & 0.00 & 0.00 & 0.00 & 0.00 \\
\hline $\mathrm{PhNH}_{2} \cdot \mathrm{Bf}_{3}$ & 0 & 169.71 & 192.45 & 131.60 & -21.58 & -17.12 & -2497.45674 & -2500.02350 & -2499.83806 & 23.95 & 24.86 & $11.83^{-}$ & 10.91 \\
\hline $2 \mathrm{PhNH}_{2}+\mathrm{Bf}_{3}$ & 0 & 240.41 & 267.36 & 171.42 & -34.53 & -24.09 & -2785.21484 & -2788.09370 & -2787.84989 & 0.00 & 0.00 & 0.00 & 0.00 \\
\hline $\mathrm{PhNH}_{3}^{+}+\mathrm{PhNHBf}_{3}^{-}$ & 0 & 242.43 & 269.55 & 186.02 & 108.78 & -98.63 & -2785.09849 & -2787.97784 & -2787.83256 & 73.01 & 72.71 & 10.88 & 11.18 \\
\hline $\mathrm{PhNH}_{2} \cdot \mathrm{Bf}_{3}+\mathbf{1 a}$ & 0 & 273.05 & 301.93 & 213.85 & -32.86 & -24.41 & -2884.76903 & -2887.75817 & -2887.45026 & 0.00 & 0.00 & 0.00 & 0.00 \\
\hline $\mathbf{1 a H}^{+}+\mathrm{PhNHBf}_{3}^{-}$ & 0 & 271.48 & 300.58 & 211.98 & -98.42 & -87.42 & -2884.62683 & -2887.61167 & -2887.40716 & 89.23 & 91.93 & 27.05 & 24.35 \\
\hline
\end{tabular}

Ph to Cy replacement of 1 a leads to 1,3-diene 1 n with $2.9 \mathrm{kcal} / \mathrm{mol}$ smaller proton affinity but more reactive 1-position within allyl cation 1 n $H^{+}$

\begin{tabular}{|c|c|c|c|c|c|c|c|c|c|c|c|c|c|}
\hline $1 \mathbf{a H}^{+}+\mathbf{1 n}$ & 0 & 259.05 & 271.84 & 216.64 & -62.83 & -52.51 & -778.61431 & -779.45039 & -779.18281 & 0.00 & 0.00 & 0.00 & 0.00 \\
\hline $1 \mathbf{n} \mathrm{H}^{+}+\mathbf{1 a}$ & 0 & 257.74 & 270.98 & 214.76 & -65.74 & -54.88 & -778.60406 & -779.43903 & -779.17822 & 6.43 & 7.13 & 2.88 & 2.19 \\
\hline \multicolumn{14}{|c|}{...and highly selective electrophilic aromatic substitution via 1-position $\left(\boldsymbol{T S} 2 \boldsymbol{n}^{+}\right)$} \\
\hline $\mathbf{2 a}+\mathbf{1 n} \mathrm{H}^{+}$ & 0 & 277.33 & 291.31 & 233.81 & -69.73 & -58.24 & -910.28961 & -911.27090 & -910.98509 & 0.00 & 0.00 & 0.00 & 0.00 \\
\hline $\mathbf{T S 2 n}^{+} \quad$ (via 1-position) & $243 \mathrm{i}$ & 277.31 & 291.42 & 247.69 & -56.09 & -47.61 & -910.32649 & -911.30268 & -910.98081 & 23.14 & 19.94 & 2.68 & -0.51 \\
\hline TS2na $^{+}$(via 3-position) & $139 \mathrm{i}$ & 276.71 & 291.08 & 246.62 & -56.39 & -47.55 & -910.31913 & -911.29273 & -910.97248 & $18.52^{-}$ & 13.70 & 7.91 & 3.09 \\
\hline \multicolumn{14}{|c|}{ Effects of multiple alkylation: } \\
\hline \multicolumn{14}{|c|}{ Tertiary amine $2 \mathrm{l}: 10.4$ and $11.5 \mathrm{kcal} / \mathrm{mol}$ higher proton affinity than amine $2 \boldsymbol{a}$ and 1,3-diene $1 \mathrm{a}$, respectively } \\
\hline $2 \mathrm{lH}^{+}+\mathbf{2 a}$ & 0 & 263.00 & 276.09 & 220.47 & -72.81 & -61.27 & -963.31546 & -964.36020 & -964.10047 & 0.00 & 0.00 & 0.00 & 0.00 \\
\hline $2 \mathbf{a} H^{+}+2 \mathbf{l}$ & 0 & 262.22 & 275.52 & 219.33 & -70.81 & -59.69 & -963.29983 & -964.34441 & -964.08397 & 9.81 & 9.91 & 10.35 & 10.24 \\
\hline
\end{tabular}

..leading to protonation of $2 \mathrm{l}$ at first, followed by proton transfer to 1 a and further electrophilic aromatic substitution via 3 or 1 position on phenyl para-position
$\mathbf{1 a}+\mathbf{2 l}+\mathrm{Hw}^{+}$
$\begin{array}{llllll}0 & 239.15 & 252.78 & 188.00 & 3.41 & 12.23\end{array}$
$-831.71211 \quad-832.60897$
$-832.28084$
$\begin{array}{llll}0.00 & 0.00 & 0.00 & 0.00\end{array}$
$2 \mathrm{IH}^{+}+\mathbf{1 a}$
$\begin{array}{llllll}0 & 243.41 & 255.76 & 201.43 & -68.82 & -57.91\end{array}$
$-831.62991$
$-832.52834$
$\begin{array}{lllll}-832.29359 & 51.58 & 50.60 & -8.01 & -7.02\end{array}$ 


\begin{tabular}{|c|c|c|c|c|c|c|c|c|c|c|c|c|c|}
\hline $1 \mathbf{a H}^{+}+\mathbf{2 l}$ & 0 & 242.29 & 254.74 & 200.22 & -65.48 & -54.62 & -831.61793 & -832.51332 & -832.27527 & 59.10 & 60.02 & 3.49 & 2.57 \\
\hline $\mathbf{1} \mathbf{a H}^{+} \mathbf{2 l} \quad(\mathrm{N}-\mathrm{C}$ adduct $)$ & 0 & 244.86 & 257.05 & 217.55 & -57.39 & -48.64 & -831.65687 & -832.55261 & -832.28041 & 34.66 & 35.37 & 0.26 & -0.44 \\
\hline $\mathbf{T S 2 I}^{+} \mathbf{0}$ (on ortho-position) & $379 \mathrm{i}$ & 241.75 & 254.61 & 213.50 & -55.10 & -46.80 & -831.64572 & -832.53646 & -832.26780 & 41.66 & 45.50 & 8.18 & 4.34 \\
\hline $\mathbf{T S 2 I}^{+}$(on para-position) & $351 \mathrm{i}$ & 241.82 & 254.68 & 213.69 & -54.37 & -46.20 & -831.65365 & -832.54460 & -832.27468 & 36.69 & 40.39 & 3.86 & 0.16 \\
\hline $3 \mathrm{IH}^{+} \quad$ (via 3-position) & 0 & 246.35 & 258.07 & 219.40 & -59.26 & -50.09 & -831.65600 & -832.55263 & -832.27983 & 35.21 & 35.35 & 0.63 & 0.50 \\
\hline TS2la $^{+}$ & $163 \mathrm{i}$ & 242.38 & 255.03 & 214.55 & -53.87 & -45.86 & -831.65814 & -832.54728 & -832.27544 & 33.87 & 38.71 & 3.39 & -1.46 \\
\hline $3 \mathrm{HHa}^{+}$(via 1-position) & 0 & 246.77 & 258.34 & 219.96 & -58.77 & -49.67 & -831.65621 & -832.55310 & -832.27871 & 35.08 & 35.06 & 1.34 & 1.36 \\
\hline \multicolumn{14}{|c|}{.facile deprotonation with amine $\mathbf{2 l}$} \\
\hline $\mathbf{1 a}+2 * \mathbf{2 l}+\mathrm{Hw}^{+}$ & 0 & 369.38 & 389.37 & 297.11 & -9.00 & 3.82 & -1275.65386 & -1277.02961 & -1276.53799 & 0.00 & 0.00 & 0.00 & 0.00 \\
\hline $1 \mathrm{a}+\mathbf{2 l}+\mathbf{2 l \mathrm { H } ^ { + }}$ & 0 & 373.63 & 392.34 & 310.54 & -81.22 & -66.32 & -1275.57166 & -1276.94898 & -1276.55075 & 51.58 & 50.60 & -8.01 & -7.02 \\
\hline $\mathrm{TS3I}^{+}$ & $1085 \mathrm{i}$ & 370.31 & 389.68 & 335.66 & -60.12 & -50.00 & -1275.61244 & -1276.98229 & -1276.52406 & 25.99 & 29.69 & 8.74 & 5.04 \\
\hline $2 \mathrm{IH}^{+}+13$ & 0 & 375.62 & 394.23 & 327.01 & -78.50 & -65.50 & -1275.59450 & -1276.97416 & -1276.55140 & 37.25 & 34.79 & -8.41 & -5.96 \\
\hline TS3la $^{+}$ & $1127 \mathrm{i}$ & 370.94 & 389.64 & 337.00 & -61.50 & -51.13 & -1275.61416 & -1276.98393 & -1276.52535 & 24.91 & 28.66 & 7.93 & 4.18 \\
\hline $2 \mathrm{IH}^{+}+13 \mathrm{a}$ & 0 & 376.94 & 395.15 & 328.61 & -79.26 & -66.03 & -1275.59908 & -1276.97863 & -1276.55417 & 34.37 & 31.99 & 10.15 & -7.76 \\
\hline
\end{tabular}

The use of cyclo-1,3-hexadiene (1w) instead: $2.2 \mathrm{kcal} / \mathrm{mol}$ higher overall barrier $\left(\mathbf{T S 1} \boldsymbol{w}^{+}\right)$due to lower proton affinity than $1 \mathrm{a}$

\begin{tabular}{|c|c|c|c|c|c|c|c|c|c|c|c|c|c|}
\hline $1 w$ & 0 & 75.96 & 79.73 & 58.35 & -6.78 & -4.17 & -233.57446 & -233.82791 & -233.73856 & & & & \\
\hline $\mathbf{1 w}+\mathbf{2 a}+\mathrm{Hw}^{+}$ & 0 & 204.48 & 216.26 & 156.28 & 5.05 & 13.12 & -753.03037 & -753.84810 & -753.56911 & 0.00 & 0.00 & 0.00 & 0.00 \\
\hline $\mathbf{2 a H}++\mathbf{1 w}$ & 0 & 207.95 & 218.67 & 168.57 & -65.18 & -55.45 & -752.93254 & -753.75168 & -753.56538 & 61.39 & 60.51 & 2.34 & 3.22 \\
\hline TS1w $^{+}$ & $1208 \mathrm{i}$ & 204.18 & 215.27 & 177.48 & -56.03 & -47.98 & -752.94057 & -753.75021 & -753.54084 & 56.35 & 61.43 & 17.74 & 12.66 \\
\hline $\begin{array}{l}\text { 2a.1wH } H^{+}(\text {loose } \pi- \\
\text { complex) }\end{array}$ & 0 & 208.98 & 219.71 & 182.90 & -56.83 & -48.59 & -752.95307 & -753.76235 & -753.54530 & 48.51 & 53.81 & 14.94 & 9.64 \\
\hline $\mathbf{1} \mathbf{w} H^{+}+\mathbf{2 a}$ & 0 & 206.62 & 217.30 & 167.35 & -73.33 & -62.50 & -752.91028 & -753.72781 & -753.55470 & 75.36 & 75.49 & 9.05 & 8.92 \\
\hline \multicolumn{14}{|c|}{.. forming stable $\mathrm{N}-\mathrm{C}$ adduct of reactive allyl cation $\mathbf{1} \mathbf{w} \mathrm{H}^{+}$that may favor electrophilic aromatic substitution on ortho-position and deprotonation with 2 a } \\
\hline $\mathbf{1} \mathbf{w} \mathrm{H}^{+} \mathbf{2 a}$ & 0 & 209.76 & 220.21 & 183.89 & -55.22 & -47.27 & -752.97535 & -753.79261 & -753.57187 & 34.52 & 34.82 & -1.73 & -2.03 \\
\hline TS2 $\mathbf{w}^{+}$(on ortho-position) & -122 & 207.05 & 217.78 & 180.93 & -55.41 & -47.58 & -752.95635 & -753.76639 & -753.55087 & 46.45 & 51.27 & 11.44 & 6.62 \\
\hline $3 \mathbf{w H}^{+}$ & 0 & 209.37 & 219.82 & 183.64 & -55.08 & -47.27 & -752.96769 & -753.78328 & -753.56294 & 39.33 & 40.68 & 3.87 & 2.53 \\
\hline $\mathbf{1} \mathbf{w}+2 * \mathbf{2 a}+\mathrm{Hw}^{+}$ & 0 & 327.41 & 346.08 & 257.59 & -10.22 & 2.47 & -1272.02821 & -1273.41464 & -1272.98818 & 0.00 & 0.00 & 0.00 & 0.00 \\
\hline TS3 $^{+}$ & -1188 & 329.06 & 346.54 & 295.87 & -59.37 & -49.70 & -1271.98004 & -1273.35873 & -1272.96342 & 30.23 & 35.09 & 15.53 & 10.67 \\
\hline $35+2 \mathbf{a H}^{+}$ & 0 & 332.79 & 350.30 & 285.00 & -78.11 & -65.51 & -1271.96184 & -1273.35116 & -1272.99535 & 41.65 & 39.84 & -4.50 & -2.69 \\
\hline
\end{tabular}


14.4 Table S3. TPSS-D3/def2-TZVP + COSMO optimized Cartesian coordinates (in Å) in DCE solution. Each structure is labeled by the specific name (See Figure S2, Table S2 and main-text Figure 2), followed by the number of atoms, the total energy (in hartrees), and the atomic coordinates (in double-column text list).

\begin{tabular}{|c|c|c|c|c|c|c|c|}
\hline \multicolumn{4}{|c|}{$\mathbf{1 0 a}: 4,1$-hydroarylation of $\mathbf{1 a}$ with $\mathbf{2} \mathbf{h} \mathrm{Ph}_{2} \mathrm{NMe}$} & $\mathrm{H}$ & 2.0042542 & -2.6965527 & 4.4147282 \\
\hline \multicolumn{4}{|c|}{47} & $\mathrm{H}$ & 3.7583260 & -4.2231090 & 3.5289310 \\
\hline \multicolumn{4}{|c|}{ Energy $=-945.6166594500$} & $\mathrm{C}$ & -3.4525441 & 2.5338412 & -0.2906731 \\
\hline $\mathrm{N}$ & -2.9407068 & 1.1690881 & -0.1578752 & $\mathrm{H}$ & -3.4634531 & 3.0550684 & 0.6775547 \\
\hline $\mathrm{C}$ & -3.8763359 & 0.1101345 & -0.0593025 & $\mathrm{H}$ & -2.8358616 & 3.1047008 & -0.9919101 \\
\hline $\mathrm{C}$ & -1.5899614 & 0.9915468 & 0.1615401 & $\mathrm{H}$ & -4.4733832 & 2.4925012 & -0.6671824 \\
\hline $\mathrm{C}$ & -4.9188243 & 0.0024204 & -0.9941632 & & & & \\
\hline $\mathrm{C}$ & -3.8005670 & -0.8327812 & 0.9793068 & \multicolumn{4}{|c|}{$10: 4,3$-hydroarylation of $\mathbf{1 a}$ with $\mathbf{2} \mathbf{h} \mathrm{Ph}_{2} \mathrm{NMe}$} \\
\hline $\mathrm{C}$ & -0.7915826 & 2.0715652 & 0.5790854 & \multicolumn{4}{|l|}{47} \\
\hline $\mathrm{C}$ & -0.9644071 & -0.2648700 & 0.0089383 & \multicolumn{4}{|c|}{ Energy $=-945.6218235385$} \\
\hline $\mathrm{C}$ & -5.8606646 & -1.0198539 & -0.8881046 & $\mathrm{~N}$ & -3.5964665 & 0.6716927 & -0.1124197 \\
\hline $\mathrm{H}$ & -4.9823041 & 0.7130074 & -1.8131508 & $\mathrm{C}$ & -3.9489274 & -0.6951209 & 0.0015918 \\
\hline $\mathrm{C}$ & -4.7359180 & -1.8611811 & 1.0693378 & $\mathrm{C}$ & -2.2826270 & 1.1153835 & 0.0698602 \\
\hline $\mathrm{H}$ & -3.0069850 & -0.7512657 & 1.7154405 & $\mathrm{C}$ & -4.9153280 & -1.2476243 & -0.8545143 \\
\hline $\mathrm{C}$ & 0.5665784 & 1.8906706 & 0.8504554 & $\mathrm{C}$ & -3.3663558 & -1.5157776 & 0.9817154 \\
\hline $\mathrm{H}$ & -1.2238433 & 3.0582072 & 0.7029269 & $\mathrm{C}$ & -2.0091797 & 2.4427438 & 0.4451343 \\
\hline $\mathrm{C}$ & 0.3830727 & -0.4270374 & 0.2940118 & $\mathrm{C}$ & -1.1821654 & 0.2679159 & -0.1867848 \\
\hline $\mathrm{H}$ & -1.5436629 & -1.1134428 & -0.3399389 & $\mathrm{C}$ & -5.2884853 & -2.5850198 & -0.7294741 \\
\hline $\mathrm{C}$ & -5.7744327 & -1.9618884 & 0.1395736 & $\mathrm{H}$ & -5.3654966 & -0.6308841 & -1.6269096 \\
\hline $\mathrm{H}$ & -6.6570741 & -1.0874359 & -1.6243669 & $\mathrm{C}$ & -3.7328292 & -2.8552333 & 1.0902930 \\
\hline $\mathrm{H}$ & -4.6616541 & -2.5784906 & 1.8824299 & $\mathrm{H}$ & -2.6300716 & -1.0946539 & 1.6590537 \\
\hline $\mathrm{C}$ & 1.1817469 & 0.6446641 & 0.7252102 & $\mathrm{C}$ & -0.6925540 & 2.8929242 & 0.5760971 \\
\hline $\mathrm{H}$ & 1.1549759 & 2.7467176 & 1.1705614 & $\mathrm{H}$ & -2.8197993 & 3.1354120 & 0.6414298 \\
\hline $\mathrm{H}$ & 0.8282529 & -1.4117055 & 0.1750958 & $\mathrm{C}$ & 0.1159755 & 0.7306260 & -0.0415746 \\
\hline $\mathrm{H}$ & -6.5052015 & -2.7615836 & 0.2156661 & $\mathrm{H}$ & -1.3544155 & -0.7540206 & -0.5075224 \\
\hline $\mathrm{C}$ & 4.2392278 & 2.3881822 & 1.3511782 & $\mathrm{C}$ & -4.6978839 & -3.4000587 & 0.2387980 \\
\hline $\mathrm{C}$ & 4.8620936 & 3.4956286 & 2.1489182 & $\mathrm{H}$ & -6.0343428 & -2.9942449 & -1.4055362 \\
\hline $\mathrm{H}$ & 4.6095951 & 2.2541269 & 0.3327817 & $\mathrm{H}$ & -3.2726556 & -3.4724057 & 1.8573501 \\
\hline $\mathrm{C}$ & 3.2795004 & 1.5725513 & 1.7947397 & $\mathrm{C}$ & 0.3967452 & 2.0525978 & 0.3428095 \\
\hline $\mathrm{H}$ & 5.9455593 & 3.3474736 & 2.2482141 & $\mathrm{H}$ & -0.5324424 & 3.9263653 & 0.8691224 \\
\hline $\mathrm{H}$ & 4.4285479 & 3.5556449 & 3.1522696 & $\mathrm{H}$ & 0.9410639 & 0.0495787 & -0.2387599 \\
\hline $\mathrm{H}$ & 4.7225579 & 4.4648278 & 1.6523264 & $\mathrm{H}$ & -4.9854841 & -4.4432499 & 0.3295635 \\
\hline $\mathrm{C}$ & 2.6745289 & 0.4459510 & 0.9899511 & $\mathrm{C}$ & 1.8515231 & 2.4871788 & 0.5070278 \\
\hline $\mathrm{H}$ & 2.8959290 & 1.6914380 & 2.8097911 & $\mathrm{C}$ & 2.0288131 & 4.0069905 & 0.6579565 \\
\hline $\mathrm{H}$ & 3.1929751 & 0.4186995 & 0.0213166 & $\mathrm{H}$ & 2.3973067 & 2.1665067 & -0.3908357 \\
\hline $\mathrm{C}$ & 2.9596768 & -0.8784826 & 1.6911632 & $\mathrm{C}$ & 2.4482645 & 1.7624013 & 1.6863282 \\
\hline $\mathrm{C}$ & 3.9455778 & -1.7419349 & 1.2038119 & $\mathrm{H}$ & 3.0940322 & 4.2541370 & 0.6909589 \\
\hline $\mathrm{C}$ & 2.2652094 & -1.2381941 & 2.8536439 & $\mathrm{H}$ & 1.5701405 & 4.3704707 & 1.5842527 \\
\hline $\mathrm{C}$ & 4.2336644 & -2.9415850 & 1.8589567 & $\mathrm{H}$ & 1.5730669 & 4.5402143 & -0.1834918 \\
\hline $\mathrm{H}$ & 4.4900080 & -1.4730866 & 0.3011243 & $\mathrm{C}$ & 3.4566954 & 0.8801312 & 1.6008490 \\
\hline $\mathrm{C}$ & 2.5524936 & -2.4316412 & 3.5143914 & $\mathrm{H}$ & 1.9880355 & 1.9861737 & 2.6493498 \\
\hline $\mathrm{H}$ & 1.4845184 & -0.5834981 & 3.2330791 & $\mathrm{H}$ & 3.8885286 & 0.6890332 & 0.6169660 \\
\hline $\mathrm{C}$ & 3.5382948 & -3.2896815 & 3.0181205 & $\mathrm{C}$ & 4.0654649 & 0.1227520 & 2.6991454 \\
\hline $\mathrm{H}$ & 4.9985821 & -3.6039703 & 1.4621307 & $\mathrm{C}$ & 5.1709176 & -0.7030900 & 2.4237255 \\
\hline
\end{tabular}




$\begin{array}{rrrr}\mathrm{C} & 3.5976305 & 0.1752234 & 4.0266586 \\ \mathrm{C} & 5.7908104 & -1.4433679 & 3.4301579 \\ \mathrm{H} & 5.5442320 & -0.7590646 & 1.4034962 \\ \mathrm{C} & 4.2153742 & -0.5625954 & 5.0317187 \\ \mathrm{H} & 2.7403635 & 0.7950400 & 4.2733401 \\ \mathrm{C} & 5.3165683 & -1.3761867 & 4.7412019 \\ \mathrm{H} & 6.6433259 & -2.0729523 & 3.1897999 \\ \mathrm{H} & 3.8357915 & -0.5073318 & 6.0485772 \\ \mathrm{H} & 5.7951882 & -1.9512781 & 5.5287359 \\ \mathrm{C} & -4.6708249 & 1.6645982 & -0.1514404 \\ \mathrm{H} & -4.8028997 & 2.1498072 & 0.8260895 \\ \mathrm{H} & -4.4548323 & 2.4335606 & -0.9001945 \\ \mathrm{H} & -5.6023877 & 1.1649813 & -0.4122101\end{array}$

13a : 4,3-hydroarylation of $\mathbf{1 a}$ with $\mathbf{2 l}$ 44

Energy $=-831.2393569533$

$\begin{array}{lrrc}\mathrm{N} & -2.6205138 & 0.1026684 & 1.2866072 \\ \mathrm{C} & -1.5445858 & -0.7216172 & 0.9792072 \\ \mathrm{C} & -0.7354266 & -1.2751932 & 1.9926902 \\ \mathrm{C} & -1.2430276 & -1.0558153 & -0.3693420 \\ \mathrm{C} & 0.3073824 & -2.1521539 & 1.6901707 \\ \mathrm{H} & -0.9174848 & -1.0221189 & 3.0312702 \\ \mathrm{C} & -0.2001842 & -1.9370815 & -0.6317930 \\ \mathrm{C} & 0.5991649 & -2.5048594 & 0.3714690 \\ \mathrm{H} & 0.8970035 & -2.5518464 & 2.5100110 \\ \mathrm{H} & 0.0002915 & -2.1972121 & -1.6706055 \\ \mathrm{C} & 1.7113927 & -3.4763670 & -0.0220383 \\ \mathrm{C} & 2.5881508 & -3.9182172 & 1.1611285 \\ \mathrm{H} & 2.3574767 & -2.9707725 & -0.7533531 \\ \mathrm{C} & 1.1026403 & -4.6734566 & -0.7048295 \\ \mathrm{H} & 3.3962038 & -4.5632375 & 0.8035993 \\ \mathrm{H} & 2.0049053 & -4.4826483 & 1.8973866 \\ \mathrm{H} & 3.0322046 & -3.0532034 & 1.6654942 \\ \mathrm{C} & 1.3208594 & -5.0038664 & -1.9879026 \\ \mathrm{H} & 0.4334572 & -5.2713231 & -0.0850989 \\ \mathrm{H} & 2.0021968 & -4.3792326 & -2.5682047 \\ \mathrm{C} & 0.7427580 & -6.1332851 & -2.7230048 \\ \mathrm{C} & 1.1583510 & -6.3660609 & -4.0467954 \\ \mathrm{C} & -0.2164490 & -7.0047712 & -2.1717669 \\ \mathrm{C} & 0.6452618 & -7.4277139 & -4.7913387 \\ \mathrm{H} & 1.8962192 & -5.7013306 & -4.4906716 \\ \mathrm{C} & -0.7285924 & -8.0649158 & -2.9131742 \\ \mathrm{H} & -0.5675877 & -6.8482719 & -1.1557942 \\ \mathrm{C} & -0.3010306 & -8.2847840 & -4.2275221 \\ \mathrm{H} & 0.9844457 & -7.5848938 & -5.8117257 \\ \mathrm{H} & -1.4681750 & -8.7242560 & -2.4665730 \\ \mathrm{H} & -0.7040619 & -9.1132109 & -4.8030729 \\ \mathrm{C} & -2.7494667 & 0.6299531 & 2.6370657\end{array}$

$\begin{array}{rrrr}\mathrm{H} & -2.8465135 & -0.1823707 & 3.3651237 \\ \mathrm{H} & -1.8906238 & 1.2553575 & 2.9296758 \\ \mathrm{H} & -3.6550139 & 1.2380965 & 2.6827454 \\ \mathrm{C} & -3.2411471 & 0.8863888 & 0.2179199 \\ \mathrm{H} & -2.6400588 & 1.7820144 & -0.0189450 \\ \mathrm{H} & -4.2130524 & 1.2294280 & 0.5849535 \\ \mathrm{C} & -3.4095728 & 0.0303129 & -1.0333310 \\ \mathrm{H} & -3.9064623 & 0.6155071 & -1.8139007 \\ \mathrm{H} & -4.0512848 & -0.8260329 & -0.7941215 \\ \mathrm{C} & -2.0385529 & -0.4541211 & -1.5081784 \\ \mathrm{H} & -1.4862279 & 0.3968851 & -1.9316458 \\ \mathrm{H} & -2.1420888 & -1.1892959 & -2.3141197\end{array}$

13 : 4,1-hydroarylation of 1 a with 21 44

Energy $=-831.2342998280$

$\begin{array}{lll}\mathrm{N} & -3.3647445 & -0.9682875\end{array}$

C $\quad-1.9823074 \quad-0.9221257$

C $\quad-1.3916420-0.4960046$

C $\quad-1.1206444-1.3331356$

C $\quad-0.0083295 \quad-0.5113985$

H $\quad-2.0156976-0.1532672$

$-1.3406806-0.1131022$

$\begin{array}{llll}\text { C } & 0.8470709 & -0.9389844 & 1.0920258\end{array}$

H $\quad 0.4084628 \quad-0.1778923 \quad 3.0565552$

H $\quad 0.8929478 \quad-1.6818771 \quad-0.9272413$

$\begin{array}{llll}\text { C } & 3.4721571 & 0.0374126 & 3.3092258\end{array}$

$\begin{array}{llll}\text { C } & 3.9147975 & 0.0167453 & 4.7427985\end{array}$

$\begin{array}{llll}\mathrm{H} & 3.7312630 & 0.9277980 & 2.7325649\end{array}$

$\begin{array}{llll}\text { C } & 2.7932826 & -0.9405509 & 2.7043357\end{array}$

$\begin{array}{llll}\mathrm{H} & 5.0064735 & 0.1076282 & 4.8194275\end{array}$

H $\quad 3.6102222 \quad-0.9100408 \quad 5.2395152$

$\begin{array}{llll}\mathrm{H} & 3.4900897 & 0.8631673 & 5.2984472\end{array}$

$\begin{array}{llll}\text { C } & 2.3681387 & -0.9185581 & 1.2541730\end{array}$

$\mathrm{H} \quad 2.5214124 \quad-1.8366484 \quad 3.2653927$

$\begin{array}{llll}\mathrm{H} & 2.7501713 & 0.0143804 & 0.8157344\end{array}$

$\begin{array}{llll}\text { C } & 3.0450078 & -2.0695571 & 0.5178855\end{array}$

$\begin{array}{llll}\text { C } & 4.1091700 & -1.8258535 & -0.3557201\end{array}$

$\begin{array}{llll}\text { C } & 2.6361934 & -3.3937518 & 0.7254903\end{array}$

$\begin{array}{llll}\text { C } & 4.7542267 & -2.8781162 & -1.0104375\end{array}$

H $\quad 4.4331549 \quad-0.8014217 \quad-0.5267536$

$\begin{array}{llll}\text { C } & 3.2786681 & -4.4478723 & 0.0779650\end{array}$

$\begin{array}{llll}\mathrm{H} & 1.7983499 & -3.5950329 & 1.3883825\end{array}$

$\begin{array}{llll}\text { C } & 4.3413138 & -4.1937227 & -0.7944047\end{array}$

$\mathrm{H} \quad 5.5763653 \quad-2.6690093 \quad-1.6900280$

$\begin{array}{llll}\mathrm{H} & 2.9481034 & -5.4691241 & 0.2490480\end{array}$

H $\quad 4.8387989 \quad-5.0144080 \quad-1.3040516$

C $\quad-4.2059933 \quad-0.2913561 \quad 1.5564426$

H $\quad-4.0513635 \quad-0.7069729 \quad 2.5576934$ 


$\begin{array}{lrrr}\mathrm{H} & -4.0152824 & 0.7933847 & 1.5984338 \\ \mathrm{H} & -5.2497641 & -0.4519449 & 1.2788315 \\ \mathrm{C} & -3.9230933 & -1.1170931 & -0.7649616 \\ \mathrm{H} & -3.8971349 & -0.1594588 & -1.3148935 \\ \mathrm{H} & -4.9728556 & -1.4052246 & -0.6539891 \\ \mathrm{C} & -3.1513373 & -2.1806886 & -1.5393379 \\ \mathrm{H} & -3.6012107 & -2.3142206 & -2.5286315 \\ \mathrm{H} & -3.2271556 & -3.1344806 & -1.0033643 \\ \mathrm{C} & -1.6860826 & -1.7588688 & -1.6625368 \\ \mathrm{H} & -1.6132168 & -0.9229910 & -2.3730829 \\ \mathrm{H} & -1.0796127 & -2.5722067 & -2.0770363\end{array}$

$\mathbf{1} \mathrm{aH}^{+} \mathbf{2 l}$ : $\mathrm{N}-\mathrm{C}$ adduct of allyl cation $\mathbf{1} \mathrm{aH}^{+}$and $\mathbf{2 l}$ 45

Energy $=-831.6673760664$

$\begin{array}{lrrr}\mathrm{N} & -1.9562828 & -1.0914094 & -0.0754723 \\ \mathrm{C} & -1.5015465 & -1.0604665 & 1.3562286 \\ \mathrm{C} & -0.5956133 & -0.0653613 & 1.7354052 \\ \mathrm{C} & -1.9582160 & -2.0061073 & 2.2840640 \\ \mathrm{C} & -0.0801711 & -0.0438699 & 3.0272411 \\ \mathrm{H} & -0.2766005 & 0.6968857 & 1.0386755 \\ \mathrm{C} & -1.4180899 & -1.9653992 & 3.5771481 \\ \mathrm{C} & -0.4759669 & -1.0129260 & 3.9484538 \\ \mathrm{H} & 0.6265909 & 0.7310642 & 3.3050440 \\ \mathrm{H} & -1.7771170 & -2.6882978 & 4.3047320 \\ \mathrm{H} & -0.0767531 & -1.0065406 & 4.9579859 \\ \mathrm{C} & -0.7412951 & -1.6010058 & -1.0078166 \\ \mathrm{C} & -1.1774558 & -1.6552128 & -2.4707044 \\ \mathrm{H} & -0.5787802 & -2.6040063 & -0.6069178 \\ \mathrm{C} & 0.4895674 & -0.7831003 & -0.8496840 \\ \mathrm{H} & -0.3772354 & -2.1581556 & -3.0204461 \\ \mathrm{H} & -1.2881990 & -0.6528326 & -2.8922422 \\ \mathrm{H} & -2.0967761 & -2.2206663 & -2.6313619 \\ \mathrm{C} & 1.5799135 & -1.2560129 & -0.2207467 \\ \mathrm{H} & 0.4960081 & 0.1936856 & -1.3300651 \\ \mathrm{H} & 1.5199775 & -2.2506608 & 0.2220684 \\ \mathrm{C} & 2.8569892 & -0.5738845 & -0.0324724 \\ \mathrm{C} & 3.8759124 & -1.2409033 & 0.6710066 \\ \mathrm{C} & 3.1082567 & 0.7290383 & -0.5031574 \\ \mathrm{C} & 5.1078485 & -0.6304813 & 0.8979024 \\ \mathrm{H} & 3.6916449 & -2.2469596 & 1.0398966 \\ \mathrm{C} & 4.3372695 & 1.3379705 & -0.2763041 \\ \mathrm{H} & 2.3400202 & 1.2684528 & -1.0494755 \\ \mathrm{C} & 5.3424901 & 0.6612408 & 0.4243576 \\ \mathrm{H} & 5.8827631 & -1.1614939 & 1.4430014 \\ \mathrm{H} & 4.5164426 & 2.3436921 & -0.6454432 \\ \mathrm{H} & 6.3013067 & 1.1408795 & 0.5981781 \\ \mathrm{C} & -2.4188127 & 0.2818189 & -0.4972416 \\ \mathrm{H} & -3.2278958 & 0.5804214 & 0.1686737\end{array}$

$\begin{array}{lrrr}\mathrm{H} & -1.5941176 & 0.9857419 & -0.4244001 \\ \mathrm{H} & -2.7728242 & 0.2247225 & -1.5245029 \\ \mathrm{C} & -3.1129380 & -2.0773982 & -0.3216869 \\ \mathrm{H} & -2.6520862 & -3.0184150 & -0.6281930 \\ \mathrm{H} & -3.6793933 & -1.6714453 & -1.1578518 \\ \mathrm{C} & -3.9680237 & -2.3190365 & 0.9027154 \\ \mathrm{H} & -4.7834395 & -2.9840901 & 0.6035759 \\ \mathrm{H} & -4.4150028 & -1.3888557 & 1.2709715 \\ \mathrm{C} & -3.0843110 & -2.9567507 & 1.9694910 \\ \mathrm{H} & -2.7001230 & -3.9170461 & 1.5998313 \\ \mathrm{H} & -3.6421355 & -3.1655691 & 2.8861798\end{array}$

$1 \mathbf{a H}^{+} \mathrm{HOBf}_{3}{ }^{-}$: contact ion pair of $1 \mathbf{a H}^{+}$ 57

Energy $=-2673.329303618$

$\begin{array}{lrrc}\text { B } & -0.2794197 & 0.1325010 & 0.8266418 \\ \text { C } & -1.4625917 & 0.2473192 & 1.9541171 \\ \text { C } & -0.0930393 & -1.3105042 & 0.1166874 \\ \text { C } & 1.0623242 & 0.7622897 & 1.5322630 \\ \text { O } & -0.5519384 & 1.1120348 & -0.4138369 \\ \text { C } & -1.3836133 & -0.5838384 & 3.0764119 \\ \text { C } & -2.4990244 & 1.1761776 & 2.0087627 \\ \text { C } & 0.9981181 & -1.5307389 & -0.7303945 \\ \text { C } & -1.0099478 & -2.3600213 & 0.1618188 \\ \text { C } & 1.1535355 & 2.1321050 & 1.7767363 \\ \text { C } & 2.1308681 & 0.0207929 & 2.0426710 \\ \text { H } & -0.6055705 & 2.0363396 & -0.0932145 \\ \text { C } & -2.2618157 & -0.5315112 & 4.1506893 \\ \text { F } & -0.3982756 & -1.5121622 & 3.1431776 \\ \text { C } & -3.4060314 & 1.2704785 & 3.0627310 \\ \text { F } & -2.6825391 & 2.0800328 & 1.0028981 \\ \text { C } & 1.1962772 & -2.6990221 & -1.4548150 \\ \text { F } & 1.9259427 & -0.5574227 & -0.9026898 \\ \text { C } & -0.8551641 & -3.5469670 & -0.5505480 \\ \text { F } & -2.1582426 & -2.2560370 & 0.8756909 \\ \text { C } & 2.2010691 & 2.7540158 & 2.4422732 \\ \text { F } & 0.1422812 & 2.9593195 & 1.3465536 \\ \text { C } & 3.2034929 & 0.5937720 & 2.7234669 \\ \text { F } & 2.1783766 & -1.3227748 & 1.8995356 \\ \text { C } & -3.2897128 & 0.4075378 & 4.1443879 \\ \text { F } & -2.1303292 & -1.3729489 & 5.1933624 \\ \text { F } & -4.3860885 & 2.1939899 & 3.0426339 \\ \text { C } & 0.2590056 & -3.7220960 & -1.3618132 \\ \text { F } & 2.2684892 & -2.8432835 & -2.2562242 \\ \text { F } & -1.7901062 & -4.5141943 & -0.4845648 \\ \text { C } & 3.2435491 & 1.9690370 & 2.9244115 \\ \text { F } & 2.2163579 & 4.0863775 & 2.6270211 \\ \text { F } & 4.2068653 & -0.1742954 & 3.1870092 \\ \text { F } & -4.1513390 & 0.4807589 & 5.1714041\end{array}$




$\begin{array}{lrrl}\text { F } & 0.4144523 & -4.8507496 & -2.0671026 \\ \text { F } & 4.2734518 & 2.5326753 & 3.5740526 \\ \text { C } & -1.9159801 & 2.2764480 & -2.0484907 \\ \text { H } & -2.2281485 & 2.9867310 & -1.2765210 \\ \text { H } & -1.0269419 & 2.6554091 & -2.5611793 \\ \text { H } & -2.7256117 & 2.1917806 & -2.7777403 \\ \text { C } & -1.6489774 & 0.8983738 & -1.4646573 \\ \text { C } & -1.1509819 & -0.0756365 & -2.4703763 \\ \text { H } & -2.5117869 & 0.5113972 & -0.9197982 \\ \text { H } & -0.2160484 & 0.1836033 & -2.9625572 \\ \text { C } & -1.8574408 & -1.1648544 & -2.8128815 \\ \text { H } & -2.7886981 & -1.3590005 & -2.2796808 \\ \text { C } & -1.5025222 & -2.1624805 & -3.8215113 \\ \text { C } & -2.3401674 & -3.2795752 & -3.9866734 \\ \text { C } & -0.3371371 & -2.0774753 & -4.6060643 \\ \text { C } & -2.0226991 & -4.2852121 & -4.8974045 \\ \text { H } & -3.2413702 & -3.3576795 & -3.3833319 \\ \text { C } & -0.0196438 & -3.0821395 & -5.5130652 \\ \text { H } & 0.3274949 & -1.2247440 & -4.5022797 \\ \text { C } & -0.8596785 & -4.1910617 & -5.6627348 \\ \text { H } & -2.6797316 & -5.1433268 & -5.0060434 \\ \text { H } & 0.8869495 & -3.0042685 & -6.1063238 \\ \text { H } & -0.6064526 & -4.9743460 & -6.3712447\end{array}$

$\mathbf{1 a H}^{+}$: allyl cation after 4-protonation of $\mathbf{1 a}$ 21

Energy $=-387.7179768055$

$\begin{array}{lrrr}\mathrm{C} & -4.4343469 & 0.3217941 & 0.0129677 \\ \mathrm{C} & -3.1651782 & -0.4180413 & 0.0798339 \\ \mathrm{H} & -5.0429685 & -0.0754083 & -0.8127290 \\ \mathrm{H} & -4.2973900 & 1.3973513 & -0.1058789 \\ \mathrm{H} & -3.2266043 & -1.5003586 & 0.1932232 \\ \mathrm{C} & -1.9162712 & 0.1480963 & 0.0112690 \\ \mathrm{C} & -0.7844968 & -0.6735384 & 0.0889095 \\ \mathrm{H} & -1.8189900 & 1.2238878 & -0.1018817 \\ \mathrm{H} & -0.9652364 & -1.7420795 & 0.2011061 \\ \mathrm{C} & 0.5708759 & -0.2704190 & 0.0366461 \\ \mathrm{C} & 1.5701885 & -1.2772621 & 0.1331572 \\ \mathrm{C} & 0.9644024 & 1.0887072 & -0.1073198 \\ \mathrm{C} & 2.9109430 & -0.9384678 & 0.0868449 \\ \mathrm{H} & 1.2632743 & -2.3130782 & 0.2432585 \\ \mathrm{C} & 2.3057466 & 1.4158458 & -0.1522950 \\ \mathrm{H} & 0.2145070 & 1.8684872 & -0.1816052 \\ \mathrm{C} & 3.2775949 & 0.4061413 & -0.0556133 \\ \mathrm{H} & 3.6739453 & -1.7057105 & 0.1603191 \\ \mathrm{H} & 2.6113942 & 2.4506481 & -0.2626533 \\ \mathrm{H} & 4.3292415 & 0.6733784 & -0.0918886 \\ \mathrm{H} & -5.0212198 & 0.1147857 & 0.9202928\end{array}$

\section{1a $: \mathrm{PhCH}=\mathrm{CHCH}=\mathrm{CH}_{2}$}

20

Energy $=-387.2947304513$

$\begin{array}{llll}\text { C } & -7.8837161 & -0.4239420 & 0.0619242\end{array}$

$\begin{array}{llll}\text { C } & -6.5418411 & -0.5388658 & 0.0610881\end{array}$

$\begin{array}{llll}\mathrm{H} & -8.3708224 & 0.5462907 & 0.0614759\end{array}$

$\begin{array}{llll}\mathrm{H} & -8.5262022 & -1.3019043 & 0.0630590\end{array}$

$\begin{array}{llll}\mathrm{H} & -5.9287485 & 0.3632065 & 0.0597450\end{array}$

$\begin{array}{llll}\text { C } & -5.8340171 & -1.7989711 & 0.0616168\end{array}$

$\begin{array}{llll}\text { C } & -4.4829807 & -1.8912061 & 0.0606557\end{array}$

$\mathrm{H} \quad-6.4456457 \quad-2.7009946 \quad 0.0630071$

$\begin{array}{llll}\mathrm{H} & -3.9100348 & -0.9629009 & 0.0600698\end{array}$

C $\quad-3.6833892 \quad-3.1120144 \quad 0.0607895$

$\begin{array}{llll}\text { C } & -2.2788810 & -3.0025799 & 0.0612043\end{array}$

$\begin{array}{llll}\text { C } & -4.2453079 & -4.4054777 & 0.0606274\end{array}$

$\begin{array}{llll}\text { C } & -1.4660657 & -4.1345898 & 0.0614533\end{array}$

$\mathrm{H} \quad-1.8286291 \quad-2.0125744 \quad 0.0615666$

C $\quad-3.4336798 \quad-5.5347570 \quad 0.0608842$

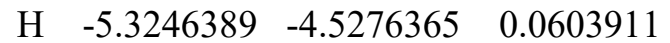

C $\quad-2.0396368 \quad-5.4074457 \quad 0.0612901$

$\mathrm{H} \quad-0.3852543 \quad-4.0232008 \quad 0.0618495$

$\mathrm{H} \quad-3.8876099 \quad-6.5219937 \quad 0.0607705$

H $\quad-1.4101229-6.2926244 \quad 0.0612616$

$\mathbf{1 n} \mathrm{H}^{+}$: allyl cation after 4-protonation of $\mathbf{1 a}$ 27

Energy $=-391.3313268072$

$\begin{array}{lrrr}\mathrm{C} & -0.7435798 & -1.1215169 & -1.0322830 \\ \mathrm{C} & 0.6954299 & -1.3171830 & -1.2112287 \\ \mathrm{H} & -1.1208329 & -0.4936179 & -1.8548298 \\ \mathrm{H} & -1.3064040 & -2.0531345 & -0.9743617 \\ \mathrm{H} & 1.3156212 & -0.4228248 & -1.2742462 \\ \mathrm{C} & 1.3281475 & -2.5410472 & -1.3112381 \\ \mathrm{C} & 2.7034435 & -2.5809819 & -1.4951390 \\ \mathrm{H} & 0.7558317 & -3.4636795 & -1.2559003 \\ \mathrm{H} & 3.2324186 & -1.6274211 & -1.5457648 \\ \mathrm{C} & 3.5099109 & -3.7858935 & -1.5931192 \\ \mathrm{C} & 4.4265473 & -3.7545687 & -2.8531860 \\ \mathrm{C} & 4.4158869 & -3.8420757 & -0.3025507 \\ \mathrm{H} & 2.8758196 & -4.6785130 & -1.6099142 \\ \mathrm{C} & 5.3320675 & -4.9889595 & -2.8843259 \\ \mathrm{H} & 5.0313325 & -2.8395058 & -2.8270467 \\ \mathrm{H} & 3.7977254 & -3.7133325 & -3.7475164 \\ \mathrm{C} & 5.3180830 & -5.0794904 & -0.3627899 \\ \mathrm{H} & 5.0204088 & -2.9287514 & -0.2576866 \\ \mathrm{H} & 3.7752799 & -3.8691918 & 0.5831024 \\ \mathrm{C} & 6.1929326 & -5.0916559 & -1.6203027 \\ \mathrm{H} & 5.9674549 & -4.9334648 & -3.7756149 \\ \mathrm{H} & 4.7132302 & -5.8902035 & -2.9856477\end{array}$




\begin{tabular}{|c|c|c|c|}
\hline $\mathrm{H}$ & 5.9421523 & -5.0853743 & 0.5388056 \\
\hline & 4.6956016 & -5.9828244 & -0.3321147 \\
\hline & 6.7960448 & -6.0054287 & -1.6478431 \\
\hline H & 6.8909392 & -4.2442520 & -1.5854582 \\
\hline & -0.9099713 & -0.5122210 & -0.1301954 \\
\hline 1n : $\mathrm{CyCH}=\mathrm{CHCH}=\mathrm{CH}_{2}$ & $\mathrm{CyCH}=\mathrm{CH}$ & 149327081 & \\
\hline ( & -0.7571927 & -1.2300277 & -1.3131778 \\
\hline & 0.5863874 & -1.2698611 & -1.3489314 \\
\hline $\mathrm{H}$ & -1.2974045 & -0.2894633 & -1.2655096 \\
\hline $\mathrm{H}$ & -1.3506733 & -2.1417449 & -1.3311473 \\
\hline H & 1.1476615 & -0.3347240 & -1.3299170 \\
\hline C & 1.3724588 & -2.4884895 & -1.4130653 \\
\hline C & 2.7176650 & -2.5145250 & -1.4491555 \\
\hline $\mathrm{H}$ & 0.8211457 & -3.4303939 & -1.4333876 \\
\hline $\mathrm{H}$ & 3.2612167 & -1.5665592 & -1.4310336 \\
\hline $\mathrm{C}$ & 3.5580897 & -3.7513940 & -1.5142392 \\
\hline C & 4.4072805 & -3.7762942 & -2.8057445 \\
\hline C & 4.4809875 & -3.8601688 & -0.2790283 \\
\hline $\mathrm{H}$ & 2.8938755 & -4.6276346 & -1.5238574 \\
\hline $\mathrm{C}$ & 5.3017794 & -5.0212056 & -2.8720629 \\
\hline $\mathrm{H}$ & 5.0337455 & -2.8730171 & -2.8323930 \\
\hline $\mathrm{H}$ & 3.7449097 & -3.7331625 & -3.6782347 \\
\hline $\mathrm{C}$ & 5.3776890 & -5.1034008 & -0.3477765 \\
\hline $\mathrm{H}$ & 5.1083562 & -2.9585675 & -0.2280316 \\
\hline 11 & 3.8705379 & -3.8777914 & 0.6314397 \\
\hline $\mathrm{C}$ & 6.2073433 & -5.1228622 & -1.6380221 \\
\hline $\mathrm{H}$ & 5.9050409 & -4.9974195 & -3.7876539 \\
\hline $\mathrm{H}$ & 4.6680008 & -5.9174676 & -2.9300434 \\
\hline 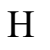 & 6.0351577 & -5.1364402 & 0.5293553 \\
\hline H & 4.7483191 & -6.0038284 & -0.3093809 \\
\hline $\mathrm{H}$ & 6.8140760 & -6.0351217 & -1.6858946 \\
\hline & 6.9050399 & -4.2733278 & -1.6313073 \\
\hline
\end{tabular}

$\mathbf{1} \mathbf{w} \mathrm{H}^{+} \mathbf{2 a}: \mathrm{N}-\mathrm{C}$ adduct of allyl cation $\mathbf{1} \mathbf{w} \mathrm{H}^{+}$ 39

Energy $=-752.9887510798$

$\begin{array}{lrrc}\mathrm{N} & 0.0893856 & -0.0997787 & 0.1298821 \\ \mathrm{H} & 0.4271285 & -0.4279015 & 1.0433992 \\ \mathrm{C} & 0.4041060 & 1.3525131 & 0.0658410 \\ \mathrm{C} & 0.1766033 & 2.0651460 & -1.1058688 \\ \mathrm{C} & 0.9227347 & 1.9489488 & 1.2087201 \\ \mathrm{C} & 0.4843586 & 3.4247806 & -1.1237389 \\ \mathrm{H} & -0.2271089 & 1.5862993 & -1.9905418 \\ \mathrm{C} & 1.2242602 & 3.3110720 & 1.1781159 \\ \mathrm{H} & 1.0902620 & 1.3674413 & 2.1114734 \\ \mathrm{C} & -1.3778814 & -0.3626616 & 0.1240970\end{array}$

$\begin{array}{lrrr}\mathrm{C} & 1.0048499 & 4.0470158 & 0.0136439 \\ \mathrm{H} & 0.3151407 & 3.9958541 & -2.0306503 \\ \mathrm{H} & 1.6298303 & 3.7889739 & 2.0636903 \\ \mathrm{C} & -1.9903088 & -0.5710741 & 1.3531721 \\ \mathrm{C} & -2.0898778 & -0.3726375 & -1.0695304 \\ \mathrm{H} & 1.2396266 & 5.1063283 & -0.0096231 \\ \mathrm{C} & -3.3660101 & -0.7979948 & 1.3909783 \\ \mathrm{H} & -1.4075883 & -0.5553878 & 2.2697522 \\ \mathrm{C} & -3.4652228 & -0.5991403 & -1.0184314 \\ \mathrm{H} & -1.6018305 & -0.2221909 & -2.0255923 \\ \mathrm{C} & -4.1025158 & -0.8096942 & 0.2060409 \\ \mathrm{H} & -3.8545122 & -0.9657170 & 2.3451060 \\ \mathrm{H} & -4.0349040 & -0.6137680 & -1.9417456 \\ \mathrm{H} & -5.1727849 & -0.9871968 & 0.2351241 \\ \mathrm{C} & 1.3807099 & -3.2664735 & -0.0828049 \\ \mathrm{C} & 0.5321230 & -2.4133992 & -0.6716906 \\ \mathrm{C} & 0.8902853 & -0.9776512 & -0.9107913 \\ \mathrm{C} & 2.3810546 & -0.6983997 & -0.7493094 \\ \mathrm{C} & 2.9404438 & -1.3767915 & 0.5048914 \\ \mathrm{C} & 2.7396900 & -2.8939444 & 0.4316390 \\ \mathrm{H} & 0.5272288 & -0.6275390 & -1.8782741 \\ \mathrm{H} & -0.4352463 & -2.7557157 & -1.0263851 \\ \mathrm{H} & 1.0857585 & -4.3094326 & 0.0157309 \\ \mathrm{H} & 2.5719694 & 0.3784486 & -0.7455055 \\ \mathrm{H} & 3.9998102 & -1.1327490 & 0.6201034 \\ \mathrm{H} & 2.4400215 & -0.9859012 & 1.4035335 \\ \mathrm{H} & 3.4940155 & -3.3455153 & -0.2303393 \\ \mathrm{H} & 2.8961682 & -3.3540874 & 1.4143223 \\ \mathrm{H} & 2.8697811 & -1.1043008 & -1.6421049\end{array}$

1wH $\mathrm{w}^{+}$: allyl cation $c-\mathrm{CH}_{2} \mathrm{CH}_{2} \mathrm{CH}_{2} \mathrm{CHCHCH}^{+}$ 15

Energy $=-233.9737835884$

$\begin{array}{lrrr}\mathrm{C} & -0.0735373 & 0.7759935 & 1.1901215 \\ \mathrm{C} & 0.0248231 & 1.4819601 & 0.0000069 \\ \mathrm{C} & -0.0738920 & 0.7759736 & -1.1900894 \\ \mathrm{C} & -0.0915173 & -0.6911753 & -1.2605965 \\ \mathrm{C} & 0.4525003 & -1.3657440 & -0.0000061 \\ \mathrm{C} & -0.0913648 & -0.6911690 & 1.2606425 \\ \mathrm{H} & -0.2145354 & 1.3320345 & -2.1152520 \\ \mathrm{H} & 0.0257388 & 2.5658584 & -0.0000254 \\ \mathrm{H} & -0.2149931 & 1.3320916 & 2.1151285 \\ \mathrm{H} & 0.4227480 & -1.0189760 & -2.1724295 \\ \mathrm{H} & 0.2081119 & -2.4293442 & 0.0000132 \\ \mathrm{H} & 1.5445175 & -1.2780971 & -0.0000294 \\ \mathrm{H} & -1.1526952 & -0.9531228 & 1.4516239 \\ \mathrm{H} & 0.4228528 & -1.0190061 & 2.1724872 \\ \mathrm{H} & -1.1528437 & -0.9531251 & -1.4515955\end{array}$


1w : cyclo-1,3-hexadiene

14

Energy $=-233.5621699697$

$\begin{array}{rrrr}\mathrm{C} & 0.0998143 & -1.4211403 & -0.0989276 \\ \mathrm{C} & 1.2486710 & -0.7206182 & -0.1288628 \\ \mathrm{C} & 1.2486982 & 0.7206699 & 0.1285079 \\ \mathrm{C} & 0.0997902 & 1.4211304 & 0.0991199 \\ \mathrm{C} & -1.2018200 & 0.7321016 & -0.2339441 \\ \mathrm{C} & -1.2018503 & -0.7320754 & 0.2338300 \\ \mathrm{H} & 2.1977638 & 1.2167211 & 0.3188296 \\ \mathrm{H} & 2.1977599 & -1.2167395 & -0.3189304 \\ \mathrm{H} & 0.1000910 & -2.4985325 & -0.2488219 \\ \mathrm{H} & 0.0999575 & 2.4984348 & 0.2497209 \\ \mathrm{H} & -2.0495667 & 1.2681112 & 0.2053917 \\ \mathrm{H} & -1.3451702 & 0.7707633 & -1.3275669 \\ \mathrm{H} & -1.3454715 & -0.7707800 & 1.3274137 \\ \mathrm{H} & -2.0495033 & -1.2680936 & -0.2056752\end{array}$

2a.1aH $\mathrm{H}^{+}$: loose $\pi$-complex of $\mathbf{2 a}$ and $\mathbf{1} \mathbf{a H} H^{+}$ 45

Energy $=-906.7171755430$

$\begin{array}{lrrr}\mathrm{N} & 1.5942672 & -0.1943372 & 0.1921418 \\ \mathrm{C} & 1.7697510 & -1.5708333 & -0.0145284 \\ \mathrm{C} & 1.0775801 & 0.8070835 & -0.5926042 \\ \mathrm{H} & 1.9516278 & 0.1262458 & 1.0860469 \\ \mathrm{C} & 2.0638558 & -2.3405302 & 1.1256813 \\ \mathrm{C} & 1.7234303 & -2.1974847 & -1.2694803 \\ \mathrm{C} & 1.2667577 & 2.1377243 & -0.1368043 \\ \mathrm{C} & 0.3463893 & 0.6035375 & -1.7863974 \\ \mathrm{C} & 2.2627273 & -3.7124050 & 1.0200767 \\ \mathrm{H} & 2.1357363 & -1.8501139 & 2.0920737 \\ \mathrm{C} & 1.9180374 & -3.5746945 & -1.3593658 \\ \mathrm{H} & 1.5820870 & -1.6249963 & -2.1761383 \\ \mathrm{C} & 0.8363070 & 3.2172513 & -0.8913701 \\ \mathrm{H} & 1.8055396 & 2.3010135 & 0.7932236 \\ \mathrm{C} & -0.0920853 & 1.6928223 & -2.5199641 \\ \mathrm{H} & 0.1023791 & -0.3949072 & -2.1212399 \\ \mathrm{C} & 2.1750047 & -4.3434385 & -0.2224529 \\ \mathrm{H} & 2.4859415 & -4.2884662 & 1.9132095 \\ \mathrm{H} & 1.8824477 & -4.0456098 & -2.3373967 \\ \mathrm{C} & 0.1458488 & 3.0087797 & -2.0884815 \\ \mathrm{H} & 1.0241997 & 4.2253138 & -0.5351511 \\ \mathrm{H} & -0.6503791 & 1.5191697 & -3.4348418 \\ \mathrm{H} & 2.3236199 & -5.4153662 & -0.3067726 \\ \mathrm{H} & -0.1723156 & 3.8503062 & -2.6940403 \\ \mathrm{C} & -2.3533889 & 3.1134625 & -0.7359928 \\ \mathrm{C} & -3.2003698 & 3.4735022 & -1.8989968 \\ \mathrm{H} & -1.8980113 & 3.9292822 & -0.1775349 \\ \mathrm{C} & -2.2132209 & 1.8364702 & -0.2435265\end{array}$

$\begin{array}{cccc}\mathrm{H} & -4.1087558 & 3.9741608 & -1.5294436 \\ \mathrm{H} & -3.4991996 & 2.5980852 & -2.4799270 \\ \mathrm{H} & -2.7008777 & 4.2001223 & -2.5478920 \\ \mathrm{C} & -1.4765677 & 1.5842940 & 0.9146778 \\ \mathrm{H} & -2.6681336 & 1.0138269 & -0.7885342 \\ \mathrm{H} & -1.0785364 & 2.4458387 & 1.4467752 \\ \mathrm{C} & -1.2626441 & 0.3090472 & 1.5232864 \\ \mathrm{C} & -0.5649515 & 0.2540556 & 2.7535450 \\ \mathrm{C} & -1.6951848 & -0.9035263 & 0.9333838 \\ \mathrm{C} & -0.3292589 & -0.9615391 & 3.3809555 \\ \mathrm{H} & -0.2250907 & 1.1810699 & 3.2075339 \\ \mathrm{C} & -1.4485047 & -2.1131923 & 1.5581435 \\ \mathrm{H} & -2.2142241 & -0.8886581 & -0.0189564 \\ \mathrm{C} & -0.7669861 & -2.1472777 & 2.7828175 \\ \mathrm{H} & 0.1990381 & -0.9916732 & 4.3285630 \\ \mathrm{H} & -1.7751721 & -3.0388531 & 1.0956523 \\ \mathrm{H} & -0.5760487 & -3.0999224 & 3.2668337\end{array}$

2a.1wH $\mathrm{w}^{+}$: loose $\pi$-complex of $\mathbf{2 a}$ and $\mathbf{1} \mathbf{w} \mathrm{H}^{+}$ 39

Energy $=-752.9691214331$

H $\quad 2.0836637 \quad-0.9561679 \quad-0.3983160$

$\begin{array}{llll}\mathrm{N} & 1.0699910 & -0.9568762 & -0.4551201\end{array}$

$\begin{array}{llll}\mathrm{H} & 0.4830838 & 0.0596422 & 1.4523857\end{array}$

C $\quad 0.5450202 \quad 0.1646184-1.0670600$

$\begin{array}{llll}\text { C } & 0.4900299 & -2.2257009 & -0.2375088\end{array}$

$\begin{array}{llll}\text { C } & -0.7900435 & 0.2634292 & -1.5280871\end{array}$

C $\quad 1.4054354 \quad 1.2713388 \quad-1.2366194$

$\begin{array}{llll}\text { C } & -0.8384243 & -2.3978084 & 0.1763502\end{array}$

$\begin{array}{llll}\text { C } & 1.3203524 & -3.3487048 & -0.3764894\end{array}$

$\begin{array}{llll}\text { C } & -1.2218350 & 1.4261146 & -2.1712885\end{array}$

H $\quad-1.4511355 \quad-0.5921233 \quad-1.4720925$

C $\quad 0.9446396 \quad 2.4315730 \quad-1.8309856$

H $\quad 2.4371725 \quad 1.1958568 \quad-0.9028129$

$\begin{array}{llll}\text { C } & -1.3302395 & -3.6804267 & 0.4134688\end{array}$

$\begin{array}{llll}\mathrm{H} & -1.4818357 & -1.5435937 & 0.3437711\end{array}$

C $\quad 0.8218875 \quad-4.6236578-0.1277309$

$\mathrm{H} \quad 2.3536793 \quad-3.2135448 \quad-0.6853530$

$\begin{array}{llll}\text { C } & -0.3785634 & 2.5220278 & -2.3023217\end{array}$

$\mathrm{H} \quad-2.2392214 \quad 1.4686990 \quad-2.5471284$

$\mathrm{H} \quad 1.6196182 \quad 3.2736820 \quad-1.9508191$

$\begin{array}{llll}\text { C } & -0.5093625 & -4.7982551 & 0.2595131\end{array}$

$\mathrm{H} \quad-2.3610122 \quad-3.8002814 \quad 0.7330579$

$\mathrm{H} \quad 1.4751603 \quad-5.4832154 \quad-0.2436088$

$\mathrm{H} \quad-0.7249890 \quad 3.4311186 \quad-2.7825107$

$\begin{array}{llll}\mathrm{H} & -0.8993616 & -5.7935592 & 0.4475811\end{array}$

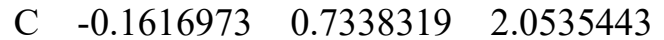

$\begin{array}{llll}\text { C } & -1.2449238 & 1.2306014 & 1.1809460\end{array}$

H $\quad-0.5977589 \quad 0.0689416 \quad 2.8103845$ 


$\begin{array}{lrrl}\mathrm{H} & -2.0647459 & 0.5592274 & 0.9431281 \\ \mathrm{C} & -1.3235431 & 2.5521884 & 0.7435571 \\ \mathrm{C} & -0.2648626 & 3.4094434 & 0.9479328 \\ \mathrm{H} & -2.1828153 & 2.8767999 & 0.1685571 \\ \mathrm{H} & -0.3019138 & 4.4094188 & 0.5233967 \\ \mathrm{C} & 0.9371264 & 3.0098450 & 1.7213884 \\ \mathrm{H} & 1.3523118 & 3.8826370 & 2.2364910 \\ \mathrm{H} & 1.7067576 & 2.7112425 & 0.9893061 \\ \mathrm{C} & 0.6445487 & 1.8669497 & 2.7034522 \\ \mathrm{H} & 1.5775669 & 1.4785943 & 3.1184989 \\ \mathrm{H} & 0.0594326 & 2.2707127 & 3.5375438\end{array}$

2a. $\mathrm{Bf}_{3}: \mathrm{B}-\mathrm{N}$ adduct of amine $\mathbf{2 a}$ and $\mathrm{Bf}_{3}$ 58

$\begin{array}{lccc}\text { Energy }=-2728.513210611 & \\ \mathrm{~B} & 0.0958400 & 0.0327574 & 0.0489781 \\ \mathrm{C} & -1.1848352 & -0.2060811 & -0.9493813 \\ \mathrm{C} & 0.7459629 & 1.5544213 & -0.0610837 \\ \mathrm{C} & 1.3995220 & -0.9463785 & -0.1752222 \\ \mathrm{C} & -1.4685542 & -1.3822852 & -1.6524352 \\ \mathrm{C} & -2.1473187 & 0.7973913 & -1.1450385 \\ \mathrm{C} & 1.5060543 & 2.1430906 & 0.9535036 \\ \mathrm{C} & 0.7485865 & 2.3033576 & -1.2471784 \\ \mathrm{C} & 2.2330724 & -1.4639705 & 0.8209407 \\ \mathrm{C} & 1.8735141 & -1.1797298 & -1.4730929 \\ \mathrm{C} & -2.5969731 & -1.5737861 & -2.4462378 \\ \mathrm{~F} & -0.6299332 & -2.4523363 & -1.6083713 \\ \mathrm{C} & -3.2872243 & 0.6529657 & -1.9273529 \\ \mathrm{~F} & -2.0006396 & 2.0156636 & -0.5753356 \\ \mathrm{C} & 2.1617058 & 3.3653434 & 0.8608821 \\ \mathrm{~F} & 1.6591075 & 1.5079162 & 2.1608168 \\ \mathrm{C} & 1.3966669 & 3.5275646 & -1.3951148 \\ \mathrm{~F} & 0.1065738 & 1.8678268 & -2.3545381 \\ \mathrm{C} & 3.3830727 & -2.2094704 & 0.5712338 \\ \mathrm{~F} & 1.9724641 & -1.2467028 & 2.1379093 \\ \mathrm{C} & 3.0143411 & -1.9156040 & -1.7718028 \\ \mathrm{~F} & 1.2163613 & -0.6620887 & -2.5378159 \\ \mathrm{C} & -3.5220056 & -0.5480115 & -2.5848863 \\ \mathrm{~F} & -2.7931724 & -2.7438462 & -3.0833040 \\ \mathrm{~F} & -4.1650940 & 1.6665040 & -2.0499358 \\ \mathrm{C} & 2.1073634 & 4.0727436 & -0.3331944 \\ \mathrm{~F} & 2.8525134 & 3.8557146 & 1.9067044 \\ \mathrm{~F} & 1.3393303 & 4.1889493 & -2.5656913 \\ \mathrm{C} & 3.7786922 & -2.4455695 & -0.7383259 \\ \mathrm{~F} & 4.1210637 & -2.6868905 & 1.5917785 \\ \mathrm{~F} & 3.3899818 & -2.1098632 & -3.0502583 \\ \mathrm{~F} & -4.6161955 & -0.7108727 & -3.3438160 \\ \mathrm{~F} & 2.7346662 & 5.2514386 & -0.4601700 \\ \mathrm{~F} & 4.8871075 & -3.1554432 & -1.0016726 \\ & & & \\ & & & \\ & \end{array}$

$\begin{array}{lrrl}\mathrm{N} & -0.5022305 & -0.1439022 & 1.6363764 \\ \mathrm{H} & 0.2512480 & 0.2697409 & 2.1956764 \\ \mathrm{C} & -1.7064905 & 0.6790952 & 1.9145191 \\ \mathrm{C} & -0.7088955 & -1.4964593 & 2.2363919 \\ \mathrm{C} & -2.9666101 & 0.1557224 & 1.6363992 \\ \mathrm{C} & -1.5546683 & 1.9470498 & 2.4642399 \\ \mathrm{C} & -0.8075520 & -2.6450764 & 1.4704235 \\ \mathrm{C} & -0.8153468 & -1.5431029 & 3.6263972 \\ \mathrm{C} & -4.0929410 & 0.9389757 & 1.8784793 \\ \mathrm{H} & -3.0668301 & -0.8500690 & 1.2437228 \\ \mathrm{C} & -2.6894536 & 2.7217825 & 2.7085582 \\ \mathrm{H} & -0.5706058 & 2.3305424 & 2.7104373 \\ \mathrm{C} & -1.0175785 & -3.8711444 & 2.1100029 \\ \mathrm{H} & -0.6993632 & -2.6111471 & 0.3985019 \\ \mathrm{C} & -1.0184399 & -2.7669326 & 4.2562784 \\ \mathrm{H} & -0.7442413 & -0.6299772 & 4.2108684 \\ \mathrm{C} & -3.9568115 & 2.2241630 & 2.4075694 \\ \mathrm{H} & -5.0777245 & 0.5397301 & 1.6570756 \\ \mathrm{H} & -2.5769884 & 3.7120853 & 3.1383923 \\ \mathrm{C} & -1.1240452 & -3.9360142 & 3.4969953 \\ \mathrm{H} & -1.0930695 & -4.7742126 & 1.5121482 \\ \mathrm{H} & -1.0943981 & -2.8060225 & 5.3384216 \\ \mathrm{H} & -4.8375103 & 2.8300701 & 2.5965240 \\ \mathrm{H} & -1.2856765 & -4.8908514 & 3.9876478\end{array}$

$\mathbf{2} \mathbf{a} \mathrm{H}^{+} \mathrm{HOBf}_{3}{ }^{-}$: contact ion pair of $\mathbf{2} \mathbf{a} \mathrm{H}^{+}$ 61

Energy $=-2805.014475965$

$\begin{array}{lrrc}\text { B } & 0.1413923 & 0.3472974 & 0.5838982 \\ \mathrm{C} & -1.3113004 & -0.1141700 & 1.2263462 \\ \mathrm{C} & 0.8116965 & -0.7613528 & -0.4246899 \\ \mathrm{C} & 1.1116118 & 0.7373076 & 1.8709821 \\ \mathrm{O} & 0.0107038 & 1.5822536 & -0.2770400 \\ \mathrm{C} & -1.3629569 & -1.1042326 & 2.2107941 \\ \mathrm{C} & -2.5519376 & 0.4381815 & 0.9169300 \\ \mathrm{C} & 2.0275395 & -0.4583919 & -1.0462278 \\ \mathrm{C} & 0.2811033 & -2.0010130 & -0.7813719 \\ \mathrm{C} & 0.8205464 & 1.8835863 & 2.6092478 \\ \mathrm{C} & 2.2081863 & 0.0194040 & 2.3462532 \\ \mathrm{H} & -0.2937628 & 2.3401050 & 0.2469259 \\ \mathrm{H} & -0.6125949 & 1.5675093 & -1.6004691 \\ \mathrm{C} & -2.5263622 & -1.5180847 & 2.8472174 \\ \mathrm{~F} & -0.2226260 & -1.7485151 & 2.5683732 \\ \mathrm{C} & -3.7487191 & 0.0590448 & 1.5227667 \\ \mathrm{~F} & -2.6774751 & 1.4125120 & -0.0360858 \\ \mathrm{C} & 2.6781869 & -1.2989343 & -1.9417995 \\ \mathrm{~F} & 2.6625616 & 0.7084116 & -0.7619617 \\ \mathrm{C} & 0.9066803 & -2.8851674 & -1.6589253 \\ \mathrm{~F} & -0.9056024 & -2.4298492 & -0.2850679\end{array}$




\begin{tabular}{|c|c|c|c|}
\hline $\mathrm{C}$ & 1.5439420 & 2.3214993 & 3.7119137 \\
\hline 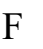 & -0.2561899 & 2.6555029 & 2.2554345 \\
\hline$C$ & 2.9683054 & 0.4123071 & 3.4460736 \\
\hline F & 2.5928990 & -1.1388473 & 1.7544455 \\
\hline $\mathrm{N}$ & -1.0607694 & 1.6196975 & -2.6266167 \\
\hline C & -3.7378524 & -0.9271253 & 2.5004169 \\
\hline $\mathrm{F}$ & -2.5002178 & -2.4881675 & 3.7838754 \\
\hline$F$ & -4.9151651 & 0.6394043 & 1.1688940 \\
\hline $\mathrm{C}$ & 2.1123511 & -2.5316362 & -2.2484220 \\
\hline F & 3.8483656 & -0.9387449 & -2.5099101 \\
\hline $\mathrm{F}$ & 0.3405110 & -4.0714282 & -1.9598892 \\
\hline $\mathrm{C}$ & 2.6377305 & 1.5737653 & 4.1345969 \\
\hline $\mathrm{F}$ & 1.2020420 & 3.4475161 & 4.3710883 \\
\hline F & 4.0223092 & -0.3246858 & 3.8540391 \\
\hline $\mathrm{H}$ & -2.0074353 & 1.9877171 & -2.4904398 \\
\hline $\mathrm{C}$ & -1.1817090 & 0.2541345 & -3.1917117 \\
\hline $\mathrm{C}$ & -0.2863864 & 2.5713555 & -3.4463931 \\
\hline $\mathrm{F}$ & -4.8803468 & -1.3082447 & 3.0996312 \\
\hline $\mathrm{F}$ & 2.7132353 & -3.3597850 & -3.1220205 \\
\hline $\mathrm{F}$ & 3.3633043 & 1.9679081 & 5.1963500 \\
\hline $\mathrm{C}$ & -2.3121240 & -0.4893199 & -2.8696187 \\
\hline $\mathrm{C}$ & -0.1667475 & -0.2585344 & -3.9900485 \\
\hline $\mathrm{C}$ & -0.8682175 & 3.1020290 & -4.5935057 \\
\hline $\mathrm{C}$ & 1.0114360 & 2.8869974 & -3.0596658 \\
\hline $\mathrm{C}$ & -2.4270014 & -1.7875284 & -3.3655811 \\
\hline $\mathrm{H}$ & -3.0912638 & -0.0647540 & -2.2435197 \\
\hline $\mathrm{C}$ & -0.2956934 & -1.5574106 & -4.4821118 \\
\hline $\mathrm{H}$ & 0.7031179 & 0.3417466 & -4.2321640 \\
\hline $\mathrm{C}$ & -0.1242516 & 3.9849517 & -5.3744409 \\
\hline $\mathrm{H}$ & -1.8815513 & 2.8294073 & -4.8738032 \\
\hline $\mathrm{C}$ & 1.7477849 & 3.7687405 & -3.8540336 \\
\hline $\mathrm{H}$ & 1.4321731 & 2.4579145 & -2.1562386 \\
\hline $\mathrm{C}$ & -1.4216240 & -2.3214107 & -4.1725229 \\
\hline $\mathrm{H}$ & -3.3043960 & -2.3774113 & -3.1213025 \\
\hline $\mathrm{H}$ & 0.4896096 & -1.9699278 & -5.1076149 \\
\hline $\mathrm{C}$ & 1.1828622 & 4.3153802 & -5.0066803 \\
\hline $\mathrm{H}$ & -0.5658018 & 4.4119244 & -6.2692450 \\
\hline $\mathrm{H}$ & 2.7619281 & 4.0279788 & -3.5668885 \\
\hline $\mathrm{H}$ & -1.5143634 & -3.3315705 & -4.5578373 \\
\hline $\mathrm{H}$ & 1.7597986 & 5.0008836 & -5.6196965 \\
\hline
\end{tabular}

2aH ${ }^{+}$: ammonium $\mathrm{Ph}_{2} \mathrm{NH}_{2}{ }^{+}$

25

Energy $=-519.4015153536$

$\begin{array}{lrrr}\mathrm{N} & 0.0539200 & 0.0000027 & -1.4525163 \\ \mathrm{H} & -0.7421779 & -0.0905123 & -2.0950566 \\ \mathrm{C} & -0.0886451 & 1.2443639 & -0.6433376 \\ \mathrm{C} & -1.1994571 & 1.3756181 & 0.1805508 \\ \mathrm{C} & 0.9055360 & 2.2073884 & -0.7314685\end{array}$ $\begin{array}{llll}\text { C } & -1.3157634 & 2.5354671 & 0.9445339\end{array}$

$\begin{array}{llll}\mathrm{H} & -1.9528792 & 0.5955779 & 0.2312835\end{array}$

$\begin{array}{llll}\text { C } & 0.7767096 & 3.3622540 & 0.0435453\end{array}$

H $\quad 1.75910892 .0690244 \quad-1.3888922$

C $\quad 0.1528611 \quad-1.2450591 \quad-0.6377964$

$\begin{array}{llll}\text { C } & -0.3299816 & 3.5242343 & 0.8770969\end{array}$

$\begin{array}{llll}\mathrm{H} & -2.1760331 & 2.6634868 & 1.5931997\end{array}$

$\begin{array}{llll}\mathrm{H} & 1.5425029 & 4.1290250 & -0.0087657\end{array}$

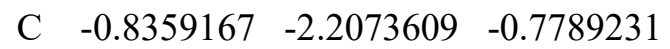

$\begin{array}{llll}\text { C } & 1.2183492 & -1.3774177 & 0.2436791\end{array}$

$\begin{array}{llll}\mathrm{H} & -0.4264860 & 4.4232035 & 1.4775124\end{array}$

$\begin{array}{llll}\text { C } & -0.7495237 & -3.3621803 & 0.0020041\end{array}$

$\mathrm{H} \quad-1.6532163 \quad-2.0685528 \quad-1.4808985$

$\begin{array}{llll}\text { C } & 1.2930558 & -2.5373607 & 1.0126302\end{array}$

$\mathrm{H} \quad 1.9685003 \quad-0.5978695 \quad 0.3347065$

$\begin{array}{llll}\text { C } & 0.3108757 & -3.5248050 & 0.8935892\end{array}$

$\mathrm{H} \quad-1.5118269-4.1285540 \quad-0.0912746$

$\begin{array}{llll}\mathrm{H} & 2.1178848 & -2.6663258 & 1.7056199\end{array}$

$\begin{array}{llll}\mathrm{H} & 0.3739814 & -4.4233209 & 1.4990936\end{array}$

$\mathrm{H} \quad 0.8833742 \quad 0.0910895 \quad-2.0513208$

2a : amine $\mathrm{Ph}_{2} \mathrm{NH}$

24

Energy $=-518.9759278348$

$\begin{array}{llll}\mathrm{N} & 0.0000340 & 0.0000127 & -1.0237770\end{array}$

$\mathrm{H} \quad 0.0001905 \quad 0.0000575 \quad-2.0363386$

$\begin{array}{llll}\text { C } & -0.0209054 & 1.2630260 & -0.4307484\end{array}$

C $\quad-0.4783854 \quad 1.4920706 \quad 0.8794629$

$\begin{array}{llll}\text { C } & 0.3849002 & 2.3662198 & -1.2066866\end{array}$

$\begin{array}{llll}\text { C } & -0.5037328 & 2.7874983 & 1.3945917\end{array}$

$\begin{array}{llll}\mathrm{H} & -0.8411223 & 0.6680879 & 1.4828992\end{array}$

C $\quad 0.3430965 \quad 3.6550316-0.6840935$

$\mathrm{H} \quad 0.7340993 \quad 2.1991411 \quad-2.2231015$

C $\quad 0.0211741 \quad-1.2630576 \quad-0.4308486$

$\begin{array}{llll}\text { C } & -0.0915982 & 3.8781787 & 0.6259839\end{array}$

$\begin{array}{llll}\mathrm{H} & -0.8634217 & 2.9418878 & 2.4084762\end{array}$

H $\quad 0.6611839 \quad 4.4892954 \quad-1.3035695$

C $\quad-0.3847337 \quad-2.3662180 \quad-1.2067807$

$\begin{array}{llll}\text { C } & 0.4786762 & -1.4921438 & 0.8793296\end{array}$

$\mathrm{H} \quad-0.1140564 \quad 4.8829834 \quad 1.0366429$

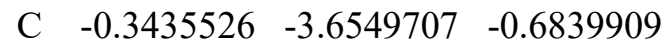

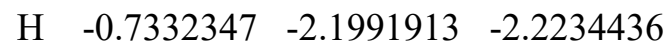

$\begin{array}{llll}\text { C } & 0.5035746 & -2.7875040 & 1.3946079\end{array}$

$\begin{array}{llll}\mathrm{H} & 0.8418684 & -0.6682586 & 1.4826080\end{array}$

$\begin{array}{llll}\text { C } & 0.0914895 & -3.8781753 & 0.6259634\end{array}$

$\mathrm{H} \quad-0.6627433 \quad-4.4890949 \quad-1.3030870$

$\mathrm{H} \quad 0.8626131 \quad-2.9418227 \quad 2.4087314$

H $\quad 0.1145864 \quad-4.8830538 \quad 1.0364089$ 
2g. $\mathrm{Bf}_{3} \mathrm{c}: \mathrm{B}-\mathrm{C}$ adduct of $\mathrm{Bf}_{3}$ on para-position 68

Energy $=-2959.685177997$

$\begin{array}{lrcc}\text { B } & -1.3180066 & 0.2428645 & -0.2972539 \\ \mathrm{C} & -2.1656909 & 1.5741343 & 0.1665385 \\ \mathrm{C} & -2.4661614 & -0.8206808 & -0.8116141 \\ \mathrm{C} & -0.2671187 & -0.3436148 & 0.8002173 \\ \mathrm{C} & -2.4828224 & 1.8744720 & 1.4990036 \\ \mathrm{C} & -2.7722146 & 2.4381642 & -0.7536987 \\ \mathrm{C} & -2.8811025 & -1.0964063 & -2.1166417 \\ \mathrm{C} & -3.2367799 & -1.4689976 & 0.1631486 \\ \mathrm{C} & 0.5193639 & 0.4806308 & 1.6140632 \\ \mathrm{C} & 0.0344505 & -1.7041089 & 0.9243285 \\ \mathrm{C} & -3.2736254 & 2.9532633 & 1.8878224 \\ \mathrm{~F} & -2.0386412 & 1.1036023 & 2.5191327 \\ \mathrm{C} & -3.5619963 & 3.5314562 & -0.4116189 \\ \mathrm{~F} & -2.6292207 & 2.2340911 & -2.0924798 \\ \mathrm{C} & -3.9388539 & -1.9475123 & -2.4372053 \\ \mathrm{~F} & -2.2623962 & -0.5434794 & -3.1964792 \\ \mathrm{C} & -4.2960903 & -2.3262471 & -0.1060918 \\ \mathrm{~F} & -2.9526981 & -1.2793078 & 1.4753905 \\ \mathrm{C} & 1.4868901 & 0.0146115 & 2.4998312 \\ \mathrm{~F} & 0.3652433 & 1.8274667 & 1.5853377 \\ \mathrm{C} & 0.9738208 & -2.2181787 & 1.8138050 \\ \mathrm{~F} & -0.5828514 & -2.6238468 & 0.1414345 \\ \mathrm{C} & -3.8148186 & 3.7981521 & 0.9277715 \\ \mathrm{~F} & -3.5223310 & 3.1824532 & 3.1928872 \\ \mathrm{~F} & -4.0920033 & 4.3223947 & -1.3658273 \\ \mathrm{C} & -4.6556617 & -2.5717942 & -1.4266672 \\ \mathrm{~F} & -4.2718952 & -2.1645425 & -3.7258903 \\ \mathrm{~F} & -4.9740761 & -2.9217378 & 0.8953811 \\ \mathrm{C} & 1.7114423 & -1.3514426 & 2.6124118 \\ \mathrm{~F} & 2.2120764 & 0.8756388 & 3.2421839 \\ \mathrm{~F} & 1.2046974 & -3.5462856 & 1.8803184 \\ \mathrm{~F} & -4.5782077 & 4.8437897 & 1.2866192 \\ \mathrm{~F} & -5.6785358 & -3.3936859 & -1.7171070 \\ \mathrm{~F} & 2.6478844 & -1.8257968 & 3.4520805 \\ \mathrm{H} & 2.5437099 & 2.4323106 & -0.5736459 \\ \mathrm{C} & 1.9649972 & 1.6130275 & -0.9813658 \\ \mathrm{C} & 2.5965097 & 0.3513265 & -1.2059903 \\ \mathrm{C} & 0.6379559 & 1.7742607 & -1.2527244 \\ \mathrm{C} & 1.8369072 & -0.6968106 & -1.8127826 \\ \mathrm{~N} & 3.9039690 & 0.1689894 & -0.8764955 \\ \mathrm{C} & -0.1950580 & 0.6870754 & -1.7060621 \\ \mathrm{H} & 0.1743568 & 2.7368763 & -1.0608052 \\ & 0.5144698 & -0.5120824 & -2.0897160 \\ & 2.3304122 & -1.6224355 & -2.0830650 \\ \mathrm{H} & 4362361 & -1.1231300 & -0.8605960\end{array}$

$\begin{array}{cccc}\mathrm{H} & -0.9874396 & 0.9807804 & -2.3875466 \\ \mathrm{H} & -0.0337600 & -1.3057935 & -2.5852345 \\ \mathrm{C} & 5.0182230 & 2.2950603 & -1.4084430 \\ \mathrm{C} & 5.2918133 & 1.2986279 & 0.7907457 \\ \mathrm{C} & 3.9482825 & -2.1858146 & -0.1716636 \\ \mathrm{C} & 5.7710408 & -1.2843763 & -1.4937119 \\ \mathrm{C} & 5.8458256 & 3.3508707 & -1.0273753 \\ \mathrm{H} & 4.5901880 & 2.2534578 & -2.4053047 \\ \mathrm{C} & 6.1197408 & 2.3565198 & 1.1621580 \\ \mathrm{H} & 5.0693331 & 0.4948139 & 1.4857171 \\ \mathrm{C} & 4.5871573 & -3.4228179 & -0.1343478 \\ \mathrm{H} & 3.0008967 & -2.0332432 & 0.3315677 \\ \mathrm{C} & 6.4071984 & -2.5237920 & -1.4470105 \\ \mathrm{H} & 6.2255347 & -0.4475162 & -2.0143089 \\ \mathrm{C} & 6.3969008 & 3.3825716 & 0.2554352 \\ \mathrm{H} & 6.0648891 & 4.1431942 & -1.7365445 \\ \mathrm{H} & 6.5442946 & 2.3811596 & 2.1609711 \\ \mathrm{C} & 5.8176102 & -3.5953761 & -0.7720815 \\ \mathrm{H} & 4.1279893 & -4.2462707 & 0.4041159 \\ \mathrm{H} & 7.3649168 & -2.6513032 & -1.9422131 \\ \mathrm{H} & 7.0437461 & 4.2041609 & 0.5476936 \\ \mathrm{H} & 6.3173173 & -4.5585337 & -0.7382233\end{array}$

2g. $\mathrm{Bf}_{3}$ : loose $\pi$-complex of $\mathrm{Ph}_{3} \mathrm{~N}$ and $\mathrm{Bf}_{3}$ 68

Energy $=-2959.697545601$

$\begin{array}{lccc}\text { B } & 0.0026773 & -0.0036215 & -1.5692086 \\ \text { C } & 1.5410338 & -0.2976551 & -1.5707261 \\ \text { C } & -1.0194600 & -1.1903812 & -1.5668547 \\ \text { C } & -0.5127130 & 1.4749252 & -1.5652114 \\ \text { C } & 2.4578515 & 0.5098692 & -2.2640142 \\ \text { C } & 2.1095689 & -1.3802635 & -0.8805510 \\ \text { C } & -2.2413389 & -1.1427150 & -0.8764426 \\ \text { C } & -0.7754702 & -2.3899542 & -2.2562211 \\ \text { C } & 0.1478834 & 2.5098819 & -0.8834259 \\ \text { C } & -1.6823667 & 1.8633232 & -2.2399713 \\ \text { C } & 3.8264423 & 0.2771263 & -2.2713675 \\ F & 2.0214088 & 1.5545061 & -2.9983899 \\ \text { C } & 3.4731160 & -1.6390464 & -0.8530514 \\ F & 1.3288363 & -2.2235140 & -0.1757406 \\ \text { C } & -3.1450381 & -2.1959915 & -0.8465185 \\ F & -2.5845073 & -0.0433900 & -0.1758486 \\ \text { C } & -1.6585833 & -3.4611963 & -2.2598790 \\ F & 0.3473110 & -2.5339888 & -2.9909379 \\ \text { C } & -0.3138231 & 3.8184742 & -0.8458627 \\ \text { F } & 1.2805948 & 2.2587658 & -0.1970810 \\ \text { C } & -2.1702023 & 3.1629804 & -2.2357203 \\ \text { F } & -2.3773117 & 0.9629751 & -2.9661522 \\ \text { C } & 4.3381367 & -0.8012070 & -1.5520535\end{array}$




$\begin{array}{lrrl}\mathrm{F} & 4.6583730 & 1.0709898 & -2.9646674 \\ \mathrm{~F} & 3.9641461 & -2.6807396 & -0.1663051 \\ \mathrm{C} & -2.8488973 & -3.3655955 & -1.5416304 \\ \mathrm{~F} & -4.2936344 & -2.1010709 & -0.1612892 \\ \mathrm{~F} & -1.3836817 & -4.5807613 & -2.9483442 \\ \mathrm{C} & -1.4845313 & 4.1460205 & -1.5246288 \\ \mathrm{~F} & 0.3500562 & 4.7652346 & -0.1672731 \\ \mathrm{~F} & -3.2864287 & 3.4840774 & -2.9093093 \\ \mathrm{~F} & 5.6541999 & -1.0339634 & -1.5378626 \\ \mathrm{~F} & -3.7064374 & -4.3906641 & -1.5250683 \\ \mathrm{~F} & -1.9459890 & 5.4000196 & -1.4987246 \\ \mathrm{H} & -0.0933794 & -2.3448614 & 1.7052979 \\ \mathrm{C} & -0.9868129 & -2.1284267 & 2.2784613 \\ \mathrm{C} & -1.0741871 & -0.9227394 & 2.9905501 \\ \mathrm{C} & -2.0365901 & -3.0426308 & 2.3132324 \\ \mathrm{C} & -2.2342379 & -0.6522928 & 3.7320847 \\ \mathrm{~N} & -0.0006896 & 0.0056930 & 2.9613650 \\ \mathrm{C} & -3.1976619 & -2.7659323 & 3.0381112 \\ \mathrm{H} & -1.9492598 & -3.9724040 & 1.7573406 \\ \mathrm{C} & -3.2876951 & -1.5639014 & 3.7437461 \\ \mathrm{H} & -2.3076593 & 0.2735112 & 4.2936627 \\ \mathrm{C} & 1.3404413 & -0.4592871 & 2.9871337 \\ \mathrm{C} & -0.2678622 & 1.3998177 & 2.9911438 \\ \mathrm{H} & -4.0194840 & -3.4751457 & 3.0524816 \\ \mathrm{H} & -4.1795261 & -1.3368577 & 4.3213851 \\ \mathrm{C} & 1.6887218 & -1.5997184 & 3.7265673 \\ \mathrm{C} & 2.3386283 & 0.2206581 & 2.2733088 \\ \mathrm{C} & -1.3543153 & 1.9276819 & 2.2775761 \\ \mathrm{C} & 0.5459385 & 2.2682972 & 3.7341512 \\ \mathrm{C} & 3.0052538 & -2.0553078 & 3.7346466 \\ \mathrm{H} & 0.9253611 & -2.1272072 & 4.2894964 \\ \mathrm{C} & 3.6555864 & -0.2307625 & 2.3039457 \\ \mathrm{H} & 2.0771237 & 1.1033641 & 1.7023075 \\ \mathrm{C} & -1.6199016 & 3.2942723 & 2.3120851 \\ \mathrm{H} & -1.9886139 & 1.2625796 & 1.7040170 \\ \mathrm{C} & 0.2843699 & 3.6366025 & 3.7458518 \\ \mathrm{H} & 1.3831660 & 1.8678449 & 4.2967992 \\ \mathrm{C} & 3.9990331 & -1.3750078 & 3.0271187 \\ \mathrm{H} & 3.2564654 & -2.9416806 & 4.3106747 \\ \mathrm{H} & 4.4152170 & 0.3107547 & 1.7465071 \\ \mathrm{C} & -0.8000366 & 4.1605682 & 3.0383529 \\ \mathrm{H} & -2.4675442 & 3.6843970 & 1.7549907 \\ \mathrm{H} & 0.9262741 & 4.2947323 & 4.3249916 \\ \mathrm{H} & -1.0244329 & -1.7313941 & 3.0388035 \\ & -1.0025431 & 5.2270505 & 3.0528312\end{array}$

$2 \mathrm{gcBf}_{3}{ }^{-}$: anion of deprotonated adduct $\mathbf{2 g} \cdot \mathrm{Bf}_{3} \mathrm{c}$ 67

Energy $=-2959.235489022$

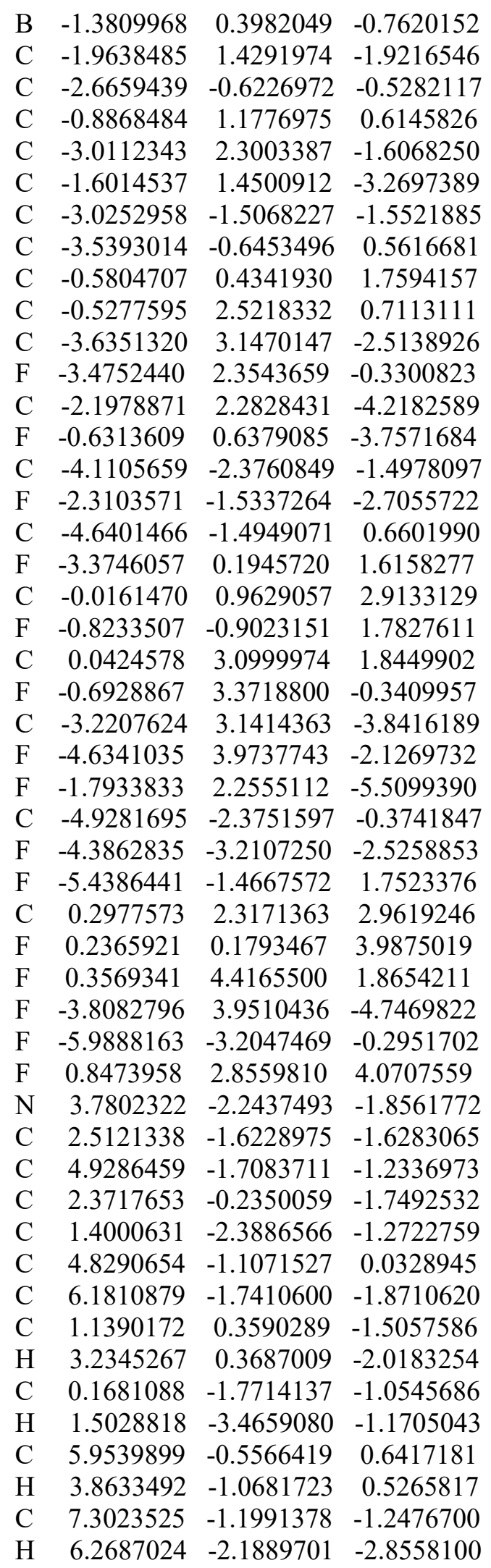




$\begin{array}{lrrr}\mathrm{C} & -0.0068379 & -0.3853882 & -1.1681698 \\ \mathrm{H} & 1.0622812 & 1.4416947 & -1.5920899 \\ \mathrm{H} & -0.6732794 & -2.3953178 & -0.7631787 \\ \mathrm{C} & 7.2007708 & -0.6012458 & 0.0117890 \\ \mathrm{H} & 5.8562445 & -0.0977787 & 1.6221370 \\ \mathrm{H} & 8.2606657 & -1.2315112 & -1.7595798 \\ \mathrm{H} & 8.0766042 & -0.1745282 & 0.4916462 \\ \mathrm{C} & 3.8511768 & -3.3854131 & -2.6835440 \\ \mathrm{C} & 4.7681831 & -4.4171420 & -2.4186908 \\ \mathrm{C} & 2.9804515 & -3.5173498 & -3.7788755 \\ \mathrm{C} & 4.8201826 & -5.5405145 & -3.2402522 \\ \mathrm{H} & 5.4348042 & -4.3347775 & -1.5661357 \\ \mathrm{C} & 3.0317491 & -4.6508498 & -4.5860562 \\ \mathrm{H} & 2.2613823 & -2.7304456 & -3.9830655 \\ \mathrm{C} & 3.9536664 & -5.6695893 & -4.3293075 \\ \mathrm{H} & 5.5344828 & -6.3284968 & -3.0158128 \\ \mathrm{H} & 2.3506804 & -4.7329562 & -5.4291213 \\ \mathrm{H} & 3.9934754 & -6.5500058 & -4.9639820\end{array}$

$2 \mathrm{gHc}^{+}$: para-protonated $\mathrm{Ph}_{2} \mathrm{NPhH}^{+}$

35

Energy $=-750.5809335832$

$\begin{array}{lrrr}\mathrm{N} & 0.0636162 & 0.0000336 & -0.0003960 \\ \mathrm{C} & -0.7038026 & -1.2223264 & 0.0335315 \\ \mathrm{C} & 1.4080713 & -0.0003064 & 0.0000317 \\ \mathrm{C} & -1.6986198 & -1.4239734 & -0.9246728 \\ \mathrm{C} & -0.4746646 & -2.1514357 & 1.0503675 \\ \mathrm{C} & 2.1359760 & 1.2053979 & 0.3044800 \\ \mathrm{C} & 2.1354420 & -1.2063103 & -0.3048873 \\ \mathrm{C} & -2.4596476 & -2.5894536 & -0.8733278 \\ \mathrm{H} & -1.8660606 & -0.6816855 & -1.6983717 \\ \mathrm{C} & -1.2435048 & -3.3130264 & 1.0918407 \\ \mathrm{H} & 0.2831062 & -1.9576380 & 1.8029125 \\ \mathrm{C} & 3.4869514 & 1.2007736 & 0.3258453 \\ \mathrm{H} & 1.5825723 & 2.0998742 & 0.5633243 \\ \mathrm{C} & 3.4863606 & -1.2026183 & -0.3262703 \\ \mathrm{H} & 1.5811212 & -2.1002914 & -0.5634992 \\ \mathrm{C} & -2.2327944 & -3.5339600 & 0.1312761 \\ \mathrm{H} & -3.2287699 & -2.7597158 & -1.6199216 \\ \mathrm{H} & -1.0760537 & -4.0375567 & 1.8824009 \\ \mathrm{C} & 4.2871210 & -0.0009773 & -0.0005536 \\ \mathrm{H} & 4.0218969 & 2.1021261 & 0.6097509 \\ \mathrm{H} & 4.0209294 & -2.1040991 & -0.6104293 \\ \mathrm{H} & -2.8317660 & -4.4384634 & 0.1691920 \\ \mathrm{C} & -0.7034398 & 1.2224778 & -0.0337970 \\ \mathrm{C} & -0.4729531 & 2.1528633 & -1.0491485 \\ \mathrm{C} & -1.6990717 & 1.4234491 & 0.9237590 \\ \mathrm{C} & -1.2412905 & 3.3148033 & -1.0899265 \\ \mathrm{H} & 0.2855651 & 1.9598647 & -1.8011482\end{array}$

$\begin{array}{rrrr}\mathrm{C} & -2.4596733 & 2.5892083 & 0.8730794 \\ \mathrm{H} & -1.8671715 & 0.6805664 & 1.6967364 \\ \mathrm{C} & -2.2315048 & 3.5348878 & -0.1301375 \\ \mathrm{H} & -1.0727284 & 4.0402532 & -1.8794055 \\ \mathrm{H} & -3.2295285 & 2.7587559 & 1.6190788 \\ \mathrm{H} & -2.8303760 & 4.4394695 & -0.1677347 \\ \mathrm{H} & 4.9802167 & -0.2193231 & 0.8305633 \\ \mathrm{H} & 4.9762596 & 0.2179517 & -0.8351408\end{array}$

2g $\mathrm{gH}^{+}$: N-protonated ammonium $\mathrm{Ph}_{3} \mathrm{NH}^{+}$ 35

Energy $=-750.5822054832$

$\begin{array}{llll}\mathrm{N} & -1.2093291 & -0.2255446 & 1.3466192\end{array}$

$\begin{array}{llll}\text { C } & -1.2857724 & 1.0390964 & 2.1535716\end{array}$

$\begin{array}{llll}\text { C } & -1.7478191 & -1.4247617 & 2.0733126\end{array}$

$\begin{array}{llll}\text { C } & -1.4160945 & 2.2283217 & 1.4467279\end{array}$

$\begin{array}{llll}\text { C } & -1.1959068 & 0.9920469 & 3.5370277\end{array}$

$\begin{array}{llll}\text { C } & -0.8811963 & -2.3196421 & 2.6834788\end{array}$

$\begin{array}{llll}\text { C } & -3.1298061 & -1.5610801 & 2.1224185\end{array}$

$\begin{array}{llll}\text { C } & -1.4612681 & 3.4235032 & 2.1626911\end{array}$

$\begin{array}{llll}\mathrm{H} & -1.4782640 & 2.2265222 & 0.3623261\end{array}$

$\begin{array}{llll}\text { C } & -1.2352551 & 2.1985645 & 4.2393201\end{array}$

$\mathrm{H} \quad-1.1024261 \quad 0.0478450 \quad 4.0613173$

C $-1.4327723 \quad-3.3984450 \quad 3.3779472$

$\begin{array}{llll}\mathrm{H} & 0.1933986 & -2.1909253 & 2.6213348\end{array}$

C $\quad-3.6646017 \quad-2.6471191 \quad 2.8135109$

$\begin{array}{llll}\mathrm{H} & -3.7774291 & -0.8366487 & 1.6370333\end{array}$

C $\quad-1.3679218 \quad 3.4079438 \quad 3.5563340$

$\mathrm{H} \quad-1.5699539 \quad 4.3620853 \quad 1.6294359$

H $\quad-1.1654433 \quad 2.1848537 \quad 5.3219339$

C $\quad-2.8168874 \quad-3.5619323 \quad 3.4425497$

$\mathrm{H} \quad-0.7731824 \quad-4.1102273 \quad 3.8631682$

H $\quad-4.7410976-2.7749725 \quad 2.8574525$

$\begin{array}{llll}\mathrm{H} & -1.4030873 & 4.3404977 & 4.1103642\end{array}$

$\begin{array}{llll}\text { C } & 0.1448018 & -0.4596323 & 0.7401278\end{array}$

$\begin{array}{llll}\text { C } & 0.1848139 & -1.1785625 & -0.4482742\end{array}$

$\begin{array}{llll}\text { C } & 1.2859465 & 0.0058479 & 1.3766944\end{array}$

C $\quad 1.4272505 \quad-1.4384110 \quad-1.0243514$

H $\quad-0.7299071 \quad-1.5327038 \quad-0.9146899$

$\begin{array}{llll}\text { C } & 2.5234658 & -0.2689346 & 0.7907557\end{array}$

$\begin{array}{llll}\mathrm{H} & 1.2223763 & 0.5708264 & 2.2996702\end{array}$

$\begin{array}{llll}\text { C } & 2.5941925 & -0.9866254 & -0.4036344\end{array}$

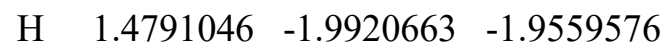

$\begin{array}{llll}\mathrm{H} & 3.4288918 & 0.0851906 & 1.2723126\end{array}$

$\mathrm{H} \quad 3.5598085 \quad-1.1915946 \quad-0.8546575$

$\mathrm{H} \quad-3.2369391 \quad-4.4056213 \quad 3.9806763$

$\begin{array}{llll}\mathrm{H} & -1.8433372 & -0.0808841 & 0.5513093\end{array}$

2g : amine $\mathrm{Ph}_{3} \mathrm{~N}$ 
34

Energy $=-750.1646787371$

$\begin{array}{lll}\mathrm{N} & 0.0042349 & 0.0023798\end{array}$

C $\quad-1.0459399 \quad-0.9509810$

C $\quad 1.3542360-0.4314608$

C $\quad-0.2964069 \quad 1.3879813$

C $\quad-0.9387045 \quad-2.1261102$

C $\quad-2.2034224 \quad-0.7303809$

C $\quad 1.7409480-1.5494340$

$\begin{array}{lll}\text { C } & 2.3191734 & 0.2516208\end{array}$

C $\quad-1.3738870 \quad 1.8802824$

C $\quad 0.4794666 \quad 2.2831638$

$\mathrm{H} \quad-0.0470308 \quad-2.2983073$

C $\quad-1.9681050 \quad-3.0645960$

C $-3.2346952-1.6669850$

$\mathrm{H} \quad-2.2894701 \quad 0.1755884$

C $3.0665967-1.9773291$

$\mathrm{H} \quad 0.9991884 \quad-2.0785310$

C $3.6456008 \quad-0.1740164$

$\begin{array}{lll}\mathrm{H} & 2.0243669 & 1.1143667\end{array}$

C $\quad-1.6707519 \quad 3.2413044$

$\mathrm{H} \quad-1.9742890 \quad 1.1924957$

C $\quad 0.1849739 \quad 3.6446760$

$\mathrm{H} \quad 1.3109265 \quad 1.9068703$

C $\quad-3.1240804-2.8415313$

$\mathrm{H} \quad-1.8708534 \quad-3.9689453$

H $\quad-4.1239665-1.4827223$

C $\quad 4.0290643 \quad-1.2922581$

H $\quad 3.3504122 \quad-2.8436716$

H $\quad 4.3804348 \quad 0.3645694$

C $\quad-0.8929008 \quad 4.1338163$

$\mathrm{H} \quad-2.5072046 \quad 3.6069957$

$\mathrm{H} \quad 0.7944461 \quad 4.3247086$

$\mathrm{H} \quad-3.9270574 \quad-3.5725784$

$\mathrm{H} \quad 5.0627661 \quad-1.6249187$

$\mathrm{H} \quad-1.1231992 \quad 5.1950285$

$\mathbf{2} \mathbf{h} \mathrm{H}^{+} \mathrm{HOBf}_{3}{ }^{-}$: contact ion pair of $\mathbf{2} \mathbf{h} \mathrm{H}^{+}$

64

Energy $=-2844.344295869$

$\begin{array}{lrrc}\text { B } & 0.0067481 & 0.3677563 & 0.5900421 \\ \text { C } & -1.3409607 & -0.2715635 & 1.3087400 \\ \text { C } & 0.7476589 & -0.6420888 & -0.4738748 \\ \text { C } & 0.9840088 & 0.8246378 & 1.8543702 \\ \text { O } & -0.3014855 & 1.5851656 & -0.2418918 \\ \text { C } & -1.2184789 & -1.3566202 & 2.1804420 \\ \text { C } & -2.6402481 & 0.2174066 & 1.1978828 \\ \text { C } & 1.9320002 & -0.2201172 & -1.0853406 \\ \text { C } & 0.2923257 & -1.8858987 & -0.9104962\end{array}$

$\begin{array}{llll}\text { C } & 0.6052267 & 1.9171385 & 2.6332654\end{array}$

$\begin{array}{llll}\text { C } & 2.1557743 & 0.2020083 & 2.2831891\end{array}$

$\begin{array}{llll}\mathrm{H} & -0.6800716 & 2.2933297 & 0.3015692\end{array}$

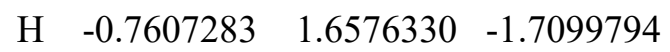

C $\quad-2.2756033 \quad-1.9281455 \quad 2.8768016$

$\begin{array}{llll}\text { F } & -0.0010208 & -1.9302627 & 2.3607622\end{array}$

$\begin{array}{llll}\text { C } & -3.7365977 & -0.3191620 & 1.8718837\end{array}$

$\begin{array}{llll}\text { F } & -2.9291757 & 1.2879012 & 0.3982282\end{array}$

$\begin{array}{llll}\text { C } & 2.6131496 & -0.9438066 & -2.0566286\end{array}$

$\begin{array}{lllll}\text { F } & 2.5064428 & 0.9533557 & -0.7115356\end{array}$

$\begin{array}{llll}\text { C } & 0.9561618 & -2.6615484 & -1.8602968\end{array}$

F $\quad-0.8522900 \quad-2.4274105 \quad-0.4258982$

$\begin{array}{llll}\text { C } & 1.3134754 & 2.3924502 & 3.7303671\end{array}$

$\begin{array}{llll}\text { F } & -0.5527272 & 2.5863790 & 2.3338753\end{array}$

$\begin{array}{llll}\text { C } & 2.9045967 & 0.6363084 & 3.3752213\end{array}$

$\begin{array}{llll}\text { F } & 2.6385969 & -0.8940740 & 1.6459335\end{array}$

$\begin{array}{llll}\mathrm{N} & -1.1286925 & 1.7454901 & -2.7363824\end{array}$

C $\quad-3.5549086-1.4024723 \quad 2.7211055$

$\begin{array}{llll}\text { F } & -2.0825365 & -2.9861682 & 3.6918158\end{array}$

$\begin{array}{llll}\text { F } & -4.9698490 & 0.2068194 & 1.7107382\end{array}$

$\begin{array}{llll}\text { C } & 2.1198424 & -2.1840307 & -2.4472277\end{array}$

F $\quad 3.7475366 \quad-0.4674782 \quad-2.6117884$

F $\quad 0.4681106 \quad-3.8629150 \quad-2.2318667$

$\begin{array}{llll}\text { C } & 2.4842630 & 1.7420449 & 4.1048984\end{array}$

F $\quad 0.8830821 \quad 3.4616739 \quad 4.4321263$

$\begin{array}{llll}\text { F } & 4.0360004 & -0.0062175 & 3.7349991\end{array}$

$\begin{array}{llll}\text { C } & -1.3676924 & 0.3494036 & -3.2114941\end{array}$

$\begin{array}{llll}\text { C } & -0.1122238 & 2.4848805 & -3.5227372\end{array}$

$\begin{array}{llll}\text { F } & -4.5977089 & -1.9344465 & 3.3848528\end{array}$

$\begin{array}{llll}\text { F } & 2.7546287 & -2.9049154 & -3.3899142\end{array}$

F $\quad 3.1974765 \quad 2.1751258 \quad 5.1605420$

C $\quad-2.4409164 \quad-0.3634320 \quad-2.6806466$

$\begin{array}{llll}\text { C } & -0.4924640 & -0.2402786 & -4.1181942\end{array}$

$\begin{array}{llll}\text { C } & -0.3808444 & 2.8242712 & -4.8468559\end{array}$

$\begin{array}{llll}\text { C } & 1.0818235 & 2.8292428 & -2.9029711\end{array}$

C $\quad-2.6549290 \quad-1.6774358 \quad-3.0932198$

$\mathrm{H} \quad-3.1021658 \quad 0.0797544 \quad-1.9462744$

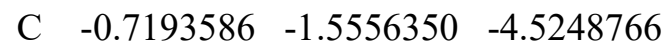

$\begin{array}{llll}\mathrm{H} & 0.3554839 & 0.3097923 & -4.5067702\end{array}$

C $\quad 0.5849043 \quad 3.5229414 \quad-5.5684249$

$\mathrm{H} \quad-1.3158008 \quad 2.5370570 \quad-5.3170349$

$\begin{array}{llll}\text { C } & 2.0425440 & 3.5282641 & -3.6377270\end{array}$

$\begin{array}{llll}\mathrm{H} & 1.2557225 & 2.5563851 & -1.8682344\end{array}$

$\begin{array}{llll}\text { C } & -1.8018495 & -2.2742324 & -4.0209043\end{array}$

$\begin{array}{llll}\mathrm{H} & -3.4893839 & -2.2338422 & -2.6790047\end{array}$

$\mathrm{H} \quad-0.0387325 \quad-2.0158128 \quad-5.2342449$

$\begin{array}{llll}\text { C } & 1.7967327 & 3.8728546 & -4.9661474\end{array}$

$\begin{array}{llll}\mathrm{H} & 0.3902451 & 3.7896743 & -6.6022953\end{array}$

$\mathrm{H} \quad 2.9812067 \quad 3.8008830 \quad-3.1657810$ 


$\begin{array}{crrr}\text { H } & -1.9722690 & -3.2976868 & -4.3383542 \\ \mathrm{H} & 2.5467718 & 4.4139654 & -5.5346054 \\ \mathrm{C} & -2.3987421 & 2.5630919 & -2.6435453 \\ \mathrm{H} & -3.0514755 & 2.1192565 & -1.8965982 \\ \mathrm{H} & -2.1118071 & 3.5680910 & -2.3370591 \\ \mathrm{H} & -2.8832564 & 2.5803740 & -3.6180733\end{array}$

\begin{tabular}{|c|c|c|c|}
\hline & & \\
\hline $\mathrm{N}$ & -2.7761487 & 1.1915912 & -0.6458884 \\
\hline C & -3.8255391 & 0.2195124 & -0.2054062 \\
\hline $\mathrm{C}$ & -1.4477979 & 0.8937038 & -0.0220519 \\
\hline $\mathrm{C}$ & -4.5295058 & -0.4685414 & -1.1832752 \\
\hline $\mathrm{C}$ & -4.0638426 & 0.0518983 & 1.1528143 \\
\hline $\mathrm{C}$ & -0.9568009 & 1.6712736 & 1.0168853 \\
\hline C & -0.7651705 & -0.2148816 & -0.5118317 \\
\hline 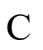 & -5.5199127 & -1.3665776 & -0.7799016 \\
\hline $\mathrm{H}$ & -4.3161539 & -0.3119673 & -2.2369602 \\
\hline C & -5.0576078 & -0.8454346 & 1.5400893 \\
\hline $\mathrm{H}$ & -3.4861287 & 0.5935801 & 1.8946469 \\
\hline$C$ & 0.2713635 & 1.3186756 & 1.5828427 \\
\hline $\mathrm{H}$ & -1.4953268 & 2.5342776 & 1.3892003 \\
\hline $\mathrm{C}$ & 0.4599436 & -0.5511019 & 0.0591320 \\
\hline $\mathrm{H}$ & -1.1813020 & -0.8094046 & -1.3201238 \\
\hline $\mathrm{C}$ & -5.7836246 & -1.5516271 & 0.5772340 \\
\hline $\mathrm{H}$ & -6.0782033 & -1.9181386 & -1.5289778 \\
\hline $\mathrm{H}$ & -5.2576262 & -0.9957710 & 2.5957964 \\
\hline $\mathrm{C}$ & 0.9761841 & 0.2135661 & 1.1083828 \\
\hline 1 & 0.6715463 & 1.9180354 & 2.3938509 \\
\hline H & 1.0078327 & -1.4086027 & -0.3171654 \\
\hline $\mathrm{H}$ & -6.5542202 & -2.2499182 & 0.8875820 \\
\hline $\mathrm{H}$ & 1.9295888 & -0.0522833 & 1.5534433 \\
\hline $\mathrm{C}$ & -3.2514789 & 2.6191003 & -0.4821581 \\
\hline $\mathrm{H}$ & -3.4934044 & 2.7848209 & 0.5651842 \\
\hline $\mathrm{H}$ & -2.4581036 & 3.2842108 & -0.8193817 \\
\hline 11 & -4.1443362 & 2.7274962 & -1.0956497 \\
\hline & -2.6552441 & 1.0435833 & -1.6539537 \\
\hline
\end{tabular}

2h : amine $\mathrm{Ph}_{2} \mathrm{NMe}$

27

Energy $=-558.3022197299$

$\begin{array}{lrrr}\mathrm{N} & -2.8112990 & 1.1478501 & -0.1493348 \\ \mathrm{C} & -3.8494865 & 0.1888795 & -0.0324290 \\ \mathrm{C} & -1.5159817 & 0.8964430 & 0.3089526 \\ \mathrm{C} & -4.7723144 & 0.0216932 & -1.0765931 \\ \mathrm{C} & -3.9917681 & -0.5870417 & 1.1291492 \\ \mathrm{C} & -0.6403648 & 1.9577711 & 0.6170584 \\ \mathrm{C} & -1.0209433 & -0.4223678 & 0.4105685\end{array}$ $\begin{array}{llll}\text { C } & -5.8132943 & -0.8983255 & -0.9580456\end{array}$

$\begin{array}{llll}\mathrm{H} & -4.6646739 & 0.6038252 & -1.9872524\end{array}$

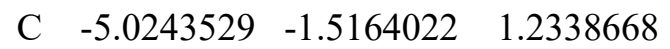

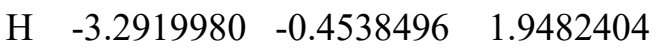

$\begin{array}{llll}\text { C } & 0.6709179 & 1.7024247 & 1.0210152\end{array}$

$\begin{array}{llll}\mathrm{H} & -0.9804732 & 2.9847614 & 0.5482470\end{array}$

$\begin{array}{llll}\text { C } & 0.2855947 & -0.6605068 & 0.8200173\end{array}$

$\begin{array}{llll}\mathrm{H} & -1.6646237 & -1.2570744 & 0.1544262\end{array}$

$\begin{array}{llll}\text { C } & -5.9442155 & -1.6768903 & 0.1939634\end{array}$

$\mathrm{H} \quad-6.5149757 \quad-1.0168953 \quad-1.7792029$

$\begin{array}{llll}\mathrm{H} & -5.1200070 & -2.1057713 & 2.1418257\end{array}$

C $\quad 1.1477272 \quad 0.3968117 \quad 1.1330553$

$\begin{array}{llll}\mathrm{H} & 1.3207457 & 2.5420704 & 1.2540713\end{array}$

$\begin{array}{llll}\mathrm{H} & 0.6379245 & -1.6868777 & 0.8836721\end{array}$

$\mathrm{H} \quad-6.7523337 \quad-2.3970189 \quad 0.2815447$

$\mathrm{H} \quad 2.1680440 \quad 0.2039055 \quad 1.4503379$

C $\quad-3.1739228 \quad 2.5161412 \quad-0.5223192$

$\mathrm{H} \quad-3.1987176 \quad 3.1777341 \quad 0.3551946$

$\mathrm{H} \quad-2.4591800 \quad 2.9183337 \quad-1.2474478$

$\mathrm{H} \quad-4.1666983 \quad 2.5052980 \quad-0.9695870$ 


\begin{tabular}{|c|c|c|c|}
\hline $\mathrm{N}$ & -1.3665774 & 0.9927279 & -2.7227535 \\
\hline$C$ & -3.6179965 & 0.2212154 & 3.3387865 \\
\hline 4 & -2.6930613 & -1.7022176 & 4.3708922 \\
\hline F & -4.4849561 & 2.1450463 & 2.2620703 \\
\hline $\mathrm{C}$ & 0.6977513 & -3.3096004 & -2.0090628 \\
\hline $\mathrm{F}$ & 2.8369376 & -2.4104609 & -2.4846364 \\
\hline $\mathrm{F}$ & -1.4767554 & -4.1206975 & -1.5444124 \\
\hline C & 3.1862076 & 1.2864172 & 3.5921104 \\
\hline $\mathrm{F}$ & 2.3375341 & 3.5016002 & 3.6123383 \\
\hline$F$ & 3.9751739 & -0.9441949 & 3.5367645 \\
\hline $\mathrm{C}$ & -0.6629324 & 0.0368136 & -3.6011382 \\
\hline $\mathrm{F}$ & -4.6636935 & 0.1977087 & 4.1852394 \\
\hline $\mathrm{F}$ & 0.9341746 & -4.3559933 & -2.8193213 \\
\hline $\mathrm{F}$ & 4.1285352 & 1.6159907 & 4.4943728 \\
\hline $\mathrm{C}$ & -1.2359228 & -1.1577994 & -4.0189426 \\
\hline $\mathrm{C}$ & 0.6156973 & 0.4354948 & -4.0075291 \\
\hline $\mathrm{C}$ & -0.5028596 & -1.9915313 & -4.8670597 \\
\hline $\mathrm{H}$ & -2.2287307 & -1.4528691 & -3.7015539 \\
\hline $\mathrm{C}$ & 1.3266093 & -0.4102667 & -4.8601345 \\
\hline $\mathrm{C}$ & 0.7740107 & -1.6197219 & -5.2846712 \\
\hline $\mathrm{H}$ & -0.9363870 & -2.9307749 & -5.1948871 \\
\hline $\mathrm{H}$ & 2.3201083 & -0.1171363 & -5.1874830 \\
\hline $\mathrm{H}$ & 1.3411351 & -2.2721341 & -5.9413973 \\
\hline $\mathrm{C}$ & -2.7576754 & 0.5989468 & -2.3470121 \\
\hline $\mathrm{H}$ & -2.7280908 & -0.3505843 & -1.8138427 \\
\hline $\mathrm{H}$ & -3.1515320 & 1.3759149 & -1.6937025 \\
\hline $\mathrm{H}$ & -3.3682117 & 0.5171121 & -3.2464740 \\
\hline $\mathrm{C}$ & -1.3662249 & 2.3752090 & -3.3650946 \\
\hline $\mathrm{H}$ & -1.8654401 & 3.0336525 & -2.6523930 \\
\hline $\mathrm{H}$ & -1.9892392 & 2.2847191 & -4.2561814 \\
\hline $\mathrm{C}$ & 0.0591912 & 2.8575539 & -3.6978902 \\
\hline $\mathrm{H}$ & 0.0704444 & 3.1944627 & -4.7373930 \\
\hline $\mathrm{H}$ & 0.3127523 & 3.7159770 & -3.0712694 \\
\hline $\mathrm{C}$ & 1.1271862 & 1.7556780 & -3.5074745 \\
\hline $\mathrm{H}$ & 1.3583224 & 1.6667455 & -2.4391309 \\
\hline $\mathrm{H}$ & 2.0496446 & 2.0325081 & -4.0227431 \\
\hline \multicolumn{4}{|c|}{$\mathbf{2} \mathbf{I H}^{+}$: tertiary ammonium } \\
\hline \multicolumn{4}{|c|}{25} \\
\hline \multicolumn{4}{|c|}{ Energy $=-444.3617204927$} \\
\hline $\mathrm{H}$ & 1.1486216 & -1.6669754 & -1.1184335 \\
\hline $\mathrm{N}$ & 1.1630431 & -0.9968271 & -0.3436577 \\
\hline $\mathrm{C}$ & -0.2243913 & -0.4667484 & -0.2050840 \\
\hline $\mathrm{C}$ & -1.2605579 & -1.3904043 & -0.3272674 \\
\hline $\mathrm{C}$ & -0.4391279 & 0.8860257 & 0.0551394 \\
\hline $\mathrm{C}$ & -2.5738691 & -0.9596767 & -0.1749868 \\
\hline $\mathrm{H}$ & -1.0433515 & -2.4328378 & -0.5447663 \\
\hline $\mathrm{C}$ & -1.7746922 & 1.2901753 & 0.2083236 \\
\hline $\mathrm{C}$ & -2.8273300 & 0.3873872 & 0.0997489 \\
\hline
\end{tabular}

$\begin{array}{crrc}\mathrm{H} & -3.3893296 & -1.6690633 & -0.2683970 \\ \mathrm{H} & -1.9782190 & 2.3374679 & 0.4147853 \\ \mathrm{H} & -3.8486916 & 0.7327665 & 0.2260494 \\ \mathrm{C} & 1.5841579 & -1.7662562 & 0.8893174 \\ \mathrm{H} & 1.5832369 & -1.0823258 & 1.7351471 \\ \mathrm{H} & 0.8633212 & -2.5660851 & 1.0497151 \\ \mathrm{H} & 2.5797648 & -2.1711482 & 0.7097166 \\ \mathrm{C} & 2.1733878 & 0.0784904 & -0.7166227 \\ \mathrm{H} & 1.9355210 & 0.3707777 & -1.7415849 \\ \mathrm{H} & 3.1515111 & -0.4026940 & -0.6937253 \\ \mathrm{C} & 2.0604400 & 1.2521107 & 0.2398088 \\ \mathrm{H} & 2.8450525 & 1.9700725 & -0.0124441 \\ \mathrm{H} & 2.2505491 & 0.9253241 & 1.2672741 \\ \mathrm{C} & 0.6801031 & 1.9000401 & 0.1199084 \\ \mathrm{H} & 0.6404076 & 2.5075904 & -0.7938181 \\ \mathrm{H} & 0.5035874 & 2.5839258 & 0.9554196\end{array}$

2l : tertiary amine with only one aryl

24

Energy $=-443.9199259592$

$\begin{array}{llll}\text { C } & 2.4839571 & 0.9806320 & -0.1334334\end{array}$

$\begin{array}{llll}\text { C } & 1.1496540 & 1.3827963 & -0.1491460\end{array}$

$\begin{array}{llll}\text { C } & 0.1008724 & 0.4383880 & -0.0681385\end{array}$

$\begin{array}{llll}\text { C } & 0.4405193 & -0.9362916 & 0.0612895\end{array}$

$\begin{array}{llll}\text { C } & 1.7850292 & -1.3061166 & 0.0680227\end{array}$

$\begin{array}{llll}\text { C } & 2.8182561 & -0.3696356 & -0.0280235\end{array}$

$\begin{array}{llll}\mathrm{H} & 3.2633393 & 1.7355562 & -0.2022368\end{array}$

$\mathrm{H} \quad 0.9207709 \quad 2.4396005 \quad-0.2286217$

$\begin{array}{llll}\mathrm{H} & 2.0247920 & -2.3644908 & 0.1575983\end{array}$

H $\quad 3.8562999 \quad-0.6886563 \quad-0.0158011$

$\begin{array}{llll}\mathrm{N} & -1.2267583 & 0.8403538 & -0.1386884\end{array}$

C $\quad-1.5533030 \quad 2.2538089 \quad-0.0106802$

$\begin{array}{llll}\mathrm{H} & -1.2407379 & 2.6719579 & 0.9595608\end{array}$

$\mathrm{H} \quad-1.0785836 \quad 2.8370871 \quad-0.8067802$

$\mathrm{H} \quad-2.6352414 \quad 2.3643679 \quad-0.1061378$

C $\quad-0.6402815 \quad-1.9879206 \quad 0.1963095$

$\mathrm{H} \quad-0.3289910 \quad-2.9046965 \quad-0.3170161$

$\mathrm{H} \quad-0.7642341 \quad-2.2509070 \quad 1.2566369$

C $\quad-1.9776800 \quad-1.4854496 \quad-0.3502001$

$\mathrm{H} \quad-2.7865295 \quad-2.1768539 \quad-0.0923378$

H $\quad-1.9350464 \quad-1.4092144 \quad-1.4433402$

$\begin{array}{llll}\text { C } & -2.2795861 & -0.1077781 & 0.2302245\end{array}$

$\mathrm{H} \quad-3.2224782 \quad 0.2801647 \quad-0.1666873$

$\begin{array}{llll}\mathrm{H} & -2.3834029 & -0.1772191 & 1.3272798\end{array}$

35p : 4,3-hydroarylation of $\mathbf{1} \mathbf{w}$ and $\mathbf{2 a}$ on para 38

Energy $=-752.5679684578$

$\mathrm{N} \quad-2.0087555 \quad-0.9780400 \quad-0.6672487$ 


$\begin{array}{lrrc}\mathrm{H} & -2.1263498 & -1.6956298 & -1.3717866 \\ \mathrm{C} & -0.7448247 & -0.9338076 & -0.0704455 \\ \mathrm{C} & -0.5122120 & -0.4232084 & 1.2169128 \\ \mathrm{C} & 0.3420403 & -1.4815696 & -0.7782209 \\ \mathrm{C} & 0.7734025 & -0.4472497 & 1.7572055 \\ \mathrm{H} & -1.3336609 & -0.0390116 & 1.8106868 \\ \mathrm{C} & 1.6132772 & -1.5089461 & -0.2175361 \\ \mathrm{H} & 0.1762954 & -1.8881570 & -1.7734737 \\ \mathrm{C} & -3.1571451 & -0.2467580 & -0.3676712 \\ \mathrm{C} & 1.8598743 & -0.9822150 & 1.0583963 \\ \mathrm{H} & 0.9281229 & -0.0491920 & 2.7581263 \\ \mathrm{H} & 2.4335712 & -1.9441299 & -0.7841285 \\ \mathrm{C} & -3.1243398 & 1.0159046 & 0.2521761 \\ \mathrm{C} & -4.4036577 & -0.7729642 & -0.7599829 \\ \mathrm{C} & -4.3096247 & 1.7117266 & 0.4856045 \\ \mathrm{H} & -2.1760788 & 1.4649232 & 0.5239283 \\ \mathrm{C} & -5.5778697 & -0.0623756 & -0.5313599 \\ \mathrm{H} & -4.4390423 & -1.7462405 & -1.2442845 \\ \mathrm{C} & -5.5446248 & 1.1827026 & 0.1040792 \\ \mathrm{H} & -4.2601720 & 2.6873650 & 0.9619756 \\ \mathrm{H} & -6.5263314 & -0.4900013 & -0.8452563 \\ \mathrm{H} & -6.4623982 & 1.7312518 & 0.2925989 \\ \mathrm{C} & 3.2594419 & -0.9624133 & 1.6438094 \\ \mathrm{C} & 3.9708372 & -2.2846761 & 1.4532400 \\ \mathrm{C} & 5.2550581 & -2.3999407 & 1.0963280 \\ \mathrm{C} & 6.1720436 & -1.2236313 & 0.8844524 \\ \mathrm{C} & 5.5677556 & 0.0714431 & 1.4437775 \\ \mathrm{C} & 4.0940551 & 0.1912017 & 1.0435208 \\ \mathrm{H} & 5.6814290 & -3.3932795 & 0.9579903 \\ \mathrm{H} & 3.3731716 & -3.1819850 & 1.6113778 \\ \mathrm{H} & 3.1529112 & -0.7798468 & 2.7244482 \\ \mathrm{H} & 6.3791244 & -1.1102782 & -0.1909873 \\ \mathrm{H} & 5.6444730 & 0.0636336 & 2.5393179 \\ \mathrm{H} & 6.1334089 & 0.9393253 & 1.0860995 \\ \mathrm{H} & 3.6733799 & 1.1501613 & 1.3662161 \\ \mathrm{H} & 4.0078155 & 0.1509113 & -0.0506826 \\ \mathrm{H} & 7.1431795 & -1.4279055 & 1.3540480\end{array}$

35 : 4,3-hydroarylation of $\mathbf{1} \mathbf{w}$ and $\mathbf{2 a}$ on ortho 38

Energy $=-752.5660269961$

$\begin{array}{rrrr}\mathrm{H} & -0.4337029 & -1.0544854 & -0.7028747 \\ \mathrm{~N} & -0.9629601 & -0.2750737 & -0.3358673 \\ \mathrm{H} & 2.8816889 & -0.2344233 & -1.5431672 \\ \mathrm{C} & -0.2733120 & 0.9431856 & -0.2301077 \\ \mathrm{C} & -2.3179937 & -0.5253867 & -0.1254501 \\ \mathrm{C} & -0.9185639 & 2.1668746 & -0.4725238 \\ \mathrm{C} & 1.1053051 & 0.9284706 & 0.0860638 \\ \mathrm{C} & -3.1234547 & 0.2887170 & 0.6912174\end{array}$

$\begin{array}{lrrc}\mathrm{C} & -2.8880256 & -1.6735371 & -0.7072340 \\ \mathrm{C} & -0.2152843 & 3.3660755 & -0.3993023 \\ \mathrm{H} & -1.9691742 & 2.1711864 & -0.7433145 \\ \mathrm{C} & 1.7916660 & 2.1456588 & 0.1279247 \\ \mathrm{C} & -4.4625363 & -0.0369394 & 0.8983395 \\ \mathrm{H} & -2.6974018 & 1.1594631 & 1.1774019 \\ \mathrm{C} & -4.2238718 & -1.9934671 & -0.4828363 \\ \mathrm{H} & -2.2723006 & -2.3115644 & -1.3375980 \\ \mathrm{C} & 1.1490296 & 3.3624201 & -0.1020324 \\ \mathrm{H} & -0.7340034 & 4.3013409 & -0.5915887 \\ \mathrm{H} & 2.8533751 & 2.1323440 & 0.3629442 \\ \mathrm{C} & -5.0275845 & -1.1738592 & 0.3149351 \\ \mathrm{H} & -5.0665536 & 0.6040911 & 1.5351688 \\ \mathrm{H} & -4.6401213 & -2.8857713 & -0.9427553 \\ \mathrm{H} & 1.7056850 & 4.2936298 & -0.0500464 \\ \mathrm{H} & -6.0720764 & -1.4188750 & 0.4817854 \\ \mathrm{C} & 2.3315258 & -1.0045060 & -0.9873327 \\ \mathrm{C} & 1.8303590 & -0.3817205 & 0.3377951 \\ \mathrm{H} & 1.4823290 & -1.2988639 & -1.6171353 \\ \mathrm{H} & 1.1002558 & -1.0726834 & 0.7898803 \\ \mathrm{C} & 2.9737555 & -0.2301367 & 1.3149710 \\ \mathrm{C} & 4.1607089 & -0.8308749 & 1.1774244 \\ \mathrm{H} & 2.7898785 & 0.4090227 & 2.1777013 \\ \mathrm{H} & 4.9326967 & -0.6572646 & 1.9265102 \\ \mathrm{C} & 4.5013685 & -1.7617769 & 0.0434552 \\ \mathrm{H} & 5.0361129 & -2.6359658 & 0.4368686 \\ \mathrm{H} & 5.2062798 & -1.2638158 & -0.6401520 \\ \mathrm{C} & 3.2474149 & -2.2032336 & -0.7224766 \\ \mathrm{H} & 3.5278993 & -2.6840311 & -1.6662544 \\ \mathrm{H} & 2.7026382 & -2.9487231 & -0.1279412 \\ & & & \\ & & & \end{array}$

$\mathbf{3 a H} \mathrm{H}^{+} 1$ : adduct of $\mathbf{1} \mathbf{a H}^{+} \mathrm{C} 1$ on $\mathbf{2 a}$ para 45

\begin{tabular}{lccc}
\multicolumn{4}{l}{ Energy $=-906.7066857594$} \\
$\mathrm{~N}$ & -3.1813948 & 1.2489558 & 0.2080011 \\
$\mathrm{C}$ & -4.2381211 & 0.2975190 & 0.1077846 \\
$\mathrm{C}$ & -1.8881775 & 1.0374492 & 0.4474597 \\
$\mathrm{H}$ & -3.4559085 & 2.2188726 & 0.0661897 \\
$\mathrm{C}$ & -5.1897643 & 0.4810703 & -0.9000747 \\
$\mathrm{C}$ & -4.3542641 & -0.7512782 & 1.0255244 \\
$\mathrm{C}$ & -1.0395680 & 2.1867112 & 0.6225078 \\
$\mathrm{C}$ & -1.3168243 & -0.2801784 & 0.5060543 \\
$\mathrm{C}$ & -6.2489076 & -0.4166076 & -1.0084836 \\
$\mathrm{H}$ & -5.0906552 & 1.3100416 & -1.5944380 \\
$\mathrm{C}$ & -5.4158688 & -1.6449524 & 0.9022692 \\
$\mathrm{H}$ & -3.6476508 & -0.8462333 & 1.8429778 \\
$\mathrm{C}$ & 0.2761203 & 2.0329201 & 0.8797491 \\
$\mathrm{H}$ & -1.4895924 & 3.1737440 & 0.5615302 \\
$\mathrm{C}$ & 0.0048343 & -0.4290940 & 0.7463726
\end{tabular}




$\begin{array}{cccc}\mathrm{H} & -1.9466263 & -1.1424083 & 0.3253150 \\ \mathrm{C} & -6.3594753 & -1.4844677 & -0.1146811 \\ \mathrm{H} & -6.9857578 & -0.2820898 & -1.7939423 \\ \mathrm{H} & -5.5128024 & -2.4579753 & 1.6147705 \\ \mathrm{C} & 0.9314451 & 0.7071098 & 0.9526073 \\ \mathrm{H} & 0.9047886 & 2.9063284 & 1.0226002 \\ \mathrm{H} & 0.4337212 & -1.4266311 & 0.7622087 \\ \mathrm{H} & -7.1864877 & -2.1820689 & -0.2020664 \\ \mathrm{H} & 1.4077883 & 0.6022863 & 1.9422517 \\ \mathrm{C} & 3.4288076 & 2.7073968 & -0.6673947 \\ \mathrm{C} & 4.4342037 & 3.7939135 & -0.4366902 \\ \mathrm{H} & 2.8983759 & 2.7301915 & -1.6212541 \\ \mathrm{C} & 3.1486564 & 1.7280518 & 0.1971998 \\ \mathrm{H} & 5.2127141 & 3.7722372 & -1.2100007 \\ \mathrm{H} & 4.9127289 & 3.6957907 & 0.5422457 \\ \mathrm{H} & 3.9600111 & 4.7819058 & -0.4974898 \\ \mathrm{C} & 2.1604240 & 0.6274040 & -0.0763753 \\ \mathrm{H} & 3.6673787 & 1.6842610 & 1.1558927 \\ \mathrm{H} & 1.7386902 & 0.7631938 & -1.0771845 \\ \mathrm{C} & 2.8134942 & -0.7408296 & -0.0037663 \\ \mathrm{C} & 2.8355018 & -1.5628635 & -1.1354396 \\ \mathrm{C} & 3.4059635 & -1.2008118 & 1.1794773 \\ \mathrm{C} & 3.4399804 & -2.8204287 & -1.0898378 \\ \mathrm{H} & 2.3766832 & -1.2149425 & -2.0578546 \\ \mathrm{C} & 4.0082769 & -2.4575406 & 1.2287113 \\ \mathrm{H} & 3.4001369 & -0.5786182 & 2.0715642 \\ \mathrm{C} & 4.0269875 & -3.2715340 & 0.0933311 \\ \mathrm{H} & 3.4499166 & -3.4466036 & -1.9774939 \\ \mathrm{H} & 4.4636395 & -2.8010639 & 2.1531601 \\ \mathrm{H} & 4.4955633 & -4.2506185 & 0.1320831\end{array}$

3aH.OHBf ${ }_{3}: \mathrm{H}$-bonded $\mathbf{3 a H}{ }^{+}$and $\mathrm{HOBf}_{3}{ }^{-}$ 81

Energy $=-3192.299955693$

$\begin{array}{rrrr}\mathrm{N} & -3.4656108 & 2.7341401 & 1.1578297 \\ \mathrm{H} & -3.5236174 & 3.1696678 & 2.0745491 \\ \mathrm{C} & -3.1870411 & 1.4288761 & 1.1210631 \\ \mathrm{C} & -3.5606281 & 3.6042732 & 0.0215714 \\ \mathrm{C} & -2.9702219 & 0.7450337 & -0.1205540 \\ \mathrm{C} & -3.0765930 & 0.7060209 & 2.3550997 \\ \mathrm{C} & -4.5627815 & 3.4094798 & -0.9305150 \\ \mathrm{C} & -2.6357225 & 4.6416171 & -0.1056824 \\ \mathrm{C} & -2.5118976 & -0.5270530 & -0.1059597 \\ \mathrm{H} & -3.1446379 & 1.2690869 & -1.0520936 \\ \mathrm{C} & -2.5941599 & -0.5590770 & 2.3580701 \\ \mathrm{H} & -3.3434575 & 1.2109423 & 3.2789795 \\ \mathrm{C} & -4.6257393 & 4.2592131 & -2.0341255 \\ \mathrm{H} & -5.2866225 & 2.6109897 & -0.8008767 \\ \mathrm{C} & -2.7137826 & 5.4907638 & -1.2082668\end{array}$

\begin{tabular}{|c|c|c|c|}
\hline & -1.8586149 & 4.7685778 & \\
\hline C & -2.1430035 & -1.2344428 & 1.1302711 \\
\hline П & -2.3302936 & -1.0435412 & -1.0441768 \\
\hline $\mathrm{H}$ & -2.4730966 & -1.0791830 & 3.3032233 \\
\hline $\mathrm{C}$ & -3.7049195 & 5.2993995 & -2.1725370 \\
\hline $\mathrm{H}$ & -5.3992856 & 4.1099112 & -2.7808441 \\
\hline P & -1.9907577 & 6.2925161 & -1.3184481 \\
\hline & -2.3674805 & -2.7810760 & 1.0593430 \\
\hline $\mathrm{H}$ & -3.7617273 & 5.9626081 & -3.0300547 \\
\hline$C$ & -1.7056748 & -3.4996051 & 50988 \\
\hline $\mathrm{H}$ & -1.8722637 & -3.1083209 & 0.1369275 \\
\hline $\mathrm{C}$ & -3.8331747 & -3.0901460 & 0.9508126 \\
\hline $\mathrm{H}$ & -0.6574228 & -3.1974957 & 2.3421000 \\
\hline$H_{3}$ & -2.2202208 & -3.2807335 & 3.1875692 \\
\hline $\mathrm{H}$ & -1.7475052 & -4.58 & 2437 \\
\hline $\mathrm{C}$ & -4.4000381 & -3.65 & 538 \\
\hline $\mathrm{H}$ & -4.4388971 & -2.8149281 & 3390 \\
\hline $\mathrm{H}$ & -3.7534445 & -3.9192896 & -0.9648541 \\
\hline$C$ & -5.8176527 & -3.9789406 & -0.3166035 \\
\hline $\mathrm{C}$ & -6.2098713 & -4.6274810 & -1.5010878 \\
\hline C & -6.8126751 & -3.6709066 & 3600 \\
\hline$c$ & -7.5435739 & -4.96 & -1.7 \\
\hline $\mathrm{H}$ & -5.4534088 & -4.8728085 & -2.2 \\
\hline $\mathrm{C}$ & -8.1432385 & -4.0049310 & 0.4000348 \\
\hline $\mathrm{H}$ & -6.5444105 & -3.1640568 & 1.5529504 \\
\hline $\mathrm{C}$ & -8.5171146 & -4.6528358 & -0.7827837 \\
\hline $\mathrm{H}$ & -7.8215439 & -5.4627367 & -2.6569342 \\
\hline $\mathrm{H}$ & -8.8955969 & -3.7589578 & 5681 \\
\hline $\mathrm{H}$ & -9.5573761 & -4.9114024 & -0.9587759 \\
\hline $\mathrm{H}$ & -1.0044039 & -1.1330599 & 1.1349857 \\
\hline $\mathrm{H}$ & 0.7254768 & -2.0278051 & 0.0985173 \\
\hline 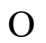 & 0.7991939 & -1.3193434 & 0.7554398 \\
\hline B & 1.7821636 & -0.2988307 & 0.2679742 \\
\hline $\mathrm{C}$ & 3.0957733 & -1.0912733 & -0.3509888 \\
\hline $\mathrm{C}$ & 3.6274844 & -2.1979137 & 0.3218601 \\
\hline $\mathrm{C}$ & 3.7455498 & -0.7911928 & -1.5485390 \\
\hline $\mathrm{C}$ & 4.6862930 & -2.9642888 & -0.1532442 \\
\hline $\mathrm{C}$ & 4.8108605 & -1.5282049 & -2.0638105 \\
\hline G & 5.2843878 & -2.6292942 & -1.3631017 \\
\hline $\mathrm{C}$ & 1.0437498 & 0.6371226 & -0.9002825 \\
\hline $\mathrm{C}$ & 1.1017505 & 2.0268639 & -0.9981357 \\
\hline $\mathrm{C}$ & 0.2178730 & 0.0434411 & -1.8526151 \\
\hline $\mathrm{C}$ & 0.3618475 & 2.7774160 & -1.9091351 \\
\hline $\mathrm{C}$ & -0.5408275 & 0.7426765 & -2.7854723 \\
\hline 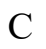 & -0.4793281 & 2.1312453 & -2.8076413 \\
\hline $\mathrm{C}$ & 2.1358733 & 0.6109976 & 1.5992318 \\
\hline $\mathrm{C}$ & 3.3862554 & 0.7998038 & 2.1846177 \\
\hline$C$ & 1.0966352 & 1.2620931 & 2.2644137 \\
\hline $\mathrm{C}$ & 3.5943051 & 1.5496528 & 3.3425297 \\
\hline
\end{tabular}




$\begin{array}{lrrr}\text { C } & 1.2493122 & 2.0194800 & 3.4189332 \\ \text { C } & 2.5178212 & 2.1627091 & 3.9703203 \\ \text { F } & 3.1418128 & -2.5588862 & 1.5358788 \\ \text { F } & 5.1522923 & -4.0187955 & 0.5519310 \\ \text { F } & 6.3115333 & -3.3565077 & -1.8423999 \\ \text { F } & 5.3893873 & -1.1822728 & -3.2350612 \\ \text { F } & 3.3770699 & 0.2844880 & -2.2950418 \\ \text { F } & 0.1188986 & -1.3199476 & -1.9211789 \\ \text { F } & -1.3474506 & 0.0924969 & -3.6553216 \\ \text { F } & -1.2132094 & 2.8349631 & -3.6904081 \\ \text { F } & 0.4573915 & 4.1243097 & -1.9354975 \\ \text { F } & 1.9252937 & 2.7458950 & -0.1890077 \\ \text { F } & -0.1625723 & 1.2148927 & 1.7541325 \\ \text { F } & 0.1896551 & 2.6275932 & 3.9990358 \\ \text { F } & 2.6998616 & 2.8905641 & 5.0892017 \\ \text { F } & 4.8343433 & 1.6870729 & 3.8613701 \\ \text { F } & 4.5099961 & 0.2665620 & 1.6375545\end{array}$

$\mathbf{3 a} \mathrm{aH}^{+}$: adduct of $\mathbf{1} \mathrm{aH}^{+} \mathrm{C} 3$ on $\mathbf{2 a}$ para-position 45

Energy $=-906.7109398510$

$\begin{array}{lrrr}\mathrm{N} & -3.7537563 & 0.7359561 & -0.3573989 \\ \mathrm{C} & -4.3569761 & -0.5343618 & -0.1245444 \\ \mathrm{C} & -2.5068569 & 1.1261281 & -0.0962064 \\ \mathrm{H} & -4.3593491 & 1.4324433 & -0.7867375 \\ \mathrm{C} & -5.2038865 & -1.0482465 & -1.1114082 \\ \mathrm{C} & -4.1562289 & -1.2175983 & 1.0791707 \\ \mathrm{C} & -2.1784741 & 2.5126334 & -0.2936739 \\ \mathrm{C} & -1.4881717 & 0.2153016 & 0.3497033 \\ \mathrm{C} & -5.8281445 & -2.2754470 & -0.9017387 \\ \mathrm{H} & -5.3588522 & -0.4962502 & -2.0335843 \\ \mathrm{C} & -4.7828429 & -2.4466327 & 1.2736319 \\ \mathrm{H} & -3.5494011 & -0.7794338 & 1.8642783 \\ \mathrm{C} & -0.9331403 & 2.9661423 & -0.0349356 \\ \mathrm{H} & -2.9651138 & 3.1877166 & -0.6192732 \\ \mathrm{C} & -0.2370204 & 0.6696656 & 0.5811765 \\ \mathrm{H} & -1.7247629 & -0.8358723 & 0.4592913 \\ \mathrm{C} & -5.6131013 & -2.9799134 & 0.2853245 \\ \mathrm{H} & -6.4802728 & -2.6816609 & -1.6683691 \\ \mathrm{H} & -4.6333745 & -2.9785617 & 2.2078346 \\ \mathrm{C} & 0.1742712 & 2.0807337 & 0.3955396 \\ \mathrm{H} & -0.7246019 & 4.0255843 & -0.1392880 \\ \mathrm{H} & 0.5375164 & -0.0320227 & 0.8768140 \\ \mathrm{H} & -6.1019479 & -3.9357437 & 0.4454449 \\ \mathrm{H} & 0.5806435 & 2.4663187 & 1.3464175 \\ \mathrm{C} & 1.4269292 & 2.1483424 & -0.6055946 \\ \mathrm{C} & 1.8525786 & 3.6046071 & -0.8518539 \\ \mathrm{H} & 1.1003995 & 1.6968182 & -1.5484835 \\ \mathrm{C} & 2.5640376 & 1.3536142 & -0.0390523\end{array}$

$\begin{array}{lrrr}\mathrm{H} & 2.7534932 & 3.6134667 & -1.4719443 \\ \mathrm{H} & 2.0828844 & 4.1107053 & 0.0922657 \\ \mathrm{H} & 1.0782092 & 4.1722173 & -1.3748575 \\ \mathrm{C} & 3.0563958 & 0.2431968 & -0.6117644 \\ \mathrm{H} & 2.9990920 & 1.7439378 & 0.8817803 \\ \mathrm{H} & 2.5839933 & -0.1119378 & -1.5291871 \\ \mathrm{C} & 4.1809325 & -0.5718862 & -0.1439501 \\ \mathrm{C} & 4.5330820 & -1.7186153 & -0.8783507 \\ \mathrm{C} & 4.9285860 & -0.2652595 & 1.0092418 \\ \mathrm{C} & 5.5907916 & -2.5342021 & -0.4778740 \\ \mathrm{H} & 3.9663562 & -1.9665277 & -1.7728768 \\ \mathrm{C} & 5.9840565 & -1.0783988 & 1.4089473 \\ \mathrm{H} & 4.6848049 & 0.6155585 & 1.5962387 \\ \mathrm{C} & 6.3210734 & -2.2173442 & 0.6684176 \\ \mathrm{H} & 5.8446175 & -3.4150798 & -1.0608206 \\ \mathrm{H} & 6.5498728 & -0.8249030 & 2.3011429 \\ \mathrm{H} & 7.1466805 & -2.8486288 & 0.9839557\end{array}$

3gH.OHBf 3 : H-bonded $\mathbf{3 g H}{ }^{+}$and $\mathrm{HOBf}_{3}{ }^{-}$ 91

Energy $=-3423.491354426$

$\begin{array}{lrrr}\mathrm{N} & -3.5193202 & 2.6844420 & 0.9821808 \\ \mathrm{C} & -3.1681498 & 1.3788504 & 0.9952738 \\ \mathrm{C} & -4.0302303 & 3.3210917 & -0.2047858 \\ \mathrm{C} & -3.3324991 & 3.5252386 & 2.1369563 \\ \mathrm{C} & -2.9525763 & 0.6657441 & -0.2320183 \\ \mathrm{C} & -2.9979750 & 0.6863210 & 2.2378632 \\ \mathrm{C} & -5.1435474 & 2.7803254 & -0.8521920 \\ \mathrm{C} & -3.4210078 & 4.4875183 & -0.6698602 \\ \mathrm{C} & -4.3757496 & 4.3516060 & 2.5581375 \\ \mathrm{C} & -2.0937056 & 3.5375957 & 2.7837511 \\ \mathrm{C} & -2.4282449 & -0.5813961 & -0.2005110 \\ \mathrm{H} & -3.1540839 & 1.1612298 & -1.1735529 \\ \mathrm{C} & -2.5151171 & -0.5802388 & 2.2572237 \\ \mathrm{H} & -3.2697394 & 1.1892384 & 3.1582977 \\ \mathrm{C} & -5.6351017 & 3.4046668 & -1.9969255 \\ \mathrm{H} & -5.6208328 & 1.8899903 & -0.4538012 \\ \mathrm{C} & -3.9215593 & 5.1042466 & -1.8139507 \\ \mathrm{H} & -2.5623440 & 4.8955162 & -0.1476735 \\ \mathrm{C} & -4.1784320 & 5.1856541 & 3.6568175 \\ \mathrm{H} & -5.3261601 & 4.3350485 & 2.0343855 \\ \mathrm{C} & -1.9102028 & 4.3715110 & 3.8840826 \\ \mathrm{H} & -1.2882031 & 2.9050521 & 2.4260007 \\ \mathrm{C} & -2.0582597 & -1.2748123 & 1.0449481 \\ \mathrm{H} & -2.2116038 & -1.0926098 & -1.1347533 \\ \mathrm{H} & -2.4088367 & -1.0876816 & 3.2108199 \\ \mathrm{C} & -5.0240146 & 4.5638599 & -2.4800089 \\ \mathrm{H} & -6.5022457 & 2.9923269 & -2.5032261 \\ \mathrm{H} & -3.4450195 & 6.0046723 & -2.1881226\end{array}$




\begin{tabular}{|c|c|c|c|}
\hline $\mathrm{C}$ & -2.9499724 & 5.1946777 & 4.3225506 \\
\hline H & -4.9875134 & 5.8254632 & 3.9948449 \\
\hline $\mathrm{H}$ & -0.9506787 & 4.3836238 & 4.3914007 \\
\hline $\mathrm{C}$ & -2.3416507 & -2.8181672 & 0.9918427 \\
\hline H & -5.4103593 & 5.0486512 & -3.3711073 \\
\hline H & -2.8013008 & 5.8466700 & 5.1776829 \\
\hline$C$ & -1.7172675 & -3.5449708 & 2.1926843 \\
\hline H & -1.8580507 & -3.1765324 & 0.0746613 \\
\hline C & -3.8195138 & -3.0618489 & 0.8810191 \\
\hline $\mathrm{H}$ & -0.6596744 & -3.2817365 & 2.2964855 \\
\hline $\mathrm{H}$ & -2.2322307 & -3.2927376 & 3.1255330 \\
\hline $\mathrm{H}$ & -1.8010815 & -4.6263644 & 2.0512799 \\
\hline $\mathrm{C}$ & -4.4155682 & -3.5666229 & -0.2099259 \\
\hline H & -4.4097108 & -2.7788705 & 1.7537608 \\
\hline $\mathrm{H}$ & -3.7841438 & -3.8407915 & -1.0569335 \\
\hline $\mathrm{C}$ & -5.8491980 & -3.8021066 & -0.4091352 \\
\hline $\mathrm{C}$ & -6.2773265 & -4.3889839 & -1.6131125 \\
\hline $\mathrm{C}$ & -6.8258321 & -3.4639221 & 0.5466887 \\
\hline $\mathrm{C}$ & -7.6282408 & -4.6346610 & -1.8561576 \\
\hline $\mathrm{H}$ & -5.5354902 & -4.6548602 & -2.3628982 \\
\hline $\mathrm{C}$ & -8.1737088 & -3.7087356 & 0.3054144 \\
\hline $\mathrm{H}$ & -6.5293052 & -3.0036674 & 1.4849542 \\
\hline $\mathrm{C}$ & -8.5834024 & -4.2961690 & -0.8968561 \\
\hline $\mathrm{H}$ & -7.9339700 & -5.0901660 & -2.7940974 \\
\hline $\mathrm{H}$ & -8.9114591 & -3.4396484 & 1.0565445 \\
\hline $\mathrm{H}$ & -9.6371032 & -4.4851565 & -1.0813731 \\
\hline $\mathrm{H}$ & -0.9179006 & -1.2186392 & 1.0800456 \\
\hline $\mathrm{O}$ & 0.9155824 & -1.4542801 & 0.9914375 \\
\hline $\mathrm{H}$ & 0.9324754 & -2.1480969 & 0.3166664 \\
\hline B & 1.8753444 & -0.3671986 & 0.6152281 \\
\hline $\mathrm{C}$ & 3.1662786 & -1.0026979 & -0.2049846 \\
\hline $\mathrm{C}$ & 1.0608640 & 0.6878100 & -0.3902889 \\
\hline $\mathrm{C}$ & 2.3307413 & 0.3338969 & 2.0384483 \\
\hline $\mathrm{C}$ & 3.6689175 & -2.2764532 & 0.0759111 \\
\hline $\mathrm{C}$ & 3.8503897 & -0.3391186 & -1.2256470 \\
\hline $\mathrm{C}$ & 0.8890532 & 2.0613715 & -0.2326780 \\
\hline $\mathrm{C}$ & 0.4320830 & 0.1683962 & -1.5221188 \\
\hline $\mathrm{C}$ & 3.6325975 & 0.5101410 & 2.5054670 \\
\hline $\mathrm{C}$ & 1.3498678 & 0.7827389 & 2.9231280 \\
\hline $\mathrm{C}$ & 4.7274844 & -2.8667720 & -0.6096467 \\
\hline $\mathrm{F}$ & 3.1455514 & -3.0194121 & 1.0874334 \\
\hline $\mathrm{C}$ & 4.9126723 & -0.8864625 & -1.9395822 \\
\hline $\mathrm{F}$ & 3.5261645 & 0.9392190 & -1.5600683 \\
\hline $\mathrm{C}$ & 0.1415368 & 2.8570300 & -1.1000688 \\
\hline $\mathrm{F}$ & 1.4821863 & 2.7287960 & 0.7928567 \\
\hline $\mathrm{C}$ & -0.3132113 & 0.9177161 & -2.4251546 \\
\hline $\mathrm{F}$ & 0.5305518 & -1.1651343 & -1.8023826 \\
\hline $\mathrm{C}$ & 3.9424706 & 1.0713174 & 3.7452553 \\
\hline $\mathrm{F}$ & 4.7081267 & 0.1487600 & 1.7576857 \\
\hline
\end{tabular}

$\begin{array}{cccc}\text { C } & 1.6038426 & 1.3508845 & 4.1644963 \\ \text { F } & 0.0414104 & 0.7244017 & 2.5586989 \\ \text { C } & 5.3545136 & -2.1679976 & -1.6338084 \\ \text { F } & 5.1572607 & -4.1057302 & -0.2851778 \\ \text { F } & 5.5248522 & -0.1849828 & -2.9182615 \\ \text { C } & -0.4671334 & 2.2825328 & -2.2094568 \\ \text { F } & 0.0138285 & 4.1845462 & -0.8812980 \\ \text { F } & -0.9182139 & 0.3347231 & -3.4839194 \\ \text { C } & 2.9215843 & 1.4952928 & 4.5852552 \\ \text { F } & 5.2278661 & 1.2074503 & 4.1376621 \\ F & 0.5941304 & 1.7822263 & 4.9536895 \\ \text { F } & 6.3787138 & -2.7190375 & -2.3120345 \\ F & -1.2010076 & 3.0291362 & -3.0554323 \\ \text { F } & 3.2012940 & 2.0423795 & 5.7834430\end{array}$

$\mathbf{3 g \mathrm { gH } ^ { + }}$ : adduct of $\mathbf{1 a H} \mathrm{H}^{+} \mathrm{C} 3$ on $\mathbf{2} \mathbf{g} \mathrm{Ph}_{3} \mathrm{~N}$ para 55

Energy $=-1137.898392186$

$\begin{array}{lrrr}\mathrm{N} & -2.6717915 & 0.1452930 & -0.1784049 \\ \mathrm{C} & -2.9288041 & 1.5632030 & -0.2527054 \\ \mathrm{C} & -1.4826440 & -0.3833360 & -0.5188740 \\ \mathrm{C} & -3.4505779 & 2.2167078 & 0.8651871 \\ \mathrm{C} & -2.6987783 & 2.2429257 & -1.4507754 \\ \mathrm{C} & -1.3577954 & -1.7931010 & -0.7775142 \\ \mathrm{C} & -0.3162723 & 0.4522682 & -0.6392803 \\ \mathrm{C} & -3.7233739 & 3.5801834 & 0.7842899 \\ \mathrm{H} & -3.6314292 & 1.6647192 & 1.7818354 \\ \mathrm{C} & -2.9766264 & 3.6068012 & -1.5194445 \\ \mathrm{H} & -2.3228979 & 1.7063579 & -2.3162059 \\ \mathrm{C} & -0.1613235 & -2.3256445 & -1.1139221 \\ \mathrm{H} & -2.2493795 & -2.4086276 & -0.7601207 \\ \mathrm{C} & 0.8855575 & -0.0938508 & -0.9254938 \\ \mathrm{H} & -0.4078740 & 1.5137631 & -0.4437861 \\ \mathrm{C} & -3.4856279 & 4.2758096 & -0.4042439 \\ \mathrm{H} & -4.1198598 & 4.0990856 & 1.6511525 \\ \mathrm{H} & -2.8063088 & 4.1414790 & -2.4484301 \\ \mathrm{C} & 1.0950441 & -1.5428725 & -1.1511312 \\ \mathrm{H} & -0.1129879 & -3.3748478 & -1.3857765 \\ \mathrm{H} & 1.7675481 & 0.5397916 & -0.9415626 \\ \mathrm{H} & -3.7038369 & 5.3375370 & -0.4629656 \\ \mathrm{H} & 1.5692648 & -1.6947567 & -2.1354736 \\ \mathrm{C} & 2.1889281 & -2.0971071 & -0.1146403 \\ \mathrm{C} & 2.3891167 & -3.6104211 & -0.2926150 \\ \mathrm{H} & 1.7999938 & -1.8939706 & 0.8892618 \\ \mathrm{C} & 3.4802107 & -1.3631855 & -0.3077373 \\ \mathrm{H} & 3.1867439 & -3.9480328 & 0.3752235 \\ \mathrm{H} & 2.6811026 & -3.8483871 & -1.3214731 \\ \mathrm{H} & 1.4826388 & -4.1706485 & -0.0472150 \\ \mathrm{C} & 4.0241264 & -0.5536616 & 0.6155846\end{array}$




$\begin{array}{lrrr}\mathrm{H} & 3.9823232 & -1.5361939 & -1.2603257 \\ \mathrm{H} & 3.4817501 & -0.4069422 & 1.5511189 \\ \mathrm{C} & 5.2916686 & 0.1753734 & 0.5190458 \\ \mathrm{C} & 5.6824560 & 0.9932167 & 1.5945631 \\ \mathrm{C} & 6.1408287 & 0.1000014 & -0.6016936 \\ \mathrm{C} & 6.8755520 & 1.7137491 & 1.5554766 \\ \mathrm{H} & 5.0379126 & 1.0595770 & 2.4680908 \\ \mathrm{C} & 7.3312689 & 0.8183893 & -0.6410037 \\ \mathrm{H} & 5.8698229 & -0.5256704 & -1.4472603 \\ \mathrm{C} & 7.7054192 & 1.6294549 & 0.4366185 \\ \mathrm{H} & 7.1568121 & 2.3393499 & 2.3979202 \\ \mathrm{H} & 7.9736906 & 0.7468280 & -1.5142881 \\ \mathrm{H} & 8.6362816 & 2.1880110 & 0.4016107 \\ \mathrm{C} & -3.7658797 & -0.6725286 & 0.2845848 \\ \mathrm{C} & -5.0193224 & -0.5301390 & -0.3136312 \\ \mathrm{C} & -3.5657544 & -1.5520440 & 1.3509451 \\ \mathrm{C} & -6.0816396 & -1.3013904 & 0.1517293 \\ \mathrm{H} & -5.1536638 & 0.1680412 & -1.1334454 \\ \mathrm{C} & -4.6366096 & -2.3171386 & 1.8085871 \\ \mathrm{H} & -2.5905295 & -1.6201101 & 1.8225566 \\ \mathrm{C} & -5.8921534 & -2.1949917 & 1.2093348 \\ \mathrm{H} & -7.0571928 & -1.2049280 & -0.3139647 \\ \mathrm{H} & -4.4912543 & -2.9977756 & 2.6413888 \\ \mathrm{H} & -6.7247323 & -2.7901849 & 1.5710436\end{array}$

3hHa.OHBf 3 : $\mathrm{H}$-bonded $\mathbf{3 h H a}{ }^{+}$and $\mathrm{HOBf}_{3}{ }^{-}$ 84

\begin{tabular}{lrrr}
\multicolumn{4}{l}{ Energy $=-3231.635424669$} \\
$\mathrm{~N}$ & -3.4507013 & 2.6445950 & 1.3447438 \\
$\mathrm{C}$ & -3.0386835 & 1.3714531 & 1.3670444 \\
$\mathrm{C}$ & -3.6114875 & 3.3590416 & 0.0997986 \\
$\mathrm{C}$ & -2.9314716 & 0.6152507 & 0.1483146 \\
$\mathrm{C}$ & -2.7121185 & 0.7272885 & 2.6090641 \\
$\mathrm{C}$ & -4.8838429 & 3.7735464 & -0.2937806 \\
$\mathrm{C}$ & -2.4841945 & 3.6514381 & -0.6699860 \\
$\mathrm{C}$ & -2.3976099 & -0.6273479 & 0.1675722 \\
$\mathrm{H}$ & -3.2972040 & 1.0519854 & -0.7733745 \\
$\mathrm{C}$ & -2.1606330 & -0.5118201 & 2.6095082 \\
$\mathrm{H}$ & -2.8695298 & 1.2507723 & 3.5436501 \\
$\mathrm{C}$ & -5.0276162 & 4.4867845 & -1.4838221 \\
$\mathrm{H}$ & -5.7478665 & 3.5333584 & 0.3182849 \\
$\mathrm{C}$ & -2.6397730 & 4.3631043 & -1.8589669 \\
$\mathrm{H}$ & -1.5047597 & 3.3256112 & -0.3352812 \\
$\mathrm{C}$ & -1.8257756 & -1.2368678 & 1.3767138 \\
$\mathrm{H}$ & -2.3615979 & -1.2063998 & -0.7503783 \\
$\mathrm{H}$ & -1.8802285 & -0.9605199 & 3.5574268 \\
$\mathrm{C}$ & -3.9085000 & 4.7801940 & -2.2660369 \\
$\mathrm{H}$ & -6.0153182 & 4.8071564 & -1.8004898 \\
$\mathrm{H}$ & -1.7684177 & 4.5926212 & -2.4629716
\end{tabular}

\begin{tabular}{|c|c|c|c|}
\hline $\mathrm{C}$ & -1.9678110 & -2.7965544 & \\
\hline H & -4.0253171 & 5.3356149 & -3.1915152 \\
\hline $\mathrm{C}$ & -1.2872051 & -3.4081262 & 2.6441566 \\
\hline $\mathrm{H}$ & -1.4542677 & -3.1584277 & 0.5106974 \\
\hline $\mathrm{C}$ & -3.4163949 & -3.1838133 & 1.3223048 \\
\hline $\mathrm{H}$ & -0.2729407 & -3.0159059 & 2.7736234 \\
\hline H & -1.8534419 & -3.1986529 & 3.5574707 \\
\hline $\mathrm{H}$ & -1.2293409 & -4.4943560 & 2.5319754 \\
\hline $\mathrm{C}$ & -3.9586798 & -3.8242850 & 0.2751331 \\
\hline $\mathrm{H}$ & -4.0328110 & -2.8946477 & 6300 \\
\hline $\mathrm{H}$ & -3.3021455 & -4.1010677 & -0.5519103 \\
\hline $\mathrm{C}$ & -5.3623373 & -4.2119910 & 0.1028962 \\
\hline $\mathrm{C}$ & -5.7290772 & -4.9335116 & -1.0470169 \\
\hline $\mathrm{C}$ & -6.3690882 & -3.8940899 & 1.0341314 \\
\hline $\mathrm{C}$ & -7.0489955 & -5.3294831 & -1.26 \\
\hline $\mathrm{H}$ & -4.9637762 & -5.1856086 & -1.7776767 \\
\hline $\mathrm{C}$ & -7.6859751 & -4.2890445 & 0.8225151 \\
\hline $\mathrm{H}$ & -6.1209452 & -3.3309527 & 1.9292526 \\
\hline $\mathrm{C}$ & -8.0342986 & -5.0096455 & -0.3255517 \\
\hline $\mathrm{H}$ & -7.3069470 & -5.8875239 & -2.1566011 \\
\hline $\mathrm{H}$ & -8.4476543 & -4.0334232 & 1.5541966 \\
\hline $\mathrm{H}$ & -9.0639559 & -5.3156738 & -0.4 \\
\hline $\mathrm{H}$ & -0.6862639 & -1.0792058 & 9109 \\
\hline $\mathrm{H}$ & 1.2834407 & -2.0227866 & 1.1509512 \\
\hline $\mathrm{O}$ & 1.0693748 & -1.0882810 & 1.2853901 \\
\hline B & 1.8558812 & -0.2336659 & 0.3475030 \\
\hline $\mathrm{C}$ & 3.2163839 & -0.9790022 & -0.2291487 \\
\hline $\mathrm{C}$ & 3.5695541 & -2.3109248 & -0.0308822 \\
\hline $\mathrm{C}$ & 4.0855887 & -0.2856850 & -1.0747118 \\
\hline $\mathrm{C}$ & 4.6867772 & -2.9192126 & -0.5997209 \\
\hline $\mathrm{C}$ & 5.2140028 & -0.8400491 & -1.6652615 \\
\hline $\mathrm{C}$ & 5.5199734 & -2.1765967 & -1.4260623 \\
\hline $\mathrm{C}$ & 0.9651932 & 0.0709215 & -1.0350367 \\
\hline $\mathrm{C}$ & 0.7788475 & 1.2944205 & -1.6785121 \\
\hline $\mathrm{C}$ & 0.3508912 & -1.0036912 & -1.6803665 \\
\hline $\mathrm{C}$ & 0.0193755 & 1.4552531 & -2.8358167 \\
\hline $\mathrm{C}$ & -0.4320285 & -0.8941277 & -2.8251755 \\
\hline $\mathrm{C}$ & -0.6037762 & 0.3546495 & -3.4115866 \\
\hline $\mathrm{C}$ & 2.1636705 & 1.1178832 & 1.2436719 \\
\hline $\mathrm{C}$ & 3.3793827 & 1.4543493 & 1.8355769 \\
\hline $\mathrm{C}$ & 1.1100670 & 1.9562275 & 1.6051607 \\
\hline $\mathrm{C}$ & 3.5541001 & 2.5525948 & 2.6782940 \\
\hline $\mathrm{C}$ & 1.2280601 & 3.0605042 & 2.4386286 \\
\hline $\mathrm{C}$ & 2.4706544 & 3.3664927 & 2.9832455 \\
\hline $\mathrm{F}$ & 2.8085993 & -3.1310177 & 0.7621612 \\
\hline 1 & 4.9642278 & -4.2182542 & -0.3600692 \\
\hline 1 & 6.6055628 & -2.7401056 & -1.9876115 \\
\hline $\mathrm{F}$ & 6.0166952 & -0.1025294 & -2.4612194 \\
\hline F & 3.8474986 & 1.0237137 & -1.3438760 \\
\hline
\end{tabular}




$\begin{array}{crrr}\text { F } & 0.4962603 & -2.2676042 & -1.1886346 \\ \text { F } & -1.0304735 & -1.9788455 & -3.3652384 \\ \text { F } & -1.3528343 & 0.4935549 & -4.5211660 \\ \text { F } & -0.1159288 & 2.6708190 & -3.4097264 \\ \text { F } & 1.3391215 & 2.4333332 & -1.1960411 \\ \text { F } & -0.1379960 & 1.7140459 & 1.1192384 \\ \text { F } & 0.1563005 & 3.8355742 & 2.7248449 \\ \text { F } & 2.6179432 & 4.4274613 & 3.7991845 \\ \text { F } & 4.7634463 & 2.8249698 & 3.2146973 \\ \text { F } & 4.4924261 & 0.7021953 & 1.6302558 \\ \text { C } & -3.6375646 & 3.4239212 & 2.5860127 \\ \text { H } & -2.6705326 & 3.5945383 & 3.0686085 \\ \text { H } & -4.0776796 & 4.3807006 & 2.3125211 \\ \text { H } & -4.3066028 & 2.8981852 & 3.2699730\end{array}$

$\mathbf{3} \mathbf{h H a}{ }^{+}$: adduct of $\mathbf{1} \mathbf{a H} H^{+} \mathrm{Cl}$ on $\mathbf{2} \mathbf{h} \mathrm{Ph}_{2} \mathrm{NMe}$ para 48

Energy $=-946.0361831182$

$\begin{array}{lrrc}\mathrm{N} & -2.9372847 & 1.2070754 & 0.0454022 \\ \mathrm{C} & -3.8642728 & 0.0976402 & 0.0258473 \\ \mathrm{C} & -1.6381133 & 1.0325739 & 0.2897278 \\ \mathrm{C} & -4.5133582 & -0.2272270 & -1.1655012 \\ \mathrm{C} & -4.1197142 & -0.5997238 & 1.2071588 \\ \mathrm{C} & -0.7659926 & 2.1707235 & 0.4415104 \\ \mathrm{C} & -1.0747787 & -0.2924932 & 0.3853192 \\ \mathrm{C} & -5.4256284 & -1.2816051 & -1.1719529 \\ \mathrm{H} & -4.2990949 & 0.3280164 & -2.0734186 \\ \mathrm{C} & -5.0348776 & -1.6517571 & 1.1882649 \\ \mathrm{H} & -3.6133762 & -0.3161437 & 2.1244850 \\ \mathrm{C} & 0.5533430 & 1.9955350 & 0.6742904 \\ \mathrm{H} & -1.1771881 & 3.1709320 & 0.3891390 \\ \mathrm{C} & 0.2473777 & -0.4599936 & 0.6007925 \\ \mathrm{H} & -1.7255008 & -1.1492674 & 0.2611215 \\ \mathrm{C} & -5.6861393 & -1.9931990 & 0.0014688 \\ \mathrm{H} & -5.9282870 & -1.5482446 & -2.0962160 \\ \mathrm{H} & -5.2411543 & -2.1981064 & 2.1031420 \\ \mathrm{C} & 1.1972422 & 0.6662509 & 0.7479285 \\ \mathrm{H} & 1.1934894 & 2.8637823 & 0.7978391 \\ \mathrm{H} & 0.6563887 & -1.4648371 & 0.6456018 \\ \mathrm{H} & -6.3988215 & -2.8119222 & -0.0089828 \\ \mathrm{H} & 1.7030628 & 0.5777129 & 1.7247350 \\ \mathrm{C} & 3.6682022 & 2.6116175 & -0.9705371 \\ \mathrm{C} & 4.6963470 & 3.6846316 & -0.7835861 \\ \mathrm{H} & 3.1047719 & 2.6348861 & -1.9053313 \\ \mathrm{C} & 3.4039670 & 1.6426320 & -0.0893605 \\ \mathrm{H} & 5.4473716 & 3.6462383 & -1.5830925 \\ \mathrm{H} & 5.2075646 & 3.5880129 & 0.1788607 \\ \mathrm{H} & 4.2347542 & 4.6791018 & -0.8364699 \\ \mathrm{C} & 2.3892316 & 0.5560703 & -0.3183430\end{array}$

$\begin{array}{lrrr}\mathrm{H} & 3.9561270 & 1.5981359 & 0.8504566 \\ \mathrm{H} & 1.9371419 & 0.6892214 & -1.3064039 \\ \mathrm{C} & 3.0200157 & -0.8229638 & -0.2535636 \\ \mathrm{C} & 2.9840067 & -1.6588648 & -1.3745476 \\ \mathrm{C} & 3.6478162 & -1.2798746 & 0.9124786 \\ \mathrm{C} & 3.5659193 & -2.9271966 & -1.3355519 \\ \mathrm{H} & 2.4982011 & -1.3132374 & -2.2839442 \\ \mathrm{C} & 4.2278862 & -2.5472175 & 0.9551883 \\ \mathrm{H} & 3.6869891 & -0.6470758 & 1.7962084 \\ \mathrm{C} & 4.1884367 & -3.3751771 & -0.1695263 \\ \mathrm{H} & 3.5306914 & -3.5639916 & -2.2149474 \\ \mathrm{H} & 4.7111835 & -2.8881244 & 1.8663095 \\ \mathrm{H} & 4.6394130 & -4.3626523 & -0.1357578 \\ \mathrm{C} & -3.5157714 & 2.5516734 & -0.1760094 \\ \mathrm{H} & -3.5032748 & 3.1210992 & 0.7573061 \\ \mathrm{H} & -2.9537346 & 3.0827045 & -0.9461457 \\ \mathrm{H} & -4.5454495 & 2.4175412 & -0.4998848\end{array}$

$\mathbf{3} \mathbf{h} \mathrm{H}^{+} \mathrm{HOBf}_{3}^{-}$: contact ion pair of $\mathbf{3} \mathbf{h} \mathrm{H}^{+}$ 84

Energy $=-3231.633306900$

$\begin{array}{lrrr}\mathrm{N} & 0.9930800 & 4.5047881 & 1.2160941 \\ \mathrm{C} & 0.0017493 & 3.6051219 & 1.2489543 \\ \mathrm{C} & 1.5872861 & 4.9209098 & -0.0308219 \\ \mathrm{C} & -0.6722416 & 3.2145054 & 0.0394570 \\ \mathrm{C} & -0.4173086 & 3.0179312 & 2.4919584 \\ \mathrm{C} & 1.5970902 & 6.2739703 & -0.3717939 \\ \mathrm{C} & 2.1755574 & 3.9632271 & -0.8605268 \\ \mathrm{C} & -1.5620819 & 2.1983768 & 0.0576980 \\ \mathrm{H} & -0.4556880 & 3.7478771 & -0.8780926 \\ \mathrm{C} & -1.3032617 & 1.9918434 & 2.4944469 \\ \mathrm{H} & 0.0215124 & 3.3567562 & 3.4218644 \\ \mathrm{C} & 2.1961416 & 6.6687914 & -1.5676838 \\ \mathrm{H} & 1.1306575 & 7.0049336 & 0.2816443 \\ \mathrm{C} & 2.7673002 & 4.3684846 & -2.0554146 \\ \mathrm{H} & 2.1676116 & 2.9189425 & -0.5669500 \\ \mathrm{C} & -1.8542218 & 1.4064131 & 1.2630707 \\ \mathrm{H} & -2.0774618 & 1.9286877 & -0.8588733 \\ \mathrm{H} & -1.5520746 & 1.5129278 & 3.4368342 \\ \mathrm{C} & 2.7789346 & 5.7190822 & -2.4098391 \\ \mathrm{H} & 2.1995988 & 7.7188476 & -1.8430095 \\ \mathrm{H} & 3.2232526 & 3.6277457 & -2.7043356 \\ \mathrm{C} & -3.8737178 & -1.2953002 & 2.3306191 \\ \mathrm{H} & 3.2440245 & 6.0320875 & -3.3395280 \\ \mathrm{C} & -4.1489067 & -2.2466612 & 3.4550795 \\ \mathrm{H} & -3.8820333 & -1.7190679 & 1.3245610 \\ \mathrm{C} & -3.6254670 & 0.0104268 & 2.4711555 \\ \mathrm{H} & -3.4093358 & -3.0577377 & 3.4658660 \\ \mathrm{H} & -4.1222747 & -1.7395811 & 4.4243432\end{array}$




\begin{tabular}{|c|c|c|c|}
\hline & -5.1318238 & -2.7198917 & 3.3346451 \\
\hline & -3.3546018 & 0.9314936 & 1.3122315 \\
\hline & -3.6311578 & 0.4519295 & 3.4681565 \\
\hline & -3.5039607 & 0.3607354 & 0.3897238 \\
\hline & -4.2927104 & 2.1270515 & 1.2765274 \\
\hline & -5.1152357 & 2.3298094 & 0.1630773 \\
\hline & -4.3422124 & 3.0515025 & 2.3271488 \\
\hline & -5.9667320 & 3.4341216 & 0.0945724 \\
\hline & -5.0876895 & 1.6162518 & -0.6576676 \\
\hline & -5.1908521 & 4.1562180 & 2.2629164 \\
\hline $\mathrm{H}$ & -3.7150727 & 861 & 3569 \\
\hline & -6.0056159 & 4.3518753 & 1.1449699 \\
\hline & -6.5977521 & 3.5761424 & -0.7784580 \\
\hline & -5.2167260 & 4.8649179 & 3.0860852 \\
\hline & 56094 & 5.2132507 & 1.0944849 \\
\hline & $-1.2^{\prime}$ & 0017 & 7638 \\
\hline $\mathrm{H}$ & -1.2 & -1.7 & \\
\hline $\mathrm{O}$ & -0.4689521 & 1823 & 5129 \\
\hline B & 0.4554951 & -1.6145378 & 0.1970481 \\
\hline $\mathrm{C}$ & 0.2733077 & -3.1828719 & -0.2929521 \\
\hline C & -0.6562177 & -4.0955096 & 0.1974411 \\
\hline C & 1.0414021 & $-3.6^{\prime}$ & -1.3 \\
\hline 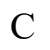 & -0.8 & -5.3 & -0.2 \\
\hline $\mathrm{C}$ & 0.92 & -4.9 & -1.8 \\
\hline $\mathrm{C}$ & -0.0245314 & 2022 & 21135 \\
\hline $\mathrm{C}$ & 0.0927462 & -0.7216648 & -1.1736176 \\
\hline $\mathrm{C}$ & 0.9496791 & 0.0517418 & -1.9549519 \\
\hline $\mathrm{C}$ & -1.2270903 & -0.7 & -1.6 \\
\hline $\mathrm{C}$ & 5773 & 0.7 & -3.0 \\
\hline $\mathrm{C}$ & $-1.6 S$ & -0.0 & -2.7 \\
\hline $\mathrm{C}$ & -0.7967851 & 1037 & -3.4536321 \\
\hline $\mathrm{C}$ & 1.9521772 & -1.2902612 & 0.8195601 \\
\hline 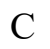 & 2.9299608 & -2.2145790 & 1.1825155 \\
\hline $\mathrm{C}$ & 2.2625013 & 0.0228983 & 1.1723038 \\
\hline $\mathrm{C}$ & 4.1279937 & 1455 & 5203 \\
\hline $\mathrm{C}$ & 3.43 & 0.4 & $1.7 \mathrm{C}$ \\
\hline C & 4.3873197 & -0.5420528 & 2.1223748 \\
\hline $\mathrm{F}$ & -1.4953217 & -3.7601975 & 1.2257625 \\
\hline $\mathrm{F}$ & -1.7458255 & -6.2183942 & 0.2256738 \\
\hline $\mathrm{F}$ & -0.1650225 & -7.0620532 & -1.8502775 \\
\hline & 1.7083302 & -5.3565368 & -2.9027120 \\
\hline 1 & 1.9930212 & -2.8738634 & -1.9060697 \\
\hline $\mathrm{F}$ & -2.1623743 & -1.4840472 & -0.9755896 \\
\hline $\mathrm{F}$ & -2.9910975 & -0.0696217 & -3.0941638 \\
\hline $\mathrm{F}$ & -1.2159155 & 1.4705709 & -4.5167142 \\
\hline $\mathrm{F}$ & 1.4178560 & 1.5342053 & -3.7661565 \\
\hline & 2.2762964 & 0.1288531 & -1.6733930 \\
\hline $\mathrm{F}$ & 1.3831752 & 1.0194547 & 0.8817723 \\
\hline & 3.6643020 & 1.7212206 & 2.0917318 \\
\hline
\end{tabular}

$\begin{array}{crrr}\text { F } & 5.5355431 & -0.1918468 & 2.7327288 \\ \text { F } & 5.0347223 & -2.8185178 & 2.1315547 \\ \text { F } & 2.7648202 & -3.5436942 & 0.9530707 \\ \text { C } & 1.6259913 & 5.0027866 & 2.4550206 \\ \text { H } & 0.8681893 & 5.3713332 & 3.1484094 \\ \text { H } & 2.2045823 & 4.2018440 & 2.9254388 \\ \text { H } & 2.2955058 & 5.8158432 & 2.1825049\end{array}$

$\mathbf{3} \mathbf{h} \mathrm{H}^{+}$: adduct of $\mathbf{1} \mathbf{a H}{ }^{+} \mathrm{C} 3$ on $\mathbf{2} \mathbf{h} \mathrm{Ph}_{2} \mathrm{NMe}$ para 48

Energy $=-946.0405126156$

$\begin{array}{llll}\mathrm{N} & -3.6091575 & 0.6966957 & -0.2381513\end{array}$

$\begin{array}{llll}\text { C } & -4.0089522 & -0.6693038 & 0.0143335\end{array}$

$\begin{array}{llll}\text { C } & -2.3799049 & 1.1310301 & 0.0423733\end{array}$

C $\quad-4.3682738 \quad-1.4848583 \quad-1.0591013$

$\begin{array}{llll}\text { C } & -4.0618092 & -1.1330753 & 1.3294383\end{array}$

$\begin{array}{llll}\text { C } & -2.0611537 & 2.5332369 & -0.0537459\end{array}$

$\begin{array}{llll}\text { C } & -1.3409194 & 0.2092653 & 0.4341909\end{array}$

C $\quad-4.7721214 \quad-2.7951728 \quad-0.8065672$

$\mathrm{H} \quad-4.3202743 \quad-1.1042538 \quad-2.0746790$

C $\quad-4.4680829 \quad-2.4452775 \quad 1.5694599$

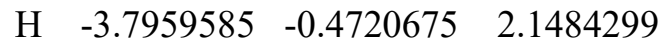

C $\quad-0.8085550 \quad 2.9713301 \quad 0.2047814$

$\mathrm{H} \quad-2.8418028 \quad 3.2435235 \quad-0.2957541$

C $\quad-0.0867611 \quad 0.6513807 \quad 0.6633721$

H $\quad-1.5770559 \quad-0.8449324 \quad 0.5121973$

$\begin{array}{llll}\text { C } & -4.8208785 & -3.2758271 & 0.5041217\end{array}$

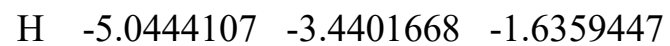

$\mathrm{H} \quad-4.5135274 \quad-2.8133846 \quad 2.5895170$

C $\quad 0.3185682 \quad 2.0708162 \quad 0.5353910$

$\begin{array}{llll}\mathrm{H} & -0.6117718 & 4.0379337 & 0.1735078\end{array}$

$\begin{array}{llll}\mathrm{H} & 0.6916899 & -0.0631677 & 0.9140087\end{array}$

$\begin{array}{llll}\mathrm{H} & -5.1350556 & -4.2972712 & 0.6946797\end{array}$

$\begin{array}{llll}\mathrm{H} & 0.7621281 & 2.4066584 & 1.4884852\end{array}$

C $\quad 1.5226099 \quad 2.2035365 \quad-0.5144500$

C $\quad 1.9485214 \quad 3.6714248 \quad-0.6757329$

$\mathrm{H} \quad 1.1485654 \quad 1.8228529 \quad-1.4711118$

$\begin{array}{llll}\text { C } & 2.6781609 & 1.3629919 & -0.0627317\end{array}$

$\mathrm{H} \quad 2.8145759 \quad 3.7220514 \quad-1.3417209$

$\begin{array}{llll}\mathrm{H} & 2.2341479 & 4.1047311 & 0.2892283\end{array}$

H $\quad 1.1522315 \quad 4.2808545 \quad-1.1117272$

$\begin{array}{lllll}\text { C } & 3.1337015 & 0.2978677 & -0.7417612\end{array}$

$\begin{array}{llll}\mathrm{H} & 3.1588978 & 1.6791852 & 0.8638262\end{array}$

$\mathrm{H} \quad 2.6195123 \quad 0.0223274 \quad-1.6640589$

C $\quad 4.2682600 \quad-0.5604368-0.3901972$

$\begin{array}{llll}\text { C } & 4.5735989 & -1.6495290 & -1.2263451\end{array}$

$\begin{array}{llll}\text { C } & 5.0703005 & -0.3509431 & 0.7481609\end{array}$

$\begin{array}{llll}\text { C } & 5.6381167 & -2.5029171 & -0.9385330\end{array}$

H $3.9647993 \quad-1.8227065 \quad-2.1108293$ 


$\begin{array}{lrrr}\mathrm{C} & 6.1322444 & -1.2021929 & 1.0358465 \\ \mathrm{H} & 4.8640981 & 0.4842091 & 1.4114747 \\ \mathrm{C} & 6.4219951 & -2.2830898 & 0.1950347 \\ \mathrm{H} & 5.8554993 & -3.3373949 & -1.5992620 \\ \mathrm{H} & 6.7399345 & -1.0239888 & 1.9186520 \\ \mathrm{H} & 7.2522295 & -2.9449743 & 0.4236992 \\ \mathrm{C} & -4.6535580 & 1.5998600 & -0.7719535 \\ \mathrm{H} & -4.9997206 & 2.2753060 & 0.0152818 \\ \mathrm{H} & -4.2619310 & 2.1731073 & -1.6134263 \\ \mathrm{H} & -5.4847583 & 0.9813731 & -1.1037012\end{array}$

$\mathbf{3} \mathrm{H}^{+}$: adduct of $\mathbf{1} \mathbf{a H} H^{+} \mathrm{C} 3$ on $\mathbf{2} \mathbf{a} \mathrm{Ph}_{2} \mathrm{NH}$ para 45

Energy $=-906.7106998180$

$\begin{array}{lrrr}\mathrm{N} & -3.2130111 & -1.2453017 & -0.4358844 \\ \mathrm{H} & 1.4043853 & 3.6710875 & 0.3927455 \\ \mathrm{C} & -4.5850936 & -0.9638500 & -0.1666295 \\ \mathrm{C} & -2.1755400 & -0.4098266 & -0.4307957 \\ \mathrm{C} & -5.2132081 & 0.1526619 & -0.7272247 \\ \mathrm{C} & -5.2977414 & -1.8701023 & 0.6240506 \\ \mathrm{C} & -2.2829859 & 0.9561183 & 0.0017265 \\ \mathrm{C} & -0.8994035 & -0.9177360 & -0.8603257 \\ \mathrm{C} & -6.5612767 & 0.3784832 & -0.4573744 \\ \mathrm{H} & -4.6662809 & 0.8136945 & -1.3910086 \\ \mathrm{C} & -6.6464741 & -1.6355546 & 0.8808363 \\ \mathrm{H} & -4.7958433 & -2.7391050 & 1.0387464 \\ \mathrm{C} & -1.1904048 & 1.7530580 & -0.0057343 \\ \mathrm{H} & -3.2372541 & 1.3314743 & 0.3499612 \\ \mathrm{C} & 0.1842222 & -0.1145196 & -0.8785035 \\ \mathrm{H} & -0.8393685 & -1.9524893 & -1.1861218 \\ \mathrm{C} & -7.2775500 & -0.5082120 & 0.3495607 \\ \mathrm{H} & -7.0546146 & 1.2412008 & -0.8936985 \\ \mathrm{H} & -7.2022699 & -2.3329544 & 1.4994795 \\ \mathrm{C} & 0.1553622 & 1.2951528 & -0.4206184 \\ \mathrm{H} & -1.2929507 & 2.7815492 & 0.3240787 \\ \mathrm{H} & 1.1390110 & -0.5067172 & -1.2150212 \\ \mathrm{H} & -8.3286258 & -0.3285063 & 0.5525190 \\ \mathrm{H} & 0.5103875 & 1.9434123 & -1.2404571 \\ \mathrm{C} & 1.2335767 & 2.9785250 & 1.2244307 \\ \mathrm{C} & 1.2434900 & 1.5210224 & 0.7364156 \\ \mathrm{H} & 0.2907350 & 3.2408081 & 1.7118703 \\ \mathrm{H} & 2.0376508 & 3.1159673 & 1.9529768 \\ \mathrm{H} & 0.9685951 & 0.8545274 & 1.5609118 \\ \mathrm{C} & 2.6013756 & 1.1456505 & 0.2242794 \\ \mathrm{C} & 3.3278481 & 0.1286193 & 0.7149020 \\ \mathrm{H} & 2.9911762 & 1.7705055 & -0.5802893 \\ \mathrm{H} & 2.8922478 & -0.4720283 & 1.5151480 \\ \mathrm{C} & 4.6691198 & -0.2866163 & 0.2946450 \\ \mathrm{C} & 5.2570129 & -1.4001902 & 0.9212996\end{array}$

$\begin{array}{rrrr}\mathrm{C} & 5.4012076 & 0.3737191 & -0.7107367 \\ \mathrm{C} & 6.5282874 & -1.8435543 & 0.5584189 \\ \mathrm{H} & 4.7042916 & -1.9194259 & 1.7009153 \\ \mathrm{C} & 6.6693304 & -0.0683563 & -1.0733382 \\ \mathrm{H} & 4.9768495 & 1.2389342 & -1.2120520 \\ \mathrm{C} & 7.2403457 & -1.1788735 & -0.4410178 \\ \mathrm{H} & 6.9621055 & -2.7063256 & 1.0560495 \\ \mathrm{H} & 7.2187430 & 0.4543883 & -1.8513479 \\ \mathrm{H} & 8.2316645 & -1.5190886 & -0.7260367 \\ \mathrm{H} & -3.0023484 & -2.2175555 & -0.6516576\end{array}$

$31 \mathrm{Ha}^{+}$: adduct of $1 \mathrm{aH}^{+} \mathrm{Cl}$ on amine 21 para 45

Energy $=-831.6666734673$

$\begin{array}{llll}\mathrm{N} & -2.2691053 & 0.4854583 & 1.1688061\end{array}$

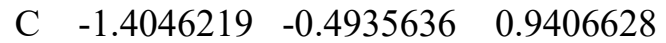

$\begin{array}{llll}\text { C } & -0.3977742 & -0.8199969 & 1.9237077\end{array}$

$\begin{array}{llll}\text { C } & -1.4598653 & -1.2564758 & -0.2969053\end{array}$

$\begin{array}{llll}\text { C } & 0.5021398 & -1.7988752 & 1.6952093\end{array}$

$\mathrm{H} \quad-0.3948363 \quad-0.2985587 \quad 2.8727956$

C $\quad-0.5480269-2.2330747 \quad-0.4999804$

$\begin{array}{llll}\text { C } & 0.5529954 & -2.5706012 & 0.4333232\end{array}$

$\mathrm{H} \quad 1.2196537 \quad-2.0446523 \quad 2.4713513$

$\mathrm{H} \quad-0.5795368 \quad-2.7903178 \quad-1.4332958$

H $\quad 0.4948529 \quad-3.6439454 \quad 0.6826585$

C $\quad 1.9642199 \quad-2.4210495 \quad-0.3042438$

$\begin{array}{llll}\text { C } & 3.1203678 & -2.7746839 & 0.6456894\end{array}$

H $\quad 2.0491760 \quad-1.3731895 \quad-0.6126336$

C $\quad 1.9931306 \quad-3.2999266 \quad-1.5185207$

$\mathrm{H} \quad 4.0645569-2.7322895 \quad 0.0952765$

$\begin{array}{llll}\mathrm{H} & 3.0025307 & -3.7887096 & 1.0439429\end{array}$

$\mathrm{H} \quad 3.1863157 \quad-2.0749816 \quad 1.4834095$

C $\quad 2.0712818 \quad-2.8340705 \quad-2.7752241$

$\mathrm{H} \quad 1.9666665 \quad-4.3730939-1.3243023$

$\mathrm{H} \quad 2.1032956 \quad-1.7526483 \quad-2.9186580$

C $\quad 2.1254081 \quad-3.6200329-4.0114722$

$\begin{array}{llll}\text { C } & 2.1974622 & -2.9395620 & -5.2401499\end{array}$

$\begin{array}{llll}\text { C } & 2.1074529 & -5.0277714 & -4.0319867\end{array}$

$\begin{array}{llll}\text { C } & 2.2498865 & -3.6348953 & -6.4477079\end{array}$

$\mathrm{H} \quad 2.2136581 \quad-1.8519713 \quad-5.2404636$

C $\quad 2.1615136 \quad-5.7221686 \quad-5.2361223$

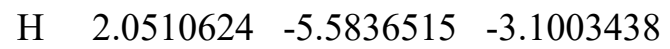

C $\quad 2.2336667 \quad-5.0303455 \quad-6.4507566$

$\mathrm{H} \quad 2.3035038 \quad-3.0874150 \quad-7.3846274$

$\mathrm{H} \quad 2.1492464 \quad-6.8086635 \quad-5.2310522$

$\mathrm{H} \quad 2.2793233 \quad-5.5769852 \quad-7.3882923$

$\begin{array}{llll}\text { C } & -2.1726456 & 1.3733503 & 2.3402431\end{array}$

$\begin{array}{llll}\mathrm{H} & -2.6330779 & 0.8994113 & 3.2122691\end{array}$

$\mathrm{H} \quad-1.1318615 \quad 1.6174938 \quad 2.5495935$ 


$\begin{array}{rrrr}\mathrm{H} & -2.7128765 & 2.2895453 & 2.1023243 \\ \mathrm{C} & -3.4298230 & 0.7622294 & 0.2870304 \\ \mathrm{H} & -3.1837456 & 1.6474123 & -0.3114325 \\ \mathrm{H} & -4.2579293 & 1.0247258 & 0.9503794 \\ \mathrm{C} & -3.7769253 & -0.4261932 & -0.5926283 \\ \mathrm{H} & -4.5463422 & -0.1186438 & -1.3051891 \\ \mathrm{H} & -4.1908695 & -1.2331104 & 0.0225514 \\ \mathrm{C} & -2.5173047 & -0.9078925 & -1.3125803 \\ \mathrm{H} & -2.1454676 & -0.1064425 & -1.9649393 \\ \mathrm{H} & -2.7214275 & -1.7757298 & -1.9447581\end{array}$

$3 \mathrm{IH}^{+}$: adduct of $1 \mathrm{aH}^{+} \mathrm{C} 3$ on amine $\mathbf{2 l}$ para 45

Energy $=-831.6670543264$

$\begin{array}{lrrr}\mathrm{N} & -4.4461868 & 1.0167163 & 0.2704901 \\ \mathrm{C} & -3.2273669 & 0.4919688 & 0.2635333 \\ \mathrm{C} & -2.0809029 & 1.3213156 & 0.5578226 \\ \mathrm{C} & -3.0300894 & -0.9190857 & -0.0305659 \\ \mathrm{C} & -0.8355834 & 0.8032815 & 0.5558548 \\ \mathrm{H} & -2.2312723 & 2.3620589 & 0.8163345 \\ \mathrm{C} & -1.7718433 & -1.4149617 & -0.0139354 \\ \mathrm{C} & -0.5529539 & -0.6120604 & 0.2348770 \\ \mathrm{H} & 0.0060160 & 1.4440067 & 0.8030436 \\ \mathrm{H} & -1.6241026 & -2.4716156 & -0.2290302 \\ \mathrm{H} & -0.0049649 & -1.0559912 & 1.0812792 \\ \mathrm{C} & 0.4682385 & -0.7617708 & -0.9894968 \\ \mathrm{C} & -0.1475424 & -0.2123863 & -2.2830965 \\ \mathrm{H} & 0.6309232 & -1.8408478 & -1.0879952 \\ \mathrm{C} & 1.7755410 & -0.1011461 & -0.6728278 \\ \mathrm{H} & 0.5309048 & -0.3906255 & -3.1222002 \\ \mathrm{H} & -0.3199049 & 0.8666497 & -2.2076596 \\ \mathrm{H} & -1.1033202 & -0.6987542 & -2.5031406 \\ \mathrm{C} & 2.8846466 & -0.7785395 & -0.3333235 \\ \mathrm{H} & 1.7964662 & 0.9861151 & -0.7448126 \\ \mathrm{H} & 2.8246783 & -1.8671047 & -0.2830830 \\ \mathrm{C} & 4.2032109 & -0.2201612 & -0.0199947 \\ \mathrm{C} & 5.2482753 & -1.1025204 & 0.3083117 \\ \mathrm{C} & 4.4787041 & 1.1608815 & -0.0297132 \\ \mathrm{C} & 6.5229723 & -0.6279411 & 0.6155441 \\ \mathrm{H} & 5.0509114 & -2.1719933 & 0.3213849 \\ \mathrm{C} & 5.7502258 & 1.6348807 & 0.2760742 \\ \mathrm{H} & 3.6940380 & 1.8696693 & -0.2788767 \\ \mathrm{C} & 6.7796616 & 0.7437260 & 0.6005100 \\ \mathrm{H} & 7.3144922 & -1.3287303 & 0.8658167 \\ \mathrm{H} & 5.9429476 & 2.7040684 & 0.2621194 \\ \mathrm{H} & 7.7710097 & 1.1183657 & 0.8384198 \\ \mathrm{C} & -4.6802933 & 2.4604785 & 0.4507287 \\ \mathrm{H} & -4.5862775 & 2.7316237 & 1.5064836 \\ \mathrm{H} & -3.9759802 & 3.0381675 & -0.1480878 \\ & & & \\ & & & \end{array}$

$\begin{array}{lrrr}\mathrm{H} & -5.6938422 & 2.6745838 & 0.1139529 \\ \mathrm{C} & -5.6789032 & 0.2036613 & 0.1282694 \\ \mathrm{H} & -6.0457502 & 0.3384907 & -0.8961610 \\ \mathrm{H} & -6.4131542 & 0.6436102 & 0.8077529 \\ \mathrm{C} & -5.4376153 & -1.2619818 & 0.4450032 \\ \mathrm{H} & -6.3409109 & -1.8247145 & 0.1963973 \\ \mathrm{H} & -5.2518327 & -1.3824922 & 1.5183063 \\ \mathrm{C} & -4.2355372 & -1.7626535 & -0.3554605 \\ \mathrm{H} & -4.4555009 & -1.6825037 & -1.4287316 \\ \mathrm{H} & -4.0188688 & -2.8129639 & -0.1453269\end{array}$

$\mathbf{3} \mathbf{w H} \mathrm{H}^{+} \mathrm{p}$ : adduct of $\mathbf{1} \mathbf{w} \mathrm{H}^{+} \mathrm{C} 3$ on $\mathbf{2 a} \mathrm{Ph}_{2} \mathrm{NH}$ para 39

Energy $=-752.9840861855$

$\begin{array}{llll}\mathrm{N} & -1.9667134 & -0.9168022 & -0.7922877\end{array}$

H $\quad-2.0462693 \quad-1.4862920 \quad-1.6322856$

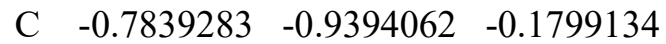

$\begin{array}{llll}\text { C } & -0.5676158 & -0.3256472 & 1.1020789\end{array}$

$\begin{array}{llll}\text { C } & 0.2971714 & -1.6334085 & -0.8301694\end{array}$

C $\quad 0.6612351 \quad-0.3641606 \quad 1.6633679$

$\begin{array}{llll}\mathrm{H} & -1.4026134 & 0.1286084 & 1.6214998\end{array}$

C $\quad 1.5285969 \quad-1.6427061 \quad-0.2789519$

$\mathrm{H} \quad 0.1005303 \quad-2.1183467 \quad-1.7824345$

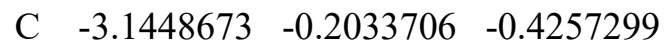

$\begin{array}{llll}\text { C } & 1.8381565 & -0.9812380 & 1.0107914\end{array}$

$\mathrm{H} \quad 0.8139341 \quad 0.0753966 \quad 2.6455254$

H $\quad 2.3485635 \quad-2.1334938 \quad-0.7958813$

C $\quad-3.0792108 \quad 1.1309905 \quad-0.0122298$

$\begin{array}{llll}\text { C } & -4.3736910 & -0.8586041 & -0.5504780\end{array}$

$\begin{array}{llll}\mathrm{H} & 2.2319811 & -1.7503647 & 1.6989847\end{array}$

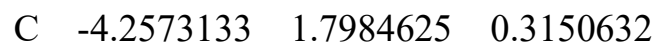

$\begin{array}{llll}\mathrm{H} & -2.1268095 & 1.6494411 & 0.0190754\end{array}$

C $\quad-5.5443548 \quad-0.1780546 \quad-0.2248856$

$\begin{array}{llll}\mathrm{H} & -4.4058769 & -1.8905168 & -0.8869284\end{array}$

$\begin{array}{llll}\text { C } & -5.4883838 & 1.1464555 & 0.2156648\end{array}$

$\mathrm{H} \quad-4.2116038 \quad 2.8354870 \quad 0.6320182$

H $\quad-6.4999028 \quad-0.6850053 \quad-0.3136891$

$\begin{array}{llll}\mathrm{H} & -6.4030664 & 1.6735867 & 0.4680336\end{array}$

$\begin{array}{llll}\text { C } & 3.0338431 & 0.0539526 & 0.8788601\end{array}$

$\begin{array}{lllll}\text { C } & 4.2768397 & -0.6566121 & 0.4097848\end{array}$

$\begin{array}{llll}\text { C } & 5.1033174 & -0.1611018 & -0.5178329\end{array}$

$\begin{array}{llll}\text { C } & 4.9161303 & 1.1837193 & -1.1668121\end{array}$

$\begin{array}{llll}\text { C } & 3.9054472 & 2.0462141 & -0.4000050\end{array}$

$\begin{array}{llll}\text { C } & 2.6615379 & 1.2282052 & -0.0375015\end{array}$

$\begin{array}{llll}\mathrm{H} & 5.9750429 & -0.7425082 & -0.8148447\end{array}$

H $\quad 4.4926567 \quad-1.6216544 \quad 0.8683266$

$\begin{array}{llll}\mathrm{H} & 3.1959007 & 0.4235723 & 1.9012354\end{array}$

$\mathrm{H} \quad 4.5851216 \quad 1.0434907 \quad-2.2073033$

$\mathrm{H} \quad 4.3731254 \quad 2.4194657 \quad 0.5203490$ 


$\begin{array}{lrrr}\mathrm{H} & 3.6211853 & 2.9203757 & -0.9953490 \\ \mathrm{H} & 1.9104920 & 1.8588941 & 0.4504849 \\ \mathrm{H} & 2.2079998 & 0.8370780 & -0.9584205 \\ \mathrm{H} & 5.8851070 & 1.6944872 & -1.2300700\end{array}$

$\mathbf{3} \mathbf{w H} \mathrm{H}^{+}$: adduct of $\mathbf{1} \mathbf{w H} \mathrm{H}^{+} \mathrm{C} 3$ on $\mathbf{2 a} \mathrm{Ph}_{2} \mathrm{NH}$ ortho 39

Energy $=-752.9822644727$

$\begin{array}{lrcc}\mathrm{N} & 0.8369762 & -0.5233523 & 0.1951717 \\ \mathrm{H} & 1.4913477 & -0.2911356 & -0.5527421 \\ \mathrm{C} & 0.3850763 & 0.5045368 & 0.9110639 \\ \mathrm{C} & -0.6905287 & 0.3927508 & 1.8285445 \\ \mathrm{C} & 1.0074519 & 1.8371421 & 0.6532215 \\ \mathrm{C} & -1.0276778 & 1.4664780 & 2.6102522 \\ \mathrm{H} & -1.2397481 & -0.5373821 & 1.9068810 \\ \mathrm{C} & 0.6647741 & 2.8826542 & 1.6502607 \\ \mathrm{H} & 2.0961667 & 1.7310090 & 0.5909180 \\ \mathrm{C} & 0.4011334 & -1.8796922 & 0.2227770 \\ \mathrm{C} & -0.3131573 & 2.7112584 & 2.5656787 \\ \mathrm{H} & -1.8653488 & 1.3704839 & 3.2946228 \\ \mathrm{H} & 1.1979422 & 3.8246180 & 1.5626672 \\ \mathrm{C} & 0.1935171 & -2.5269145 & -0.9992944 \\ \mathrm{C} & 0.2414272 & -2.5615779 & 1.4327460 \\ \mathrm{H} & -0.5826636 & 3.5025622 & 3.2565030 \\ \mathrm{C} & -0.2039920 & -3.8615853 & -1.0064932 \\ \mathrm{H} & 0.3388435 & -1.9868342 & -1.9298198 \\ \mathrm{C} & -0.1622640 & -3.8949881 & 1.4116812 \\ \mathrm{H} & 0.4614720 & -2.0674706 & 2.3728446 \\ \mathrm{C} & -0.3907078 & -4.5452573 & 0.1971033 \\ \mathrm{H} & -0.3710610 & -4.3650949 & -1.9533312 \\ \mathrm{H} & -0.2825204 & -4.4293333 & 2.3487132 \\ \mathrm{H} & -0.7016099 & -5.5851226 & 0.1882680 \\ \mathrm{C} & 0.5937000 & 2.4055523 & -0.8148322 \\ \mathrm{C} & 1.2577568 & 1.6012445 & -1.8907191 \\ \mathrm{C} & 0.5846051 & 0.9298833 & -2.8376099 \\ \mathrm{C} & -0.9131893 & 0.8543449 & -2.9052676 \\ \mathrm{C} & -1.5740452 & 1.2781990 & -1.5881897 \\ \mathrm{C} & -0.9225347 & 2.5460557 & -1.0213409 \\ \mathrm{H} & 1.1458456 & 0.4308377 & -3.6262958 \\ \mathrm{H} & 2.3471956 & 1.6208746 & -1.9108653 \\ \mathrm{H} & 1.0502413 & 3.4012918 & -0.8062283 \\ \mathrm{H} & -1.2188783 & -0.1630286 & -3.1798216 \\ \mathrm{H} & -2.6432705 & 1.4533612 & -1.7423225 \\ \mathrm{H} & -1.4975989 & 0.4585481 & -0.8652682 \\ \mathrm{H} & -1.0786067 & 3.3699263 & -1.7282534 \\ \mathrm{H} & -1.4059883 & 2.8430663 & -0.0848171 \\ \mathrm{H} & -1.2561788 & 1.4953559 & -3.7313761\end{array}$

3 : 4,3-hydroarylation of $\mathbf{1 a}$ and $\mathbf{2 a}$
44

Energy $=-906.2956332458$

$\mathrm{N} \quad-0.9012472 \quad-0.2879282$

$\begin{array}{llll}\text { C } & -1.1261846 & 1.0355158 & 2.4487395\end{array}$

$\begin{array}{llll}\text { C } & -1.5467798 & -1.4532003 & 2.4935588\end{array}$

$\begin{array}{llll}\text { C } & -0.6455053 & 2.0576508 & 1.6068348\end{array}$

$\begin{array}{llll}\text { C } & -1.7617972 & 1.3965673 & 3.6508067\end{array}$

$\begin{array}{llll}\text { C } & -0.8741853 & -2.6787668 & 2.3558590\end{array}$

$\begin{array}{llll}\text { C } & -2.8610004 & -1.4682557 & 2.9953288\end{array}$

$\begin{array}{llll}\text { C } & -0.8034909 & 3.3956984 & 1.9536655\end{array}$

$\begin{array}{llll}\mathrm{H} & -0.1493858 & 1.7886452 & 0.6770689\end{array}$

C $\quad-1.9256362 \quad 2.7415676 \quad 3.9790719$

$\mathrm{H} \quad-2.1007253 \quad 0.6311046 \quad 4.3391514$

$\begin{array}{llll}\text { C } & -1.4893241 & -3.8761242 & 2.7153401\end{array}$

H $\quad 0.1398918 \quad-2.6875966 \quad 1.9625972$

C $\quad-3.4509951 \quad-2.6705678 \quad 3.3680141$

H $\quad-3.4292816 \quad-0.5480556 \quad 3.0685571$

C $\quad-1.4546263 \quad 3.7524518 \quad 3.1385314$

$\begin{array}{llll}\mathrm{H} & -0.4198205 & 4.1647639 & 1.2886493\end{array}$

$\mathrm{H} \quad-2.4197048 \quad 2.9971383 \quad 4.9127321$

C $\quad-2.7866107 \quad-3.8982966 \quad 3.2391160$

$\mathrm{H} \quad-0.9357754 \quad-4.8009965 \quad 2.5849276$

$\mathrm{H} \quad-4.4636150 \quad-2.6557609 \quad 3.7647567$

$\mathrm{H} \quad-1.5842978 \quad 4.7970137 \quad 3.4044788$

C $\quad-2.7914162 \quad-6.4647452 \quad 3.2304161$

C $\quad-3.4883837 \quad-5.1758090 \quad 3.6964856$

$\mathrm{H} \quad-3.3895185 \quad-7.3338118 \quad 3.5196140$

$\mathrm{H} \quad-1.8018580 \quad-6.5698982 \quad 3.6887975$

$\begin{array}{llll}\mathrm{H} & -4.5026427 & -5.1641850 & 3.2754982\end{array}$

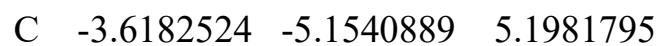

C $\quad-4.7886873 \quad-5.1254934 \quad 5.8553825$

$\mathrm{H} \quad-2.6750886 \quad-5.1447320 \quad 5.7456335$

H $\quad-5.7074545 \quad-5.1357182 \quad 5.2665238$

$\begin{array}{llll}\text { C } & -4.9873140 & -5.0836935 & 7.3078342\end{array}$

$\begin{array}{llll}\text { C } & -6.2983828 & -5.1386072 & 7.8155268\end{array}$

$\begin{array}{llll}\text { C } & -3.9273667 & -4.9878374 & 8.2304123\end{array}$

$\begin{array}{llll}\text { C } & -6.5455699 & -5.1067082 & 9.1877375\end{array}$

H $\quad-7.1300454 \quad-5.2084455 \quad 7.1179054$

C $\quad-4.1726632 \quad-4.9569813 \quad 9.5997106$

$\mathrm{H} \quad-2.9030955 \quad-4.9315116 \quad 7.8730426$

$\begin{array}{llll}\text { C } & -5.4828357 & -5.0181529 & 10.0881538\end{array}$

$\mathrm{H} \quad-7.5681400 \quad-5.1494518 \quad 9.5529272$

$\mathrm{H} \quad-3.3389160 \quad-4.8815634 \quad 10.2926574$

$\begin{array}{llll}\mathrm{H} & -5.6696676 & -4.9952498 & 11.1579538\end{array}$

$\mathrm{H} \quad-2.6690168 \quad-6.4729854 \quad 2.1418928$

$\begin{array}{llll}\mathrm{H} & -0.1569079 & -0.4252225 & 1.3996673\end{array}$

9 : 4,3-hydroarylation of $\mathbf{1 a}$ and $\mathbf{2 g} \mathrm{Ph}_{3} \mathrm{~N}$ 54 


\begin{tabular}{|c|c|c|c|}
\hline \multicolumn{4}{|c|}{ Energy $=-1137.484559148$} \\
\hline & -2.6564207 & 0.1710346 & \\
\hline & & & \\
\hline & & & \\
\hline & & & \\
\hline & -2.1 & & \\
\hline & -1.27 & & \\
\hline & -0.21 & & \\
\hline & & & \\
\hline & & & \\
\hline & & & \\
\hline & -1.4 & & \\
\hline & -0.0 & -2 & \\
\hline & -2.1 & -2. & \\
\hline & 1.0 & & \\
\hline & -0.2 & & \\
\hline & -3.1 & & \\
\hline & -4. & & \\
\hline & -1.7 & & \\
\hline & & & \\
\hline & & & \\
\hline & 1.9 & & \\
\hline & -3.2 & & \\
\hline & & & \\
\hline & & & \\
\hline & & & \\
\hline & & & \\
\hline & 3.5 & & \\
\hline & & & \\
\hline & & & \\
\hline & & & \\
\hline & & & \\
\hline & & & \\
\hline & & & \\
\hline & & & \\
\hline & & & \\
\hline & & & \\
\hline & & & \\
\hline & & & \\
\hline & & & \\
\hline & & & \\
\hline & & & \\
\hline & & & \\
\hline & & & \\
\hline & -3.7 & -0. & \\
\hline & -5.03 & -0.3 & -0 . \\
\hline & & & \\
\hline & & & \\
\hline & & & \\
\hline
\end{tabular}

$\begin{array}{lccc}\mathrm{C} & -4.7048374 & -2.6063679 & 1.2587276 \\ \mathrm{H} & -2.6312324 & -2.0289319 & 1.3756468 \\ \mathrm{C} & -5.9717909 & -2.2976677 & 0.7560943 \\ \mathrm{H} & -7.1067805 & -0.9145309 & -0.4502758 \\ \mathrm{H} & -4.5668017 & -3.4807194 & 1.8889632 \\ \mathrm{H} & -6.8229281 & -2.9323393 & 0.9840490\end{array}$

$\mathrm{Bf}_{3}$ : Lewis-acidic borane $\mathrm{B}\left(\mathrm{C}_{6} \mathrm{~F}_{5}\right)_{3}$ 34

Energy $=-2209.516675314$

$\begin{array}{llll}\text { B } & -0.0001016 & 0.0008045 & -0.0006333\end{array}$

$\begin{array}{llll}\text { C } & -0.0001801 & 1.5634586 & -0.0005958\end{array}$

$\begin{array}{llll}\text { C } & 1.3522102 & -0.7827958 & -0.0035299\end{array}$

$\begin{array}{llll}\text { C } & -1.3524183 & -0.7827862 & 0.0028896\end{array}$

$\begin{array}{llll}\text { C } & -0.9416191 & 2.3140682 & 0.7208581\end{array}$

$\begin{array}{llll}\text { C } & 0.9415179 & 2.3142697 & -0.7215026\end{array}$

$\begin{array}{llll}\text { C } & 2.4664577 & -0.3605799 & 0.7368735\end{array}$

$\begin{array}{llll}\text { C } & 1.5346001 & -1.9571833 & -0.7490138\end{array}$

$\begin{array}{llll}\text { C } & -1.5346709 & -1.9567415 & 0.7490302\end{array}$

$\begin{array}{llll}\text { C } & -2.4667663 & -0.3608365 & -0.7374543\end{array}$

$\begin{array}{llll}\text { C } & -0.9500339 & 3.7022183 & 0.7427237\end{array}$

$\begin{array}{llll}\text { C } & 0.9504412 & 3.7024303 & -0.7423427\end{array}$

$\begin{array}{llll}\text { C } & 3.6716043 & -1.0495868 & 0.7533276\end{array}$

$\begin{array}{llll}\text { C } & 2.7325470 & -2.6585408 & -0.7787205\end{array}$

$\begin{array}{llll}\text { C } & -2.7325318 & -2.6582390 & 0.7790745\end{array}$

$\begin{array}{llll}\text { C } & -3.6719237 & -1.0498484 & -0.7533881\end{array}$

$\begin{array}{llll}\text { C } & 0.0003325 & 4.4001814 & 0.0004627\end{array}$

$\begin{array}{llll}\text { C } & 3.8062504 & -2.2031571 & -0.0164994\end{array}$

$\begin{array}{llll}\text { C } & -3.8062656 & -2.2033966 & 0.0165463\end{array}$

$\begin{array}{llll}\text { F } & -1.8755305 & 1.6897508 & 1.4685544\end{array}$

$\begin{array}{llll}\text { F } & 1.8753412 & 1.6901384 & -1.4694782\end{array}$

$\begin{array}{llll}\text { F } & 2.3860451 & 0.7441975 & 1.5080182\end{array}$

F $\quad 0.5303473 \quad-2.4351907 \quad-1.5136696$

$\begin{array}{llll}\text { F } & -0.5305536 & -2.4337952 & 1.5144791\end{array}$

$\begin{array}{llll}\text { F } & -2.3865259 & 0.7438964 & -1.5087074\end{array}$

$\begin{array}{llll}\text { F } & -1.8577099 & 4.3770968 & 1.4659196\end{array}$

$\begin{array}{lllll}\text { F } & 1.8586958 & 4.3775254 & -1.4646227\end{array}$

$\begin{array}{llll}\text { F } & 4.7044422 & -0.6196228 & 1.4954508\end{array}$

F $\quad 2.8684764 \quad-3.7639754 \quad-1.5283882$

F $\quad-2.8682402 \quad-3.7634966 \quad 1.5290634$

F $\quad-4.7048145 \quad-0.6203316 \quad-1.4957030$

$\begin{array}{llll}\text { F } & 0.0004853 & 5.7363190 & 0.0008567\end{array}$

F $\quad 4.9632735 \quad-2.8717301 \quad-0.0230722$

$\begin{array}{llll}\text { F } & -4.9631129 & -2.8723109 & 0.0231667\end{array}$

$\mathrm{H}_{2} \mathrm{O} \cdot \mathrm{Bf}_{3}$ : B-O adduct of $\mathrm{H}_{2} \mathrm{O}$ and $\mathrm{Bf}_{3}$ 37

Energy $=-2286.012914771$

B $\quad-1.6172784 \quad-0.7060328 \quad-0.1462413$ 


$\begin{array}{lccc}\mathrm{C} & -0.9173596 & -1.5570544 & 1.0420188 \\ \mathrm{C} & -1.8141680 & 0.8750476 & 0.1739063 \\ \mathrm{C} & -2.9269879 & -1.3980410 & -0.8231156 \\ \mathrm{C} & -1.4842717 & -2.6539986 & 1.6941333 \\ \mathrm{C} & 0.3517823 & -1.1926735 & 1.5066408 \\ \mathrm{C} & -1.8912928 & 1.8202892 & -0.8466792 \\ \mathrm{C} & -1.9733683 & 1.4005965 & 1.4574594 \\ \mathrm{C} & -2.8082909 & -2.5358727 & -1.6210361 \\ \mathrm{C} & -4.2386147 & -0.9518064 & -0.6507208 \\ \mathrm{C} & -0.8448007 & -3.3532093 & 2.7155601 \\ \mathrm{~F} & -2.7245771 & -3.0930350 & 1.3703438 \\ \mathrm{C} & 1.0279177 & -1.8654902 & 2.5172675 \\ \mathrm{~F} & 0.9777417 & -0.1111134 & 0.9817147 \\ \mathrm{C} & -2.0859763 & 3.1811159 & -0.6522509 \\ \mathrm{~F} & -1.7766555 & 1.4069941 & -2.1536029 \\ \mathrm{C} & -2.1756929 & 2.7553418 & 1.7088169 \\ \mathrm{~F} & -1.9706093 & 0.5889461 & 2.5401416 \\ \mathrm{C} & -3.8709128 & -3.1890166 & -2.2292208 \\ \mathrm{~F} & -1.5655391 & -3.0849288 & -1.8208266 \\ \mathrm{C} & -5.3413787 & -1.5728629 & -1.2338400 \\ \mathrm{~F} & -4.5085974 & 0.1169954 & 0.1352838 \\ \mathrm{C} & 0.4230863 & -2.9601350 & 3.1273511 \\ \mathrm{~F} & -1.4425991 & -4.4051050 & 3.3062170 \\ \mathrm{~F} & 2.2502369 & -1.4671199 & 2.9170743 \\ \mathrm{C} & -2.2294724 & 3.6543019 & 0.6484618 \\ \mathrm{~F} & -2.1392171 & 4.0329695 & -1.6908254 \\ \mathrm{~F} & -2.3262597 & 3.2033345 & 2.9678879 \\ \mathrm{C} & -5.1581905 & -2.6968039 & -2.0317245 \\ \mathrm{~F} & -3.6758541 & -4.2788415 & -2.9917953 \\ \mathrm{~F} & -6.5825976 & -1.0964928 & -1.0306994 \\ \mathrm{~F} & 1.0554677 & -3.6250290 & 4.1062052 \\ \mathrm{~F} & -2.4195864 & 4.9618503 & 0.8761896 \\ \mathrm{~F} & -6.2088947 & -3.3012253 & -2.6047085 \\ \mathrm{O} & -0.4608841 & -0.7126387 & -1.3073893 \\ \mathrm{H} & -0.3830005 & -1.5921714 & -1.7330475 \\ \mathrm{H} & -0.6482255 & -0.0512800 & -2.0077371 \\ & & & \\ \mathrm{H} 2 \mathrm{O} & : \mathrm{water} & & \\ 3 & & & \\ \mathrm{E} & & & \\ \mathrm{O} & 0.059 y & -76.47484731353 & \\ \mathrm{H} & 1.0151520 & -0.0669314 & 0.0000000 \\ \mathrm{H} & -0.0669312 & 1.0151531 & 0.0000000\end{array}$

HOBf3- : aa

36

Energy $=-2285.569322315$

B $\quad-1.3200437 \quad-0.8934790 \quad-0.1957754$

$\begin{array}{llll}\text { C } & -0.8453076 & -1.6556602 & 1.1900766\end{array}$

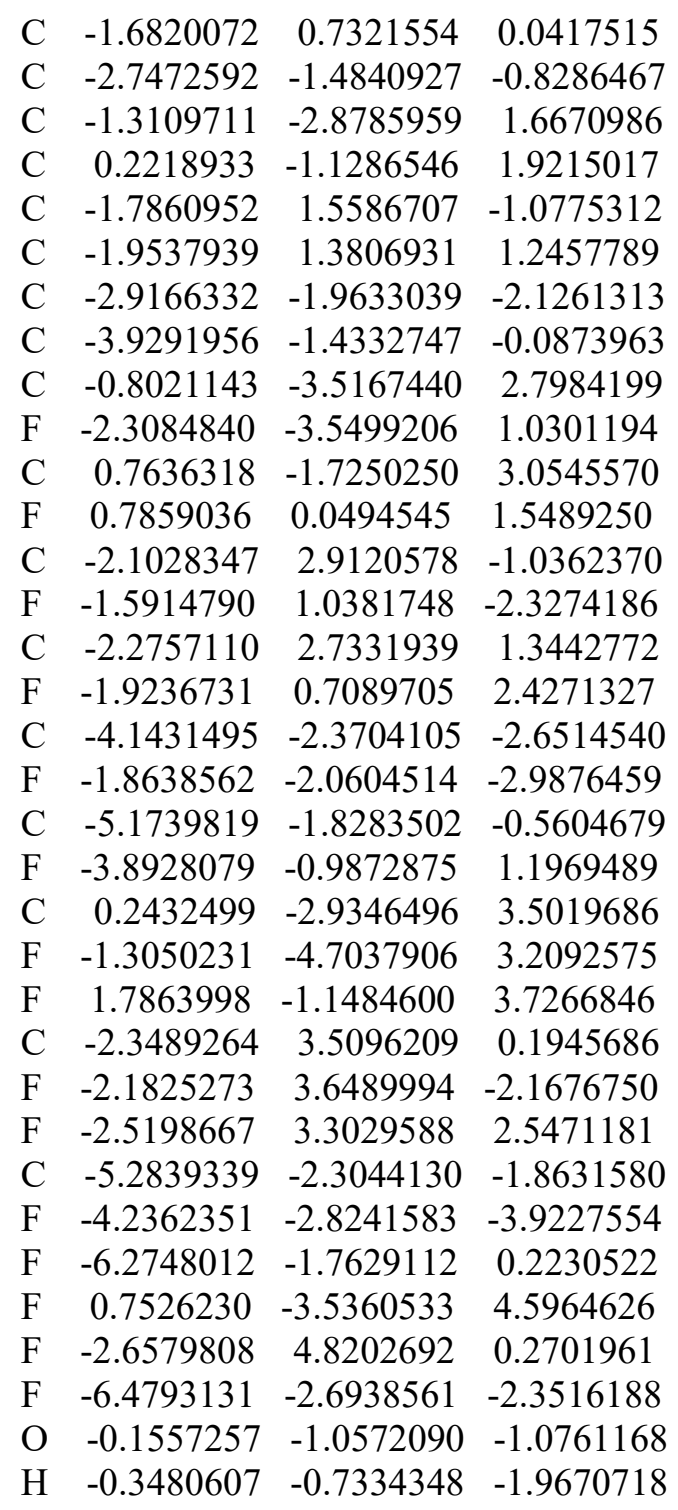

$\mathrm{Ph}_{2} \mathrm{NBf}_{3}^{-}$: borate anion 57

Energy $=-2728.048857568$

B $\quad 0.0118233 \quad-0.0057852 \quad 0.0886463$

$\begin{array}{llll}\text { C } & -1.1939334 & -0.3175461 & -1.0290301\end{array}$

C $\quad 0.6636956 \quad 1.5202966 \quad-0.0845165$

$\begin{array}{llll}\text { C } & 1.3924422 & -0.9119105 & -0.1091214\end{array}$

C $\quad-1.3878400 \quad-1.5118972 \quad-1.7259583$

$\begin{array}{llll}\text { C } & -2.2571128 & 0.5840303 & -1.1827860\end{array}$

$\begin{array}{llll}\text { C } & 1.5258387 & 2.0300465 & 0.8937036\end{array}$

$\begin{array}{llll}\text { C } & 0.6023559 & 2.3176777 & -1.2328499\end{array}$

$\begin{array}{llll}\text { C } & 2.1915890 & -1.4632618 & 0.9001117\end{array}$

C $\quad 1.9585938 \quad-1.0173421 \quad-1.3850701$

$\begin{array}{llll}\text { C } & -2.5317014 & -1.8240046 & -2.4584899\end{array}$ 


$\begin{array}{lccc}\text { F } & -0.4277663 & -2.4824444 & -1.7536137 \\ \text { C } & -3.4165388 & 0.3205587 & -1.9027902 \\ \text { F } & -2.1906706 & 1.8288998 & -0.6458913 \\ \text { C } & 2.2060203 & 3.2392783 & 0.7997810 \\ \text { F } & 1.7554632 & 1.3289120 & 2.0333535 \\ \text { C } & 1.2694175 & 3.5342207 & -1.3746776 \\ \text { F } & -0.1146541 & 1.9474749 & -2.3227734 \\ \text { C } & 3.4011652 & -2.1157497 & 0.6583746 \\ \text { F } & 1.8482626 & -1.3969994 & 2.2064459 \\ \text { C } & 3.1576630 & -1.6584285 & -1.6713276 \\ \text { F } & 1.3330754 & -0.4540511 & -2.4508543 \\ \text { C } & -3.5655209 & -0.9032274 & -2.5436915 \\ \text { F } & -2.6406058 & -3.0135254 & -3.0953541 \\ \text { F } & -4.3983735 & 1.2465488 & -1.9966238 \\ \text { C } & 2.0748726 & 4.0086935 & -0.3493525 \\ \text { F } & 2.9991630 & 3.6675672 & 1.8077449 \\ \text { F } & 1.1460015 & 4.2554167 & -2.5119792 \\ \text { C } & 3.8911533 & -2.2226503 & -0.6350521 \\ \text { F } & 4.1109943 & -2.6413109 & 1.6831768 \\ \text { F } & 3.6219281 & -1.7298455 & -2.9396123 \\ \text { F } & -4.6861791 & -1.1850637 & -3.2407677 \\ \text { F } & 2.7268583 & 5.1818879 & -0.4709804 \\ \text { F } & 5.0606456 & -2.8473051 & -0.8805038 \\ \text { N } & -0.6939046 & -0.2212235 & 1.4793923 \\ \text { C } & -1.6703043 & 0.7342487 & 1.9045763 \\ \text { C } & -0.8906693 & -1.4929658 & 2.0468980 \\ \text { C } & -3.0351344 & 0.3989852 & 1.8325764 \\ \text { C } & -1.3243629 & 2.0023517 & 2.3862260 \\ \text { C } & -0.6634003 & -2.6969603 & 1.3555403 \\ \text { C } & -1.3048459 & -1.5927291 & 3.3956837 \\ \text { C } & -4.0191993 & 1.3102105 & 2.2000700 \\ \text { H } & -3.3099382 & -0.5889085 & 1.4752039 \\ \text { C } & -2.3131791 & 2.9190193 & 2.7485585 \\ \text { H } & -0.2826154 & 2.2663394 & 2.5039673 \\ \text { C } & -0.8263462 & -3.9314831 & 1.9840564 \\ \text { H } & -0.3409260 & -2.6772408 & 0.3260346 \\ \text { C } & -1.4786227 & -2.8260347 & 4.0110043 \\ \text { H } & -1.4795231 & -0.6820853 & 3.9600145 \\ \text { C } & -3.6641325 & 2.5841344 & 2.6541432 \\ \text { H } & -5.0670058 & 1.0314165 & 2.1198532 \\ \text { H } & -2.0206204 & 3.8980035 & 3.1204110 \\ \text { C } & -1.2407339 & -4.0161988 & 3.3129804 \\ \text { H } & -0.6372380 & -4.8384746 & 1.4139826 \\ \text { H } & -1.7916164 & -2.8584119 & 5.0523618 \\ \text { H } & -4.4300097 & 3.3012594 & 2.9368084 \\ \text { H } & -1.3737621 & -4.9804197 & 3.7956388\end{array}$

$\mathrm{PhNH}_{2} \cdot \mathrm{Bf}_{3}: \mathrm{B}-\mathrm{N}$ adduct of $\mathrm{PhNH}_{2}$ and $\mathrm{Bf}_{3}$ 48

\begin{tabular}{|c|c|c|c|}
\hline \multicolumn{4}{|c|}{ Energy $=-2497.342003392$} \\
\hline B & 0.1249196 & 0.1655055 & -0.0170172 \\
\hline $\mathrm{C}$ & -1.1596569 & -0.1488880 & -0.9752135 \\
\hline $\mathrm{C}$ & 0.7842978 & 1.6638623 & -0.1359677 \\
\hline $\mathrm{C}$ & 1.3881553 & -0.8708378 & -0.2087672 \\
\hline $\mathrm{C}$ & -1.4329649 & -1.4207415 & -1.4876630 \\
\hline $\mathrm{C}$ & -2.1520723 & 0.8008617 & -1.2476892 \\
\hline $\mathrm{C}$ & 1.5986298 & 2.1668165 & 0.8823317 \\
\hline $\mathrm{C}$ & 0.7455559 & 2.4771599 & -1.2745779 \\
\hline $\mathrm{C}$ & 2.0197112 & -1.6323048 & 0.7728517 \\
\hline $\mathrm{C}$ & 1.9861030 & -0.9661090 & -1.4697212 \\
\hline $\mathrm{C}$ & -2.5602485 & -1.7285851 & -2.2459861 \\
\hline $\mathrm{F}$ & -0.5955066 & -2.4611975 & -1.2453545 \\
\hline $\mathrm{C}$ & -3.2933362 & 0.5382239 & -1.9971472 \\
\hline $\mathrm{F}$ & -2.0525547 & 2.0581857 & -0.7586407 \\
\hline $\mathrm{C}$ & 2.2693407 & 3.3828028 & 0.8386077 \\
\hline $\mathrm{F}$ & 1.7884695 & 1.4349281 & 2.0247047 \\
\hline $\mathrm{C}$ & 1.4057661 & 3.7008003 & -1.3707207 \\
\hline $\mathrm{F}$ & 0.0619218 & 2.1062888 & -2.3794798 \\
\hline $\mathrm{C}$ & 3.1401468 & -2.4283708 & 0.5456554 \\
\hline $\mathrm{F}$ & 1.5486777 & -1.6453448 & 2.0560535 \\
\hline $\mathrm{C}$ & 3.1011396 & -1.7462668 & -1.7495236 \\
\hline $\mathrm{F}$ & 1.4597346 & -0.2743592 & -2.5089462 \\
\hline $\mathrm{C}$ & -3.4992365 & -0.7385164 & -2.5077541 \\
\hline $\mathrm{F}$ & -2.7523495 & -2.9756717 & -2.7167523 \\
\hline $\mathrm{F}$ & -4.2090606 & 1.5012924 & -2.2159799 \\
\hline $\mathrm{C}$ & 2.1682446 & 4.1656011 & -0.3058686 \\
\hline $\mathrm{F}$ & 3.0159586 & 3.7991415 & 1.8782900 \\
\hline $\mathrm{F}$ & 1.3141305 & 4.4375453 & -2.4934646 \\
\hline $\mathrm{C}$ & 3.6889998 & -2.4873833 & -0.7287156 \\
\hline $\mathrm{F}$ & 3.6919389 & -3.1383347 & 1.5481828 \\
\hline $\mathrm{F}$ & 3.6149800 & -1.7945547 & -2.9930215 \\
\hline $\mathrm{F}$ & -4.5955289 & -1.0136105 & -3.2316934 \\
\hline $\mathrm{F}$ & 2.8061656 & 5.3433523 & -0.3842335 \\
\hline $\mathrm{F}$ & 4.7689024 & -3.2469516 & -0.9723097 \\
\hline $\mathrm{N}$ & -0.4846484 & -0.0408545 & 1.5201475 \\
\hline $\mathrm{C}$ & -1.6815510 & 0.7293025 & 1.8769694 \\
\hline $\mathrm{C}$ & -2.9340089 & 0.1566882 & 1.6774118 \\
\hline $\mathrm{C}$ & -1.5440911 & 2.0180632 & 2.3821535 \\
\hline $\mathrm{C}$ & -4.0755162 & 0.9024391 & 1.9701208 \\
\hline $\mathrm{H}$ & -3.0187199 & -0.8573502 & 1.2979252 \\
\hline $\mathrm{C}$ & -2.6912713 & 2.7553131 & 2.6734925 \\
\hline $\mathrm{H}$ & -0.5610570 & 2.4426744 & 2.5547487 \\
\hline $\mathrm{C}$ & -3.9555166 & 2.2033543 & 2.4607266 \\
\hline $\mathrm{H}$ & -5.0558137 & 0.4628519 & 1.8160073 \\
\hline $\mathrm{H}$ & -2.5934157 & 3.7615993 & 3.0683090 \\
\hline $\mathrm{H}$ & -4.8453924 & 2.7831182 & 2.6851590 \\
\hline $\mathrm{H}$ & -0.6986451 & -1.0355239 & 1.6262867 \\
\hline 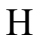 & 0.2549825 & 0.1541971 & 2.1996115 \\
\hline
\end{tabular}


$\mathrm{PhNH}_{2}$ : aniline

14

Energy $=-287.7864448520$

$\begin{array}{lrrr}\mathrm{C} & 0.9414573 & 0.0000537 & -0.0143627 \\ \mathrm{C} & 0.2221069 & -1.2095604 & -0.0082362 \\ \mathrm{C} & -1.1710645 & -1.2034256 & 0.0001770 \\ \mathrm{C} & -1.8824289 & -0.0001084 & 0.0030053 \\ \mathrm{C} & -1.1711977 & 1.2032897 & -0.0002259 \\ \mathrm{C} & 0.2219670 & 1.2095751 & -0.0079320 \\ \mathrm{H} & 0.7643080 & -2.1524756 & -0.0106488 \\ \mathrm{H} & -1.7044152 & -2.1505216 & 0.0078158 \\ \mathrm{H} & -2.9680853 & -0.0001624 & 0.0103187 \\ \mathrm{H} & -1.7046740 & 2.1503227 & 0.0063269 \\ \mathrm{H} & 0.7640424 & 2.1525568 & -0.0092717 \\ \mathrm{~N} & 2.3343817 & 0.0001235 & -0.0937716 \\ \mathrm{H} & 2.7871490 & 0.8385342 & 0.2532204 \\ \mathrm{H} & 2.7872352 & -0.8381556 & 0.2534239\end{array}$

$\mathrm{PhNH}_{3}{ }^{+}$: ammonium

15

Energy $=-288.2213701460$

N $\quad 2.3127260 \quad 0.0013032$

$\begin{array}{llll}\text { C } & 0.8292196 & -0.0010073 & 0.0007324\end{array}$

$\begin{array}{llll}\text { C } & 0.1666789 & -1.2222446 & -0.0001169\end{array}$

$\begin{array}{llll}\text { C } & 0.1643686 & 1.2168601 & 0.0015244\end{array}$

C $\quad-1.2279944-1.2142765 \quad-0.0005750$

H $\quad 0.7191518 \quad-2.1570239 \quad-0.0014410$

$\begin{array}{llll}\text { C } & -1.2318335 & 1.2083389 & 0.0005935\end{array}$

$\begin{array}{llll}\mathrm{H} & 0.7160916 & 2.1525602 & 0.0028463\end{array}$

C $\quad-1.9242418-0.0030490 \quad-0.0007020$

$\begin{array}{llll}\mathrm{H} & -1.7671812 & -2.1558754 & -0.0004486\end{array}$

$\begin{array}{llll}\mathrm{H} & -1.7716112 & 2.1495519 & 0.0008376\end{array}$

$\mathrm{H} \quad-3.0096122 \quad-0.0046842 \quad-0.0012429$

$\begin{array}{llll}\mathrm{H} & 2.6974834 & -0.4656970 & 0.8290388\end{array}$

$\begin{array}{llll}\mathrm{H} & 2.6817226 & 0.9581374 & -0.0059651\end{array}$

H $\quad 2.6963309 \quad-0.4750937 \quad-0.8247821$

$\mathrm{PhNHBf}_{3}{ }^{-}: \mathrm{B}-\mathrm{N}$ borate anion

47

Energy $=-2496.867237478$

$\begin{array}{rrrr}\text { B } & -0.1559690 & 0.1520630 & -0.2209311 \\ \mathrm{C} & -1.2018822 & -0.1172278 & -1.4947114 \\ \mathrm{C} & 0.6009296 & 1.6295718 & -0.2226012 \\ \mathrm{C} & 1.1501157 & -0.8848353 & -0.2694372 \\ \mathrm{C} & -1.3756606 & -1.3755636 & -2.0763431 \\ \mathrm{C} & -2.1374425 & 0.8252121 & -1.9283729 \\ \mathrm{C} & 1.2522832 & 2.0612133 & 0.9402888 \\ \mathrm{C} & 0.7826228 & 2.4797142 & -1.3166956\end{array}$ $\begin{array}{llll}\text { C } & 1.6397475 & -1.6635198 & 0.7813717\end{array}$

$\begin{array}{llll}\text { C } & 1.9432332 & -0.9421487 & -1.4197526\end{array}$

$\begin{array}{llll}\text { C } & -2.3430217 & -1.6751746 & -3.0327976\end{array}$

F $\quad-0.5884896 \quad-2.4285632-1.7145165$

$\begin{array}{llll}\text { C } & -3.1223907 & 0.5767300 & -2.8790031\end{array}$

$\begin{array}{llll}\text { F } & -2.1390469 & 2.0849725 & -1.4171253\end{array}$

$\begin{array}{llll}\text { C } & 1.9652464 & 3.2510911 & 1.0433348\end{array}$

$\begin{array}{llll}\text { F } & 1.2354119 & 1.2972556 & 2.0594772\end{array}$

$\begin{array}{llll}\text { C } & 1.4913988 & 3.6790722 & -1.2595582\end{array}$

$\begin{array}{llll}\text { F } & 0.2734348 & 2.1816757 & -2.5381132\end{array}$

$\begin{array}{llll}\text { C } & 2.8015987 & -2.4327236 & 0.7052655\end{array}$

$\begin{array}{llll}\text { F } & 0.9938711 & -1.7435084 & 1.9777967\end{array}$

C $\quad 3.1052372 \quad-1.6929769-1.5454296$

$\begin{array}{llll}\text { F } & 1.5800061 & -0.2337686 & -2.5202027\end{array}$

C $\quad-3.2274158 \quad-0.6878311 \quad-3.4450594$

F $\quad-2.4389279 \quad-2.9200716 \quad-3.5556149$

F $\quad-3.9815946 \quad 1.5510125 \quad-3.2587579$

$\begin{array}{llll}\text { C } & 2.0840329 & 4.0757897 & -0.0687104\end{array}$

$\begin{array}{llll}\text { F } & 2.5544487 & 3.6079552 & 2.2069071\end{array}$

$\begin{array}{llll}\text { F } & 1.6164868 & 4.4584337 & -2.3579517\end{array}$

$\begin{array}{llll}\text { C } & 3.5448074 & -2.4510364 & -0.4660950\end{array}$

$\begin{array}{llll}\text { F } & 3.2110384 & -3.1657326 & 1.7655260\end{array}$

F $\quad 3.8091307 \quad-1.6982150 \quad-2.6997171$

F $\quad-4.1785054 \quad-0.9546872 \quad-4.3642054$

F $\quad 2.7750365 \quad 5.2311658 \quad 0.0033176$

F $\quad 4.6696771 \quad-3.1884371 \quad-0.5565554$

$\begin{array}{llll}\mathrm{N} & -0.9880248 & -0.1042603 & 1.0417106\end{array}$

$\begin{array}{llll}\text { C } & -1.7801698 & 0.6879450 & 1.8288174\end{array}$

$\begin{array}{llll}\text { C } & -2.4600210 & 0.1045905 & 2.9302591\end{array}$

$\begin{array}{llll}\text { C } & -1.9595295 & 2.0781380 & 1.6325309\end{array}$

$\begin{array}{llll}\text { C } & -3.2579388 & 0.8603952 & 3.7796471\end{array}$

$\begin{array}{llll}\mathrm{H} & -2.3397188 & -0.9631207 & 3.1069330\end{array}$

$\begin{array}{llll}\text { C } & -2.7602368 & 2.8241737 & 2.4930683\end{array}$

$\begin{array}{llll}\mathrm{H} & -1.4761678 & 2.5682470 & 0.7987005\end{array}$

C $\quad-3.4214650 \quad 2.2362777 \quad 3.5754399$

$\mathrm{H} \quad-3.7590555 \quad 0.3692230 \quad 4.6113628$

$\begin{array}{llll}\mathrm{H} & -2.8708798 & 3.8908225 & 2.3078091\end{array}$

$\mathrm{H} \quad-4.0449365 \quad 2.8288978 \quad 4.2388327$

H $\quad-0.9991084 \quad-1.0674796 \quad 1.3516304$

$\mathrm{PhNHC}_{6} \mathrm{H}_{4} \mathrm{Bf}_{3}{ }^{-}$: B-C borate anion 57

Energy $=-2728.045730602$

B $\quad-1.5602249 \quad 0.4796043 \quad-2.2032602$

$\begin{array}{llll}\text { C } & -2.0183581 & 1.8506448 & -3.0168754\end{array}$

$\begin{array}{llll}\text { C } & -2.5199839 & -0.6735748 & -2.9095257\end{array}$

$\begin{array}{llll}\text { C } & -1.7559041 & 0.6015420 & -0.5620768\end{array}$

C $\quad-3.3138738 \quad 2.3501528 \quad-2.8512333$

$\begin{array}{llll}\text { C } & -1.2760771 & 2.5268724 & -3.9865719\end{array}$ 


\begin{tabular}{|c|c|c|c|}
\hline $\mathrm{C}$ & -2.2874232 & -1.0227544 & -4.2446931 \\
\hline 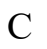 & -3.6431178 & -1.2944335 & -2.3579796 \\
\hline C & -1.6260069 & -0.5392826 & 0.2375456 \\
\hline $\mathrm{C}$ & -1.8289355 & 1.7873451 & 0.1684508 \\
\hline $\mathrm{C}$ & -3.8391060 & 3.4325919 & -3.5450646 \\
\hline $\mathrm{F}$ & -4.1471609 & 1.7625017 & -1.9518248 \\
\hline $\mathrm{C}$ & -1.7590103 & 3.6194374 & -4.7089975 \\
\hline $\mathrm{F}$ & -0.0124028 & 2.1510483 & -4.3040140 \\
\hline $\mathrm{C}$ & -3.0489877 & -1.9375633 & -4.9650024 \\
\hline $\mathrm{F}$ & -1.2759855 & -0.4330041 & -4.9335239 \\
\hline $\mathrm{C}$ & -4.4381271 & -2.2145149 & -3.0400072 \\
\hline $\mathrm{F}$ & -4.0518724 & -1.0191445 & -1.0927603 \\
\hline $\mathrm{C}$ & -1.6131143 & -0.5293025 & 1.6264596 \\
\hline $\mathrm{F}$ & -1.4863104 & -1.7594452 & -0.3432275 \\
\hline $\mathrm{C}$ & -1.8152695 & 1.8510246 & 1.5615034 \\
\hline $\mathrm{F}$ & -1.8911182 & 2.9968113 & -0.4568888 \\
\hline $\mathrm{C}$ & -3.0489918 & 4.0804522 & -4.4887092 \\
\hline $\mathrm{F}$ & -5.1013701 & 3.8645125 & -3.3178310 \\
\hline $\mathrm{F}$ & -0.9823647 & 4.2314303 & -5.6337580 \\
\hline $\mathrm{C}$ & -4.1374709 & -2.5478505 & -4.3539156 \\
\hline $\mathrm{F}$ & -2.7508342 & -2.2317696 & -6.2514023 \\
\hline $\mathrm{F}$ & -5.5087876 & -2.7820322 & -2.4377997 \\
\hline $\mathrm{C}$ & -1.7122061 & 0.6823840 & 2.3024028 \\
\hline $\mathrm{F}$ & -1.5004282 & -1.6796713 & 2.3309913 \\
\hline $\mathrm{F}$ & -1.8923274 & 3.0425792 & 2.1999163 \\
\hline $\mathrm{F}$ & -3.5312083 & 5.1332640 & -5.1812547 \\
\hline $\mathrm{F}$ & -4.8944996 & -3.4375658 & -5.0282361 \\
\hline $\mathrm{F}$ & -1.6990553 & 0.7200778 & 3.6519565 \\
\hline $\mathrm{N}$ & 4.1824539 & -0.8297008 & -2.0898987 \\
\hline $\mathrm{H}$ & 4.4627796 & -1.5555762 & -2.7374510 \\
\hline $\mathrm{C}$ & 2.8165755 & -0.4933944 & -2.1162926 \\
\hline $\mathrm{C}$ & 5.1638194 & -0.4177743 & -1.1982694 \\
\hline $\mathrm{C}$ & 2.3389182 & 0.7759118 & -1.7517434 \\
\hline $\mathrm{C}$ & 1.8940661 & -1.4426500 & -2.5765488 \\
\hline $\mathrm{C}$ & 4.8795653 & 0.2463037 & 0.0113105 \\
\hline $\mathrm{C}$ & 6.5108777 & -0.7103156 & -1.5003947 \\
\hline $\mathrm{C}$ & 0.9778250 & 1.0524394 & -1.8266875 \\
\hline $\mathrm{H}$ & 3.0314915 & 1.5470174 & -1.4294084 \\
\hline $\mathrm{C}$ & 0.5375912 & -1.1298923 & -2.6617987 \\
\hline $\mathrm{H}$ & 2.2468469 & -2.4328297 & -2.8603816 \\
\hline $\mathrm{C}$ & 5.9140927 & 0.6153228 & 0.8694392 \\
\hline $\mathrm{H}$ & 3.8509394 & 0.4503522 & 0.2856729 \\
\hline $\mathrm{C}$ & 7.5317218 & -0.3427017 & -0.6303165 \\
\hline $\mathrm{H}$ & 6.7436922 & -1.2298563 & -2.4274793 \\
\hline $\mathrm{C}$ & 0.0302239 & 0.1225588 & -2.2909093 \\
\hline $\mathrm{H}$ & 0.6427781 & 2.0426691 & -1.5214575 \\
\hline $\mathrm{H}$ & -0.1435730 & -1.9038785 & -3.0078344 \\
\hline $\mathrm{C}$ & 7.2470646 & 0.3326031 & 0.5614171 \\
\hline $\mathrm{H}$ & 5.6686959 & 1.1241592 & 1.7983523 \\
\hline
\end{tabular}

\section{H $\quad 8.5600643 \quad-0.5811839 \quad-0.8897757$ \\ $\begin{array}{llll}\mathrm{H} & 8.0457357 & 0.6268173 & 1.2355852\end{array}$}

TS0a : $\mathrm{Bf}_{3}$-induced $\mathrm{N}$-site $\mathrm{H}^{+}$transfer of two 2a 82

Energy $=-3247.469095316$

B $\quad 0.1425372 \quad-0.0590434 \quad-0.8134978$

$\begin{array}{llll}\text { C } & -0.8282873 & -0.5155199 & -2.0753857\end{array}$

$\begin{array}{llll}\text { C } & 0.6487049 & 1.5216403 & -0.9117872\end{array}$

$\begin{array}{llll}\text { C } & 1.6294916 & -0.8031637 & -0.8286687\end{array}$

$\begin{array}{llll}\text { C } & -0.7565973 & -1.7482573 & -2.7367426\end{array}$

$\begin{array}{llll}\text { C } & -1.8793146 & 0.2913804 & -2.5424955\end{array}$

$\begin{array}{llll}\text { C } & 1.3196412 & 2.1025614 & 0.1680077\end{array}$

$\begin{array}{llll}\text { C } & 0.6875692 & 2.3194888 & -2.0644755\end{array}$

$\begin{array}{llll}\text { C } & 2.3473267 & -1.3049536 & 0.2532732\end{array}$

$\begin{array}{llll}\text { C } & 2.3745083 & -0.7969778 & -2.0174390\end{array}$

$\begin{array}{llll}\text { C } & -1.6625760 & -2.1825314 & -3.7018615\end{array}$

F $\quad 0.2587905 \quad-2.6234736 \quad-2.5053040$

C $\quad-2.8057663 \quad-0.0998842 \quad-3.5038381$

$\begin{array}{llll}\text { F } & -2.0400081 & 1.5595205 & -2.0996918\end{array}$

$\begin{array}{llll}\text { C } & 1.8774597 & 3.3740187 & 0.1869597\end{array}$

$\begin{array}{llll}\text { F } & 1.4745270 & 1.3878333 & 1.3166349\end{array}$

C $\quad 1.2407975 \quad 3.6002507 \quad-2.1015993$

F $\quad 0.2150015 \quad 1.8904623 \quad-3.2561662$

$\begin{array}{llll}\text { C } & 3.6517909 & -1.7895769 & 0.1941923\end{array}$

$\begin{array}{llll}\text { F } & 1.7759484 & -1.3746574 & 1.4881961\end{array}$

$\begin{array}{llll}\text { C } & 3.6757704 & -1.2709645 & -2.1351128\end{array}$

$\begin{array}{llll}\text { F } & 1.8314932 & -0.3029341 & -3.1546778\end{array}$

C $\quad-2.7093472-1.3566870 \quad-4.0853079$

F $\quad-1.5201199 \quad-3.3946682 \quad-4.2779754$

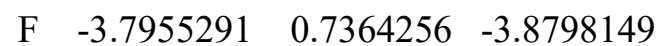

C $\quad 1.8298768 \quad 4.1444428 \quad-0.9687240$

$\begin{array}{llll}\text { F } & 2.4742273 & 3.8524875 & 1.2967286\end{array}$

F $\quad \begin{array}{llll}1.2201652 & 4.3136901 & -3.2455037\end{array}$

C $\quad 4.3297182 \quad-1.7757233 \quad-1.0161873$

$\begin{array}{llll}\text { F } & 4.2539115 & -2.2682288 & 1.3016619\end{array}$

F $\quad 4.3115354 \quad-1.2416624 \quad-3.3229671$

F $\quad-3.5958197 \quad-1.7549601 \quad-5.0145048$

F $\quad 2.3642586 \quad 5.3768456-0.9947990$

F $\quad 5.5887058 \quad-2.2357583 \quad-1.1060448$

$\begin{array}{llll}\mathrm{N} & -0.8116563 & -0.3300530 & 0.5661771\end{array}$

$\begin{array}{llll}\mathrm{H} & -0.3738106 & -0.0824811 & 1.9116392\end{array}$

$\begin{array}{llll}\text { C } & -1.9842651 & 0.5559343 & 0.4963011\end{array}$

$\begin{array}{llll}\text { C } & -1.2101991 & -1.7199980 & 0.8351656\end{array}$

$\begin{array}{llll}\text { C } & -3.2273498 & 0.1082758 & 0.0313246\end{array}$

$\begin{array}{llll}\text { C } & -1.8452836 & 1.8914778 & 0.8723548\end{array}$

$\begin{array}{llll}\text { C } & -2.2458305 & -1.9693624 & 1.7514618\end{array}$

$\begin{array}{llll}\text { C } & -0.5214867 & -2.8108107 & 0.3112387\end{array}$

$\begin{array}{llll}\text { C } & -4.3069684 & 0.9842331 & -0.0446163\end{array}$ 


$\begin{array}{lrrc}\mathrm{H} & -3.3512064 & -0.9237653 & -0.2745641 \\ \mathrm{C} & -2.9255860 & 2.7694405 & 0.7897537 \\ \mathrm{H} & -0.8963907 & 2.2523489 & 1.2445912 \\ \mathrm{C} & -2.5864108 & -3.2674077 & 2.1124794 \\ \mathrm{H} & -2.7906230 & -1.1425276 & 2.1914122 \\ \mathrm{C} & -0.8611558 & -4.1151581 & 0.6767490 \\ \mathrm{H} & 0.2931635 & -2.6631568 & -0.3781405 \\ \mathrm{C} & -4.1628578 & 2.3205504 & 0.3330855 \\ \mathrm{H} & -5.2618318 & 0.6195616 & -0.4118731 \\ \mathrm{H} & -2.7949427 & 3.8034405 & 1.0953708 \\ \mathrm{C} & -1.8956887 & -4.3549113 & 1.5755233 \\ \mathrm{H} & -3.3840057 & -3.4235026 & 2.8320332 \\ \mathrm{H} & -0.3029926 & -4.9427054 & 0.2482429 \\ \mathrm{H} & -5.0060526 & 3.0021396 & 0.2719486 \\ \mathrm{H} & -2.1574584 & -5.3695189 & 1.8601546 \\ \mathrm{H} & 0.9930447 & -0.1740976 & 2.9845615 \\ \mathrm{~N} & -0.0070843 & -0.0289596 & 3.1419557 \\ \mathrm{C} & -0.4534118 & -1.1582970 & 3.9932877 \\ \mathrm{C} & 0.2684859 & -2.3497535 & 3.9352574 \\ \mathrm{C} & -1.5589631 & -1.0426355 & 4.8322692 \\ \mathrm{C} & -0.1443633 & -3.4428196 & 4.6934856 \\ \mathrm{H} & 1.1329979 & -2.4396671 & 3.2894215 \\ \mathrm{C} & -1.9626617 & -2.1426590 & 5.5887979 \\ \mathrm{H} & -2.1091316 & -0.1141393 & 4.9072742 \\ \mathrm{C} & -1.2644229 & -3.3473436 & 5.5191100 \\ \mathrm{H} & 0.4153784 & -4.3709593 & 4.6334626 \\ \mathrm{H} & -2.8297777 & -2.0483691 & 6.2353932 \\ \mathrm{H} & -1.5861262 & -4.2015245 & 6.1067369 \\ \mathrm{C} & -0.1403777 & 1.2934477 & 3.7685556 \\ \mathrm{C} & 1.0079528 & 1.9046936 & 4.2653820 \\ \mathrm{C} & -1.3804708 & 1.9199455 & 3.8639610 \\ \mathrm{C} & 0.9180825 & 3.1640418 & 4.8563824 \\ \mathrm{H} & 1.9701939 & 1.4069271 & 4.1813479 \\ \mathrm{C} & -1.4655772 & 3.1746276 & 4.4664079 \\ \mathrm{H} & -2.2666974 & 1.4560737 & 3.4483068 \\ \mathrm{C} & -0.3199804 & 3.7993721 & 4.9611758 \\ \mathrm{H} & 1.8157151 & 3.6441094 & 5.2329358 \\ \mathrm{H} & -2.4309522 & 3.6659364 & 4.5371910 \\ \mathrm{H} & -0.3912712 & 4.7798752 & 5.4217230\end{array}$

TS0b : $\mathrm{Bf}_{3}$-induced para $\mathrm{H}^{+}$transfer of two $\mathbf{2 a}$ 82

Energy $=-3247.452071286$

$\begin{array}{lrrr}\text { B } & -0.9852057 & 0.0472129 & 0.6342715 \\ \text { C } & -1.7426458 & 1.4975820 & 0.3937057 \\ \text { C } & -2.1474894 & -1.0511508 & 0.2078660 \\ \text { C } & -0.4468056 & -0.1007450 & 2.2011210 \\ \text { C } & 0.4800502 & -0.2146125 & -0.1783774 \\ \text { C } & -2.8834758 & 1.7841293 & 1.1536773\end{array}$

C $\quad-1.4506762 \quad 2.4772601 \quad-0.5496093$

C $\quad-2.5563458-1.1026844 \quad-1.1266112$

$\begin{array}{llll}\text { C } & -2.8947195 & -1.8860266 & 1.0456532\end{array}$

$\begin{array}{llll}\text { C } & -0.0268190 & -1.3279053 & 2.7272832\end{array}$

$\begin{array}{llll}\text { C } & -0.1569177 & 0.9829069 & 3.0354307\end{array}$

$\begin{array}{llll}\text { C } & 0.9066437 & -1.5820054 & -0.3071474\end{array}$

$\begin{array}{llll}\text { C } & 1.5372551 & 0.6868879 & 0.2095768\end{array}$

$\begin{array}{llll}\text { C } & -3.6652845 & 2.9227821 & 1.0049671\end{array}$

$\begin{array}{llll}\text { F } & -3.2745191 & 0.9207116 & 2.1254285\end{array}$

$\begin{array}{llll}\text { C } & -2.2003565 & 3.6363359 & -0.7440521\end{array}$

$\begin{array}{llll}\text { F } & -0.3670570 & 2.3578511 & -1.3713608\end{array}$

C $\quad-3.5363896 \quad-1.9471864 \quad-1.6344802$

F $\quad-2.0100553 \quad-0.2347803 \quad-2.0230768$

C $\quad-3.8873102 \quad-2.7526843 \quad 0.5889539$

$\begin{array}{llll}\text { F } & -2.7273619 & -1.8766289 & 2.3893085\end{array}$

$\begin{array}{llll}\text { C } & 0.5919776 & -1.4881521 & 3.9618738\end{array}$

$\begin{array}{llll}\text { F } & -0.2002498 & -2.4752320 & 2.0191453\end{array}$

$\begin{array}{llll}\text { C } & 0.4563761 & 0.8736761 & 4.2821777\end{array}$

$\begin{array}{llll}\text { F } & -0.4351212 & 2.2596251 & 2.6533539\end{array}$

C $\quad 2.2143143 \quad-1.9812559-0.1567172$

$\mathrm{H} \quad 0.1612091 \quad-2.3296570 \quad-0.5625617$

C $\quad 2.8435209 \quad 0.3075040 \quad 0.3936871$

$\begin{array}{llll}\mathrm{H} & 1.2813339 & 1.7267770 & 0.3928471\end{array}$

$\begin{array}{llll}\text { C } & -3.3221528 & 3.8651178 & 0.0403548\end{array}$

F $\quad-4.7472384 \quad 3.1283260 \quad 1.7852773$

F $\quad-1.8473249 \quad 4.5328271 \quad-1.6906212$

C $\quad-4.2052656 \quad-2.7980440 \quad-0.7625615$

F $\quad-3.8575930 \quad-1.9338040 \quad-2.9447496$

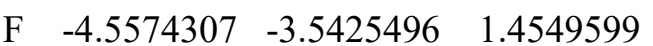

C $\quad 0.8349006 \quad-0.3744606 \quad 4.7566561$

$\begin{array}{llll}\text { F } & 0.9613926 & -2.7130674 & 4.3952017\end{array}$

F $\quad 0.6961044 \quad 1.9735251 \quad 5.0292045$

$\begin{array}{llll}\text { C } & 3.2179741 & -1.0467597 & 0.2011448\end{array}$

H $\quad 2.4925510 \quad-3.0237647 \quad-0.2942880$

$\begin{array}{llll}\mathrm{H} & 3.5785068 & 1.0351912 & 0.7137932\end{array}$

F $\quad-4.0640698 \quad 4.9754895 \quad-0.1277702$

F $\quad-5.1615303 \quad-3.6294904 \quad-1.2151844$

F $\quad 1.4319582 \quad-0.5030905 \quad 5.9571558$

$\begin{array}{llll}\mathrm{N} & 4.4961625 & -1.5044219 & 0.3606992\end{array}$

$\begin{array}{llll}\mathrm{H} & 4.5934667 & -2.5136567 & 0.3882777\end{array}$

$\begin{array}{llll}\text { C } & 5.6958283 & -0.7884063 & 0.5575351\end{array}$

$\begin{array}{llll}\text { C } & 5.9334287 & 0.4635325 & -0.0270291\end{array}$

$\begin{array}{llll}\text { C } & 6.7051242 & -1.3994601 & 1.3159506\end{array}$

$\begin{array}{llll}\text { C } & 7.1559334 & 1.1013136 & 0.1752952\end{array}$

$\begin{array}{llll}\mathrm{H} & 5.1828781 & 0.9253137 & -0.6572176\end{array}$

$\begin{array}{llll}\text { C } & 7.9277566 & -0.7591707 & 1.4997412\end{array}$

$\begin{array}{llll}\mathrm{H} & 6.5235155 & -2.3744625 & 1.7607057\end{array}$

$\begin{array}{llll}\text { C } & 8.1575158 & 0.4996415 & 0.9394240\end{array}$

H $\quad 7.3289146 \quad 2.0710946 \quad-0.2825617$ 


\begin{tabular}{lrrr}
$\mathrm{H}$ & 8.6995931 & -1.2442100 & 2.0900655 \\
$\mathrm{H}$ & 9.1084353 & 1.0014825 & 1.0890798 \\
$\mathrm{H}$ & 0.4373894 & 0.0229769 & -1.5652811 \\
$\mathrm{~N}$ & 0.5420474 & 0.2418548 & -2.9465767 \\
$\mathrm{H}$ & -0.2759877 & 0.8355861 & -3.0854626 \\
$\mathrm{C}$ & 1.7219226 & 1.0343411 & -3.2658907 \\
$\mathrm{C}$ & 0.3423317 & -0.9821003 & -3.7123868 \\
$\mathrm{C}$ & 1.5386132 & 2.2991903 & -3.8254319 \\
$\mathrm{C}$ & 3.0056101 & 0.5569055 & -3.0064364 \\
$\mathrm{C}$ & -0.7333306 & -1.0393580 & -4.5998756 \\
$\mathrm{C}$ & 1.1774177 & -2.0862954 & -3.5447663 \\
$\mathrm{C}$ & 2.6461314 & 3.0882617 & -4.1343375 \\
$\mathrm{H}$ & 0.5345656 & 2.6667403 & -4.0196378 \\
$\mathrm{C}$ & 4.1086242 & 1.3430642 & -3.3351396 \\
$\mathrm{H}$ & 3.1502315 & -0.4095474 & -2.5407180 \\
$\mathrm{C}$ & -0.9761033 & -2.2054957 & -5.3220685 \\
$\mathrm{H}$ & -1.3831618 & -0.1773226 & -4.7203073 \\
$\mathrm{C}$ & 0.9332542 & -3.2480979 & -4.2778687 \\
$\mathrm{H}$ & 1.9974352 & -2.0576803 & -2.8398844 \\
$\mathrm{C}$ & 3.9348984 & 2.6094624 & -3.8963630 \\
$\mathrm{H}$ & 2.4979118 & 4.0720204 & -4.5688432 \\
$\mathrm{H}$ & 5.1081908 & 0.9629484 & -3.1470730 \\
$\mathrm{C}$ & -0.1416904 & -3.3136630 & -5.1645145 \\
$\mathrm{H}$ & -1.8165541 & -2.2441536 & -6.0081042 \\
$\mathrm{H}$ & 1.5842136 & -4.1068541 & -4.1443361 \\
$\mathrm{H}$ & 4.7980215 & 3.2193481 & -4.1444262 \\
$\mathrm{H}$ & -0.3303886 & -4.2231284 & -5.7266670 \\
& & & \\
\hline
\end{tabular}

TS0g : $\mathrm{Bf}_{3}$-induced para $\mathrm{H}^{+}$of $\mathbf{2 g}$ to $\mathbf{1 a} \mathrm{C} 4$ 88

\begin{tabular}{lrrr}
\multicolumn{4}{l}{ Energy $=-3346.956917129$} \\
B & -2.0492900 & -0.0411248 & 0.1210950 \\
C & -2.8475261 & 0.8767848 & -1.0152232 \\
C & -2.6676759 & -1.5731355 & 0.0324940 \\
C & -2.1928643 & 0.6295314 & 1.6311473 \\
C & -4.1710612 & 0.6135962 & -1.3932955 \\
C & -2.3146053 & 1.9906502 & -1.6665136 \\
C & -2.5506772 & -2.2859356 & -1.1639551 \\
C & -3.3465817 & -2.2720508 & 1.0320111 \\
C & -1.4908954 & 0.0752343 & 2.7093063 \\
C & -2.8881700 & 1.7942869 & 1.9613863 \\
C & -4.8704992 & 1.3231362 & -2.3663550 \\
$F$ & -4.8961650 & -0.3564976 & -0.7777279 \\
C & -2.9680694 & 2.7331197 & -2.6461273 \\
$F$ & -1.0775820 & 2.4590558 & -1.3384268 \\
C & -3.0213187 & -3.5776944 & -1.3694895 \\
$F$ & -1.9425773 & -1.7031704 & -2.2357369 \\
C & -3.8340072 & -3.5692281 & 0.8789014 \\
$F$ & -3.6187743 & -1.7003180 & 2.2324551
\end{tabular}

\begin{tabular}{|c|c|c|c|}
\hline C & -1.4456784 & 0.6179389 & 3.9861557 \\
\hline F & -0.8072682 & -1.0863688 & 2.5422337 \\
\hline $\mathrm{C}$ & -2.8755577 & 2.3778179 & 3.2297900 \\
\hline $\mathrm{F}$ & -3.6439844 & 2.4544898 & 1.0450455 \\
\hline $\mathrm{C}$ & -4.2624032 & 2.3910374 & -3.0130434 \\
\hline $\mathrm{F}$ & -6.1419138 & 0.9928575 & -2.6765165 \\
\hline $\mathrm{F}$ & -2.3579923 & 3.7861872 & -3.2322565 \\
\hline $\mathrm{C}$ & -3.6682420 & -4.2341679 & -0.3291096 \\
\hline $\mathrm{F}$ & -2.8567742 & -4.1970231 & -2.5576688 \\
\hline $\mathrm{F}$ & -4.4809369 & -4.1837142 & 1.8921739 \\
\hline $\mathrm{C}$ & -2.1458078 & 1.7904211 & 4.2529167 \\
\hline $\mathrm{F}$ & -0.7414669 & 0.0188202 & 4.9709525 \\
\hline $\mathrm{F}$ & -3.5678020 & 3.5124081 & 3.4697789 \\
\hline $\mathrm{F}$ & -4.9184700 & 3.0886710 & -3.9577616 \\
\hline $\mathrm{F}$ & -4.1324433 & -5.4868580 & -0.4920809 \\
\hline $\mathrm{F}$ & -2.1202302 & 2.3390656 & 5.4820478 \\
\hline $\mathrm{N}$ & 3.7749468 & -0.6472346 & 0.7675889 \\
\hline $\mathrm{C}$ & 2.4248172 & -0.5115848 & 0.5408809 \\
\hline $\mathrm{C}$ & 4.4949748 & 0.3495709 & 1.5127883 \\
\hline $\mathrm{C}$ & 1.7933966 & 0.7562643 & 0.6816026 \\
\hline $\mathrm{C}$ & 1.6176407 & -1.6155948 & 0.1715584 \\
\hline $\mathrm{C}$ & 4.0506092 & 0.7299983 & 2.7822497 \\
\hline $\mathrm{C}$ & 5.6534971 & 0.9066110 & 0.9666847 \\
\hline $\mathrm{C}$ & 0.4618857 & 0.9051406 & 0.4036849 \\
\hline $\mathrm{H}$ & 2.3782761 & 1.6109446 & 1.0001863 \\
\hline $\mathrm{C}$ & 0.2836119 & -1.4396982 & -0.1014182 \\
\hline $\mathrm{H}$ & 2.0486914 & -2.6095638 & 0.1450446 \\
\hline $\mathrm{C}$ & 4.7621273 & 1.6909449 & 3.4982156 \\
\hline $\mathrm{H}$ & 3.1595483 & 0.2715459 & 3.2005260 \\
\hline $\mathrm{C}$ & 6.3607697 & 1.8628384 & 1.6918710 \\
\hline $\mathrm{H}$ & 5.9910687 & 0.5913943 & -0.0146709 \\
\hline $\mathrm{C}$ & -0.3720608 & -0.1556950 & -0.1104978 \\
\hline $\mathrm{H}$ & 0.0303779 & 1.8922273 & 0.5195491 \\
\hline $\mathrm{H}$ & -0.2947997 & -2.3244517 & -0.3459476 \\
\hline $\mathrm{C}$ & 5.9169886 & 2.2600006 & 2.9553461 \\
\hline $\mathrm{H}$ & 4.4211716 & 1.9860019 & 4.4860059 \\
\hline $\mathrm{H}$ & 7.2562205 & 2.3026894 & 1.2636770 \\
\hline $\mathrm{H}$ & -0.1120452 & 0.1134581 & -1.4941352 \\
\hline $\mathrm{H}$ & 6.4705397 & 3.0061726 & 3.5172192 \\
\hline $\mathrm{C}$ & 0.2472566 & 0.2266962 & -2.8792677 \\
\hline $\mathrm{C}$ & 1.2915886 & 1.1436540 & -2.8865693 \\
\hline $\mathrm{H}$ & -0.7395541 & 0.5519425 & -3.1996707 \\
\hline $\mathrm{H}$ & 0.4739538 & -0.8217159 & -3.0637373 \\
\hline $\mathrm{H}$ & 1.0463622 & 2.2040929 & -2.8445124 \\
\hline $\mathrm{C}$ & 2.6662482 & 0.8055474 & -2.8472181 \\
\hline $\mathrm{C}$ & 3.6464065 & 1.7461193 & -2.6991542 \\
\hline $\mathrm{H}$ & 2.9247123 & -0.2486042 & -2.9198513 \\
\hline $\mathrm{H}$ & 3.3343026 & 2.7856307 & -2.5996365 \\
\hline $\mathrm{C}$ & 5.0671506 & 1.5045321 & -2.5838283 \\
\hline
\end{tabular}




$\begin{array}{lrrr}\mathrm{C} & 5.9048807 & 2.5696071 & -2.1881947 \\ \mathrm{C} & 5.6538069 & 0.2390109 & -2.8054125 \\ \mathrm{C} & 7.2680384 & 2.3728820 & -1.9939350 \\ \mathrm{H} & 5.4659679 & 3.5493566 & -2.0190048 \\ \mathrm{C} & 7.0137318 & 0.0440579 & -2.6039683 \\ \mathrm{H} & 5.0423093 & -0.5899768 & -3.1436097 \\ \mathrm{C} & 7.8267409 & 1.1075178 & -2.1926008 \\ \mathrm{H} & 7.8953426 & 3.2023654 & -1.6814995 \\ \mathrm{H} & 7.4461777 & -0.9386311 & -2.7667046 \\ \mathrm{H} & 8.8894820 & 0.9496683 & -2.0352220 \\ \mathrm{C} & 4.5323657 & -1.7681089 & 0.3034485 \\ \mathrm{C} & 5.5358693 & -2.2989993 & 1.1228825 \\ \mathrm{C} & 4.3211508 & -2.3031041 & -0.9724247 \\ \mathrm{C} & 6.3176139 & -3.3563828 & 0.6649079 \\ \mathrm{H} & 5.7004997 & -1.8812957 & 2.1105538 \\ \mathrm{C} & 5.1010203 & -3.3664751 & -1.4192114 \\ \mathrm{H} & 3.5503684 & -1.8861858 & -1.6095073 \\ \mathrm{C} & 6.1046858 & -3.8955292 & -0.6059938 \\ \mathrm{H} & 7.0917743 & -3.7637782 & 1.3081142 \\ \mathrm{H} & 4.9316503 & -3.7713688 & -2.4123671 \\ \mathrm{H} & 6.7159873 & -4.7200095 & -0.9596703\end{array}$

TS0 : $\mathrm{H}^{+}$from $\mathrm{H}_{2} \mathrm{O} \cdot \mathrm{Bf}_{3}$ to $\mathbf{2 a} \mathrm{Ph}_{2} \mathrm{NH} \mathrm{N}$-site 61

\begin{tabular}{lrrc}
\multicolumn{4}{l}{ Energy $=-2805.009863155$} \\
B & -0.0340765 & 0.1675685 & 0.6474181 \\
C & -1.4959483 & 0.0545976 & 1.4000651 \\
C & 0.3588749 & -1.1393087 & -0.2522107 \\
C & 1.1508812 & 0.5473890 & 1.7333010 \\
O & -0.0668419 & 1.3380035 & -0.3557995 \\
C & -1.6498508 & -0.3480296 & 2.7298708 \\
C & -2.7018139 & 0.3513850 & 0.7627397 \\
C & 1.4935637 & -1.1007948 & -1.0690453 \\
C & -0.3205301 & -2.3573249 & -0.2690687 \\
C & 1.2179881 & 1.8305508 & 2.2713825 \\
C & 2.1459817 & -0.3136951 & 2.1970304 \\
H & -0.3632481 & 2.1601304 & 0.0723480 \\
H & -0.5160263 & 1.2698788 & -1.5495172 \\
C & -2.8757117 & -0.4170584 & 3.3865666 \\
F & -0.5761252 & -0.7377074 & 3.4620869 \\
C & -3.9510079 & 0.3004278 & 1.3724472 \\
F & -2.7216024 & 0.6839312 & -0.5632952 \\
C & 1.9340449 & -2.1688681 & -1.8402870 \\
F & 2.2576129 & 0.0208123 & -1.1171353 \\
C & 0.0829047 & -3.4540975 & -1.0272967 \\
F & -1.4375498 & -2.5480108 & 0.4778729 \\
C & 2.1848878 & 2.2639894 & 3.1709100 \\
F & 0.2736013 & 2.7569362 & 1.9212864 \\
C & 3.1387631 & 0.0675369 & 3.0966800
\end{tabular}

$\begin{array}{lrrc}\text { F } & 2.1761728 & -1.6147123 & 1.8123896 \\ \text { N } & -0.9037220 & 1.3310066 & -2.7270149 \\ \mathrm{C} & -4.0401302 & -0.0826879 & 2.7052649 \\ \mathrm{~F} & -2.9467464 & -0.8107198 & 4.6737973 \\ \mathrm{~F} & -5.0698334 & 0.6060142 & 0.6851895 \\ \mathrm{C} & 1.2184295 & -3.3605045 & -1.8205575 \\ \mathrm{~F} & 3.0414345 & -2.0657114 & -2.6014029 \\ \mathrm{~F} & -0.6308185 & -4.5988859 & -1.0172862 \\ \mathrm{C} & 3.1634860 & 1.3683139 & 3.5868942 \\ \mathrm{~F} & 2.1837192 & 3.5278058 & 3.6411374 \\ \mathrm{~F} & 4.0733279 & -0.8148448 & 3.5053735 \\ \mathrm{H} & -1.9232912 & 1.3397854 & -2.6488103 \\ \mathrm{C} & -0.5281445 & 0.1336535 & -3.4975879 \\ \mathrm{C} & -0.4649581 & 2.6123144 & -3.2923237 \\ \mathrm{~F} & -5.2331327 & -0.1398143 & 3.3210586 \\ \mathrm{~F} & 1.6110998 & -4.4015133 & -2.5743163 \\ \mathrm{~F} & 4.1153423 & 1.7529569 & 4.4548468 \\ \mathrm{C} & -1.3462891 & -0.9893485 & -3.3941251 \\ \mathrm{C} & 0.6226344 & 0.1214163 & -4.2786192 \\ \mathrm{C} & -1.2552331 & 3.2440664 & -4.2482753 \\ \mathrm{C} & 0.7421304 & 3.1601356 & -2.8653171 \\ \mathrm{C} & -1.0058797 & -2.1448839 & -4.0949157 \\ \mathrm{H} & -2.2390811 & -0.9633031 & -2.7752150 \\ \mathrm{C} & 0.9535261 & -1.0417987 & -4.9751598 \\ \mathrm{H} & 1.2495403 & 1.0027376 & -4.3526036 \\ \mathrm{C} & -0.8302637 & 4.4601971 & -4.7821630 \\ \mathrm{H} & -2.1890883 & 2.7933629 & -4.5733643 \\ \mathrm{C} & 1.1629522 & 4.3735045 & -3.4121996 \\ \mathrm{H} & 1.3414553 & 2.6432607 & -2.1224594 \\ \mathrm{C} & 0.1429518 & -2.1732812 & -4.8879196 \\ \mathrm{H} & -1.6418535 & -3.0213347 & -4.0195754 \\ \mathrm{H} & 1.8504818 & -1.0574913 & -5.5858559 \\ \mathrm{C} & 0.3785800 & 5.0232053 & -4.3662589 \\ \mathrm{H} & -1.4425293 & 4.9635852 & -5.5235399 \\ \mathrm{H} & 2.1028620 & 4.8098168 & -3.0887152 \\ \mathrm{H} & 0.4054254 & -3.0750189 & -5.4318551 \\ \mathrm{H} & 0.7081413 & 5.9686056 & -4.7859328\end{array}$

TS1g : $\mathrm{H}^{+}$from $\mathrm{H}_{2} \mathrm{O}_{2} \mathrm{Bf}_{3}$ to 1a C4-site 57

\begin{tabular}{lrrr}
\multicolumn{4}{l}{ Energy $=-2673.308662006$} \\
$\mathrm{~B}$ & -0.9755340 & 0.0353333 & 0.6625380 \\
$\mathrm{C}$ & -1.7486178 & 1.4020738 & 0.1575285 \\
$\mathrm{C}$ & 0.4849846 & -0.2290830 & -0.0402776 \\
$\mathrm{C}$ & -2.0620952 & -1.1919474 & 0.4142222 \\
$\mathrm{O}$ & -0.6648652 & 0.0430545 & 2.1543940 \\
$\mathrm{C}$ & -2.1818076 & 1.4977184 & -1.1675530 \\
$\mathrm{C}$ & -2.1105287 & 2.4877029 & 0.9518973 \\
$\mathrm{C}$ & 1.1729262 & -1.4128235 & 0.2501868
\end{tabular}




$\begin{array}{lrcc}\text { C } & 1.1466868 & 0.6089553 & -0.9370753 \\ \mathrm{C} & -3.1962947 & -1.2586830 & 1.2245541 \\ \mathrm{C} & -2.0143052 & -2.1717108 & -0.5778294 \\ \mathrm{H} & -1.4520835 & 0.2490817 & 2.6871928 \\ \mathrm{C} & -2.8984602 & 2.5701432 & -1.6823012 \\ \mathrm{~F} & -1.8781111 & 0.5018149 & -2.0389596 \\ \mathrm{C} & -2.8292652 & 3.5877620 & 0.4856054 \\ \mathrm{~F} & -1.7797973 & 2.5305664 & 2.2751421 \\ \mathrm{C} & 2.3802133 & -1.7781202 & -0.3345087 \\ \mathrm{~F} & 0.6368040 & -2.3083950 & 1.1143273 \\ \mathrm{C} & 2.3514406 & 0.2802058 & -1.5579159 \\ \mathrm{~F} & 0.6510469 & 1.8321943 & -1.2549538 \\ \mathrm{C} & -4.2001954 & -2.2119682 & 1.1037556 \\ \mathrm{~F} & -3.3756494 & -0.3257533 & 2.2107143 \\ \mathrm{C} & -2.9953210 & -3.1479250 & -0.7442752 \\ \mathrm{~F} & -0.9879266 & -2.2205044 & -1.4628689 \\ \mathrm{C} & -3.2271923 & 3.6317877 & -0.8441445 \\ \mathrm{~F} & -3.2705667 & 2.6002291 & -2.9783306 \\ \mathrm{~F} & -3.1464342 & 4.6048892 & 1.3138233 \\ \mathrm{C} & 2.9677310 & -0.9307624 & -1.2679747 \\ \mathrm{~F} & 2.9774154 & -2.9467459 & -0.0239191 \\ \mathrm{~F} & 2.9364307 & 1.1363742 & -2.4214250 \\ \mathrm{C} & -4.0962738 & -3.1730933 & 0.1037733 \\ \mathrm{~F} & -5.2647936 & -2.2137389 & 1.9312367 \\ \mathrm{~F} & -2.8885692 & -4.0710433 & -1.7220863 \\ \mathrm{~F} & -3.9199966 & 4.6836186 & -1.3154688 \\ \mathrm{~F} & 4.1222710 & -1.2686138 & -1.8633400 \\ \mathrm{~F} & -5.0490599 & -4.1109225 & -0.0418470 \\ \mathrm{C} & 1.1940576 & 1.5846080 & 3.2336577 \\ \mathrm{H} & 0.5795975 & 2.4383555 & 3.5152057 \\ \mathrm{H} & 1.4529379 & 0.9058169 & 4.0473489 \\ \mathrm{H} & 0.3100882 & 0.8628383 & 2.5983185 \\ \mathrm{C} & 2.1635690 & 1.7989005 & 2.2474516 \\ \mathrm{H} & 7.1095666 & -2.3155774 & 1.3346886 \\ \mathrm{C} & 3.2685237 & 0.9608060 & 2.0031115 \\ \mathrm{H} & 2.0295466 & 2.6399979 & 1.5661234 \\ \mathrm{H} & 3.4185760 & 0.1014935 & 2.6517333 \\ \mathrm{C} & 4.1489137 & 1.2476187 & 0.9918672 \\ \mathrm{H} & 3.9477179 & 2.1373355 & 0.3954967 \\ \mathrm{C} & 5.3286428 & 0.5069069 & 0.6263160 \\ \mathrm{C} & 6.1294003 & 0.9977168 & -0.4277334 \\ \mathrm{C} & 5.7008717 & -0.6997942 & 1.2603115 \\ \mathrm{C} & 7.2653788 & 0.3090334 & -0.8346534 \\ \mathrm{H} & 5.8424553 & 1.9225291 & -0.9206932 \\ \mathrm{H} & 6.8320408 & -1.3863830 & 0.8468538 \\ & 7.0970408 & -1.0987824 & 2.0690486 \\ \mathrm{H} & -1.4276584 & -0.5183243\end{array}$

$\mathbf{T S 1 h}^{+}: \mathrm{H}^{+}$from ammonium $\mathbf{2} \mathbf{h} \mathrm{H}^{+}$to diene $\mathbf{1 a}$ 48 Energy $=-946.0264375808$

N $\quad 0.8149519 \quad-2.0592764$

$\begin{array}{llll}\mathrm{H} & 0.2014401 & -1.5900625 & 1.4608703\end{array}$

$\begin{array}{llll}\text { C } & 0.4318741 & -1.0187923 & -0.5714681\end{array}$

$\begin{array}{llll}\text { C } & 0.2786926 & -3.3962190 & 0.0680732\end{array}$

$\begin{array}{llll}\text { C } & -0.9251281 & -0.9237997 & -0.9077059\end{array}$

$\begin{array}{llll}\text { C } & 1.3393135 & -0.0453009 & -0.9973755\end{array}$

$\begin{array}{llll}\text { C } & -0.0952993 & -4.2088774 & 1.1360138\end{array}$

$\begin{array}{llll}\text { C } & 0.1993492 & -3.8488236 & -1.2479240\end{array}$

$\begin{array}{llll}\text { C } & -1.3713354 & 0.1530725 & -1.6668786\end{array}$

H $\quad-1.6204372 \quad-1.6872929 \quad-0.5746874$

C $\quad 0.8790534 \quad 1.0291541 \quad-1.7546709$

H $\quad 2.3911731 \quad-0.1122826 \quad-0.7483135$

$\begin{array}{llll}\text { C } & -0.5691832 & -5.4955281 & 0.8787327\end{array}$

H $\quad-0.0155690 \quad-3.8478449 \quad 2.1565567$

C $\quad-0.2763837 \quad-5.1363220 \quad-1.4946589$

$\mathrm{H} \quad 0.5013208 \quad-3.2046545 \quad-2.0673300$

C $\quad-0.4741983 \quad 1.1405204 \quad-2.0781220$

$\mathrm{H} \quad-2.4233643 \quad 0.2270846 \quad-1.9230013$

$\mathrm{H} \quad 1.5828435 \quad 1.7868566 \quad-2.0838018$

C $\quad-0.6629498 \quad-5.9590606 \quad-0.4346742$

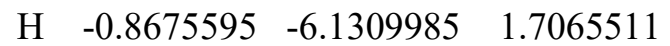

$\mathrm{H} \quad-0.3406111 \quad-5.4946998 \quad-2.5173552$

$\begin{array}{llll}\mathrm{H} & -0.8259035 & 1.9888941 & -2.6563995\end{array}$

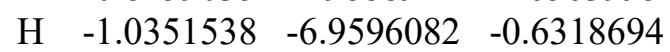

$\begin{array}{llll}\text { C } & -0.3367695 & -0.9301940 & 2.5562227\end{array}$

$\begin{array}{llll}\text { C } & -0.9439700 & 0.1636627 & 1.9355100\end{array}$

$\mathrm{H} \quad 0.5921003 \quad-0.7564884 \quad 3.1017704$

$\mathrm{H} \quad-0.9853932 \quad-1.6999440 \quad 2.9692023$

$\begin{array}{llll}\mathrm{H} & -1.9837375 & 0.0687739 & 1.6254153\end{array}$

$\begin{array}{llll}\text { C } & -0.2971536 & 1.3806342 & 1.6122124\end{array}$

C $\quad-0.9482465 \quad 2.3705151 \quad 0.9299258$

$\begin{array}{llll}\mathrm{H} & 0.7408457 & 1.5028058 & 1.9134682\end{array}$

$\begin{array}{llll}\mathrm{H} & -1.9887168 & 2.1910042 & 0.6615490\end{array}$

$\begin{array}{llll}\text { C } & -0.4096791 & 3.6496187 & 0.5219020\end{array}$

$\begin{array}{llll}\text { C } & -1.2552847 & 4.5490318 & -0.1598160\end{array}$

$\begin{array}{llll}\text { C } & 0.9298112 & 4.0273360 & 0.7546645\end{array}$

$\begin{array}{llll}\text { C } & -0.7822645 & 5.7821068 & -0.5958391\end{array}$

$\mathrm{H} \quad-2.2878998 \quad 4.2642764 \quad-0.3442355$

$\begin{array}{llll}\text { C } & 1.4000040 & 5.2582554 & 0.3172427\end{array}$

$\begin{array}{llll}\mathrm{H} & 1.6026433 & 3.3529300 & 1.2751542\end{array}$

$\begin{array}{lllll}\text { C } & 0.5474674 & 6.1397769 & -0.3594398\end{array}$

$\mathrm{H} \quad-1.4456161 \quad 6.4637057 \quad-1.1197652$

$\begin{array}{llll}\mathrm{H} & 2.4332686 & 5.5373411 & 0.5002132\end{array}$

$\mathrm{H} \quad 0.9211016 \quad 7.1008813 \quad-0.6999500$

$\begin{array}{llll}\text { C } & 2.2573858 & -2.1138288 & 0.7375871\end{array}$ 


\section{H $\quad 2.3686669 \quad-2.9019215 \quad 1.4809293$ \\ H $\quad 2.8686019 \quad-2.3420051 \quad-0.1383669$ \\ H $2.5503566 \quad-1.1560909 \quad 1.1694853$}

TS1 $\mathbf{w}^{+}: \mathrm{H}^{+}$from ammonium $\mathbf{2} \mathrm{aH}^{+}$to diene $\mathbf{1 w}$ 39

Energy $=-752.9558033030$

H $\quad 1.8117389-0.5104309$

$\mathrm{N} \quad 0.7993545 \quad-0.5869706 \quad 0.1538883$

$\begin{array}{llll}\mathrm{H} & 0.2428073 & 0.1782957 & 1.1629509\end{array}$

C $\quad 0.3834198 \quad 0.1541696 \quad-1.0133815$

$\begin{array}{llll}\text { C } & 0.3842638 & -1.9767762 & 0.3025446\end{array}$

$\begin{array}{llll}\text { C } & -0.9369959 & 0.0837430 & -1.4629450\end{array}$

$\begin{array}{llll}\text { C } & 1.2923076 & 1.0467328 & -1.5859576\end{array}$

$\begin{array}{llll}\text { C } & -0.8773851 & -2.2799581 & 0.8121593\end{array}$

$\begin{array}{llll}\text { C } & 1.2795371 & -2.9836487 & -0.0554819\end{array}$

C $\quad-1.3455338 \quad 0.9251696 \quad-2.4991639$

H $\quad-1.6308568 \quad-0.6291108 \quad-1.0325742$

$\begin{array}{llll}\text { C } & 0.8701971 & 1.8858691 & -2.6120729\end{array}$

$\mathrm{H} \quad 2.3201673 \quad 1.0773316 \quad-1.2349318$

C $\quad-1.2506462-3.6174863 \quad 0.9486780$

$\mathrm{H} \quad-1.5630095 \quad-1.4932870 \quad 1.1076388$

C $\quad 0.9002188 \quad-4.3168980 \quad 0.0925552$

H $\quad 2.2599289 \quad-2.7277311 \quad-0.4475296$

C $\quad-0.4518861 \quad 1.8325248 \quad-3.0653544$

$\mathrm{H} \quad-2.3687915 \quad 0.8696731 \quad-2.8563741$

$\mathrm{H} \quad 1.5753123 \quad 2.5766341 \quad-3.0632469$

C $\quad-0.3655206 \quad-4.6347614 \quad 0.5897524$

$\begin{array}{llll}\mathrm{H} & -2.2326130 & -3.8596699 & 1.3425394\end{array}$

H $\quad 1.5942155 \quad-5.1044289-0.1833207$

$\mathrm{H} \quad-0.7776764 \quad 2.4891229 \quad-3.8660051$

$\begin{array}{llll}\mathrm{H} & -0.6593609 & -5.6736148 & 0.7016034\end{array}$

C $\quad-0.3103950 \quad 1.0066648 \quad 2.0849205$

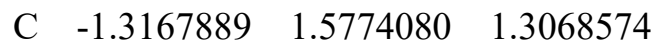

$\mathrm{H} \quad-0.6192423 \quad 0.2152896 \quad 2.7717330$

$\begin{array}{llll}\mathrm{H} & -2.2325546 & 1.0186516 & 1.1269057\end{array}$

C $\quad-1.1905712 \quad 2.8759221 \quad 0.7163835$

$\begin{array}{llll}\text { C } & -0.0243059 & 3.5600105 & 0.8192937\end{array}$

$\begin{array}{llll}\mathrm{H} & -2.0277979 & 3.2813913 & 0.1579428\end{array}$

$\begin{array}{llll}\mathrm{H} & 0.0800444 & 4.5267007 & 0.3328010\end{array}$

C $\quad 1.1750128 \quad 2.9793265 \quad 1.5006928$

$\mathrm{H} \quad 1.7849576 \quad 3.7731910 \quad 1.9419742$

$\begin{array}{llll}\mathrm{H} & 1.8043897 & 2.5319207 & 0.7122484\end{array}$

$\begin{array}{llll}\text { C } & 0.8066951 & 1.9281772 & 2.5623159\end{array}$

$\mathrm{H} \quad 1.6940640 \quad 1.3544573 \quad 2.8443911$

$\mathrm{H} \quad 0.4557005 \quad 2.4335936 \quad 3.4723879$

TS1 $^{+}: \mathrm{H}^{+}$from ammonium $2 \mathbf{a H}^{+}$to diene $\mathbf{1 a}$ 45

\begin{tabular}{|c|c|c|c|}
\hline \multicolumn{4}{|c|}{ Energy $=-906.6947198096$} \\
\hline $\mathrm{H}$ & 2.0509869 & -2.3114064 & -0.0408756 \\
\hline $\mathrm{N}$ & 1.0429356 & -2.2198046 & 0.0988461 \\
\hline $\mathrm{H}$ & 0.8964628 & -1.6722054 & 1.3301454 \\
\hline $\mathrm{C}$ & 0.5259916 & -1.1504889 & -0.7258686 \\
\hline $\mathrm{C}$ & 0.4077011 & -3.5303643 & 0.0598483 \\
\hline $\mathrm{C}$ & -0.8439177 & -1.0177133 & -0.9510806 \\
\hline $\mathrm{C}$ & 1.4273628 & -0.1696795 & -1.1526595 \\
\hline $\mathrm{C}$ & -0.7507688 & -3.7629591 & 0.7993747 \\
\hline $\mathrm{C}$ & 0.9911583 & -4.5384306 & -0.7055689 \\
\hline $\mathrm{C}$ & -1.3136965 & 0.1257916 & -1.5962798 \\
\hline $\mathrm{H}$ & -1.5343103 & -1.7902571 & -0.6328061 \\
\hline $\mathrm{C}$ & 0.9465388 & 0.9630831 & -1.7985552 \\
\hline $\mathrm{H}$ & 2.4908803 & -0.2914963 & -0.9653065 \\
\hline $\mathrm{C}$ & -1.3407710 & -5.0265240 & 0.7564678 \\
\hline $\mathrm{H}$ & -1.1881984 & -2.9777369 & 1.4061914 \\
\hline $\mathrm{C}$ & 0.3980202 & -5.8001023 & 3790 \\
\hline $\mathrm{H}$ & 1.8961906 & -4.3392402 & -1.2721899 \\
\hline $\mathrm{C}$ & -0.4268960 & 1.1220404 & -2.0048906 \\
\hline $\mathrm{H}$ & -2.3789092 & 0.2381163 & -1.7715520 \\
\hline $\mathrm{H}$ & 1.6409290 & 1.7293384 & -2.1274579 \\
\hline $\mathrm{C}$ & -0.7694402 & -6.0436929 & -0.0093670 \\
\hline $\mathrm{H}$ & -2.2440015 & -5.2128674 & 1.3284013 \\
\hline $\mathrm{H}$ & 0.8488697 & -6.5891916 & -1.3287144 \\
\hline $\mathrm{H}$ & -0.8020406 & 2.0161720 & -2.4925812 \\
\hline $\mathrm{H}$ & -1.2302258 & -7.0261258 & -0.0364953 \\
\hline $\mathrm{C}$ & 0.7258300 & -0.9771163 & 2.5085300 \\
\hline $\mathrm{C}$ & -0.1297725 & 0.0395687 & 2.0753945 \\
\hline $\mathrm{H}$ & 1.7618203 & -0.7148983 & 2.7249792 \\
\hline $\mathrm{H}$ & 0.3046383 & -1.7723626 & 3.1213827 \\
\hline $\mathrm{H}$ & -1.2014771 & -0.1605879 & 2.0584330 \\
\hline $\mathrm{C}$ & 0.2816004 & 1.3022249 & 1.5893999 \\
\hline $\mathrm{C}$ & -0.6228805 & 2.2059109 & 1.1028952 \\
\hline $\mathrm{H}$ & 1.3438212 & 1.5341611 & 1.6105819 \\
\hline $\mathrm{H}$ & -1.6727253 & 1.9140713 & 1.1060272 \\
\hline $\mathrm{C}$ & -0.3429493 & 3.5284237 & 0.5889118 \\
\hline $\mathrm{C}$ & -1.4229173 & 4.3223100 & 0.1499813 \\
\hline $\mathrm{C}$ & 0.9643900 & 4.0511881 & 0.4896895 \\
\hline $\mathrm{C}$ & -1.2074092 & 5.5929452 & -0.3727236 \\
\hline $\mathrm{H}$ & -2.4323104 & 3.9253165 & 0.2219114 \\
\hline $\mathrm{C}$ & 1.1766211 & 5.3195821 & -0.0330219 \\
\hline $\mathrm{H}$ & 1.8134463 & 3.4603175 & 0.8191594 \\
\hline $\mathrm{C}$ & 0.0932842 & 6.0950518 & -0.4658237 \\
\hline $\mathrm{H}$ & -2.0488075 & 6.1923463 & -0.7072000 \\
\hline $\mathrm{H}$ & 2.1867554 & 5.7115605 & -0.1058273 \\
\hline $\mathrm{H}$ & 0.2657754 & 7.0863230 & -0.8743193 \\
\hline
\end{tabular}

TS2 $\mathbf{a}^{+}$: addition of $\mathbf{1 a H}{ }^{+} \mathrm{C} 1$ to amine $\mathbf{2 a}$ para 45 
Energy $=-906.6973814946$

$\begin{array}{lrcc}\mathrm{N} & -2.8068634 & 1.1952793 & 0.0274894 \\ \mathrm{C} & -3.8434734 & 0.2359415 & -0.1002668 \\ \mathrm{C} & -1.5444700 & 1.0275558 & 0.4760574 \\ \mathrm{H} & -3.0436979 & 2.1331051 & -0.2836063 \\ \mathrm{C} & -4.6920079 & 0.3307815 & -1.2102472 \\ \mathrm{C} & -4.0623146 & -0.7424179 & 0.8773146 \\ \mathrm{C} & -0.7351464 & 2.1931080 & 0.6480973 \\ \mathrm{C} & -0.9820538 & -0.2544953 & 0.7536508 \\ \mathrm{C} & -5.7417872 & -0.5725203 & -1.3539500 \\ \mathrm{H} & -4.5199915 & 1.1023618 & -1.9553285 \\ \mathrm{C} & -5.1101806 & -1.6466382 & 0.7167188 \\ \mathrm{H} & -3.4465002 & -0.7733914 & 1.7694371 \\ \mathrm{C} & 0.5554665 & 2.0810095 & 1.0760057 \\ \mathrm{H} & -1.1748957 & 3.1671603 & 0.4527978 \\ \mathrm{C} & 0.3154067 & -0.3507444 & 1.1720848 \\ \mathrm{H} & -1.5787996 & -1.1472324 & 0.6153670 \\ \mathrm{C} & -5.9483710 & -1.5696292 & -0.3975312 \\ \mathrm{H} & -6.3956150 & -0.4992670 & -2.2175061 \\ \mathrm{H} & -5.2810342 & -2.4022342 & 1.4773571 \\ \mathrm{C} & 1.1665926 & 0.7989400 & 1.2868388 \\ \mathrm{H} & 1.1518328 & 2.9758478 & 1.2202403 \\ \mathrm{H} & 0.7342949 & -1.3311637 & 1.3743842 \\ \mathrm{H} & -6.7659052 & -2.2743690 & -0.5132204 \\ \mathrm{H} & 2.0034618 & 0.7406654 & 1.9771943 \\ \mathrm{C} & 3.2640846 & 2.8725579 & -0.8349825 \\ \mathrm{C} & 4.2692548 & 3.9718620 & -0.7730072 \\ \mathrm{H} & 2.3892343 & 3.0439570 & -1.4640821 \\ \mathrm{C} & 3.3701827 & 1.6944112 & -0.1900027 \\ \mathrm{H} & 4.6502774 & 4.2003229 & -1.7767582 \\ \mathrm{H} & 5.1105448 & 3.7177986 & -0.1229240 \\ \mathrm{H} & 3.8007450 & 4.8947428 & -0.4055130 \\ \mathrm{C} & 2.3912430 & 0.6286145 & -0.3429063 \\ \mathrm{H} & 4.2373357 & 1.5045389 & 0.4391414 \\ \mathrm{H} & 1.6200527 & 0.8254186 & -1.0854799 \\ \mathrm{C} & 2.8382018 & -0.7741384 & -0.3064579 \\ \mathrm{C} & 2.2177016 & -1.7054164 & -1.1580564 \\ \mathrm{C} & 3.8624757 & -1.2133921 & 0.5503246 \\ \mathrm{C} & 2.6208700 & -3.0373160 & -1.1659036 \\ \mathrm{H} & 1.4224771 & -1.3722475 & -1.8197077 \\ \mathrm{C} & 4.2559398 & -2.5485099 & 0.5497873 \\ \mathrm{H} & 4.3448522 & -0.5155012 & 1.2283583 \\ \mathrm{C} & 3.6394253 & -3.4632957 & -0.3089657 \\ \mathrm{H} & 2.1420136 & -3.7436386 & -1.8373945 \\ \mathrm{H} & 5.0451649 & -2.8775185 & 1.2190024 \\ & 3.9507787 & -4.5035884 & -0.3089181\end{array}$

TS2 $g^{+}$: addition of $\mathbf{1} \mathrm{aH}^{+} \mathrm{C} 3$ to $\mathbf{2 g} \mathrm{Ph}_{3} \mathrm{~N}$ para 55
Energy $=-1137.887562409$

N $\quad-2.5093437 \quad-0.1639171$

$\begin{array}{llll}\text { C } & -2.7114670 & -1.5869299 & 0.2406685\end{array}$

$\begin{array}{llll}\text { C } & -1.3705839 & 0.4001027 & 0.7280339\end{array}$

$\begin{array}{llll}\text { C } & -3.1162391 & -2.2237816 & -0.9353505\end{array}$

$\begin{array}{llll}\text { C } & -2.5433655 & -2.3081599 & 1.4261573\end{array}$

$\begin{array}{llll}\text { C } & -1.3414802 & 1.7797685 & 1.0906821\end{array}$

$\begin{array}{llll}\text { C } & -0.1856657 & -0.3829311 & 0.9035947\end{array}$

$\begin{array}{llll}\text { C } & -3.3375193 & -3.5991275 & -0.9243911\end{array}$

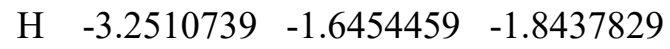

C $\quad-2.7628165 \quad-3.6844268 \quad 1.4247648$

$\begin{array}{llll}\mathrm{H} & -2.2563558 & -1.7919617 & 2.3370072\end{array}$

$\begin{array}{llll}\text { C } & -0.1923202 & 2.3430705 & 1.5729584\end{array}$

$\begin{array}{llll}\mathrm{H} & -2.2542097 & 2.3610547 & 1.0347204\end{array}$

$\begin{array}{llll}\text { C } & 0.9628475 & 0.2019244 & 1.3485594\end{array}$

H $\quad-0.2030152 \quad-1.4359811 \quad 0.6490456$

$\begin{array}{llll}\text { C } & -3.1591176 & -4.3315962 & 0.2521339\end{array}$

$\mathrm{H} \quad-3.6461475 \quad-4.0992872 \quad-1.8371500$

H $\quad-2.6375517 \quad-4.2471745 \quad 2.3445464$

$\begin{array}{llll}\text { C } & 1.0433320 & 1.6124021 & 1.6245724\end{array}$

$\mathrm{H} \quad-0.2132372 \quad 3.3714975 \quad 1.9177008$

$\begin{array}{llll}\mathrm{H} & 1.8651369 & -0.3956815 & 1.4339742\end{array}$

$\mathrm{H} \quad-3.3342253 \quad-5.4031927 \quad 0.2560736$

$\begin{array}{llll}\mathrm{H} & 1.7908003 & 1.9447697 & 2.3402726\end{array}$

$\begin{array}{llll}\text { C } & 2.0926000 & 2.2806518 & 0.0332990\end{array}$

$\begin{array}{llll}\text { C } & 2.0486592 & 3.7829563 & 0.1709530\end{array}$

$\begin{array}{llll}\mathrm{H} & 1.4338566 & 1.8693590 & -0.7305273\end{array}$

$\begin{array}{llll}\text { C } & 3.3903211 & 1.6508322 & 0.1232898\end{array}$

$\begin{array}{llll}\mathrm{H} & 2.6230471 & 4.2151112 & -0.6602901\end{array}$

$\mathrm{H} \quad 2.5150242 \quad 4.1178062 \quad 1.1026794$

$\begin{array}{llll}\mathrm{H} & 1.0307427 & 4.1727793 & 0.1154076\end{array}$

$\begin{array}{llll}\text { C } & 3.7067901 & 0.5260720 & -0.5671879\end{array}$

$\begin{array}{llll}\mathrm{H} & 4.1247410 & 2.1278547 & 0.7695875\end{array}$

$\mathrm{H} \quad 2.9320710 \quad 0.0896621 \quad-1.1989089$

$\begin{array}{llll}\text { C } & 4.9797675 & -0.1723642 & -0.5786749\end{array}$

$\begin{array}{llll}\text { C } & 5.1115416 & -1.3148088 & -1.3940951\end{array}$

$\begin{array}{llll}\text { C } & 6.0900672 & 0.2365855 & 0.1900722\end{array}$

$\begin{array}{llll}\text { C } & 6.3065597 & -2.0267875 & -1.4411617\end{array}$

$\mathrm{H} \quad 4.2632524 \quad-1.6353054 \quad-1.9939590$

$\begin{array}{llll}\text { C } & 7.2811762 & -0.4758717 & 0.1422362\end{array}$

$\begin{array}{llll}\mathrm{H} & 6.0180105 & 1.1136783 & 0.8262435\end{array}$

C $\quad 7.3957556 \quad-1.6090158-0.6733780$

$\mathrm{H} \quad 6.3901332 \quad-2.9043111 \quad-2.0752452$

H $\quad 8.1279333 \quad-0.1512502 \quad 0.7397902$

H $\quad 8.3304076 \quad-2.1608896 \quad-0.7071509$

$\begin{array}{llll}\text { C } & -3.5688467 & 0.6352315 & -0.3157420\end{array}$

$\begin{array}{llll}\text { C } & -4.8828794 & 0.4088977 & 0.1014406\end{array}$

$\begin{array}{llll}\text { C } & -3.2819112 & 1.5956063 & -1.2900183\end{array}$

$\begin{array}{llll}\text { C } & -5.9137956 & 1.1649038 & -0.4517346\end{array}$ 


$\begin{array}{lrrr}\mathrm{H} & -5.0888451 & -0.3473809 & 0.8519977 \\ \mathrm{C} & -4.3195593 & 2.3491006 & -1.8337355 \\ \mathrm{H} & -2.2588572 & 1.7368985 & -1.6239005 \\ \mathrm{C} & -5.6354008 & 2.1368414 & -1.4158896 \\ \mathrm{H} & -6.9352040 & 0.9967515 & -0.1248938 \\ \mathrm{H} & -4.1006488 & 3.0915064 & -2.5948292 \\ \mathrm{H} & -6.4422624 & 2.7224131 & -1.8451274\end{array}$

TS2 $\mathbf{h a}^{+}$: addition of $\mathbf{1} \mathbf{a H}^{+} \mathrm{C} 1$ to amine $\mathbf{2 h}$ para 48

Energy $=-946.0265943000$

\begin{tabular}{lrrc}
$\mathrm{N}$ & -2.7124473 & 1.1539728 & -0.0756293 \\
$\mathrm{C}$ & -3.6246175 & 0.0422190 & -0.1068220 \\
$\mathrm{C}$ & -1.4597505 & 1.0343145 & 0.4140632 \\
$\mathrm{C}$ & -4.1436919 & -0.3869408 & -1.3303590 \\
$\mathrm{C}$ & -4.0083902 & -0.5717798 & 1.0881928 \\
$\mathrm{C}$ & -0.6386923 & 2.1930351 & 0.5968884 \\
$\mathrm{C}$ & -0.9101446 & -0.2493201 & 0.7382528 \\
$\mathrm{C}$ & -5.0480385 & -1.4478688 & -1.3547367 \\
$\mathrm{H}$ & -3.8353976 & 0.0980041 & -2.2515960 \\
$\mathrm{C}$ & -4.9095825 & -1.6349966 & 1.0530433 \\
$\mathrm{H}$ & -3.6089923 & -0.2125108 & 2.0316226 \\
$\mathrm{C}$ & 0.6421220 & 2.0657324 & 1.0559389 \\
$\mathrm{H}$ & -1.0415567 & 3.1776478 & 0.3960621 \\
$\mathrm{C}$ & 0.3742339 & -0.3577560 & 1.1840639 \\
$\mathrm{H}$ & -1.5222733 & -1.1344023 & 0.6166699 \\
$\mathrm{C}$ & -5.4299195 & -2.0741349 & -0.1661033 \\
$\mathrm{H}$ & -5.4484434 & -1.7880930 & -2.3047129 \\
$\mathrm{H}$ & -5.2112400 & -2.1120410 & 1.9804767 \\
$\mathrm{C}$ & 1.2388066 & 0.7839731 & 1.2917903 \\
$\mathrm{H}$ & 1.2423727 & 2.9572905 & 1.2061646 \\
$\mathrm{H}$ & 0.7733591 & -1.3394702 & 1.4172656 \\
$\mathrm{H}$ & -6.1341364 & -2.9001196 & -0.1898663 \\
$\mathrm{H}$ & 2.0686253 & 0.7283075 & 1.9906176 \\
$\mathrm{C}$ & 3.4023697 & 2.8192966 & -0.8223815 \\
$\mathrm{C}$ & 4.4281321 & 3.8991314 & -0.7524615 \\
$\mathrm{H}$ & 2.5387527 & 3.0055803 & -1.4626067 \\
$\mathrm{C}$ & 3.4766776 & 1.6423592 & -0.1709863 \\
$\mathrm{H}$ & 4.8236902 & 4.1181520 & -1.7526898 \\
$\mathrm{H}$ & 5.2577560 & 3.6299477 & -0.0934880 \\
$\mathrm{H}$ & 3.9743473 & 4.8317543 & -0.3910999 \\
$\mathrm{C}$ & 2.4796590 & 0.5950468 & -0.3320142 \\
$\mathrm{H}$ & 4.3334751 & 1.4374684 & 0.4675066 \\
$\mathrm{H}$ & 1.7167841 & 0.8058182 & -1.0792794 \\
$\mathrm{C}$ & 2.8955955 & -0.8164224 & -0.2887018 \\
$\mathrm{C}$ & 2.2557529 & -1.7372799 & -1.1375838 \\
$\mathrm{C}$ & 3.9068001 & -1.2756924 & 0.5731268 \\
$\mathrm{C}$ & 2.6267725 & -3.0785158 & -1.1372179 \\
$\mathrm{H}$ & 1.4701776 & -1.3881831 & -1.8025433 \\
& & & \\
\hline
\end{tabular}

$\begin{array}{lrrr}\mathrm{C} & 4.2682756 & -2.6197064 & 0.5804268 \\ \mathrm{H} & 4.4036810 & -0.5861398 & 1.2491621 \\ \mathrm{C} & 3.6324219 & -3.5241860 & -0.2752409 \\ \mathrm{H} & 2.1330978 & -3.7767659 & -1.8064131 \\ \mathrm{H} & 5.0473239 & -2.9641325 & 1.2538711 \\ \mathrm{H} & 3.9188724 & -4.5716193 & -0.2687447 \\ \mathrm{C} & -3.2243884 & 2.4530822 & -0.5507854 \\ \mathrm{H} & -3.3789249 & 3.1324414 & 0.2935043 \\ \mathrm{H} & -2.5251757 & 2.9001123 & -1.2610253 \\ \mathrm{H} & -4.1790023 & 2.2763031 & -1.0421140\end{array}$

TS2 $\mathbf{h}^{+}$: addition of $\mathbf{1} \mathbf{a H} H^{+} \mathrm{C} 3$ to amine $\mathbf{2 h}$ para 48

Energy $=-946.0278922285$

$\begin{array}{llll}\mathrm{N} & -3.2801737 & 0.6203006 & -0.3356353\end{array}$

C $\quad-3.5914823 \quad-0.7639612 \quad-0.1232306$

$\begin{array}{llll}\text { C } & -2.1687042 & 1.1944717 & 0.1928189\end{array}$

C $\quad-3.7872626 \quad-1.6045448 \quad-1.2225783$

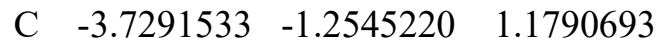

$\begin{array}{llll}\text { C } & -2.0009565 & 2.6097394 & 0.1687963\end{array}$

$\begin{array}{llll}\text { C } & -1.1251760 & 0.3972857 & 0.7626268\end{array}$

C $\quad-4.1132399 \quad-2.9438835 \quad-1.0135107$

$\mathrm{H} \quad-3.6741148 \quad-1.2153740 \quad-2.2299243$

C $\quad-4.0485609 \quad-2.5966455 \quad 1.3785612$

$\mathrm{H} \quad-3.5947237 \quad-0.5851252 \quad 2.0232226$

C $\quad-0.8576631 \quad 3.1826549 \quad 0.6724447$

H $\quad-2.7930832 \quad 3.2428454 \quad-0.2109594$

$\begin{array}{llll}\text { C } & 0.0133869 & 0.9848451 & 1.2403261\end{array}$

H $\quad-1.2397387 \quad-0.6794881 \quad 0.7961016$

$\begin{array}{llll}\text { C } & -4.2412595 & -3.4430699 & 0.2846187\end{array}$

$\mathrm{H} \quad-4.2590557 \quad-3.5986074 \quad-1.8672453$

$\mathrm{H} \quad-4.1581903 \quad-2.9761460 \quad 2.3899240$

$\begin{array}{llll}\text { C } & 0.2237147 & 2.3918908 & 1.1503392\end{array}$

$\mathrm{H} \quad-0.7775552 \quad 4.2645799 \quad 0.6949638$

$\begin{array}{llll}\mathrm{H} & 0.8017102 & 0.3599806 & 1.6482287\end{array}$

$\mathrm{H} \quad-4.4938713 \quad-4.4870454 \quad 0.4429566$

$\begin{array}{llll}\mathrm{H} & 0.9879702 & 2.8560777 & 1.7644519\end{array}$

$\begin{array}{llll}\text { C } & 1.5690426 & 2.4709747 & -0.5909350\end{array}$

$\begin{array}{llll}\text { C } & 1.7989469 & 3.9459329 & -0.7228490\end{array}$

$\begin{array}{llll}\mathrm{H} & 0.8037730 & 2.0355657 & -1.2298329\end{array}$

$\begin{array}{llll}\text { C } & 2.6651981 & 1.6271524 & -0.2518886\end{array}$

$\begin{array}{llll}\mathrm{H} & 2.4480878 & 4.1019057 & -1.5970094\end{array}$

$\begin{array}{llll}\mathrm{H} & 2.3227722 & 4.3562566 & 0.1455049\end{array}$

$\begin{array}{llll}\mathrm{H} & 0.8745794 & 4.4982715 & -0.8929316\end{array}$

$\begin{array}{lllll}\text { C } & 2.6975262 & 0.3084107 & -0.6053320\end{array}$

$\begin{array}{llll}\mathrm{H} & 3.4957287 & 2.0765724 & 0.2883846\end{array}$

$\begin{array}{llll}\mathrm{H} & 1.8353388 & -0.0843674 & -1.1453630\end{array}$

$\begin{array}{llll}\text { C } & 3.7574547 & -0.6411354 & -0.3557842\end{array}$

$\begin{array}{llll}\text { C } & 3.5999899 & -1.9628613 & -0.8259131\end{array}$ 


$\begin{array}{lrrc}\mathrm{C} & 4.9402209 & -0.3093082 & 0.3422156 \\ \mathrm{C} & 4.5834895 & -2.9207935 & -0.6055920 \\ \mathrm{H} & 2.6953513 & -2.2252836 & -1.3685422 \\ \mathrm{C} & 5.9198619 & -1.2678043 & 0.5597998 \\ \mathrm{H} & 5.0882964 & 0.7002885 & 0.7124119 \\ \mathrm{C} & 5.7463476 & -2.5757208 & 0.0879446 \\ \mathrm{H} & 4.4472556 & -3.9333853 & -0.9726985 \\ \mathrm{H} & 6.8246993 & -1.0021403 & 1.0980017 \\ \mathrm{H} & 6.5171975 & -3.3206527 & 0.2614256 \\ \mathrm{C} & -4.2589764 & 1.4264094 & -1.0844548 \\ \mathrm{H} & -4.8058149 & 2.0915235 & -0.4073576 \\ \mathrm{H} & -3.7552585 & 2.0185984 & -1.8521843 \\ \mathrm{H} & -4.9659333 & 0.7459494 & -1.5553805\end{array}$

TS2 $\mathbf{l a}^{+}$: addition of $\mathbf{1 a H}{ }^{+} \mathrm{C} 3$ to amine $\mathbf{2 l}$ para 45

Energy $=-831.6645133299$

$\begin{array}{lrrr}\mathrm{N} & 0.7580244 & 0.3553798 & 1.8173308 \\ \mathrm{C} & 0.7458907 & -0.7645826 & 1.0770976 \\ \mathrm{C} & 1.8773730 & -1.1229798 & 0.2693517 \\ \mathrm{C} & -0.3996937 & -1.6446409 & 1.1009013 \\ \mathrm{C} & 1.8434219 & -2.2363705 & -0.5230091 \\ \mathrm{H} & 2.7830597 & -0.5315422 & 0.3260543 \\ \mathrm{C} & -0.4006364 & -2.7464141 & 0.2923778 \\ \mathrm{C} & 0.6479213 & -3.0002025 & -0.6541788 \\ \mathrm{H} & 2.7145708 & -2.5036357 & -1.1127071 \\ \mathrm{H} & -1.2623265 & -3.4095423 & 0.3103116 \\ \mathrm{H} & 0.7070592 & -3.9846761 & -1.1072924 \\ \mathrm{C} & -0.1483856 & -2.1073632 & -2.3811158 \\ \mathrm{C} & -1.1414142 & -3.1391090 & -2.8419124 \\ \mathrm{H} & 0.7992262 & -2.1017213 & -2.9160033 \\ \mathrm{C} & -0.6094375 & -0.8348719 & -1.9233762 \\ \mathrm{H} & -1.4996892 & -2.8480446 & -3.8393912 \\ \mathrm{H} & -2.0086193 & -3.1914981 & -2.1777690 \\ \mathrm{H} & -0.6914605 & -4.1307970 & -2.9289606 \\ \mathrm{C} & 0.1997473 & 0.2593299 & -1.8563771 \\ \mathrm{H} & -1.6298911 & -0.7820382 & -1.5500525 \\ \mathrm{H} & 1.2046813 & 0.1725513 & -2.2671081 \\ \mathrm{C} & -0.1419211 & 1.5618754 & -1.3098008 \\ \mathrm{C} & 0.7782447 & 2.6187033 & -1.4522189 \\ \mathrm{C} & -1.3381664 & 1.8044687 & -0.6041364 \\ \mathrm{C} & 0.5174972 & 3.8725038 & -0.9071206 \\ \mathrm{H} & 1.7047374 & 2.4415006 & -1.9931398 \\ \mathrm{C} & -1.5970945 & 3.0560119 & -0.0585833 \\ \mathrm{H} & -2.0629299 & 1.0063710 & -0.4756309 \\ \mathrm{C} & -0.6694083 & 4.0942055 & -0.2040045 \\ \mathrm{H} & 1.2392285 & 4.6750977 & -1.0250163 \\ \mathrm{H} & -2.5215550 & 3.2276410 & 0.4853721 \\ \mathrm{H} & -0.8734413 & 5.0691410 & 0.2286634\end{array}$

$\begin{array}{lrrr}\mathrm{C} & 1.8494313 & 1.3318412 & 1.7478054 \\ \mathrm{H} & 2.6618957 & 1.0564766 & 2.4293256 \\ \mathrm{H} & 2.2292909 & 1.4087948 & 0.7303779 \\ \mathrm{H} & 1.4478019 & 2.3020123 & 2.0440284 \\ \mathrm{C} & -0.2796386 & 0.6632021 & 2.8237811 \\ \mathrm{H} & -0.9469783 & 1.4253908 & 2.4021279 \\ \mathrm{H} & 0.2373528 & 1.1105621 & 3.6775645 \\ \mathrm{C} & -1.0526218 & -0.5770278 & 3.2449482 \\ \mathrm{H} & -1.8814246 & -0.2729103 & 3.8894860 \\ \mathrm{H} & -0.4001019 & -1.2378451 & 3.8270677 \\ \mathrm{C} & -1.5588655 & -1.3090683 & 2.0019609 \\ \mathrm{H} & -2.2652815 & -0.6603124 & 1.4646979 \\ \mathrm{H} & -2.1006552 & -2.2223303 & 2.2646502\end{array}$

$\mathbf{T S 2 I}^{+} \mathbf{0}$ : addition of $\mathbf{1} \mathrm{aH}^{+} \mathrm{C} 1$ to amine $\mathbf{2 l}$ ortho 45

Energy $=-831.6525481960$

$\begin{array}{lrrr}\mathrm{N} & -2.1915167 & 0.9310888 & -0.5494825 \\ \mathrm{C} & -1.4510608 & 0.5873902 & 0.5145884 \\ \mathrm{C} & -0.0519461 & 0.9698747 & 0.6019770 \\ \mathrm{C} & -1.9877985 & -0.2653480 & 1.5376038 \\ \mathrm{C} & 0.5972211 & 0.8618165 & 1.8905596 \\ \mathrm{H} & 0.2810731 & 1.7895007 & -0.0247165 \\ \mathrm{C} & -1.2494452 & -0.4600880 & 2.6809198 \\ \mathrm{C} & 0.0288208 & 0.1360451 & 2.8910970 \\ \mathrm{H} & 1.5811577 & 1.3032438 & 2.0009894 \\ \mathrm{H} & -1.6748810 & -1.0775171 & 3.4681543 \\ \mathrm{H} & 0.5331951 & 0.0002449 & 3.8417251 \\ \mathrm{C} & -0.9042187 & -1.3597904 & -1.9222514 \\ \mathrm{C} & -1.5216712 & -1.5401475 & -3.2669485 \\ \mathrm{H} & -1.3737838 & -1.8896532 & -1.0924327 \\ \mathrm{C} & 0.2057431 & -0.6354986 & -1.6744541 \\ \mathrm{H} & -1.5140115 & -2.5999721 & -3.5521190 \\ \mathrm{H} & -0.9995731 & -0.9627356 & -4.0343434 \\ \mathrm{H} & -2.5783278 & -1.2383570 & -3.2525746 \\ \mathrm{C} & 0.8047513 & -0.5341500 & -0.3524039 \\ \mathrm{H} & 0.6952776 & -0.1143227 & -2.4949211 \\ \mathrm{H} & 0.4761124 & -1.3049087 & 0.3439591 \\ \mathrm{C} & 2.2546937 & -0.2428396 & -0.2632284 \\ \mathrm{C} & 3.0327755 & -0.9340157 & 0.6784406 \\ \mathrm{C} & 2.8729442 & 0.7083516 & -1.0899069 \\ \mathrm{C} & 4.4010423 & -0.6922642 & 0.7811912 \\ \mathrm{H} & 2.5581617 & -1.6671471 & 1.3248351 \\ \mathrm{C} & 4.2386800 & 0.9566067 & -0.9804001 \\ \mathrm{H} & 2.2864228 & 1.2663595 & -1.8149254 \\ \mathrm{C} & 5.0070445 & 0.2554355 & -0.0465359 \\ \mathrm{H} & 4.9935336 & -1.2410676 & 1.5071544 \\ \mathrm{H} & 4.7052101 & 1.6969453 & -1.6232051 \\ \mathrm{H} & 6.0723814 & 0.4491849 & 0.0359642\end{array}$




$\begin{array}{cccc}\mathrm{C} & -1.7295626 & 1.8835456 & -1.5682055 \\ \mathrm{H} & -1.9265980 & 2.9083156 & -1.2339360 \\ \mathrm{H} & -0.6702418 & 1.7592753 & -1.7776224 \\ \mathrm{H} & -2.2880095 & 1.6925575 & -2.4851326 \\ \mathrm{C} & -3.6281391 & 0.5789237 & -0.6718257 \\ \mathrm{H} & -3.7135483 & -0.2317592 & -1.4048850 \\ \mathrm{H} & -4.1254915 & 1.4567814 & -1.0917730 \\ \mathrm{C} & -4.2510084 & 0.1843215 & 0.6575789 \\ \mathrm{H} & -5.2504665 & -0.2155337 & 0.4672163 \\ \mathrm{H} & -4.3579300 & 1.0703945 & 1.2927611 \\ \mathrm{C} & -3.3629878 & -0.8476786 & 1.3554528 \\ \mathrm{H} & -3.3061958 & -1.7552298 & 0.7380127 \\ \mathrm{H} & -3.7759597 & -1.1419195 & 2.3236293\end{array}$

TS2I ${ }^{+}$: addition of $\mathbf{1 a H}{ }^{+} \mathrm{C} 1$ to amine $\mathbf{2 l}$ para 45

\begin{tabular}{lrcc}
\multicolumn{4}{c}{ Energy $=-831.6598342943$} \\
$\mathrm{~N}$ & -2.7077412 & -0.2898564 & 0.3850293 \\
$\mathrm{C}$ & -1.4800376 & -0.7127983 & 0.7065945 \\
$\mathrm{C}$ & -0.9093121 & -0.3827596 & 1.9842982 \\
$\mathrm{C}$ & -0.7139501 & -1.5223418 & -0.2180419 \\
$\mathrm{C}$ & 0.3899723 & -0.6826632 & 2.2556823 \\
$\mathrm{H}$ & -1.5261115 & 0.0912975 & 2.7373522 \\
$\mathrm{C}$ & 0.5848708 & -1.8031007 & 0.0886444 \\
$\mathrm{C}$ & 1.2409894 & -1.2412160 & 1.2408528 \\
$\mathrm{H}$ & 0.8139832 & -0.4289918 & 3.2224621 \\
$\mathrm{H}$ & 1.1749252 & -2.3929061 & -0.6084425 \\
$\mathrm{H}$ & 2.1651193 & -1.7078900 & 1.5660807 \\
$\mathrm{C}$ & 4.4495911 & -0.2729428 & 0.0618468 \\
$\mathrm{C}$ & 5.5764597 & -0.7688921 & -0.7831858 \\
$\mathrm{H}$ & 4.6914951 & -0.0075977 & 1.0923447 \\
$\mathrm{C}$ & 3.1809231 & -0.1252907 & -0.3584496 \\
$\mathrm{H}$ & 6.3777954 & -0.0207075 & -0.8363470 \\
$\mathrm{H}$ & 5.2472229 & -1.0056795 & -1.7988176 \\
$\mathrm{H}$ & 6.0221031 & -1.6679956 & -0.3372991 \\
$\mathrm{C}$ & 2.1197139 & 0.3927585 & 0.5050598 \\
$\mathrm{H}$ & 2.9251119 & -0.3907308 & -1.3822467 \\
$\mathrm{H}$ & 2.4867896 & 0.7625975 & 1.4613193 \\
$\mathrm{C}$ & 1.0334702 & 1.2089828 & -0.0545488 \\
$\mathrm{C}$ & 0.4033192 & 2.1668314 & 0.7613797 \\
$\mathrm{C}$ & 0.5616197 & 1.0382289 & -1.3692868 \\
$\mathrm{C}$ & -0.6497393 & 2.9391238 & 0.2776564 \\
$\mathrm{H}$ & 0.7546424 & 2.3073824 & 1.7798390 \\
$\mathrm{C}$ & -0.4962967 & 1.8039241 & -1.8508937 \\
$\mathrm{H}$ & 1.0226654 & 0.3029255 & -2.0206342 \\
$\mathrm{C}$ & -1.1093136 & 2.7520048 & -1.0276893 \\
$\mathrm{H}$ & -1.1141995 & 3.6819856 & 0.9192261 \\
$\mathrm{H}$ & -0.8441420 & 1.6610107 & -2.8696623 \\
$\mathrm{H}$ & -1.9357161 & 3.3472731 & -1.4049240
\end{tabular}

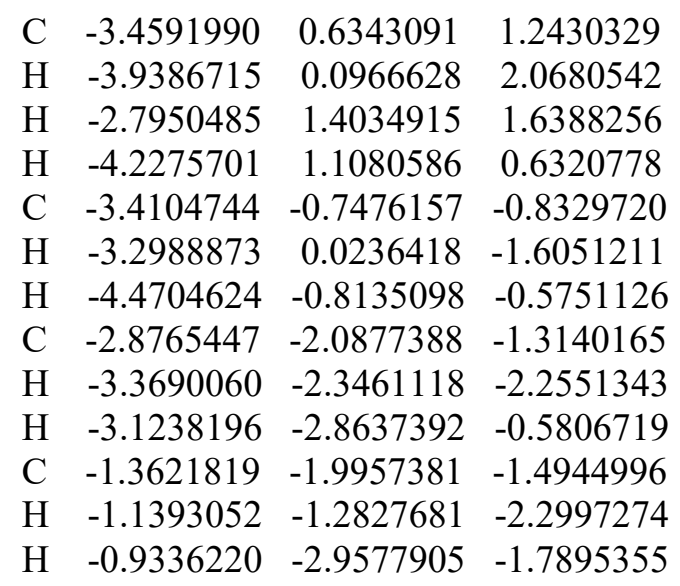

TS2 $^{+} \mathbf{m}$ : addition of $1 \mathrm{aH}^{+} \mathrm{C} 3$ to amine $2 \mathbf{a}$ meta 45

Energy $=-906.6751465456$

$\begin{array}{lrrc}\mathrm{N} & 3.4622968 & 0.9067919 & -0.0841772 \\ \mathrm{C} & 4.6700935 & 0.1947435 & 0.0772021 \\ \mathrm{C} & 2.2162711 & 0.4013054 & -0.3818634 \\ \mathrm{H} & 3.5016241 & 1.8966040 & 0.1262811 \\ \mathrm{C} & 5.5925927 & 0.6462567 & 1.0311911 \\ \mathrm{C} & 4.9914726 & -0.9055444 & -0.7302124 \\ \mathrm{C} & 1.1844535 & 1.2652126 & -0.7458985 \\ \mathrm{C} & 1.9153632 & -0.9839616 & -0.3133632 \\ \mathrm{C} & 6.8186681 & 0.0003882 & 1.1772897 \\ \mathrm{H} & 5.3453294 & 1.5039410 & 1.6511043 \\ \mathrm{C} & 6.2118937 & -1.5574135 & -0.5608604 \\ \mathrm{H} & 4.3088867 & -1.2325393 & -1.5084547 \\ \mathrm{C} & -0.1568230 & 0.7928617 & -0.9824415 \\ \mathrm{H} & 1.3828984 & 2.3291651 & -0.8285322 \\ \mathrm{C} & 0.6586308 & -1.4895779 & -0.6705416 \\ \mathrm{H} & 2.6779964 & -1.6731849 & 0.0325734 \\ \mathrm{C} & 7.1304475 & -1.1119635 & 0.3922472 \\ \mathrm{H} & 7.5265509 & 0.3625187 & 1.9170883 \\ \mathrm{H} & 6.4503305 & -2.4081234 & -1.1925538 \\ \mathrm{C} & -0.3600066 & -0.6384032 & -1.0307268 \\ \mathrm{H} & -0.6781459 & 1.3590151 & -1.7589346 \\ \mathrm{H} & 0.4956952 & -2.5612546 & -0.6312329 \\ \mathrm{H} & 8.0819987 & -1.6200123 & 0.5144377 \\ \mathrm{H} & -1.3509615 & -1.0150066 & -1.2589862 \\ \mathrm{C} & -1.1758762 & 1.3253780 & 0.3776002 \\ \mathrm{C} & -0.8790700 & 2.8020832 & 0.6119897 \\ \mathrm{H} & -0.8286862 & 0.6980230 & 1.1991579 \\ \mathrm{C} & -2.5788210 & 1.0642836 & 0.0245901 \\ \mathrm{H} & -1.5788257 & 3.1670214 & 1.3715348 \\ \mathrm{H} & -1.0287604 & 3.3937768 & -0.2961697 \\ \mathrm{H} & 0.1373026 & 2.9552061 & 0.9797000 \\ \mathrm{C} & -3.3055380 & 0.0837570 & 0.6031323\end{array}$




$\begin{array}{rrrr}\text { H } & -3.0270140 & 1.7396090 & -0.7027828 \\ \mathrm{H} & -2.7975407 & -0.5769677 & 1.3074801 \\ \mathrm{C} & -4.7155135 & -0.2105764 & 0.3889962 \\ \mathrm{C} & -5.2721763 & -1.3319334 & 1.0350253 \\ \mathrm{C} & -5.5475837 & 0.5711025 & -0.4386502 \\ \mathrm{C} & -6.6101413 & -1.6713916 & 0.8522788 \\ \mathrm{H} & -4.6408327 & -1.9361786 & 1.6817505 \\ \mathrm{C} & -6.8821518 & 0.2308016 & -0.6198743 \\ \mathrm{H} & -5.1478099 & 1.4473915 & -0.9400193 \\ \mathrm{C} & -7.4192556 & -0.8912376 & 0.0236220 \\ \mathrm{H} & -7.0220309 & -2.5410331 & 1.3555164 \\ \mathrm{H} & -7.5118880 & 0.8412069 & -1.2604393 \\ \mathrm{H} & -8.4639701 & -1.1508531 & -0.1196372\end{array}$

TS2na ${ }^{+}$: addition of $\mathbf{1} \mathrm{nH}^{+} \mathrm{C} 3$ to amine $2 \mathrm{a}$ para 51

Energy $=-910.3186513868$

$\begin{array}{lrrr}\mathrm{C} & 0.9426936 & 3.6714744 & 0.9426548 \\ \mathrm{C} & 1.1877718 & 2.2319046 & 0.6258614 \\ \mathrm{H} & 1.4850653 & 3.8959123 & 1.8739828 \\ \mathrm{H} & 1.3476214 & 4.3340968 & 0.1720901 \\ \mathrm{H} & 0.5485666 & 1.4995984 & 1.1133218 \\ \mathrm{C} & 2.5095051 & 1.8035894 & 0.2948569 \\ \mathrm{C} & 2.9308827 & 0.5306141 & 0.5046841 \\ \mathrm{H} & 3.2034288 & 2.5451173 & -0.0988302 \\ \mathrm{H} & 2.2122800 & -0.1938890 & 0.8941169 \\ \mathrm{C} & 4.2985407 & 0.0205514 & 0.2409369 \\ \mathrm{C} & 4.9146736 & -0.6065751 & 1.5155063 \\ \mathrm{C} & 4.2458373 & -1.0397034 & -0.8940203 \\ \mathrm{H} & 4.9374243 & 0.8493949 & -0.0913568 \\ \mathrm{C} & 6.3043956 & -1.1889812 & 1.2305807 \\ \mathrm{H} & 4.2466436 & -1.4016151 & 1.8750709 \\ \mathrm{H} & 4.9702625 & 0.1521893 & 2.3040266 \\ \mathrm{C} & 5.6396924 & -1.6186354 & -1.1690723 \\ \mathrm{H} & 3.5619502 & -1.8454834 & -0.5936059 \\ \mathrm{H} & 3.8351764 & -0.5826635 & -1.8013465 \\ \mathrm{C} & 6.2568090 & -2.2224929 & 0.0982622 \\ \mathrm{H} & 6.7041014 & -1.6422369 & 2.1451935 \\ \mathrm{H} & 6.9868247 & -0.3740843 & 0.9518019 \\ \mathrm{H} & 5.5672031 & -2.3758354 & -1.9586451 \\ \mathrm{H} & 6.2919774 & -0.8200043 & -1.5482717 \\ \mathrm{H} & 7.2644790 & -2.5974586 & -0.1147233 \\ \mathrm{H} & 5.6541991 & -3.0840140 & 0.4192721 \\ \mathrm{H} & -0.1110924 & 3.8929419 & 1.1102871 \\ \mathrm{~N} & -2.7173745 & -1.0641220 & -0.4033792 \\ \mathrm{H} & -2.3152549 & -1.9930409 & -0.4779622 \\ \mathrm{C} & -4.0622736 & -1.0246223 & 0.0337549 \\ \mathrm{C} & -4.9796874 & -0.0975023 & -0.4780522 \\ \mathrm{C} & -4.4811712 & -1.9903086 & 0.9591491\end{array}$

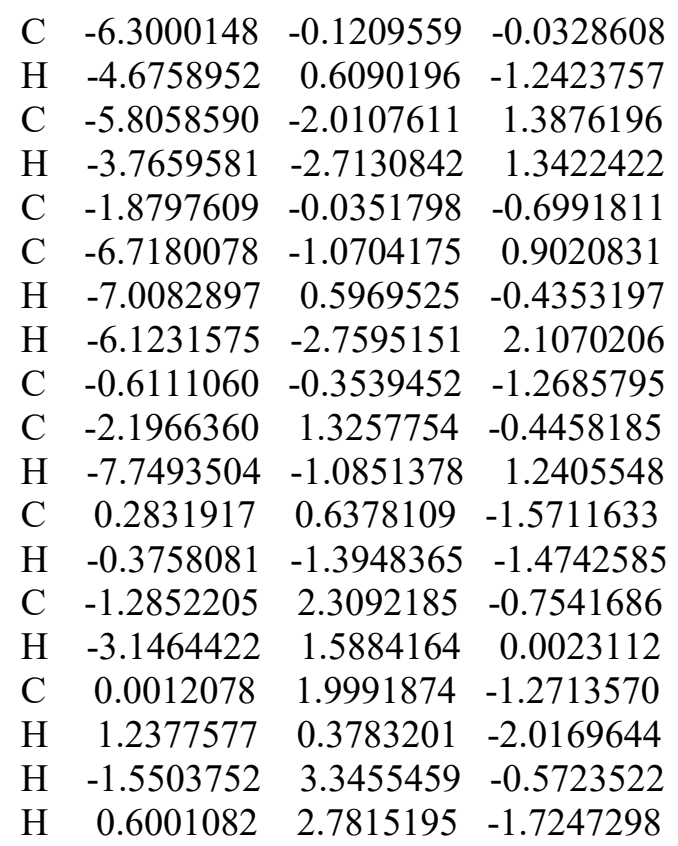

TS2 $\mathbf{n}^{+}$: addition of $\mathbf{1} \mathbf{n} \mathrm{H}^{+} \mathrm{C} 1$ to amine $\mathbf{2 a}$ para 51

\begin{tabular}{lrrr}
\multicolumn{4}{l}{ Energy $=-910.3275346240$} \\
$\mathrm{C}$ & -0.5875094 & 2.2614823 & 2.0346140 \\
$\mathrm{C}$ & 0.4541449 & 1.1981379 & 1.9521899 \\
$\mathrm{H}$ & -0.3180696 & 3.0081345 & 2.7923045 \\
$\mathrm{H}$ & -0.7322466 & 2.7638492 & 1.0745618 \\
$\mathrm{H}$ & 0.6598653 & 0.6409374 & 2.8656874 \\
$\mathrm{C}$ & 1.1679426 & 0.9068101 & 0.8436075 \\
$\mathrm{C}$ & 2.1596508 & -0.1417693 & 0.7786474 \\
$\mathrm{H}$ & 0.9665792 & 1.4548550 & -0.0762086 \\
$\mathrm{H}$ & 2.4952789 & -0.5225129 & 1.7433040 \\
$\mathrm{C}$ & 3.2613709 & 0.0023513 & -0.2491557 \\
$\mathrm{C}$ & 4.0983550 & 1.2407190 & 0.1895023 \\
$\mathrm{C}$ & 4.1855841 & -1.2162559 & -0.3961901 \\
$\mathrm{H}$ & 2.8116894 & 0.2387918 & -1.2234376 \\
$\mathrm{C}$ & 5.2538516 & 1.5104511 & -0.7829177 \\
$\mathrm{H}$ & 4.4981712 & 1.0545335 & 1.1958152 \\
$\mathrm{H}$ & 3.4438178 & 2.1158585 & 0.2589229 \\
$\mathrm{C}$ & 5.3523640 & -0.9383081 & -1.3544109 \\
$\mathrm{H}$ & 4.5770379 & -1.4856153 & 0.5950760 \\
$\mathrm{H}$ & 3.6260050 & -2.0812156 & -0.7679054 \\
$\mathrm{C}$ & 6.1640447 & 0.2865056 & -0.9201369 \\
$\mathrm{H}$ & 5.8232483 & 2.3782716 & -0.4298785 \\
$\mathrm{H}$ & 4.8426479 & 1.7730349 & -1.7673939 \\
$\mathrm{H}$ & 5.9941356 & -1.8247638 & -1.4116058 \\
$\mathrm{H}$ & 4.9526542 & -0.7679622 & -2.3640547 \\
$\mathrm{H}$ & 6.9644134 & 0.4862603 & -1.6419497 \\
$\mathrm{H}$ & 6.6452666 & 0.0813702 & 0.0465013 \\
& & &
\end{tabular}




$\begin{array}{crrr}\mathrm{H} & -1.5455806 & 1.8328580 & 2.3583290 \\ \mathrm{~N} & -2.7855809 & -0.4385961 & 0.8459987 \\ \mathrm{H} & -3.0948592 & -0.4537817 & 1.8131893 \\ \mathrm{C} & -3.6993394 & 0.1339663 & -0.0824276 \\ \mathrm{C} & -3.9697088 & -0.4842882 & -1.3078670 \\ \mathrm{C} & -4.3686525 & 1.3068401 & 0.2828628 \\ \mathrm{C} & -4.8875340 & 0.0978457 & -2.1805759 \\ \mathrm{H} & -3.4917131 & -1.4243672 & -1.5621347 \\ \mathrm{C} & -5.2939811 & 1.8726253 & -0.5914546 \\ \mathrm{H} & -4.1584275 & 1.7721015 & 1.2415635 \\ \mathrm{C} & -1.5557203 & -0.9411442 & 0.6091015 \\ \mathrm{C} & -5.5486201 & 1.2762968 & -1.8286244 \\ \mathrm{H} & -5.0977393 & -0.3836513 & -3.1306669 \\ \mathrm{H} & -5.8104817 & 2.7842085 & -0.3071396 \\ \mathrm{C} & -0.8707913 & -1.5768836 & 1.6929582 \\ \mathrm{C} & -0.9241752 & -0.8679837 & -0.6693423 \\ \mathrm{H} & -6.2665867 & 1.7218136 & -2.5101606 \\ \mathrm{C} & 0.3952023 & -2.0555632 & 1.5210306 \\ \mathrm{H} & -1.3913748 & -1.6961890 & 2.6390209 \\ \mathrm{C} & 0.3487576 & -1.3393914 & -0.8211648 \\ \mathrm{H} & -1.4468734 & -0.4202596 & -1.5049949 \\ \mathrm{C} & 1.1026727 & -1.8287668 & 0.2982131 \\ \mathrm{H} & 0.9022934 & -2.5534677 & 2.3416027 \\ \mathrm{H} & 0.8316088 & -1.2686145 & -1.7909876 \\ \mathrm{H} & 1.9753170 & -2.4416440 & 0.1024517\end{array}$

$\mathbf{T S 2}^{+} \mathbf{p}$ : addition of $\mathbf{1 w H ^ { + }}$ to amine $2 \mathbf{a}$ para 39

\begin{tabular}{lrcc}
\multicolumn{4}{l}{ Energy $=-752.9721195171$} \\
$\mathrm{~N}$ & 1.0137024 & -1.6765718 & -0.3446186 \\
$\mathrm{H}$ & 1.7601059 & -1.5925856 & -1.0281301 \\
$\mathrm{C}$ & 0.7432725 & -0.5628904 & 0.3713353 \\
$\mathrm{C}$ & -0.3927103 & -0.4456586 & 1.2294399 \\
$\mathrm{C}$ & 1.6294455 & 0.5477423 & 0.2352291 \\
$\mathrm{C}$ & -0.6051073 & 0.7180697 & 1.9168174 \\
$\mathrm{H}$ & -1.0791936 & -1.2770767 & 1.3335701 \\
$\mathrm{C}$ & 1.4003238 & 1.7068026 & 0.9214180 \\
$\mathrm{H}$ & 2.5011266 & 0.4448799 & -0.4055134 \\
$\mathrm{C}$ & 0.3757087 & -2.9434649 & -0.2980730 \\
$\mathrm{C}$ & 0.2299845 & 1.8678105 & 1.7298264 \\
$\mathrm{H}$ & -1.4654848 & 0.7962671 & 2.5756326 \\
$\mathrm{H}$ & 2.0919654 & 2.5382799 & 0.8238169 \\
$\mathrm{C}$ & 0.1770481 & -3.6245491 & -1.5050324 \\
$\mathrm{C}$ & 0.0123084 & -3.5362653 & 0.9168309 \\
$\mathrm{H}$ & 0.2379345 & 2.6256243 & 2.5065746 \\
$\mathrm{C}$ & -0.4052536 & -4.8897313 & -1.4954140 \\
$\mathrm{H}$ & 0.4733091 & -3.1601024 & -2.4412153 \\
$\mathrm{C}$ & -0.5812914 & -4.7972244 & 0.9125635 \\
$\mathrm{H}$ & 0.2231890 & -3.0345366 & 1.8548861
\end{tabular}

$\begin{array}{lrrr}\mathrm{C} & -0.7961221 & -5.4754037 & -0.2891394 \\ \mathrm{H} & -0.5600808 & -5.4147634 & -2.4328160 \\ \mathrm{H} & -0.8585911 & -5.2569662 & 1.8560311 \\ \mathrm{H} & -1.2513705 & -6.4607621 & -0.2844420 \\ \mathrm{C} & -1.0087073 & 3.0284969 & 0.5273207 \\ \mathrm{C} & -0.4709638 & 4.3705644 & 0.6257536 \\ \mathrm{C} & -0.0624044 & 5.0490338 & -0.4695041 \\ \mathrm{C} & -0.0622989 & 4.4665053 & -1.8478837 \\ \mathrm{C} & -0.0651724 & 2.9352114 & -1.8211382 \\ \mathrm{C} & -1.0976114 & 2.3890392 & -0.8286044 \\ \mathrm{H} & 0.2205893 & 6.0944795 & -0.3615496 \\ \mathrm{H} & -0.4791893 & 4.8567293 & 1.5976813 \\ \mathrm{H} & -1.8428486 & 2.8235962 & 1.1931296 \\ \mathrm{H} & 0.8046150 & 4.8408458 & -2.4043541 \\ \mathrm{H} & -0.2635893 & 2.5331903 & -2.8196777 \\ \mathrm{H} & 0.9306679 & 2.5899928 & -1.5283458 \\ \mathrm{H} & -2.1189544 & 2.5784758 & -1.1926437 \\ \mathrm{H} & -1.0196987 & 1.2979207 & -0.7415489 \\ \mathrm{H} & -0.9428860 & 4.8533094 & -2.3831001\end{array}$

TS2 $\mathbf{w}^{+}$: addition of $1 \mathbf{w H}^{+}$to amine $2 \mathrm{a}$ ortho 39

Energy $=-752.9719281582$

$\begin{array}{lrrr}\mathrm{N} & 0.5529356 & 0.3790700 & -1.1883063 \\ \mathrm{H} & 0.5392738 & -0.0082042 & -2.1270273 \\ \mathrm{C} & -0.6087840 & 0.9291472 & -0.7614495 \\ \mathrm{C} & -0.8496874 & 1.2508494 & 0.6225744 \\ \mathrm{C} & -1.6661977 & 1.1106422 & -1.6863771 \\ \mathrm{C} & -2.0230549 & 2.0003027 & 0.9582840 \\ \mathrm{H} & -0.0040565 & 1.3135061 & 1.2949104 \\ \mathrm{C} & -2.8336432 & 1.7253206 & -1.2923742 \\ \mathrm{H} & -1.5242511 & 0.8052539 & -2.7190868 \\ \mathrm{C} & 1.8358386 & 0.4330777 & -0.5756203 \\ \mathrm{C} & -3.0140839 & 2.2018397 & 0.0348871 \\ \mathrm{H} & -2.1309406 & 2.3518065 & 1.9794825 \\ \mathrm{H} & -3.6222468 & 1.8763288 & -2.0232962 \\ \mathrm{C} & 2.7070156 & -0.6448803 & -0.7650294 \\ \mathrm{C} & 2.2550645 & 1.5577655 & 0.1427314 \\ \mathrm{H} & -3.9244670 & 2.7284204 & 0.2995022 \\ \mathrm{C} & 3.9842533 & -0.6084732 & -0.2125708 \\ \mathrm{H} & 2.3748739 & -1.5100178 & -1.3314975 \\ \mathrm{C} & 3.5298849 & 1.5769586 & 0.7070467 \\ \mathrm{H} & 1.6061916 & 2.4218578 & 0.2373965 \\ \mathrm{C} & 4.3966867 & 0.4962248 & 0.5371449 \\ \mathrm{H} & 4.6547798 & -1.4494896 & -0.3607861 \\ \mathrm{H} & 3.8498237 & 2.4517099 & 1.2649806 \\ \mathrm{H} & 5.3894382 & 0.5186379 & 0.9755542 \\ \mathrm{C} & -0.0865348 & -2.5345092 & 0.5263011 \\ \mathrm{C} & -0.1495739 & -1.3876242 & 1.2436649\end{array}$




$\begin{array}{lrrr}\mathrm{C} & -1.3780443 & -0.6439703 & 1.3639615 \\ \mathrm{C} & -2.6076823 & -1.1705580 & 0.6868098 \\ \mathrm{C} & -2.3061370 & -2.0441942 & -0.5359538 \\ \mathrm{C} & -1.2420725 & -3.1021402 & -0.2304856 \\ \mathrm{H} & -1.5440786 & -0.1729487 & 2.3287680 \\ \mathrm{H} & 0.7151261 & -1.0500575 & 1.8074998 \\ \mathrm{H} & 0.8302514 & -3.1197822 & 0.5587507 \\ \mathrm{H} & -3.2861802 & -0.3487309 & 0.4288929 \\ \mathrm{H} & -3.2276084 & -2.5184410 & -0.8850866 \\ \mathrm{H} & -1.9488045 & -1.4086186 & -1.3517090 \\ \mathrm{H} & -1.6631514 & -3.9242094 & 0.3709272 \\ \mathrm{H} & -0.8846787 & -3.5726374 & -1.1537352 \\ \mathrm{H} & -3.1413487 & -1.7559011 & 1.4529165\end{array}$

TS2 $^{+}$: addition of $1 \mathbf{a H}^{+} \mathrm{C} 3$ to amine 2 a para 45

Energy $=-906.7023018157$

$\begin{array}{lrrc}\mathrm{N} & -3.5026222 & 0.9082647 & -0.2396499 \\ \mathrm{C} & -4.0777565 & -0.3815177 & -0.1227744 \\ \mathrm{C} & -2.2857114 & 1.3400879 & 0.1670893 \\ \mathrm{H} & -4.0745615 & 1.5948529 & -0.7221635 \\ \mathrm{C} & -4.8650489 & -0.8477792 & -1.1834574 \\ \mathrm{C} & -3.9253022 & -1.1539804 & 1.0354102 \\ \mathrm{C} & -2.0036205 & 2.7359939 & 0.0609360 \\ \mathrm{C} & -1.2787253 & 0.4647834 & 0.6688820 \\ \mathrm{C} & -5.4754663 & -2.0961623 & -1.0940682 \\ \mathrm{H} & -4.9874833 & -0.2352108 & -2.0722957 \\ \mathrm{C} & -4.5321793 & -2.4064130 & 1.1083576 \\ \mathrm{H} & -3.3707822 & -0.7667744 & 1.8830907 \\ \mathrm{C} & -0.7859291 & 3.2266746 & 0.4397940 \\ \mathrm{H} & -2.7825156 & 3.4006082 & -0.3026281 \\ \mathrm{C} & -0.0597413 & 0.9701831 & 1.0389052 \\ \mathrm{H} & -1.4732351 & -0.5978050 & 0.7418666 \\ \mathrm{C} & -5.3031347 & -2.8842681 & 0.0465652 \\ \mathrm{H} & -6.0817185 & -2.4549788 & -1.9201697 \\ \mathrm{H} & -4.4149770 & -3.0015373 & 2.0088709 \\ \mathrm{C} & 0.2623462 & 2.3521173 & 0.8694202 \\ \mathrm{H} & -0.5934582 & 4.2935167 & 0.3790797 \\ \mathrm{H} & 0.7004504 & 0.2967075 & 1.4233169 \\ \mathrm{H} & -5.7769365 & -3.8587132 & 0.1126332 \\ \mathrm{H} & 1.0793797 & 2.7650815 & 1.4494099 \\ \mathrm{C} & 1.4870120 & 2.2761434 & -0.8442123 \\ \mathrm{C} & 0.6146119 & 1.7318232 & -1.9317916 \\ \mathrm{H} & 2.1649705 & 1.5620840 & -0.3806094 \\ \mathrm{C} & 1.9970039 & 3.6132891 & -0.9645131 \\ \mathrm{H} & 1.2476345 & 1.5617243 & -2.8135895 \\ \mathrm{H} & -0.1684242 & 2.4388428 & -2.2181837 \\ \mathrm{H} & 0.1644310 & 0.7752199 & -1.6589704 \\ \mathrm{C} & 3.1559098 & 4.0065513 & -0.3693704\end{array}$

$\begin{array}{lrrr}\mathrm{H} & 1.4180694 & 4.3113717 & -1.5660973 \\ \mathrm{H} & 3.6907281 & 3.2691025 & 0.2304041 \\ \mathrm{C} & 3.7831052 & 5.3108656 & -0.4506705 \\ \mathrm{C} & 5.0020227 & 5.5139227 & 0.2293674 \\ \mathrm{C} & 3.2300619 & 6.3874801 & -1.1774886 \\ \mathrm{C} & 5.6454248 & 6.7471326 & 0.1896464 \\ \mathrm{H} & 5.4364303 & 4.6899441 & 0.7897549 \\ \mathrm{C} & 3.8749233 & 7.6164942 & -1.2161247 \\ \mathrm{H} & 2.2947696 & 6.2589613 & -1.7135402 \\ \mathrm{C} & 5.0839453 & 7.8021465 & -0.5333916 \\ \mathrm{H} & 6.5825832 & 6.8870868 & 0.7199013 \\ \mathrm{H} & 3.4391448 & 8.4366927 & -1.7788490 \\ \mathrm{H} & 5.5834923 & 8.7657819 & -0.5675251\end{array}$

TS3a : $\mathrm{H}^{+}$from adduct $\mathbf{3 a H} \mathrm{H}^{+}$to $\mathrm{HOBf}_{3}^{-}$ 81

\begin{tabular}{lrrr}
\multicolumn{4}{l}{ Energy $=-3192.297704247$} \\
$\mathrm{~N}$ & -3.5252109 & 2.7776842 & 1.4821982 \\
$\mathrm{H}$ & -3.4043397 & 3.1384516 & 2.4246016 \\
$\mathrm{C}$ & -3.0977257 & 1.5253403 & 1.2673004 \\
$\mathrm{C}$ & -4.0704805 & 3.6899627 & 0.5327839 \\
$\mathrm{C}$ & -3.0389240 & 0.9508737 & -0.0435508 \\
$\mathrm{C}$ & -2.6517035 & 0.7601437 & 2.3921931 \\
$\mathrm{C}$ & -5.1002389 & 3.2976399 & -0.3278982 \\
$\mathrm{C}$ & -3.5819795 & 4.9993500 & 0.5112853 \\
$\mathrm{C}$ & -2.5208454 & -0.2918008 & -0.2063092 \\
$\mathrm{H}$ & -3.3737548 & 1.5315021 & -0.8944530 \\
$\mathrm{C}$ & -2.1419158 & -0.4845278 & 2.2162759 \\
$\mathrm{H}$ & -2.7297317 & 1.1994033 & 3.3824953 \\
$\mathrm{C}$ & -5.6166909 & 4.2164886 & -1.2394217 \\
$\mathrm{H}$ & -5.5100516 & 2.2949310 & -0.2616709 \\
$\mathrm{C}$ & -4.1139742 & 5.9132300 & -0.3960252 \\
$\mathrm{H}$ & -2.7867345 & 5.2914734 & 1.1908323 \\
$\mathrm{C}$ & -1.9572609 & -1.0797550 & 0.8920542 \\
$\mathrm{H}$ & -2.4519971 & -0.7138496 & -1.2063165 \\
$\mathrm{H}$ & -1.8081787 & -1.0466516 & 3.0832019 \\
$\mathrm{C}$ & -5.1230295 & 5.5220838 & -1.2789897 \\
$\mathrm{H}$ & -6.4159864 & 3.9133681 & -1.9082133 \\
$\mathrm{H}$ & -3.7315409 & 6.9289686 & -0.4179002 \\
$\mathrm{C}$ & -2.2218309 & -2.6227124 & 0.7724757 \\
$\mathrm{H}$ & -5.5311704 & 6.2353015 & -1.9882979 \\
$\mathrm{C}$ & -1.4477746 & -3.4147759 & 1.8389772 \\
$\mathrm{H}$ & -1.8502319 & -2.9145760 & -0.2178197 \\
$\mathrm{C}$ & -3.6967725 & -2.8987020 & 0.8251950 \\
$\mathrm{H}$ & -0.3932720 & -3.1214932 & 1.8493922 \\
$\mathrm{H}$ & -1.8674348 & -3.2567682 & 2.8378745 \\
$\mathrm{H}$ & -1.5150301 & -4.4838805 & 1.6174821 \\
$\mathrm{C}$ & -4.3904510 & -3.4480546 & -0.1835398 \\
$\mathrm{H}$ & -4.1945148 & -2.6269701 & 1.7571313
\end{tabular}




\begin{tabular}{|c|c|c|c|}
\hline $\mathrm{H}$ & -3.8460039 & -3.7242023 & -1.0884978 \\
\hline c & -5.8238049 & -3.7559522 & -0.2076636 \\
\hline $\mathrm{C}$ & -6.3392089 & -4.4939869 & -1.2879880 \\
\hline $\mathrm{C}$ & -6.7155477 & -3.3495235 & 0.8027928 \\
\hline $\mathrm{C}$ & -7.6912685 & -4.8284492 & -1.3536616 \\
\hline $\mathrm{H}$ & -5.6639533 & -4.8114890 & -2.0794260 \\
\hline$c$ & -8.0649585 & -3.6814998 & 0.7375610 \\
\hline $\mathrm{H}$ & -6.3516556 & -2.7642549 & 1.6424657 \\
\hline & -8.5608048 & -4.4248754 & -0.3393859 \\
\hline 1 & -8.0647948 & -5.4034129 & -2.1964166 \\
\hline 1 & -8.7370532 & -3.3571015 & 1.5274595 \\
\hline $\mathrm{H}$ & -9.6152028 & -4.6819021 & -0.3864118 \\
\hline$H$ & -0.8083819 & -1.0269507 & 0.6946759 \\
\hline $\mathrm{H}$ & 0.6688031 & -2.0122181 & -0.4126513 \\
\hline 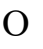 & 0.7740547 & -1.3542204 & 0.2909572 \\
\hline B & 1.9301565 & -0.4532292 & -0.0433509 \\
\hline$c$ & 3.2401012 & -1.4249361 & -0.3157777 \\
\hline $\mathrm{C}$ & 3.4913908 & -2.5118026 & 0.5310826 \\
\hline $\mathrm{C}$ & 4.1310085 & -1.3236034 & -1.3853007 \\
\hline $\mathrm{C}$ & 4.5083944 & -3.4383357 & 0.3293795 \\
\hline $\mathrm{C}$ & 5.1650274 & -2.2270831 & -1.6273188 \\
\hline $\mathrm{C}$ & 5.3541870 & -3.2989715 & -0.7659333 \\
\hline $\mathrm{C}$ & 1.5571169 & 0.4548462 & -1.3850243 \\
\hline $\mathrm{C}$ & 1.9892136 & 1.7659758 & -1.5839450 \\
\hline $\mathrm{C}$ & 0.7175856 & -0.0192368 & -2.3903457 \\
\hline $\mathrm{C}$ & 1.5918367 & 2.5659147 & -2.6505355 \\
\hline $\mathrm{C}$ & 0.2838917 & 0.7380009 & -3.4751410 \\
\hline C & 0.7217024 & 2.0503408 & -3.6050653 \\
\hline $\mathrm{C}$ & 2.0682532 & 0.5339484 & 1.2728180 \\
\hline $\mathrm{C}$ & 3.1307138 & 0.6007861 & 2.1723985 \\
\hline $\mathrm{C}$ & 1.0092128 & 1.3907450 & 1.5816721 \\
\hline $\mathrm{C}$ & 3.1393876 & 1.4219600 & 3.3002704 \\
\hline $\mathrm{C}$ & 0.9686890 & 2.2260168 & 2.6917744 \\
\hline $\mathrm{C}$ & 2.0478900 & 2.2389083 & 3.5683255 \\
\hline $\mathrm{F}$ & 2.7472166 & -2.6951013 & 1.6500752 \\
\hline $\mathrm{F}$ & 4.6950342 & -4.4631897 & 1.1896615 \\
\hline $\mathrm{F}$ & 6.3441206 & -4.1856257 & -0.9825372 \\
\hline $\mathrm{F}$ & 5.9838203 & -2.0712343 & -2.6904578 \\
\hline $\mathrm{F}$ & 4.0464154 & -0.2995989 & -2.2756199 \\
\hline $\mathrm{F}$ & 0.2630808 & -1.3114217 & -2.3683947 \\
\hline $\mathrm{F}$ & -0.5495534 & 0.2132156 & -4.4001060 \\
\hline $\mathrm{F}$ & 0.3209946 & 2.8057043 & -4.6450767 \\
\hline F & 2.0438268 & 3.8327198 & -2.7759066 \\
\hline $\mathrm{F}$ & 2.8691728 & 2.3349789 & -0.7169763 \\
\hline $\mathrm{F}$ & -0.0620767 & 1.4669032 & 0.7486901 \\
\hline $\mathrm{F}$ & -0.0954252 & 3.0287077 & 2.9215898 \\
\hline $\mathrm{F}$ & 2.0391929 & 3.0360748 & 4.6533397 \\
\hline $\mathrm{F}$ & 4.1994483 & 1.4322413 & 4.1370460 \\
\hline & 4.2550846 & -0.1371631 & 1.989592 \\
\hline
\end{tabular}

TS3a $^{+}: \mathrm{H}^{+}$from adduct $\mathbf{3 a H} \mathrm{H}^{+}$to neutral diene $\mathbf{1 a}$ 65 Energy $=-1294.003657597$

$\begin{array}{llll}\mathrm{N} & 1.6983981 & 0.9734194 & 0.6585108\end{array}$

$\mathrm{H} \quad 2.2180132 \quad 0.6906235 \quad 1.4847339$

$\begin{array}{llll}\text { C } & 0.5582871 & 0.2742751 & 0.4480124\end{array}$

$\begin{array}{llll}\text { C } & 2.2609345 & 2.0893374 & -0.0034721\end{array}$

$\begin{array}{llll}\text { C } & -0.2373389 & 0.3708022 & -0.7318656\end{array}$

$\begin{array}{llll}\text { C } & 0.1763646 & -0.6555062 & 1.4588573\end{array}$

$\begin{array}{llll}\text { C } & 1.5147785 & 2.9972324 & -0.7666103\end{array}$

$\begin{array}{llll}\text { C } & 3.6330817 & 2.3029240 & 0.1916829\end{array}$

C $\quad-1.3125548 \quad-0.4591662 \quad-0.8943760$

$\mathrm{H} \quad 0.0168326 \quad 1.0784515 \quad-1.5086017$

C $\quad-0.8891903 \quad-1.4898124 \quad 1.2781777$

$\begin{array}{llll}\mathrm{H} & 0.7430918 & -0.6809367 & 2.3854670\end{array}$

C $\quad 2.1599589 \quad 4.0748853 \quad-1.3715704$

H $\quad 0.4402980 \quad 2.9038029 \quad-0.8550298$

C $\quad 4.2637414 \quad 3.3833894 \quad-0.4154206$

$\mathrm{H} \quad 4.1992642 \quad 1.6161190 \quad 0.8138716$

C $\quad-1.5861494 \quad-1.5408431 \quad 0.0157280$

$\begin{array}{llll}\mathrm{H} & -1.9030954 & -0.3937529 & -1.8036928\end{array}$

H $\quad-1.1521419 \quad-2.1964462 \quad 2.0582052$

C $\quad 3.5332019 \quad 4.2673955-1.2129852$

$\mathrm{H} \quad 1.5750882 \quad 4.7767344 \quad-1.9586014$

H $\quad 5.3281791 \quad 3.5331037 \quad-0.2645220$

C $\quad-2.9449332 \quad-2.2527140 \quad-0.1086503$

$\begin{array}{llll}\mathrm{H} & 4.0254137 & 5.1093492 & -1.6896452\end{array}$

C $\quad-2.9948722 \quad-3.5833594 \quad 0.6604490$

$\begin{array}{llll}\text { H } & -3.0965092 & -2.4587941 & -1.1754429\end{array}$

C $\quad-4.0311805 \quad-1.3134996 \quad 0.3462355$

$\mathrm{H} \quad-2.1681774 \quad-4.2412341 \quad 0.3726283$

$\mathrm{H} \quad-2.9489222 \quad-3.4236516 \quad 1.7425392$

$\begin{array}{llll}\mathrm{H} & -3.9348239 & -4.0962357 & 0.4409497\end{array}$

C $\quad-4.9970238-0.8404795 \quad-0.4563556$

$\mathrm{H} \quad-3.9909584 \quad-1.0268459 \quad 1.3975941$

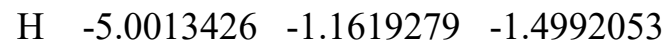

C $\quad-6.0852048 \quad 0.0703603 \quad-0.0874553$

$\begin{array}{llll}\text { C } & -7.0436730 & 0.4082572 & -1.0600272\end{array}$

$\begin{array}{llll}\text { C } & -6.2214739 & 0.6268607 & 1.1986020\end{array}$

$\begin{array}{llll}\text { C } & -8.1017289 & 1.2667686 & -0.7631363\end{array}$

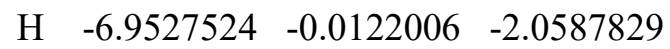

$\begin{array}{llll}\text { C } & -7.2766936 & 1.4831551 & 1.4957858\end{array}$

$\begin{array}{llll}\mathrm{H} & -5.4961732 & 0.3895227 & 1.9720233\end{array}$

$\begin{array}{llll}\text { C } & -8.2232872 & 1.8083926 & 0.5171940\end{array}$

$\mathrm{H} \quad-8.8303428 \quad 1.5124559 \quad-1.5310362$

$\begin{array}{llll}\mathrm{H} & -7.3631011 & 1.9014368 & 2.4947175\end{array}$

$\mathrm{H} \quad-9.0455316 \quad 2.4776589 \quad 0.7529221$

$\mathrm{H} \quad-0.6945425 \quad-2.3894702 \quad-0.5731265$ 


$\begin{array}{rrrr}\mathrm{C} & 0.2802477 & -3.3135224 & -1.1686764 \\ \mathrm{C} & 1.3510936 & -2.4680880 & -1.4329700 \\ \mathrm{H} & -0.3692616 & -3.6056892 & -1.9916038 \\ \mathrm{H} & 0.3570605 & -4.0191782 & -0.3435294 \\ \mathrm{H} & 1.3349241 & -1.8899673 & -2.3562395 \\ \mathrm{C} & 2.4661858 & -2.2651776 & -0.5792236 \\ \mathrm{C} & 3.4590750 & -1.3860793 & -0.9056934 \\ \mathrm{H} & 2.5099555 & -2.8411161 & 0.3430148 \\ \mathrm{H} & 3.3659592 & -0.8573940 & -1.8544350 \\ \mathrm{C} & 4.6474395 & -1.0768266 & -0.1400365 \\ \mathrm{C} & 5.6212269 & -0.2369400 & -0.7188275 \\ \mathrm{C} & 4.8611012 & -1.5478726 & 1.1733326 \\ \mathrm{C} & 6.7679284 & 0.1186534 & -0.0153834 \\ \mathrm{H} & 5.4625163 & 0.1357454 & -1.7269454 \\ \mathrm{C} & 6.0023661 & -1.1840673 & 1.8759016 \\ \mathrm{H} & 4.1258304 & -2.1921176 & 1.6455295 \\ \mathrm{C} & 6.9604673 & -0.3500848 & 1.2856660 \\ \mathrm{H} & 7.5081331 & 0.7646288 & -0.4771587 \\ \mathrm{H} & 6.1509877 & -1.5475990 & 2.8880553 \\ \mathrm{H} & 7.8505903 & -0.0689234 & 1.8402504\end{array}$

$\mathbf{T S 3}^{+}$: deprotonation of $\mathrm{C} 1$ adduct with $\mathbf{1 a}$ 69

\begin{tabular}{lrrr}
\multicolumn{4}{l}{ Energy $=-1425.686147266$} \\
$\mathrm{~N}$ & 1.6864135 & 2.4480378 & 1.7198574 \\
$\mathrm{H}$ & 2.1716659 & 2.2721670 & 2.5932576 \\
$\mathrm{C}$ & 0.8883338 & 1.4441740 & 1.2745862 \\
$\mathrm{C}$ & 2.0541057 & 3.6392324 & 1.0458901 \\
$\mathrm{C}$ & 0.3076878 & 1.4323207 & -0.0208441 \\
$\mathrm{C}$ & 0.6630685 & 0.3326178 & 2.1349282 \\
$\mathrm{C}$ & 1.1107770 & 4.4277187 & 0.3772333 \\
$\mathrm{C}$ & 3.3952911 & 4.0387800 & 1.0944766 \\
$\mathrm{C}$ & -0.3935824 & 0.3352438 & -0.4460550 \\
$\mathrm{H}$ & 0.4631764 & 2.2669520 & -0.6918937 \\
$\mathrm{C}$ & -0.0503348 & -0.7537122 & 1.7030791 \\
$\mathrm{H}$ & 1.0676581 & 0.3595456 & 3.1431092 \\
$\mathrm{C}$ & 1.5221133 & 5.5911647 & -0.2715051 \\
$\mathrm{H}$ & 0.0616429 & 4.1525522 & 0.3892331 \\
$\mathrm{C}$ & 3.7931269 & 5.2107333 & 0.4557276 \\
$\mathrm{H}$ & 4.1190453 & 3.4254494 & 1.6239504 \\
$\mathrm{C}$ & -0.5250853 & -0.8613797 & 0.3470654 \\
$\mathrm{H}$ & -0.8200538 & 0.3370266 & -1.4460221 \\
$\mathrm{H}$ & -0.2068638 & -1.5889951 & 2.3783567 \\
$\mathrm{C}$ & 2.8611609 & 5.9849668 & -0.2392986 \\
$\mathrm{H}$ & 0.7864018 & 6.1996174 & -0.7887858 \\
$\mathrm{H}$ & 4.8350109 & 5.5141198 & 0.4949499 \\
$\mathrm{C}$ & -1.2445387 & -4.2927286 & 0.1664416 \\
$\mathrm{H}$ & 3.1742950 & 6.8944740 & -0.7426558 \\
$\mathrm{C}$ & -1.1031285 & -5.5999399 & 0.8836025
\end{tabular}

$\begin{array}{cccc}\mathrm{H} & -1.0520932 & -4.3072502 & -0.9084333 \\ \mathrm{C} & -1.5752174 & -3.1346161 & 0.7429953 \\ \mathrm{H} & -0.0806663 & -5.9837773 & 0.7807136 \\ \mathrm{H} & -1.3284483 & -5.4960048 & 1.9491157 \\ \mathrm{H} & -1.7723332 & -6.3571306 & 0.4558692 \\ \mathrm{C} & -1.6871899 & -1.8242959 & 0.0016604 \\ \mathrm{H} & -1.7907982 & -3.1084822 & 1.8114820 \\ \mathrm{H} & -1.6137719 & -2.0404235 & -1.0722375 \\ \mathrm{C} & -3.0472814 & -1.1764323 & 0.2393772 \\ \mathrm{C} & -4.0718455 & -1.3672992 & -0.6946138 \\ \mathrm{C} & -3.3132150 & -0.4230234 & 1.3879232 \\ \mathrm{C} & -5.3375110 & -0.8174532 & -0.4870013 \\ \mathrm{H} & -3.8759160 & -1.9519778 & -1.5906741 \\ \mathrm{C} & -4.5783739 & 0.1252336 & 1.6011923 \\ \mathrm{H} & -2.5277263 & -0.2526826 & 2.1200917 \\ \mathrm{C} & -5.5950780 & -0.0701034 & 0.6640672 \\ \mathrm{H} & -6.1203172 & -0.9708519 & -1.2247065 \\ \mathrm{H} & -4.7685925 & 0.7088773 & 2.4976469 \\ \mathrm{H} & -6.5787932 & 0.3605978 & 0.8275607 \\ \mathrm{H} & 0.4958573 & -1.5547626 & -0.1778926 \\ \mathrm{H} & 1.1693306 & -2.5319768 & -1.7144821 \\ \mathrm{~N} & 1.5906421 & -2.1812373 & -0.8521296 \\ \mathrm{C} & 2.4328345 & -1.0441377 & -1.1736972 \\ \mathrm{C} & 3.4115516 & -0.5804965 & -0.2955653 \\ \mathrm{C} & 2.1375292 & -0.3446520 & -2.3458106 \\ \mathrm{C} & 4.0662466 & 0.6157809 & -0.5823212 \\ \mathrm{H} & 3.6569576 & -1.1326454 & 0.6035296 \\ \mathrm{C} & 2.8017582 & 0.8481783 & -2.6246256 \\ \mathrm{H} & 1.3821606 & -0.7245349 & -3.0286071 \\ \mathrm{C} & 3.7552301 & 1.3419988 & -1.7340905 \\ \mathrm{H} & 4.8227510 & 0.9821269 & 0.1046370 \\ \mathrm{H} & 2.5649017 & 1.3918664 & -3.5337574 \\ \mathrm{H} & 4.2614773 & 2.2796655 & -1.9396444 \\ \mathrm{C} & 2.1183401 & -3.3002815 & -0.0955127 \\ \mathrm{C} & 2.3451366 & -4.5061903 & -0.7587673 \\ \mathrm{C} & 2.3392098 & -3.1880608 & 1.2769903 \\ \mathrm{C} & 2.8041168 & -5.6101482 & -0.0418578 \\ \mathrm{H} & 2.1639542 & -4.5804644 & -1.8277030 \\ \mathrm{C} & 2.8031725 & -4.2962641 & 1.9854250 \\ \mathrm{H} & 2.1423376 & -2.2547027 & 1.7913636 \\ \mathrm{H} & 3.0367810 & -5.5063720 & 1.3308027 \\ \mathrm{H} & 2.9801230 & -6.5485180 & -0.5584484 \\ & 3.3936969 & -6.3664276 & 1.8887182\end{array}$

TS3ha ${ }^{+}: \mathrm{H}^{+}$from adduct $\mathbf{3 h \mathbf { h a } ^ { + }}$ to amine $\mathbf{2 h}$ 75

Energy $=-1504.345507374$

$\mathrm{N} \quad-4.2122348 \quad 0.0434525 \quad 0.9954226$ 


\begin{tabular}{|c|c|c|c|}
\hline C & -4.8802262 & 0.9620830 & 0.1157784 \\
\hline$C$ & -2.8746715 & 0.1037904 & 1.2133428 \\
\hline C & -5.8840565 & 1.7928227 & 0.6185158 \\
\hline $\mathrm{C}$ & -4.5504109 & 0.9934647 & -1.2419964 \\
\hline $\mathrm{C}$ & -2.2147898 & -0.9042546 & 1.9742691 \\
\hline $\mathrm{C}$ & -2.0866346 & 1.1807505 & 0.7020533 \\
\hline $\mathrm{C}$ & -6.5546342 & 2.6608795 & -0.2434237 \\
\hline $\mathrm{H}$ & -6.1309660 & 1.7636296 & 1.6754697 \\
\hline C & -5.2175511 & 1.8702381 & -2.0958436 \\
\hline $\mathrm{H}$ & -3.7794063 & 0.3317271 & -1.6245809 \\
\hline $\mathrm{C}$ & -0.8592626 & -0.8536565 & 2.1775594 \\
\hline $\mathrm{H}$ & -2.7830195 & -1.7242912 & 2.3951278 \\
\hline $\mathrm{C}$ & -0.7347329 & 1.2086665 & 0.9095867 \\
\hline $\mathrm{H}$ & -2.5735664 & 1.9938684 & 0.1765688 \\
\hline $\mathrm{C}$ & -6.2211535 & 2.7045258 & -1.5985855 \\
\hline $\mathrm{H}$ & -7.3327768 & 3.3088661 & 0.1483270 \\
\hline $\mathrm{H}$ & -4.9611844 & 1.8922566 & -3.1506546 \\
\hline $\mathrm{C}$ & -0.0187683 & 0.1579752 & 1.5918967 \\
\hline $\mathrm{H}$ & -0.3980634 & -1.6367795 & 2.7710371 \\
\hline $\mathrm{H}$ & -0.1637052 & 2.0624735 & 0.5527526 \\
\hline $\mathrm{H}$ & -6.7433656 & 3.3834864 & -2.2656434 \\
\hline $\mathrm{H}$ & 0.4799180 & -0.5690406 & 0.5038868 \\
\hline $\mathrm{C}$ & 1.2512166 & 0.6526843 & 2.3731944 \\
\hline $\mathrm{C}$ & 1.7774177 & -0.3575758 & 3.4017908 \\
\hline $\mathrm{H}$ & 0.9102068 & 1.5386837 & 2.9227050 \\
\hline $\mathrm{C}$ & 2.3433135 & 1.0809699 & 1.4358983 \\
\hline $\mathrm{H}$ & 2.6421858 & 0.0725933 & 3.9154527 \\
\hline $\mathrm{H}$ & 2.0993322 & -1.2873436 & 2.9235889 \\
\hline $\mathrm{H}$ & 1.0196556 & -0.5926705 & 4.1548816 \\
\hline $\mathrm{C}$ & 2.5789460 & 2.3496947 & 1.0643824 \\
\hline $\mathrm{H}$ & 2.9608607 & 0.2776225 & 1.0357628 \\
\hline $\mathrm{H}$ & 1.9460668 & 3.1396762 & 1.4718815 \\
\hline $\mathrm{C}$ & 3.6013793 & 2.7950260 & 0.1117436 \\
\hline $\mathrm{C}$ & 3.5456084 & 4.1093823 & -0.3845014 \\
\hline $\mathrm{C}$ & 4.6338268 & 1.9550193 & -0.3472236 \\
\hline $\mathrm{C}$ & 4.4730672 & 4.5646575 & -1.3208920 \\
\hline $\mathrm{H}$ & 2.7602153 & 4.7741676 & -0.0325051 \\
\hline $\mathrm{C}$ & 5.5590780 & 2.4084440 & -1.2820170 \\
\hline $\mathrm{H}$ & 4.7223456 & 0.9457626 & 0.0439997 \\
\hline $\mathrm{C}$ & 5.4819204 & 3.7146657 & -1.7770923 \\
\hline $\mathrm{H}$ & 4.4089020 & 5.5833600 & -1.6928347 \\
\hline $\mathrm{H}$ & 6.3511160 & 1.7462499 & -1.6208931 \\
\hline $\mathrm{H}$ & 6.2076290 & 4.0667201 & -2.5043894 \\
\hline $\mathrm{C}$ & -5.0333577 & -0.9949987 & 1.6412908 \\
\hline $\mathrm{H}$ & -4.7378241 & -1.9887585 & 1.2876674 \\
\hline $\mathrm{H}$ & -4.9292463 & -0.9501286 & 2.7288050 \\
\hline $\mathrm{H}$ & -6.0721929 & -0.8189286 & 1.3688164 \\
\hline 18 & 0.9934459 & -1.2016891 & -0.6579026 \\
\hline C & -0.0246380 & -2.1515983 & -1.1282612 \\
\hline
\end{tabular}

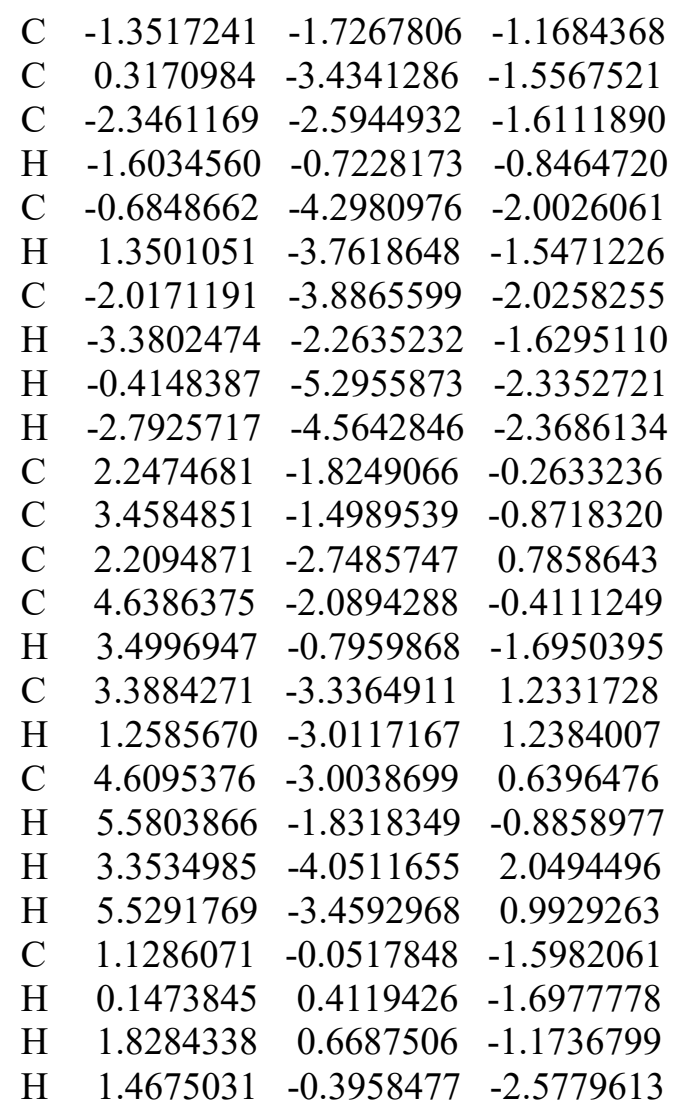

$\mathbf{T S 3 h}^{+}: \mathrm{H}^{+}$from adduct $\mathbf{3 h} \mathrm{H}^{+}$to $\mathbf{2 h}$ 75

Energy $=-1504.339718223$

$\begin{array}{lrrr}\mathrm{N} & -3.6442244 & 1.2084465 & 0.2537462 \\ \mathrm{C} & -4.7321038 & 0.4352946 & -0.2752644 \\ \mathrm{C} & -2.3750121 & 1.0618439 & -0.2012977 \\ \mathrm{C} & -5.0441341 & 0.5079133 & -1.6356274 \\ \mathrm{C} & -5.4901235 & -0.3650825 & 0.5833474 \\ \mathrm{C} & -1.3500170 & 1.9643728 & 0.2076690 \\ \mathrm{C} & -2.0114111 & -0.0181665 & -1.0638330 \\ \mathrm{C} & -6.1088117 & -0.2385653 & -2.1390231 \\ \mathrm{H} & -4.4591868 & 1.1494134 & -2.2877661 \\ \mathrm{C} & -6.5592487 & -1.1013677 & 0.0741976 \\ \mathrm{H} & -5.2433584 & -0.4126405 & 1.6397825 \\ \mathrm{C} & -0.0573145 & 1.7827971 & -0.2075932 \\ \mathrm{H} & -1.5981280 & 2.8240572 & 0.8182716 \\ \mathrm{C} & -0.7081785 & -0.2061342 & -1.4353481 \\ \mathrm{H} & -2.7746262 & -0.7087001 & -1.4026093 \\ \mathrm{C} & -6.8677658 & -1.0434293 & -1.2866214 \\ \mathrm{H} & -6.3511826 & -0.1810686 & -3.1958607 \\ \mathrm{H} & -7.1455563 & -1.7258969 & 0.7412593 \\ \mathrm{C} & 0.3680557 & 0.6328903 & -0.9638387 \\ \mathrm{H} & 0.6959532 & 2.5192234 & 0.0585486\end{array}$




\begin{tabular}{|c|c|c|c|}
\hline $\mathrm{H}$ & -0.4633684 & -1.0482362 & -2.0756195 \\
\hline & -7.6990924 & -1.6200628 & -1.6805726 \\
\hline & 0.8161257 & -0.1462022 & 0.1053957 \\
\hline 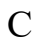 & 2.9869254 & 2.9115730 & -1.6065677 \\
\hline & 4.1255043 & 3.6910969 & -1.0213306 \\
\hline 1 & 2.3502437 & 3.4406466 & -2.3183401 \\
\hline & 2.7101895 & 1.6358019 & -1.3231895 \\
\hline & 4.7912618 & 4.0600561 & -1.8117590 \\
\hline & 4.7106274 & 3.0798574 & -0.3287095 \\
\hline & 3.7599128 & 4.5747284 & -0.4824436 \\
\hline & 1.5723022 & 0.8768135 & -1.9514266 \\
\hline 1 & 3.3372666 & 1.1067943 & -0.6098577 \\
\hline & 1.1422323 & 1.5289995 & -2.7225568 \\
\hline & 2.0287502 & -0.3866576 & -2.6698427 \\
\hline C & 3.1322374 & -1.1355925 & -2.2448832 \\
\hline C & 1.3480878 & -0.8063621 & -3.8210862 \\
\hline $\mathrm{C}$ & 3.5235453 & -2.2883926 & -2.9281077 \\
\hline $\mathrm{H}$ & 3.7061119 & -0.8123326 & -1.3826278 \\
\hline 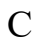 & 1.7326529 & -1.9584841 & -4.5068866 \\
\hline $\mathrm{H}$ & 0.5128760 & -0.2155253 & -4.1905224 \\
\hline C & 2.8199163 & -2.7099039 & -4.0567312 \\
\hline 11 & 4.3850368 & -2.8523050 & -2.5813944 \\
\hline $\mathbf{I}$ & 1.1885951 & -2.2640454 & -5.3960641 \\
\hline $\mathrm{H}$ & 3.1251650 & -3.6051689 & -4.5905070 \\
\hline $\mathrm{C}$ & -3.9639047 & 2.2184407 & 1.2775327 \\
\hline $\mathrm{H}$ & -3.2879054 & 2.1203093 & 2.1315786 \\
\hline H & -3.8852250 & 3.2287967 & 0.8621354 \\
\hline $\mathrm{H}$ & -4.9883572 & 2.0518450 & 1.6053158 \\
\hline $\mathrm{N}$ & 1.3093793 & -0.9487735 & 1.1769244 \\
\hline $\mathrm{C}$ & 0.3622953 & -0.9326987 & 2.3033378 \\
\hline $\mathrm{C}$ & -0.9999905 & -1.0390079 & 2.0301728 \\
\hline $\mathrm{C}$ & 0.8124488 & -0.8753046 & 3.6228207 \\
\hline . & -1.9189706 & -1.0495808 & 3.0762201 \\
\hline $\mathrm{H}$ & -1.3416936 & -1.1101155 & 1.0046874 \\
\hline $\mathrm{C}$ & -0.1141317 & -0.8877233 & 4.6665280 \\
\hline $\mathrm{H}$ & 1.8728035 & -0.8194314 & 3.8408885 \\
\hline $\mathrm{C}$ & -1.4808393 & -0.9666338 & 4.3991172 \\
\hline $\mathrm{H}$ & -2.9789928 & -1.1283781 & 2.8548267 \\
\hline $\mathrm{H}$ & 0.2409110 & -0.8404079 & 5.6914540 \\
\hline $\mathrm{H}$ & -2.1974802 & -0.9732235 & 5.2142620 \\
\hline $\mathrm{C}$ & 2.5948369 & -0.3443356 & 1.4821205 \\
\hline $\mathrm{C}$ & 3.7993359 & -1.0041837 & 1.2453516 \\
\hline $\mathrm{C}$ & 2.5927829 & 0.9686655 & 1.9681276 \\
\hline $\mathrm{C}$ & 5.0054882 & -0.3375049 & 1.4814667 \\
\hline-1 & 3.8159512 & -2.0260405 & 0.8857121 \\
\hline C & 3.7971452 & 1.6188594 & 2.2119600 \\
\hline 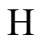 & 1.6502916 & 1.4698391 & 2.1617884 \\
\hline $\mathrm{C}$ & 5.0098675 & 0.9703894 & 1.9622015 \\
\hline $\mathrm{H}$ & 5.9416309 & -0.8537419 & 1.2927733 \\
\hline
\end{tabular}

$\begin{array}{rrrr}\mathrm{H} & 3.7880146 & 2.6368940 & 2.5883740 \\ \mathrm{H} & 5.9500384 & 1.4806197 & 2.1462195 \\ \mathrm{C} & 1.3586629 & -2.3061262 & 0.5602414 \\ \mathrm{H} & 0.3396888 & -2.5788928 & 0.2863794 \\ \mathrm{H} & 1.9759495 & -2.2681538 & -0.3374022 \\ \mathrm{H} & 1.7513708 & -3.0359115 & 1.2725040\end{array}$

TS3Ia $^{+}: \mathrm{H}^{+}$from adduct $3 \mathrm{IHa}^{+}$to $\mathbf{2 l}$ 69

Energy $=-1275.592174913$

$\mathrm{N}-3.2312652-1.1889847$

$\begin{array}{llll}\text { C } & -0.9771573 & -0.3796756 & 1.1585417\end{array}$

$\begin{array}{llll}\text { C } & -2.0121423 & -0.4298970 & -1.0767223\end{array}$

C $\quad 0.1882602 \quad 0.0719343 \quad 0.6056509$

H $\quad-1.0429162 \quad-0.5098072 \quad 2.2316071$

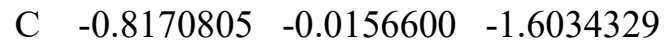

$\begin{array}{llll}\text { C } & 0.3821580 & 0.1739916 & -0.8203175\end{array}$

$\mathrm{H} \quad 1.0189778 \quad 0.3270066 \quad 1.2598275$

$\mathrm{H} \quad-0.7621360 \quad 0.1441912 \quad-2.6779433$

H $\quad 1.0326810 \quad-0.9814212 \quad-1.0209726$

C $\quad 1.4198669 \quad 1.2294782 \quad-1.2950729$

C $\quad 1.7638356 \quad 1.1108933 \quad-2.7882196$

H $\quad 2.3358415 \quad 1.0710121 \quad-0.7133383$

C $\quad 0.8977940 \quad 2.6029602 \quad-0.9704468$

$\mathrm{H} \quad 2.5687876 \quad 1.8081132 \quad-3.0365305$

$\mathrm{H} \quad 0.9039042 \quad 1.3587626-3.4180276$

H $\quad 2.0942607 \quad 0.1001303 \quad-3.0479413$

C $\quad 1.4811274 \quad 3.4427713 \quad-0.1020815$

$\mathrm{H} \quad-0.0234070 \quad 2.8832209 \quad-1.4821823$

$\begin{array}{llll}\mathrm{H} & 2.4036531 & 3.1191487 & 0.3822932\end{array}$

$\begin{array}{llll}\text { C } & 1.0186404 & 4.7796974 & 0.2842909\end{array}$

C $\quad 1.8119942 \quad 5.5445756 \quad 1.1572566$

$\begin{array}{llll}\text { C } & -0.1886711 & 5.3404105 & -0.1733119\end{array}$

C $\quad 1.4216903 \quad 6.8227889 \quad 1.5557674$

$\begin{array}{llll}\mathrm{H} & 2.7464961 & 5.1253907 & 1.5222301\end{array}$

$\begin{array}{llll}\text { C } & -0.5789295 & 6.6155648 & 0.2232536\end{array}$

H $\quad-0.8292852 \quad 4.7730566 \quad-0.8412904$

$\begin{array}{llll}\text { C } & 0.2238609 & 7.3653387 & 1.0894534\end{array}$

$\begin{array}{llll}\mathrm{H} & 2.0525855 & 7.3942118 & 2.2298848\end{array}$

$\mathrm{H} \quad-1.5148694 \quad 7.0291681 \quad-0.1410658$

$\begin{array}{llll}\mathrm{H} & -0.0851206 & 8.3600881 & 1.3973147\end{array}$

C $\quad-3.3359073-1.4078152 \quad 2.3216929$

$\mathrm{H} \quad-2.5841243 \quad-2.1333537 \quad 2.6551644$

$\mathrm{H} \quad-3.2009186 \quad-0.4717397 \quad 2.8722552$

$\begin{array}{llll}\mathrm{H} & -4.3292727 & -1.8003131 & 2.5361078\end{array}$

C $\quad-4.4102132 \quad-1.5528574 \quad 0.0676752$

$\mathrm{H} \quad-5.1494865 \quad-0.7429200 \quad 0.1351665$

$\mathrm{H} \quad-4.8506268 \quad-2.4417052 \quad 0.5277887$ 


$\begin{array}{lrrc}\mathrm{C} & -4.0440871 & -1.8230344 & -1.3850256 \\ \mathrm{H} & -4.9647234 & -1.9681861 & -1.9572702 \\ \mathrm{H} & -3.4594562 & -2.7476361 & -1.4534764 \\ \mathrm{C} & -3.2299243 & -0.6519266 & -1.9383189 \\ \mathrm{H} & -3.8547110 & 0.2522072 & -1.9363344 \\ \mathrm{H} & -2.9264002 & -0.8304781 & -2.9739498 \\ \mathrm{~N} & 1.7868678 & -2.2091483 & -1.0057243 \\ \mathrm{C} & 0.9866111 & -3.0324057 & -0.1151316 \\ \mathrm{C} & -0.3102919 & -3.3704407 & -0.5238285 \\ \mathrm{C} & 1.4582931 & -3.4223231 & 1.1481005 \\ \mathrm{C} & -1.1637467 & -4.0481946 & 0.3358938 \\ \mathrm{H} & -0.6642344 & -3.0723556 & -1.5043630 \\ \mathrm{C} & 0.5744026 & -4.0971181 & 2.0018723 \\ \mathrm{C} & -0.7268565 & -4.3996543 & 1.6164622 \\ \mathrm{H} & -2.1687592 & -4.2986033 & 0.0115805 \\ \mathrm{H} & 0.9328766 & -4.4005478 & 2.9825461 \\ \mathrm{H} & -1.3884350 & -4.9276697 & 2.2964239 \\ \mathrm{C} & 1.9183397 & -2.7610769 & -2.3786779 \\ \mathrm{H} & 2.4566125 & -3.7139624 & -2.3587591 \\ \mathrm{H} & 0.9297412 & -2.9142772 & -2.8101478 \\ \mathrm{H} & 2.4669010 & -2.0427348 & -2.9889881 \\ \mathrm{C} & 3.0985303 & -1.7749650 & -0.4382436 \\ \mathrm{H} & 2.8963067 & -0.9186035 & 0.2161488 \\ \mathrm{H} & 3.7092591 & -1.4294704 & -1.2738206 \\ \mathrm{C} & 3.7715496 & -2.8728733 & 0.3729736 \\ \mathrm{H} & 4.7627783 & -2.5342411 & 0.6873861 \\ \mathrm{H} & 3.9066733 & -3.7705971 & -0.2418899 \\ \mathrm{C} & 2.8848593 & -3.1717291 & 1.5800483 \\ \mathrm{H} & 2.9173943 & -2.3198162 & 2.2736359 \\ \mathrm{H} & 3.2496309 & -4.0418554 & 2.1356976\end{array}$

TS3I ${ }^{+}: \mathrm{H}^{+}$from adduct $\mathbf{3} \mathrm{IH}^{+}$to $\mathbf{2 I}$ 69

\begin{tabular}{lrrc}
\multicolumn{4}{l}{ Energy $=-1275.588054082$} \\
$\mathrm{~N}$ & -3.3852967 & -1.4653354 & 0.3478147 \\
$\mathrm{C}$ & -2.3030071 & -0.6680853 & 0.2331658 \\
$\mathrm{C}$ & -1.4358208 & -0.4581448 & 1.3481768 \\
$\mathrm{C}$ & -1.9887844 & -0.0237951 & -1.0123450 \\
$\mathrm{C}$ & -0.2982861 & 0.2884537 & 1.2182077 \\
$\mathrm{H}$ & -1.6822463 & -0.8891025 & 2.3105043 \\
$\mathrm{C}$ & -0.8188403 & 0.6816384 & -1.1210042 \\
$\mathrm{C}$ & 0.1432872 & 0.8081834 & -0.0518056 \\
$\mathrm{H}$ & 0.3247169 & 0.4595227 & 2.0933076 \\
$\mathrm{H}$ & -0.5836139 & 1.1332278 & -2.0820357 \\
$\mathrm{H}$ & 1.0185334 & -0.1420259 & -0.3788912 \\
$\mathrm{C}$ & 2.9679762 & 2.5233058 & -1.5613791 \\
$\mathrm{C}$ & 3.5867178 & 2.9213862 & -2.8671604 \\
$\mathrm{H}$ & 3.6589342 & 2.3100724 & -0.7432644 \\
$\mathrm{C}$ & 1.6548357 & 2.4257912 & -1.3331920
\end{tabular}

\begin{tabular}{|c|c|c|c|}
\hline H & 4.1990470 & 3.8244938 & -2.7483725 \\
\hline $\mathrm{H}$ & 2.8245210 & 3.1157912 & -3.6279964 \\
\hline $\mathrm{H}$ & 4.2581187 & 2.1361004 & -3.2391060 \\
\hline & 1.0552881 & 2.0632192 & 0.0024341 \\
\hline H & 0.9568698 & 2.6723412 & -2.1338169 \\
\hline H & 1.8824126 & 1.8351108 & 0.6842893 \\
\hline$C$ & 0.3136273 & 3.2592141 & 0.5955121 \\
\hline C & 0.9225137 & 4.0111372 & 1.6063420 \\
\hline $\mathrm{C}$ & -0.9512984 & 3.6458142 & 0.1382757 \\
\hline $\mathrm{C}$ & 0.2826912 & 5.1246018 & 2.1529079 \\
\hline $\mathrm{H}$ & 1.9060751 & 3.7209111 & 1.9690077 \\
\hline 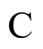 & -1.5928515 & 4.7600855 & 0.6791015 \\
\hline П & -1.4460874 & 3.0698624 & -0.6392813 \\
\hline $\mathrm{C}$ & -0.9783973 & 5.5036506 & 1.6892898 \\
\hline 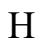 & 0.7682336 & 5.6934408 & 2.9410965 \\
\hline $\mathrm{H}$ & -2.5753643 & 5.0463868 & 0.3140107 \\
\hline $\mathrm{H}$ & -1.4800393 & 6.3688035 & 2.1134396 \\
\hline $\mathrm{C}$ & -3.7131975 & -2.1037637 & 1.6250722 \\
\hline H & -2.8986128 & -2.7652929 & 1.9424928 \\
\hline $\mathrm{H}$ & -3.8946929 & -1.3569712 & 2.4048410 \\
\hline 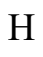 & -4.6176447 & -2.6949739 & 1.4881669 \\
\hline $\mathrm{C}$ & -4.3048353 & -1.7211320 & -0.77 \\
\hline $\mathrm{H}$ & -5.1719193 & -1.0535538 & -0.6852426 \\
\hline $\mathrm{H}$ & -4.6642788 & -2.7474734 & -0.6690599 \\
\hline $\mathrm{C}$ & -3.6228552 & -1.5299763 & -2.1244688 \\
\hline $\mathrm{H}$ & -4.3726031 & -1.6247952 & -2.9147914 \\
\hline $\mathrm{H}$ & -2.8781509 & -2.3193413 & -2.2762345 \\
\hline 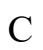 & -2.9457253 & -0.1592897 & -2.1699528 \\
\hline $\mathrm{H}$ & -3.7141495 & 0.6233999 & -2.1060007 \\
\hline I & -2.4138289 & -0.0089929 & -3.1137143 \\
\hline $\mathrm{N}$ & 1.9842840 & -1.1874742 & -0.5653154 \\
\hline $\mathrm{C}$ & 1.2536261 & -2.3725238 & -0.1509505 \\
\hline 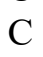 & 0.1149325 & -2.7320106 & -0.8843615 \\
\hline 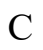 & 1.6196639 & -3.0994750 & 0.9929215 \\
\hline $\mathrm{C}$ & -0.6952221 & -3.7759565 & -0.4595918 \\
\hline $\mathrm{H}$ & -0.1564808 & -2.1717739 & -1.7721220 \\
\hline $\mathrm{C}$ & 0.7820806 & -4.1453033 & 1.4054847 \\
\hline $\mathrm{C}$ & -0.3712310 & -4.4792528 & 0.7042914 \\
\hline 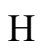 & -1.5792895 & -4.0395810 & -1.0316258 \\
\hline $\mathrm{H}$ & 1.0607071 & -4.7122100 & 2.2905216 \\
\hline 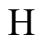 & -0.9999322 & -5.2959632 & 1.0456321 \\
\hline $\mathrm{C}$ & 2.4091929 & -1.1925067 & -1.9887697 \\
\hline $\mathrm{H}$ & 3.1765681 & -1.9545900 & -2.1571692 \\
\hline $\mathrm{H}$ & 1.5506765 & -1.3999233 & -2.6260672 \\
\hline $\mathrm{H}$ & 2.7989994 & -0.2021094 & -2.2262481 \\
\hline & 3.0917347 & -0.7896165 & 0.3530524 \\
\hline $\mathrm{H}$ & 2.6364248 & -0.2498257 & 1.1919358 \\
\hline $\mathrm{H}$ & 3.7256394 & -0.0943079 & -0.1987114 \\
\hline & 3.8573628 & -1.9901919 & 0.8892224 \\
\hline
\end{tabular}




$\begin{array}{llll}\mathrm{H} & 4.7110349 & -1.6422852 & 1.4776337 \\ \mathrm{H} & 4.2483067 & -2.5907205 & 0.0592116 \\ \mathrm{C} & 2.8978431 & -2.8115823 & 1.7468777 \\ \mathrm{H} & 2.6692358 & -2.2571551 & 2.6679076 \\ \mathrm{H} & 3.3527206 & -3.7582730 & 2.0559062\end{array}$

TS3 $\mathbf{w}^{+} \mathbf{p}: \mathrm{H}^{+}$from adduct $\mathbf{3} \mathbf{w} \mathrm{H}^{+} \mathrm{p}$ to $\mathbf{2 a}$ 63

Energy $=-1271.958011495$

$\begin{array}{lrrr}\mathrm{N} & -3.2287678 & -1.6471673 & -0.7024712 \\ \mathrm{H} & -3.1780436 & -2.6401691 & -0.9041165 \\ \mathrm{C} & -2.0955023 & -0.9356770 & -0.9308969 \\ \mathrm{C} & -1.9388277 & 0.4198882 & -0.5276419 \\ \mathrm{C} & -1.0082613 & -1.5890168 & -1.5814484 \\ \mathrm{C} & -0.7525087 & 1.0707292 & -0.7534744 \\ \mathrm{H} & -2.7548404 & 0.9348185 & -0.0359810 \\ \mathrm{C} & 0.1708483 & -0.9281700 & -1.8027259 \\ \mathrm{H} & -1.1428762 & -2.6104825 & -1.9273385 \\ \mathrm{C} & -4.4764074 & -1.1873219 & -0.2067956 \\ \mathrm{C} & 0.4016260 & 0.3975629 & -1.2893259 \\ \mathrm{H} & -0.6433600 & 2.1035870 & -0.4326549 \\ \mathrm{H} & 0.9839079 & -1.4299815 & -2.3207504 \\ \mathrm{C} & -5.0358477 & 0.0228895 & -0.6336560 \\ \mathrm{C} & -5.1732710 & -2.0065903 & 0.6895659 \\ \mathrm{H} & 0.8517230 & 0.0157852 & -0.0714679 \\ \mathrm{C} & -6.2734850 & 0.4228760 & -0.1321845 \\ \mathrm{H} & -4.5263558 & 0.6298914 & -1.3741721 \\ \mathrm{C} & -6.4162400 & -1.6039789 & 1.1726157 \\ \mathrm{H} & -4.7364170 & -2.9494372 & 1.0070848 \\ \mathrm{C} & -6.9658163 & -0.3837065 & 0.7728401 \\ \mathrm{H} & -6.7041263 & 1.3617682 & -0.4669871 \\ \mathrm{H} & -6.9512635 & -2.2424640 & 1.8689993 \\ \mathrm{H} & -7.9316818 & -0.0688327 & 1.1555566 \\ \mathrm{C} & 1.5310460 & 1.2450968 & -1.8958144 \\ \mathrm{C} & 2.8288796 & 0.4767056 & -1.9893341 \\ \mathrm{C} & 3.6451734 & 0.5280782 & -3.0475045 \\ \mathrm{C} & 3.3977063 & 1.3896133 & -4.2559603 \\ \mathrm{C} & 2.2948619 & 2.4252596 & -3.9995193 \\ \mathrm{C} & 1.1080694 & 1.7799346 & -3.2791959 \\ \mathrm{H} & 4.5532353 & -0.0735081 & -3.0433071 \\ \mathrm{H} & 3.0969333 & -0.1528597 & -1.1423873 \\ \mathrm{H} & 1.6730949 & 2.1069104 & -1.2257337 \\ \mathrm{H} & 3.1264088 & 0.7482259 & -5.1082742 \\ \mathrm{H} & 2.6963285 & 3.2364808 & -3.3779417 \\ \mathrm{H} & 1.9677208 & 2.8741704 & -4.9434520 \\ \mathrm{H} & 0.2835348 & 2.4915774 & -3.1599764 \\ \mathrm{H} & 0.7285875 & 0.9398989 & -3.8769367 \\ \mathrm{H} & 4.3321544 & 1.8872906 & -4.5445357 \\ \mathrm{~N} & 1.1567537 & -0.4309823 & 1.2417044\end{array}$

$\begin{array}{crcc}\mathrm{H} & 0.3926522 & -1.0923886 & 1.3905748 \\ \mathrm{C} & 0.9693911 & 0.7202549 & 2.0966227 \\ \mathrm{C} & 0.0503710 & 0.6494113 & 3.1423232 \\ \mathrm{C} & 1.6405606 & 1.9102319 & 1.8111067 \\ \mathrm{C} & -0.1913626 & 1.7808022 & 3.9206128 \\ \mathrm{H} & -0.4702808 & -0.2827492 & 3.3469530 \\ \mathrm{C} & 1.4014024 & 3.0335385 & 2.6012128 \\ \mathrm{H} & 2.3412502 & 1.9589457 & 0.9840979 \\ \mathrm{C} & 0.4861154 & 2.9719738 & 3.6539677 \\ \mathrm{H} & -0.9061813 & 1.7285529 & 4.7357600 \\ \mathrm{H} & 1.9252282 & 3.9595487 & 2.3861632 \\ \mathrm{H} & 0.2980422 & 3.8513881 & 4.2618320 \\ \mathrm{C} & 2.4125533 & -1.1583104 & 1.2223167 \\ \mathrm{C} & 2.4088951 & -2.4022137 & 0.5853847 \\ \mathrm{C} & 3.5895326 & -0.6259586 & 1.7440683 \\ \mathrm{C} & 3.5992596 & -3.1149119 & 0.4625807 \\ \mathrm{H} & 1.4818468 & -2.8089299 & 0.1897979 \\ \mathrm{C} & 4.7762733 & -1.3488831 & 1.6127509 \\ \mathrm{H} & 3.5874649 & 0.3304003 & 2.2537959 \\ \mathrm{C} & 4.7884396 & -2.5872004 & 0.9701242 \\ \mathrm{H} & 3.5947990 & -4.0822823 & -0.0298220 \\ \mathrm{H} & 5.6938397 & -0.9371146 & 2.0214146 \\ \mathrm{H} & 5.7167395 & -3.1408401 & 0.8709170\end{array}$

$\mathbf{T S 3}^{+}: \mathrm{H}^{+}$from adduct $\mathbf{3} \mathbf{w} \mathrm{H}^{+}$to $\mathbf{2 a}$ to ortho-35 63

Energy $=-1271.956688941$

$\begin{array}{lrrr}\mathrm{N} & -0.1020485 & -1.8587474 & 0.2127095 \\ \mathrm{H} & 0.2088795 & -1.9488383 & -0.7559703 \\ \mathrm{C} & -1.0905991 & -0.9416421 & 0.4088448 \\ \mathrm{C} & -1.8232970 & -0.8678967 & 1.6139681 \\ \mathrm{C} & -1.3552356 & 0.0367351 & -0.6420438 \\ \mathrm{C} & -2.7879632 & 0.1012408 & 1.8032698 \\ \mathrm{H} & -1.6592489 & -1.6150567 & 2.3800592 \\ \mathrm{C} & -2.4330100 & 0.9624265 & -0.4013303 \\ \mathrm{H} & -0.2887679 & 0.8382421 & -0.3849020 \\ \mathrm{C} & 0.4462283 & -2.8155266 & 1.0973870 \\ \mathrm{C} & -3.0893597 & 1.0463943 & 0.8002123 \\ \mathrm{H} & -3.3400330 & 0.1144892 & 2.7380909 \\ \mathrm{H} & -2.6992092 & 1.6293130 & -1.2172216 \\ \mathrm{C} & 0.8797091 & -4.0263513 & 0.5367592 \\ \mathrm{C} & 0.6364473 & -2.5804569 & 2.4660277 \\ \mathrm{H} & -3.8586030 & 1.7933789 & 0.9627776 \\ \mathrm{C} & 1.4822104 & -4.9934756 & 1.3361591 \\ \mathrm{H} & 0.7368990 & -4.2061742 & -0.5255755 \\ \mathrm{C} & 1.2317503 & -3.5605659 & 3.2591735 \\ \mathrm{H} & 0.3482487 & -1.6344073 & 2.9073859 \\ \mathrm{C} & 1.6540374 & -4.7694834 & 2.7041743 \\ \mathrm{H} & 1.8104611 & -5.9268082 & 0.8888894\end{array}$




$\begin{array}{lrrr}\mathrm{H} & 1.3739652 & -3.3688431 & 4.3186261 \\ \mathrm{H} & 2.1170933 & -5.5265861 & 3.3291615 \\ \mathrm{C} & -1.1223436 & -0.2504928 & -2.1590495 \\ \mathrm{C} & 0.2164859 & -0.8374298 & -2.5440154 \\ \mathrm{C} & 0.3669759 & -2.0658213 & -3.0626129 \\ \mathrm{C} & -0.7579213 & -3.0462447 & -3.2421593 \\ \mathrm{C} & -2.0486819 & -2.5962637 & -2.5439265 \\ \mathrm{C} & -2.2732464 & -1.0962871 & -2.7523835 \\ \mathrm{H} & 1.3571622 & -2.3839537 & -3.3845190 \\ \mathrm{H} & 1.0888318 & -0.1884718 & -2.4702501 \\ \mathrm{H} & -1.1700608 & 0.7446149 & -2.6213044 \\ \mathrm{H} & -0.4434614 & -4.0345286 & -2.8819450 \\ \mathrm{H} & -2.9006180 & -3.1623687 & -2.9325719 \\ \mathrm{H} & -1.9912900 & -2.8162803 & -1.4708534 \\ \mathrm{H} & -2.3214898 & -0.8870428 & -3.8281906 \\ \mathrm{H} & -3.2243701 & -0.7780789 & -2.3140750 \\ \mathrm{H} & -0.9329681 & -3.1737572 & -4.3207980 \\ \mathrm{~N} & 0.8589269 & 1.5757064 & -0.1870392 \\ \mathrm{H} & 1.6157948 & 0.9167247 & -0.3776402 \\ \mathrm{C} & 0.8822332 & 1.8875921 & 1.2344935 \\ \mathrm{C} & 0.3717806 & 3.0761645 & 1.7504497 \\ \mathrm{C} & 1.3815819 & 0.8940494 & 2.0783445 \\ \mathrm{C} & 0.3549986 & 3.2583585 & 3.1336652 \\ \mathrm{H} & 0.0058183 & 3.8581339 & 1.0967361 \\ \mathrm{C} & 1.3629073 & 1.0883628 & 3.4569554 \\ \mathrm{H} & 1.7895322 & -0.0212459 & 1.6569062 \\ \mathrm{C} & 0.8406937 & 2.2685185 & 3.9894467 \\ \mathrm{H} & -0.0383894 & 4.1853115 & 3.5394198 \\ \mathrm{H} & 1.7612004 & 0.3201030 & 4.1126965 \\ \mathrm{H} & 0.8222726 & 2.4198265 & 5.0640449 \\ \mathrm{C} & 0.8804219 & 2.6183848 & -1.1955007 \\ \mathrm{C} & 1.9879250 & 2.7018130 & -2.0381714 \\ \mathrm{C} & -0.2230631 & 3.4539538 & -1.3747926 \\ \mathrm{C} & 1.9988438 & 3.6423337 & -3.0669682 \\ \mathrm{H} & 2.8353393 & 2.0378835 & -1.8889987 \\ \mathrm{C} & -0.1985781 & 4.3974736 & -2.4017213 \\ \mathrm{H} & -1.0915341 & 3.3689172 & -0.7322259 \\ \mathrm{C} & 0.9076927 & 4.4938994 & -3.2470884 \\ \mathrm{H} & 2.8608187 & 3.7081681 & -3.7227761 \\ \mathrm{H} & -1.0518321 & 5.0530413 & -2.5425162 \\ \mathrm{H} & 0.9175599 & 5.2279896 & -4.0465603\end{array}$

$\mathbf{T S 3}^{+}: \mathrm{H}^{+}$from adduct $\mathbf{3 a} \mathrm{H}^{+}$to $\mathbf{2 a}$ to form $\mathbf{3}$ 69

Energy $=-1425.689634525$

$\begin{array}{llll}\mathrm{N} & 1.5669447 & 2.3063436 & 1.7727125 \\ \mathrm{H} & 2.0108051 & 2.0871128 & 2.6582987 \\ \mathrm{C} & 0.7493888 & 1.3449383 & 1.2693248 \\ \mathrm{C} & 2.0315626 & 3.4834517 & 1.1344859\end{array}$ $\begin{array}{llll}\text { C } & 0.2175855 & 1.3884185 & -0.0453903\end{array}$

$\begin{array}{llll}\text { C } & 0.4463725 & 0.2226046 & 2.0913790\end{array}$

$\begin{array}{llll}\text { C } & 1.1678776 & 4.3311658 & 0.4316546\end{array}$

$\begin{array}{llll}\text { C } & 3.3886375 & 3.8075745 & 1.2543203\end{array}$

$\begin{array}{llll}\text { C } & -0.5023010 & 0.3258008 & -0.5254918\end{array}$

$\mathrm{H} \quad 0.4240631 \quad 2.2344765 \quad-0.6875097$

C $\quad-0.2790585 \quad-0.8314027 \quad 1.6009315$

$\begin{array}{llll}\mathrm{H} & 0.8039840 & 0.2136650 & 3.1177588\end{array}$

$\begin{array}{llll}\text { C } & 1.6753205 & 5.4758620 & -0.1818775\end{array}$

$\mathrm{H} \quad 0.1054175 \quad 4.1168506 \quad 0.3907820$

$\begin{array}{llll}\text { C } & 3.8826467 & 4.9610160 & 0.6500785\end{array}$

$\begin{array}{llll}\mathrm{H} & 4.0500431 & 3.1500627 & 1.8116350\end{array}$

$\begin{array}{llll}\text { C } & -0.6949744 & -0.8901571 & 0.2234814\end{array}$

$\mathrm{H} \quad-0.8932543 \quad 0.3687978 \quad-1.5388780$

$\mathrm{H} \quad-0.4943804 \quad-1.6720466 \quad 2.2528951$

C $\quad 3.0311180 \quad 5.7929325-0.0806222$

$\mathrm{H} \quad 1.0009063 \quad 6.1298993 \quad-0.7263757$

$\begin{array}{llll}\mathrm{H} & 4.9367002 & 5.2046073 & 0.7440250\end{array}$

C $\quad-1.8634835 \quad-1.8138264 \quad-0.1994622$

$\begin{array}{llll}\mathrm{H} & 3.4192569 & 6.6879381 & -0.5571665\end{array}$

C $\quad-1.7665241 \quad-3.2141987 \quad 0.4302083$

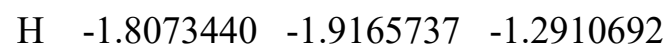

C $\quad-3.1713362-1.1519656 \quad 0.1404124$

H $\quad-0.7885551 \quad-3.6718245 \quad 0.2457915$

$\mathrm{H} \quad-1.9261560 \quad-3.1768106 \quad 1.5125023$

$\begin{array}{llll}\mathrm{H} & -2.5361733 & -3.8626894 & 0.0036842\end{array}$

C $\quad-4.0743664 \quad-0.7609517 \quad-0.7715062$

H $\quad-3.3490031 \quad-0.9909879 \quad 1.2042599$

H $\quad-3.8561300 \quad-0.9498392 \quad-1.8238872$

$\begin{array}{llll}\text { C } & -5.3586754 & -0.1006508 & -0.5166751\end{array}$

$\begin{array}{llll}\text { C } & -6.2197315 & 0.1462926 & -1.6008438\end{array}$

$\begin{array}{llll}\text { C } & -5.7766549 & 0.3023231 & 0.7659877\end{array}$

$\begin{array}{llll}\text { C } & -7.4554715 & 0.7654247 & -1.4148198\end{array}$

H $\quad-5.9106412 \quad-0.1560758 \quad-2.5989429$

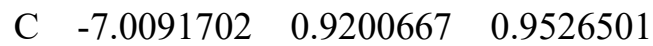

$\mathrm{H} \quad-5.1313370 \quad 0.1355560 \quad 1.6236428$

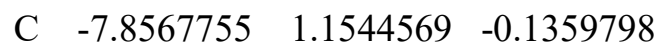

$\mathrm{H} \quad-8.1038512 \quad 0.9437928 \quad-2.2680912$

$\mathrm{H} \quad-7.3123028 \quad 1.2238130 \quad 1.9509616$

$\mathrm{H} \quad-8.8180168 \quad 1.6375576 \quad 0.0142011$

$\mathrm{H} \quad 0.3541008 \quad-1.5660354 \quad-0.3036061$

$\mathrm{H} \quad 1.0334946 \quad-2.5389034 \quad-1.8252057$

$\begin{array}{llll}\mathrm{N} & 1.4491151 & -2.1705916 & -0.9670308\end{array}$

$\begin{array}{llll}\text { C } & 2.2853906 & -1.0321897 & -1.3059213\end{array}$

$\begin{array}{llll}\text { C } & 3.2688717 & -0.5568782 & -0.4397654\end{array}$

$\begin{array}{llll}\text { C } & 1.9789291 & -0.3469769 & -2.4832254\end{array}$

$\begin{array}{llll}\text { C } & 3.9200797 & 0.6365598 & -0.7450321\end{array}$

$\begin{array}{llll}\mathrm{H} & 3.5233071 & -1.0985464 & 0.4631386\end{array}$

$\begin{array}{llll}\text { C } & 2.6386634 & 0.8445580 & -2.7794739\end{array}$ 


$\begin{array}{lrrr}\mathrm{H} & 1.2199188 & -0.7362337 & -3.1567943 \\ \mathrm{C} & 3.5984622 & 1.3494978 & -1.9020539 \\ \mathrm{H} & 4.6813478 & 1.0119149 & -0.0685437 \\ \mathrm{H} & 2.3940507 & 1.3778025 & -3.6926702 \\ \mathrm{H} & 4.1021707 & 2.2854192 & -2.1220094 \\ \mathrm{C} & 1.9807378 & -3.2725195 & -0.1876977 \\ \mathrm{C} & 2.1997935 & -4.4952002 & -0.8226672 \\ \mathrm{C} & 2.2044971 & -3.1279971 & 1.1821426 \\ \mathrm{C} & 2.6546354 & -5.5836065 & -0.0798354 \\ \mathrm{H} & 2.0182435 & -4.5925925 & -1.8894294 \\ \mathrm{C} & 2.6675928 & -4.2205408 & 1.9154925 \\ \mathrm{H} & 2.0104366 & -2.1817658 & 1.6737224 \\ \mathrm{C} & 2.8924112 & -5.4472371 & 1.2892746 \\ \mathrm{H} & 2.8250332 & -6.5351354 & -0.5737197 \\ \mathrm{H} & 2.8448778 & -4.1110560 & 2.9808543 \\ \mathrm{H} & 3.2478953 & -6.2951220 & 1.8664243\end{array}$




\subsection{References}

[S1] Lishchynskyi, A.; Muniz, K. An Approach to the Regioselective Diamination of Conjugated Di- and Trienes. Chem. - Eur. J. 2012, 18, 2212-2216.

[S2] Hilt, G.; Danz, M. Regioselective Cobalt-catalyzed Diels-Alder Reaction Towards 1, 3disubstituted and 1, 2, 3-trisubstituted Benzene Derivatives. Synthesis 2008, 14, 22572263.

[S3] Marcum, J. S.; Roberts, C. C.; Manan, R. S.; Cervarich, T. N.; Meek, S. J. Chiral Pincer Carbodicarbene Ligands for Enantioselective Rhodium-Catalyzed Hydroarylation of Terminal and Internal 1,3-Dienes with Indoles. J. Am. Chem. Soc. 2017, 139, 1558015583.

[S4] [a]. Liao, L.; Guo, R.; Zhao, X. Organoselenium-Catalyzed Regioselective C-H Pyridination of 1, 3-Dienes and Alkenes. Angew. Chem., Int. Ed. 2017, 56, 3201-3205. [b]. Sardini, S. R.; Brown, M. K. Catalyst Controlled Regiodivergent Arylboration of Dienes. J. Am. Chem. Soc. 2017, 139, 9823-9826.

[S5] Qiao, C.; Chen, A.; Gao, B.; Liu, Y.; Huang, H. Palladium Catalyzed Cascade Double CN Bond Activation: A New Strategy for Aminomethylation of 1,3-Dienes with Aminals. Chin. J. Chem. 2018, 36, 929-933.

[S6] [a]. H. Lebel, D. Guay, V. Paquet, K. Huard, Highly Efficient Synthesis of Terminal Alkenes from Ketones. Org. Lett. 2004, 6, 3047-3050. [b]. Katritzky, A. R.; Denisko, O. V. 1, 2-vs 1, 4-Addition of Acylbenzotriazoles to $\alpha, \beta$-Unsaturated Aldehydes and Ketones. A Novel Route to 3-Alkyl-4, 6-diaryl-3, 4-dihydropyran-2-ones. J. Org. Chem. 2002, 67, 31043108. [c]. Liu, C.; Wang, Q. Alkenylation of C(sp3 )-H Bonds by Zincation/CopperCatalyzed Cross-Coupling with lodonium Salts. Angew. Chem. Int. Ed. 2018, 57, 47274731.

[S7] Sharma, U.; Kancherla, R.; Naveen, T.; Agasti, S.; Maiti, D. Palladium-Catalyzed Annulation of Diarylamines with Olefins through $\mathrm{C}-\mathrm{H}$ Activation: Direct Access to $\mathrm{N}$ Arylindoles. Angew. Chem. Int. Ed. 2014, 53, 11895-11899.

[S8] [a]. Solé, D.; Amenta, A.; Mariani, F.; Bennasar, M.-L.; Fernández, I. Transition MetalCatalysed Intramolecular Carbenoid $\mathrm{C}-\mathrm{H}$ Insertion for Pyrrolidine Formation by 
Decomposition of a-Diazoesters. Adv. Synth. Catal. 2017, 359, 3654-3664. [b]. Barzanò, G.; Mao, R.; Garreau, M.; Waser, J.; Hu, X. Tandem Photoredox and Copper-Catalyzed Decarboxylative $\mathrm{C}(\mathrm{sp} 3)-\mathrm{N}$ Coupling of Anilines and Imines Using an Organic Photocatalyst. Org. Lett. 2020, 22, 5412-5416.

[S9] [a]. Danopoulos, A. A.; Galsworthy, J. R.; Green, Malcolm L. H.; Cafferkey, S.; Doerrera, L. $\mathrm{H}$.; Hursthouse, M. B. Equilibria in the $\mathrm{B}\left(\mathrm{C}_{6} \mathrm{~F}_{5}\right)_{3}-\mathrm{H}_{2} \mathrm{O}$ system: synthesis and crystal structures of $\mathrm{H}_{2} \mathrm{O} \cdot \mathrm{B}\left(\mathrm{C}_{6} \mathrm{~F}_{5}\right)_{3}$ and the anions $\left[\mathrm{HOB}\left(\mathrm{C}_{6} \mathrm{~F}_{5}\right)_{3}\right]_{2}$ and $\left[\left(\mathrm{F}_{5} \mathrm{C}_{6}\right)_{3} \mathrm{~B}(\mathrm{~m}-\mathrm{OH}) \mathrm{B}\left(\mathrm{C}_{6} \mathrm{~F}_{5}\right)_{3}\right]_{2}$. Chem Commun. 1998, 2529-2530 [b]. Bergquist, C.; Bridgewater, B. M.; Harlan, C. J.; Norton, J. R.; Friesner, R. A.; Parkin, G. Aqua, Alcohol, and Acetonitrile Adducts of Tris(perfluorophenyl)borane: Evaluation of Brønsted Acidity and Ligand Lability with Experimental and Computational Methods. J. Am. Chem. Soc. 2000, 122, 10581-10590. [c]. Beringhelli, T.; Maggioni, D.; D'Alfonso, G. ${ }^{1} \mathrm{H}$ and ${ }^{19} \mathrm{~F}$ NMR Investigation of the Reaction of $\mathrm{B}\left(\mathrm{C}_{6} \mathrm{~F}_{5}\right)_{3}$ with Water in Toluene Solution. Organometallics 2001, 20, 49274938. [d]. Focante, F.; Camurati, I.; Nanni, D.; Leardini, R.; Resconi, L. Synthesis and Reactivity of $\mathrm{N}$-Heterocycle-B $\left(\mathrm{C}_{6} \mathrm{~F}_{5}\right)_{3}$ Complexes. 3. Generation of $\mathrm{N}$-Methylpyrrol-2-yl and N-Methylindol-2-yl Borate Zwitterions with Acidic sp ${ }^{3}$ Carbons. Organometallics 2004, 23, 5135-5141.

[S10] CCDC $2111194\left((2 \mathrm{a})_{2} \mathrm{H}^{+}(\mathrm{HOBCF})_{2} \mathrm{H}^{-}\right)$contain the supplementary crystallographic data for this paper. These data can be obtained free of charge from The Cambridge Crystallographic Data Centre www.ccdc.cam.ac.uk/data_request/cif.

[S11] Ban, K.; Yamamoto, Y.; Sajiki, H.; Sawama, Y. Arylation of Indoles using Cyclohexanones Dually-Catalyzed by Niobic Acid and Palladium-on-Carbons. Org. Biomol. Chem. 2020, 18, 3898-3902.

[S12] Davies, S. G.; Fletcher, A. M.; Roberts, P. M.; Thomson, J. E. The Hancock Alkaloids Angustureine, Cuspareine, Galipinine, and Galipeine: A Review of their Isolation, Synthesis, and Spectroscopic Data. Eur. J. Org. Chem. 2019, 5093-5119.

[S13] [a]. Hadziz, S. Infrared Absmption Bands Associated with the NH Group. Part I. Some Secondary Aromatic Amines. J. Chem. Soc., Perkin Trans. 1957, 843-845. [b]. Hwang, J. Y.; Ji, A. Y.; Lee, S. H.; Kang, E. J. Redox-Selective Iron Catalysis for a-Amino C-H Bond Functionalization via Aerobic Oxidation. Org. Lett. 2020, 22, 16-21. 
[S14] TURBOMOLE V7.4, 2019, a development of University of Karlsruhe and Forschungszentrum Karlsruhe GmbH, 1989-2007, TURBOMOLE GmbH, since 2007; available from http://www.turbomole.com.

[S15] Tao, J.; Perdew, J. P.; Staroverov, V. N.; Scuseria, G. E. Climbing the Density Functional Ladder: Nonempirical Metal-generalized Gradient Approximation Designed for Molecules and Solides. Phys. Rev. Lett. 2003, 91, 146401.

[S16] Grimme, S.; Antony, J.; Ehrlich, S.; Krieg, H. A Consistent and Accurate ab initio Parametrization of Density Functional Dispersion Correction (DFT-D) for the 94 Elements H-Pu. J. Chem. Phys. 2010, 132, 154104-154119.

[S17] Grimme, S.; Ehrlich, S.; Goerigk, L. Effect of the Damping Function in Dispersion Corrected Density Functional Theory. J. Comput. Chem. 2011, 32, 1456-1465.

[S18] Weigend, F.; Haser, M.; Patzelt, H.; Ahlrichs, R. R1-MP2: Optimized Auxiliary Basis Sets and Demonstration of Efficiency. Chem. Phys. Lett. 1998, 294, 143-152.

[S19] Weigend, F.; Ahlrichs, R. Balanced Basis Sets of Split Valance, Triple Zeta Valance Quality and Quadruple Zeta Valance Quality for H to Rn: Design and Assessment of Accuracy. Phys. Chem. Chem. Phys. 2005, 7, 3297-3305.

[S20] Klamt, A.; Schüürmann, G. COSMO: A New Approach to Dielectric Screening in Solvents with Explicit Expressions for the Screening Energy and its Gradient. J. Chem. Soc., Perkin Trans. 2, 1993, 799-805.

[S21] Eichkorn, K.; Weigend, F.; Treutler, O.; Ahlrichs, R. Auxiliary Basis Sets for Main Row Atoms and Metals and their use to Approximate Coulomb Potentials. Theor. Chem. Acc. 1997, 97, 119-124.

[S22] Weigend, F. Accurate Coulomb-fitting Basis Sets for $\mathrm{H}$ to Rn. Phys. Chem. Chem. Phys. 2006, 8, 1057-1065.

[S23] Deglmann, P.; May, K.; Furche, F.; Ahlrichs, R. Nuclear Second Analytical Derivative Calculations Using Auxiliary Basis Set Expansions. Chem. Phys. Lett. 2004, 384, 103107.

[S24] Grimme, S. Supramolecular Binding Thermodynamics by Dispersion Corrected Density Functional Theory. Chem. - Eur. J. 2012, 18, 9955-9964.

[S25] Eckert, F.; Klamt, A. Fast Solvent Screening via Quantum Chemistry: COSMO-RS Approach. A/ChE J. 2002, 48, 369-385. 
[S26] F. Eckert, A. Klamt, COSMOtherm, Version C3.0, Release 16.01, 2015, COSMOlogic $\mathrm{GmbH} \&$ Co., Leverkusen, Germany.

[S27] Zhao, Y.; Truhlar, D. G. Design of Density Functionals that are broadly Accurate for Thermochemistry, Thermochemical Kinetics, and Nonbonded Interaction. J. Phys. Chem. A 2005, 109, 5656-5667.

[S28] Weigend, F.; Furche, F.; Ahlrichs, R. Gaussian Basis Sets of Quadruple Zeta Valance Quality for Atoms H-Kr. J. Chem. Phys. 2003, 119, 12753-12762.

[S29] Goerigk, L.; Hansen, A.; Bauer, C.; Ehrlich, S.; Najibi, A. Grimme, S. A look at the Density Dunctional Theory Zoo with the Advanced GMTKN55 Database for General Main Group Thermochemistry, Kinetics and Noncovalent Interactions. Phys. Chem. Chem. Phys. 2017, 19, 23184-32215. 